\title{
MASTER
}

\section{Analytical Calculations of Neutron Slowing Down and Transport in the Constant-Cross-Section Problem}

D. G. Cacuci 


\section{DISCLAIMER}

This report was prepared as an account of work sponsored by an agency of the United States Government. Neither the United States Government nor any agency Thereof, nor any of their employees, makes any warranty, express or implied, or assumes any legal liability or responsibility for the accuracy, completeness, or usefulness of any information, apparatus, product, or process disclosed, or represents that its use would not infringe privately owned rights. Reference herein to any specific commercial product, process, or service by trade name, trademark, manufacturer, or otherwise does not necessarily constitute or imply its endorsement, recommendation, or favoring by the United States Government or any agency thereof. The views and opinions of authors expressed herein do not necessarily state or reflect those of the United States Government or any agency thereof. 


\section{DISCLAIMER}

Portions of this document may be illegible in electronic image products. Images are produced from the best available original document. 


\section{Printed in the United States of America. Available from National Technical Information Service U.S. Department of Commerce 5285 Port Royal Road, Springfield, Virginia 22161 \\ Price: Printed Copy $\$ 10.75$; Microfiche $\$ 3.00$}

This report was prepared as an account of work sponsored by an agency of the United States Government. Neither the United States Government nor any agency thereof, nor any of their employees, contractors, subcontractors, or their employees, makes any warranty, express or implied, nor assumes any legal liability or responsibility for any third party's use or the results of such use of any information, apparatus, product or process disclosed in this report, nor represents that its use by such third party would not infringe privately owned rights. 
Contract No. W-7405-eng-26

Neutron Physics Division

ANALYTICAL CALCULATIONS OF NEUTRON SLOWING DOWN AND TRANSPORT IN THE CONSTANT-CROSS-SECTION PROBLEM*

D. G. Cacuci

* Submitted to Columbia University as a doctoral dissertation in the Faculty of Pure Science.

DATE PUBLISHED-APRIL 1978

OAK RIDGE NATIONAL LABORATORY This report was prepared as an accourit of work
sponsored by the United States Government. Neither the
United States nor the United States Department of
Energy, nor any of their employees, nor any of their
contractors, subcontractors, or their employees. makes
sny warranry, express or implier, n assumes any legui
liability or responsibility for the accuracy, completeness
or usefulness of any information, apparatus, product or
process disclosed, or represents that its use would nut
infringe privately owned rights.

Oak Ridge, Tennessee 37830

operated by

UNION CARBIDE CORPORATION

for the

DEPARTMENT OF ENERGY 
THIS PAGE

WAS INTENTIONALLY

LEFT BLANK 


\section{PREFACE AND ACKNOWLEDGMENTS}

This report describes work performed by the author in partial fulfillment of the requirements for the degree of Doctor of Philosophy in the Faculty of Pure Science at Columbia University.

I consider myself most fortunate to have been doing research under the supervision of Professor Herbert Goldstéin, who was so much more than merely a dissertation adviser. I am greatly indebted to him not only for suggesting what proved to be a fascinating research area for me, but also for his guidance, invaluable suggestions, and everlasting human understanding which marked, reassuringly, the entire course of this work.

I am also particularly grateful to Mr. Waldo Patton, who wholeheartedly made available the benefits of his profound experience in the fields of computers and applied mathematics. Many an incipient idea crystallized as a result of his constructive criticism while guiding me through the intricacies of FORMAC, or during enlightening discussions on combinatorial analysis and other areas of mathematics.

Mention must also be made of the faculty of the former Division of Nuclear Science and Engineering--Professors W. W. Havens, Jr., J. P. Felvinci, E. Melkonian, and L. J. Lidofsky--both in a general sense, for it has been a privilege to work within the intellectual climate they provided, and in a special sense, for their kind help and encouragement during my four years as their graduate teaching and research assistant.

Last, but not least, it was the firm friendship of fellow doctoral candidates J. C. Arbo and S. L. Ostrow that provided additional moral support at critical stages during the course of this work at Columbia. 
Furthermore, the author wishes to express his greatful appreciation to the Neutron Physics Division of the Oak Ridge National Laboratory, to Union Carbide Corporation, and to the U. S. Department of Energy for their support and assistance in the publication of this work. I am particularly indebted to $\mathrm{Dr}$. Charles Weisbin for his invaluable support and suggestions, and to Mrs. Lorraine Abbott and Mrs. LaWanda Klobe for their help, understanding, and patience with a meddling author while they accomplished the transmutation from scribbled manuscript to printed page in miraculously short time.

Finally, I am deeply indebted to my wife, for it was her sacrifice and inspiration which made this work a reality. 
TABLE OF CONTENTS

Page

PREFACE AND ACKNOWLEDGMENTS ................. i i

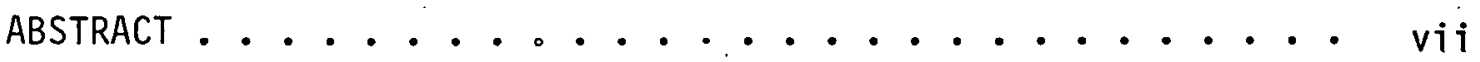

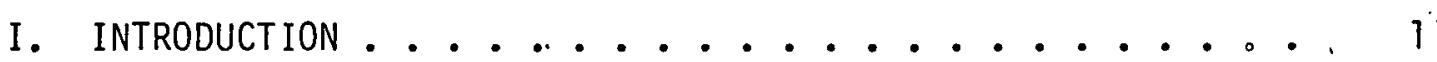

II. MATHEMATICAL TREATMENT OF THE BOLTZMANN EQUATION .... 11

1. The Singular Eigenfunction Method Applied to the Lethargy-Laplace Transformed Boltzmann Equation . . 11

A. Mathematical Preliminaries ......... 11

B. Orthogonality and Completeness Properties of the Eigenfunctions .......... 20

C. Further Aspects of the Singular Eigenfunction Formalism: Transport Theory and the Theory of Orthogonal Polynomials .......... 27

2. Advances in the Formalism: The "Eigenvalue Space" Moments $m_{k}(n)$ and Their Properties ....... 34

III. THE SPATIAL MOMENTS OF THE GREEN'S FUNCTION FOR A PLANE ISOTROPIC MONOENERGETIC SOURCE IN AN

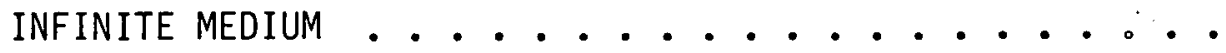

1. Introduction. The Infinite Medium -

Green's function ................ 45

2. The Spatial Moments $M_{k}(u)$ of the Scalar Flux .... 50

A. The Spatial Moments $M_{k}(n)$ and Their Relation to the "Eigenvalue Space" Moments $m_{k}(n) \ldots 50$

B. The Exact Analytical Expressions of the Space-Angle Integrated Flux $\mathrm{M}_{2}(u)$ and the Second Spatial Moment $M_{0}(u)$.......。

C. The Laplace Inversion of $M_{2 n}(n)$. Form of the Lethargy-Dependent Spatial Moments $M_{2 n}(u)$ for $c=1 \ldots \ldots 2$

IV. CONCLUSIONS AND DIRECTIONS OF FURTHER RESEARCH . . . . 74 APPENDICES :

A. THE FUNCTIONS $g_{n}(n) \ldots \ldots 7 . \ldots \ldots$

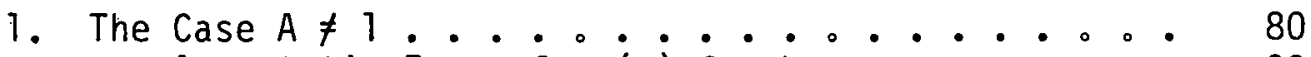

a. Asymptotic Form of $g_{n}(n)$ for Large $n \ldots 80$

b. Asymptotic Form of $g_{n}(n)$ for Small $n$.... 85

2. The Case $A=1$ (Hydrogen) ........ 89 
Page

APPENDICES (Continued)

A. 3. Convergence Properties of Infinite

Series Involving the Functions $g_{n}(n) \ldots . . .92$

B. THE "COMPLETENESS THEOREM" FOR THE EIGENFUNCTIONS $\phi(\nu, \mu, n)$, FOR FINITE ANISOTROPY ........ 96

c. ORTHOGONALITY PROPERTIES OF THE POLYNOMIALS $\phi_{n}(\nu, n)$ FOR INFINITE ORDER ANISOTROPY: PROPERTIES OF THE DISCRETE EIGENVALUES $j(n) \ldots \ldots . \ldots 105$

D. CONVERGENCE CONDITION FOR THE FUNCTIONS $g_{n}(n) \ldots \ldots$

E. LISTING OF THE "EIGENVALUE-SPACE" MOMENTS $m_{2 n}(n)$, FOR $n=0$ TO 9 , AS GENERATED BY FORMAC ..? . . . 125

F. METHODS OF COMBINATORIAL ANALYSIS; DERIVATION OF THE COEFFICIENTS WK $(n)$, FOR $k=n$ TO $n-5 \ldots 139$

G. LIST OF COEFFICIENTS $B_{r, s}(n)$ FOR $r, s=0$ TO $r, s=5 \ldots \ldots 147$

H. DERIVATION OF THE EXACT ANALYTICAL EXPRESSION FOR THE SECOND SPATIAL MOMENT $M_{2}(u)$ OF THE SCALAR FLUX ....................... 149

I. THE COEFFICIENTS $t_{m, j} ; z_{m, j} ; x_{m, j} ; Y_{m, j}$ AND $c_{m, j}$ PROOFS OF EQUATIONS (III.72-76) ....... 164

1. Proof of Eq. (III.72) for $t_{m, j} \ldots \ldots 164$

2. Proof of Eq. (III.74) for $x_{m, j} \ldots \ldots 166$

3. Proof of Eq. (III.75) for $Y_{m, j} \ldots \ldots 167$

4. Proof of Eq. (III.73) for $z_{m, j} \ldots \ldots 168$

5. Proof of Eq. (III.76) for $c_{m, j} \ldots \ldots 170$

J. LISTING OF COEFFICIENTS $C_{m, j}$ FOR $m, j=0$ TO $m, j=5$ AS GENERATED BY FORMAC ......... 175

REFERENCES . . . . . . . . . . . . 252

DISTRIBUTION . . . . . . . . . . . . . 257 


\section{ABSTRACT}

Some aspects of the problem of neutron slowing down and transport in an infinite medium consisting of a single nuclide that scatters elastically and isotropically and has energy-independent cross sections have been investigated. The method of singular eigenfunctions has been applied to the Boltzmann Equation governing the Laplace transform (with respect to the lethargy variable) of the neutron flux. A new sufficient condition for the convergence of the coefficients of the expansion of the scattering kernel in Legendre polynomials has been rigorously derived for this energy-dependent probiem. Formulae have been obtained for the lethargy-dependent spatial moments of the scalar flux that are valid for medium to large lethargies. In deriving these formulae, use has been made of the well-known connection between the spatial moments of the Laplace-transformed scalar flux and the moments of the flux in the "eigenvalue space." The calculations have been greatly aided by the construction of a closed general expression for these "eigenvalue space" moments. Extensive use has also been made of the methods of combinatorial analysis and of computer evaluation, via FORMAC, of complicated sequences of manipulations. For the case of no absorption it has been possible to obtain for materials of any atomic weight explicit corrections to the age-theory formulae for the spatial moments $M_{2 n}(u)$ of the scalar flux that are valid through terms of the order of $u^{-5}$. Higher order correction terms could be obtained at the expense of 
additional computer time. The evaluation of the coefficients of the powers of $n$, as explicit functions of the nuclear mass, represent one of the end products of this investigation. In addition, an exact expression for the second spatial moment, $M_{2}(u)$, valid for arbitrary (constant) absorption, has been derived. Combined with the previously known exact expression for the zeroth spatial moment, it is now possible to calculate analytically and rigorously the "age" for the constant-cross-section problem for arbitrary (constant) absurption and nuclear mass. 
CHAPTER I

INTRODUCTION

Some forty years have elapsed since the first neutron transport problems were formulated. During this period, linear transport problems have been very extensively investigated; this, of course, was largely a consequence of (and in response to) the compelling needs of technology in search of new sources of energy-fission and fusion.

Along the way, it was realized that the structure of the linear transport equation is different from, and in some aspects more complicated than those of classical mathematical physics. One has to exercise caution in carrying over familiar and intuitive ideas from the study of classical boundary value problems.

The class of linear transport problems soluble in closed form turns out to be extremely limited. In fact, closed-form analytic solutions have been obtained for only very simple and highly idealized situations. Therefore people faced with practical questions had to resort to drastic approximations or to rely on numerical procedures.

Perhaps the most "complex" class of problems for which exact, closed-form analytic solutions have been obtained, are the so-called one-speed (or monoenergetic) stationary transport problems in onedimensional geometry. This was possible mainly because of the drastic underlying idealizations and simplifications, e.g., the neutron flux density reduces to a function of only two variables (one-angle and one-position) -an enormous departure from the seven variables $(\bar{r}, \bar{\Omega}$, 
$E, t)$ of "real life." Solutions of monoenergetic problems, either by the older Fourier transform technique or by the method of singular eigenfunctions applied by Case in 1960, provide extremely valuable insight into the structure of solutions of linear transport boundaryvalue problems. However the methods are far from trivial and require the application of highly sophisticated mathematical techniques in order to obtain useful solutions. The next natural step, that of introducing. energy as an additional variable, therefore leads to extreme difficulties when trying to find analytic solutions to even idealized energy-dependent problems.

Perhaps the simplest problem of linear transport theory involving transport combined with energy change is the slowing down and transport of neutrons by purely elastic scattering in an infinite homogeneous medium with cross-sections independent of energy with plane or spherical geometry.* Even here, and even with the further restriction to scattering isotropic in the center-of-mass system, no one has yet found a closed form solution for neutron flux density valid over the entire range of the space and energy variables. The many attempts ${ }^{1-13}$ at deriving such a solution have produced so far only approximate expressions valid for certain limiting cases.

Thus, consider a neutron that has suffered many collisions and thereby slowed down to an energy small compared to the suurce energy (i.e., to large lethargy $u$ ). Its track in phase space then corresponds

*To avoid long winded repetition later on, we shall refer to this problem as the "constant-cross section problem." 
to a random walk with many steps. The law of large numbers may therefore be used to describe the energy and spatial distribution of such neutrons, at least not far from the source. The resultant predictions, known as "age-theory," have been widely used in practical applications .

Very little has been done to define precisely the limits of validity of "age-theory" or to obtain corrections that can be used for a wider range of conditions. It is likely that the requirements for "age-theory" as given in the older literature, ${ }^{1-8}$ mostly dating from studies during World War II, are not quite correct. Thus Davison ${ }^{9}$ states explicitly that "age theory . . . is nowhere valid in hydrogen" [p. 407], while in more recent times, McInerneyl1 and Amster ${ }^{12-13}$ have shown that even in hydrogen, age-theory is the correct limiting form for large lethargy.

One of the earliest, and most significant, attempts at extending and improving on age-theory was by Placzek. Some of his remarkable results were briefly mentioned by Marshak in a 1947 review paper on neutron transport, ${ }^{1}$ but a full presentation of Placzek's method of approach and details of the results can only be found in an unpublished 1942 report. 6 Placzek concentrated on the set of equations, obtained from the Boltzmann equation, for the spatial moments of the various components of the angular flux density. By working with the Laplace transform of the lethargy dependent Fourier space transform of the flux, he was able to derive formulae for the spatial moments of the scalar flux which incorporate the first correction term in the inverse lethargy. Other attempts at extending age-theory were made by Marshak ${ }^{1}$ who used 
a low order spherical harmonics solution, Bethe, Tonks and Hurwitz, 5 who pioneered what is now known as "the $B_{L}$ method," and Holte, 7-8 who extended Waller's approach, ${ }^{3}$-based on the use of continued fractions.

The second category of limiting cases for the neutron flux distribution comprises what we shall refer to as "Wick-type" asymptotic solutions. These approximate solutions attempt to describe the distribution of neutrons at the opposite extreme in the phase-space, i.e., at "large" distances $x$ from the source and at small lethargies $u$. The main difficulty associated with finding analytical solutions valid in this region of phase-space is that the neutron angular flux density becomes strongly peaked in the forward direction, approaching a deltafunction of the form $\delta(\mu-1)$. The physical reason is obvious: the region of phase-space being considered is populated by neutrons which travelled far from the source spatially, but have not lost much energy in the process. In penetrating to large distances, the neutron may still have undergone many scattering collisions. But the collisions must then be such that little energy is lost per scattering, i.e., they must therefore all be of small angle scattering. Thus, for any given velocity close to the source velocity, the neutrons moving directly away from the source predominate at sufficiently large distances and this effect is the more pronounced the greater the distance from the source. The result is that the neutron distribution can no longer be represented in the space variable $x$ by a Gaussian, but rather by a decaying exponential. It is quite clear that a method of successive approximations which would start with age-theory as a first approximation is not likely to yield the spatial dependence of the asymptotic neutron flux in this region. 
Alternate methods of tackling this problem had to be devised and, after less-than-successful attempts by Wigner, ${ }^{1}$ Breit ${ }^{1}$ and Placzek, 1 Wick ${ }^{4}$ succeeded in deducing what appeared to be the correct asymptotic space and lethargy dependence for "very large" $x$.

A few years later, Holte ${ }^{8}$ devised a generalization of Wick's solution, keeping basically the same functional shape as Wick's, but calculating the first few correction terms. Unfortunately, his method of calculating the mass-dependent constants appearing in his formula, is purely numerical. Holte himself calculated coefficients only for Deuterium and Carbon. The fact that the method in practice requires an analytical continuation to be performed numerically for each nuclide mass (with its uncertain inherent errors and assumptions) severely limits its usefulness.

About fifteen years later, by applying the method of singular eigenfunctions ${ }^{14,15}$ to the transport equation transformed with respect to lethargy, McInerneyll in 1965 obtained a new solution valid for small lethargy and only in hydrogen. McInerney gave the range of his formula as valid for " $x, u>0$ " and also performed Monte-Carlo calculations to demonstrate $i$ ts reliability, Furthermore, for large $x$ and $x \gg u_{\text {, }}$ McInerney's formula takes on the same functional form as Wick's, but the value of his constants differ from those given by Wick. ${ }^{4}$

Another region of phase-space where there are known discrepancies among the various approximations to the neutron flux density is the region around the source, i.e., $u$ and $x$ small. Thus, for exalliple, as the independent variables lethargy. and position approach the values of 
the source, the exact solution for the scattered flux reaches a finite maximum. On the other hand, in the same limit, the $P-1$ approximation 10 goes to zero, and the "age-theory" result becomes infinite. Such extreme errors occur despite the fact that these functions are asymptotically equal at large lethargies. By the same token; as $u \rightarrow 0$ (i.e., as we approach the source lethargy), McInerney's formula takes on the exact value (for hydrogen), while Wick's solution becomes infinite. (of course-both are in error at large lechargles.)

An attempt to display graphically the state of the present knowledge about the neutron distribution in phase-space, is given by the following diagram:

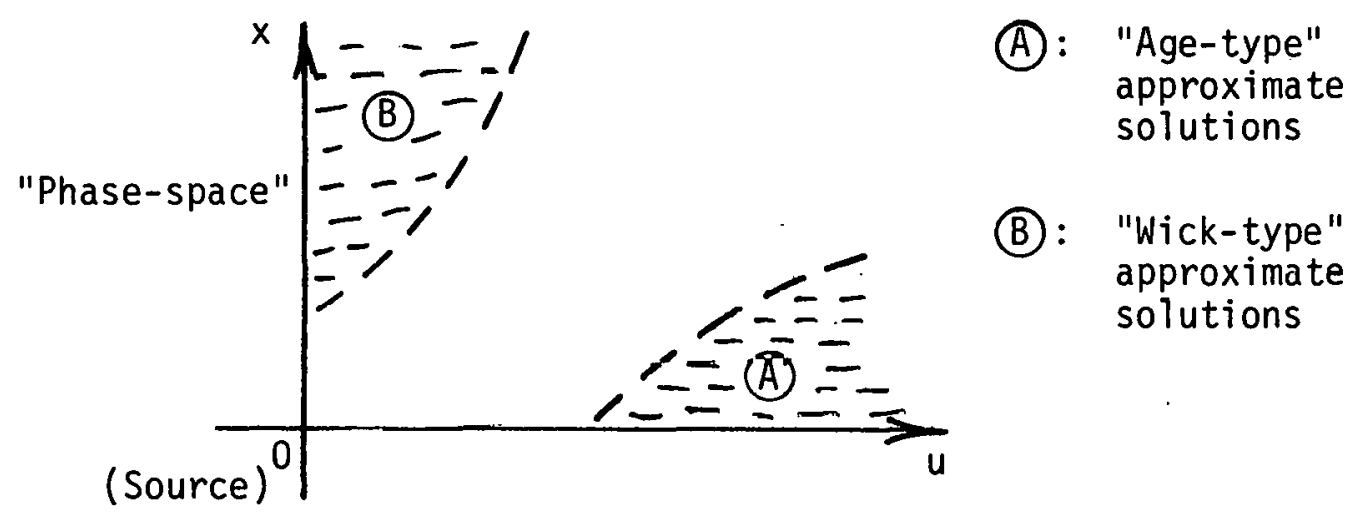

i.e., the presently available approximate solutions cover only two relatively small "wedges," with their two respective apexes oriented tuward the origin, of the entire phase-space. The two "wedges" have the $x$ and, respectively, the $u$ axes as one of their respective boundaries, but their "inside" boundaries are by no means well-defined and do not overlap, leaving quite a gap in between.

A similar situation exists in the case of the spatial moments of the scalar flux: only the zeroth spatial moment (i.e., the space and 
angle integrated flux) is known exactly at all lethargies, in the special case of a nonabsorbing medium. ${ }^{64-65}$ The expression given by Placzek 6 for the general term $M_{2 n}(u)$ is valid only for large lethargies and represents essentially the "age-theory" limiting behavior of the neutrons.

This brief introductory discussion has indicated the state of knowledge, at the start of the investigation described here, of the constant cross-section transport problem. The major new results presented in this thesis are:

1. A new exact analytical expression for the second spatial moment $M_{2}(u)$ (and therefore of the "age" of the neutrons) valid at all lethargies and for arbitrary absorption (constant at all lethargies).

2. A new method for calculating the spatial moments of the scalar fluxes valid for moderate to large lethargies, giving corrections to the age-theory formulae. Placzek's expressions for the moments plus some later extensions, include these corrections to second order in the reciprocal lethargy. The method presented here can in principle be carried out to all orders in $1 / 4$. Correction terms through $u^{-5}$ have been explicitly calculated here.

In the process of obtaining these results it has been possible to obtain clarification of a number of the mathematical aspects of the transport theory. New points of particular interest include:

(a) The mathematical convergence conditions that have to be satisfied by the Legendre components of the scattering kernel have been reexamined. It is here shown that a sufficient condition derived previously by Case ${ }^{16}$ is not satisfactory, and we have obtained a new 
(and considerably weaker) one, using a more rigorous approach than Case's.

(b) A key advance in the formalism, one that provided a new insight into the analytical structure of the expression for the neutron flux density, was the discovery of a closed form expression of what we shall refer to as the "eigenvalue-space" moments. It will be shown that this type of moments plays a crucial role throughout our investigation.

Ihe basic technique used in the investigation is the powerfur method of singular eigenfunctions expansion as applied to the Laplacetransform of the Boltzmann equation with respect to lethargy.

Chapter II is devoted to the necessary mathematical treatment of the Boltzmann equation. The material presented in this chapter is divided into two parts. In Part II.1, the Boltzmann equation for time independent neutron slowing-down and transport is considered, under the following conditions: infinite medium, plane geometry, azimuthally independent scattering and constant cross sections. By means of a Laplace transform with respect to lethargy, this equation is transformed into an equation of the same form as the one-velocity linear transport equation with scattering anisotropic to all orders of a Legendre polynomial expansion. The only difference from the standard one-velocity form is that the scattering coefficients now depend upon an additional parameter, the transform variable $n$. Case's singular eigenfunctions method 11,15 is applied to this equation, and the spectrum of this Laplace-transformed operator is derived. The mathematical formalism of the singular eigenfunctions method is then reviewed. It is shown that most of the existing proofs given in the literature can be taken 
over without substantial change for our case of the transformed energydependent transport equation. The major original result presented in this section is the treatment of the sufficiency conditions relating to convergence criteria for the expansion of the scattering kernel in Legendre polynomials. Existing conditions are shown to be either not entirely applicable or too stringent for the energy dependent problem. It was found possible however to derive rigorously a new and satisfactory sufficient condition for the convergence of these expansion coefficients.

In Part II.2, however, advances in the mathematical formalism are presented. The "eigenvalue-space" moments associated with the fundamental orthogonal set of polynomials $\phi_{n}(v, n)$ are introduced. A closedform general expression for these moments is derived, and their most important characteristics are derived by employing methods of combịnatorial analysis.

Chapter III is devoted to the investigation of the spatial moments of the angle-integrated Green's function for a plane, isotropic source, monoenergetically emitting neutrons in an infinite medium. First.. the Green's function for the Laplace transformed Boltzmann equation is introduced; its spatial moments $M_{2 n}(n)$ are defined next and the relationship between $M_{2 n}(n)$ and the "eigenvalue-space" moments $m_{2 n}(n)$ is displayed-all of these in a manner analogous to monoenergetic transport. After these preliminaries, the remainder of the chapter presents the major techniques and results of our investigation of the lethargy-dependent spatial moments of the scalar flux. Thus, an exact method of tackling the problems associated with the Laplace inversion is employed to obtain the analytical 
expression of the space and angle integrated flux, $M_{0}(u)$. The same method is used, in conjuncture with the expression for $m_{2}(n)$, to obtain the rigorous analytical expression of the second moment, $M_{2}(u)$, for slowing-down with absorption. Finally, expressions for the lethargy-dependent spatial moments $M_{2 n}(u)$, valid for medium to large. lethargies are derived, and the mass-dependent coefficients for the correction terms to age-theory through $u^{-5}$ are explicitly obtained.

The concluding Chapter IV point.s to directions for future researches and to the conclusions that may be drawn from this work. Extensions of the theory described here are now in process (and will be reported elsewhere), chiefly on obtaining generalized expressions for the scalar flux density itself, as distinguished from the spatial moments. It is possible to derive expressions which are generalizations of the age theory results on the one hand and of McInerney's formula on the other. Derivation of a "universal" formula for the scalar flux valid for all regions of phase space is sometime in the future. It will require elucidation of a number of mathematical sore spots, e.g., with regard to the behavior for large n of the "eigenvalue-space". moments of the "transient" flux, $T_{2 n}(n)$. Finally, some thoughts are offered on the significance of the results obtained for the constant cross section problem in relation to transport problems of the "real world." 
CHAPTER II

MATHEMATICAL TREATMENT OF THE BOLTZMANN EQUATION

Part II.1. The Singular Eigenfunction Method Appl ied to the Lethargy-Laplace Transformed Boltżmanin Equation

\section{Section II.1.A. Mathematical Preliminaries}

We consider the time independent, homogeneous Boltzmann equation for the case of neutron slowing down and transport under the following conditions: infinite medium, plane geometry, azimuthally independent scattering, and cross-sections that are constant in energy. It will further be assumed that the scattering is isotropic in the center-ofmass system. The explicit form of the Boltzmann equation under these conditions is:

$$
\left(\mu \frac{\partial}{\partial x}+1\right) \Psi(x, \mu, u)=c \int_{\Omega^{-}} \int_{u^{-}} f\left(\bar{\Omega} \cdot \bar{\Omega}^{-}, u^{-} u^{-}\right) \Psi\left(x, \mu^{-}, u^{-}\right) d u^{-} d \bar{\Omega}^{-}
$$

where:

$\Psi(x, \mu, u)$ is the angular flux density of neutrons per unit solid angle and lethargy.

$x \equiv \Sigma_{\top} Z$ is the position variable measured in optical units.

$u$ is the neutron lethargy (i.e., $u \equiv \ln \frac{E_{0}}{E}$, $E$ being the energy variable).

$\mu$ is the cosine of the angle between the neutron velocity vector and the $x$-axis.

$c$ is the mean number of secondaries, emitted in a neutron-nucleous 
interaction (i.e., $\mathrm{c} \equiv \Sigma_{S} / \Sigma_{T}$, where $\Sigma_{S}$ is the macroscopic scattering cross-section).

$\Omega$ is the solid angle.

The scattering kernel for isotropic scattering in the $\mathrm{CM}$ system by a single element of mass $A$ is

$f\left(\bar{\Omega} \cdot \bar{\Omega}^{\circ}, u^{\prime} u^{\prime}\right)=\left\{\begin{array}{cl}\frac{e^{-\left(u-u^{-}\right)}}{8 \pi} \frac{(A+1)^{2}}{A} \times \delta\left[\bar{\Omega} \cdot \bar{\Omega}^{-}-\left(\frac{A+1}{2} e^{-\frac{u-u^{\prime}}{2}}-\frac{A-1}{2} e^{\left.\frac{u-u^{\prime}}{2}\right)}\right)\right] \\ \text { for }\left(u-u^{-}\right) \in[0, \varepsilon] \\ \text { for }\left(u-u^{-}\right)>\varepsilon\end{array}\right.$

where $\varepsilon \equiv \ln \left(\frac{A+1}{A-1}\right)^{2}$

By means of a Laplace transform over lethargy, Eq. (II.I) can be transformed into an equation of the same form as the one-velocity linear transport equation. The structure of the Laplace-transformed transport operator will subsequently be studied by employing Case's singular eigenfunction method.

This approach to seeking solutions to the Boltzmann Equation has been used before, and a detailed description of the mathematical derivations can be found, for example, in the work of McInerney. ${ }^{21}$ However, since our investigation will subsequently be carried well beyond McInerney's, a brief and concise review of the mathematical preliminaries will be given in this introductory section, for ease of reference and for the sake of completeness.

Thus, Eq. (II.1) will be transformed into an equation of the same form as the one-speed transport equation with scattering that is 
anisotropic to all orders in terms of Legendre polynomials (i.e., what we shall henceforth refer to as "infinite order anisotropy"), by means of the following sequence of manipulations:

The scattering kernel can, with the aid of the spherical harmonics addition theorem, ${ }^{17}$ be put in a degenerate form via an expansion in Legendre polynomials. Thus

$$
\int_{0}^{2 \pi} f\left(\bar{\Omega} \cdot \bar{\Omega}^{\prime}, u-u^{\prime}\right) d \phi=\sum_{n=0}^{\infty} \frac{2 n+1}{2} f_{n}\left(u-u^{\prime}\right) P_{n}(u) P_{n}\left(\mu^{\prime}\right)
$$

where $\phi$ denotes the azimuthal coordinate of $\bar{\Omega}^{-}$and where the coefficients $f_{n}$, for $\left(u-u^{-}\right)$in the nonzero range of $f\left(\bar{\Omega}^{\cdot} \cdot \bar{\Omega}^{-}, u-u^{-}\right)$, are given by

$$
f_{n}\left(u-u^{\prime}\right)=\frac{(A+1)^{2}}{4 A} e^{-\left(u-u^{\prime}\right)} P_{n}\left(\frac{A+1}{2} e^{-\frac{u-u^{\prime}}{2}}-\frac{A-1}{2} e^{\frac{u-u^{\prime}}{2}}\right)
$$

Using Eq. (II.4), Eq. (II.1) becomes

$$
\begin{aligned}
& \left(\mu \frac{\partial}{\partial x}+1\right) \Psi(x, \mu, u) \\
& \quad=c \sum_{n=0}^{\infty} \frac{2 n+1}{2} P_{n}(u) \int_{-1}^{1} P_{n}\left(\mu^{\prime}\right) \int_{u^{\prime}} f_{n}\left(u-u^{\prime}\right) \psi\left(x, \mu^{\prime}, u^{\prime}\right) d \mu^{\prime} d u^{\prime}
\end{aligned}
$$

We now introduce the one-sided Laplace transform, defined as

$$
\mathscr{L}[G(u)] \equiv \int_{0}^{\infty} e^{-n u} G(u) d u \equiv G(n)
$$


and satisfying the convolution theorem

$$
\mathscr{L}[G(u)] \mathscr{L}[H(u)]=\mathscr{L}\left[\int_{0}^{u} G\left(u^{-}\right) H\left(u-u^{-}\right) d u^{-}\right]
$$

Taking into account Eqs. (II.7) and (II.8), the Laplace transform of (II.6) becomes

$\left(\mu \frac{\partial}{\partial x}+1\right) \Psi(x, \mu, n)=c \sum_{n=0}^{m} \frac{2 n+1}{2} g_{n}(n) P_{n}(\mu) \int_{-1}^{1} P_{n}\left(\mu^{-}\right) \Psi\left(x, \mu^{-}, n\right) d \mu^{-}$

where $\Psi(x, \mu, n) \equiv \mathscr{L}[\Psi(x, \mu, u)]$

and $g_{n}(n) \equiv \mathscr{L}\left[f_{n}(u)\right]=\int_{0}^{\varepsilon} e^{-n u_{f}}(u) d u$

It can easily be seen from Eqs. (II.11) and (II.5) that the functions $g_{n}(n)$ depend on $n, n$ and the mass $A$. Since these functions will play. an essential role throughout our investigation, we have devoted Appendix $A$ to the study of their properties.

Equation (II.9) has the same form as the one-speed transport equation with anisotropic scattering to all orders in terms of Legendre polynomials depending, moreover, on the additional parameter $n$.

The method of singular eigenfunctions, originated by $\mathrm{Case}^{14}$ and extended by Mika and others, ${ }^{18^{-39}}$ provides a powerful tool for seeking solutions to equations of form (II.9). This is the approach chosen in the present investigation, and techniques parallel to theirs will now be applied. We seek a separable solution to Eq. (II.9) of the form: 


$$
\begin{aligned}
& \Psi(x, \mu, n)=e^{-\dot{x} / \nu} \phi(\nu, \mu, n) \\
& \text { Defining } \\
& \phi_{n}(\nu, n) \equiv \int_{-1}^{1} \phi(\nu, \mu, n) P_{n}(\mu) d \mu
\end{aligned}
$$

we obtain the well-known eigenvalue equation $11,15,40$ for $\phi(\nu, \mu, n)$ :

$$
(\nu-\mu) \phi(\nu, \mu, n)=\frac{c \nu}{2} M(\mu, \nu, n)
$$

where

$$
M(\mu, \nu, n)=\sum_{n=0}^{\infty}(2 n+1) g_{n}(n) P_{n}(\mu) \phi_{n}(\nu, n)
$$

We remark that since, at this stage, the normalization of $\phi(\nu, \mu, \pi)$ is arbitrary, we can assume, with no loss of generality, that

$$
\phi_{0}(\nu, n) \equiv \int_{-1}^{1} \phi(\nu, \mu, n) \mathrm{d} \mu=1
$$

and define

$$
\phi_{-1}(\nu, n) \equiv 0
$$

The quantities $\phi_{n}(\nu, n)$ are known 11,15 to satisfy a 3 term recursion relation which can be obtained by multiplying Eq. (II.14) by $P_{k}(\mu)$, and 
integrating over $\mu$ from -1 to +1 . By using the well-known Legendre polynomial recurrence relation ${ }^{17}$

$$
(2 n+1) \mu P_{n}(\mu)=(n+1) P_{n+1}(\mu)+n P_{n-1}(\mu)
$$

we then obtain

$$
\xi_{n} \nu \phi_{n}(\nu, n)=(n+1) \phi_{n+1}(\nu, n)+n \phi_{n-1}(\nu, n)
$$

where

$$
\xi_{n} \equiv \xi_{n}(n)=(2 n+1) \gamma_{n}(i)
$$

and

$$
\gamma_{n}(n)=1-\operatorname{cg}_{n}(n)
$$

Comparing Eqs. (II.19) and (II.18) [and keeping in mind the normalization (II.16)], we see that the $\phi_{n}(\nu, n)$ are $n$-degree polynomials in odd or even powers of $v$, according to whether $n$ is odd or even, respectively, i.e.,

$$
\phi_{n}(\nu, n)=(-1)^{n_{\phi_{n}}}(-v, n)
$$

The explicit form of the first few $\phi_{n}$ 's is:

$$
\begin{aligned}
& \phi_{0}(\nu, n)=1 \\
& \phi_{1}(\nu, n)=\gamma_{0}(n) \nu
\end{aligned}
$$




$$
\phi_{2}(v, n)=\frac{3}{2} \gamma_{0}(n) \gamma_{1}(n) v^{2}-\frac{1}{2}
$$

As an aside, we note here the very important fact that the 3 term recursion relation (II.19) satisfied by the polynomials $\phi_{n}$ has a form similar to recursion relations satisfied by orthogonal polynomials. ${ }^{16,40,55,58}$ This suggests that the polynomials $\phi_{n}(\nu, n)$ could provide a link between transport theory and the theory of orthogonal polynomials. One might expect that considerable light could be shed on the structure of the transport equation by studying the $\phi_{n}$ 's from the point of view of the theory of orthogonal polynomials. In the case of monoenergetic transport, the polynomials $h_{n}(v)$ [corresponding to our $\left.\phi_{n}(\nu, n)\right]$ do indeed form an orthogonal system, as has been shown by Inönü ${ }^{40}$ and by Case. ${ }^{16}$ It will be seen later that our $\phi_{n}{ }^{\prime} s$ are also orthogonal, in the Stieltjes sense. However, since it has far reaching consequences, the detailed discussion of this important property will be reserved for Part II.2, below. We shall therefore return to the presentation of the "mathematical preliminaries" for the purpose of introducing still more quantities, which will repeatedly be used throughout this work.

It is well known $15,41,42$ that if in equations of the type of Eq. (II.14) $v$ takes values located on the interval of definition of $\mu$ [i.e., between -1 and +1$]$, the eigenvalue equation is singular. Thus, for $v \in(-1,1)$, the solutions of Eq. (II.14) can be written as the sum of a delta distribution and a Cauchy principal value distribution, 


$$
\phi(\nu, \mu, \eta)=\frac{c \nu}{2} P \frac{M(\mu, \nu, \eta)}{\nu-\mu}+\lambda(\nu, \eta) \delta(\nu-\mu)
$$

where, in general, $\lambda(\nu, \eta)$ is a completely arbitrary function of $\nu$. In our case, however, the normalization condition (II.16) requires that $\lambda(\nu, n)$ be given by:

$$
\lambda(\nu, n)=1-\frac{1}{2} c \nu P \int_{-1}^{1} \frac{M(\mu, \nu, n)}{\nu-\mu} d \mu
$$

for all values of $\nu$ on the interval $-1 \leq \mu \leq 1$.

We thus conclude that the interval $(-1,1)$ constitutes the continuous part of the spectrum of the eigenvalue equation (II.14) and that the continuum eigenfunctions are singular eigendistributions of the form (II.26), with $\lambda(\nu, n)$ given in (II.27).

For all values of $v$ outside the closed interval $(-1,1)$ the solution of the eigenvalue equation (II.14) is simply the function

$$
\phi(\nu, \mu, n)=\frac{C \nu}{2(\nu-\mu)} M(\mu, \nu, n)
$$

The normalization condition (II.16) imposed on the eigenfunctions $\phi(\nu, \mu, n)$ in (II.28) restricts, however, the admissible values of $\nu$ to the roots of the following dispersion equation ${ }^{11,15,40}$ :

$$
\begin{aligned}
\Lambda(\nu, n) & \equiv 1-\frac{1}{2} c \nu \int_{-1}^{1} \frac{M(\mu, v, n)}{v-\mu} d \mu \\
& =1-c v \sum_{n=0}^{\infty}(2 n+1) g_{n}(n) \phi_{n}(\nu, n) Q_{n}(\nu)=0
\end{aligned}
$$


From Eq. (II.29), $\Lambda(v, n)$ is seen to be an analytic function of $v$ over the whole $v$ plane cut along the axis from $(-1,1)$. On the cut, a simple application of the Plemelj formulae ${ }^{41}$ yields:

$$
\Lambda^{ \pm}(\nu, n)=\lambda(\nu, n) \pm \frac{1}{2} \pi i \operatorname{cuM}(\nu, v, n)
$$

where $\Lambda^{+}$and $\Lambda^{-}$are the boundary values of $\Lambda(\nu, n)$ above and below the cut $(-1,1)$, respectively. At infinity, $\Lambda(\nu, \eta)$ is bounded and equal to 16,40

$$
\operatorname{lime}_{\nu \rightarrow \infty} \Lambda(\nu, n)=\prod_{j=0}^{\infty} \gamma_{j}(n)
$$

Furthermore, it follows from (II.29) that $\Lambda(v, n)=\Lambda(-v, n)$. An immediate consequence of the evenness of $\Lambda$ is that the discrete eigenvalues [which we denote by $v_{j}(n)$ ] occur in \pm pairs. Further consideration of the properties of the dispersion function and eigenvalues will be given in Section II.l.C, below. We conclude this introductory section with the remark that Eq. (II.9) is both linear and homogeneous. From this, as well as previous considerations, it follows that Eq. (II.9) has a solution of the form

$$
\begin{aligned}
\Psi(x, \mu, n) & =\sum_{ \pm j} A\left(\nu_{j}, n\right) \phi\left(\nu_{j}, \mu, n\right) e^{-x / \nu_{j}} \\
& +\int_{-\{}^{1} A(\nu, i i) \psi(\nu, \mu, n) e^{-x / \nu}
\end{aligned}
$$

where the coefficients $A\left(\nu_{j}, n\right)$ and $A(\nu, n)$ are arbitrary. 
The expansion (II.32) has practical meaning providing that it is unique, i.e., if the eigenfunctions (II.26) and (II.28) are complete. It will be proven in the following section that these eigenfunctions are orthogonal with respect to a suitable weight function and that they indeed form a complete set. Consequently, expansion (II.32) is a general solution of Eq. (II.9) and, furthermore, the solution of Eq. (II.6) will be the inverse Laplace transform of (II.32),

$$
\Psi(x, \mu, u)=\mathcal{L}^{-1}\{\Psi(x, \mu, n)\}
$$

Section II.l.B. Orthogonality and Completeness Properties of the Eigenfunctions

It is well known $11,15,42$ that the system of eigenfunctions, Eqs. (II.26) and (II.28) are orthogonal with weighting factor $\mu$ over the interval $-1 \leq \mu \leq 1$.

Thus, two arbitrary eigenfunctions either from the continuous or discrete spectrum satisfy the relation

$$
\int_{-1}^{1} \mu \phi(\nu, \mu, n) \phi\left(\nu^{-}, \mu, n\right) d \mu=0
$$

for all values of $v^{\prime}$ that are different from $v$. For $v^{\prime}=v k(-1,1)$ we define the discrete normalization integral as:

$$
N_{j \pm}\left( \pm v_{j}, n\right) \equiv \int_{-1}^{1} \mu \phi^{2}\left( \pm v_{j}, \mu, n\right) d \mu
$$

The evaluation of the integral in (II.35) is a straightforward, albeit lengthy calculation. Because of the complexity of the resulting 
expression for $\mathrm{N}_{\mathrm{j} \pm}$, the use of its explicit form is avoided throughout this investigation. However, it has been shown 40 that $N_{j \pm}$ can conveniently be expressed as:

$$
N_{j \pm}= \pm\left.\frac{1}{2} c v_{j}^{2} M\left(v_{j}, v_{j}, n\right) \frac{\partial \Lambda(v, n)}{\partial v}\right|_{v=v_{j}}
$$

For $\nu=v^{-} \in(-1,1)$ it can also be shown,,$^{11}$ by direct calculations, that

$$
\int_{-1}^{1} \mu \phi(\nu, \mu, n) \phi\left(v^{-}, \mu, n\right) \dot{\alpha} \mu=v \lambda^{2}(\nu, n) \delta\left(\nu-v^{-}\right)
$$

This orthogonality relation will appear only indirectly in calculations involving the determination of some unknown "expansion coefficient" $A(\nu, n)$, in integrals of the form:

$$
\int_{-1}^{1} \mu \phi\left(\nu^{-}, \mu, n\right) d \mu \int_{-1}^{1} A(\nu, n) \phi(\nu, \mu, n) d v
$$

which is a doubly singular Cauchy type integral in the range of the $\mu$ integration. Integrals of the type (II.38) can be expressed in a simple form, providing that $A(v, n)$ is an arbitrary function defined for $-1 \leq \nu \leq 1$ and satisfying the $H^{*}$ condition ${ }^{15,41}$ in this interval. For such a function the Poincare-Bertrand transformation formula15,41 can then be invoked, in the form

$$
\int_{-1}^{1} \frac{d \mu}{v-\mu} \int_{-1}^{1} \frac{F\left(\mu, v^{-}\right)}{v^{\prime}-\mu} d v^{-}=\pi^{2} F(\nu, \nu)+\int_{-1}^{1} d v^{-} \int_{-1}^{1} \frac{F\left(\mu, v^{-}\right)}{(\nu-\mu)\left(\nu^{-}-\mu\right)} d \mu
$$


where $F(\mu, \nu)$ is an arbitrary function satisfying a. Hölder condition on the interval $(-1,+1)$. Then, with the help of (II.39), Eq. (II.38) can be written as

$$
\int_{-1}^{1} \mu \phi(\nu, \mu, n) d \mu \int_{-1}^{1} A\left(v^{-}, n\right) \phi\left(v^{-}, \mu, n\right) d v^{-}=N(v, n) A(v, n)
$$

where

$$
N(v, n) \equiv v\left[\lambda^{2}(v, n)+\left(\frac{\pi C v}{2} M(v, v, n)\right)^{2}\right]
$$

is the so-called continuum normalization integral. From Eqs. (II.30) and (II.41), it also follows that $N(v, n)$ can be conveniently expressed as:

$$
N(v, n)=v \Lambda^{+}(\nu, n) \Lambda^{-}(v, n)
$$

At this stage we would like to remark that the orthogonality relations (II.34), (II.35) and (II.37) for the eiqenfunctions hold irrespective of the order of scattering $N$,* provided that the expressions for the eigenfunctions [i.e., Eqs. (II.26) and (II.28)] still have meaning in the limit as $N \rightarrow \infty$ [i.e., in the case of "infinite

*We shall denote the (finite) order of anisotropic scattering by the letter $N$; (e.g., $N=0$ for isotropic scattering, $N=1$ for anisotropic scattering, etc.). Moreover, $\mathrm{N}$ also appears as a subscript in $\Psi_{N}(\mu, \nu, u)$ and $\Psi_{N}(\mu, \nu, n)$, indicating that $\Psi_{N}$ is the solution of the (respective) Boltzmann equation for finite anisotropic scattering (of order $N$ ). It is hoped that this usage of $N$ will nnt lead to confusions with the traditiond notations $N(\nu, n)$ - for the continuous normalization integral [cf. Eq. (II.41)]-or $\mathrm{N}_{\mathrm{j} \pm}$-for the discrete normalization integral [Cf. Eq. (II.36)]. 
order anisotropy"]. From the structure of the quantities involved in Eqs. (II.26) and (II.28), one can readily anticipate that the conditions under which (II.26) and (II.28) have meaning as $N \rightarrow \infty$ will ultimately require that some sort of convergence condition be satisfied by the quantities $g_{n}(n)$ [i.e., the Laplace transforms of the Legendre. coefficients of the scattering kernel] as $n \rightarrow \infty$. The mathematical difficulties introduced when $N \rightarrow \infty$ are indeed far from trivial, as we shall see time and again the remainder of this chapter. For example, difficulties of this nature will be encountered in our next topic-the discussion of the completeness theorems for the eigenfunctions (II.26) and (II.28).

The application of the singular eigenfunction technique to initial boundary value problems has given rise to a number of difficulties associated with the rigor of the mathematical proofs concerning the completeness of the eigenfunctions and the methods for solving for the proper expansion coefficients, e.g., $A\left(v_{j}, n\right)$ and $A(\nu, n)$ that appear in (II.32).

Historically, Case's original treatment of the simplified planesymmetric, time independent one-speed transport equation with isotropic scattering, ${ }^{14}$ was extended to finite anisotropic scattering, ${ }^{18,19}$ twogroup, ${ }^{20-23}$ multigroup ${ }^{24-29}$ and continuous energy models. ${ }^{30-36}$ The associated completeness proofs were based on the reduction of the singular integral for the expansion coefficient to an inhomogeneous Hilbert problem of the form

$$
M^{+}(\mu)=G(\mu) M^{-}(\mu)+K(\mu) \quad \mu \in L
$$


where $L$ is an open arc, $G(\mu)$ and $K(\mu)$ are given and where one seeks $M(\mu)$, analytic in the complex plane along $L$ with prescribed behavior at infinity. Since in these "completeness proofs". $K(\mu)$ was allowed to depend on the unknown expansion coefficient, the ensuing analysis therefore yielded Fredholm equations rather than explicit results for the desired expansion coefficients. This approach was criticized 3 when several authors extended it to the multigroup case, where one has to sulve a system of singular integral equations and ( $i$ ) becomes d malrix version of the inhomogeneous Riemann-Hilbert problem. The most severe criticism was extended to half-range multigroup problems, where it was actually stated 43 that those "proofs" were, in fact, no proofs at all! In addition, and all along, mathematicians seem to have objected to Case and his disciples' cavalier treatment of the continuous spectrum, contending that the entire spectrum of the transport operator was deduced via merely heuristic arguments, rather than "rigorous" mathematical proofs.

An important development therefore occurred in 1973, when Larsen and Habetler 44 succeeded in setting the "completeness" proof on a rigorous mathematical footing, deducing the spectrum of the transport operator using functional analytical methods applied to the simplest transport problem [i.e., monoenergetic, isotropic scattering, $c<1$ ], by employing the so-called "resolvent integration" technique. The above-mentioned transport problem was worked out in a weighted Banach space $X$ where, even though $\mu \Psi(\mu)$ is required to satisfy a Hölder condition on $\mu \in[-1,1]$, the runctions of interest $\Psi(\mu)$ can be suitably 
singular at the origin. ${ }^{4-45}$ Later, the completeness proofs were extended by Larsen to $L_{p} 46$ and $L_{1} 47$ spaces. However the "straight" Larsen-Habetler technique fails for the conservative $(c=1)$ case because their $\mathrm{K}^{-1}$ [i.e., "transport"] operator [but acting on $\mu$ only] was not invertible on its range. In 1976, Greenberg and Zweifel 48 managed to solve the $c=1$ case, by finding a direct sum decomposition of $x_{p}$, i.e., $x_{p}=y_{p}+y_{p o}$ and proving that the restricted operator $K^{-1} Y_{p}$ is invertible. This, in turn, facilitated the construction of the entire spectrum of $K$. In the meantime, the aforementioned "resolvent integration" technique was extended to two-groups and multigroup problems. ${ }^{49-50}$

However, any application of the functional analytic techniques to the case of "infinite order anisotropy," is conspicuously absent. We have therefore devised, for our purposes, the following approach consisting of two main steps:

(a) In the remainder of this section, we establish the "completeness theorem" along the same functional analytic lines as those of Larsen's, ${ }^{45}$ i.e., when the scattering kernel is a finite sum, of order $N$, of Legendre polynomials.

(b) In the following section, we extend the "completeness theorem" to cover the case of "infinite order anisotropy" [i.e., for $N \rightarrow \infty$, using methods of the theory of orthogonal polynomials and scattering theory.

The idea of "completeness" of the eigenfunctions is well-known: one has to show that, given a boundary value problem governing the neutron 
density $\Psi_{N}(x, \mu, u)$ which satisfies certain conditions in a given $\mu$ interval, the coefficients $A(v, n)$, of its expansion in the eigenfunction space $\phi(\nu, \mu, \eta)$, exist and are uniquely determined.

Since in the Larsen technique the operator $K^{-1}$ acts on $\mu$ only, the additional n-dependence-displayed by the quantities of interest in our case-does not alter in any way the validity of his approach. We shall therefore only quote the results, as applicable to our situation. Nevertheless, for the sake of easy reference, the proof [a la Larsen] can be found in Appendix B. .

Thus, for finite order, $N$, of expansion of the scattering kernel the "full-range" "completeness theorem" can be expressed as:

$$
\Psi_{N}(\mu, \nu, n)=\sum_{ \pm n=1}^{m} A\left(\nu_{n}, n\right) \phi\left(\nu_{n}, \mu, n\right)+\int_{-1}^{1} A(\nu, n) \phi(\nu, \mu, n) d v
$$

In the Larsen formalism, the completeness theorem [i.e., Eq. (II.43)] is actually derived as the "resolution of the identity" [i.e., a family of orthogonal projections] associated with the operator $k$, by means of

$$
\Psi_{N}(\mu)=\frac{1}{2 \pi i} \oint_{\Gamma}(z I-K)^{-1} \Psi_{N}(\mu) d z
$$

In (II.44), $\Gamma$ is a closed contour which contains the spectrum of $K$. The "projection" [or - "expansion"] coefficinnts $A(\nu, n)$ and $A(\nu, n)$ appearing in (II.43) satisfy the relations

$$
A\left( \pm v_{j}, n\right)=\frac{1}{N_{j \pm}\left( \pm v_{j}, n\right)} \int_{-1}^{1} \mu \Psi_{N}(\mu, v, n) \phi\left( \pm v_{j}, \mu, n\right) d \mu
$$


with $N_{j \pm}\left( \pm v_{j}, n\right)$ given by (II.35), for $v= \pm v_{j}$, and

$$
A(v, n)=\frac{1}{N(\nu, n)} \int_{-1}^{1} \mu \psi_{N}(\mu, v, n) \phi(\nu, \mu, n) d \mu
$$

with $N(\nu, n)$ given by (II.42), for $-1<v<1$. The summation in (II.43) is taken over the entire number of discrete eigenvalues.

This is all that needs to be said for part (a) of the "completeness theorem." Part (b), the extension to the limit as $N \rightarrow \infty$, breaks new ground, and will be the major goal of the next section.

Section II.1.C. Further Aspects of the Singular Eigenfunction Formalism: Transport
Theory and the Theory of Orthogonal
Polynomials

In this section, we shall address the following points of interest:

(a) We shall verify that the polynomials $\phi_{n}(\nu, n)$ are, indeed, orthogonal in the Stieltjes sense, in the v-space.

(b) We shall confirm that the "completeness theorem," i.e., Eq. (II.43) is still valid in the limit as $N \rightarrow \infty$.

(c) We shall consider the properties of the discrete eigenvalues $v_{j}(n)$, and

(d) We shall discuss the convergence criteria (in $n$ ) that need be satisfied by lhe functions $g_{n}(n)$ in order for the statements made in (a) throuyh (c) above to hold true. In particular, we shall show that convergence criteria for the $g_{n}$ 's that were either stated 40 or derived ${ }^{16}$ by previous authors for the case of monoenergetic transport, 
are either questionable $e^{40}$ or cannot be fulfilled 16 for all values of $n$, in our case.

The above-mentioned problems will not necessarily be discussed in that order since there is a strong interdependence and interplay between both the methods used to arrive at the results of $(a)-(d)$, and the results themselves.

It was first shown by Inönü $4^{40}$ that, for the case of monoenergetic transport, the polynomials $h_{n}(v)$ [in his notation], which correspond to our $\phi_{n}(v, n)$, are orthogonal in the Stieltjes sense, over the interval $\left(-v_{1}, v_{i}\right)$, where $v_{1}=\max _{j}\left|v_{j}\right|$. Clearly, the orthogonalization interval covers both the continuous and discrete spectra in the v-space [what we shall henceforth refer to as "the eigenvalue space"]. Inönü derived the orthogonality relations for the polynomials $h_{n}(v)$ using the equivalent of the "completeness theorem," i.e., Eq. (II.43) for finite $\mathrm{N}$, for the case of monoenergetic transport. Then, he showed that these orthogonality relations hold even when $N \rightarrow \infty$ by arguing that the quantities involved in the expressions for the eigenfunction converge, in the distribution sense, as $N \rightarrow \infty$. Finally, by reversing this process of reasoning, he noted that the "completeness theorem" for the eigenfunctions can be established from the completeness of the Legendre polynomials and from the orthogonality of the $h_{n}$ 's, when $\mathrm{N} \rightarrow \infty$. This implied, therefore, that the "completeness theorem" is still valid in the limit as $N \rightarrow \infty$.

Throughout his work, Inönü's basic assumption 40 was the explicit requirement that the coefficients of the Legendre expansion of the scattering kernel satisfy the convergence condition: 


$$
\sum_{n=0}^{\infty} n\left|f_{n}\right|<\infty
$$

It should be noted that, since Inönü did not investigate the behavior of the dispersion function $\Lambda(v)$ at the points $v= \pm 1$, his proofs are somewhat in want of the rigor a mathematical purist might expect. Indeed, it will soon become clear that the behavior of the dispersion function on the cut $(-1,1)$ and, particularly, at $v= \pm 1$, requires special attention, and that consequently condition (II.47) might not be satisfactory.

About four years later, Case ${ }^{16}$ reexamined the problem of monoenergetic transport with "infinite order anisotropic" scattering from a different point of view, bringing into evidence the interrelation between transport theory, the theory of orthogonal polynomials and scattering theory. Based on this interplay, Case rigorously proved the orthogonality properties of the polynomials $h_{n}(v)$ [corresponding to our $\left.\phi_{n}(\nu, n)\right]$, arriving at a relation identical to Inönü's; he also derived the closure relations for these polynomials. The completeness of the eigenfunctions then followed immediately. Finally, he rigorously proved that the discrete eigenvalues are:

(1) simple

(2) $\geq 1$ in absolute magnitude, i.e., no discrete eigenvalue can occur in the continuum and the closest they can approach is the edge of the continuum

(3) occur in \pm pairs

(4) real, for $c \leq 1$, and

(5) finite in number. 
Indeed, all but the finiteness of the number of discrete eigenvalues was proven rigorously using rather elementary methods. To prove this last property of the $v_{j}{ }^{\prime} s$, one has to use a rather more sophisticated approach since it is not immediately obvious that the number of discrete eigenvalues remains necessarily finite when $N \rightarrow \infty$. This is where one ultimately arrives, mathematically, at the convergence condition for the Legendre coefficients of the scattering kernel. Thus, Case's method of proof led to

$$
\sum_{n=0}^{\infty} n^{2}\left|f_{n}\right|<\infty
$$

as a sufficient condition for the finiteness of the number of eigenvalues.

Case's convergence condition (II.48) is obviously stronger than Inönü's (II.47), and was arrived at precisely as a consequence of the behavior of the dispersion function on the cut $(-1,+1)$, in the $v-p l a n e$.

It turns out that Case's proofs ${ }^{16}$ can be taken over, with one major exception, to our situation of $n$-dependent quantities. This one major exception is the fact that the equivalent convergence condition to (II.48), for our $g_{n}$ 's, i.e.,

$$
\sum_{n=0}^{\infty} n^{2}|g n(n)|<m
$$

is not satisfied for all values of $n$, in the case of hydrogen. This fact is demonstrated in Appendix $A$, where we show that, for $A=1$ and 
$\operatorname{Re}\{n\} \leq 1 / 4$, the series (II.48.a) is divergent. Obviously, the failure of (II.48.a) would imply, according to Case's arguments, the occurrence of infinitely many discrete eigenvalues, with all the displeasing consequences.

However, there seems to be no obvious physical reason to expect the occurrence of infinitely many discrete eigenvalues in this region. Therefore, we have reexamined the entire problem of convergence requirements for the functions $g_{n}(n)$. Our detailed derivations, can be found in Appendix $D$.

The starting point of our proofs is based on Case's general considerations, as applicable to our polynomials $\phi_{n}(\nu, n)$. For convenience, we have therefore devoted Appendix $C$ to reproducing the main points of Case's demonstrations as applicable to our situation; this has the additional advantage of clearly showing that the additional $n$-dependence of our quantities does not affect the validity of these proofs [with the exception, of course, of the above-mentioned considerations leading to (II.48)].

Thus, as a consequence of our investigations of Appendix $D$, we have proven that

$$
\sum_{n=0}^{\infty} n \ln n\left|g_{n}(n)\right|<\infty
$$

is a sufficient condition to ensure the finiteness of the number of eigenvalues. The convergence condition (II.49) is satisfied for ali moderators, including hydrogen, and for all values of $n$ such that $\operatorname{Re}\{n\}>-1 / 4$. 
Obviously, our condition (II.49) is considerably weaker than Case's (II.48), but stronger than Inönü's (II.47). Inönü's condition is certainly necessary, as can be seen from our derivations, in Appendix D. (We also discuss, in the same appendix, the possibility that it might conceivably be sufficient.)

Therefore, based on the convergence condition (II.49), and the application of Case's approach to our case (Appendix C), we can conclude that:

(i) The "completeness theorem" for the eigenfunctions, i.e., Eq. (II.43) holds true even in the limit as $N \rightarrow \infty$.

(ii) The discrete eigenvalues enjoy properties (1), (2), (3) and (5), above, page 29; property (4) becomes, in our $n$-dependent case: "for $c g_{\theta}(n) \leq l, v_{j}(n)$ are real for real $n . "$

(iii) The polynomials $\phi_{n}(\nu, n)$ are orthogonal, in the Stieltjes sense, over the interval $\left[-v_{1}(n),+v_{1}(n)\right]$, where

$$
v_{1}(n)=\max _{j}\left|v_{j}(n)\right|
$$

The orthogonality relations for the $\phi_{n}(\nu, n)$ can be written as: $:^{16,40}$

$$
\begin{aligned}
& \int_{-1}^{1} \phi_{k}(v, n) \phi_{\ell}(v, n) \frac{v}{N(v, n)} d v+\sum_{j}\left[\phi_{k}\left(v_{j}, n\right) \phi_{\ell}\left(v_{j}, n\right) \frac{v_{j}}{N_{j+}}+\right. \\
& \left.+\phi_{k}\left(-v_{j}, n\right) \phi_{\ell}\left(-v_{j}, n\right)\left(\frac{-v_{j}}{N_{j-}}\right)\right]=\frac{2 \delta_{k \ell}}{(2 \ell+7) \gamma_{\ell}(n)} \\
& =\int_{-v_{l}(n)}^{\nu_{1}(n)} \phi_{k}(\nu, n) \phi_{\ell}(\nu, n) d \omega(\nu, n)
\end{aligned}
$$


where $\omega(\nu, \eta)$ is the corresponding weight function covering both the continuous and discrete spectra, and which can be expressed as: ${ }^{40}$

$$
\omega(\nu, n)=\int_{0}^{\nu} \frac{v}{N(v, n)} d v ; \text { for }|v| \leq 1
$$

and the sequence of horizontal lines

$$
\omega(\mp v, n)=\omega(\mp i, n) \mp \sum_{i=m}^{M} \frac{v_{i}}{N_{i}} ; \text { for } v_{m}<v<v_{m-1}
$$

with

$$
\omega(\mp \nu, n)=\omega(\mp 1, n) \mp \sum_{i=1}^{M} \frac{v_{i}}{N_{i}} ; \text { for } v_{1}<v
$$

Finally, we note that the orthogonality relations for the eigenfunctions $\phi(\nu, \mu, \eta)$, i.e., Eqs. (II.34-37) and (II.41), lead to the closure relations for the polynomials $\phi_{n}(\nu, n) 40$

$$
\begin{aligned}
\frac{N(\nu, n)}{\nu} \delta\left(\nu-v^{-}\right) & =\frac{1}{\nu} \int_{-\{}^{1} \phi(\nu, \mu, n) \phi\left(v^{-}, \mu, \eta\right) \mu d \mu \\
& =\sum_{k=0}^{\infty} \frac{\xi_{k}(n)}{2} \phi_{k}(\nu, n) \phi_{k}\left(v^{-}, n\right), \text { for }|\nu|<1
\end{aligned}
$$

and 


$$
\begin{aligned}
\frac{N_{j \pm}\left( \pm v_{j}, n\right)}{ \pm v_{j}} \delta_{j k} & =\frac{1}{ \pm \nu_{j}} \int_{-1}^{1} \phi\left( \pm v_{j}, \mu, n\right) \phi\left( \pm v_{k}, \mu, n\right) \mu d \mu \\
& =\sum_{\ell=0}^{\infty} \frac{\xi_{\ell}(n)}{2} \phi_{\ell}\left( \pm v_{j}, n\right) \phi_{\ell}\left( \pm v_{k}, n\right)
\end{aligned}
$$

Part II.2: Advances in the Formal ism: The "Eigenvalue Space" Moments $m_{k}(n)$ and Their Properties

In this last part of Chapter II, we forus our attention on whal will henceforth be referred to as the "eigenvalue space" moments, $m_{k}(n)$. These moments are defined as

$$
m_{k}(n) \equiv \int_{-\dot{v}_{1}(n)}^{v_{1}(n)} v^{k} d \omega(v, n), \text { for } k=0,1,2, \ldots
$$

where $w(v, n)$ is the weight function as defined in Eqs. (II.52-54). Obviously, the $m_{k}$ 's are associated with the set of orihogonal polynomials $\phi_{n}(\nu, n)$.

As will be seen subsequently, in Chapters III and IV, the moments $m_{k}$ play a fundamental role when seeking solutions to slowing-down and transport problems, such as determining the space-lethargy dependent Green's function (i.e., the scalar flux), and its lethargy dependent spatial moments. It is expected that they will also prove to be of equal importance to other aspects of neutron slowing-down and transport.

Inönü40 was first lu consider briefly the equivalent of our "eigenvalue space" moments $m_{k}$, for the monoenergetic transport problem. 
His presentation and treatment of these moments was essentially a specific application to monoenergetic transport, of the basic concepts of the theory of the classical moment problem55-57-as can be found in the elegant monography of Akhiezer. ${ }^{55}$. As in the general theory, Inönü's considerations led to an implicit recursion relation for the moments, in determinantal form [given below as Eq. (II.58)]. From this relation [which has the same form as Eq. (II.58), below], it can be immediately seen that the expression for the general term $m_{n}$ would involve $\Delta_{n}$, as well as all the lower order (nonzero) moments. Thus, Inönü was able to calculate explicitly the first three (nonzero) moments, but failed to determine in the general case their significant characteristics.

Fortunately, our investigations, carried out within the more general frame of energy-dependent slowing-down and transport, have led to the development of a closed form for the general expression of these "eigenvalue space" moments $m_{k}(n)$. It is thus possible to display explicitly their functional dependence on the order $k$, and [via the known functions $g_{n}(n)$ ] on $n$. This, in turn, has shed considerable light on a number of key properties of the general term $m_{k}(n)$ and, mutatis mutandis, it.led to new and more complete solutions to the relatively old but only partially solved problems discussed in the later chapters.

We begin our investigation by recalling some of the fundamentals of the so-called "moment problem," as originally defined by Stieltjes. These basic concepts are admirably developed in the classical works of Akhiezer ${ }^{55}$ and Wal $11^{58}$ and, since we shall present only certain results 
from the general theory as immediately applicable to our case, we refer the interested reader to the above-mentioned works for details.

From the general theory of the moment problem, ${ }^{55}$ and the properties (II.16-17) and (II.19) of the orthogonal polynomials $\phi_{n}(\nu, n)$, the determinants

$$
\Delta_{n}=\left|\begin{array}{llll}
m_{0} & m_{1} & \cdots & m_{n-1} \\
m_{1} & m_{2} & \cdots & m_{n} \\
\vdots & \vdots & & \vdots \\
\vdots & \vdots & & \vdots \\
m_{n-1} & m_{n} & \cdots & m_{2(n-1)}
\end{array}\right| n \geq 1, \Delta_{0}=1
$$

exist and are all positive, since $\Delta_{n}$ is the discriminant of a positivedefinite quadratic form. ${ }^{55,58}$

The polynomials $\phi_{n}(\nu, n)$ can be expressed in terms of these determinants and the moments as

$$
\phi_{n}(\nu, n)=\left[\frac{\xi_{n}(n)}{2} \Delta_{n} \Delta_{n+1}\right]^{-1 / 2}\left|\begin{array}{llll}
m_{0} & m_{1} & \cdots & m_{n} \\
m_{1} & m_{2} & \cdots & m_{n+1} \\
\vdots & \vdots & \cdots & \vdots \\
m_{n-1} & m_{n} & \cdots & m_{2 n-1} \\
1 & \nu & \cdots & \nu^{n}
\end{array}\right|
$$

From the general theory, it also follows that the $\phi_{n}{ }^{\prime} s$, being orthogonal with respect to the sequence $m_{k}$, are the denominators of the Stieltjes type continued fraction:*

*According to the general theory, 55,58 the set of polynomials $\mathrm{B}_{\mathrm{p}}(\mathrm{z})$ orthogonal with respect to the sequence $\mathrm{C}_{\mathrm{p}}$ (i.e., the moments),
satisfy the recursion relation 


$$
s(\nu, n)=\frac{1}{T \nu}-\frac{W_{0}}{T \nu}-\frac{W_{1}}{T \nu}-\ldots
$$

where

$$
w_{j} \equiv \frac{(j+1)^{2}}{\xi_{j} \xi_{j+1}} \equiv w_{j}(n)
$$

with $\xi_{j}$ as defined in (II.20). In fact, it can easily be shown 55,58 that we have the relation

$$
\frac{2 v}{\xi_{0}} S(v, n)=\sum_{i=0}^{\infty} \frac{m_{i}}{v^{i}}
$$

In view of (II.62), the moments $m_{i}$ could be obtained, recursively, in terms of the known functions $\gamma_{n}(n)$, by an application of the Stieltjes expansion theorem. ${ }^{58}$ Equation (II.62) will also be of importance in other applications to be presented below. We also remark that the Stieltjes continued fraction $S(\nu, n)$ can be shown to be related to the spatial Fourier transform of the Laplace transformed scalar flux $\Psi_{0}^{G}(x, n)$, providing thus the key link between our treatment of the

$B_{p}(z)=\left(b_{p}-z\right) B_{p-1}(z)-a_{p-1} B_{p-2}(z) \quad p=2,3,4, \ldots$

with $B_{0}=1$ and $B_{1}(z)=b_{1}+z$, and are the denominators of the J-fraction:

$$
J(z)=\frac{a_{0}}{b_{1}+z-\frac{a_{1}}{b_{2}+z}-\ldots}
$$

In our case, however, $b_{p} \equiv 0$ and the $J$-fraction becomes an $S$ fraction. This has the important consequence that the odd moments $\mathrm{C}_{2 k+1}$ are identically zero and therefore our problem is symmetric in ${ }_{v}^{+1}$ (i.e., in the "eigenvalue space"), as we could have expected all along. 
transport equation and the (apparently dissimilar) methods of Waller, ${ }^{3}$ Hol te 7,8 and Wick. ${ }^{4}$

Returning now to the general theory of the "moment problem," we note that the determinants $\Delta_{n}$ in (II.58) satisfy the recursion relation 58

$$
\Delta_{n}=m_{0} w_{0} w_{1} \ldots w_{n} \Delta_{n-1} \text { for } n \geq 1
$$

or, by iterating (II.63),

$$
\Delta_{n}=2^{n} /\left[n ! a_{n, n}\left(\begin{array}{ll}
n-1 & \\
\prod_{i=0} & a_{i, i}
\end{array}\right)^{2}\right]
$$

where

$$
a_{k, k}=\frac{1}{k !} \prod_{i=0}^{k-1} \xi_{i} ; a_{0,0}=1
$$

Relations (II.64-65), together with (II.58) were used by Inönü40 to determine (recursively) the first 3 nonzero moments $m_{k}$, in terms of the Legendre expansion coefficients of the scattering kernel $f\left(\bar{\Omega} \cdot \bar{\Omega}^{-}\right)$; for monoenergetic transport. Obviously, it is difficult, if not impossible, to obtain a closed formula for $m_{k}$, in this manner.

We instead propose a new strategy that will be seen to lead to a closed form for $m_{k}$ [i.e., expressing the general term $m_{k}$ in terms of only the $g_{k}(\eta)$; see Eq. (II.77) below] and to a number of new and important results. [We also remark that Eq. (II.58), together with (II.64-65), will serve as a useful check for our new formula for $m_{k}$.] 
From the recursion relation (II.19) and initial conditions (II.16-17), we see that $\phi_{n}(\nu, n)$ can be expressed as the determinant 40 $\phi_{n}(\nu, n)=\frac{1}{n !}\left|\begin{array}{llllllll}\xi_{0} \nu & 1 & 0 & 0 & \cdot & \cdot & \cdot & \cdot \\ 1 & \xi_{1} \nu & 2 & 0 & \cdot & \cdot & \cdot & \cdot \\ 0 & 2 & \xi_{2 \nu} & 3 & \cdot & \cdot & \cdot & \cdot \\ 0 & 0 & 3 & \xi_{3 \nu} \nu & \cdot & \cdot & \cdot & \cdot \\ \cdot & \cdot & \cdot & \cdot & \cdot & \cdot & \cdot & \cdot \\ \cdot & \cdot & \cdot & \cdot & \cdot & \cdot & \cdot & n-1 \\ 0 & 0 & 0 & 0 & \cdot & \cdot & n-1 & \xi_{n-1} \nu\end{array}\right|$ for $n \geq 1$ or, expanding the determinant

$$
\begin{aligned}
\phi_{n}(v, n) & =\frac{1}{n !} \prod_{i=0}^{n-1} \xi_{n} \times \\
& \times\left[\nu^{n}-v^{n-2} \sum_{j_{1}=0}^{n-2} w_{j_{1}}+v^{n-4} \sum_{j_{1}=0}^{n-4} w_{j_{1}} \sum_{j_{2}=j_{1}+2}^{n-2} w_{j_{2}}+\ldots+\right. \\
& +(-1)^{m} v^{n-2 m} \sum_{j_{1}=0}^{n-2 m} w_{j_{1}} \sum_{j_{2}=j_{1}+2}^{n-2 m+2} w_{j_{2}} \ldots \sum_{j_{m}=j_{m-1}+2}^{n-2} w_{j_{m}}+\ldots \\
& +\left\{\begin{array}{l}
(-1)^{\frac{n}{2}} w_{0} w_{2} \ldots w_{n-2}, \text { for } n=\text { even } \\
(-1)^{\frac{n-1}{2}} \sum_{j_{1}=0}^{1} w_{j_{1}} \sum_{j_{2}=j_{1}+2}^{3} w_{j_{2}} \ldots \sum_{j_{m}=j_{m=1}+2}^{n-2} w_{j_{m}} \text {, for } n=\text { odd } \\
m=\frac{1}{2}(n-1)
\end{array}\right]
\end{aligned}
$$

with $W_{j}$ as defined in $(I I .61)$. Now, in view of the fact that 


$$
\phi_{j}(v, n)=(-1)^{i} \phi_{j}(-v, n)
$$

we can easily see from (II.36) and (II.41) that the normalization integrals are odd functions of $v$ :

$$
\begin{aligned}
& N_{j+}\left(+v_{j}, n\right)=-N_{j-}\left(-v_{j}, n\right) \\
& N(v, n)=-N(-v, n)
\end{aligned}
$$

and, as we already know, $\Lambda(v, n)=\Lambda(-v, n)$. In view of the above "parity" properties, it follows from (II.52-54) that

$$
\omega(v, n)=\omega(-v, n)
$$

i.e., the "weight distribution" is an even function of $v$. Therefore, as we anticipated [see previous footnote],

$$
m_{k}(n)= \begin{cases}0 & \text { for } k=\text { odd } \\ 2 \int_{0}^{\nu_{1}} v^{k} d \omega(v, n) \text { for } k \text { - even }\end{cases}
$$

Also, in view of the symmetry in the $v$ space [i.e., the "eigenvalue space"], the orthogonality relation (II.51) become

$$
\int_{0}^{\nu_{l}} \phi_{k} \phi_{\ell} d \omega(\nu, n)=\frac{\delta_{k, l}}{\xi_{\ell}}
$$

We now multiply Eq. (II.67) by $d \omega(\nu, n)$ and integrate it over the interval $\left(0, v_{1}\right)$. Taking into account relations (II.71) and (II.72), we obtain: 


$$
\begin{aligned}
& m_{2 p}=m_{2(p-1)} A_{1, p}-m_{2(p-2)} A_{2, p}+m_{2(p-3)} A_{3, p}+\ldots \\
& \ldots+(-1)^{k+1} m_{2(p-k)} A_{k, p}+\ldots+(-1)^{p+1} m_{0} A_{p, p} ; \text { for } p>0
\end{aligned}
$$

with

$$
m_{0}=\frac{2}{\xi_{0}}=\frac{2}{r_{0}(n)} \text { for } p=0
$$

where

$$
A_{k, r}=\sum_{j_{1}=0}^{2(r-k)} w_{j_{1}} \sum_{j_{2}=j_{1}+2}^{2(r-k+1)} w_{j_{2}} \cdots \sum_{j_{k}=j_{k-1}+2}^{2(r-1)} w_{j_{k}}
$$

By successively setting $p=1,2, \ldots n$ in (II.73), we obtain a system of $n$ linear equations, which can be solved for $m_{2 n}$ by Cramer's rule. The determinant of this set of equations is automatically unity, since all terms on the main diagonal are +1 and all terms on one side of the main diagonal are zero. Hence, $m_{2 n}$ is given by:

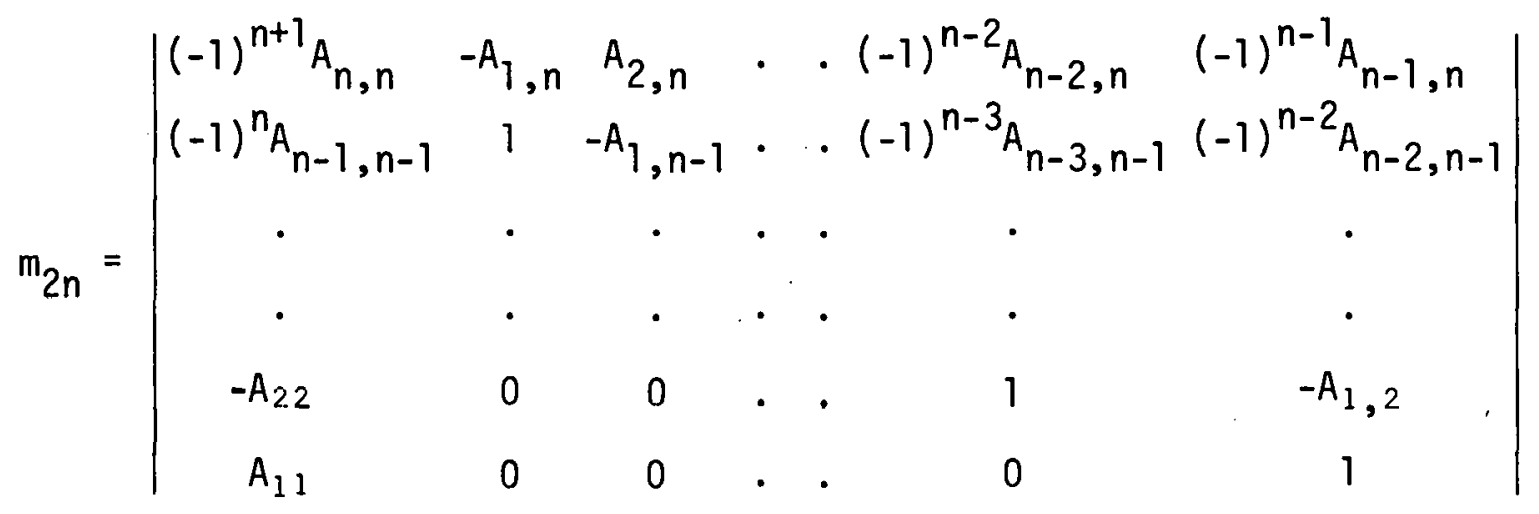


By reducing the first column to zeros, except for $A_{1,1}$, then using Laplace's theorem ${ }^{59}$ and repeating the process on the resulting determinants, Eq. (II.76) can be reduced to

$$
m_{2 n}=m_{0} \sum_{i_{0}=0}^{0} w_{i_{0}} \sum_{i_{1}=0}^{i_{0}+1} w_{i_{1}} \sum_{i_{2}=0}^{i_{1}+1} w_{i_{2}} \ldots \sum_{i_{n-1}=0}^{i_{n-2}+1} w_{i_{n-1}}
$$

where the $m_{2 n}$ 's and $W^{\prime} s$ are functions of $n$ and the nuclear mass $A$. The values for $m_{2 n}$ obtained through Eq. (II.77) satisfy identically Eq. (II.58), as can be checked by direct and tedious calculations, providing, as we have already mentioned, an immediate verification of the validity of (II.77).

Based on Eq. (II.77), we have calculated moments up to, and including $m_{18}$, using the symbolic computer language FORMAC, ${ }^{60}$ on Columbia's IBM 360/91. A listing is presented in Appendix E.

Equation (II.77) is of paramount importance insofar as it gives the dependence of $m_{2 n}(n)$ on the coefficients $g_{n}(n)$ of the Legendre expansion of the Laplace transform of the scattering kernel $f\left(\bar{\Omega} \cdot \bar{\Omega}^{\circ}, u\right)$, in a closed and structured way, facilitating the investigation of the most important characteristics of $m_{2 n}(n)$.

We shall now present some of these characteristics, in particular those pertinent to subsequent applications, such as evaluating the Green function for an infinite medium, and its spatidl moments. We also remark that these properties of $m_{2 n}(n)$ are derived from Eq. (II.77) using methods of combinatorial analysis, ${ }^{6.1}$ 
Examination of the nested sums in Eq. (II.77) indicates that the RHS of the equation can be written as a single sum (i.e., $S=\underset{k}{\sum} T_{k}$ ) of terms $T_{k}$ all of the form

$$
T_{k}=w_{j_{1}} w_{j_{2}} \ldots w_{j_{n}}
$$

where the indeces $j_{i}$ are subject to the restrictions

$$
\left.\begin{array}{l}
0 \leq j_{i} \leq i-1 \\
j_{i} \leq j_{i+1}+1
\end{array}\right\}
$$

It is obviously consistent with these restrictions for there to be terms in which an index $j_{\ell}$ can be equal to another index $j_{r}$, so that powers of various $W$ 's can occur. Indeed, all powers through $n$ can occur, e.g., $W_{0}^{n}$ is a valid term of Eq. (II.77).

In the interests of continuity of the presentation, the details of the procedure are relegated to Appendix $F$, but the following explicit formulae for the coefficients of some specific terms are given by way of illustration:

$$
\begin{aligned}
& \operatorname{coeff}\left(w_{0}^{n-k} w_{1}^{k}\right)=\frac{(n-1) !}{(n-1-k) ! k !} \\
& \operatorname{coeff}\left(w_{0}^{n-k} w_{1} w_{2} \ldots w_{k}\right)=n-k \\
& \operatorname{coeff}\left(w_{0}^{n-k} w_{1}^{k-j} w_{2} \ldots w_{j+1}\right)=\frac{(n-j-1) !}{(n-1-k) !(k-. j-1) !}
\end{aligned}
$$

[By coefficient of a term is meant the number of times the term appears in the expansion of the RHS of Eq. (II.77).] In addition, Appendix F 
presents the first 32 terms in Eq. (II.77) in order of decreasing powers of $W_{0}$, starting with $w_{0}^{n}$.

Some other properties of $m_{2 n}$ may be mentioned: Let $E_{2 n}$ be the number of terms in the fully expanded expression of $m_{2 n}(n)$ [i.e., the sum of all coefficients in Eq. (II.77)]. Then $E_{2 n}$ is given by:

$$
E_{2 n}=\sum_{i=0}^{n-1}(-1)^{n-i+1} E_{2 i} \cdot k_{n-i, 2 i+1}
$$

where $k_{i, j}$ is the $j$ 'th triangular number of $i$, i.e.,

$$
\left.\begin{array}{l}
k_{i, j}=\sum_{r=1}^{j} k_{i-1, r} \\
\text { with } k_{0, j}=1 \text { and } k_{1, j}=j
\end{array}\right\}
$$

Also, the number of distinct terms in $m_{2 n}$ is given by $2^{n-1}$. Moreover, for a particular $m_{2 n}$, the number of distinct terms that contain a particular $w_{k}^{n-r}[k=0$ to $n-1 ; r=0$ to $n]$ is given by $2^{r}$.

These properties of Eq. (II.77) enable us to recast the expansion of $m_{2 n}(n)$ in the form:

$$
m_{2 n}=m_{0} w_{0}^{n} \sum_{r=0}^{n-1} \frac{1}{w_{0}^{r}} \sum_{s=0}^{r} n^{s} B_{r, s}(n)
$$

where the coefficients $B_{r, s}(n)$ are related in an obvinus way to the coefficients of $w_{0}^{k}[k=0$ to $n]$. A listing of the coefficients $B_{r, s}(n)$ from $r, s=0$ to 5 is presented in Appendix $G$. 


\section{CHAPTER II I}

\section{THE SPATIAL MOMENTS OF THE GREEN'S FUNCTION FOR A PLANE} ISOTROPIC MONOENERGETIC SOURCE IN AN INFINITE MEDIUM

Part III.1. Introduction. The Infinite Medium Green's Function

As an explicit application of the formalism that has been developed throughout the course of Chapter II, we shall consider in this chapter some of the properties of the following "full-range" practical problem: we seek the neutron distribution $\Psi^{G}(x, \mu, u)$ [i.e., the Green's function] in space, angle and energy, due to a plane monoenergetic source $S(x, \mu, u)$ isotropically emitting neutrons with energy $E_{0}$. Without loss of generality, the source can be located at $x=0$, i.e., of the form:

$$
S(x, \mu, u)=\frac{1}{2} \delta(x) \delta(u)
$$

Following the procedure outlined in Section II.1.A up to Eq. (II.10), we find that the inhomogeneous Laplace-transformed Boltzmann Equation for our source (III.1), has the form:

$$
\begin{aligned}
\left(\mu \frac{\partial}{\partial x}+1\right) \psi^{G}(x, \mu, n) & =c \sum_{n=0}^{\infty} \frac{2 n+1}{2} g_{n}(n) P_{n}(\mu) \int_{-1}^{1} P_{n}\left(\mu^{-}\right) \psi^{G}\left(x, \mu^{-}, n\right) d \mu^{-} \\
& +\frac{1}{2} \delta(x)
\end{aligned}
$$

where

$$
\psi^{G}(x, \mu, n) \equiv \mathcal{L}\left[\psi^{G}(x, \mu, u)\right]
$$


and where all other quantities in (III.2) have the same meaning as before.

As is customary, Eq. (III.2) will be converted to a homogeneous equation by replacing the inhomogeneous part by a suitable boundary condition. ${ }^{1,11}$ This boundary condition is found by integrating (III.2) over a vanishingly small interval enclosing the origin $x=0$.

Performing this operation gives:

$$
\mu\left[\Psi^{(i}\left(0^{+}, \mu, \eta\right)-\Psi^{G}\left(0^{-}, \mu, \eta\right)\right]=\frac{1}{2}
$$

i.e., a "jump condition" at the origin. For a unique solution, we need an additional constraint specifying the behavior of the solution $\psi^{G}(x, \mu, n)$ as $x$ becomes very large. It is well known ${ }^{15}$ that for multiplying media, i.e., $c>1$, the physical interpretation of the problem is not obvious, since more neutrons are emitted per collision than are incident. We shall therefore restrict our attention to the case $c \leq 1^{*}$ for which the physical situation dictates that, at large distances from the source, the neutron flux per unit lethargy becomes vanishingly small and that therefore

$$
\lim _{|x|+\infty} \Psi^{G}(x, \mu, n)=0
$$

We are now in a position to solvc Fq. (III.2), by expanding its homogeneous part in the complete set of singular eigenfunctions

*B. C. (III.5) doesn't strictly hold for $c=1$. However, $c=1$ means, in this context, that absorption is neglected, except at very large distances $x$ from the source, such as to maintain the stationary nature of the problem. We thus employ (III.5) even for $c=1$. 
derived in Chapter II, leading to a general solution of the form (II.32).

Applying next the "growth constraint" (III.5) to the general solution prescribed in (II.32) gives

$$
\begin{gathered}
\psi^{G}(x, \mu, n)=\sum_{+j} A\left(v_{j}, n\right) \phi\left(v_{j}, \mu, n\right) e^{-x / v_{j}}+\int_{0}^{1} A(\nu, n) \phi(\nu, \mu, n) e^{-x / \nu_{d}} d v \\
\text { for } \operatorname{Re}\left[v_{j}(n)\right]>0 \text { and } x>0
\end{gathered}
$$

and

$$
\begin{gathered}
\Psi^{G}(x, \mu, n)=-\sum_{-j} A\left(-v_{j}, n\right)_{\phi}\left(-v_{j}, \mu, n\right) e^{x / v_{j}}-\int_{-1}^{0} A(v, n) \phi(v, \mu, n) e^{-x / v} d v \\
\text { for } \operatorname{Re}\left[v_{j}(n)\right]>0 \text { and } x<0
\end{gathered}
$$

where the negative signs for $x<0$ are introduced as an algebraic convenience. Only the "jump condition" (III.4) remains to be satisfied; imposing it combines Eqs. (III.6) and (III.7) in the form:

$$
\begin{gathered}
\sum_{+j} A\left(v_{j}, n\right) \phi\left(v_{j}, \mu, n\right)+\sum_{-j} A\left(-v_{j}, n\right) \phi\left(-v_{j}, \mu, n\right)+\int_{-1}^{1} A(\nu, n) \phi(\nu, \mu, n) d v \\
=\frac{1}{2 \mu}
\end{gathered}
$$

Eyuation (III.8) is a singular integral equation for the coefficients $A\left( \pm v_{j}, n\right)$ and $A(v, n)$ of prerisely the same form as was solved when the full-range completeness theorem was proved [Cf. Eq. (II.43)], i.e., Eq. (III.8) is the full-range expansion of the function 


$$
\psi(\mu)=\frac{1}{2 \mu}
$$

Thus, the expansion coefficients can be found directly by substitution of (III.9) into (II.45) and (II.46) and using the normalization condition (II.16); alternately, they can be determined using the orthogonality properties of the eigenfunctions, presented in Section II.1.B. Either way, we are led to

$$
A\left( \pm v_{j}, n\right)=\frac{1}{2 N_{j \pm}\left( \pm v_{j}, n\right)}
$$

and

$$
A(\nu, n)=\frac{1}{2 N(\nu, n)}
$$

with $N_{j \pm}$ and $N(\nu, n)$ as defined in (II.36) and (II.41), respectively. Finally, substituting the coefficients (III.10) and (III.11) into Eqs. (III.6) and (III.7) yields the solution, i.e., the angular neutron flux, as

$$
\begin{gathered}
\Psi^{G}(x, \mu, n)=\sum_{ \pm j} \frac{\phi\left( \pm v_{j}, \mu, n\right) e^{-|x| / v_{j}}}{2 N_{j \pm}\left( \pm v_{j}, n\right)}+\int_{0 \pm}^{l} \frac{\phi( \pm v, \mu, n) e^{-|x| / \nu}}{2 N( \pm v, n)} d v \\
\text { for } \operatorname{Re}\left(\nu_{j}\right)>0
\end{gathered}
$$

where the upper signs apply for $x>0$ and the lower for $x<0$. The scalar flux, i.e., the $P_{0}$ component of $\psi_{0}^{G}$, defined by:

$$
\psi_{0}^{G_{1}}(x, n) \equiv \int_{-\{}^{1} \psi^{G}(x, \mu, n) d \mu
$$


can be written down immediately from (III.12) as

$$
\begin{aligned}
& \Psi_{0}^{G}(x, n)= \sum_{j} \frac{e^{-x / v_{j}}}{2 N_{j+}\left(v_{j}, n\right)}+\int_{0}^{1} \frac{e^{-x / v}}{2 N(v, n)} d v \\
& \quad \text { for } \operatorname{Re}\left(v_{j}\right)>0 \text { and } x>0 .
\end{aligned}
$$

It is easily seen that for $x<0$, we have the relation

$$
\psi_{0}^{G}(x, n)=\psi_{0}^{G}(-x, n)
$$

from the symmetry of our problem, a fact that can also be checked by direct calculations.

Clearly, the neutron flux $\Psi_{0}^{G}(x, n)$ in (III.14) is the sum

$$
\Psi_{0}^{G}=\left(\Psi_{0}^{G}\right)_{\text {as }}+\left(\Psi_{0}^{G}\right)_{\text {nonas }}
$$

where

$$
\left(\Psi_{0}^{G}\right)_{a S} \equiv \sum_{j} \frac{e^{-x / \nu_{j}}}{2 N_{j+}}
$$

is the asymptotic flux, i.e., the predominant contribution to $\Psi_{0}^{G}$ at large distances $x$ from the source, while

$$
\left(\psi_{0}^{G}\right)_{\text {nonas }} \equiv \int_{0}^{1} \frac{e^{-x / v}}{2 N(v, n)} d \nu
$$

represents the "transient" contribution, which will be important only at very small distances, i.e., at or near the source. 
So far, the solution does not differ from that of monoenergetic transport, ${ }^{15}$ except for explicitly showing the $n$-dependence.

We have thus solved the Laplace-transformed transport equation (III.2) and obtained its solution in the form (III.12) for the angular flux, and (III.14) for the scalar flux, respectively; the solution to our original problem, i.e., lethargy-dependent slowing down and transport of neutrons due to a source $S$ of the form (III.1) will be the Laplace inverses (with respect to $n$ ) of (III.12) and (III.14), respectively.

However, before carrying out the actual inversion, we shall turn our attention to the spatial moments of the flux $\psi_{0}^{G}(x, n)$; the investigation of these moments will be seen to provide us with a deeper understanding of various properties of the quantities involved in the expression of $\Psi_{0}^{G}(x, n)$, as well as with a number of new and useful relations of practical importance.

Part III.2. The Spatial Moments $M_{k}(u)$ of the Scalar Flux

Section III.2.A. The Spatial Moments $M_{f}(n)$ and Their Relation to the "Eigenvalue Space" Moments i! $k$ (n)

Following the customary definition, ${ }^{1,6},{ }^{15}$ we introduce the spatial moments of the Laplace-transformed scalar flux $\psi_{i}^{G}(x, n)$ by

$$
M_{n}(n) \equiv \int_{-\infty}^{\infty} x^{n} \Psi_{0}^{G}(x, n) d x
$$

In view. of the symmetry of our problem, i.e., Eq. (III.15), we see that 


$$
M_{n}(n)= \begin{cases}0 & \text { for } n=2 k+1 \\ 2 \int_{0}^{\infty} x^{2 k} \Psi_{0}^{G}(x, n) d x & \text { for } n=2 k\end{cases}
$$

The integration over $x$ in (III.20) can be easily performed by using the expression (III.14) for $\psi_{0}^{G}(x, n)$, leading to

$$
M_{2 n}(n)=(2 n) !\left[\sum_{j} \frac{v_{j}^{2 n+1}}{N_{j+}\left(v_{j}, n\right)}+\int_{0}^{1} \frac{v^{2 n+1}}{N(v, n)} d v\right]
$$

since we are perfectly justified in interchanging the order of integration over $v$ and $x$ for the second term in (III.21).

It is also useful (for future purposes) to introduce the spatial moments of the asymptotic and nonasymptotic densities (III.17) and (III.18), respectively; using the traditional ${ }^{15,62}$ notations, these are defined as:

$$
\begin{aligned}
A_{2 n}(n) & \equiv \int_{0}^{\infty} x^{2 n}(\Psi G) a_{a s} d x \\
& =(2 n) ! \sum_{j} \frac{v_{j}^{2 n+1}}{N_{j+}\left(v_{j}, n\right)}
\end{aligned}
$$

and

$$
\begin{aligned}
N_{2 n}(n) & \equiv \int_{0}^{\infty} x^{2 n}\left(\psi_{0}^{G}\right)_{\text {nonas }} d x \\
& =(2 n) ! \int_{0}^{1} \frac{v^{2 n+1}}{N(v, n)} d v
\end{aligned}
$$


respectively, with (of course):

$$
M_{2 n}=A_{2 n}+N_{2 n}
$$

A simple relation exists between the spatial moments $M_{2 n}(n)$ defined by Eq. (III.19) and the "eigenvalue space" moments $m_{2 n}(n)$ defined by (II.57), as has been demonstrated by $\mathrm{Case}^{63}$ for monoenergetic transport. The proof used there depends basically on the identity of the density functions $\omega(v, n)$ appearing in the orthognnality relations for the polynomials $\phi_{n}(\nu, n)$ i.e., Eq. (II.51), and the solution representation (III.14) for the scalar flux $\psi_{0}^{G_{7}}(x, n)$. Inasmuch as $n$ appears here solely as a parameter, the derivation can be taken over without change, leading to:

$$
M_{2 n}(n)=(2 n) ! \frac{1}{2} m_{2 n}(n)
$$

with $m_{0}(n)=\frac{2}{\gamma_{0}(n)}$, as given in Eq. (II.74).

Equation (III.25) translates in a very simple fashinn the properties of the "eigenvalue space" moments $m_{2 n}(n)$-which we have extensively investigated in Part II.2 of this work-, into the properties of $M_{2 n}(n)$. Further, it then follows through Laplace inversion, that the properties of the lethargy-dependent spatial moments $M_{2 n}(u)$, of the scalar flux $\Psi_{0}^{G}(x, u)$, are in large measure dependent on those of $m_{2 n}(n)$. Indeed, as will be seen in the remainder of this work, the roots of all of our results can be traced back to the properties of these "eigenvalue space" moments. 
Section III.2.B. The Exact Analytical Expressions of the Space-Angle Integrated FTux Mo $(u)$ and the Second Spatial Moment $M_{2}(u)$

The nontrivial task of performing Laplace inversions on the various quantities of interest, lies ahead. In order to successfully accomplish it, we shall embark on a systematic search of relevant characteristics of the structure of the quantities of interest.

We shall commence with the investigation of problems associated with the Laplace inversion of the zeroth spatial moment, i.e., $M_{0}(n)$. The motivation is twofold:

(i) although the characteristics of $M_{0}(u)$ (i.e., the infinite medium spectrum) are well known, our procedure-of obtaining its analytical expression-will, hopefully, shed light on a major difficulty associated with seeking solutions to the neutron flux itself, and

(ii) furthermore, we shall not only derive an expression for $M_{0}(u)$ that is slightly more general than Placzek's $s^{64}$ and Teichmann's65by taking absorption into account, but, using the same procedure, we shall also obtain the exact expression for the second spatial moment $M_{2}(u)$-again-for slowing down with absorption. The exact expression of the well-known "Age" [which is proportional to $M_{2}(u) / M_{0}(u)$ ] can then easily be obtained from the exact expressions of $M_{2}(u)$ and $M_{0}(u)$. Historically, the infinite medium spectrum, $M_{0}(u)$, was one of the first quantities to be thoroughly investigated. Undoubtedly, the calculations were facilitated by its relatively "simple" functional dependence on lethargy and the mass of the moderator, only.

Placzek ${ }^{64}$ derived an exact analytical expression for $M_{0}(u)$, as early as 1940. Thus, by calculating the distribution of neutrons that 
have suffered 1,2 , etc., collisions, he showed that the first collision density is a discontinuous function; the second collision density has a discontinuous first derivative, and so on. In other words, close to the source energy, the infinite medium spectrum has transients which are known today as the famous "Placzek oscillations."

Placzek's expression64 for the spectrum is very involved, indeed. However, there is the well-known asymptotic behavior for large $u$ that

$$
\lim _{u \rightarrow \infty} M_{0}(u)=\frac{1}{\bar{\xi}}
$$

where

$$
\bar{\xi}=1-\frac{A-1}{2 A} \ln \frac{A+1}{A-1}
$$

is the average increase in lethargy per collision (for isotropic scattering in the C.M. system).

As is well known, slowing down in hydrogen is an exception to the above considerations. Thus, even though one might have expected the transients to extend over the widest energy range compared to any uther moderator, it is remarkable that there are no transients in hydrogen; in fact, neglecting the virgin neutrons,

$$
\left.M_{0}(u)\right|_{A=1}=1
$$

throughout the energy range.

After these preliminary considerations, we shall proceed with the Laplace inversion of $M_{0}(n)$. Equation (III.25) says that $M_{0}(n)$ is the 
reciprocal of $\gamma_{0}(n)$. Thus, using the definition of $\gamma_{0}(\eta)$ [Eq. (II.21)] and the explicit form of $g_{0}(n)$ from Appendix $A$, we have

$$
M_{0}(n)=\frac{1}{1-\frac{c \alpha}{n+1}\left[1-e^{-\varepsilon(n+1)}\right]}
$$

Eq. (III.29) can be rewritten as

$$
M_{0}(n)=\left(1+\frac{c u}{n+1-c \alpha}\right) \frac{1}{1+\frac{c \alpha \exp [-\varepsilon(n+1)]}{n+1-c \alpha}}
$$

But

$$
\left[1+\frac{c \alpha e^{-\varepsilon(n+1)}}{n+1-c \alpha}\right]^{-1}=1+\sum_{k=1}^{\infty}(-1)^{k} \frac{(c \alpha)^{k} e^{-k \varepsilon(n+1)}}{(n+1-c \alpha)^{k}}
$$

Substituting (III.31) into (III.30) and carrying out the multiplications, we obtain:

$$
\begin{aligned}
M_{0}(n) & =1+\frac{c \alpha}{n+1-c \alpha}+\sum_{k=1}^{\infty}(-1)^{k} \frac{(c \alpha)^{k} e^{-k(n+1) \varepsilon}}{(n+1-c \alpha)^{k}}+ \\
& +\sum_{k=1}^{\infty}(-1)^{k}(c \alpha)^{k+1} \frac{e^{-k(n+1) \varepsilon}}{(n+1-c \alpha)^{k+1}}
\end{aligned}
$$

Using the methods of distribution theory and transform analysis, ${ }^{54,66}$ the Laplace inversion of (III.32) can be easily performed, leading to:

$$
\begin{aligned}
M_{n}(u) & =\delta(u)+c \alpha e^{(c \alpha-1) u} 1_{+}(u) \\
& +\sum_{k=1}^{\infty}(-1)^{k}(c \alpha)^{k} e^{-k \varepsilon}(u-k \varepsilon)^{k-1} \frac{e^{-k \varepsilon(c \alpha-1)}}{(k-1) !} e^{(c \alpha-1) u} 1_{+}(u-k \varepsilon)
\end{aligned}
$$




$$
+\sum_{k=1}^{\infty}(-1)^{k}\left(c_{\alpha}\right)^{k+1} e^{-k \varepsilon}(u-k \varepsilon)^{k} \frac{e^{-k \varepsilon(c \alpha-1)}}{k !} e^{(c \alpha-1) u} 1_{+}(u-k \varepsilon)
$$

In (III.33), we employed the customary definition for the pseudofunction $1_{+}(u)$, i.e.,

$$
I_{+}(u)=\left\{\begin{array}{l}
1 \text { for } u>0 \\
\frac{1}{2} \text { for } u=0 \\
0 \text { for } u<0
\end{array}\right.
$$

It is therefore clear that the summations in (III.33) terminate, the upper limit on $k$ being the closest integer less than $u / \varepsilon$, rather than infinity. Equation (III.33) can be simplified and, after a little algebra, we obtain

$$
\begin{aligned}
& M_{0}(u)=\delta(u)+c \alpha e^{(c \alpha-1) u}\left\{\sum_{k=0}^{\frac{u}{\varepsilon}} \frac{\left(c \alpha \varepsilon e^{-c \alpha \varepsilon}\right)^{k}}{k !}\left(k-\frac{u}{\varepsilon}\right)^{k} 1_{+}(u-k \varepsilon)\right. \\
& \left.-e^{-c \alpha \varepsilon} \sum_{k=0}^{\frac{u}{\varepsilon}-1} \frac{\left(c \alpha \varepsilon e^{-c \alpha \varepsilon}\right)^{k}}{k !}\left[k-\left(\frac{u}{\varepsilon}-1\right)\right]^{k} 1_{+}[u-(k+1) \varepsilon]\right\}
\end{aligned}
$$

where $k$ goes through the closest integer less than the given upper limits.

Equation (III.35) is the exact expression for the space-angle integrated neutron flux, valid over the entire lethargy range, for arbitrary absorption. An expression having a form similar to (III.35) was obtained by Teichmann 65 -for the neutron infinite-medium spectrum, 
but for a nonabsorbing medium; his approach, however, had a different and more limited scope than ours.

It can readily be shown that (III.35) takes on the asymptotic value $1 / \bar{\xi}$, in the limit of large lethargies, and no absorption. To accomplish this, we shall make use of some well-known results obtained by Polya and Szego ${ }^{67}$ [Ref. 67, Vol. I, ex. 207-215].

Let

$$
S=\sum_{k=0}^{\infty} \frac{w^{k}}{k !}(k-\beta)^{k}
$$

Then, the radius of convergence of (III.36) is $w<1 / e$ and, employing the generalized Lagrange's expansion, ${ }^{67-70}$ one obtains:

$$
S=\frac{e^{\beta z}}{1-z}
$$

where $z$ is the solution of

$$
z e^{-z}=w
$$

which vanishes for $w=0$.

Suppose, with Eq. (III.35) in mind, we set

$$
w=\alpha \varepsilon \mathrm{e}^{-\alpha \varepsilon}
$$

Then, Eq. (III.38) has two solutions $z_{1}$ and $z_{2}$ which tend to zero and infinity, respectively, when $w$ tends to zero. After some algebraic manipulations, we find that

$$
z_{1}=\varepsilon(\alpha-1)
$$

and 


$$
z_{2}=\alpha \varepsilon
$$

Clearly, the solution of (III.38) which vanishes for $w=0$ is $z_{1}$, as given in (III.40). Therefore, using Eqs. (III.36-40) in (III.35), we obtain in the limit as $u \rightarrow \infty$, that

$$
\begin{aligned}
\lim _{u \rightarrow \infty} M_{0}(u) & =\lim _{u \rightarrow \infty} \alpha e^{(\alpha-1) u}\left[\frac{e^{-\varepsilon(\alpha-1) \frac{u}{\varepsilon}}}{1+\log e^{-\varepsilon(\alpha-1)}}-\frac{e^{-\alpha \varepsilon} e^{-\varepsilon(\alpha-1)\left(\frac{u}{\varepsilon}-1\right)}}{1+\log e^{-\varepsilon(\alpha-1)}}\right] \\
& =\frac{1}{1-\frac{(A-1)^{2}}{4 A} \varepsilon}=\frac{1}{\overline{\bar{\xi}}}
\end{aligned}
$$

as expected.

For hydrogen, the summations within the brackets of (III.35) reduce to $1_{+}(u)$ and therefore

$$
\left.M_{0}(u)\right|_{A=1}=\delta(u)+c e^{(c-1) u} 1_{+}(u)
$$

as could have been obtained by the direct inversion of $\left.M_{0}(n)\right|_{\Lambda=1}$.

We have also applied our procedure to nht.ain the exact analytical expression for the second moment, i.e., for $M_{2}(u)=\mathscr{L}^{-1}\left[M_{2}(n)\right]$, for the case of slowing-down with capture. Since the derivations are considerably more involved, we quote here only the final result for $M_{2}(u)$; the detailed proofs and considerations can be found in Appendix $H$. We have obtained:

$$
M_{2}(u)=\frac{1}{3}\left[\delta(u)+A(u)+B(u)+\int_{0}^{u} A(\tau) B(u-\tau) d \tau\right]
$$

where: 
59

$$
\begin{aligned}
A(u) & =\beta e^{(\beta-1) u}\left\{\sum_{k=0}^{\frac{u}{\varepsilon}}[(2-\beta \varepsilon) k+2+\beta u] \times\right. \\
& \times\left(\beta \varepsilon e^{-\beta \varepsilon}\right)^{k \frac{\left(k-\frac{u}{\varepsilon}\right)^{k}}{k !} 1_{+}(u-k \varepsilon)-} \\
& -e^{-\beta \varepsilon} \sum_{k=0}^{\frac{u}{\varepsilon}-1}(k+2)\left(\beta \varepsilon e^{-\beta \varepsilon}\right)^{\left.k \frac{\left[k-\left(\frac{u}{\varepsilon}-1\right)\right]^{k}}{k !} 1_{+}[u-(k+1) \varepsilon]\right\}}
\end{aligned}
$$

( I I I.45)

and

$$
\begin{aligned}
& B(u)=I_{+}(u) B e^{\left(\frac{B}{2}-1\right) u}\left[\cosh \frac{u}{2} \sqrt{\beta^{2}-2 A B+1}-\right. \\
& \left.-\frac{A-B}{\sqrt{B^{2}-2 \beta A+1}} \sinh \frac{u}{2} \sqrt{B^{2}-2 \beta A+1}\right]+ \\
& +1_{+}(u-\varepsilon) \beta e^{-\frac{\beta \varepsilon}{2}} e^{\left(\frac{\beta}{2}-1\right) u}\left[\cosh \frac{u-\varepsilon}{2} \sqrt{\beta^{2}-2 \beta A+1}+\right. \\
& +\frac{A+\beta}{\sqrt{\beta^{2}-2 \beta A+1}} \sinh \frac{u-\varepsilon}{2} \sqrt{\beta^{2}-2 \beta A+1}+ \\
& +\frac{\beta\left(2 \beta^{2}-2 \beta A+1-A^{2}\right)}{\beta^{2}-2 \beta A+1} \frac{U-\varepsilon}{2} \cosh \frac{u-\varepsilon}{2} \sqrt{\beta^{2}-2 \beta A+1}+ \\
& +\frac{2 \beta^{2}}{\sqrt{B^{2}-2 B A+1}} \frac{U-\varepsilon}{2} \sinh \frac{U-\varepsilon}{2} \sqrt{B^{2}-2 B A+T}+ \\
& \left.+\frac{\beta\left(A^{2}-2 \beta A+1\right)}{\left[\beta^{2}-2 \beta A+1\right]^{3 / 2}} \sinh \frac{u-\varepsilon}{2} \cdot \sqrt{\beta^{2}-2 \beta A+T}\right] \mp
\end{aligned}
$$

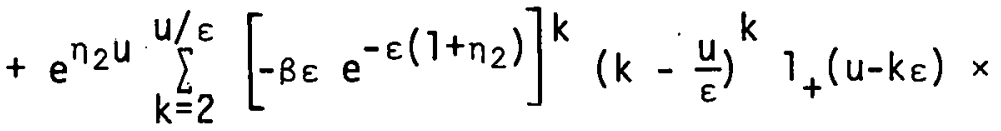

$$
\begin{aligned}
& \times \sum_{m=0}^{k}\left(\begin{array}{l}
k \\
m
\end{array}\right)\left(n_{3}-n_{2}\right)^{m} \frac{\varepsilon^{m}\left(k-\frac{u}{\varepsilon}\right)^{m}}{\Gamma(m+k)}\left\{\frac{\beta\left(n_{1}+1-\frac{A}{2}\right)}{(m+k)(m+k+1)}\left(u-k_{\varepsilon}\right) \times\right.
\end{aligned}
$$




$$
\begin{aligned}
\times{ }_{1} F_{1}\left[k+2 ; m+k+2 ;\left(n_{2}-n_{1}\right)(u-k \varepsilon)\right]+\left(\frac{\beta}{m+k}+\frac{n_{1}-n_{2}}{m+k+1}\right) \times \\
\times{ }_{1} F_{1}\left[k ; m+k+1 ;\left(n_{2}-n_{1}\right)(u-k \varepsilon)\right]+. \\
+\frac{1}{u-k \varepsilon}{ }_{1} F_{1}\left[k-1 ; m+k ;\left(n_{2}-n_{1}\right)(u-k \varepsilon)\right]
\end{aligned}
$$

In (III.45) and (III.46), we have used the following definitions:

$$
\begin{gathered}
B \equiv \mathrm{c} \alpha ; \\
n_{1} \equiv \frac{1}{2}\left[\beta-2+\sqrt{\beta^{2}-2 B A+1}\right] ; \\
n_{2} \equiv \frac{1}{2}\left[\beta-2-\sqrt{\beta^{2}-2 \beta A+1}\right] ; \eta_{3}=-\left(\frac{A}{2}+1\right) ;
\end{gathered}
$$

and ${ }_{1} F_{1}(a ; c ; z)$ is the well-known degenerate hypergeometric function It is hopefully clear by now that the major mathematical difficulty introduced by the consideration of slowing-down in moderators of mass $A>1$, is caused by the fact that the functions $\gamma_{n}(n)$ are only entire functions of $n$, as opposed to the case of hydrogen, where they are rational in $n$. (Obviously, the roots of this problem lie with the forms of the scattering kernel, which differ according to whether $A>1$ or $A=1$.

Now, when seeking solutions (to problems of slowing down with or without transport) valid for small u (i.e., in a region close to the source), one must investigate, according to well-known Tauberian. Theorems, 51-54 the behavior of the functions for large $n$, in the $n$-space. But for large $n$, terms proportional to $e^{-\varepsilon n}$ are, evidently, exponentially small compared to terms of order $1 / n$. Thus, for any $A=1$, one is raced with the question of how to treat these exponentially small terms-when seeking solutions to the neutron flux 
or its spatial moments-, valid for small values of $u$. This is an important point, and it is our hope that we succeeded to bring it into evidence during the course of our investigation of $M_{0}(u)$ and $M_{2}(u)$. For it is clear that by neglecting these $0\left(e^{-\varepsilon \eta}\right)$ terms, one obtains not only a nonuniform "solution" - in $u$ and $A-$, but one whose validity is severely limited to the narrow range $u \in[0, \varepsilon]$.

The approach described here thus makes it possible to derive the exact analytical form of $M_{2}(u)$. But it becomes increasingly difficult to carry the same method further so as to obtain the exact analytical expressions of higher order moments. In fact,-while possible in principle-, it seems extremely improbable that one could derive an exact analytical expression in closed form for the general term $M_{2 n}(u)$.

We believe, however, that our method can be used to obtain the exact analytical expression of $M_{2 n}(u)$ valid for lethargies not larger than the first few $\varepsilon$-intervals, e.g., for $u \in[0, \varepsilon]$, or $u \in[0,2 \varepsilon]$, etc . This could be done by truncating the infinite sums of terms containing $e^{-k \varepsilon n}$, and retaining only the first few of them, e.g., $k=0$, or $k=0$ and 1 , etc. It is our intention to investigate this possibility in the near future.

For the time being, however, we have chosen rather to investigate the behavior of $M_{2 n}(u)$, in the limit of large lethargies $u$, since this region of the phàse space is of considerably more interest for immediate practical applications. This investigation forms the subject of our next section. 
Section III.2.C. The Laplace Inversion of $M_{2 n}(n)$. Form of the Lethargy-Dependent Spatial Moments $\underline{M}_{2 n}(u)$ for $c=1$

The lethargy dependent spatial moments of the scalar flux $\psi_{0}^{G}(x, u)$ are given by:

$$
M_{2 n}(u)=\mathcal{L}^{-1}\left[M_{2 n}(n)\right]
$$

where $\mathcal{L}^{-1}$ denotes the Inverse Laplace transform wilh respect to $n$. In view of (III.25), Eq. (III.47) becomes

$$
M_{2 n}(u)=(2 n) ! \mathcal{L}^{-1}\left[\frac{1}{2} m_{2 n}(n)\right]
$$

where $m_{2 n}(\eta)$ is given by Eq. (II.77) or, alternatively, by Eq. (II.85). In order to carry out the Laplace inversion in (III.48) by integration around the appropriate Bromwich contour, we first need to determine the relevant poles of $m_{2 n}(n)$, as well as the behavior of $m_{2 n}(n)$ in the limit as $n \rightarrow \infty$.

It is readily seen from (II.77) that the poles of $m_{2 n}(n)$ occur at the zeros of $\gamma_{j}(n)(i=0$ to $n)$; moreover, the order of these poles can also be readily deduced from the structure of Eq. (II.77), as follows:

By simple inspection of (II.77), or from Eq. (II.85), it can be seen that the highest power $k$ of a $W_{i}(0 \leq i \leq n-1)$ that can occur in (II.77) [i.e., in the expression of $m_{2 n}(\eta)$ ] is such that, for $w_{j}^{k}$, we have

$$
i+k=n ; \quad 0 \leq i \leq n-1
$$


Therefore, if we were to use the defining form (II.61) for the $W_{i}$ 's in (II.77), and then rewrite (II.77) in a fractional form with a common denominator, the expression of this denominator would be of the form:

$$
\gamma_{0}^{n+1} \gamma_{1}^{n} \gamma_{2}^{n-1} \ldots \gamma_{i}^{n-i+1} \ldots \gamma_{n}
$$

Thus, we see that the poles due to the zeros of any $\gamma_{j}$ will be of $\operatorname{order}(n-i+1)$, where $0 \leq i \leq n$.

Next, we determine the behavior of $m_{2 n}(n)$ in the limit as $n \rightarrow \infty$. This can be done in several ways, among which the most direct is as follows:

In view of the behavior of $g_{n}(n)$ for large $n$ [i.e., $\lim _{n \rightarrow \infty} g_{n}(n)=0$, cf. Appendix A, Eq. (A.13)] and the definition (II.21) for $\gamma_{n}$, we have:

$$
\begin{aligned}
& \lim _{n \rightarrow \infty} \gamma_{n}(n)=1 \\
& \text { Thus, from }(\text { I I. 20), we have } \\
& \lim _{n \rightarrow \infty} \xi_{n}(n)=\lim _{n \rightarrow \infty}(2 n+1) \gamma_{n}(n)=(2 n+1)
\end{aligned}
$$

We thus immediately see that, in the limit as $n \rightarrow \infty$, the recursion relation (II.19) for the polynomials $\phi_{n}(\nu, n)$ takes the form

$$
(2 n+1) \cup \lim _{n \rightarrow \infty} \phi_{n}(\nu, n)=(n+1) \lim _{n \rightarrow \infty} \phi_{n+1}(\nu, n)+n \lim _{n \rightarrow \infty} \phi_{n-1}(v, n)
$$

i.e., becomes of the same form as the recursion relation (II.18) satisfied by the Legendre polynomials $P_{n}(v)$. Moreover, since 


$$
\phi_{0}(\nu, n)=1 \quad \text { (cf. II.23) }
$$

and

$$
\lim _{n \rightarrow \infty} \phi_{1}(\nu, n)=\lim _{n \rightarrow \infty} r_{0}(n) \nu=v
$$

we conclude that

$$
\lim _{n \rightarrow \infty} \phi_{n}(v, n)=P_{n}(v)
$$

[Note that Eqs. (III.51-54) hold true irrespective of the value of c.] Therefore, as $n \rightarrow \infty$, the orthogonality relations (II.5I) for the $\phi_{n}(\nu, n)$ must become identical to the orthogonality relations satisfied by the Legendre polynomials $P_{n}(v)$. Thus

$$
\lim _{n \rightarrow \infty} d \omega(v, n)=d v
$$

and

$$
\lim _{n \rightarrow \infty} v_{1}(\nu)=1
$$

Finally, from Eq. (II.7I) and Eqs. (III.55-56), we see that

$$
\lim _{n \rightarrow \infty} \frac{1}{2} m_{2 n}(n)=\lim _{n \rightarrow \infty} \int_{0}^{v_{1}(n)} v^{2 n_{d}}(v, n)=\frac{1}{2 n+1}
$$

Incidentally, relation (III.57) can also be obtained by taking the limit as $n \rightarrow \infty$ in the relation

$$
\frac{1}{2} m_{2 n}(n)=\sum_{j} \frac{v_{j}^{2 n+1}}{N_{j+}\left(v_{j}, n\right)}+\int_{0}^{1} \frac{v^{2 n+1}}{N(v, n)} d v
$$


[Relation (III.58), above, follows immediately from Eqs. (III.25) and (III.21):] Thus, the obvious interpretation of (III.57) is that, at $\eta=\infty$, we have no contribution from the "asymptotic" moments $A_{2 n}(\eta)$ [cf. Eq. (III.22)], but only-and entirely-from the "nonasymptotic" moments $\mathrm{N}_{2 n}(n)$ [cf. Eq. (III.23)]. We can translate the above discussion in terms of lethargy: mathematically, Tauberian Theorems $51-54$ dictate that the behavior of any Laplace-transformed function [in our case $\left.m_{2 n}(n)\right]$ as $n \rightarrow \infty$, is determined by the behavior of the original function as $u \rightarrow 0$ (i.e., at or close to the source lethargy), and vice versa. A direct Laplace inversion of (III.57) and use of (III.48), leads to:

$$
M_{2 n}(u) \stackrel{u \rightarrow 0}{\longrightarrow} \frac{(2 n) !}{2 n+1} \delta(u)
$$

as the limiting form for the spatial moments at source lethargy. But it is well known that, at source lethargy, the only contribution comes from the unscattered flux, of the form:

$$
\psi_{0}^{G}(x, u)=\frac{1}{2} E_{1}(x) \delta(u), \text { at } u=0
$$

with spatial moment.s identical to (III.59).

With the preliminary considerations we can proceed with the Laplace inversion for the spatial moments $M_{2 n}(n)$, i.e., we can solve (III.48).

Separating the contribution proportional to $\delta(u)$ we obtain

$$
M_{2 n}(u)=\frac{(2 n) !}{2 n+1} \delta(u)+(2 n) ! \mathcal{L}^{-1}\left[\frac{1}{2} m_{2 n}(n)-\frac{1}{2 n+7}\right] .
$$


The inversion in (III.60) can now be carried out by integration around the appropriate Bromwich contour enclosing all the poles of the integrand.

For simplicity, the ensuing derivations will be carried out for the special case of no absorption, i.e., $c=1$. The appropriate modifications for the case of scattering with absorption, i.e., $c \neq 1$ will be discussed in the concluding paragraphs of this section.

Thus, when $c=1$, the pole with the largest real part of $m_{2 n}(11)$ is at $n=0$, since $n=0$ is the zero with the largest real part of $r_{0}(n)$, as can be easily seen from (II.21). Moreover, all the other poles of $\dot{m}_{2 n}(n)$ have negative real parts less than or, at most, equal. to $\left(-\frac{1}{2}\right)$, since all the other zeros of the functions $\gamma_{j}(n)$ have been shown by Wick $^{4}$ to have negative real parts less than, or at most equal to, $\left(-\frac{1}{2}\right)$. Furthermore, since

$$
\lim _{n \rightarrow \infty}\left[\frac{1}{2} m_{2 n}(n)-\frac{1}{2 n+1}\right]=0
$$

Jordan's Lemma ${ }^{51-54}$ ensures no contribution from the integral around the arc as its radius tends to infinity. The only contributions will therefore come from the residues at the poles of $m_{2 n}(n)$; [obviousiy there will be no contributions from the constant part $\frac{1}{2 n+7}$ ]. Equation (III.60) thus becomes:

$$
M_{2 n}(u)=(2 n) !\left[\frac{\delta(u)}{2 n+1}+\sum_{\text {all poles }} \operatorname{Res}\left\{\frac{1}{2} m_{2 n}(n) e^{n u}\right\}_{\text {poles }}\right]
$$

Equation (III.62) represents the exact expression for $M_{2 n}(u)$; it is, however, of little practical value for computational purposes, since 
it requires knowledge of the exact locations of all the zeros of the entire transcendental functions $\gamma_{j}(n), i=0$ to $n$.

However, Tauberian theorems $51-54$ ensure that the asymptotic behavior of $\mathrm{M}_{2 \mathrm{n}}(u)$ for large lethargies is dictated by the contribution of the pole with the largest real part which, in our case, is located at $n=0$. Indeed, the contributions from all the other poles will be exponentially small [i.e., will vanish at least as fast as $\mathrm{e}^{-0.5 u}$ ] compared to the contributions arising from the pole at $n=0$.

The analysis that we now begin is devoted to determining the contribution arising from the "main pole," i.e., at $n=0$. The resulting expression for $M_{2 n}(u)$ will be valid for large lethargies and actually corresponds to a "generalized age-theory" formula, in the sense that the zeroth approximation will correspond to the well-known "age theory" expression for the spatial moments.

As we already know, [cf. Eq. (III.50) et seq.] $n=0$ is a pole of order $(n+1)$ of $m_{2 n}(n)$. Therefore the residue appearing in the rightmost member of Eq. (III.62), evaluated at $n=0$, is

$$
\operatorname{Res}=\frac{1}{n !} \frac{d^{n}}{d_{n}^{n}}\left[n^{n+1} e^{n u} \frac{1}{2} \cdot m_{2 n}(n)\right]_{n=0} \text {. }
$$

We expand Eq. (II.85) in a power series of $n$. The leading term in this expansion of $m_{2 n}(n)$ is of order $n^{-(n+1)}$, which cancels the corresponding factor in Eq. (III.63), so that the equation can now be written as:

$$
\text { Res }=\frac{1}{n !} \frac{1}{\gamma_{0}^{\prime}(0)}\left[\frac{1}{3 \gamma_{0}(0) \gamma_{1}(0)}\right]^{n} \frac{d^{n}}{d_{n}^{n}}\left[e^{n u_{h}(n)}\right]_{n=0}
$$

where 


$$
h(n) \equiv\left(\sum_{r=0}^{n-1} \frac{1}{w_{0}^{r}} \cdots \sum_{s=0}^{r} n^{s} B_{r, s}\right) /\left[\left(\sum_{k=0}^{\infty} a_{k} k^{k}\right)^{n+1}\left(\sum_{k=0}^{\infty} b_{k^{n}} k^{k}\right)\right]
$$

with

$$
a_{0}=1 ; a_{k}=\frac{1}{r_{0}(0)} \frac{1}{(k+1) !}\left[\frac{d^{k+1}}{d n^{k+1}} r_{0}(n)\right]_{n=0} \text { for } k=1,2, \ldots
$$

and

$$
b_{0}=1 ; b_{k}=\frac{1}{\gamma_{1}(0)} \frac{1}{k !}\left[\frac{d^{k}}{d n^{k}} \gamma_{1}(n)\right]_{n=0} \text { for } k=1,2, \ldots
$$

Next, applying Leibnitz' differentiation formula to Eq. (III.64), we obtain

$$
\text { Res }=\frac{1}{\gamma_{0}^{\prime}(0)} \frac{\tau^{n}}{n !} \sum_{m=0}^{n}\left(\begin{array}{l}
n \\
m
\end{array}\right) \frac{1}{u^{m}} h_{(0)}^{(m)}
$$

where

$$
\tau \equiv \frac{u}{3 \gamma_{0}(0)_{\gamma_{1}}(0)}
$$

and

$$
h_{(n)}^{(m)}=\left[\frac{d^{m}}{d 1^{m}} h(n)\right]_{n=0}
$$

In the following, the function $h_{(0)}^{(m)}$ appearing in (III.70) will be cast into a form suitable for practical applications. Specifically, we will show that 


$$
h_{(0)}^{(m)}=m ! C_{m}(n)=m ! \sum_{j=0}^{m} C_{m, j} n^{j}
$$

The coefficients $C_{m, j}$ in (III.71) are functions of the nuclear mass only. The values of $C_{m, j}$ can be obtained through a recursion relation which can be derived by the following sequence of steps:

(i) The numerator in (III.65) is expanded in an infinite power series in $n$, around $\eta=0$. The coefficient of $\eta^{m}$ of this power series is seen to be a polynomial in $n$ with coefficients $t_{m, j}$. The explicit form of the $t_{m, j}$, after invoking Leibmitz' differentiation rule and after some algebraic manipulations (the details of which are relegated to Appendix I], is found to be

$$
\begin{aligned}
t_{m, j}= & \frac{1}{m !} \sum_{s=j}^{m} \sum_{r=j}^{s}\left(\begin{array}{l}
m \\
s
\end{array}\right)\left[\left(\frac{1}{w_{0}^{r}}\right)^{(s)} B_{r, j}(m-s)\right]_{n=0} \\
& \text { for } m, j=0,1,2, \ldots \text { and } j \leq m \\
& \text { with } t_{0,0}=1
\end{aligned}
$$

It will be recalled that $W_{0}$ is defined in Eq. (II.61) and that the quantities $B_{r, j}$ are defined by Eq. (II.85) and examples of $B_{r, j}$ are listed in Appendix $G$. We also mention that we shall present in Appendix I [cf. Eqs. (I.6-9)] some noteworthy expressions for $t_{m, j}$, for a few selected values of the index $j$.

(ii) The same procedure is used on the denominator: a single power series in $n$ (around $n=0$ ) is formed from the two factors in the denominator. The coefficients of this power series are again polynomials in $n$ with coefficients 


$$
\left.\begin{array}{rl}
Z_{m \cdot j}= & \sum_{k=0}^{m} \sum_{s=\max }^{s=\min } \begin{array}{c}
(j, m-k) \\
(0, j-k) \\
X_{k, j-s}
\end{array} Y_{m-k, s} \\
& \text { for } m, j=0,1,2, \ldots \text { and } j \leq m \\
& \text { with } z_{0,0}=1
\end{array}\right\}
$$

Here the $x_{m, j}$ 's in Eq. (III.73) come from the first factor in the denominator of (III.65) and obey the recursion relation

$$
\left.\begin{array}{rl}
x_{m, j}= & \frac{1}{m} \sum_{k=1}^{m} a_{k}\left[k x_{m-k, j-1}+(2 k-m) x_{m-k, j}\right] \\
& \text { for } m, j=0,1,2 \ldots \text { and } j \leq m \\
& \text { with } x_{0,0}=1
\end{array}\right\}
$$

Similarly, the quantities $Y_{m, j}$ that appear in (III.73) come from an expansion of the second term of the denominator of (III.65) and obey the recursion relation

$$
\left.\begin{array}{rl}
Y_{m, j}= & \frac{1}{m} \sum_{k=1}^{m} b_{k}\left[k Y_{m-k, j-1}+(k-m) Y_{m-k, j}\right] \\
& \text { for } m, j=0,1 \ldots \text { and } j \leq m \\
& \text { with } Y_{0,0}=1
\end{array}\right\}
$$

[Note: the detailed calculations leading to Eqs. (III.73-75) were again relegated to Appendix I, in order to facilitate a continunus presentation of the main steps of the derivation.]

(iii) In consequence of the manipulations described in the previous two steps, $h(n)$ in Eq. (III.65) can be written as the ratio 
of two power series in $n$. Evaluating the $m^{\text {th }}$ derivative of $h(n)$ with respect to $n$ at $n=0$ we are led [after some algebraic manipulations that have been once again relegated to Appendix I, cf. Eqs. I.27 et seq.] to the polynomial form of $h(m)$ given in Eq. (III.71), with the coefficients $C_{m, j}$ determined by a recursion relation of the form

$$
\left.\begin{array}{c}
c_{m, j}=t_{m, j}-\sum_{k=1}^{m} \sum_{s=m a x}^{s=m i n(m-k, j)}(0, j-k) \\
\quad c_{m-k, s} z_{k, j-s} \\
\text { for } m, j=0,1,2 \ldots \text { where } j \leq m \\
\text { with } c_{0,0}=1 \text { and } c_{m, j}=0 \text { if } m<j
\end{array}\right\}
$$

Equation (III.76), in combination with Eqs. (III.72-75), has been programmed in FORMAC 60 for use on the IBM 360/91. We have by this means obtained algebraic expressions for the coefficients $C_{m, j}$ as functions of the nuclear mass $A$, from $m, j=0$ to $m, j=5$. A full listing of the results is presented in Appendix $\mathrm{J}$.

Substituting now Eq. (III.71) into (III.68), we can write

$$
\text { Res }=\frac{1}{r_{0}^{j}(0)} \frac{\tau^{n}}{n !} \sum_{m=0}^{n} \frac{n !}{(n-m) !} \frac{1}{u^{m}} \sum_{j=0}^{m} n^{j} c_{m, j}
$$

and, by Eq. (III.60), we find the expression for the spatial moments of the scalar flux, valid for large lethargies, as

$$
M_{2 n}(u)=(2 n) ! \frac{1}{\gamma_{0}^{-}(0)} \frac{\tau^{n}}{n !} \sum_{m=0}^{n} \frac{n !}{(n-m) !} \frac{1}{u^{m}} \sum_{j=0}^{m} n^{j} C_{m, j}
$$

The form of the lethargy-dependent spatial moments of the scalar flux, $M_{2 n}(u)$ given in Eq. (III.78) is similar to that of the results 
obtained by Placzek ${ }^{6}$ and our $C_{m, j}$ correspond to his $A_{m, j}$. Placzek explicitly calculated $A_{0,0}, A_{1,0}$ and $A_{1,1}$ and also obtained the special relation

$$
A_{m, m}=\frac{1}{m !}\left(A_{1,1}\right)^{m}
$$

As expected, our values agree with his for the above three coefficients and Eq. (III.76) can be simplified for the particular case $j=m$ to recover Placzek's expression (III.79) [cf. Appendix I, Eq. (I.32)].

Huang ${ }^{71}$ has used Placzek's method to extend the results ${ }^{6}$ to correction terms of the order of $u^{-2}$ for scatterers heavier than hydrogen.

It would be very difficult to carry Placzek's method ${ }^{6}$ beyond correction terms in $\mathrm{u}^{-2}$. On the other hand, the technique described here can be used in a straightforward, albeit lengthy, procedure to go to any desired order of correction. It does not seem likely, however, that significant useful information could be obtained from corrections beyond $u^{-5}$ and therefore only coefficients through this order were explicitly obtained. It may be noted that the computer time on the IBM $360 / 91$ needed to calculate the coefficients presented here wàs about seven minutes.

In principle, our prosedure for evaluating the lilust important conLribution to $M_{2 n}(u)\left(1 . e_{0}\right.$, that due to the pole at $\left.n=0\right)$ can also be used to calculate the contributions from the remaining poles of $m_{2 n}(n)$, once the locations of these poles are determined. We would just need to determine the other roots of the transcendental functions 
$\gamma_{j}(n)(i=0$ to $n)$. While $i t$ seems improbable to obtain the exact expressions of these roots as a function of the mass of the scatterer, it is, nevertheless, possible to obtain approximations to these roots which would then be used, in conjunction with our method, to calculate the corresponding contributing residue to $\mathrm{M}_{2 n}(u)$. It is likely that this same idea could be employed if absorption were present. Thus, it seems possible to determine the zero of largest absolute value of $\gamma_{0}(n)$ (at least for cases such that $|c-1|<<1$ or $c<1$ ) by using Lagrange's Expansion. 68 Then, one could calculate the residue of $M_{2 n}(n)$ at this pole using the methods outlined in this section and therefore obtain the asymptotic form of $M_{2 n}(u)$ valid for intermediate to large lethargies even when absorption is present. Such calculations, as well as an investigation of the spatial moments for realistic anisotropies of the basic scattering process, remain, however, for the future.

The results for $M_{2 n}(u)$, Eq. (III.78) and Appendix J, can be used to explore analytically and numerically the range of validity in $n-u$ space of the simple age-theory. Useful as these conclusions might be, it would be even more desirable to analyze the convergence to age theory in $x-u$ phase space. Considerable progress in this direction has already been achieved and will be reported by the author elsewhere. 


\section{CHAPTER IV}

\section{CONCLUSIONS AND DIRECTIONS OF FURTHER RESEARCH}

The specific new properties of the constant-cross-section problem presented in the previous pages have concentrated on the spatial moments of the scalar flux. In the region of large lethargy, explicit expressions have been obtained for the spatial moments which correct the "age-theory" limit through terms of the order $u^{-5}$. These expressions, and the derivations leading to them, have recently been published by the author. 80 In addition, an exact expression for the second moment, $M_{2}(u)$, has been derived. Combined with the previously known exact expression for the zeroth moment, it is now possible to calculate analytically and rigorously the "age" for the constant-crosssection problem for arbitrary (constant) absorption and nuclear mass.

It is hoped that these results can contribute towards increasing our ability to do practical neutron transport calculations in two ways. First, the analytical results for the moments provide for "gold-plated" benchmarks against which approximate numerical techniques can be tested. Secondly, a study of the corrections to age theory, and particularly of the age, can deepen our understanding of where age theory is applicable and how rapidly it is approached. For both purposes it would be desirable to carry out some numerical evaluation of the spatial moments for different nuclei and possibly for realistic anisotropies of the basic scattering process. Such calculations remain for the future.

An obvious extension of the present work, and one which would provide for more direct comparison with other approaches, would be calcu- 
lation of the space-energy distribution of the scalar flux itself, rather than the spatial moments. What is required for this purpose is the inversion of the Laplace-transform of the scalar flux, Eq. (III.14). Considerable progress has already been achieved (and will be reported by the author elsewhere), al though much remains to be done before complete, or even practically useful, solutions can be presented.

By a combination of numerical and physical arguments it has been demonstrated that for elastic scattering, isotropic in the C-0-M system, there exists only one distinct discrete eigenvalue. This simplifies considerably the form of the Laplace-transformed flux. Even so, the singularities of the Laplace transform are of so complicated a nature that a direct and complete expression for the inversion does not seem within grasp at this time. Instead some approximate limits must be considered, even for the conservative case of zero absorption.

One such limit is the region of phase space for a large (corresponding to the transformed variable $n \rightarrow 0)$. By performing the inversion through a stationary point method, it is possible to obtain a "generalized age-theory" expression for the asymptotic part of the scalar flux. In the $x-u$ plane this form for the scalar flux is valid for all $u$ greater than $x / 2 R$, where $R$ is a function of the nuclear mass only. For very large $u$ the generalized age-theory reduces to the usual age-theory formulae. Holte's large $u$ approximations can also be recovered. The magnitude and behavior of deviations from conventional age theory have not yet been explored in detail, especially for values of $x / u$ approaching the bounding line. 
In the remaining half of the $x-u$ plane, i.e., for $u$ less than $x / 2 R$ a variety of approximate methods for carrying out the Laplace inversion have been tried, none of them completely satisfactory. For example, the stationary-point method can again be used (with a different stationary point, of course). It yields a formula which approaches a Wick-type asymptotic result in the limit of large $x$. But it fails to show the expected Placzek oscillations in the scalar flux, or its derivatives, at lethargies that are integral multiples of $\varepsilon$, i.e., at $u=$ k.c. Thc approximation McInerney used for the inversion in hydrogen can be suitably generalized to other nuclei, and yields an interesting formula with connections to other previously derived results. However, it holds only in the first $\varepsilon$ region, and at that only through terms in $u^{2}$.

It appears that in order to display the Placzek discontinuities in a manifest form it is necessary to include the terms in the transform, previously ignored, of the form $e^{-k \varepsilon n}$. Thus an inversion can be performed along the lines of the McInerney approach, but including the exponential terms in the representation of the normalization integral for the discrete eigenvalue. The resulting formula for the scalar flux shows the right type of discontinuities at $u=k \varepsilon$, and gives a vigorously correct zeroth spatial moment. But the second moment is correct only. through terms in $u^{2}$, so that the error in the scalar flux can be no belter than this limit. An accurate representation is yet to be found for the flux in the small $u$, large $x$, portion of phase space. Realization of the ultimate dream-a description of the scalar flux valid for all $u$ and $x$-is clearly still a good way off in the future.

Future work (beyond what has been sketched here) should involve a two-pronged attack. The approximate formulae derived for the spatial 
moments and the flux are of value in themselves for providing standards in benchmark comparisons and for examining the rates of approach to popular simplifications, such as age theory. To be useful for these purposes, a good deal of numerical evaluation should be carried out. In that connection it would be helpful if some of the results, e.g., the spatial moments, could be generalized to arbitrary anisotropy of scattering in the C-0-M system. For example, very little is known about how the rate of convergence to the age-theory limit is affected by scattering anisotropy.

The other branch in the course of future research is, of course, an attempt to improve the range of validity of the flux inversion procedures. Here it would seem that the points to concentrate on are the treatment of the transient or non-asymptotic terms (which are only sketchily treated at present) and a more thoroughgoing analysis of the effects of the $e^{-k \varepsilon n}$ terms in the Laplace-transformed flux. The investigations described in this thesis have, it is believed, significantly improved our understanding of the constant-cross-section problem and opened up avenues for still further advances in the future. 


\section{APPENDIX A}

\section{THE FUNCTIONS $g_{n}(n)$}

We recall that the functions $g_{n}(n)$ are defined as the Laplace Transforms of the coefficients $f_{n}(u)$ of the Legendre expansion of the scattering kernel $f\left(\bar{\Omega} \cdot \bar{\Omega}^{\circ}, u\right)$, i.e.,

$$
g_{n}(n) \equiv \mathscr{L}\left[f_{n}(u)\right]
$$

Two cases have to be distinguished, depending on the nuclear mass A of the scatterer:
(1) When $A=1$ (i.e., hydrogen), the quantity
$\varepsilon \equiv 2 \ln \left(\frac{A+1}{A-1}\right)$

becomes infinite and therefore

$$
g_{n}(n)=\int_{0}^{\infty} e^{-(n+1) u} P_{n}\left(e^{-\frac{u}{2}}\right) d u
$$

(2) For any $A>1$, we have

$$
g_{n}(n)=\alpha \int_{0}^{\varepsilon} e^{-(n+1) u} P_{n}\left(\frac{A+1}{2} e^{-\frac{u}{2}}-\frac{A-1}{2} e^{\frac{u}{2}}\right) d u
$$

Using an obvious change of variable, Eq. (A.3) can be written in the equivalent form: 


$$
g_{n}(n)=2 \pi \int_{-1}^{1} d \mu P_{n}(\mu) g(\mu, n)
$$

where

$$
g(\mu, n)=\frac{\alpha}{\pi}\left(A^{2}-1+\mu^{2}\right)^{-1 / 2}\left[\frac{\mu+\left(\mu^{2}+A^{2}-1\right)^{1 / 2}}{A+1}\right]^{2(n+1)}
$$

The quantity $\alpha$ appearing in both Eqs. (A.3) and (A.5) has the usual meaning, i.e.,

$$
\alpha=\frac{(A+1)^{2}}{4 A}
$$

Clearly - in the case of hydrogen the functions $g_{n}(n)$ are rational functions of $\eta$, while for any $A>1$, they are entire functions of $n$.

It is well known ${ }^{2}, 4$ that in the case of hydrogen, the functions $g_{n}(n)$ satisfy the simple recursion relation

$$
g_{n}(n)=\frac{2 n+3-n}{2 n+2+n} g_{n-2}(n)
$$

No such simple relation exists for $A>1$. For convenience, we list here the explicit form of the first few $g_{n}{ }^{\prime} s$, i.e.,

$$
\begin{aligned}
g_{0}(n) & =\frac{\alpha}{n+1}\left[1-e^{-\varepsilon(n+1)}\right] \\
g_{1}(n) & =\frac{\alpha}{(n+1 / 2)(n+3 / 2)}\left[n+1-\frac{A}{2}+\left(n+1+\frac{A}{2}\right) e^{-\varepsilon(n+1)]}\right. \\
g_{2}(n) & =\frac{\alpha}{n(n+1)(n+2)}\left\{n^{2}+n\left(2-\frac{3}{2} A\right)+3\left(\frac{A-1}{2}\right)^{2}-\right. \\
& \left.-\left[n^{2}+n\left(2+\frac{3}{2} A\right)+3\left(\frac{A+1}{2}\right)^{2}\right] e^{-\varepsilon(n+1)}\right\}
\end{aligned}
$$




$$
\begin{aligned}
g_{3}(\eta) & =\frac{5 \alpha}{2}\left[\left(\frac{A+1}{2}\right)^{3} \frac{1-e^{-\varepsilon\left(\ddot{n}+\frac{5}{2}\right)}}{n+\frac{5}{2}}-3\left(\frac{A+1}{2}\right)^{2} \frac{A-1}{2} \frac{1-e^{-\varepsilon\left(n+\frac{3}{2}\right)}}{n+\frac{3}{2}}\right. \\
& \left.+3\left(\frac{A+1}{2}\right)\left(\frac{A-1}{2}\right)^{2} \frac{1-e^{-\varepsilon\left(n+\frac{1}{2}\right)}}{n+\frac{1}{2}}-\left(\frac{A-1}{2}\right)^{3} \frac{1-e^{-\varepsilon\left(n-\frac{1}{2}\right)}}{n-\frac{1}{2}}\right]-\frac{3}{2} g_{1}(n) .
\end{aligned}
$$

The functions $g_{n}(n)$ have received considerable attention in the earlier literature and important results concerning their properties have been obtained, among others, by Placzek, ${ }^{6}$ Wick, ${ }^{2,4}$ Bethe et a1., Marshakl and Hol te. ${ }^{7,8}$

In this appendix we wish to present some new results concerning the properties of the $g_{n}$ 's. In particular, we shall focus our attention on investigating the various asymptotic forms taken on by these functions, as one (or more) of the variables $n, n$ and $A$ become large, small or some combination thereof. In the process, we shall derive new asymptotic expressions for the $g_{i i}(n)$. which ran be used for numerical calculations and also for the purpose of examining questions related to convergence properties of infinite sums of the form $\sum_{n=0}^{\infty} h(n) g_{n}(n)$, where $h(n)$ is a simple function of the form $n^{\beta}(\beta>0$, constant) or $h(n)=\ln n$.

\section{The Case $A \neq 1$}

1.a. Asymptotic Form of $g_{n}(n)$ for Large $n$

We start from the definition of $g_{n}(n)$, i.e.,.Eq. (II.11) or, explicitly, (A.3). 
Since $\left|f_{n}(u)\right|<M_{e}^{\beta u}$ ( $M$ and $\beta$ are positive constants), we can employ Watson's Lemma ${ }^{51}$ which ensures that the main contribution to $g_{n}(n)$ for large $n$ will come from the values of $f_{n}(u)$ around the neighborhood $u=0$. Thus, writing the expression $f_{n}(u)=$ $\alpha e^{-u} P_{n}\left(\frac{A+1}{2} e^{-\frac{u}{2}}-\frac{A-1}{2} e^{\frac{u}{2}}\right)$ as

$$
f_{n}(u)=\alpha \sum_{r=0}^{\infty} C_{r} \frac{u^{r}}{r !}
$$

we obtain, by virtue of Watson's Lemma, the asymptotic expansion (for large n):

$$
g_{n}(n) \sim \alpha \sum_{r=0}^{\infty} C_{r} n^{-r-1}
$$

We have to determine the expressions of the coefficients $C_{r}$. Defining

$$
\begin{aligned}
x & \equiv \frac{1}{2}\left[1-\left(\frac{A+1}{2} e^{-\frac{u}{2}}-\frac{A-1}{2} e^{\frac{u}{2}}\right)\right] \\
& =\frac{1}{2}\left(1-\cosh \frac{u}{2}+A \sinh \frac{u}{2}\right)
\end{aligned}
$$

we obtain

$$
\begin{aligned}
P_{n}\left(\frac{A+1}{2} \cdot e^{-\frac{u}{2}}-\frac{A-1}{2} e^{\frac{u}{2}}\right) & =P_{n}(1-2 x) \\
& =F(-n, n+1 ; 1 ; x)
\end{aligned}
$$

where $F(\alpha, \beta ; \gamma ; z)$ is the well-known Gauss' hypergeometric function. Employing the customary series expansion for $F(-n, n+1 ; 1 ; x)$, (which 
is finite in our case since $\alpha=-n)$, we obtain, for $|x|<1$ :

$$
\begin{aligned}
P_{n}(1-2 x) & =1-n(n+1) x+\frac{(n+2)(n+1) n(n-1)}{(2 !)^{2}} x^{2}- \\
& -\frac{(n+3)(n+2)(n+1) n(n-1)(n-2)}{(3 !)^{2}} \dot{x}^{3}+\ldots \\
& =\sum_{i=0}^{n}(-1)^{i} b_{i} x^{i}
\end{aligned}
$$

Obviously, the coefficients $b_{i}$ in Eq. $(A, 16)$ are functions of $n$. Next, in view of (A.14), we have.

$$
\begin{aligned}
x & =\left\{\sum_{k=1}^{\infty} \frac{\left(\frac{u}{2}\right)^{2 k}}{(2 k) !}-A \sum_{k=0}^{\infty} \frac{\left(\frac{u}{2}\right)^{2 k+1}}{(2 k+1) !} \times \times\left(-\frac{1}{2}\right)\right. \\
& =\sum_{i=1}^{\infty}(-1)^{i-1} a_{i} u^{i}
\end{aligned}
$$

where, obviously:

$$
\mathrm{a}_{2 k}=\frac{1}{2^{2 k+1}(2 k) !}
$$

and

$$
a_{2 k+1}=\frac{A}{2^{2 k+2}(2 k+1) !}
$$

Substituting the series (A.17) into (A.16) yields

$$
P_{n}(1-2 x)=\sum_{j=0}^{\infty}(-1)^{j_{B}}{ }_{j}(n, A) u^{j}
$$

The explicit forms of the first few coefficients $B_{j}(n, a)$ are: 


$$
\begin{aligned}
& B_{0}=1, B_{1}=b_{1} a_{1} ; B_{2}=b_{1} a_{2}+b_{2} a_{1}^{2} \\
& B_{3}=b_{1} a_{3}+2 b_{2} a_{1} a_{2}+b_{3} \dot{a}_{1}^{3} \\
& B_{4}=b_{1} a_{4}+b_{2}\left(a_{2}^{2}+2 a_{1} a_{3}\right)+3 b_{3} a_{1}^{2} a_{2}+a b_{1} b_{4} .
\end{aligned}
$$

Finally, multiplying the series (A.19) by

$$
e^{-u}=\sum_{k=0}^{\infty}(-1)^{k} \frac{u^{k}}{k !}
$$

yields the coefficients $C_{r}$ as

$$
C_{r}=(-1)^{r} \sum_{k=0}^{r} \frac{r !}{(r-k) !} B_{k}(n, A)
$$

We note that the asymptotic expression (A.13) for $g_{n}(n)$, where the coefficients $C_{r}$ are given by (A.20), holds when $n$ is large, for any value of $n$ and any $A>1$.

If, in addition to $n$ being large, $n$ also becomes large, a much simpler asymptotic expression for $g_{n}(n)$ can be derived. In fact, Wick ${ }^{1,4}$ has shown that

$$
g_{n}(n) \sim \frac{\alpha}{n} \exp \left[-\frac{A}{4 n}(n+1 / 2)^{2}\right]
$$

which holds for values of $n, n$ and $A$ such that the relations

$$
\left\{n \gg A \text { and } \frac{A n^{2}}{4 n} \gg 1\right\}
$$

are satisfied.

However, the conditions (A.22) are not very useful for numerical evaluations of the errors associated with (A.21). Therefore, we have 
reexamined the asymptotic behavior of $g_{n}(n)$ when both $n$ and $n$ are large and obtained a considerably more refined expression than (A.21); our procedure is as follows:

As Wick ${ }^{4}$ remarked, when $n$ is large, the angle

$$
\theta \equiv \arccos \mu=\arccos \left[\frac{A+1}{2} e^{-\frac{u}{2}}-\frac{A-1}{2} e^{\frac{u}{2}}\right]
$$

is a very small angle (i.e., $\theta \approx 0$ ). When, in addition to $\theta$ being small, $n$ becomes large, the Legendre Polynomials $P_{n}(\cos \theta)$ can be expressed as: 56

$$
\left(\frac{\sin \theta}{\theta}\right)^{1 / 2} P_{n}(\cos \theta)=J_{0}\left[\left(n+\frac{1}{2}\right) \theta\right]-\frac{\theta}{24(n+1 / 2)} J_{1}\left[\left(n+\frac{1}{2}\right) \theta\right]+\sigma
$$

where

$$
|\sigma|<0.03 \theta^{4} \text { if } 0<\theta \leq \frac{\pi}{2 n}
$$

Next, expanding Eq. (A.5) for small $\theta$, yields:

$$
g(\cos \theta, n)=\frac{\alpha}{\pi A} \exp \left[-n_{1} \theta^{2}+n_{2} \theta^{4}-n_{3} \theta^{6}+0\left(\theta^{8}\right)\right]
$$

where

$$
\begin{aligned}
& n_{1}=\frac{1}{A}\left(n+1-\frac{1}{2 A}\right) \\
& n_{2}=\frac{1}{12 A}\left(1-\frac{3}{A^{2}}\right)\left(n+1-\frac{1}{A} \cdot \frac{2-3 / A^{2}}{1-3 / A^{2}}\right) \\
& n_{3}=\frac{1}{360 A}\left(1-\frac{30}{A^{2}}-\frac{45}{A^{4}}\right)\left(n+1-\frac{4}{A^{2}} \cdot \frac{2-15 / A^{2}+15 / A^{4}}{1-30 / A^{2}+45 / A^{4}}\right)
\end{aligned}
$$


[Note: Expressions (A.27) and (A.28) have also been obtained by Marshak. ${ }^{1}$ ] Using (A.24) and (A.26), and retaining consistently terms up to $O\left(\theta^{4}\right)=O\left(\frac{1}{n^{4}}\right)$, we can easily perform the integration over $\theta=\arccos \mu$ prescribed in (A.4). The result is

$$
g_{n}(n)=\frac{\alpha}{A n_{1}} e^{-\frac{\left(n+\frac{1}{2}\right)^{2}}{4 n_{1}}}\left\{1-\frac{1}{12 n_{1}}\left[\frac{5}{4}-\frac{\left(n+\frac{1}{2}\right)^{2}}{4}\right]\right\}+R
$$

where $|R|=0\left(\frac{1}{n^{4}}\right)$ or, more explicitly,

$$
R \leq \frac{1}{n^{3}}\left\{2\left(0.03+\frac{1}{1440}+n_{2}\right)+\frac{1}{576}\left[2-\frac{\left(n+\frac{1}{2}\right)^{2}}{4}\right]\right\}
$$

We note here that, in order to obtain (A.30), we also employed the well-known relation77

$$
\int_{0}^{\infty} x^{n+\frac{1}{2} \nu} e^{-\alpha x} J_{v}(2 \beta \sqrt{x}) d x=n ! \beta^{\nu} e^{-\beta^{2} / \alpha} L_{n}^{\nu}\left(\frac{\beta^{2}}{\alpha}\right)
$$

where $L_{n}^{\nu}$ are the Laguerre Polynomials, when carrying out the integration over $\theta$. It is obvious from our analysis that Eq. (A.30) holds for $n_{1}=0\left(n^{-2}\right)$, which is a more relaxed condition than wick's (A.22). It can also be seen that the leading term in (A.30) can be reduced to (A.21) under the restrictions (A.22).

\section{1.b. Asymptotic Form of $g_{n}(n)$ for Small $n$}

Since we are still considering moderators of mass $A>1$, we obviously have 
$\frac{1}{A}<1$

It has been shown ${ }^{78}$ that whenever $(A .33)$ is satisfied, $g_{n}(n)$ can be represented as

$$
g_{n}(x)=\left(\frac{A+1}{A}\right)^{2} \sqrt{\frac{\pi}{2 x}} I_{n+\frac{1}{2}}(x)+0\left(\frac{1}{A^{2}}\right)
$$

where

$$
x=\frac{2}{A}(n+1)
$$

has values up to unity: 5 Now, in view of the well-known representation

$$
I_{v}(z)=\sum_{k=0}^{\infty} \frac{\left(\frac{z}{2}\right)^{v+2 k}}{k ! \Gamma(v+k+1)}
$$

we have

$$
g_{n}(x)=\left(\frac{A+1}{A}\right)^{2} e^{-x} \frac{x^{n}}{(2 n+1) ! !}+0\left(\frac{1}{n}\right)
$$

Clearly, for $n=0$, we have

$$
g_{n}(0)=O\left(\frac{1}{A^{n}}\right)
$$

as has beeen also observed by Marshak ${ }^{1}$ and Hol te. ${ }^{8}$

Since the values of the functions $g_{n}(n)$ and their derivatives with respect to $n$, at $n=0$, are of basic importance for practical applications, e.g., the calculation of the scalar flux and of its lethargy dependent spatial moments, we shall explicitly list the expressions of the $\left\{\frac{d^{j}}{d n^{j}} g_{k}(n)\right\}_{n=0}$ which are needed 
for explicit or numerical evaluation of all the quantities appearing in this entire work.

$$
\begin{aligned}
g_{0}(0)= & \\
g_{0}^{I}(0)= & \left(\frac{A}{4}-\frac{1}{2}+\frac{1}{4 A}\right) \varepsilon \\
g_{0}^{I I}(0)= & 2-\left(\frac{A}{2}-1+\frac{1}{2 A}\right) \varepsilon-\left(\frac{A}{4}-\frac{1}{2}+\frac{1}{4 A}\right) \varepsilon^{2} \\
g_{0}^{I I I}(0)= & -6+\left(\frac{3 A}{2}-3+\frac{3}{2 A}\right) \varepsilon+\left(\frac{3 A}{4}-\frac{3}{2}+\frac{3}{4 A}\right) \varepsilon^{2} \\
& +\left(\frac{A}{4}-\frac{1}{2}+\frac{1}{4 A}\right) \varepsilon^{3} \\
g_{0}^{I V}(0)= & 24-\left(6 A-12+\frac{6}{A}\right) \varepsilon-(3 A-6+3 / A) \varepsilon^{2}- \\
& (A-2+1 / A) \varepsilon^{3}-(A / 4-1 / 2+1 / 4 A) \varepsilon^{4} \\
g_{0}^{V}(0)= & -120+(30 A-60+30 / A) \varepsilon+(15 A-30+15 / A) \varepsilon^{2}+ \\
+ & (5 A-10+5 / A) \varepsilon^{3}+(5 A / 4-5 / 2+5 / 4 A) \varepsilon^{4}+ \\
g_{1}^{I I}(0)= & \frac{-32 \Lambda}{9}+\frac{112}{27 A}+\left(\frac{8}{9} A^{2}-\frac{2}{3} A-\frac{4}{3}+\frac{10}{9 A}\right) \varepsilon+ \\
+ & (A / 4-1 / 2+1 / 4 A) \varepsilon^{5} \\
g_{1}^{I}(0)= & \frac{2}{3 A} \\
& \frac{2 A}{3}-\frac{10}{9 A}-\left(\frac{A^{2}}{6}-\frac{1}{2}+\frac{1}{3 A}\right) \varepsilon \\
& \\
&
\end{aligned}
$$




$$
\begin{aligned}
g_{1}^{I I I}(0) & =\frac{208}{9} A+\frac{656}{27 A}-\left(\frac{52}{9} A^{2}-\frac{16}{3} A-\frac{20}{3}+\frac{56}{9 A}\right) \varepsilon- \\
& -\left(\frac{4}{3} A^{2}-A-2+\frac{5}{3 A}\right) \varepsilon^{2}-\left(\frac{A^{2}}{6}-\frac{1}{2}+\frac{1}{3 A}\right) \varepsilon^{3} \\
g_{1}^{I V}(0) & =-\frac{5120}{27} A+\frac{15616}{87 A}+\left(\frac{1280}{27} A^{2}-\frac{416}{9} A-\frac{448}{9}+\frac{1312}{27 A}\right) \varepsilon \\
& +\left(\frac{104}{9} A^{2}-\frac{32}{3} A-\frac{40}{3}+\frac{112}{9 A}\right) \varepsilon^{2}+\left(\frac{16}{9} A^{2}-\frac{4}{3} A-\frac{8}{3}+\frac{20}{9 A}\right) \varepsilon^{3}+ \\
& +\left(\frac{A^{2}}{6}-\frac{1}{2}+\frac{1}{3 A}\right) \varepsilon^{4}
\end{aligned}
$$

$$
g_{2}(0)=\frac{5}{8}-\frac{3}{8} A^{2}+\left(\frac{3}{32} A^{3}-\frac{3}{16} A+\frac{3}{32 A}\right) \varepsilon
$$

$$
\begin{aligned}
g_{2}^{I}(0) & =\frac{9}{16} A^{2}-\frac{7}{16}-\left(\frac{9}{64} A^{3}-\frac{3}{16} A^{2}-\frac{5}{32} A+\frac{5}{16}-\frac{7}{64 A}\right) \varepsilon- \\
& -\left(\frac{3}{64} A^{3}-\frac{3}{32} A+\frac{3}{64 A}\right) \varepsilon^{2}
\end{aligned}
$$

$$
\begin{aligned}
g_{2}^{I I}(0) & =-\frac{21}{16} A^{2}+\frac{11}{16}+\left(\frac{21}{64} A^{3}-\frac{9}{16} A^{2}-\frac{1}{32} A+\frac{7}{16}-\frac{11}{64 A}\right) \varepsilon+ \\
& +\left(\frac{9}{64} A^{3}-\frac{3}{16} A^{2}-\frac{5}{32} A+\frac{5}{16}-\frac{7}{64 A}\right) \varepsilon^{2}+ \\
& +\left(\frac{1}{32} A^{3}-\frac{1}{16} A+\frac{1}{32 A}\right) \varepsilon^{3}
\end{aligned}
$$

$g_{2}^{I I I}(0)=\frac{135}{32} A^{2}-\frac{57}{32}-\left(\frac{135}{128} A^{3}-\frac{63}{32} A^{2}+\frac{21}{64} A+\frac{33}{32}-\frac{57}{128 A}\right) \varepsilon$

$$
-\left(\frac{63}{128} A^{3}-\frac{27}{32} A^{2}-\frac{3}{64} A+\frac{21}{32}-\frac{33}{128 A}\right) \varepsilon^{2}-
$$$$
\text { - }\left(\frac{9}{64} A^{3}-\frac{3}{16} A^{2}-\frac{5}{32} A+\frac{5}{16}-\frac{7}{64 A}\right) \varepsilon^{3}-
$$$$
-\left(\frac{3}{128} A^{3}-\frac{3}{64} A+\frac{3}{128 A}\right) \varepsilon^{4}
$$ 


$$
\begin{aligned}
g_{3}(0)= & 0 \\
g_{3}(0)= & -2 A^{3}+\frac{10}{3} A-\frac{16}{15} A+\left(\frac{A^{4}}{2}-A^{2}+\frac{1}{2}\right) \varepsilon \\
g_{3}(0)= & \frac{64}{15} A^{3}-\frac{176}{45} A-\frac{208}{225} \frac{1}{A}- \\
& -\left(\frac{16}{15} A^{4}-2 A^{3}-\frac{4}{3} A^{2}+\frac{10}{3} A-\frac{16}{15 A}\right) \varepsilon- \\
& -\left(\frac{A^{4}}{2}-A^{2}+\frac{1}{2}\right) \varepsilon^{2} \\
g_{4}(0)= & \frac{35}{32} A^{4}-\frac{95}{48} A^{2}+\frac{27}{32}-\left(\frac{35}{128} A^{5}-\frac{75}{128} A^{3}+\right. \\
g_{5}(0)= & \left.\frac{45}{128} A-\frac{5}{128 A}\right) \varepsilon \\
& \left(\frac{175}{768} A^{5}-\frac{35}{64} A^{4}-\frac{85}{256} A^{3}+\frac{95}{96} A^{2}+\frac{19}{256} A-\right. \\
& \left.-\frac{27}{64}+\frac{7}{768 A}\right) \varepsilon+\left(\frac{35}{256} A^{5}-\frac{75}{256} A^{3}+\frac{45}{256} A-\right. \\
I(0)= & -\frac{175}{192} A^{4}+\frac{295}{288} A^{2}-\frac{7}{192}+ \\
g_{4}(0) & \\
& 0 \\
&
\end{aligned}
$$

2. The Case $A=1$ (Hydrogen)

Following the change of variable $e^{-\frac{u}{2}}=x$, we can write Eq. (A.2) as

$$
g_{n}(n)=2 \int_{0}^{1} x^{\lambda} P_{n}(x) d x
$$


where

$$
\lambda=2 n+1
$$

The integration in (A.60) can be carried out exactly, by employing the well-known formulae 77

$$
\begin{aligned}
\int_{0}^{1} x^{\lambda} P_{2 m}(x) d x= & \frac{(-1)^{m} \Gamma\left(m-\frac{1}{2} \lambda\right) \Gamma\left(\frac{1}{2}+\frac{1}{2} \lambda\right)}{2 \Gamma\left(-\frac{1}{2} \lambda\right) \Gamma\left(m+\frac{3}{2}+\frac{1}{2} \lambda\right)} \\
& \text { for } \operatorname{Re} \lambda>-1
\end{aligned}
$$

and

$$
\begin{aligned}
& \int_{0}^{1} x^{\lambda} P_{2 m+1}(x) d x=\frac{(-1)^{m} \Gamma\left(m+\frac{1}{2}-\frac{1}{2} \lambda\right) \Gamma\left(1+\frac{1}{2} \lambda\right)}{2 \Gamma\left(\frac{1}{2}-\frac{1}{2} \lambda\right) \Gamma\left(m+2+\frac{1}{2} \lambda\right)} \\
& \text { for } \operatorname{Re} \lambda>-2
\end{aligned}
$$

Therefore

$$
g_{2 m}(n)=\frac{(-1)^{m} \Gamma\left(m-\frac{1}{2} \lambda\right) \Gamma\left(\frac{1}{2}+\frac{1}{2} \lambda\right)}{\Gamma\left(-\frac{1}{2} \lambda\right) \Gamma\left(m+\frac{3}{2}+\frac{1}{2} \lambda\right)} \text { for Re } \lambda>-1
$$

and

$$
g_{2 m+1}(n)=\frac{(-1)^{m} \Gamma\left(m+\frac{1}{2}-\frac{1}{2} \lambda\right) \Gamma\left(1+\frac{1}{2} \lambda\right)}{\Gamma\left(\frac{1}{2}-\frac{1}{2} \lambda\right) \Gamma\left(m+2+\frac{1}{2} \lambda\right)} \text { for } \operatorname{Re} \lambda>-2
$$

We are now ready to investigate the behavior of $g_{2 m}(n)$ and $g_{2 m+1}(n)$, for large values of $m$. For this purpose, we shall employ the well-known ${ }^{77}$ asymptotic representation of $\Gamma(z)$, valid for large $z$ : 


$$
\begin{aligned}
& \ln \Gamma(z)=z \text { ln } z-z-\frac{1}{2} \text { ln } z+\ln \sqrt{2 \pi}+ \\
& +\sum_{k=1}^{n-1} \frac{B_{2 k}}{2 k(2 k-1) z^{2 k-1}}+R_{n}(z)
\end{aligned}
$$

where

$$
\left|R_{n}(z)\right|<\frac{\left|B_{2 n}\right|}{2 n(2 n-1)|z|^{2 n-1} \cos ^{2 n-1}\left(\frac{1}{2} \arg z\right)}
$$

For our purposes, it is enough to use (A.66) in the form:

$$
\ln \Gamma(z)=z \text { ln } z-z-\frac{1}{2} \ln z+\ln \sqrt{2 \pi}+O\left(z^{-1}\right)
$$

We investigate $\left|g_{2 m}(n)\right|$ first:

We have:

$$
\ln \left|g_{2 m}(n)\right|=\ln k+\ln \Gamma\left(m-\frac{1}{2} \lambda\right)-\ln \Gamma\left(m+\frac{3}{2}+\frac{\lambda}{2}\right)
$$

where

$$
k=\frac{\Gamma\left(\frac{1}{2}+\frac{1}{2} \lambda\right)}{\Gamma\left(-\frac{1}{2} \lambda\right)}
$$

But, for large $m$

$$
\ln \Gamma\left(m-\frac{1}{2} \lambda\right)-\ln \Gamma\left(m+\frac{3}{2}+\frac{\lambda}{2}\right)=-\left(\lambda+\frac{3}{2}\right) \ln m+O\left(m^{-1}\right)
$$

To obtain (A.71) we have made use of (A.68) and of the fact that, for large $m$

$$
\ln (m+a)=\ln m+\frac{a}{m}+0\left(m^{-2}\right)
$$


where $a$ is some constant. Therefore, for large $\mathrm{m}$ :

$$
\left|g_{2 m}(n)\right| \sim k^{-\left(2 n+\frac{5}{2}\right)}
$$

Similarly, from (A.65), we obtain for large m:

$$
\left|g_{2 m+1}(n)\right| \sim k_{1} m^{-\left(2 n+\frac{5}{2}\right)}
$$

where

$$
k_{1}=\frac{\Gamma\left(1+\frac{1}{2} \lambda\right)}{\Gamma\left(\frac{1}{2}-\frac{1}{2} \lambda\right)}
$$

3. Convergence Properties of Infinite Series Involving the Functions $g_{n}(n)$

We have seen in Section II.I.C that a number of fundamental properties of the Boltzmann Equation depend on the convergence properties of infinite sums involving the $g_{n}(n)$ (or their counterparts $f_{n}-$ in monoenergetic transport). In particular, in order that the number of discrete eigenvalues be finite, the series of the form

$$
\sum_{n=0}^{\infty} h(n)\left|g_{n}(n)\right|
$$

(where $h(n)$ is a simple function of $n$, to be defined shortly), must be convergent. The function $h(n)$ was of the form

$$
h(n)=n^{\beta}
$$


with

$\left\{\begin{array}{l}\beta=1 \text { required by Inönü }{ }^{40} \text { [cf. Eq. (II.47)] } \\ \beta=2 \text { required by Case }{ }^{16} \quad \text { [cf. Eq. (II.48)] }\end{array}\right.$

Since, as discussed in Section II.I.C and Appendix D, Inönü's condition seems insufficient and since Case's condition is not satisfied by hydrogen for $n<1 / 4$ (as we shall prove shortly), we derived, in Appendix $D$, a new sufficient convergence condition of the form (A.76), but where

$$
h(n)=n \ln n
$$

We shall analyze the case of hydrogen first. Using (A.77), the study of (A.76) reduces to the investigation of the series

$$
\sum_{m=0}^{\infty}(-1)^{m} v_{m}(n)
$$

with

$$
v_{m}(n)=(2 m)^{\beta}\left|g_{2 m}(n)\right|+(2 m+1)^{\beta}\left|g_{2 m+1}(n)\right|
$$

(Note that in writing (A.79) we have explicitly shown that we deal with an alternating series.)

Now, in view of (A.73) and (A.74), we have

$$
v_{m}(n)=2^{\beta}\left(k+k_{1}\right) m^{-\left(2 n+\frac{5}{2}-\beta\right)}\left[1+O\left(m^{-1}\right)\right]
$$

for large $m$. Therefore

$$
\frac{v_{m}}{v_{m+1}}=1+\left(2 n+\frac{5}{2}-\beta\right) \frac{1}{m}+0\left(m^{-2}\right)
$$


and, obviously

$$
\lim _{m \rightarrow \infty} v_{m}(n)=0 \text {, if } B<2 n+\frac{5}{2}
$$

We thus immediately conclude from (A.82-83) that the series (A.79) [or, equivalently, (A.76)] is:

(i) absolutely convergent, if $\beta<2 \eta+\frac{3}{2}$

(by virtue of Gauss' criterion)

(ii) semiconveryent, if $\beta<2 n+\frac{5}{2}$

(by virtue of Leibnitz' criterion for al ternating series)

Relation (A.84) obviously implies that Case's requirement [i.e., $\left.\Sigma n^{2}\left|g_{n}(n)\right|<\infty\right]$ is not satisfied if Re $n \leq 1 / 4$. As a check, we see that, for example, at $n=0$

$$
\begin{aligned}
g_{n}(0) & =2 \int_{0}^{1} x P_{n}(x) d x \\
& = \begin{cases}1 & \text { for } n=0 \\
2 / 3 & \text { for } n=1 \\
0 & \text { for } n=\text { odd, } n>1 \\
\frac{(-1)^{\frac{n}{2}} n !}{2^{n-1}(n-1)(n+2)\left[\left(\frac{n}{2}\right) !\right]^{2}} \text { for } n=\text { even }\end{cases}
\end{aligned}
$$

The series

$$
\sum_{0}^{\infty} n^{2}\left|g_{n}(0)\right|=\frac{2}{3}+\sum_{m=0}^{\infty}(2 m)^{2}\left|g_{2 m}(0)\right|
$$


is divergent, since

$$
\frac{(2 m)^{2}\left|g_{2 m}(0)\right|}{[2(m+1)]^{2}\left[g_{2(m+1)}(0) \mid\right.}=1+\frac{1}{2 m}+0\left(m^{-2}\right)
$$

On the other hand, taking $h(n)=n$ en $n$ we easily see that the series

$$
\sum_{n=0}^{\infty} n \ln n\left|g_{n}(n)\right|
$$

is convergent for $\operatorname{Re} n>-\frac{1}{4}$.

For $A>1$, we easily deduce that the series $(A .76)$ is convergent for both forms (A.77) and (A.78) for $h(n)$. Thus, for small values of $n$, use of (A.37) and of the corrolary of d'Alembert's criterion, yields:

$$
\lim _{n \rightarrow \infty}\left|\frac{(n+1)^{\beta} g_{n+1}}{n^{\beta} g_{n}}\right|=\lim _{n \rightarrow \infty} \frac{x}{2 n+3} \rightarrow 0
$$

for any fixed $x$ and constant B, Q.E.D. If $n$ is large, we use (A.30) and the corrolary of d'Alembert's criterion, to obtain

$$
\lim _{n \rightarrow \infty}\left|\frac{(n+1)^{\beta} g_{n+1}}{n^{\beta} g_{n}}\right|=\lim _{n \rightarrow \infty} e^{-\frac{1}{2 n_{1}}(n+1)} \rightarrow 0
$$

for fixed $n_{1}$ and constant $B$; Q.E.D. 


\section{APPENDIX B}

THE "COMPLETENESS THEOREM" FOR THE EIGENFUnCTIONS $\phi(\nu, \mu, \eta)$, FOR FINITE ANISOTROPY

Consider the boundary value problem for $\Psi(x, \mu, \eta)$ :

$$
\begin{aligned}
\mu \frac{\partial \psi(x, \mu, n)}{\partial x} & +\psi(x, \mu, n)-\frac{1}{2} C(\eta) \int_{-1}^{1} F(\mu, s, n) \psi(\mu, s, n) d s \\
& =q(x, \mu, n)
\end{aligned}
$$

where $\Psi(x, \mu, n)$ is the neutron density and $0<x<\infty ;-1 \leq \mu \leq 1$

In $(B .1), q(x, \mu, n)$ is the source, which is continuous in $x$ and $\mu$, Hölder continuous. in $\mu$ and satisfies the growth constraint

$$
\lim _{x \rightarrow \infty} \sup _{-1 \leq 1} e^{\left(\varepsilon-\frac{1}{v_{1}}\right) x}|q(x, \mu, n)|=0
$$

for some $\varepsilon>0$ and all $n, v_{1}$ being the discrete eigenvalue of largest. modulus. The quantity $F(\mu, s, n)$ appearing in $(B .1)$ is:

$$
F(\mu, s, n)=\sum_{\ell=0}^{N}(2 \ell+1) f_{\ell}(n) P_{\ell}(\mu) P_{\ell}(s)
$$

where

$$
f_{\ell}(n) \equiv \frac{g_{l}(n)}{g_{0}(n)}
$$

Moreover

$$
C(n) \equiv c g_{0}(n)
$$


and we require that

$$
0 \leq C(n)<1
$$

We clearly recognize Eq. (B.1) to be the Laplace transformed inhomogeneous Boltzmann equation; it has the same form as the monoenergetic transport equation, but displays the additional $n$-dependence. However, it differs from Eq. (II.9), since the scattering kernel is expressed as a finite (rather than infinite) sum of Legendre Polynomials [cf. Eq. (B.3)].

We want to show that, if $\psi(x, \mu, \eta)$ satisfies certain conditions in a given $\mu$ interval, the coefficients $A(\nu, n)$, of its expansion in the eigenfunction space $\phi(\nu, \mu, \eta)$, exist and are uniquely determined. The proof follows the same functional-analytic treatment as first used by Larsen ${ }^{45}$ for the monoenergetic case since the presence of the additional parameter $n$ does not alter the validity of his methods. In fact, the only explicit manifestation of the presence of $n$ is reflected in the fact that we have to introduce the quantities $C(n)$ and $f_{\ell}(n)$ as defined in (B.5) and (B.4), respectively. Otherwise, Larsen's proofs ${ }^{45}$ can be taken over without modifications. For this reason, we shall restrict ourselves to merely underlining the basic ideas leading to the "completeness theorem" for the eigenfunctions $\phi(\nu, \mu, n)$ [cf. (II.26) and (11.28)], and shall refer the interested reader to Larsen's work ${ }^{45}$ for the additional details.

Thus, following Larsen, ${ }^{45}$ we write (B.1) as

$$
K \frac{\partial \psi(\mu)}{\partial x}+\Psi(\mu)=K q_{0}(\mu)
$$


where

$$
q_{0}(\mu) \equiv q_{0}(x, \mu, n)=\frac{q(x, \mu, n)}{\mu}
$$

and $k^{-1}$ is an operator acting on $\mu$ only, defined by:

$$
\begin{gathered}
\left(K^{-1} \Psi\right)(\mu) \equiv \frac{1}{\mu}\left[\Psi(\mu)-\frac{1}{2} C(n) \int_{-1}^{1} F(\mu, s) \Psi(s) d s\right] \\
\text { for } 0<|\mu| \leq 1
\end{gathered}
$$

Since $k^{-1}$ acts on $\mu$ only, we have suppressed any explicit dependence on $n$ and $x$; this will be done throughout this appendix, whenever convenient.

To determine the operator $k$, whose existence was implicitly assumed in writing (B.9), we set for convenience $\Phi=\left(K^{-1} \Psi\right)(\mu)$ and use (B.3) and (B.9) to obtain

$$
\mu \Phi(u)=\psi(\mu)-C(n) \sum_{\ell=0}^{N} \frac{2 \ell+1}{2} f_{\ell}(n) P_{\ell}(\mu) \int_{-1}^{1} P_{\ell}(s) \psi(s) d s
$$

Multiplying $(B .10)$ by $P_{K}(\mu)$, integrating over $\mu$ from $(-1)$ to $(+1)$, using the orthogonality relations for the Legendre Polynomials and rearranging the resulting expression yields:

$$
(K \Phi)(\mu)=\mu \Phi(\mu)+\sum_{\ell=0}^{N} \frac{2 \ell+1}{2} \frac{C(n) f_{\ell}}{1-C(n) f_{\ell}(n)} \int_{-1}^{1} s \Phi(s) P_{\ell}(s) d s
$$

In view of the properties of the functions $g_{n}(n)$ [cf. Appendix A], we see that 


$$
1-c(n) f_{\ell}(n) \neq 0
$$

Therefore, the operator $K$ exists.

Next, the domain of $K$ is to be defined. Since, for physical reasons, we must have $\int^{l} q(x, \mu, n) d \mu<\infty$ for each $x$, and since the resolution of the identity of $K$ is to be applied to $q_{0}(x, \mu, n)$ [Cf. (B.8)], we take the domain of $K$ to be the Banach space $X$ of functions $\psi$ defined for $\mu$ on $[-1,1]$ such that

$$
|| \Psi||=\int_{-1}^{1}|\mu \Psi| \mathrm{d} \mu<\infty
$$

In other words, the domain of $K$ is defined to be a weighted $L_{1}$ space. We clearly have from (B.11):

$$
\begin{aligned}
\|K \Psi\| & \leq\left[1+\sup _{-1 \leq s \leq 1} \int_{-1}^{1} \mid \mu \sum_{n=0}^{N}\left(n+\frac{1}{2}\right) \frac{C(n) f_{n}(n)}{1-C(n) f_{n}} \times\right. \\
& \left.\times P_{n}(\mu) P_{n}(s) \mid d \mu\right] \times|| \psi||
\end{aligned}
$$

Therefore, the spectrum of $k$ is a bounded, closed subset of the complex plane.

Next, we construct the resolvent operator $(z I-K)^{-1}$. Then, we shall derive the "full range completeness theorem" by integrating the resolvent along a contour surrounding the spectrum of $K$ and applying Cauchy's theorem.

Let $z \in_{\rho}(K)$ [i.e., $z$ is in the resolvent set of $K, \rho(K)$ ], and $\psi \in X$. We need to solve the following equation for $\Phi$ : 
$\psi=(z I-K) \Phi$

in order to construct the expression of the resolvent operator.

The solution

$$
\Phi=(z I-K)^{-1} \Psi
$$

of Eq. (B.15) can be found by starting with Eq. (B.11) and following the sequence of algebraic manipulations described in Reference 45; this leads to

$$
\begin{aligned}
& (z I-K)-I_{y^{\prime}}(\mu)= \\
& \quad=\frac{1}{z-\mu}\left[E(\mu, z, \eta)+\frac{1}{2} c \frac{M(\mu, z, n)}{\Lambda(z, n)} \int_{-1}^{1} \frac{s E(s, z, n)}{z-s} d s\right]
\end{aligned}
$$

where:

$$
\begin{aligned}
& M(\nu, \mu, n)=\sum_{n=0}^{N}(2 n+1) g_{n}(n) \phi_{n}(\nu, n) P_{n}(\mu) \\
& \Lambda(z, n)=1-\frac{1}{2} c z \int_{-1}^{1} \frac{M(s, z, n)}{z-s} d s
\end{aligned}
$$

and

$$
E(\mu, z, n) \equiv \psi(\mu)-\frac{1}{2} c(n) \int_{-\{}^{T} s \psi(s)\left[\sum_{n=1}^{N}(2 n+1) f_{n} b_{n}(s, z) P_{n}(\mu)\right] d s
$$

We remark that $b_{n}(s, z)$ in (B.20) is a polynomial of order $n-1$ in $s$ and $z$, with $b_{0} \equiv 0$; also, the quantities $\phi_{n}(v, n)$ in $(B .18)$ are the same 
polynomials as defined in Eq. (II.13). Thus, $\Lambda(\nu, n)$ is the usual dispersion function [see (II.29)], for "finite anisotropic scattering," whose zeros $v_{j}$ are simple, occur in \pm pairs, are finite in number, and such that

$$
1<v_{m}<\ldots<v_{1}
$$

Since the singularities of the resolvent $(z I-K)^{-1}$ constitute the spectrum of $\mathrm{K}$, we can obtain this spectrum by inspecting Eq. (B.17). We readily see that if $z \in[-1,1]$, i.e., on the domain of definition of $\mu$, the resolvent exists and is unbounded. Therefore $z \in C_{\sigma}(K)$, where $c_{\sigma}(K)$ denotes the continuous spectrum of $K$. Next, when $z=v_{j}$, we obviously have $\Lambda\left(v_{j}\right)=0$ and the resolvent $(z I-K)^{-1}$ does not exist since it has a simple pole at $v_{j}$. Consequently $z \in P_{\sigma}(K)\left[P_{\sigma}(K)\right.$ is the point spectrum of $K$ ] and $v_{j}$ is a simple eigenvalue of $k$.

The "resolution of the identity" of K, i.e., a family of orthogonal projections, is obtained by integrating (B.17) around a simple closed contour $\Gamma$ which contains the spectrum of $K$, and by using Cauchy's theorem:

$$
\Psi(\mu)=\frac{1}{2 \pi i} \oint_{\Gamma}(z I-K)^{-1} \Psi(\mu) d z
$$

The contour $\Gamma$ is now deformed into a set of $(2 m+1)$ disjoint curves $\Gamma_{n}$ such that $\Gamma_{0}$ encloses the line segment $[-1,1]$ and $\Gamma_{n}(1 \leq|n| \leq m)$ encloses $v_{n}$. Thus, Eq. (B.20) becomes

$$
\psi(\mu)=\sum_{n=-m}^{m} \frac{1}{2 \pi i} \oint_{\Gamma_{n}}(z I-K)^{-1} \Psi(\mu) d z
$$


The integrals around $\Gamma_{n}(n \neq 0)$ are evaluated by means of the residue theorem (since $v_{n}$ is a simple pole of the resolvent), to obtain

$$
\frac{1}{2 \pi i} \oint_{\Gamma_{n}}(z I-K)^{-1} \Psi(\mu) d z=A\left(\nu_{n}, n\right) \phi\left(\nu_{n}, \mu, n\right)
$$

where

$$
\phi\left(\nu_{n}, \mu, n\right)=\frac{1}{2} c \nu_{n} \frac{M\left(\mu, \nu_{n}, n\right)}{\nu_{n}^{-1 !}}
$$

is the usual "Case" eigenfunction, and $A\left(\nu_{n}, n\right)$ is the projection coefficient that exists and is defined for each $\psi \in X$ through

$$
A\left(v_{n}, n\right)=\frac{1}{v \Lambda^{\prime}\left(\nu_{n}\right)} \int_{-1}^{l} \frac{s E\left(s, v_{n}\right)}{v_{n}-s} d s
$$

It can be shown ${ }^{45}$ that

$$
A\left( \pm \nu_{j}, n\right)=\frac{1}{N_{j \pm}\left( \pm \nu_{j}, n\right)} \int_{-1}^{1} \mu \psi(\mu)_{\phi}\left( \pm \nu_{j}, \mu, n\right) d \mu
$$

which is the same as (II.45), with $N_{j \pm}$ given in (II.35).

To evaluate the integral around $\Gamma_{0}$, the $\mu$ interval $[-1,1]$ is divided into $n$ subintervals and $\mu \Psi(\mu)$ is required to satisfy a Hölder condition on each of the $n$ subintervals $\left(\mu_{k}, \mu_{k+1}\right)$. This constraint on $\Psi(\mu)$ does not alter the spectrum of $K$, but $\mu E(\mu, z)$ will now also satisfy a Hölder condition in $\mu$. Defining 


$$
N(z) \equiv \int_{-1}^{1} \frac{s E(s, z)}{z-s} d s
$$

$N(z)$ will tend uniformly to $N \pm(\dot{\mu})$ as $z \rightarrow \mu \pm i 0$ for $\mu$ in any closed subset of $[-1,1]$ not containing the points $\mu_{k}$. Next, assuming $0<|\mu|<1$, the contour $\Gamma_{0}$ is deformed into

$$
\Gamma_{0}=\bigcup_{k=0}^{n} \Gamma_{k}^{\prime} \cup \Gamma_{\mu} \cup \Gamma^{\prime}
$$

as shown below:

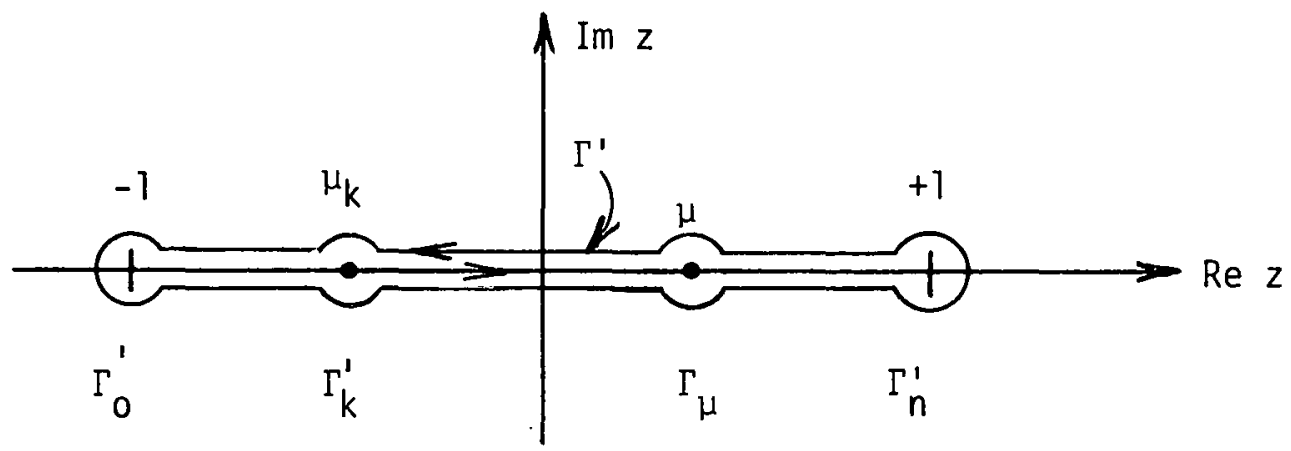

The integration over $\Gamma_{0}$ is carried out, as specified in (B.27); letting the radii of the small circles around $\mu_{k}, \mu$ and the end points tend to zero, and employing the Plemelj formulae to simplify the resulting expression, leads to:

$$
\frac{1}{2 \pi i} \oint_{\Gamma_{0}}(z I-K) \psi(\mu) d z=\int_{-1}^{1} A(\nu, n) \phi(\nu, \mu, \eta) d \nu
$$

where 


$$
\phi(v, \mu, \eta)=\lambda(\nu, n) \delta(v-\mu)+\frac{C v}{2} P \frac{M(v, \mu, n)}{\nu-\mu}
$$

is again the "Case" eigenfunction, with

$$
\lambda(\nu, n)=1-\frac{c}{2} \nu P \int_{-1}^{T} \frac{M(\nu, n, \mu)}{\nu-\mu} d \mu
$$

The projection coefficient $A(v, n)$ in $(B .28)$ is defined by

$$
A(v, n)=\frac{1}{2 \pi i v}\left[\frac{N^{-}(v)}{\Lambda^{-}(v)}-\frac{N^{+}(v)}{\Lambda^{+}(v)}\right]
$$

for $0<|\nu|<1$ and can be shown ${ }^{45}$ to satisfy

$$
A(\nu, n)=\frac{1}{N(\nu, n)} \int_{-1}^{1} \mu \Psi \phi(\nu, \mu, n) d \mu
$$

for $-1<v<1$, where $N(v, n)$ is as given in (II.42). Thus, (B.32) is identical to (II.46).

Thus, in view of (B.28) and (B.22), Eq. (B.21) is seen to be

$$
\psi(\mu)=\sum_{ \pm n=1}^{m} A\left(\nu_{n}, n\right) \phi\left(\nu_{n}, \mu, n\right)+\int_{-\{}^{l} A(\nu, n) \phi(\nu, \mu, n) d v
$$

Equation (B.33), which is "the resolution of the identity" associated with the operator $k$, is actually the "full range completeness theorem," with the "expansion" (or "projection") coefficients $A(\nu, n)$ and $A(\nu, n)$ uniquely determined. 
APPENDIX C

ORTHOGONALITY PROPERTIES OF THE POLYNOMIALS $\phi_{n}(\nu, n)$ FOR INFINITE ORDER ANISOTROPY; PROPERTIES OF THE

$$
\text { DISCRETE EIGENVALUES } v_{j}(n)
$$

As we have mentioned in Section II.1.C, it is our purpose to show in this appendix that

(a) The discrete eigenvalues $v_{j}(n)$ enjoy the following properties:

1. are simple

2. occur in \pm pairs

3. are finite in number

4. are $\geq 1$ in absolute magnitude, i.e., no discrete eigenvalue can occur in the continuum, and the closest they can approach is the edge of the continuum

5. for $\mathrm{cg}_{0}(n) \leq 1$, are real for real $n$.

(b) The "completeness theorem," i.e., Eq. (II.43), holds even in the limit of "infinite order anisotropic scattering."

(c) The polynomials $\phi_{n}(v, n)$ are orthogonal, in the Stieltjes sense, over the interval $\left[-v_{1}, v_{1}\right]$, where $v_{1}=\max _{j} v_{j}$, and satisfy the orthogonality relations (II.5I), and

(d) Provide the reader with background information, to facilitate easy transition to the developments to be presented in Appendix $D$.

The proofs of $(a),(b)$, and (c) are essentially the sallie as those developed by Case ${ }^{16}$ for the simpler problem of monoenergetic transport since, as will be seen, the additional presence of the parameter $n$ does not alter the validity of Case's considerations. ${ }^{16}$. We shall therefore 
take advantage of this fact and restrict ourselves to merely presenting the basic ideas involved in the proofs, and referring the interested reader to Case's original work (Ref. 16) for additional details.

However, as we have mentioned in Section II.1.C, our proof of the finiteness of the number of discrete eigenvalues [i.e., property (a).3] is radically different from that of Case; ${ }^{16}$ thus, as a result of our investigations presented in Appendix $D$, we require that

$$
\sum_{n=0}^{\infty} n \ln n\left|g_{n}(n)\right|<\infty
$$

be satisfied, as opposed to Case's requirement

$$
\sum_{n=0}^{\infty} n^{2}\left|g_{n}\right|<\infty
$$

The starting point is

$$
(2 n+1) \gamma_{n}(n) v \phi_{n}(\nu, n)=(n+1) \phi_{n+1}(\nu, n)+n \phi_{n-1}(\nu, n)
$$

The equivalent form of the eigenvalue problem defined by (II.14), is to find those $v$ for which the solutions of (II.19) are bounded. The solutions which are merely bounded correspond to the continuum, while those $v_{j}$ for which there are square summable solutions are the discrete eigenvalues.

Introducing the quantities

$$
\Phi(\nu, n, n) \equiv\left[\frac{2 n+1}{2} \gamma_{n}\right]^{1 / 2} \phi_{n}(\nu, n)
$$


and

$$
A(n, n) \equiv n\left[(2 n+1)(2 n-1) \gamma_{n} \gamma_{n-1}\right]^{-1 / 2}
$$

such that $A(n, n)$ is real for real $n$, (II.19) becomes:

$$
A(n+1, n) \Phi(v, n+1, n)+A(n, n) \Phi(v, n-1, n)=v \Phi(v, n, n)
$$

In view of the convergence properties of the functions $g_{n}(n)$ investigated in Appendix $A$, we have

$$
\lim _{n \rightarrow \infty} A(n, n)=\frac{1}{2}
$$

Moreover,

$$
\lim _{n \rightarrow \infty} n \ln n[A(n, n)-A(\infty, n)]
$$

exists. [Note: (C.5) is again different from the requirement set forth by Case. ${ }^{16}$ ]

Then, for large $n \rightarrow \infty$, the solutions of Equation (C.3) will tend to solutions of

$$
\frac{1}{2}\{\tilde{\Phi}(\nu, n+1, n)-\tilde{\Phi}(\nu, n-1, n)\}=\nu \tilde{\Phi}(\nu, n, n)
$$

Two linearly independent solutions of (C.6) are

$$
\tilde{\Phi}(\nu, n, n)=z^{ \pm n}
$$

where 


$$
v=\frac{1}{2}\left(z+z^{-1}\right)
$$

Thus, for $z=e^{i \theta},(\theta=$ real $)$, all solutions of $(C .6)$ are bounded as $n \rightarrow \infty$. It follows that all solutions of (C.3) are bounded for

$$
\nu=\cos \theta \quad-1 \leq \cos \theta \leq 1
$$

and, in particular, solutions $\Phi(\nu, n, n)$ subject to the initial conditions (II.16) and (II.17), i.e.,

$$
\left.\begin{array}{l}
\Phi(\nu,-1, n)=0 \\
\Phi(\nu, 0, n)=\sqrt{\frac{\gamma_{0}}{2}}
\end{array}\right\}
$$

are bounded. Thus, with these initial conditions, the spectrum of (C.3) [or, alternately, of (II.19)] has a continuous part for $-1 \leq \nu<1$, confirming what we knew already. Next, following Case, we introduce two auxiliary solutions $\Phi^{ \pm}(\nu, n, n)$ of (c.3) defined by the boundary conditions

$$
\lim _{n \rightarrow \infty}\left|\Phi^{ \pm}(v, n, n)-z^{ \pm n}\right|=0 \text { for }\left\{\begin{array}{l}
|z| \leq 1 \\
|z| \geq 1
\end{array}\right.
$$

Furthermore, using (C.3) we define the functions $f_{+}(z)$ as

$$
f_{ \pm}(z)=A(0, n) \Phi^{ \pm}(\nu,-1, n)
$$

Case ${ }^{16}$ calls $f_{+}(z)$ the "Jost function" since $f_{+}(z)$. plays the same role for the orthogonal polynomials $\phi_{n}(\nu, n)$ as the function $f_{+}(k)$ does for scattering theory. It is seen from (C.3) and (C.12) that 


$$
f_{+}(z)=\frac{z+z^{-1}}{2} \Phi^{+}(z, 0, n)-A(1, n) \Phi^{+}(z, 1, n)
$$

Next, let $\Phi_{1}(\nu, n, n)$ and $\Phi_{2}\left(\nu^{-}, n, n\right)$ be two solutions of $(c .3)$ corresponding to $v$ and $v^{-}$, respectively. Then, the following Green type identity can be shown ${ }^{16}$ to hold:

$$
\begin{aligned}
& \left(v-v^{\prime}\right) \Phi_{1}(v, n) \Phi_{2}\left(v^{\prime}, n\right)=A(n+1)\left\{\Phi_{1}(v, n+1) \Phi_{2}\left(v^{\prime}, n\right)\right. \\
& \left.-\Phi_{1}(v, n) \Phi_{2}\left(v^{\prime}, n+1\right)\right\}-A(n)\left\{\Phi_{1}(v, n) \Phi_{2}\left(v^{\prime}, n-1\right)\right. \\
& \left.-\Phi_{1}(v, n-1) \Phi_{2}\left(v^{\prime}, n\right)\right\}
\end{aligned}
$$

With these preliminaries, propositions (a), (b) and (c) can now be easily proved:

(i) Let $\nu=v^{\prime}$, with $\nu$ in the continuum $(|z|=1)$. It is seen, from (C.14) that the "Wronskian"

$$
W\left[\Phi_{1}, \Phi_{2}\right]=A(n)\left\{\Phi_{1}(v, n) \Phi_{2}(v, n-1)-\Phi_{1}(v, n-1) \Phi_{2}(v, n)\right\}
$$

is independent of $n$. Thus, for $v$ in the continuum, the solution $\Phi(\nu, n)$ of (C.3) can be written as

$$
\Phi(\nu, n)=\frac{W\left[\Phi, \Phi^{-}\right]}{W\left[\Phi^{+}, \Phi^{-}\right]} \Phi^{+}(\nu, n)-\frac{W\left[\Phi, \Phi^{+}\right)}{W\left[\Phi^{+}, \Phi^{-}\right]} \Phi^{-}(\nu, n)
$$

But, in view of (C.11)

$$
W\left[\Phi^{+}, \Phi^{-}\right]=\frac{1}{2}\left(z-z^{-1}\right)
$$

On the other hand, from (C.10) and (C.13)

$$
W\left[\Phi, \Phi^{ \pm}\right]=\sqrt{\frac{r_{0}}{2}} f_{ \pm}
$$


Therefore, using (C.17) and (C.18) in (C.16) yields

$$
\begin{aligned}
\Phi(\nu, n)= & \frac{\sqrt{2 \gamma_{0}}}{\dot{z}-z^{-1}}\left[f_{-}(z) \Phi^{+}(\nu, n)-f_{+}(z) \Phi^{-}(\nu, n)\right] \\
& \text { for } \nu \in \text { continuum }
\end{aligned}
$$

Thus, for $z=e^{i \theta}, \Phi^{+}=\left(\Phi^{-}\right) *$ and $f_{-}=\left(f_{+}\right) \star(" * \|$ denotes complex conjugation with respect to $z$ ). In particular, when $z \rightarrow z^{-1}, \Phi$ is unchanged. Therefore, the continuum is characterized by $\nu=\cos \theta$, $0 \leq \theta \leq \pi$.

(ii) Let $v=v_{i}$, i.e., $v$ is a discrete eigenvalue. Then square summable solutions of (C.3), subject to the initial conditions ( $C .10)$ exist for those $z_{j}$, with $\left|z_{j}\right| \leq 1$, such that

$$
f_{+}\left(z_{i}\right)=0
$$

The corresponding $\nu_{i}$ are the discrete eigenvalues, with eigenfunctions

$$
\Phi\left(v_{j}, n, n\right)=\sqrt{\frac{r_{0}}{2}} \frac{\Phi^{+}\left(v_{i}, n\right)}{\Phi^{+}\left(v_{i}, 0\right)}
$$

Clearly, a discrete eigenvalue cannot occur in the continuum, since $f_{+}\left(z_{j}\right)=0$ implies $f_{-}\left(z_{i}\right)=0$ and therefore $\Phi\left(v_{i}, n\right) \equiv 0$ because of (c.19). The closest $v_{i}$ can get is at the edge of the continuum (i.e., $\left.v_{i}= \pm 1\right)$, since then the denominator of (C.19) also vanishes. Next, summing (C.14) from 0 to $N$, yields:

$$
\left(v-v^{-}\right) \sum_{0}^{N} \Phi_{1}(v, n) \Phi_{2}\left(v^{-}, n\right)=A(N+1)\left\{\Phi_{1}(v, n+1) \Phi_{2}\left(v^{-}, N\right)-\right.
$$




$$
\left.-\Phi_{1}(\nu, N) \Phi_{2}\left(\nu^{\prime}, N+1\right)\right\}-A(0)\left\{\Phi_{1}(\nu, 0) \Phi_{2}\left(\nu^{\prime},-1\right)-\Phi_{1}(\nu,-1) \Phi_{2}\left(\nu^{-}, 0\right)\right\}
$$

Let $v=v_{i}$ and $v^{\prime}=v_{i}^{*}$; also, let $N \rightarrow \infty$. The terms proportional to $A(0)$ vanish due to the initial conditions, while those proportional to $A(N+1)$ vanish as $N \rightarrow \infty$ in view of the square summability of the eigenfunctions, giving

$$
2 \operatorname{Im}\left\{v_{i}\right\} \sum_{n=0}^{\infty}\left|\Phi\left(v_{i}, n\right)\right|^{2}=0
$$

Hence, the eigenvalues are real, for real $n$.

Next, let in $(C .22) \Phi_{1}(v, n)=\Phi\left(v_{i}, n\right)$ and $\Phi_{2}\left(v^{-}, n\right)=\Phi^{+}\left(v^{-}, n\right)$. Letting $N \rightarrow \infty$ and subsequently passing to the limit $v^{-} \rightarrow v_{i}$ yields:

$$
\sum_{n=0}^{\infty}\left|\Phi\left(v_{i}, n\right)\right|^{2}=\left.\frac{\gamma_{0}}{2} \frac{1}{\Phi^{+}\left(v_{i}, 0\right)} \frac{d}{d \nu^{-}} f_{+}\left(v^{-}\right)\right|_{v^{-}=v_{i}}
$$

Relation (C.24) shows that the discrete eigenvalues are simple. Indeed, if the contrary were true, $\nu_{j}$ would be a multiple root of $f_{+}$ and (C.24) would imply $\Phi^{+}\left(\nu_{j}, 0\right)=0$. But $\Phi^{+}\left(v_{j}, 0\right)$ cannot be zero since (C.3) together with $f^{+}\left(z_{j}\right)=0$ and $\Phi^{+}\left(v_{j}, 0\right)=0$ would imply $\Phi^{+}\left(v_{j}, n\right) \equiv 0$, contradicting the definition (C.11).

To show that the number of discrete eigenvalues is finite, one first relates the dispersion function $\Lambda(\nu, n)$ to the Jost function $f_{+}(z)$. We have, per Case 16

$$
\Lambda(\nu, n)=\sqrt{\frac{2 \pi \gamma_{0}(n)}{z^{-2}-1}} f_{+}(z)
$$


The proof then reduces to showing that $f_{+}(z)$ is analytic for $|z| \leq 1$, except perhaps for a single pole at $z=0$. The proof of this constitutes the subject of Appendix $D$, leading to the requirement

$$
\sum_{0}^{\infty} n \ln n\left|g_{n}\right|<\infty
$$

Finally, following Case, 16 it can be shown that the polynomials $\phi_{n}(\nu, n)$ are orthogonal in the Stieltjes sense, satisfying the orthogonality relations:

$$
\begin{aligned}
& \frac{2 \delta_{k \ell}}{(2 \ell+1) \gamma_{\ell}}=\int_{-1}^{1} \phi_{k}(\nu, n) \phi_{\ell}(\nu, n) \frac{\nu}{N(v, n)} d \nu+ \\
& \quad+\Sigma_{j}\left[\phi_{k}\left(v_{j}, n\right) \phi_{\ell}\left(\nu_{j}, n\right) \frac{v_{j}}{N_{j+}}+\phi_{k}\left(-v_{j}, n\right) \phi_{\ell}\left(-v_{j}, n\right)\left(\frac{-v_{j}}{N_{j-}}\right]\right.
\end{aligned}
$$

and the closure relations (II.55-56). This, in turn, ensures the validity of the "completeness theorem" for the eigenfunction $\phi(\nu, \mu, n)$ for arbitrary anisotropic scattering, to all orders. 


\section{APPENDIX D \\ CONVERGENCE CONDITION FOR THE FUNCTIONS $g_{n}(n)$}

In this appendix, we shall derive a sufficient convergence condition to be satisfied by the functions $g_{n}(n)$ in order to ensure the finiteness of the number of discrete eigenvalues $v_{j}(n)$. In this sense, this appendix is complementary to Appendix C. Indeed, the starting point of our derivations to be presented here is provided by Case's work, Ref. 16, part of which was presented in Appendix $C$ with the appropriate modifications to suit our purposes.

Following Case, ${ }^{16}$ we start with the equation

$$
\begin{gathered}
v^{+}(v, n, n)-a^{0}(n+1) \Psi^{+}(v, n+1, n)-a^{0}(n) \Psi^{+}(v, n-1, n)= \\
=v c g_{n}(n) \Psi^{+}(v, n, n)
\end{gathered}
$$

where

$$
\Psi^{+}(\nu, n, n) \equiv \Phi^{+}(v, n, n) / \sqrt{\gamma_{n}(n)}
$$

with $\Phi^{+}(\nu, n, n)$ as defined in Appendix $C$, and

$$
a^{0}(n) \equiv \frac{n}{\sqrt{4 n^{2}-1}}
$$

Along with Case, 16 we require that $\psi^{+}$satisfy the boundary condition

$$
\lim _{n \rightarrow \infty}\left|\Psi^{+}(v, n)-z^{n}\right|=0 \text { for }|z| \leq 1
$$

where 


$$
v=\frac{1}{2}\left(z+z^{-1}\right)
$$

(Compare with the similar B.C. satisfied by $\Phi^{+}$, cf. Appendix C.) We notice that (D.5) defines the conformal mapping:
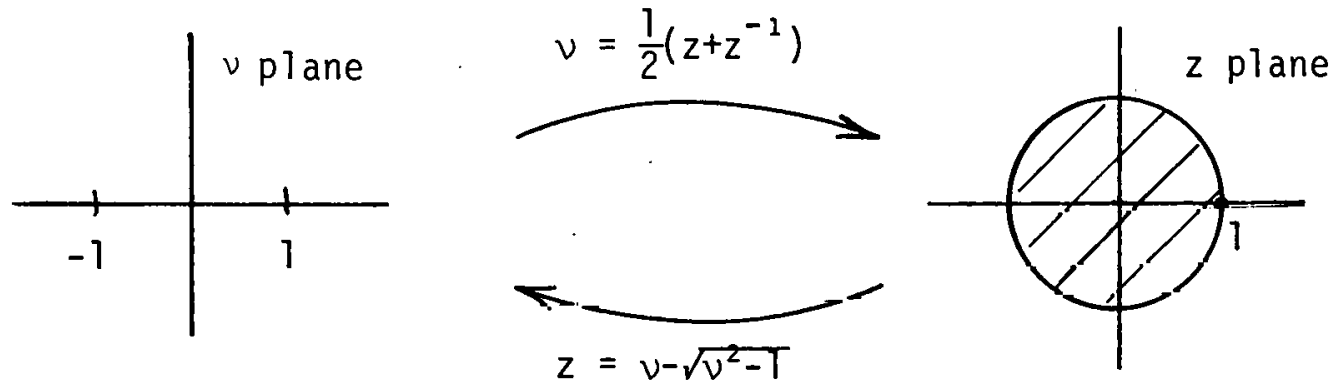

i.e., the entire $v$ plane, cut along $[-1,1]$ is mapped onto the open unit disk $K:|z|<1$, while the unit circle $\Gamma:|z|=1$ is the image of the cut $\nu \in[-1,1]$ traversed twice.

Following Case, 16 we introduce a Green's function defined by:

$$
G(v, n ; m)=\left\{\begin{array}{l}
0 \quad \text { for } n \geq m \\
\frac{\sqrt{2}}{f_{0}^{+}(z)}\left[\Psi_{0}^{+}(m) \Psi_{0}(n)-\Psi_{n}(m) \Psi_{0}^{+}(n)\right] ; n \leq m
\end{array}\right.
$$

where $\psi_{0}$ and $\psi_{0}^{+}$are the "regular" and " + " solutions of $(0.1)$ with $c=0$; explicitly

$$
\begin{aligned}
& \Psi_{0}(n)=\sqrt{\frac{2 n+1}{2}} P_{n}(\nu) \\
& \Psi_{0}^{+}(n)=\sqrt{2 n+1} f_{0}^{+}(z) Q_{n}(\nu)
\end{aligned}
$$

In (D.7) and (D.8), $P_{n}$ are the Legendre Polynomials, $Q_{n}$ are the Legendre Functions of the second kind, and 
$f_{0}^{+}(z)=\sqrt{\frac{z^{-2}-1}{2 \pi}}$

Then, according to Case, ${ }^{16}$ the solution $\psi^{+}$can be written as:

$$
\psi^{+}(v, n, n)=\psi_{0}^{+}(v, n)+\sum_{m=n+1}^{\infty} G(v, n ; m) v c g_{m}(n) \psi^{+}(v, m, n)
$$

Equation (D.10) is solved by iteration; following Case, 16 we have

$$
\Psi^{+}(v, n, n)=\sum_{i=0}^{\infty} \Psi^{+(i)}(v, n, n)
$$

where

$$
\Psi^{+(0)}(v, n)=\Psi_{0}^{+}(v, n)
$$

and

$$
\psi^{+(i)}(v, n)=\sum_{m=n+1}^{\infty} G(v, n ; m) v c g_{m} \psi^{+(i-1)}(v, m)
$$

At this stage, our program departs radically from that of Case. For convenience, we shall briefly summarize:

\section{Case's procedure:}

(i) Show that the series in (D.11) converges "uniformly" [uniformity is not actually proven], provided that $\sum_{0}^{\infty} \sqrt{m}\left|f_{m}\right|<\infty$ [where the $f_{m}$ are the equivalents of our $g_{m}(n)$, for monoenergetic transport], for $|z|<1$.

(ii) Show that the series of derivatives of (D.11) converges, provided that $\sum_{0}^{\infty} m^{3 / 2}\left|f_{m}\right|<\infty$, for $|z|<1$, so that analyticity of $\Psi^{+} \cdot(\nu, n)$ within the unit circle results. 
(iii) Finally, Case ${ }^{16}$ requires $\sum_{0}^{\infty} m^{2}\left|f_{m}\right|<\infty$ for continuity on the unit circle $\Gamma$, thus ensuring, in his opinion, the finiteness of the number of zeros $z_{i}$, of $f_{+}(z)$. Recall that

$$
f_{+}(z)=\frac{z+z^{-1}}{2} \Phi^{+}(z, 0, n)+A(1, n) \Phi^{+}(z, 1, n)
$$

\section{Our program}

In contrast to Case's procedure, we shall make use of the following sequence of steps:

Step 1: Apply the following Theorem (Ref. 68, Vol. I, p. 329): "Suppose every term of the series

$$
\sum_{i=i}^{\infty} h_{i}(z)
$$

is analytic on a domain $G$ and continuous on $\bar{G}$. Then the series is uniformly convergent on $\Gamma$, the boundary of $G . "$ [This theorem will be used with $\left.h_{j}(z) \equiv \Psi^{+(i)}(v, n):\right]$

Step 2: Apply Weierstrass' theorem on uniformly convergent series of analytic functions, i.e., "If the series

$$
\sum_{i=1}^{\infty} h_{j}(z)=h(z)
$$

is uniformly convergent on every compact subset of a domain $G$, and if every term $h_{i}$ is analytic on $G$, then the $\operatorname{sum} h(z)$ is also analytic on G. Moreover, the series $(B)$ can be differentiated term by term any number of times, i.e., $\sum_{i=1}^{\infty} h_{i}^{(k)}(z)=h^{(k)}(z), k=1,2$, etc., for all $z \in G$, 
and each differentiated series is uniformly convergent on every compact subset of G" (Ref. 68, op. cit., p. 326). [Note: in our application, $h_{j}(z) \equiv \psi^{+(i)}$, and $\left.h(z) \equiv \psi^{+}(\nu, n).\right]$

Step 3: Apply the following Theorem (Ref. 68, p. 371): "If $h(z) \equiv 0$ is analytic on a domain $G$, there can be no limit point of zeros of $h(z)$ in G." [This theorem will be applied to $f_{+}(z)$.]

Step 4: It will be shown that $f_{+}(z)$ satisfying Step 3 , has no accumulation points on the boundary of $G$, either. As a consequence, there are only a finite number of zeros of $z_{j}$, of $f_{+}(z)$, and therefore only a finite number of related discrete eigenvalues $\nu_{j}$.

We now proceed with the actual proofs: Using (D.7) and (D.8), we rewrite $G(\nu, n ; m)$ as

$$
G(\nu, n ; m)=\left\{\begin{array}{l}
0 \quad ; \quad n \geq m \\
\sqrt{(2 n+1)(2 m+1)}\left[Q_{m}(\nu) P_{n}(\nu)-P_{m}(\nu) Q_{n}(\nu)\right] ; n<m
\end{array}\right.
$$

\section{Step 1}

Our domain is the unit disk $K:|z|<1$, with boundary $\Gamma:|z|=1$. Within $|z|<1$ \{i.e., $\vee \notin[-1,1]\}$, we have ${ }^{17}$

$$
\left|Q_{n}(v)\right|<\frac{|z|^{n+1}}{[1-|z|]^{1 / 2}} \sqrt{\frac{\pi}{n}} \quad ; \quad|z|<1
$$

Also, for $|z|<1$

$$
\left|P_{n}(z)\right|<\frac{|z|^{-n}}{\sqrt{2 n+1}} \sqrt{\frac{4}{\pi}} \frac{1}{\sqrt{|1-z||1+z|\left(1-|z|^{2}\right)}}
$$

Note: Inequality (D.16) follows trivially from Eq. (7.1.9) of Ref. 56. 
Therefore, taking into account (D.9) and (D.15), we have from (D.8):

$$
\begin{aligned}
\left|\Psi_{0}^{+}(z, n)\right| & <\sqrt{2 n+1} \frac{1}{|z|} \sqrt{\frac{1-z^{2}}{2 \pi}}\left(\frac{\pi}{n}\right) \frac{|z|^{n+1}}{\sqrt{1-|z|^{2}}} \\
& \leq c_{0}|z|^{n}\left(\frac{1-z^{2}}{1-|z|^{2}}\right)^{1 / 2}
\end{aligned}
$$

where $C_{0}$ is a positive constant.

Thus, from (D.13), we have

$$
\left|\Psi^{+(i)}(v, n)\right| \leq \sum_{m=n+1}^{\infty}|G(v, n ; m)|\left|c g_{m}\right|\left|\psi^{+(i-1)}(v, m)\right|
$$

and, in particular

$$
\left|\Psi^{+(1)}(\nu, n)\right| \leq \sum_{m=n+1}^{\infty}|G(v, n ; m)|\left|\mathrm{cg}_{m}\right|\left|\Psi_{0}^{+}(\nu, m)\right|
$$

However, taking into account (D.15) and (D.16), we obtain from (D.14):

$$
\begin{aligned}
|G(v, n ; m)| & <\sqrt{(2 n+1)(2 m+1)}\left|\frac{z^{2}+1}{2 z}\right|\left\{\left|P_{m}\right|\left|Q_{n}\right|+\left|P_{n}\right|\left|Q_{m}\right|\right\} \\
& \leq C_{1}|z|^{n-m} \frac{1}{\left(1-|z|^{2}\right) \sqrt{\left|1-z^{2}\right|}}
\end{aligned}
$$

It. then follows from (D.19), (D.17) and (D.20) that

$$
\left|\Psi^{+(1)}(\nu, n)\right|<C_{0}\left(\frac{1-z^{2}}{1-|z|^{2}}\right)^{1 / 2} \frac{c_{1}|z|^{n}}{\left(1-|z|^{2}\right) \sqrt{\left|1-z^{2}\right|}} \sum_{m=n+1}^{\infty}\left|c g_{m}\right|
$$


Next, from (D.21) and (D.18), for $i=2$, we obtain

$$
\begin{aligned}
\left|\Psi^{+(2)}(\nu, n)\right| & <c_{0}\left(\frac{1-z^{2}}{1-|z|^{2}}\right)^{1 / 2}\left[\frac{c_{1}}{\left(1-|z|^{2}\right)\left|1-z^{2}\right|^{1 / 2}}\right]^{2}|z|^{n} \times \\
& \times \sum_{m=n+1}^{\infty}\left|c g_{m}\right| \sum_{m^{\wedge}=m+1}^{\infty} \mid c g_{m^{-1}}= \\
& =c_{0}\left(\frac{1-z^{2}}{1-|z|^{2}}\right)^{1 / 2}\left[\frac{c_{1}}{\left(1-|z|^{2}\right)\left|1-z^{2}\right|^{1 / 2}}\right]^{2} \frac{|z|^{n}}{2 !}\left(\sum_{m=n+1}^{\infty}\left|c g_{m}\right|\right)^{2}
\end{aligned}
$$

Continuing this process, we are led to

$$
\left|\Psi^{+(i)}(v, n)\right|<c_{0}\left(\frac{1-z^{2}}{1-|z|^{2}}\right)^{1 / 2}\left[\frac{c_{1}}{\left(1-|z|^{2}\right)\left|1-z^{2}\right|^{1 / 2}}\right]^{i} \frac{|z|^{n}}{i !}\left(\sum_{m=n+1}^{\infty}\left|c g_{m}\right|\right)^{i}
$$

Thus, the sequence $\left\{\Psi^{+(i)}(\nu, n)\right\}$ is uniformly bounded on every compact subset of $K$, if

$$
\sum_{n=1}^{\infty}\left|\operatorname{cg}_{n}\right|<\infty
$$

Moreover, each of the terms in the sum (D.13) is analytic within K. We therefore conclude that $\Psi^{+(i)}(\nu, n)$ is analytic on $K$, provided (D.24) is satisfied.

We now turn our attention to the behavior of $\psi^{+(i)}(\nu, n)$ on the boundary $r:|z|=1$. Here

$$
z=e^{i \theta}
$$

or

$$
\nu=\frac{1}{2}\left(z+z^{-1}\right)=\cos \theta
$$


We begin by investigating the behavior of $G(\cos \theta, n ; m)$ on $\Gamma:|z|=1$. Using the well-known relation 17

$$
Q_{n}(v)=P_{n}(v) Q_{0}(v)-W_{n-1}(v)
$$

where

$$
w_{n-1}(v)=\sum_{k=1}^{n} \frac{1}{k} P_{k-1}(v) P_{n-k}(v)
$$

with

$$
W_{-1} \equiv 0
$$

we obtain from (D.14)

$$
G(v, n ; m)=\left\{\begin{aligned}
\sqrt{(2 n+1)(2 m+1)}\left[P_{m}(v) W_{n-1}(v)-P_{n}(v) W_{m-1}(v)\right] \\
m>n \\
0 \quad m \leq n .
\end{aligned}\right.
$$

A crude estimate of the magnitude of $G(\cos \theta, n ; m)$ can be nhtained immediately, as follows:

It is well known that 17

$$
\left|P_{n}(\cos \theta)\right| \leq 1
$$

so that

$$
\left|W_{n}(\cos \theta)\right| \leq \sum_{k=1}^{n} \frac{1}{k}\left|P_{k-1}(\cos \theta)\right|\left|P_{n-k}(\cos \theta)\right|<\sum_{k=1}^{n} \frac{1}{k}
$$

But, it can be easily shown that 


$$
\ln (n+1) \leq \sum_{k=1}^{n} \frac{1}{k}<1+\ln n
$$

by taking the logarithm of the well-known inequality $e^{x}>\left(1+\frac{x}{y}\right)^{y}>e \frac{x y}{x+y}$, where $x=1$ and $y=k$, and summing from 1 to $n$. Therefore, in view of (D.31) and (D.33), we obtain from (D.30)

$$
|G(\cos \theta, n ; m)|<C_{2} m \text { en } m ;
$$

where $C_{2}$ is a positive constant since always $m>n$. (At this point, we wish to conjecture that it might be possible to even prove that

$$
|G(\cos \theta, n ; m)|<C_{3} m,
$$

but further work is needed to investigate some delicate estimation problems.)

We can now investigate the behavior of $\Psi_{0}^{+}\left(e^{i \theta}, n\right)$, defined in (D.8). It is well known that: 56

$$
(\sin \theta)^{1 / 2}\left|Q_{n}(\cos \theta)\right|<\left(\frac{\pi}{2 n}\right)^{1 / 2} \text { for } 0<\theta<\pi
$$

Therefore, on $\Gamma:|z|=1$,

$$
\left|\psi_{0}^{+}\left(e^{i \theta}, n\right)\right|=\sqrt{2 n+1}\left|\sqrt{\frac{e^{-2 i \theta}-1}{2 \pi}}\right|\left|Q_{n}(\cos \theta)\right|<c_{4}
$$

where $C_{4}$ is some positive constant. This holds even at $\theta=0$, $\pi$ since $\left[f_{0}^{+}\left(e^{i \theta}\right) Q_{n}(\cos \theta)\right]$ vanishes at these points.

From (D.19), (D.34) and (D.37), it follows that on $\Gamma:|z|=1$,

$$
\left|\Psi^{+(1)}(\cos \theta, n)\right|<C_{2} C_{4} \sum_{m=n+1}^{\infty} m \sin m\left|c g_{m}\right|
$$


Similarly,

$$
\left|\Psi^{+(2)}(\cos \theta, n)\right|<C_{4} \frac{C_{2}^{2}}{2 !}\left(\sum_{m=n+1}^{\infty} m \text { en } m\left|c g_{m}\right|\right)^{2}
$$

and, in general

$$
\left|\Psi^{+(i)}(\cos \theta, n)\right|<C_{4} \frac{C_{2}^{i}}{i !}\left(\sum_{m=n+1}^{\infty} m \text { ln } m\left|c g_{m}\right|\right)^{i} \equiv M_{i}
$$

Therefore, the functions $\psi^{+(i)}$ are continuous on $\Gamma$, provided that

$$
\sum_{m=1}^{w} m \ln m\left|g_{m}\right|<\infty
$$

The last condition that has to be fulfilled in order to apply the Theorem of Step 1, is to show that

$$
\sum_{i=1}^{\infty} \Psi^{+(i)}(\cos \theta, n)
$$

is uniformly convergent on $\Gamma$. This can be easily shown by employing Weierstrass' $M$ test ${ }^{68}$ since the series $\sum_{i=1}^{\infty} M_{i}$ [where $M_{i}$ are defined in (D.40)] is convergent by virtue of d'Alembert's test, i.e.,

$$
\lim _{j \rightarrow \infty} \frac{M_{j+1}}{M_{i}}=\lim _{j \rightarrow \infty} \frac{C_{2}}{i+1}\left(\sum_{m=n+1}^{\infty} m \text { en } m\left|c g_{m}\right|\right)=0
$$

since we required (D.41) to be satisfied. Thus, the series (D.42) is uniformly convergent, and therefore the theorem of Step 1 is satisfied. We can now proceed to Step 2 and use Weierstrass theorem on the uniformly convergent series of analytic functions $\psi^{+(i)}(\nu, n)$. The consequence of this is that the function 


$$
\psi^{+}(v, n)=\sum_{i=0}^{\infty} \psi^{+(i)}(v, n)
$$

is analytic on the open unit disk $k$. It then follows that

$$
f_{+}(z) \equiv \frac{z+z^{-1}}{2} \sqrt{\gamma_{0}} \Psi^{+}(z, 0)-\frac{1}{\sqrt{3 \gamma_{0}}} \Psi^{+}(z, 1)
$$

is analytic on $K$, except perhaps a simple pole at $z=0$.

Therefore, in view of the Theorem of Step $3, f_{+}(z)$ has no accumulation point of zeros in $K$. Conceivably, there could be an accumulation point of zeros on the boundary $\Gamma$. But this would require $f_{+}(z)$ to have an essential singularity on $\Gamma$, implying that $\psi^{+}$has an essential singularity on $\Gamma$. But $\psi^{+}$cannot have an essential singularity on $\Gamma$ since, in view of $(0.40)$

$$
\left|\Psi^{+}(\cos \theta, n)\right|<C_{4} \exp \left[C_{2} \sum_{m=n+1}^{\infty} m \text { en } m\left|c g_{m}\right|\right]
$$

i.e., $\Psi^{+}(\cos \theta, n)$ is bounded in view of $(0.41)$. This contradiction therefore proves that $f_{+}(z)$ can have at most a finite number of zeros $z_{i} \cdot$

We therefore conclude that the discrete eigenvalues $v_{i}$, related to $z_{j}$ via (D.5) are finite in number, provided that

$$
\sum_{m=1}^{\infty} m \ln m\left|g_{m}\right|<\infty
$$

$$
\text { Q.E.D. }
$$


As a final note, we remark that, if (D.35) is satisfied, considerations of an identical nature like above, would have led to $\sum_{m=1}^{\infty} m\left|g_{m}\right|<\infty$ as a sufficient condition for the finiteness of the number of discrete eigenvalues. This would be precisely Inönü's condition, which he required on a rather empirical basis. 
APPENDIX E

LISTING OF THE "EIGENVALUE-SPACE" MOMENTS $m_{2 n}(n)$, FOR $n=0$ TO 9 , AS GENERATED BY FORMAC

A listing (as produced by FORMAC), of $m_{2 n}\left(\equiv m_{2 n}(n)\right)$ from $m_{4}$ to $\mathrm{m}_{18}$, is presented.

The results are in the form of "equalities" (i.e., $m_{2 n}$ =expression), that should be read in the usual manner, i.e., from left to right and top to bottom. The dashed lines separating successive lines of formulae are part of the FORMAC output editing.

The FORMAC symbols appearing in this enclosure should be interpreted as follows:

1. $N$ stands for $n$ (i.e., the index of $m_{2 n}$ )

2. $N 2=2 n$

3. MOM stands for $m$; e.g., $\operatorname{MOM}(0) \equiv m_{0}$ and $\operatorname{MOM}(12) \equiv m_{12}$.

4. $W(J)$, where $J=0,1,2$, etc., stands for $W_{j}(j=0,1$, etc. $)$;

$3 w(1)^{2} \equiv 3 w_{1}^{2}$

Note that: (a) whenever a quantity is raised to a power, that power appears as shown in (4) above.

(b) no multiplication symbol appears; quantities "following" one another should be interpreted as multiplying each other, e.g.,

$3 W(1)^{2} W(0)^{2} \equiv 3 W_{1}^{2} W_{0}^{2}$ 
(c) the dependence of $m_{2 n}$ and $w_{j}$ on $n$ is not explicitly shown.

In order to facilitate the interpretation of the FORMAC results, we give below the explicit expressions for the first five even moments.

$$
\begin{aligned}
m_{0}= & 2 / r_{0}(n) \\
m_{2}= & m_{0} W_{0} \\
m_{4}= & m_{0}\left(W_{1} W_{0}+W_{0}^{2}\right) \\
m_{6}= & m_{0}\left(W_{2} W_{2} W_{0}+W_{1}^{2} W_{0}+2 W_{1} W_{0}^{2}+W_{0}^{3}\right) \\
m_{8}= & m_{0}\left(W_{3} W_{2} W_{1} W_{0}+W_{2}^{2} W_{1} W_{0}+2 W_{2} W_{1}^{2} W_{0}+W_{1}^{3} W_{0}+2 W_{2} W_{1} W_{0}^{2}+\right. \\
& \left.\quad+3 W_{1}^{2} W_{0}^{2}+3 W_{1} W_{0}^{3}+W_{0}^{4}\right) \\
m_{10}= & m_{0}\left(W_{4} W_{3} W_{2} W_{1} W_{0}+W_{3}^{2} W_{2} W_{1} W_{0}+2 W_{3} W_{2}^{2} W_{1} W_{0}+W_{2}^{3} W_{1} W_{0}+\right. \\
& \quad+2 W_{3} W_{2} W_{1}^{2} W_{0}+3 W_{2}^{2} W_{1}^{2} W_{0}+3 W_{2} W_{1}^{3} W_{0}+W_{1}^{4} W_{0}+ \\
& \quad+2 W_{3} W_{2} W_{1} W_{0}^{2}+2 W_{2}^{2} W_{1} W_{0}^{2}+6 W_{2} W_{1}^{2} W_{0}^{2}+4 W_{1}^{3} W_{0}^{2}+ \\
& \left.\quad+3 W_{2} W_{1} W_{0}^{3}+6 W_{1}^{2} W_{0}^{3}+4 W_{1} W_{0}^{4}+W_{0}^{5}\right)
\end{aligned}
$$

Computer listings follow. 


\section{DEN1OOI DENONT UNABLE TO OPEN SYSUT 1}

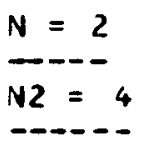

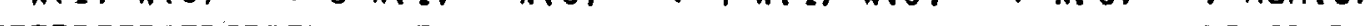


$N=6$
$N 2=12$

$M O M(12)=1 H(3) W(2) W(1) W(0) W(5) W(4)+2 W(3)^{2} W(2) W(1) W(0) W(4)$

2

$+2 W(3) W(2)^{2} W(1) W(0) W(4)+2 W(3) w(2) w(1)^{2} w(0) w(4)+2 W(3)$

2

$W(2) W(1) w(0)^{2} W(4)+w(3)^{3} w(2) w(1) w(0)+3 w(3)^{2} w(2)^{2} w(1) w(0$

-

$1+3 W(3) W(2)^{3} w(1) W(0)+W(2)^{4} W(1) W(0)+2 W(3)^{2} w(2) w(1)^{2} w($

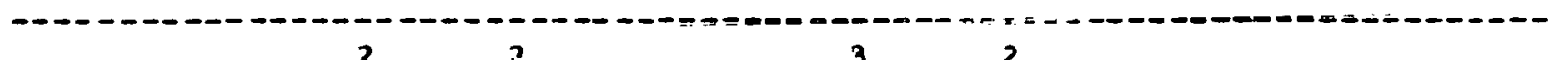
$0)^{+} 6 W(3) W(2)^{2} H(1)^{2} w(0)+4 W(2)^{3} w(1)^{2} H(0)+3 w(3) w(2) w(1)$

-

$W(0)+6 W(2)^{2} W(1)^{3} w(0)+4 H(2) W(1)^{4} w(0)+w(1)^{5} w(0)+W(3)$

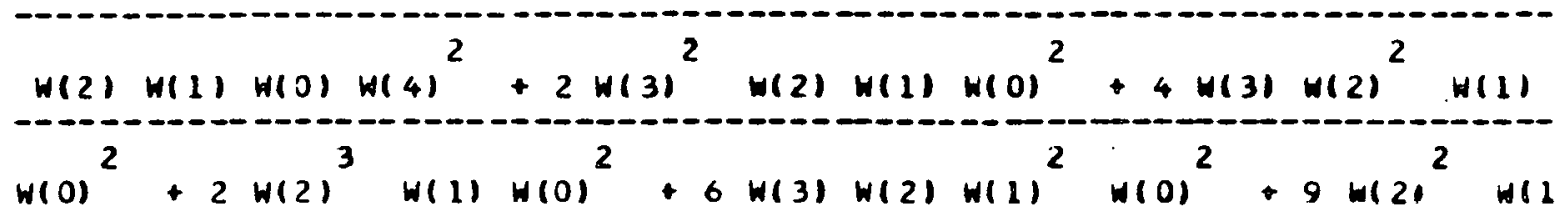

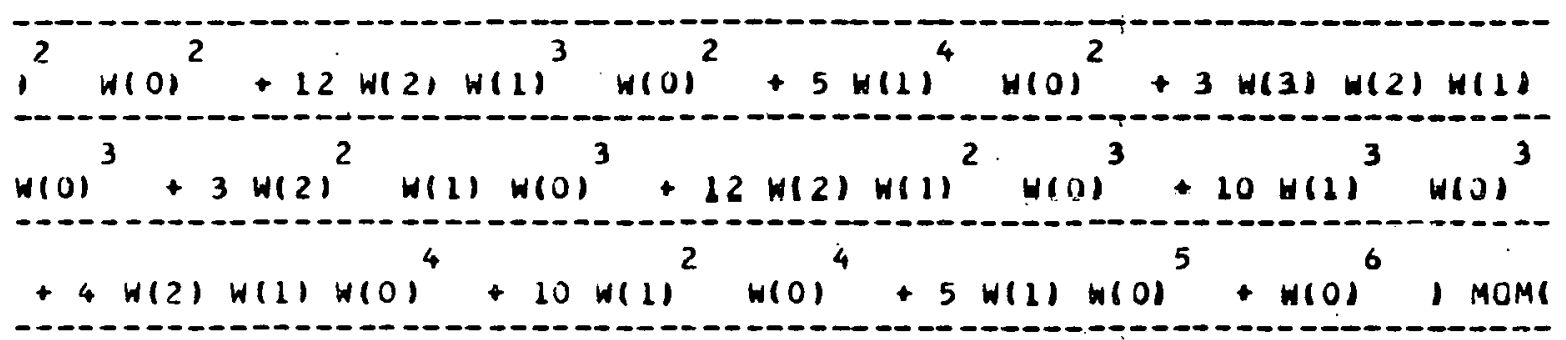

$0)$

$--$

$N=7$

N-D-

$N 2=14$

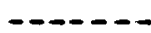

MOM(14) $1 W(3) W(2) W(1) W(0) W(6) W(5) W(4)+2 W(3)^{2} H(2) W(1) W(0)$ МММ

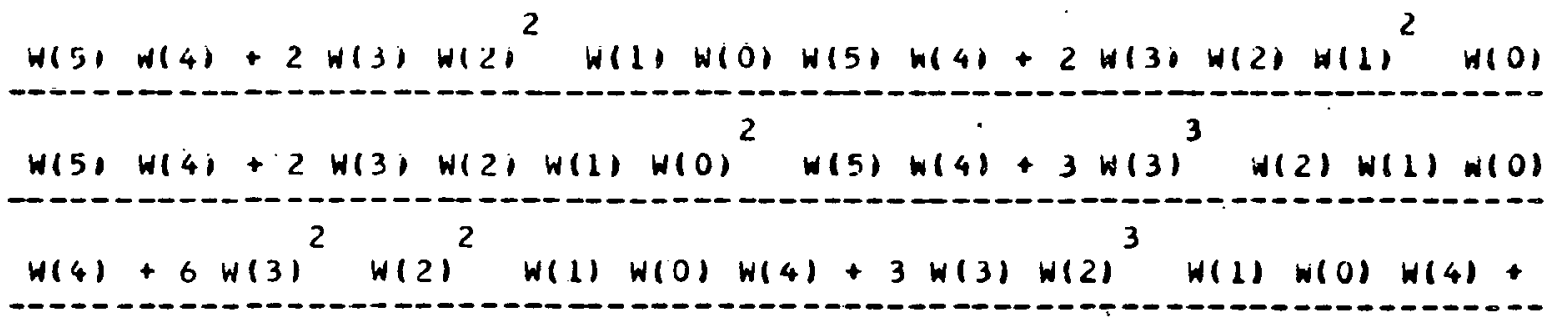


$4 w(3)^{2} w(2) w(1)^{2} w(0) w(4)+6 w(3) w(2)^{2} w(1)^{2} w(0) w(4)+3 w(3)$

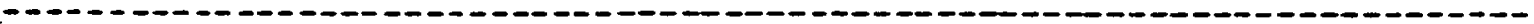

32.2

$w(2) W(1)^{3} w(0) w(4)+w(3) w(2) w(1) w(0) w(5)^{2} w(4)+4 w(3)^{2} w(2)$

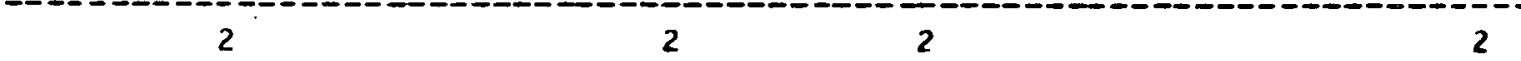

$W(1) W(0)^{2} H(4)+4 W(3) H(2)^{2} W(1) H(0)^{2} W(4)+6 H(3) W(2) W(1)^{2}$

2

$H(0)^{2} W(4)^{\circ}+3 w(3) W(2) W(1) w(0)^{3} w(4)+W(3)^{4} W(2) W(1) W(0)+4 W$

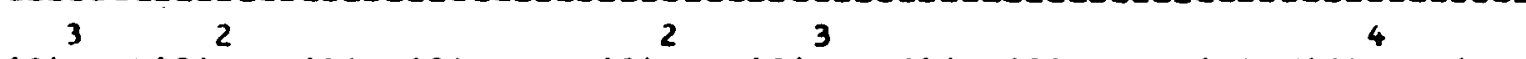
$(3)^{2} w(2)^{2} w(1) w(0)+6 w(3)^{2} w(2)^{3} w(1) H(0)+4 H(3) H(2) W(1)$ $w(0)+w(2)^{5} w(1) w(0)+2 w(3)^{3} w(2) w(1)^{2} w(0)+9 w(3)^{2} w(2)^{2} w 1$ 2

$11^{2} W(0)+12 H(3) W(2)^{3} w(1)^{2} w(0)+5 W(2) w(1)+W(0)+3 w(3)$

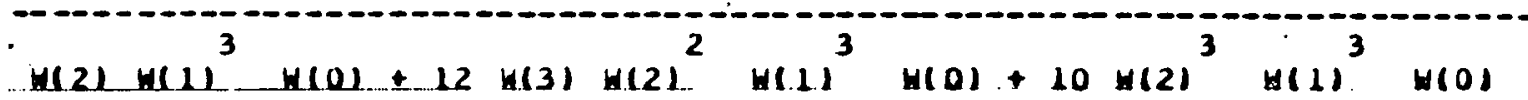
(1) +H(3)H(2)H(1) $)^{4} w(0)+10 H(2)^{2} w(1)^{4} w(0)+5 H(2) H(1)^{5} w(0)$ $+A(1)^{6} W(0)+2 W(3) W(2) W(1) w(0) W(5) w(4)^{2}+3 W(3)^{2} w(2) W(1)$ $w(0) H(4)^{2}+2 W(3) w(2)^{2} w(1) w(0) w(4)^{2}+2 w(3) w(2) w(1)^{2} w(0) w$ 2 $(4)^{2}+2 W(3) W(2) W(1) W(0)^{2} W(4)^{2}+W(3) W(2) W(1) W(0) W(4)^{3}+2$ $w(3)^{3} w(2) w(1) w(0)^{2}+6 w(3)^{2} w(2)^{2} w(1) w(0)^{2}+6 w(3) w(2)^{3} w 1$ $\mathrm{W}$ $11 w(0)^{2}+2 w(2)^{4} w(1) w(0)^{2}+6 w(3)^{2} w(2) w(1)^{2} w(0)^{2}+18 w(3)$

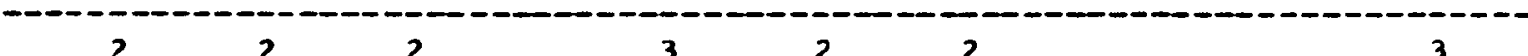
$W(2)^{2} w(1)^{2} w(0)^{2}+12 W(2)^{3} w(1)^{2} w(0)^{2}+12 W(3) \omega(2) W(1)^{3} w 1$ $0)^{2}+24 W(2)^{2} w(1)^{3} w(0)^{2}+20 w(2) w(1)^{4} w(0)^{2}+6 w(1)^{5} w(0)^{2}$ $+3 w(3)^{2} w(2) w(1) w(0)^{3}+6 w(3) w(2)^{2} w(1) w(0)^{3}+3 w(2)^{3} w(1)$

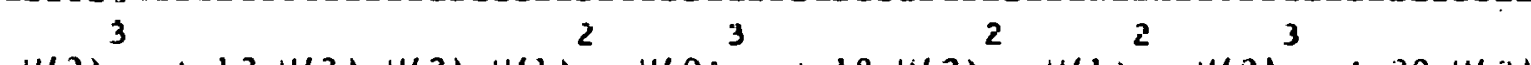
$W(0)+12 W(3) W(2) w(1)^{2} w(0)+18 w(2)^{2} w(1)^{2} w(0)^{3}+30 w(2)$ $w(1)^{3} w(0)^{3}+15 W(1)^{4} w(0)^{3}+4 W(3) W(2) W(1) w(0)^{4}+4 W(2)^{2} w$ - W $(1) w(0)^{4}+20 w(2) w(1)^{2} w(0)^{4}+20 w(1)^{3} w(0)^{4}+5 w(2) w(1) w(0)$ (1) 5. $\left.+15 W(1)^{2} w(0)^{5}+6 H(1) W(0)^{6}+W(0)^{7}\right) \mathrm{MCH}(0)$ 
$\mathbf{N}=8$

$---$

$N 2=16$

$M 04(16)=(W(3) W(2) W(1) W(0) W(7) W(6) W(5) W(4)+2 W(3) W(2) W(1)$ $H(J) W(6) W(5) W(4)+2 W(3) W(2)^{2} W(1) W(0) W(6) W(5) W(4)+2 W(3) W$ Н 2 (2) $(1,9) 2$

(2)W(1) W(0)W(6)W(5)W(4)+2W(3)W(2)W(1)W(0) W(6)W(5)W(4)

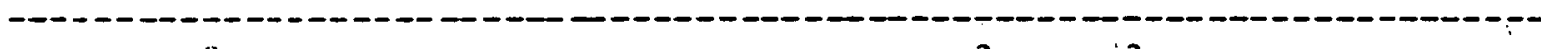
$+3 w(3)^{3} w(2) W(1) w(0) W(5) w(4)+6 w(3)^{2} W(2)^{2} W(1) W(0) w(5)^{2} w$

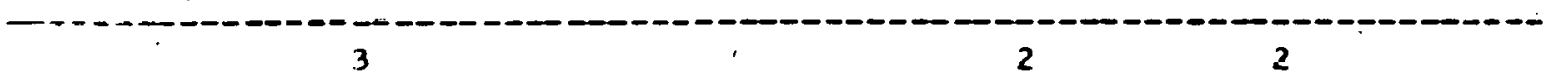
$4)^{+} 3 W(3) W(2)^{3} W(1) W(0) W(5) W(4)+4 W(3)^{2} H(2) W(1)^{2} W(0) W(5)$

41 \% $\mathrm{W}$ W $\mathrm{W}$ ( )
$W(4)+6 W(3) W(2)^{2} W(1)^{2} W(0) W(5) w(4)+3 W(3) W(2) W(1)^{3} W(0) W(5$ $1 N(4)+N(3) W(2) W(1) W(0) W(6)^{2} w(5) w(4)+4 W(3)^{2} w(2) W(1) W(0)$ $W(5) W(4)+4 W(3) W(2)^{2} w(1) W(0)^{2} w(5) W(4)+6 W(3) W(2) W(1)^{2}$ W( $0^{2} W(5) W(4)+3 W(3) W(2) W(1) W(0)^{3} W(5) W(4)+4 W(3)^{4} W(2) W(1)$ O) W(5)W(4)+3W(3)W(2)W(1)W(0) W(5)W(4)+4W(3) W(2)W(1) $W(0) W(4)+12 W(3)^{3} W(2)^{2} W(1) W(0) W(4)+12 W(3)^{2} W(2)^{3} w(1) W(0)$ $W(t)+4 W(3) w(2)^{4} w(1) W(0) W(4)+6 W(3) w(2) W(1)^{2} w(0) w(4)+$ $13 w(3)^{2} w(2)^{2} w(1)^{2} w(0) w(4)+12 W(3) w(2)^{3} w(1)^{2} w(0) W(4)+6$

-
$w(3)^{2} w(2) w(1)^{3} w(0) w(4)+12 w(3) w(2)^{2} w(1)^{3} w(0) w(4)+4 w(3)$

4

$W(2) W(1)^{4} W(0) w(4)+2 W(3) W(2) W(1) W(0) W(6) W(5)^{2} W(4)+2 W(3)$

W(2) W(1
$W(2) W(1) W(0) W(5)^{2} w(4)+2 W(3) W(2)^{2} w(1) W(0) W(5)^{2} W(4)+2 W($ $3)^{2} W(2) W(1)^{2} H(0) W(3)^{2} w(4)+2 W(3) H(2) W(1) W(0)^{2} W(5)^{2} W(4)^{+}$ $w(3) W(2) W(1) W(0) W(5)^{3} w(4)^{\circ}+6 W(3)^{3} w(2) w(1) w(0)^{2} w(4)+12 w 1$ $3)^{2} w(2)^{2} W(1) W(0)^{2} W(4)+6 W(3) w(2)^{3} W(1) W(0)^{2} W(4)+12 W(3)^{2}$ $w(?) W(1)^{2} w(0)^{2} W(4)+18 w(3) W(2)^{2} W(1)^{2} w(0)^{2} w(4)+12 W(31 W$ 3 $(2) W(1)^{2} W(0)^{2} W(4)+6 W(3)$ W(2)W(1)W(0) W(4) $W W(3) W(2)$

(


$H(1) W(0) W(4)+12 W(3) W(2) W(1) W(0) W(4)+4 H(3) W(2) W(1) W($

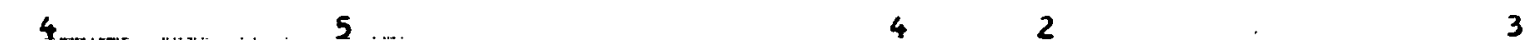
$0)^{4} H(4)+W(3)^{2} H(2) W(1) W(0)+5 W(3)^{4} W(2)^{2} W(1) W(0)+10 W(3)$

3

$w(2) W(1) W(0)+10 w(3) \quad W(2) W(1) W(0)+5 W(3) W(2) \quad W(1) W(0)$

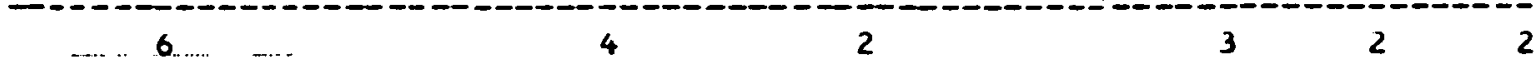

$+N(2) W(1) W(0)+2 w(3) W(2) W(1) W(0)+12 W(3) W(2) W(1)$

-
$w(3)+24 h(3)^{2} w(2)^{3} w(1)^{2} w(0)+20 h(3) w(2)^{4} w(1)^{2} w(0)+6 w($

-

$2)^{5} w(1)^{2} w(0)+3 w(3)^{3} w(2) w(1)^{3} w(0)+18 w(3)^{2} w(2)^{2} w(1)^{3} w$

-

$(0)+30 w(3) w(2)^{3} w(1)^{3} w(0)+15 w(2)^{4} w(1)^{3} w(0)+4 w(3)^{2} w(2)$

-

$w(1)^{4} w(0)+20 w(3) w(2)^{2} w(1)^{4} w(0)+20 w(2)^{3} w(1)^{4} w(0)+5 w($

5

$31 W(2) W(1)^{5} w(0)+15 w(2)^{2} w(1)^{5} w(0)+6 w(2) w(1)^{6} w(0)+w(1)$

W(0)

$W(3)+2 W(3) W(2) W(1) W(0) W(6) W(5) W(4)+6 W(3)+W(2) W(1) W(0)$ $W(5) W(4)^{2}+4 W(3) W(2)^{2} W(1) w(0) W(5) w(4)^{2}+4 W(3) w(2) W(1)^{2}$

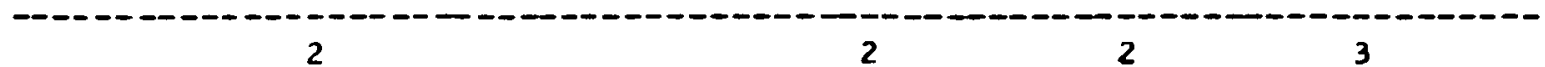
$W\left(0, W(5) W(4)^{2}+4 W(3) W(2) W(1) W(0)^{2} W(5) W(4)^{2}+6 W(3)^{3} w(2) W\right.$ $(1) W(0) W(4)^{2}+9 w(3)^{2} w(2)^{2} w(1) W(0) w(4)^{2}+3 W(3) W(2)^{3} W(1)$ $w(0) W(4)^{2}+6 w(3)^{2} W(2) w(1)^{2} w(0) w(4)^{2}+6 w(3) W(2)^{2} w(1)^{2} w($ 2 . 3 2 2 2 0) $W(4)^{2}+3 W(3) W(2) W(1) W(0) W(4)+3 W(3) W(2) W(1) W(0) W(5)^{2}$ ${ }^{2}+62^{2} H(2) H(1) H(0)^{2} H(4)^{2}+H(3) H(2)^{2} H(1) H(0)^{2}$

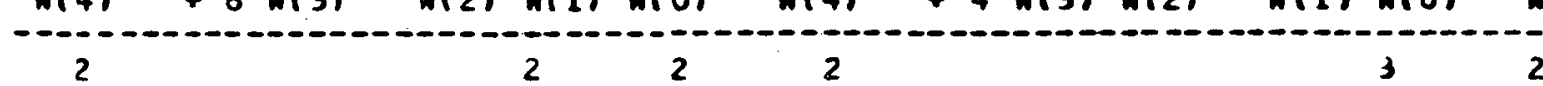
$(4)^{2}+6 W(3) W(2) W(1)^{2} w(0)^{2} W(4)^{2}+3 w(3) W(2) W(1) W(0) W(4)$ (4) $+3 W(3) W(2) W(1) W(0) W(5) w(4)^{3}+4 w(3)^{2} w(2) w(1) w(0) w(4)^{3}$.

$2 W(3) W(2)^{2} W(1) W(0) W(4)^{3}+2 W(3) W(2) W(1)^{2} W(0) W(4)^{3}+2 W(3)$

-

$\frac{H(2) W(1) w(0)^{2} w(4)^{3}+W(3) w(2) w(1) w(0) w(4)^{4}+2 w(3)^{4} w(2) w(1)}{2}$


$11 W(0)+8 W(3) W(2) W(1) W(0)+12 W(3) W(2) W(1) W(0)$

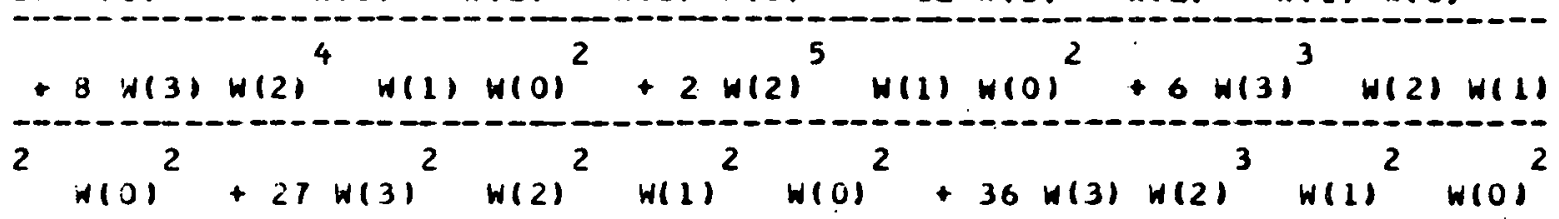

-

$+15 W(2)^{4} w(1)^{2} w(0)^{2}+12 W(3)^{2} w(2) w(1)^{3} w(0)^{2}+48 w(3) W(2)$

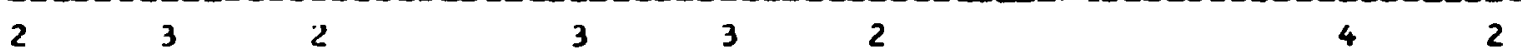

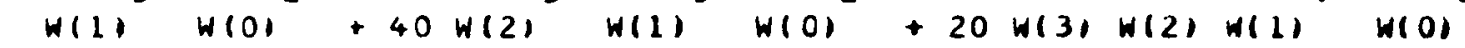

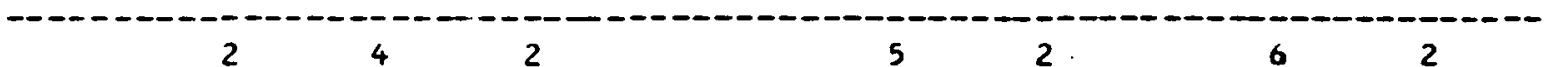

$+50 w(2)^{2} w(1)^{4} w(0)^{2}+30 w(2) w(1)^{5} w(0)^{2}+7 w(1)^{6} w(0)^{2}+3$

$w(3)^{3} w(2) w(1) w(0)^{3}+9 w(3)^{2} w(2)^{2} w(1) w(0)^{3}+9 w(3) w(2)^{3} W$

$(1) w(0)^{3}+3 w(2)^{4} w(1) w(0)^{3}+12 w(3)^{2} w(2) w(1)^{2} w(0)^{3}+36 w($
$\left.31 w(2)^{2} w(1)^{2} w(0)^{3}+24 w(2)^{3} w(1)^{2} w 10\right)^{3}+30 w(3) w(2) w(1)^{3}$

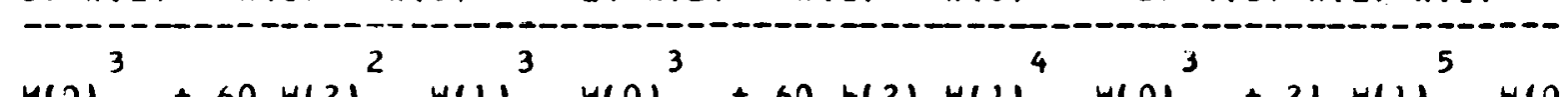
$w(0)^{3}+60 w(2)^{2} w(1)^{3} w(0)^{3}+60 w(2) w(1)^{4} w(0)^{3}+21 w(1)^{5} w(0$ $1^{3}+4 w(3)^{2} w(2) w(1) w(0)^{4}+8 w(3) w(2)^{2} w(1) w(0)^{4}+4 w(2)^{3} w$ $1+4 w(3) W(2) W(1) w(0)+9 w(3) w(2) w(1) W(0)+4 w(2) w$ $(1) w(0)^{4}+20 w(3) w(2) w(1)^{2} w(0)^{4}+30 w(2)^{2} w(1)^{2} w(0)^{4}+60 w$ 3 - 4 ( $\left.(2)^{2} w(1)^{3} w(3)\right)^{4}+35 w(1)^{4} w(0)^{4}+5 w(3) w(2) w(1) w(0)^{2}+5 w(2)^{2}$ $w(1) w(0)^{5}+30 w(2) w(1)^{2} w(0)^{5}+35 w(1)^{3} w(0)^{5}+6 w(2) w(1) w 1$ $0)^{6}+21 w(1)^{2} w(0)^{6}+7 w(1) w(0)^{7}+w(0)^{8}$ IMCM(O)

$N=9$

$---$

$N 2=18$

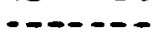

MOM(18)=1W(3) W(2)W(1)W(0)W(8)W(7)W(6)W(5)W(4)+2W(3) ${ }^{2} W(2)$

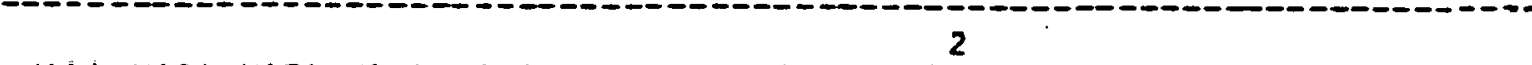

$W(1) W(0) W(7) W(6) W(5) W(4)+2 W(3) W(2) \quad W(1) W(0) W(7) W(6) W(5)$ M $W(4)+2 W(3) W(2) W(1)^{2} w(0) W(7) W(6) W(5) W(4)+2 W(3) W(2) W(1) W($ H4) + 2 W $0)^{2} W(7) W(6) W(5) W(4)^{+}+3 w(3)^{3} w(2) w(1) w(0) w(6) w(3) W(4)+6 w 1$ 
$3)^{2} W(2)^{2} W(1) W(0) W(6) W(5) W(4)+3 W(3) W(2)^{3} W(1) W(0) W(6) W(5)$ $W(4)+4 W(3)^{2} w(2) W(1)^{2} w(0) w(6) W(5) w(4)+6 w(3) w(2)^{2} W(1)^{2}$

-
$W(0) W(6) W(5) W(4)+3 W(3) W(2) W(1)^{3} W(0) W(6) W(5) W(4)+W(3) W(2)$

W
$W(1) W(0) w(7)^{2} w(6) W(5) W(4)+4 W(3)^{2} w(2) W(1) W(0)^{2} W(6) W(5) W$ 20 $(4)+4 W(3) W(2) \quad W(1) w(0)$. W(6) W(5) W(4)+6W(3)W(2)W(1) W(0)

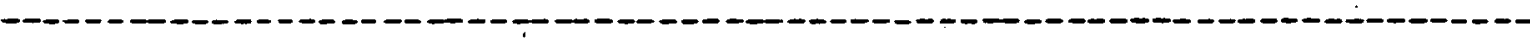

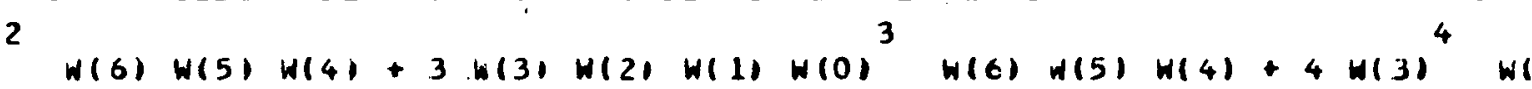
N(6) W(5) H(4) $2) W(1) W(0) W(5) W(4)+12 W(3) W(2) W(1) W(0) W(5) \cdot W(4)+12 W(3)$

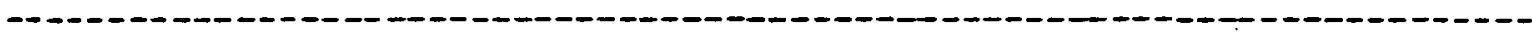
$W(2)^{3} W(1) W(0) w(5) w(4)+4 w(3) W(2)^{4} w(1) w(0) w(5) w(4)+6 w(3)$ 3 - 2 ( $3 *(2) W(1)^{2} w(0) w(5) w(4)+10 w(3)^{2} w(2)^{2} w(1)^{2} w(0) w(5)^{2} w(4)^{2}$ $+12 W(3) W(2)^{3} W(1)^{2} W(0) W(5) W(4)+6 W(3)^{2} W(2) W(1)^{3} W(0) W(5)$

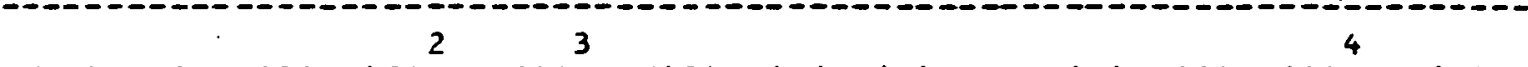

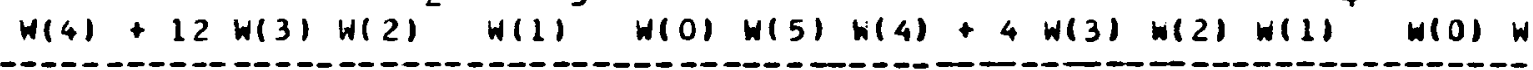
(5) H(4)+2H(3)H(2)W(1)H(0)H(7)W(6) W(5)W(4)+2H(3) H(2) W ----- - - - - - - - - - $(1) W(0) W(6)^{2} w(5) w(4)+2 W(3) w(2)^{2} w(1) W(0) W(6)^{2} w(5) W(4)+2$ (1) W(3) $W(2) W(1)^{2} H(0) W(6)^{2} W(5) w(4)+2 W(3) w(2) W(1) W(0)^{2} W(6)^{2}$

(-)-

$W(5) W(4)+W(3) W(2) W(1) W(0) w(6)^{3} w(5) W(4)+6 w(3) \quad w(2) w(1) W$

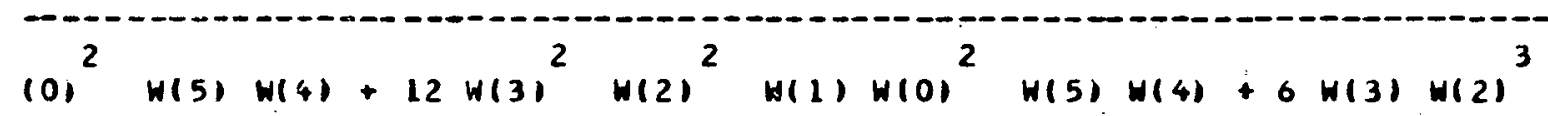

(10)

$H(1) W(0)^{2} W(5) W(4)+12 W(3)^{2} H(2) W(1)^{2} H(0)^{2} H(5) W(4)+18 H(3$

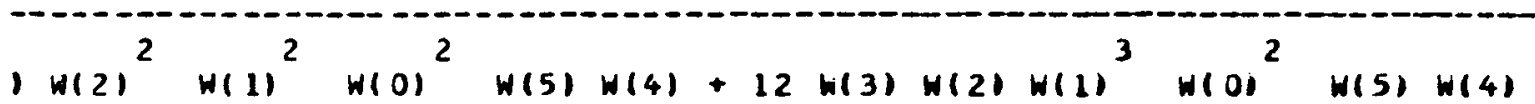

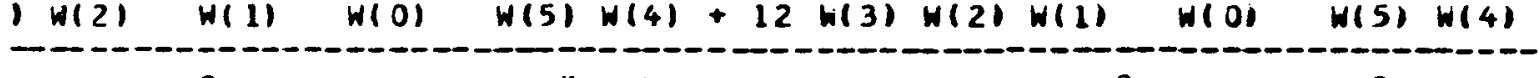
$+6 w(3)^{2} w(2) w(1) w(0)^{3} w(5) w(4)^{2}+6 w(3) w(2)$ w(1) w(0) $3(5)$ - 6 N(3) $W(4)+12 W(3) H(2) W(1)^{2} w(0)^{3} W(5) W(4)+4 H(3) W(2) W(1) W(0)^{4} W($

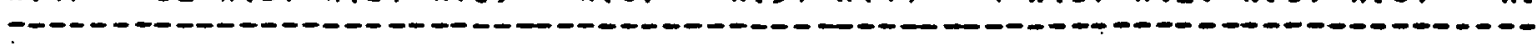

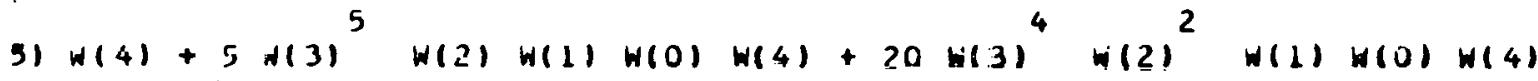

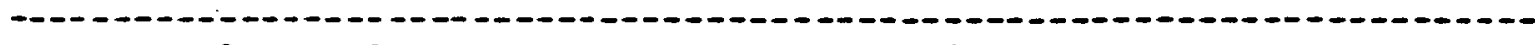
$+30 W(3)^{3} W(2)^{3} W(1) H(0) W(4)+20 H(3)^{2} H(2)^{4} H(1) \omega(0) H(4)+5$

-


$w(3) w(2)^{5} w(1) w(0) w(4)+8 w(3)^{4} w(2) w(1)^{2} w(0) w(4)+36 w(3)^{3}$

-

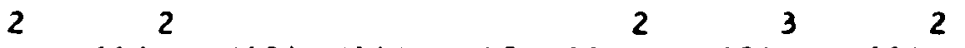

$W(2)^{2} W(1)^{2} W(0) W(4)+48 w(3)^{2} W(2)^{3} W(1)^{2} W(0) W(4)+20 w(3) W$

H

$(2)^{4} w(1)^{2} w(0) w(4)+g w(3)^{3} w(2) w(1)^{3} w(0) w(4)+36 w(3)^{2} w(2)$

-

$2 W(1)^{3} w(0) w(4)+30 w(3) w(2)^{3} w(1)^{3} w(0) w(4)+8 w(3)^{2} w(2) w($

-

$1)^{4} w(0) w(4)+20 w(3) w(2)^{2} w(1)^{4} w(0) w(4)+5 w(3) w(2) w(1)^{3} w(1$

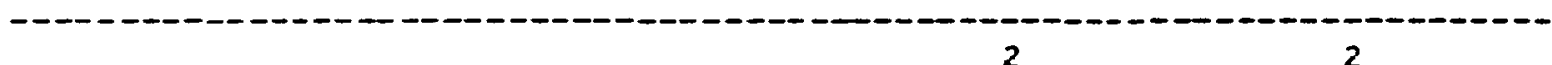

$0) w(4)+2 w(3) w(2) w(1) w(0) w(7) w(6) w(5)^{2} w(4)+4 w(3)^{2} w(2) w 1$

$(1) W(0) w(6) w(5)^{2} w(4)+4 w(3) w(2)^{2} w(1) w(0) w(6) w(5)^{2} w(4)+4$ $w(3) w(2) w(1)^{2} w(0) w(6) w(5)^{2} w(4)+4 w(3) w(2) w(1) w(0)^{2} w(6) w 1$

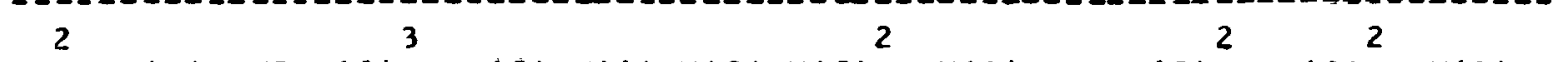
$5) w(4)+3 w(3) w(2) w(1) w(0) w(5) w(4)+6 w(3) w(2) w(1) w$

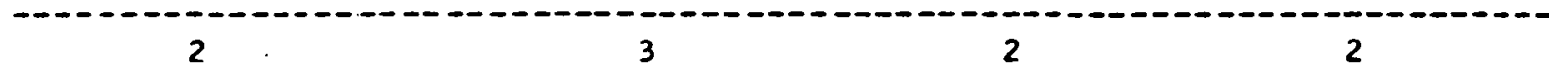
(0)W(5) W(4)+3W(3)W(2) W(1)W(0)W(5) W(4)+4W(3) W(2)W( $1)^{2} w(0) w(5)^{2} w(4)+6 w(3) w(2)^{2} h(1)^{2} w(0) w(5)^{2} w(4)+3 w(3) w$

-

$(2) W(1)^{3} w(3) w(5)^{2} w(4)+3 w(3) w(2) w(1) w(0) w(6)^{2} w(5)^{2} w(4)$

$+4 W(3)^{2} w(2) W(1) w(0)^{2} w(5)^{2} w(4)+4 W(3) w(2)^{2} w(1) w(0)^{2} W(5$
$1^{2} w(4)+6 w(3) w(2) w(1)^{2} w(0)^{2} w(5)^{2} w(4)+3 w(3) w(2) w(1) w(0)$ $3^{2} W(5)^{2} W(4)+3 W(3) W(2) W(1) W(0) W(6) W(5)^{3} W(4)+2 W(3)^{2} W(2)$

- W(5) W(4) + 3 W(3) W(2) W(1) W(O) W(6) W(5) $W(4)+2$ W(3) $W(2)$

$W(1) W(0) W(5)^{3} W(4)+2 W(3) W(2)^{2}, H(1) W(0) W(5)^{3} W(4)+2 W(3) W(2$

H

$1 w(1)^{2} w(0) w(5)^{3} w(4)+2 w(3) w(2) w(1) w(0)^{2} w(5)^{3} w(4)+w(3) w$

(2) $W(1) W(0) W(5)^{4} w(4)+8 w(3)^{4} w(2) W(1) w(0)^{2} w(4)+24 w(3)^{3} W$
$(2)^{2} w(1) w(3)^{2} w(4)+24 w(3)^{2} w(2)^{3} w(1) w(0)^{2} w(4)+8 w(3) w(2)$ 4 $4 w(1) w(0)^{2} w(4)+18 w(3)^{3} w(2) w(1)^{2} w(0)^{2} w(4)+54 w(3)^{2} w(2)$

\begin{tabular}{|c|}
\hline 2 \\
\hline
\end{tabular}
${ }^{2} w(1)^{2} w(0)^{2} w(4)+36 w(3) w(2)^{3} w(1)^{2} w(0)^{2} w(4)+24 w(3)^{2} w($ $21 w(1)^{3} w(0)^{2} w(4)+48 w(3) w(2)^{2} w(1)^{3} w(0)^{2} w(4)+20 w(3) w(2)$ $21 W(1)$ W 
$w(1)^{4} w(0)^{2} w(4)+9 w(3)^{3} w(2) w(1) w(0)^{3} w(4)+18 w(3)^{2} w(2)^{2}$ $w(1) w(0)^{3} w(4)+9 w(3) w(2)^{3} w(1) w(0)^{3} w(4)+24 w(3)^{2} w(2)^{3} w\left(1 !^{2}\right.$

$w(0)^{3} w(4)+36 w(3) w(2)^{2} w(1)^{2} w(0)^{3} w(4)+30 w(3) w(2) w(1)^{3} w$

$(0)^{3} w(4)+8 w(3)^{2} w(2) W(1) w(0)^{4} w(4)+8 w(3) w(2)^{2} w(1)^{2} w(0)^{4}$

$W(4)+20 W(3) W(2) W(1)^{2} W(0)^{4} W(4)+5 W(3) W(2) W(1) W(0)^{5} w(4)+$

W(4) + 20 W(

$W(3)^{5} W(2) W(1) W(0)+6 W(3)^{5} W(2)^{2} w(1) W(0)+15^{2} W(3)^{4} W(2)^{3} W(1$

-

$1 W(0)+20 W(3)^{3} W(2)^{4} w(1) W(0)+15 W(3)^{2} w(2)^{5} w(1) w(0)+6 w(3)$

-

$1 H(2)^{t} H(1) H(0)+H(2)^{7} W(1) W(0)+2 W(3)^{5} H(2) W(1)^{2} W(0)+15 H$

$(3)^{4} W(2)^{2} H(1)^{2} W(0)+40 W(3)^{3} W(2)^{3} w(1)^{2} W(0)+50 W(3)^{2} W(2)$

$(3) W(2) W(1) W(0)+40 W(3) W(2) W(1) W(0)+50 W(3) W(2)$

$4 W(1)^{2} H(0)+30 H(3) W(2)^{5} W(1)^{2} H(0)+7 w(2)^{6} W(1)^{2} w(0)+3 w$

$-4$

$(3)^{4} H(2) W(1)^{3} H(0)+24 W(3)^{3} W(2)^{2} w(1)^{3} w(0)+60 w(3)^{2} w(2)^{3}$

131

$3 \quad 4 \quad 3 \quad 503\left(\begin{array}{llll}3 & 3\end{array}\right.$

$H(1)^{3} W(0)+60 W(3) W(2)^{4} H(1)^{3} W(0)+21 W(2)^{5} W(1)^{3} w(0)+4 W($ МІІ $3)^{3} w(2) w(1)^{4} w(0)+30 w(3)^{2} w(2)^{2} w(1)^{4} w(0)+60 w(3) w(2)^{3} w($

4

$1)^{4} W(0)+35 W(2)^{4} W(1)^{4} W(0)+5 W(3)^{2} W(2) W(1)^{5} W(0)+30 H(3)$ 1) $W(2)^{2} H(1)^{5} H(O)+35 w(2)^{3} H(1)^{5} w(0), 6 H(3) W(2) W(1)^{6} W(0)+$

- 6 (6)

$21 W(2)^{2} H(1)^{6} W(0)+7 W(2) W(1) W(0)+H(1, W(0)+2 H(3) W(2) H$ -

$(1) W(0) W(7) W(6) W(5) W(4)^{2}+6 W(3)^{2} W(2) W(1) W(0) W(6) W(5)^{2} W(4)^{2}$ (1) $+4 W(3) W(2)^{2} W(1) W(0) W(6) W(5) W(4)^{2}+4 W(3) W(2) W(1)^{2} W(0) W($

(2)

6). H(5)W(4) $+4 W(3) W(2) W(1) w(0)^{2} W(6) W(5) W(4)^{2}+12 W(3)^{2} W($

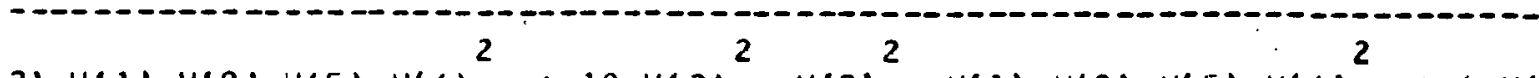
2) W(1) W(0)W(5)W(4) $)^{2}+18 W(3)^{2} W(2)^{2} W(1) W(0) W(5) W(4)^{2}+6 w($ 32022

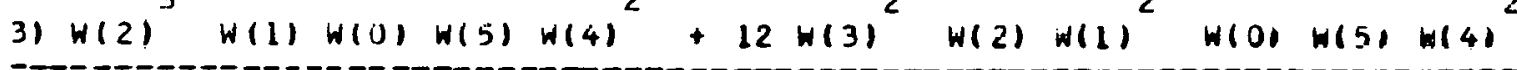


$+12 w(3) w(2)^{2} w(1)^{2} w(0) w(5) w(4)^{2}+6 w(3) w(2) w(1)^{3} w(0) w(5)$

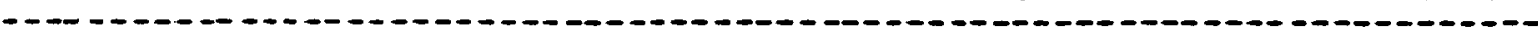
$W(4)^{?}+2 W(3) W(2) W(1) W(0) W(6)^{2} w(5) W(4)^{2}+12 W(3)^{2} w(2) W(1)$ H41 $w(0)^{2} w(5) w(4)^{2}+8 w(3) w(2)^{2} w(1) w(0)^{2} w(5) w(4)^{2}+12 w(3) w($ W $21 w(1)^{2} w(0)^{2} w(5) w(4)^{2}+6 w(3) W(2) w(1) w(0)^{3} W(5) w(4)^{2}+10$ $w(3)^{4} w(2) w(1) w(0) w(4)^{2}+24 w(3)^{2} w(2)^{2} w(1) w(0) w(4)^{2}+18 w($ $W(3) W(2) W(1) W(0) W(4)+24 W(3) W(2) W(1) W(0) W(4)^{2}+18 W($ $3)^{2} w(2)^{3} w(1) w(0) w(4)^{2}+4 w(3) w(2)^{4} w(1) w(0) w(4)^{2}+12 w(3)^{3}$ - - $w(2) w(1)^{2} w(0) w(4)^{2}+27 w(3)^{2} w(2)^{2} w(1)^{2} w(0) w(4)^{2}+12 w(3)$ ${ }_{3} \mathrm{~W}^{2} \mathrm{~W}$ $w(2)^{3} w(1)^{2} w(0) w(4)^{2}+9 w(3)^{2} w(2) W(1)^{3} w(0) W(4)^{2}+12 H(3)$ $w(2)^{2} w(1)^{3} w(0) w(4)^{2}+4 w(3) w(2) w(1)^{4} w(0) w(4)^{2}+6 w(3) w(2)$ $w(1) w(0) w(6) w(5)^{2} w(4)^{2}+9 w(3)^{2} w(2) w(1) w(0) W(5)^{2} w(4)^{2}+$ $-$ $6 w(3) w(2)^{2} w(1) w(0) w(5)^{2} w(4)^{2}+6 w(3) w(2) w(1)^{2} w(0) w(5)^{2} w$

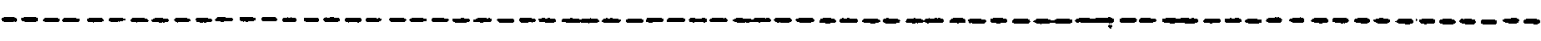
$(4)^{2}+6 w(3) w(2) w(1) w(0)^{2} w(5)^{2} w(4)^{2}+4 w(3) w(2) w(1) w(0) w 1$ $-3)^{3}$ $5)^{3} w(4)^{2}+12 w(3)^{3} w(2)^{2} w(1) w(0)^{2} w(4)^{2}+18 w(3)^{2} w(2)^{2} w(1)$ $w(0)^{2} w(4)^{2}+6 w(3) w(2)^{3} w(1) w(0)^{2} w(4)^{2}+18 w(3)^{2} w(2) w(1)^{2}$ $w(0)^{2} w(4)^{2}+18 w(3) w(2)^{2} w(1)^{2} w(0)^{2} w(4)^{2}+12 w(3) w(2) w(1)$ $3 w(0)^{2} w(4)^{2}+9 w(3)^{2} w(2) w(1) w(0)^{3} w(4)^{2}+6 w(3) w(2)^{2} w(1)$ W(10) $W(4)$ $w(0)^{3} w(4)^{2}+12 w(3) w(2) w(1)^{2} w(0)^{3} w(4)^{2}+4 w(3) w(2) w(1) w(1)$ $0)^{4} w(4)^{2}+3 w(3) w(2) w(1) w(0) w(6) w(3) w(4)^{3}+12 w(3)^{2} w(2) w($ $11 W(0) W(5) W(4)^{3}+6 W(3) W(2)^{2} w(1) W(0) W(5) w(4)^{3}+6 W(3) W(2)$

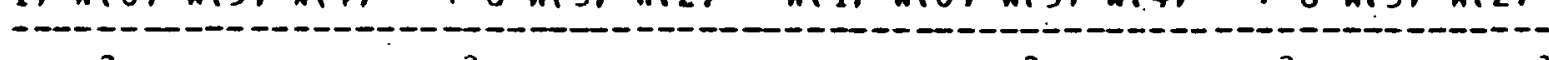
$w(1)^{2} w(0) w(5) w(4)^{3}+6 w(3) w(2) w(1) w(0)^{2} w(5) w(4)^{3}+10 w(3)^{3}$ W(1) $w(2) w(1) w(0) w(4)^{3}+12 w(3)^{2} w(2)^{2} w(1) w(0) w(4)^{3}+3 w(3) w(2)$ $3 w(1) w(0) w(4)^{3}+3 w(3)^{2} w(2) w(1)^{2} w(0) w(4)^{3}+o w(3) w(2)^{2} w$ $W(1) W(0) W(4)+0 W(3) W(2) W(1) W(0) W(4)+0 w(3) w(2){ }^{2} \mathrm{w}$ 
$(1)^{2} w(0) w(4)^{3}+3 w(3) w(2) w(1)^{3} w(0) w(4)^{3}+6 w(3) w(2) w(1) w($

-

$0 w_{(5)^{2}} \cdot w(4)^{3}+8 w(3)^{2} w(2) w(1) w(0)^{2} w(4)^{3}+4 w(3) w(2)^{2} w(1)$

W(0)

$w(0)^{2} w(4)^{3}+6 w(3) w(2) w(1)^{2} w(0)^{2} w(4)^{3}+3 w(3) w(2) w(1) w(0$

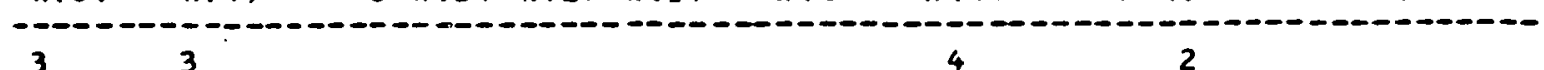

$1^{3} w(4)^{3}+4 W(3) W(2) W(1) W(0) W(5) W(4)^{4}+j W(3)^{2} w(2) w(1) W(0)$

${ }_{4}$ $w(4)^{4}+2 W(3) W(2)^{2} w(1) w(0) W(4)^{4}+2 w(3) w(2) w(1)^{2} w(0) W(4)^{4}$

$+2 W(3) W(2) W(1) W(0)^{2} w(4)^{4}+w(3) w(2) w(1) w(0) w(4)^{5}+2 W(3)^{5}$

$W(2) W(1) W(0)^{2}+10 W(3)^{4} W(2)^{2} H(1) W(c)^{2}+20 w(3)^{3} W(2)^{3} W(1)$

$W(0)^{2}+20 W(3)^{2} H(2)^{4} w(1) w(0)^{2}+10 w(3) w(2)^{5} w(1) w(0)^{2}+2$

$H(2)^{6} H(1) W(0)^{2}+6 H(3)^{4} W(2) W(1)^{2} H(0)^{2}+36 W(3)^{3} W(2)^{2} H(1)$

\begin{tabular}{|c|}
\hline . \\
\hline
\end{tabular}

$w(0)+72 w(3) w(2)^{3} w(1) w(0)+60 w(3) w(2) w(1) w(0)^{2}$

$+18 w(2)^{5} w(1)^{2} w(0)^{2}+12 w(3)^{3} w(2) w(1)^{3} w(0)^{2}+72 w(3)^{2} w 1$ $2)^{2} w(1)^{3} w(0)^{2}+120 w(3) w(2)^{3} w(1)^{3} w(0)^{2}+60 w(2)^{4} w(1)^{3} w(1$ $-0$ $0)^{2}+20 w(3)^{2} w(2) w(1)^{4} w(0)^{2}+100 w(3) W(2)^{2} w(1)^{4} w(0)^{2}+$ $100 w(2)^{3} w(1)^{4} w(0)^{2}+30 w(3) w(2) w(1)^{5} w(0)^{2}+90 w(2)^{2} w(1)^{5}$

$100 \mathrm{~W}$
$W(0)^{2}+42 W(2) W(1)^{6} w(0)^{2}+8 W(1)^{7} H(0)^{2}+3 H(3)^{4} H(2) W(1) H$ 33 $(0)^{3}+12 w(3)^{3} w(2)^{2} w(1) w(0)^{3}+18 w(3)^{2} w(2)^{3} w(1) w(0)^{3}+12$ W(3) $\mathrm{W}(2)^{4} \mathrm{H}(1) \mathrm{W}(0)$ $w(3) W(2)^{4} w(1) w(0)^{3}+3 w(2)^{5} w(1) w(0)^{3}+12 w(3)^{3} w(2) w(1)^{2}$

$w(0)^{3}+54 w(3)^{2} w(2)^{2} w(1)^{2} w(0)^{3}+72 w(3) w(2)^{3} w(1)^{2} w(0)^{3}$
$+30 w(2)^{4} w(1)^{2} w(0)^{3}+30 w(3)^{2} w(2) w(1)^{3} w(0)^{3}+120 w(3) w(2$
$1^{2} w(1)^{3} w(1)^{3}+100 w(2)^{3} w(1)^{3} w(0)^{3}+60 w(3) w(2) w(1)^{4} w(0)^{3}$
$+150 w(2)^{2} w(1)^{4} w(10)^{3}+105 w(2) w(1)^{5} w(0)^{3}+28 w(1)^{6} w(0)^{3}$




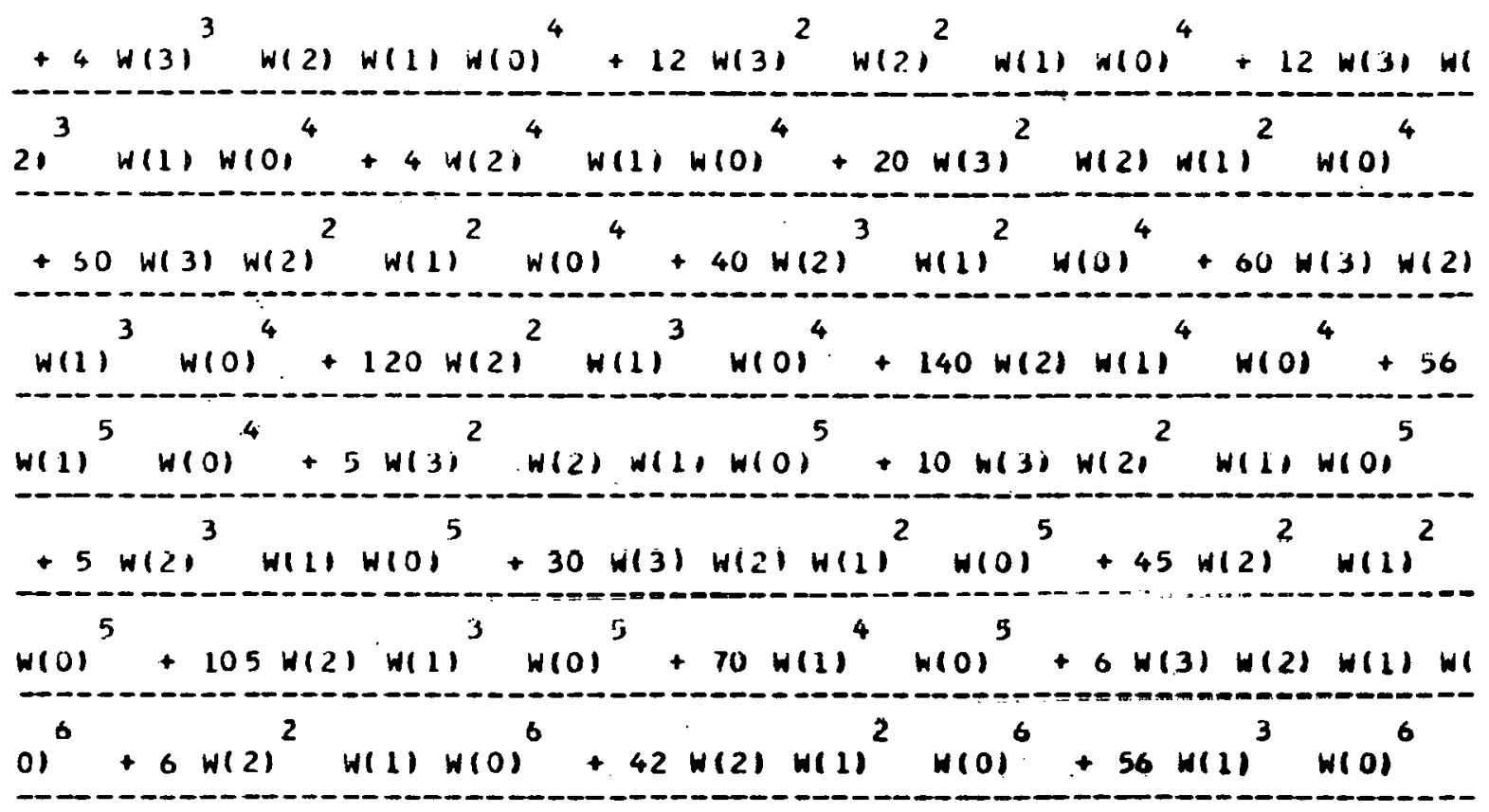

$+7 W(2) w(1) w(0)^{7}+28 w(1)^{2} w(0)^{7}+8 W(1) W(0)^{8}+W(0)^{9}$ IMUM(0) 


\section{APPENDIX $F$}

METHODS OF COMBINATORIAL ANALYSIS; DERIVATION OF THE COEFFICIENTS $w_{0}^{k}(n)$, FOR $k=n$ TO $n-5$

In Part II.2 of the main body of this work, we mentioned that the nested sums of the RHS of Eq. (II.77) can be written as a single sum $S=\sum_{k}: T_{k}$, of terms $T_{k}$ all of the form:

$$
T_{k}=w_{j_{1}} w_{j_{2}} w_{j_{3}} \ldots w_{j_{n}}
$$

where the indeces $j_{i}$ obey the restrictions:

$$
\left.\begin{array}{l}
0 \leq j_{i} \leq i-1 \\
j_{i} \leq j_{i-1}+1
\end{array}\right\}
$$

In the sum $S$ we add together terms $T_{i}$ and $T_{k}$ if and only if they have the same power of every $w_{k}$.

For this purpose, the set of terms in the sum $S$ is divided into equivalence classes. Let $\tilde{S}$ be the set of terms in the sum $S$. To say that $T_{m}$ and $T_{n}$ are members of the same class means that the set of indeces $\left\{j_{j}\right\}_{m}$ arld $\left\{j_{i}\right\}_{n}$ are permutations of each other.

We now wish to examine the implications of the restrictions (II.79) on the indeces $j_{j}$, as far as the powers of the W's occurring in a valid term $T_{k} \in \tilde{S}$, are concerned.

It is seen that a member of the equivalence class for the term

$$
w_{0}^{r_{0}} w_{1}^{r_{1}} \cdots w_{s}^{r_{s}}
$$


is the set of subscripts

$$
K=\left\{r_{0} * 0, r_{1} * 1, \ldots, r_{s}^{*} s\right\}
$$

i.e., $K$ is the set of subscripts of a legal term in the given complete expansion S. (Note that the symbol "*" denotes "occurrences," i.e., $r_{0} \star_{0}$ is a short way of writing $r_{0}$ zeros separated by commas.) Therefore, this set of subscripts corresponds to the ordered set of powers

$$
\left\{r_{0}, r_{1}, r_{2}, \ldots, r_{s}\right\}
$$

and it is easily seen that the restrictions (II.79) on the $j_{i}$ 's imply the following restrictions on the powers $r_{i}$ :

$$
\left.\begin{array}{l}
r_{i} \geq 0 ; r_{0} \geq 1 \\
r_{i}>0 \Rightarrow r_{i-1}>0 \text { for } i>0 \\
\sum_{i=1}^{s} r_{i}=n
\end{array}\right\}
$$

Furthermore, the coefficient of this set of powers is the number of members of the corresponding equivalence class, or, equivalently, the coefficient is the number of permutations of the set $K$ [defined in (F.2)] that appear in the set

$$
\left.J=\left\{i j_{i}\right\}_{k}\right\}
$$

i.e., the set of sets of $j_{j}$ 's that satisfy restrictions (II.79). 
We shall now illustrate, by means of a few examples, the practical applications of the above ideas:

Ex. $1 ; w_{0}^{n}$

The set of subscripts is

$$
K=\{0,0,0, \ldots, 0\}
$$

There can be only 1 permutation of the set $k$, therefore there is only 1 member in this equivalence class. Thus

$$
\operatorname{coeff}\left[W_{0}^{n}\right]=1
$$

Ex. $2 ; w_{0}^{n-1} W_{1}$.

Here,

$$
K=\{0,0, \ldots, 0,1\}
$$

There are $(n-1)$ permutations of the set $k$, or, equivalently, there are $(n-1)$ such members in this equivalence class. Therefore

$$
\operatorname{coeff}\left[W_{0}^{n-1} W_{1}\right]=(n-1)
$$

Obviousiy, there can be no other terms containing $w_{0}^{n-1}$, because of the restrictions (II.79).

Ex. 3; Terms containing $w_{0}^{n-2}$

Because of the restrictions (II.79), $W_{0}^{n-2}$ can occur in two ways, i.e., in terms of the form $w_{0}^{n-2} w_{1}^{2}$, and of the form $w_{0}^{n-2} w_{1} W_{2}$. (3a) $w_{0}^{n-2} w_{1}^{2}$;

Here,

$$
K=\{0,0, \ldots, 1,1\}
$$


There are $\frac{1}{2}(n-1)(n-2)$ permutations of the set $k$.

This can be seen as follows: there are $(n-1)$ "places" to "put" the first "l" and $(n-2)$ to "place" the second "l;" but since the two "l"s are indistinguishable, the total number of permutations is $\frac{1}{2 !}(n-1)(n-2)$. Thus

$$
\operatorname{coeff}\left[W_{0}^{n-2} W_{1}^{2}\right]=\frac{1}{2}(n-1)(n-2)
$$

(3b) $W_{0}^{n-2} W_{1} w_{2}$

Here

$$
K=\{0,0, \ldots, 0,1,2\}
$$

Obviously, there are $(n-2)$ permutations of the set $k$. Therefore

$$
\operatorname{coeff}\left[W_{0}^{n-2} W_{1} W_{2}\right]=(n-2)
$$

Ex. 4; Terms containing $W_{0}^{n-5} W^{3} W_{2}^{2}$

As a final example, we shall calculate the (more complicated) coefficient of $W_{0}^{n-5} W_{1}^{3} W_{2}^{2}$. For convenience. we shall illustrate cvcry detail by means of an elementary graphical model.

Here

$$
K=\{0,0, \ldots, 0,1,1,1,2,2\}
$$

We split the pattern of $K$ into 2 "subcases":

(4a) $\ldots 12 \ldots 12 \ldots 1$

Let us imagine an array of $n$ "boxes" or "places," initially filled with "zeros," in which we are allowed to place the above "nonzero" indeces such that the restrictions (II.79) are fulfilled. 
Then, for the first string "12," we obviously have $(n-2)$ boxes to place it into, i.e.,

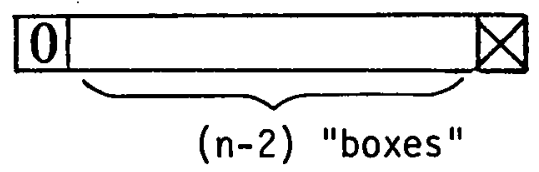

since the first box is necessarily occupied by a "zero," and we cannot place the string "12" in the last box, either. [Note that "forbidden" "boxes," i.e., boxes forbidden for the first element in the string, e.g., the "1" of the "12" string, are "crossed out," i.e., marked with the symbol " $x:$ "]

Next, we have to find the allowed "places" for the second string "12." But, because of the restrictions (II.79), placing the second string "12" will "subdivide" the (n-2) "places" for the first "12" string as follows:

(4.a.L); ["L" stands for "left".; see Fig. (4.a.L)]

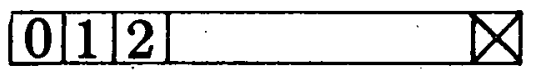

Fig. (4.a.L)

We have placed the first "12" string as far to the left as possible, and there is obviously only 1 such "place" for this string. This then leaves ( $n-4)$ "places" to place the second string "12." (4.a.M); ["M" stands for "middle"; see Fig. (4.a.M)]

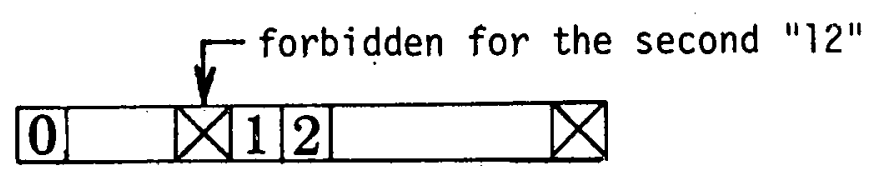

Fig. (4.a.M) 
If we place the first string "12" in the "middle," we clearly have (n-4) such possibilities. When "placing" the second string "12," the first "box" in front of the first "12" string is forbidden, because of the restrictions (II.79). We are therefore left with $n-5$ possibilities to "place" the second string "12."

(4.a.R); ["R". stands for "right"; see Fig. (4.a.R)]

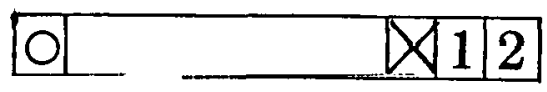

Fig. (4.a.R)

There is obviously only 1 way to place, in this manner, the first string "12." Again, as above, the first "box" in front of the first string "12" is forbidden for the second string "12." We are thus left with $(n-4)$ ways to place the second string "12."

Summing up, we have

$$
[(n-4)+(n-4)(n-5)+(n-4)]=(n-3)(n-4)
$$

ways to place both strings "12." However, since the 2 strings "12" are indistinguishable from one another, we have only $\frac{1}{2 !}(n-3)(n-4)$ possible "permutations" for the "12" strings.

We still have to place the "l"; obviously, in all of the above situations, i.e. (4.d.L), (4.a.M) and (4.a.R), we have $(n-5)$ possibilities to place the " $1: "$

Therefore, for the "pattern" (4.a), we have a total of

$$
\frac{1}{2}(n-3)(n-4)(n-5) \text { "permutations" }
$$


(4.b) .. $122 \ldots 11$

The only other "subpattern" for the set $K$ is .. $122 \ldots 11$.

Analysis along the same lines as for (4.a) above yield that:

the total number of "permutations" for case (4.b) =

[(n-3) "places" for the string "122"]

$\times[(n-4)$ "places" for the first "1"] $\times$

$\times[(n-5)$ "places" for the second "l" $] \times$

$\times\left[\left(\frac{1}{2 !}\right)\right.$, since the two "l" are indistinguishable $]$

$=\frac{1}{2}(n-3)(n-4)(n-5)$

Finally, the coefficient of $W_{0}^{n-5} W_{1}^{3} W_{2}^{2}$ will be the sum of $(F \cdot 10)$ and (F.11), i.e.,

$$
\begin{aligned}
\operatorname{coeff}\left[W^{n-5} W_{1}^{3} W_{2}^{2}\right] & =\frac{1}{2}(n-3)(n-4)(n-5)+\frac{1}{2}(n-3)(n-4)(n-5) \\
& =(n-3)(n-4)(n-5)
\end{aligned}
$$

Using these methods, we have calculated the coefficients of the first 32 terms of Eq. (II.77), in order of decreasing powers of $W_{0}$, starting with $w_{0}^{n}$. These coefficients are presented in Table F.l, below.

In addition, based on the same concepts of combinatorial analysis, we have derived formulae (II.80) through (II.82). 
Table F.1

\begin{tabular}{|c|c|c|c|c|c|}
\hline $\begin{array}{l}\text { Power } \\
W_{0}\end{array}$ & Multiplied by & Coefficient & $\begin{array}{c}\text { Power } \\
W_{0}\end{array}$ & Multiplied by & Coefficient \\
\hline$w_{0}^{n}$ & 1 & 1 & $w_{0}^{n-5}$ & WI & $\frac{(n-1)(n-2)(n-3)(n-4)(n-5)}{5 !}$ \\
\hline$w_{0}^{n-1}$ & $W_{1}$ & $(n-1)$ & $w_{0}^{n-5}$ & $W_{1}^{4} W_{2}$ & $\frac{1}{3 !}(n-2)(n-3)(n-4)(n-5)$ \\
\hline$w_{0}^{n-2}$ & $W_{1}^{2}$ & $\frac{1}{2}(n-1)(n-2)$ & $w_{0}^{n-5}$ & $W^{3} W_{L}^{2}$ & $(n-3)(n-4)(n-5)$ \\
\hline$w_{0}^{n-2}$ & $W: W_{2}$ & $(n-2)$ & $w_{0}^{n-5}$ & $W_{1}^{3} W_{2} W_{3}$ & $\frac{1}{2}(n-3)(n-4)(n-5)$ \\
\hline$w_{0}^{n-3}$ & $W^{\beta}$ & $\frac{1}{3 !}(n-1)(n-2)(n-3)$ & $w_{0}^{n-5}$ & $W_{1}^{2} W_{2}^{3}$ & $2(n-4)(n-5)$ \\
\hline$w_{0}^{n-3}$ & Wîn 2 & $(n-2)(n-3)$ & $w_{0}^{n-5}$ & $W_{1}^{2} W_{2}^{2} W_{3}$ & $3(n-4)(n-5)$ \\
\hline$w_{0}^{n-3}$ & $W_{1} H_{2}^{2}$ & $(n-3)$ & $w_{0}^{n-5}$ & $W_{2}^{2} W_{2} W_{3}^{2}$ & $(n-4)(n-5)$ \\
\hline$w_{0}^{n-3}$ & $W_{1} W_{2} W_{3}$ & $(n-3)$ & $w_{0}^{n-5}$ & $W_{1}^{2} W_{2} W_{3} W_{4}$ & $(n-4)(n-5)$ \\
\hline$w_{0}^{n-4}$ & WI & $\frac{1}{4 !}(n-1)(n-2)(n-3)(n-4)$ & $w_{0}^{n-5}$ & $W_{1} W_{2}^{4}$ & $(n-5)$ \\
\hline$w_{0}^{n-4}$ & $W^{3} \mathrm{~W}_{2}$ & $\frac{1}{2}(n-2)(n-3)(n-4)$ & $w_{0}^{n-5}$ & $W_{1} W_{2} W_{3}$ & $3(n-5)$ \\
\hline$w_{0}^{n-4}$ & $W_{1}^{2} N_{2}^{2}$ & $\frac{3}{2}(n-3)(n-4)$ & $w_{0}^{n-5}$ & $W_{1} W_{2}^{2} W_{3}^{2}$ & $3(n-5)$ \\
\hline$w_{0}^{n-4}$ & $W_{1}^{2} W_{2} W_{3}$ & $(n-3)(n-4)$ & $w_{0}^{n-5}$ & $W_{1} W_{2}^{2} W_{3} W_{4}$ & $2(n-5)$ \\
\hline$w_{0}^{n-4}$ & $W_{1} N_{2}^{3}$ & $(n-4)$ & $w_{0}^{n-5}$ & $W_{1} W_{2} W_{3}^{3}$ & $(n-5)$ \\
\hline$w_{0}^{n-4}$ & $W_{1} W_{2} W_{3}$ & $2(n-4)$ & $w_{0}^{n-5}$ & $W_{1} W_{2} W_{3}^{2} W_{4}$ & $2(n-5)$ \\
\hline$w_{0}^{n-4}$ & $W_{1} W_{2} W_{\frac{2}{3}}^{2}$ & $(n-4)$ & $w_{0}^{n-5}$ & $W_{1} W_{2} W_{3} W_{4}^{2}$ & $(n-5)$ \\
\hline$w_{0}^{n-4}$ & $W_{1} W_{2} W_{3} W_{4}$ & $(n-4)$ & $w_{0}^{n-5}$ & $W_{1} W_{2} W_{3} W_{4} W_{5}$ & $(n-5)$ \\
\hline
\end{tabular}




\section{APPENDIX G}

LIST OF COEFFICIENTS $B_{r, s}(n)$ FOR $r, s=0$ TO $r, s=5$

$B_{0,0}=1 ; \quad B_{1,0}=-W_{1} ; \quad B_{1,1}=W_{1}$

$B_{2,0}=W_{1}^{2}-W_{1} W_{2} ; \quad B_{2,1}=-\frac{3}{2} W_{1}^{2}+W_{1} W_{2} ; \quad B_{22}=\frac{1}{2 !} W_{1}^{2}$

$B_{3,0}=-W_{1}^{3}+6 W_{1}^{2} W_{2}-3 W_{1} W_{2}^{2}-3 W_{1} W_{2} W_{3}$

$B_{3,1}=\frac{11}{6} W_{1}^{3}-5 W_{1}^{2} W_{2}+W_{1} W_{2}^{2}+W_{1} W_{2} W_{3}$

$B_{3,2}=-W_{1}^{3}+W_{1}^{2} W_{2}$

$B_{3,3}=\frac{1}{3 !} W_{1}^{3}$

$B_{4}, 0=W_{1}^{4}-12 W_{1}^{3} W_{2}+18 W_{1}^{2} W_{2}^{2}+12 W_{1}^{2} W_{2} W_{3}-$

$-4\left(W_{1} W_{2}^{3}+2 W_{1} W_{2}^{2} W_{3}+W_{1} W_{2} W_{3}^{2}+W_{1} W_{2} W_{3} W_{4}\right)$

$B_{4,1}=-\frac{25}{12} W_{1}^{4}+13 W_{1}^{3} W_{2}-\frac{21}{2} W_{1}^{?} W_{2}^{?}-7 W_{1}^{2} W_{2} W_{3}+$

$+W_{1} W_{2}^{3}+2 W_{1} W_{2}^{2} W_{3}+W_{1} W_{2} W_{3}^{2}+W_{1} W_{2} W_{3} W_{4}$

$B_{4,2}=\frac{35}{4 !} W_{1}^{4}-\frac{9}{2} W_{1}^{3} W_{2}+\frac{3}{2} W_{1}^{2} W_{2}^{2}+W_{1}^{2} W_{2} W_{3}$

$B_{4,3}=-\frac{5}{12} W_{1}^{4}+\frac{1}{2} W_{1}^{3} W_{2}$ 
$B_{4,4}=\frac{1}{4 !} W_{1}^{4}$

Let:

$$
\begin{aligned}
E_{1}= & W_{1}^{3} W_{2}^{2}+\frac{1}{2} W_{1}^{3} W_{2} W_{3} \\
E_{2}= & 2 W_{1}^{2} W_{2}^{3}+3 W_{1}^{2} W_{2}^{2} W_{3}+W_{1}^{2} W_{2} W_{3}^{2}+W_{1}^{2} W_{2} W_{3} W_{4} \\
E_{3}= & W_{1} W_{2}^{4}+3 W_{1} W_{2}^{3} W_{3}+3 W_{1} W_{2}^{2} W_{3}^{2}+2 W_{1} W_{2}^{2} W_{3} W_{4}+ \\
& +W_{1} W_{2} W_{3}^{3}+2 W_{1} W_{2} W_{3}^{2} W_{4}+W_{1} W_{2} W_{3} W_{4}^{2}+W_{1} W_{2} W_{3} W_{4} W_{5}
\end{aligned}
$$

Then:

$$
\begin{aligned}
& B_{5,0}=-W_{1}^{5}+20 W_{1}^{4} W_{2}-60 E_{1}+20 E_{2}-5 E_{3} \\
& B_{5,1}=\frac{274}{5 !} W_{1}^{5}-\frac{154}{3 !} W_{1}^{4} W_{2}+47 E_{1}-9 E_{2}+E_{3} \\
& B_{5,2}=-\frac{225}{5 !} W_{1}^{5}+\frac{71}{3 !} W_{1}^{4} W_{2}-12 E_{1}+E_{2} \\
& B_{5,3}=\frac{85}{5 !} W_{1}^{5}-\frac{14}{3 !} W_{1}^{4} W_{2}+E_{1} \\
& B_{5,4}=-\frac{15}{5 !} W_{1}^{5}+\frac{1}{3 !} W_{1}^{4} \\
& B_{5,5}=\frac{1}{5 !} W_{1}^{5}
\end{aligned}
$$




\section{APPENDIX H}

DERIVATION OF THE EXACT ANALYTICAL EXPRESSION FOR THE SECOND SPATIAL MOMENT $M_{2}(u)$ OF THE SCALAR FLUX

We start with the exact expression of the Laplace-transformed second moment of the scalar flux, i.e., $M_{2}(n)$. From Eqs. (III.25) and (II.77), we have

$$
M_{2}(n)=\frac{1}{3 \gamma \gamma^{2}(n) \gamma_{1}(n)}
$$

From the definition $\gamma_{n}(n) \equiv 1-\operatorname{cg}_{n}(n)$, and using the explicit expressions of $g_{0}(n)$ and $g_{1}(n)$ from Appendix $A$, we have:

$$
r_{0}(n)=1-\frac{c \alpha}{n+1}\left[1-e^{-\varepsilon(n+1)}\right]
$$

and

$$
\gamma_{1}(n)=1-\frac{c \alpha}{(n+1 / 2)(n+3 / 2)}\left[n+1-\frac{A}{2}+\left(n+1+\frac{A}{2}\right) e^{-\varepsilon(n+1)}\right]
$$

In Eqs. (H.2) and (H.3), $A$ is the nuclear mass of the lividerator, and $\alpha$ and $\varepsilon$ have their usual meanings, i.e.,

$$
\alpha \equiv \frac{(A+1)^{2}}{4 A}
$$

and

$$
\varepsilon \equiv 2 \log \left(\frac{A+1}{A-1}\right)
$$


We define

$$
\beta \equiv C \alpha
$$

Then, we can write

$$
\frac{1}{\gamma_{0}(n)}=\left(1+\frac{\beta}{\eta+1-\beta}\right) \frac{1}{1+\frac{\beta e^{-\varepsilon(n+1)}}{n+1-\beta}}
$$

and, using the same procedure,

$$
\begin{aligned}
\frac{1}{\gamma_{1}(n)} & =\frac{\left(n+\frac{1}{2}\right)\left(n+\frac{3}{2}\right)}{\left(n+\frac{1}{2}\right)\left(n+\frac{3}{2}\right)-\beta\left(n+1-\frac{A}{2}\right)} \times \\
& \times\left[1-\frac{B\left(n+1+\frac{A}{2}\right) e^{-\varepsilon(n+1)}}{\left(n+\frac{1}{2}\right)\left(n+\frac{3}{2}\right)-B\left(n+1-\frac{A}{2}\right)}\right]^{-1}
\end{aligned}
$$

But

$$
\left(n+\frac{1}{2}\right)\left(n+\frac{3}{2}\right)-B\left(n+1-\frac{A}{2}\right) \equiv\left(n-n_{1}\right)\left(n-n_{2}\right)
$$

where

$$
n_{1} \equiv \frac{1}{2}\left[\beta-2+\sqrt{\beta^{2}-2 \beta A+1}\right]
$$

and

$$
n_{2} \equiv \frac{1}{2}\left[e-2-\sqrt{\beta^{2}-2 \beta \Lambda+1}\right]
$$

Using $(H .7-10)$ in $(H .1)$, we obtain

$$
M_{2}(n)=\frac{1}{3} F(n) G(n)
$$

where 


$$
F(n) \equiv\left(1+\frac{\beta}{n+1-\beta}\right)^{2}\left[\frac{1}{1+\frac{\beta e^{-\varepsilon(n+1)}}{n+1-\beta}}\right]^{2}
$$

and

$$
G(n) \equiv\left[1+\frac{B\left(n+1-\frac{A}{2}\right)}{\left(n-n_{1}\right)\left(n-n_{2}\right)}\right]\left[1-\frac{\beta\left(n+1+\frac{A}{2}\right) e^{-\varepsilon(n+1)}}{\left(n-n_{1}\right)\left(n-n_{2}\right)}\right]-1
$$

The expression of $M_{2}(u)$ will be obtained by Laplace Inversion of (H.12), making use of the convolution theorem. For this purpose, we shall have to obtain the Laplace inverses of $F(n)$ and $G(n)$.

We investigate $F(n)$ first. Using

$$
(1+q)^{-2}=\sum_{k=0}^{\infty}(-1)^{k}(k+1) q^{k}
$$

for $|q|<1$, we can write $(H .13)$ as

$$
\begin{aligned}
F(n) & =\left[1+2 \frac{\beta}{n+1-\beta}+\frac{\beta^{2}}{(n+1-\beta)^{2}}\right]\left[\sum_{k=0}^{\infty}(-1)^{k}(k+1) \beta^{k} e^{-\varepsilon k} \frac{e^{-\varepsilon n k}}{(\tilde{n}+1-\beta)^{k}}\right] \\
& =1+\sum_{k=1}^{\infty}(-1)^{k}(k+1) \beta^{k} e^{-\varepsilon k} \frac{e^{-k n \varepsilon}}{(n+1-\beta)^{k}}+ \\
& +2 \sum_{k=0}^{\infty}(-1)^{k}(k+1) \beta^{k+1} e^{-\varepsilon k} \frac{e^{-k \varepsilon n}}{(n+1-\beta)^{k+1}}+ \\
& +\sum_{k=0}^{\infty}(-1)^{k}(k+1) \beta^{k+2} e^{-\varepsilon k} \frac{e^{-k \varepsilon \eta}}{(n+1-\beta)^{k+2}}
\end{aligned}
$$

Using 


$$
\mathcal{L}^{-1}\left[\frac{1}{(s-\delta)^{k}}\right]=\frac{t^{k-1}}{(k-1) !} e^{\delta t} 1_{+}(t)
$$

and

$$
\mathscr{L}^{-1}\left[e^{-s \tau} F(s)\right]=f(t-\tau)
$$

we can easily carry out the Laplace inversion of (H.16), to obtain:

$$
\begin{aligned}
F(u) & =\delta(u)+\sum_{k=1}^{\infty}(-1)^{k}(k+1) \beta^{k} e^{-\varepsilon k} \frac{(u-k \varepsilon)^{k-1} e^{(\beta-1)(u-k \varepsilon)}}{(k-1) !} 1_{+}(u-k \varepsilon) \\
& +2 \sum_{k=0}^{\infty}(-1)^{k}(k+1) \beta^{k+1} e^{-\varepsilon k} \frac{(u-k \varepsilon)^{k} e^{(\beta-1)(u-k \varepsilon)}}{k !} 1_{+}(u-k \varepsilon) \\
& +\sum_{k=0}^{\infty}(-1)^{k}(k+1) \beta^{k+2} e^{-\varepsilon k} \frac{(u-k \varepsilon)^{k+1} e^{(\beta-1)(u-k \varepsilon)}}{(k+1) !} 1_{+}(u-k \varepsilon)
\end{aligned}
$$

Equation (H.19) can be rearranged and, after a little algebra, we obtain:

$$
\begin{aligned}
F(u) & =\delta(u)+\beta e^{(\beta-1) u}\left\{\sum_{k=0}^{\frac{u}{\varepsilon}}[(2-\beta \varepsilon) k+2+\beta u] \times\right. \\
& \times\left(\beta \varepsilon e^{-\beta \varepsilon}\right)^{k} \frac{\left(k-\frac{u}{\varepsilon}\right)^{k}}{k !} 1_{+}(u-k \varepsilon)- \\
& \left.-e^{-\beta \varepsilon} \sum_{k=0}^{\frac{u}{\varepsilon}-1}(k+2)\left[\beta \varepsilon e^{-\beta \varepsilon}\right]^{k} \frac{\left[k-\left(\frac{u}{\varepsilon}-1\right)\right]^{k}}{k !} 1_{+}[u-(k+1) \varepsilon]\right\}
\end{aligned}
$$

In Eq. (H:20), $u$ is the lelhargy vartable, and we have also used the customary definition for the pseudo-function $r_{+}(u)$, i.e., 


$$
I_{+}(u)=\left\{\begin{array}{cc}
1 ; & u>0 \\
\frac{1}{2} ; & u=0 \\
0 ; & u<0
\end{array}\right.
$$

We next address the problem of finding the Laplace inverse $G(u)$, of $G(n)$. We write

$$
\left[1-\frac{\beta\left(\eta+1+\frac{A}{2}\right) e^{-\varepsilon(n+1)}}{\left(n-n_{1}\right)\left(n-n_{2}\right)}\right]^{-1}=\sum_{k=0}^{\infty} \beta^{k} e^{-k \varepsilon}\left[\frac{\eta+1+\frac{A}{2}}{\left(n-n_{1}\right)\left(n-n_{2}\right)}\right] k e^{-k \varepsilon n}
$$

Since the Laplace Inverses of the $k=0$ and $k=1$ terms of Eq. (H.22) are of a different nature than the $k \geq 2$ terms, we write them out explicitly; we then substitute (H.22) into (H.14) and perform the multiplication. This gives:

$$
\begin{aligned}
G(n) & =1+\frac{\beta\left(n+1+\frac{A}{2}\right)}{\left(n-n_{1}\right)\left(n-n_{2}\right)}+\beta e^{-\varepsilon} \frac{\left(n+1+\frac{A}{2}\right)}{\left(n-n_{1}\right)\left(n-n_{2}\right)} e^{-\varepsilon n}+ \\
& +\beta^{2} e^{-\varepsilon} \frac{\left(n+1-\frac{A}{2}\right)\left(n+1+\frac{A}{2}\right)}{\left(n-n_{1}\right)^{2}\left(n-n_{2}\right)^{2}} e^{-\varepsilon n}+ \\
& +\sum_{k=2}^{\infty} \beta^{k} e^{-k \varepsilon\left[\frac{n+1+\frac{A}{2}}{\left(n-n_{1}\right)\left(n-n_{2}\right)}\right] k} e^{-k \varepsilon n}+ \\
& +\sum_{k=2}^{\infty} \beta^{k+1} e^{-k \varepsilon} \frac{n+1-\frac{A}{2}}{\left(n-n_{1}\right)\left(n-n_{2}\right)}\left[\frac{n+1+\frac{A}{2}}{\left(n-n_{1}\right)\left(n-n_{2}\right)}\right] k e^{-k \varepsilon n}
\end{aligned}
$$

But

$$
\mathscr{L}-1\left[\frac{\beta\left(n+1-\frac{A}{2}\right)}{\left(n-n_{1}\right)\left(n-n_{2}\right)}\right]=\beta\left[\frac{\frac{A}{2}-1-n_{1}}{n_{2}-n_{1}} e^{n_{1} u}-\frac{\frac{A}{2}-1-n_{2}}{n_{2}-n_{1}} e^{n_{2} u}\right] 1_{+}(u)
$$


Making use of Eqs. (H.10) and (H.11), Eq. (H.24) becomes, after some algebraic manipulations:

$$
\begin{aligned}
\mathscr{L}-1\left[\frac{\beta\left(n+1-\frac{A}{2}\right)}{\left(n-n_{1}\right)\left(n-n_{2}\right)}\right]=I_{+}(u) B e^{\left(\frac{B}{2}-1\right) u}\left[\cosh \frac{u}{2} \sqrt{B^{2}-2 \beta A+1}-\right. \\
\left.-\frac{A-B}{\sqrt{\beta^{2}-2 \beta+1}} \sinh \frac{u}{2} \sqrt{B^{2}-2 B A+1}\right]
\end{aligned}
$$

Next, we have:

$$
\begin{aligned}
& \mathscr{L}^{-1}\left[\beta \mathrm{e}^{-\varepsilon} \frac{\left(n+1+\frac{A}{2}\right) e^{-\varepsilon n}}{\left(n-\eta_{1}\right)\left(n-n_{2}\right)}\right]=1_{+}(u-\varepsilon) \beta \mathrm{e}^{-\frac{\beta \varepsilon}{2}} e^{\left(\frac{\beta}{2}-1\right) u} \times \\
& \quad \times\left[\cosh \frac{u-\varepsilon}{2} \sqrt{\beta^{2}-2 \beta A+1}+\frac{A+B}{\sqrt{\beta^{2}-2 \beta A+1}} \sinh \frac{u-\varepsilon}{2} \sqrt{\beta^{2}-2 \beta A+1}\right]
\end{aligned}
$$

Furthermore:

$$
\begin{aligned}
\mathscr{L}^{-1}\left[\beta^{2} e^{-\varepsilon} \frac{\left(\eta+1-\frac{A}{2}\right)\left(\eta+1+\frac{A}{2}\right) e^{-\varepsilon \eta}}{\left(\eta-\eta_{1}\right)^{2}\left(\eta-n_{2}\right)^{2}}\right]= \\
\quad=1_{+}(u-\varepsilon) \beta^{2} e^{-\frac{\beta \varepsilon}{2}} e^{\left(\frac{B}{2}-1\right) u} \times \\
\quad \times\left[\frac{2 \beta^{2}-2 \beta A+1-A^{2}}{\beta^{2}-2 \beta A+\varepsilon} \frac{u-1}{2} \cosh \frac{u-\varepsilon}{2} \sqrt{\beta^{2}-2 \beta A+1}+\right. \\
\quad+\frac{2 \beta}{\sqrt{\beta^{2}-2 \beta A+T}} \frac{u-\varepsilon}{2} \sinh \frac{u-\varepsilon}{2} \sqrt{\beta^{2}-2 \beta A+1}+ \\
\left.\quad+\frac{A^{2}-2 \beta A+1}{\left(\beta^{2}-2 \beta A+1\right)^{3 / 2}} \sinh \frac{u-\varepsilon}{2} \sqrt{\beta^{2}-2 \beta A 1 T}\right]
\end{aligned}
$$

Next, we perform: 


$$
\mathcal{L}^{-1}\left\{\sum_{k=2}^{\infty} \beta^{k} e^{-k \varepsilon}\left[\frac{n+1+\frac{A}{2}}{\left(n-n_{1}\right)\left(n-n_{2}\right)}\right] k e^{-k \varepsilon n}\right\}
$$

We can perform the inversion term by term. Let

$$
H(n) \equiv\left[\frac{n+1+\frac{A}{2}}{\left(n-n_{1}\right)\left(n-n_{2}\right)}\right] k
$$

Then, since $k \geq 2$, we can use the following procedure: we rewrite $H(n)$ as

$$
H(n)=X(n) Y(n)
$$

where

$$
x(n)=\frac{n-n_{2}}{\left(n-n_{1}\right)^{k}}
$$

and

$$
Y(n)=\frac{1}{n-n_{2}}\left(\frac{n-n_{3}}{n-n_{2}}\right)^{k}
$$

with

$$
n_{3} \equiv-\left(\frac{A}{2}+1\right)
$$

We shall calculate the inverses of $X(n)$ and $Y(n)$ and then use the convolution theorem to obtain the inverse of $H(n)$. We have, since $k \geq 2$

$$
\begin{aligned}
\mathscr{L}^{-1}\{x(n)\}=\mathscr{L}^{-1}\left[\frac{1}{\left(n-1_{1}\right)^{k-1}}+\frac{n_{1}-n_{2}}{\left(n-n_{1}\right)^{k}}\right]= \\
\quad=\left[\frac{u^{k-2}}{(k-2) !}+\left(n_{1}-n_{2}\right) \frac{u^{k-1}}{(k-1) !}\right] e^{n_{1} u} 1_{+}(u)
\end{aligned}
$$


Also, since: [see e.g., Erdelyi et al., Tables of Integral Transforms, Vol. 1, p. 175, Eq. (31)]

$$
\begin{gathered}
t^{\alpha} e^{\lambda t} L_{n}^{\alpha}(k t)=\mathscr{L}^{-1}\left[\frac{\Gamma(\alpha+n+1)}{n !} \frac{(p-k-\lambda)^{n}}{(p-\lambda)^{\alpha+n+1}}\right] \\
\text { for } \operatorname{Re} \alpha>-1, \operatorname{Re}(p-\lambda)>0
\end{gathered}
$$

we have:

$$
\mathscr{L}^{-1}[Y(n)]=e^{n_{2} u} L_{k}\left[\left(n_{3}-n_{2}\right) u\right] I_{+}(u)
$$

where $L_{k}(x)$ is the Laguerre Polynomial. Using Eqs. (H.34) and (H.36), together with the convolution theorem, we obtain

$$
\begin{aligned}
\mathscr{L}^{-1}[H(n)] & =\int_{0}^{u} e^{n_{2} \tau} L_{k}\left[\left(n_{3}-n_{2}\right) \tau\right] 1_{+}(\tau) e^{n_{1}(u-\tau)} 1_{+}(u-\tau) \times \\
& \times\left[\frac{(u-\tau)^{k-2}}{(k-2) !}+\left(n_{1}-n_{2}\right) \frac{(u-\tau)^{k-1}}{(k-1) !}\right] d \tau
\end{aligned}
$$

In order to evaluate the integrals in (H.37), we shall make use of the definition of the Laguerre Polynomials, i.e.,

$$
L_{n}(x)=\sum_{m=0}^{n}\left(\begin{array}{l}
n \\
m
\end{array}\right)(-1)^{m} \frac{x^{m}}{m !}={ }_{1} F_{1}(-n ; 1 ; x)
$$

Eq. (H.37) becomes

$$
\mathscr{L}^{-1}\{H(n)\}=e^{n_{1} u} 1_{+}(u)\left\{\frac{1}{(k-2) !} \sum_{m=0}^{k}(-1)^{m}\left(\begin{array}{l}
k \\
m
\end{array}\right) \frac{\left(n_{3}-n_{2}\right)^{m}}{m !} \times\right.
$$




$$
\begin{aligned}
& \times \int_{0}^{u} e^{\left(n_{2}-n_{1}\right)}(u-\tau)^{k-2} \tau^{m} d \tau+\frac{n_{1}-n_{2}}{(k-1) !} x \\
& \left.\times \sum_{m=0}^{k}(-1)^{m}\left(\begin{array}{l}
k \\
m
\end{array}\right) \frac{\left(n_{3}-n_{2}\right)^{m}}{m !} \int_{0}^{u} e^{\left(n_{2}-n_{1}\right) \tau}(u-\tau)^{k-1} \tau^{m} d \tau\right\}
\end{aligned}
$$

The integrals in (H.39) can now be evaluated in closed form, using the following result [see: e.g., Gradshteyn and Ryzhik, Tables of Integrals, p. 318, Eq. 3.383-1]:

$$
\int_{0}^{u} x^{\nu-1}(u-x)^{\mu-1} e^{\beta x} d x=B(\mu, v) u^{\mu+\nu-1}{ }_{1} F_{1}(\nu ; \mu+v ; B u)
$$

$$
\text { for } \operatorname{Re} \nu>0, \operatorname{Re} \mu>0
$$

where

$$
B(\mu, \nu) \triangleq \int_{0}^{1} t^{\mu-1}(1-t)^{\nu-1} d t=\frac{\Gamma(\mu) \Gamma(\nu)}{\Gamma(\mu+\nu)}
$$

is the well-known Beta Function, and

$$
{ }_{1} F_{1}(a ; c ; z) \triangleq \frac{\Gamma(c)}{\Gamma(a)} \sum_{n=0}^{\infty} \frac{\Gamma(a+n)}{\Gamma(c+n)} \frac{z^{n}}{n !}
$$

is the well-known degenerate hypergeometric function. Using (H.41), Equation (H.40) becomes, after some algebraic manipulations,

$$
\begin{aligned}
& \mathscr{L}^{-1}[H(n)]=e^{n_{1} u} 1_{+}(u)\left\{\frac{1}{(k-2) !} \sum_{m=0}^{k}(-1)^{m}\left(\begin{array}{l}
k \\
m
\end{array}\right) \frac{\left(n_{3}-n_{2}\right)^{m}}{m !} u^{m+k-1}\right. \\
& \quad \times B(k-1, m+1){ }_{1} F_{1}\left(m+1 ; m+k ;\left(n_{2}-n_{1}\right) u\right]+
\end{aligned}
$$




$$
\begin{aligned}
& +\frac{\eta_{1}-n_{2}}{(k-1) !} \sum_{m=0}^{k}(-1)^{m}\left(\begin{array}{l}
k \\
m
\end{array}\right) \frac{\left(n_{3}-n_{2}\right)^{m}}{m !} u^{m+k} B(k, m+1) \times \\
& \left.\times{ }_{1} F_{1}\left[m+1 ; m+k+1 ;\left(n_{2}-n_{1}\right) u\right]\right\}
\end{aligned}
$$

Next, we use the well-known relation

$$
{ }_{1} F_{1}(a ; c ; z)=e^{z}{ }_{1} F_{1}(c-a ; c ; z)
$$

and Eq. (H.41), to simplify (H.43). After some algebra, we obtain, finally:

$$
\begin{aligned}
& \mathscr{L}^{-1}\{H(n)\}=e^{n_{2} u} 1_{+}(u)\left\{\sum_{m=0}^{k}(-1)^{m}\left(\begin{array}{l}
k \\
m
\end{array}\right) \frac{\left(n_{3}-n_{2}\right)^{m}}{\Gamma(m+k)} u^{m+k-1} \times\right. \\
& \left.\left.\quad \times\left[{ }_{1} F_{1}\left[k-1 ; m+k ;\left(n_{2}-n_{1}\right) u\right]+u \frac{n_{1}-n_{2}}{k+m}{ }_{1} F_{1}\left[k ; m+k+1 ;\left(n_{2}-n_{1}\right) u\right)\right]\right]\right\}
\end{aligned}
$$

Thus, expression (H.28) ultimately becomes, in view of (H.45) and (H.18):

$$
\begin{aligned}
& \mathcal{E}^{-1}\left\{\sum_{k=2}^{\infty} \beta^{k} e^{-k \varepsilon}\left[\frac{n+1+\frac{A}{2}}{\left(n-n_{1}\right)\left(n-n_{2}\right)}\right] k e^{-k \in n}\right\}= \\
& =(-) e^{n_{2} u} \sum_{k=2}^{\frac{u}{\varepsilon}}(-1)^{k}\left[\beta \varepsilon e^{-\varepsilon\left(1+n_{2}\right)}\right]^{k}\left[k-\frac{u}{\varepsilon}\right]^{k} 1_{+}(u-k \varepsilon) \times \\
& \left.\times \sum_{m=0}^{k}\left(\begin{array}{l}
k \\
i 11
\end{array}\right) \frac{\left(n_{3}-n_{2}\right.}{\Gamma(m+k}\right)^{m}-\varepsilon^{m-1}\left[k-\frac{u}{\varepsilon}\right]^{m-1}\left\{{ }_{1} F_{1}\left[k-1 ; n+k ;\left(n_{2}-n_{1}\right)(u-k \varepsilon)\right]\right. \\
& \left.+\frac{n_{1}-n_{2}}{m+k}(u-k \varepsilon){ }_{1} F_{1}\left[k ; m+k+1 ;\left(n_{2}-n_{1}\right)(u-k \varepsilon)\right]\right\}
\end{aligned}
$$


The last term of $G(n)$ to be inverted is

$$
\mathscr{L}^{-1}\left\{\sum_{k=2}^{\infty} \beta^{k+1} e^{-k \varepsilon} \frac{n+1-\frac{A}{2}}{\left(n-n_{1}\right)\left(n-n_{2}\right)}\left[\frac{n+1+\frac{A}{2}}{\left(n-n_{1}\right)\left(n-n_{2}\right)}\right] k e^{-k \in n}\right\}
$$

Let

$$
W(n) \equiv \frac{n+1-\frac{A}{2}}{\left(n-n_{1}\right)\left(n-n_{2}\right)}\left[\frac{n+1+\frac{A}{2}}{\left(n-n_{1}\right)\left(n-n_{2}\right)}\right] k
$$

Then

$$
W(n) \equiv Y(n) Z(n)
$$

where $Y(n)$ is as defined in (H.32) and

$$
Z(n) \equiv \frac{n+1-\frac{A}{2}}{\left(n-n_{1}\right)^{k+1}}
$$

then

$$
\begin{aligned}
\mathscr{L}^{-1}\{Z(n)\} & =\mathscr{L}^{-1}\left\{\frac{1}{\left(n-n_{1}\right)^{k}}+\frac{n_{1}+1-\frac{A}{2}}{\left(n-n_{1}\right)^{k+1}}\right\}= \\
& =\left[\frac{u^{k-1}}{(k-1) !}+\left(n_{1}+1-\frac{A}{2}\right) \frac{u^{k}}{k !}\right] e^{n_{1} u} I_{+}(u)
\end{aligned}
$$

Using the convolution theorem for (H.51) and (H.36) we find the Laplace inverse of (H.49) to be

$$
\mathscr{E}=1\{W(n)\}=\int_{0}^{u} e^{n_{2} \tau} L_{k}\left[\left(n_{3}-n_{2}\right) \tau\right] 1_{+}(\tau) e^{n_{1}(u-\tau)} 1_{+}(u-\tau) \times
$$




$$
\times\left[\frac{(u-\tau)^{k-1}}{(k-1) !}+\left(n_{1}+1-\frac{A}{2}\right) \frac{(u-\tau)^{k}}{k !}\right] d \tau
$$

Using (H.38), (H.52) becomes:

$$
\begin{aligned}
& \mathcal{E}^{-1}\{W(n)\}=e^{n_{1} u} \tau_{+}(u)\left\{\frac{1}{(k-1) !} \sum_{m=0}^{k}(-1)^{m}\left(\begin{array}{l}
k \\
m
\end{array}\right) \frac{\left(n_{3}-n_{2}\right)^{m}}{m !} \times\right. \\
& \times \int_{0}^{u} e^{\left(n_{2}-n_{1}\right) \tau}(u-\tau)^{k-1} m_{\tau} d \tau+\frac{n+1-\frac{A}{2}}{k !} \sum_{m=0}^{k}(-1)^{m}\left(\begin{array}{l}
k \\
m
\end{array}\right) \times \\
& \left.\times \frac{\left(n_{3}-n_{2}\right)^{m}}{m !} \int_{0}^{u} e^{\left(n_{2}-n_{1}\right) \tau}(u=\tau)^{k} \tau^{m} d \tau\right\}
\end{aligned}
$$

Using (H.40), (H.4l), and $(\mathrm{H.44)}$ and following the same procedure that led to (H.46) above, we obtain for (H.47) the expression:

$$
\begin{aligned}
& \sum^{-1}\left\{\sum_{k=2}^{\infty} \beta^{k+1} e^{-k \varepsilon} W(n) e^{-k \varepsilon n}\right\}= \\
& =e^{n_{2} u} \sum_{k=2}^{\frac{u}{\varepsilon}} \beta^{k+1} e^{-k \varepsilon} e^{-n_{2} k \varepsilon} 1_{+}(u-k \varepsilon) \sum_{m=0}^{k}(-1)^{m}\left(\begin{array}{l}
k \\
m
\end{array}\right) \\
& \times \frac{\left(n_{3}-n_{2}\right)^{m}}{\Gamma(k+m+1)}(u-k \varepsilon)^{k+m}\left\{{ }_{1} F_{1}\left[k ; k+m+7 ;\left(n_{2}-n_{1}\right)(u-k \varepsilon)\right]+\right. \\
& \left.+\frac{n_{1}+1-\frac{A}{2}}{m+k+1}(u-k \varepsilon)_{1} F_{1}\left[k+1 ; m+k+2 ;\left(n_{2}-n_{1}\right)\left(u-k_{c} c\right)\right]\right\} \\
& =B e^{n_{2} u} \sum_{k=2}^{\frac{u}{\varepsilon}}\left[\beta \varepsilon e^{-\varepsilon\left(1+n_{2}\right)}\right]^{k}(-1)^{k}\left[k-\frac{u}{\varepsilon}\right]^{k} 1_{+}(u-k \varepsilon) \times \\
& \times \sum_{m=0}^{k}\left(\begin{array}{l}
k \\
m
\end{array}\right) \varepsilon^{m} \cdot \frac{\left(\eta_{3}-n_{2}\right)^{m}}{\Gamma(k+m+1)}\left[k-\frac{u}{\varepsilon}\right]^{m}\left\{{ }_{1} F_{1}\left[k ; k+m+1 ;\left(n_{2}-\eta_{1}\right)(u-k \varepsilon)\right]\right.
\end{aligned}
$$




$$
\left.+\frac{n_{1}+1-\frac{A}{2}}{m+k+1}(u-k \varepsilon){ }_{1} F_{1}\left[k+1 ; m+k+2 ;\left(n_{2}-n_{1}\right)(u-k \varepsilon)\right]\right\}
$$

Expression (H.53) can be combined with $(H .46)$ to yield:

$$
\begin{aligned}
& \mathscr{Z}^{-1}\left\{\sum_{k=2}^{\infty} \beta^{k} e^{-k \varepsilon}\left[\frac{n+1+\frac{A}{2}}{\left(n-n_{1}\right)\left(n-n_{2}\right)}\right] k e^{-k \varepsilon n}\left[1+\beta \frac{n+1-\frac{A}{2}}{\left(n-n_{1}\right)\left(n-n_{2}\right)}\right]\right\}= \\
& =e^{n_{2} u} \sum_{k=2}^{\frac{u}{\varepsilon}}\left[-\beta \varepsilon e^{-\varepsilon\left(1+n_{2}\right)}\right]^{k}\left(k-\frac{u}{\varepsilon}\right)^{k} 1_{+}(u-k \varepsilon) \times \\
& \times \sum_{m=0}^{k}\left(\begin{array}{l}
k \\
m
\end{array}\right)\left(n_{3}-n_{2}\right)^{m} \frac{\varepsilon^{m}\left(k-\frac{u}{\varepsilon}\right)^{m}}{\Gamma(m+k)}\left\{\frac{\beta\left(n_{1}+1-\frac{A}{2}\right)}{(m+k)(m+k+1)}(u-k \varepsilon) \times\right. \\
& \left.\quad \times{ }_{1} F_{1}\left[k+1 ; m+k+2 ;\left(n_{2}-n_{1}\right)(u-k \varepsilon)\right]+\left(\frac{\beta}{m+k}+\frac{n_{1}-n_{2}}{m+k+1}\right) \times \quad . \quad \text { (H.54) }\right)
\end{aligned}
$$

We have thus completed the inversion of $G(n)$ of Eq. (H.23). Collecting Eqs. (H.25), (H.26), (H.27) and (H.54), we see that the Laplace Inverse of $G(n)$ is:

$$
\begin{aligned}
G(u) & =\mathscr{L}^{-1}\{G(n)\}= \\
& =\delta(u)+1_{+}(u) \beta e^{\left(\frac{\beta}{2}-1\right) u}\left[\cosh \frac{u}{2} \sqrt{\beta^{2}-2 \beta A+1}\right. \\
& \left.-\frac{A-\beta}{\sqrt{\beta^{2}-2 \beta A+T}} \sinh \frac{u}{2} \sqrt{\beta^{2}-2 \beta A+1}\right]+ \\
& +1_{+}(u-\varepsilon)\left\{\beta e^{-\frac{\beta \varepsilon}{2}} e^{\left(\frac{\beta}{2}-1\right) u\left[\cosh \frac{u-\varepsilon}{2} \sqrt{\beta^{2}-2 \beta A+1}+\right.}\right.
\end{aligned}
$$




$$
\begin{aligned}
& +\frac{A+\beta}{\sqrt{\beta^{2}-2 \beta A+T}} \sinh \frac{u-\varepsilon}{2} \sqrt{\beta^{2}-2 \beta A+1}+ \\
& +\beta \frac{2 \beta^{2}-2 \beta A+1-A^{2}}{\beta^{2}-2 \beta A+1} \frac{u-\varepsilon}{2} \cosh \frac{u-\varepsilon}{2} \sqrt{\beta^{2}-2 \beta A+1}+ \\
& +\frac{2 \beta^{2}}{\sqrt{\beta^{2}-2 \beta A+1}} \frac{u-\varepsilon}{2} \sinh \frac{u-\varepsilon}{2} \sqrt{\beta^{2}-2 \beta A+1}+ \\
& \left.+\beta \frac{A^{2}-2 \beta A+1}{\left(\beta^{2}-2 \beta A+1\right)^{3 / 2}} \sinh \frac{u-\varepsilon}{2} \sqrt{\beta^{2}-2 B A+T}\right]+ \\
& +E q .(H .54)
\end{aligned}
$$

We can now write the final expression for $M_{2}(u)$. For this purpose, we notice that Eq. (H.19) for $F(u)$ can be written as:

$$
F(u)=\delta(u)+f_{1}(u)
$$

and, similarly, Eq. $(H .55)$ for $G(u)$ can be written as:

$$
G(u)=\delta(u)+g_{1}(u)
$$

Since the Laplace Inverse of $M_{2}(\eta)$ of Eq. (H.12) is

$$
M_{2}(u)=\frac{1}{3} \int_{0}^{u} F(\tau) G(u-\tau) d \tau
$$

we see that

$$
\begin{aligned}
M_{2}(u) & =\frac{1}{3}\left\{\delta(u)+f_{1}(u)+y_{1}(u)+\right. \\
& \left.+\int_{0}^{u} f_{1}(\tau) g_{1}(u-\tau) d \tau\right\}
\end{aligned}
$$


where $f_{1}(u)$ is given by $\left(H_{.19}\right)$, excluding the $\delta(u)$ term, and, similarly, $g_{1}(u)$ is given by $(H .55)$, excluding the $\delta(u)$. 


\section{APPENDIX I}

\section{THE COEFFICIENTS $t_{m, j} ; Z_{m, j} ; X_{m, j} ; Y_{m, j}$ AND $C_{m, j}$ PROOFS OF EQUATIONS (III.72-76)}

1. Proof of Eq. (III.72) for $t_{m, j}$

We expand the numerator of Eq. (III.65) in an infinite power series in $\eta$, around $n=0$, i i.e.,

$$
\sum_{r=0}^{n-1} \frac{1}{w_{0}^{r}} \sum_{j=0}^{r} n^{j} B_{r, j}(n)=\sum_{m=0}^{\infty} t_{m} n^{m}
$$

where

$$
t_{m}=\frac{1}{m !} \frac{d^{m}}{d n^{m}}\left[\sum_{r=0}^{n-1} \frac{1}{w_{0}^{r}} \sum_{j=0}^{r} n^{j} B_{r, j}\right]_{n=0}
$$

Interchanging the order of summation and invoking Leibnitz' differentiation rule, (I.2) becomes

$$
t_{m}=\frac{1}{m !} \sum_{s=0}^{m}\left(\begin{array}{l}
m \\
s
\end{array}\right) \sum_{j=0}^{n-1} n^{j} \sum_{r=j}^{n-1}\left\{\left(\frac{1}{w_{0}^{r}}\right)^{(s)} B_{r, j}(m-s)\right\}_{n=0}
$$

But since $\frac{1}{w_{0}^{r}}=\left[3 \gamma_{0}(n) \gamma_{1}(n)\right]^{r}$ and since $\gamma_{0}(0)=0$, we see that:

$$
\frac{d^{s}}{d n^{s}}\left[\frac{1}{W_{0}^{r}}\right]_{n=0}=0 \text { for } r>s
$$

Thus, in view of (I.4), the summation on $r$ in (I.3) can be reduced by replacing the upper limit $(n-1)$ by $s$, i.e., 


$$
t_{m}=\frac{1}{m !} \sum_{j=0}^{m} n^{j} \sum_{s=j}^{m} \sum_{r=j}^{s}\left(\begin{array}{l}
m \\
s
\end{array}\right)\left\{\left(\frac{1}{w_{0}^{r}}\right)^{(s)} B_{r, j}(m-s)\right\}_{n=0}
$$

Obviously, $t_{m}$ is a polynomial in $n$ with coefficients $t_{m, j}$, i.e.,

$$
t_{m}=\sum_{j=0}^{m} t_{m, j} n^{j}
$$

where

$$
\left.\begin{array}{r}
t_{m, j}=\frac{1}{m !} \sum_{s=j}^{m} \sum_{r=j}^{s}\left(\begin{array}{l}
m \\
s
\end{array}\right)\left\{\left.\left(\frac{1}{w_{0}^{r}}\right)^{(s)} B_{r, j}(m-s)\right|_{n=0}\right. \\
\text { for } m, j=0,1,2, \ldots \text { and } j \leq m \\
\text { with } t_{0,0}=1 \text { and } t_{m, j}=0 \text { for } j>m
\end{array}\right\}
$$

The first few $t_{m, j}$ are

$$
\begin{aligned}
& t_{0,0}=1 ; t_{1,0}=\left(\frac{1}{W_{0}}\right)(1)_{B_{1}, 0}=-\frac{4 r j o}{5 \gamma_{2}(0)} \\
& t_{1,1}=\frac{4 r_{0}(0)}{5 \gamma_{2}(0)}=-t_{1,0}, \text { etc. }
\end{aligned}
$$

- for $j=0$, we have

$$
\left.\left.t_{m, 0}=\frac{1}{m !} \sum_{s=1}^{m} \sum_{r=1}^{s}\left(\begin{array}{l}
m \\
s
\end{array}\right)\right\}\left(\frac{1}{w_{0}^{r}}\right)^{(s)} B_{r, 0}(m-s)\right\}_{n=0}
$$

- for $j=1$, we have

$$
t_{m, 1}=\frac{1}{m !} \sum_{s=1}^{m} \sum_{r=1}^{s}\left(\begin{array}{l}
m \\
s
\end{array}\right)\left\{\left(\frac{1}{w_{0}^{r}}\right)^{(s)} B_{r, 1}(m-s)\right\}_{n=0}
$$


- for $j=m$, we have the simple relation

$$
t_{m, m}=\left(\frac{1}{m !}\right)\left(t_{1}, 1\right)^{m}=\left(\frac{1}{m !}\right)\left[\frac{4 \gamma_{0}(0)}{5 \gamma_{2}(0)}\right]^{m}
$$

since

$$
B_{m, m}=\frac{1}{m !} W_{1}^{m}=\frac{1}{m !}\left[\frac{4}{3 \times 5 \gamma_{1} \gamma_{2}}\right]^{m}
$$

and

$$
\left(\frac{1}{w_{0}^{m}}\right)_{n=0}^{(m)}=\left[3 \gamma_{0}^{\prime}(0) \gamma_{1}(0)\right]^{m}(m !)
$$

2. Proof of Eq. (III.74) for $x_{m, j}$

We recall that the $x_{m, j}$ arise from the first factor in the denominator of (III.65), i.e., from $\left[\sum_{k=0}^{\infty} a_{k} k^{k}\right]^{n+1}$, where the $a_{k}{ }^{\prime} s$ are defined in (III.66). The above expression can be written as

$$
\left[\sum_{k=0}^{\infty} a_{k^{n}}\right]^{n+1}=\sum_{m=0}^{\infty} x_{m i n} n^{m}
$$

where the $X_{m}$ obey the recursion relation

$$
\left.\begin{array}{c}
x_{m}=\frac{1}{m} \sum_{k=1}^{m} \begin{array}{rl}
(k n+2 k-m) a_{k} & x_{m-k} \\
\text { for } m \geq 1 & \geq 1
\end{array} \\
\text { with } x_{0}=1
\end{array}\right\}
$$

We see that $x_{m}$ is again a polynomial in $n$, of degree $m$, i.e.,

$$
x_{m}=\sum_{j=0}^{m} x_{m, j} n^{j}
$$


where the $x_{m, j}$ can be found by substituting (1.12) in (I.11) and equating the powers of $n$. The result of this operation is the recursion formula for the $x_{m, j}$, i.e.,

$$
\left.\begin{array}{c}
x_{m, j}=\frac{1}{m} \sum_{k=1}^{m} a_{k}\left[k x_{m-k, j-1}+(2 k-m) x_{m-k, j}\right] \\
\text { for } j, m=0,1,2, \ldots \text { and } j \leq m \\
\text { with } x_{0,0}=1 \text { and } x_{m, j}=0 \text { for } j>m
\end{array}\right\}
$$

The first few $x_{m, j}$ are:

$$
\left.\begin{array}{l}
x_{0,0}=1 ; x_{1,1}=a_{1} ; x_{1,0}=a_{1} \\
x_{2,2}=\frac{a_{1}^{2}}{2} ; x_{2,1}=\frac{a_{1}^{2}}{2}+a_{1} ; x_{2,0}=a_{2}, \text { etc. }
\end{array}\right\}
$$

Also, for $j=m$ we have $x_{m, m}=\frac{1}{m} a_{1} x_{m-1, m-1}$, or

$$
x_{m, m}=\frac{1}{m !} a_{1}^{m}
$$

\section{Proof of Eq. (III.75) for $Y, j$}

We recall that the $Y_{m, j}$ arise from the second term of the denominator of (III.65), i.e., from $\left(\Sigma b_{k} n^{k}\right)^{n}$, where the $b_{k}{ }^{\prime} s$ are defined in (III.67). We use the same procedure to obtain the $Y_{m, j}$ as we did for the $x_{m, j}$, i.e., we write

$$
\left(\sum_{k=0}^{\infty} b_{k} n^{k}\right)^{n}=\sum_{m=0}^{\infty} Y_{m} n^{m}
$$

where 


$$
\left.\begin{array}{c}
Y_{m}=\frac{1}{m} \sum_{k=1}^{m}(k n-m+k) b_{k} Y_{m-k} ; k \geq 1 \\
\text { with } Y_{0}=1
\end{array}\right\}
$$

It is obvious that $Y_{m}$ is a polynomial in $n$, of degree $m$, i.e.,

$$
Y_{m}=\sum_{j=0}^{m} Y_{m, j} n^{j}
$$

where the coefficients $Y_{m, j}$ satisfy the recursion relation

$$
\begin{gathered}
Y_{m, j}=\frac{1}{m} \sum_{k=1}^{m} b_{k}\left[k Y_{m-k, j-1}+(k-m) Y_{m-k, j}\right] \\
\text { for } m, j=0,1,2, \ldots \text { and } j \leq m \\
\text { with } Y_{0,0}=1 \text { and } Y_{m, j}=0 \text { for } j>m \\
\text { Q.E.D. }
\end{gathered}
$$

The first few $Y_{m, j}$ are

$$
\left.\begin{array}{l}
Y_{0,0}=1 ; Y_{1,1}=b_{1} ; Y_{1,0}=0 \\
Y_{2,2}=\frac{1}{2} b_{1}^{2} ; Y_{2,1}=b_{2}-\frac{1}{2} b_{1}^{2} ; Y_{2,0}=0
\end{array}\right\}
$$

and, for $m=j$, we have

$$
Y_{m, m}=\frac{1}{m} b_{1} Y_{m-1, m-1}=\frac{1}{m !} b_{1}^{m}
$$

4. Proof of Eq. (III.73) for $Z_{m, j}$

The coefficient $z_{m, j}$ arise as a result of carrying out the multiplication of the two factors in the denominator of (III.65), i.e., from the multiplication of (1.10) by (I.15). We have: 


$$
\left(\sum_{m=0}^{\infty} X_{m} n^{m}\right)\left(\sum_{m=0}^{\infty} Y_{m^{n}}{ }^{m}\right)=\sum_{m=0}^{\infty} Z_{m^{n}}{ }^{m}
$$

where $z_{m}$ obeys the relation

$$
Z_{m}=\sum_{k=0}^{m} X_{k} Y_{m-k}
$$

Since $X_{k}$ is a polynomial in $n$ of degree $k$ and $Y_{m-k}$ also a polynomial in $n$ of degree $(m-k), z_{m}$ will also be a polynomial in $n$, of degree $m$, i.e.,

$$
z_{m}=\sum_{j=0}^{m} z_{m, j} n^{j}
$$

Substituting (I.12) for $X_{k}$ and (I.17) for $Y_{m-k}$ into (I.21) and equating the coefficients of $n^{j}$ of the resulting expression with the $z_{m, j}$ of (1.22), we obtain the recursion relation for the $z_{m, j}$ in the form:

$$
\left.\begin{array}{r}
Z_{m, j}=\sum_{k=0}^{m} \sum_{s=\max (0, j-k)}^{s=m i n(j, m-k)} X_{k, j-s} Y_{m-k, s} \\
\text { for } m, j=0,1,2, \ldots \text { and } j \leq m \\
\text { with } Z_{0,0}=1 \text { and } z_{m, j}=0 \text { for } j>m \\
\text { Q.E.D. }
\end{array}\right\}
$$

For a geometric visualization of the double sums in (III.73) we refer the reader to Fig. I.1, below. The first few $z_{m, j}$ are: 


$$
\left.\begin{array}{l}
z_{0,0}=1 ; z_{1,1}=a_{1}+b_{1} ; z_{1,0}=a_{1} ; z_{2,0}=a_{2} \\
z_{2,2}=\frac{1}{2} z_{1,1}^{2} ; z_{2,1}=-\frac{b_{1}^{2}}{2}+b_{2}+a_{1} b_{1}+\frac{a_{1}^{2}}{2}+a_{2}
\end{array}\right\}
$$

Also

$$
\begin{aligned}
& Z_{m, 0}=\sum_{k=0}^{m} X_{k, 0} Y_{m-k, 0} \\
& Z_{m, 1}=\sum_{k=0}^{I I N} \sum_{s=0}^{1} X_{k, 1-s} Y_{m-k, s}
\end{aligned}
$$

For $j=m$, we have

$$
Z_{m, m}=\sum_{k=0}^{m} X_{k, k} Y_{m-k, m-k}
$$

Using (I.14) and (I.19), the above relation becomes

$$
z_{m, m}=\sum_{k=0}^{m} \frac{1}{k !} a_{1}^{k} \frac{h_{1}^{m-k}}{(m-k) !}=\frac{1}{m !}\left(a_{1}+b_{1}\right)^{m}
$$

5. Proof of Eq. (III.76) for $C_{m, j}$

Using Eqs. (I.1) and (I.20), Equation (III.65) can be written as:

$$
h(n)=\frac{\sum_{m=0}^{\infty} t_{m} m^{m}}{\sum_{m=0}^{\infty} z_{m} n^{m}}=\sum_{m=0}^{\infty} c_{m} n^{m}
$$

where the coefficients $C_{m}$ satisfy the relation 


$$
C_{m} \equiv C_{m}(n)=t_{m}-\sum_{k=1}^{m} C_{m-k} Z_{k}
$$

But since both $t_{m}$ and $Z_{m}$ are polynomials in $n$ of degree $m$ [cf. Eqs. (I.5a) and (I.22)], we can write

$$
c_{m}=\sum_{j=0}^{m} C_{m, j} n^{j}
$$

where the coefficients $C_{m, j}$ depend on the nuclear mass $A$ only. To find these coefficients, we use Eqs. (I.5a) and (I.22) in (I.28) and equate the resulting expression to (I.29). We obtain

$$
\sum_{j=0}^{m} c_{m, j} n^{j}=\sum_{j=0}^{m} t_{m, j} n^{j}-\sum_{k=1}^{m}\left(\sum_{s=0}^{m-k} c_{m-k, s} n^{s}\right)\left(\sum_{j=0}^{k} z_{k, j} n^{j}\right)
$$

By equating the coefficients of identical powers of $n$ in (I.30) we obtain the recursion relation

$$
\left.\begin{array}{c}
c_{m, j}=t_{m, j}-\sum_{k=1}^{m} \sum_{s=m a x}^{s=m i n}(m-k, j) \\
\text { for } m, j=0, j-k) \\
\text { with } C_{00}=1 \text { and } C_{m, j}=0 \text { if } j>m
\end{array}\right\}
$$

The scheme below provides a geometrical interpretation and facilitates the understanding of the summations involved in (III.76) for the $c_{m, j}$ and, by analogy, for the summations involved in (III.73) for the $z_{m, j}$ 's; this scheme was used to rearrange the sums on $s$ and $j$ occurring in the RHS of Eq. (I.30): 


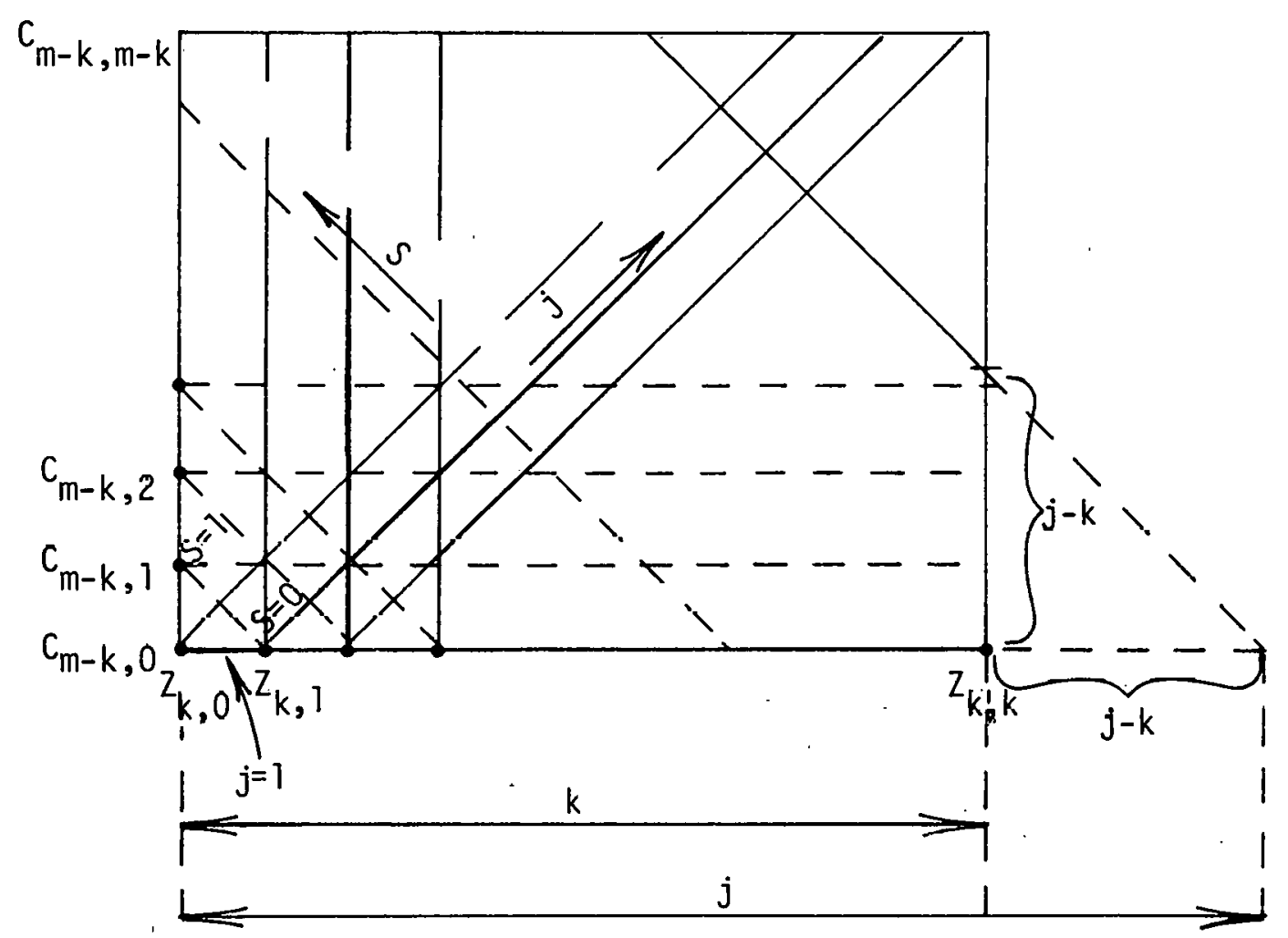

Fig. I.1

The first few $C_{m, j}$ are

$$
\begin{aligned}
& \left.C_{0,0}=1 ; C_{1,1}=t_{1,1}=\left(a_{1}+b_{1}\right)=\frac{4 \gamma \hat{n}(U)}{5 \gamma_{2}(0)}-\left(\frac{\gamma_{\hat{0}}-(0)}{\gamma_{1}(0)}+\frac{\gamma_{i}}{\gamma_{1}}\right)\right) \\
& C_{1,0}=-\frac{4}{5} \frac{\gamma \hat{0}(0)}{\gamma_{2}(0)}-\frac{\gamma_{0}-(0)}{\gamma_{0}(0)} \\
& c_{2,2}=\frac{1}{2} c_{1,1}^{2}
\end{aligned}
$$

Last, we shall prove the relation

$$
C_{m, m}=\frac{1}{m !} C_{1}^{m}, 1
$$

We shall do this by induction 
(i) If $m=1, C_{1}, 1=C_{1}, 1$, i.e., (I.32) is satisfied.

(ii) Assuming that (I.32) holds for $m=p$, i.e.,

$$
c_{p, p}=\frac{1}{p !} c_{1,1}^{p}
$$

we have to

(iii) show that (I.32) is also true for $m=p+l$.

But, from the recursion relation (III.76) we have for $j=m$

$$
c_{m, m}=t_{m, m}-\sum_{k=1}^{m} c_{m-k, m-k} z_{k, k}
$$

since, obviously $\max (0, m-k)=m-k$ and $\min (m-k, m)=m-k$. Using Eq. (I.9) for $t_{m, m}$ and (I.26) for $z_{m, m}$ in (I.34), we obtain

$$
C_{m, m}=\frac{1}{m !}\left[t_{1,1}^{m}-\sum_{k=1}^{m} \frac{m !}{k !}\left(a_{1}+b_{1}\right)^{k} C_{m-k, m-k}\right]
$$

Writing (I.35) for $m=p+1$ and employing (I.33) we obtain, successively:

$$
\begin{aligned}
c_{p+1, p+1} & =\frac{1}{(p+1) !}\left[t_{1}^{p+1}-\sum_{k=1}^{p+1} \frac{(p+1) !}{k !}\left(a_{1}+b_{1}\right)^{k} c_{p+1-k, p+1-k}\right] \\
& =\frac{1}{(p+1) !}\left[t t_{1}^{p+1}-\sum_{k=1}^{p+1} \frac{(p+1) !}{k !}\left(a_{1}+b_{1}\right)^{k} \frac{c_{1,1}^{p+1}-k}{(p+1-k) !}\right] \\
& =\frac{1}{(p+1) !}\left[t_{1}^{p+1}-\left(c_{1,1}+a_{1}+b_{1}\right)^{p+1}+\frac{(p+1) !}{(p+1) !} c_{1}^{p+1}\right] \\
& =\frac{1}{(p+1) !}\left[t_{1,1}^{p+1}-\left(t_{1,1}-a_{1}-b_{1}+a_{1}+b_{1}\right)^{p+1}+c_{1,1}^{p+1}\right] \\
& =\frac{1}{(p+1) !} c_{1}^{p+1}
\end{aligned}
$$


Since (I.36) is the same as one would obtain by taking $m=p+1$ in (I.33), the proof of (I.32) by induction is complete; Q.E.D. 


\section{APPENDIX J \\ LISTING OF COEFFICIENTS $C_{m, j}$ FOR $m, j=0$ TO $m, j=5$ AS GENERATED BY FORMAC}

The coefficients $C_{m, j}$ will be given in terms of the quantities $\gamma_{j}^{(n)}$ defined as:

$$
\gamma_{i}^{(n)}=\frac{d^{n}}{d n^{n}}\left[\gamma_{i}(n)\right]_{n=0} ; \begin{aligned}
& n=1,2,3, \ldots \\
& i=0,1,2,3, \ldots
\end{aligned}
$$

Since $\gamma_{i}^{(n)}$, as defined above, are functions of the nuclear mass $A$ only, the coefficients $C_{m ; j}$ will themselves be functions of the nuclear mass $A$ only. In order to facilitate the interpretation of the FORMAC results, we give below the "algebraic" expressions of the first few $c_{m, j}$. Thus:

$$
\begin{aligned}
& C_{0,0}=1 \\
& C_{1,0}=-\frac{4}{5} \gamma_{0}^{(1)} / \gamma_{2}-\frac{1}{2} \gamma_{0}^{(2)} / \gamma_{0}^{(1)} \\
& C_{1,1}=-r_{1}^{(1)} / \gamma_{1}+\frac{4}{5} r_{0}^{(1)} / \gamma_{2}-\frac{1}{2} r_{0}^{(2)} / \gamma_{0}^{(1)} \\
& \left.C_{2,0}=-\frac{216}{175}\left(\frac{\gamma \delta(1)}{\gamma_{2}}\right)^{2} \frac{\gamma_{1}}{\gamma_{3}}+\frac{16}{25}\left(\frac{\gamma_{0}^{(1)}}{\gamma_{2}}\right)^{2}+\frac{4}{5} \frac{\gamma_{2}^{(1)}}{\left(\gamma_{2}\right)^{2}}\right)^{(1)}+\frac{1}{4}\left(\frac{\gamma_{0}^{(2)}}{\gamma_{0}}\right)^{2}-\frac{1}{6} \frac{\gamma_{0}^{(3)}}{\gamma_{0}(1)} \\
& C_{2,1}=\frac{108}{175}\left(\frac{\gamma_{0}^{(1)}}{\gamma_{2}}\right)^{2} \frac{\gamma_{1}}{\gamma_{3}}+\frac{1}{2}\left(\frac{\gamma_{1}^{(1)}}{\gamma_{1}}\right)^{2}+\frac{4}{5} \frac{\gamma_{1}^{(1)} \gamma_{\gamma}(1)}{\gamma_{2} \gamma_{1}}+\frac{1}{2} \frac{\gamma_{1}^{(1)} \gamma_{0}^{(2)}}{\gamma_{1} \gamma_{0}^{(1)}}- \\
& -\frac{1}{2} \frac{\gamma_{1}^{(1)}}{\gamma_{1}}-\frac{24}{25}\left(\frac{\gamma_{0}^{(1)}}{\gamma_{2}}\right)^{2}-\frac{4}{5} \frac{\gamma_{2}^{(1)} \gamma_{0}^{(1)}}{\left(\gamma_{2}\right)^{2}}+\frac{2}{5} \frac{\gamma_{0}^{(2)}}{\gamma_{2}}+\frac{3}{8}\left(\frac{\gamma_{0}^{(2)}}{\gamma_{0}(1)}\right)^{2}-
\end{aligned}
$$




$$
\begin{aligned}
& -\frac{1}{6} \frac{\gamma_{0}^{(3)}}{\gamma_{0}^{(i)}} \\
C_{2}, 2 & =\frac{1}{2}\left(\frac{\gamma_{1}^{(1)}}{\gamma_{1}}\right)^{2}-\frac{4}{5} \frac{\gamma_{1}^{(1)} \gamma_{0}^{(1)}}{\gamma_{2} \gamma_{1}}+\frac{1}{2} \frac{r_{1}^{(1)} \gamma_{0}^{(2)}}{\gamma_{1} \gamma_{0}^{(1)}}+\frac{8}{25}\left(\frac{\gamma_{0}^{(1)}}{\gamma_{2}}\right)^{2} \\
& -\frac{2}{5} \frac{\gamma_{0}^{(2)}}{\gamma_{2}}+\frac{1}{8}\left(\frac{\gamma_{0}^{(2)} \gamma_{0}^{(1)}}{\gamma_{0}^{2}}\right. \\
& =\frac{1}{2 !}\left(C_{1}, 1\right)^{2}
\end{aligned}
$$

The 11sting (produced with the aid of FORMAC) of $c_{m, j}$, for values of $\mathrm{m}$ from 1 to 5 and $\mathrm{j}$ from 0 to 5 , will be presented below.

Again, the results are in the form of equations (e.g., $c(3,2)=$ expression) that should be read as usual, from left to right and top to bottom. The dashed lines separating successive lines of formulae are part of FORMAC output editing.

The FORMAC symbols appearing in this appendix should be interpreted as follows:

1. The slash (/) slands for "diviston," i.e., quantities preceding it should be thought of as constituting the numerator, and quantities following the slash as constituting the denominator, respectively, of a fraction, the slash itself playing the role of a "fraction line." A fractional coefficient appears always in front of the quantity (usually a fraction itself) that it multiplies.

2. The FORMAC symbol $C(M, J)$ stands for $C_{m, j}$, e.g., $C(3,1) \equiv C_{3}, 1$.

3. The symbol G. (ARG], ARG2) stands for the functions $\gamma_{j}(n)$ ( $i=0,1$, etc.) as defined in Eq. (II.21), in the main body of this thesis; note that ARG1 takes the numerical value of $i$ and ARG2, the numerical 
value of $n$, which in this case is always zero (since all quantities are evaluated at $n=0$ ).

For example:

$$
\text { G. }(3,0) \equiv \gamma_{3}(0)
$$

Since derivatives of $\gamma_{i}$ with respect to $n$, at $n=0$, also appear, the following general interpretation of FORMAC symbolism applies:

$$
G^{\left(2^{N}\right) M(J, 0)} \quad \equiv\left\{\left[\frac{d^{N}}{d_{n}^{N}}\left(\gamma_{j}(n)\right)\right]_{n=0}\right\}^{M}
$$

where:

$$
\begin{aligned}
& J \equiv \text { index of } \gamma_{J}(n) \quad(J=0,1,2 \text {, etc.) } \\
& M \equiv \text { the power to which that quantity (derivative) is raised; } \\
& M=2,3 \text {, etc. Note that if } M=1 \text {, it is not written explicitly. } \\
& N \equiv \text { order of derivation; } N=2,3 \text {, etc. Note that if } N=1 \text {, it does not } \\
& \text { appear, i.e., } \\
& G(2) \cdot(J, 0) \quad \equiv \frac{d}{d n}\left[\gamma_{J}(n)\right]_{n=0}
\end{aligned}
$$

Note that the digit $\underline{2}$ appearing in (J.1) or (J.2) signifies "derivative with respect to the $\underline{2}^{\text {nd }}$ argument of the function G.," in our case-with respect to $n$.

We now present, in the remainder of this appendix, the listing of coefficients $C_{m, j}$, as produced by FORMAC: 

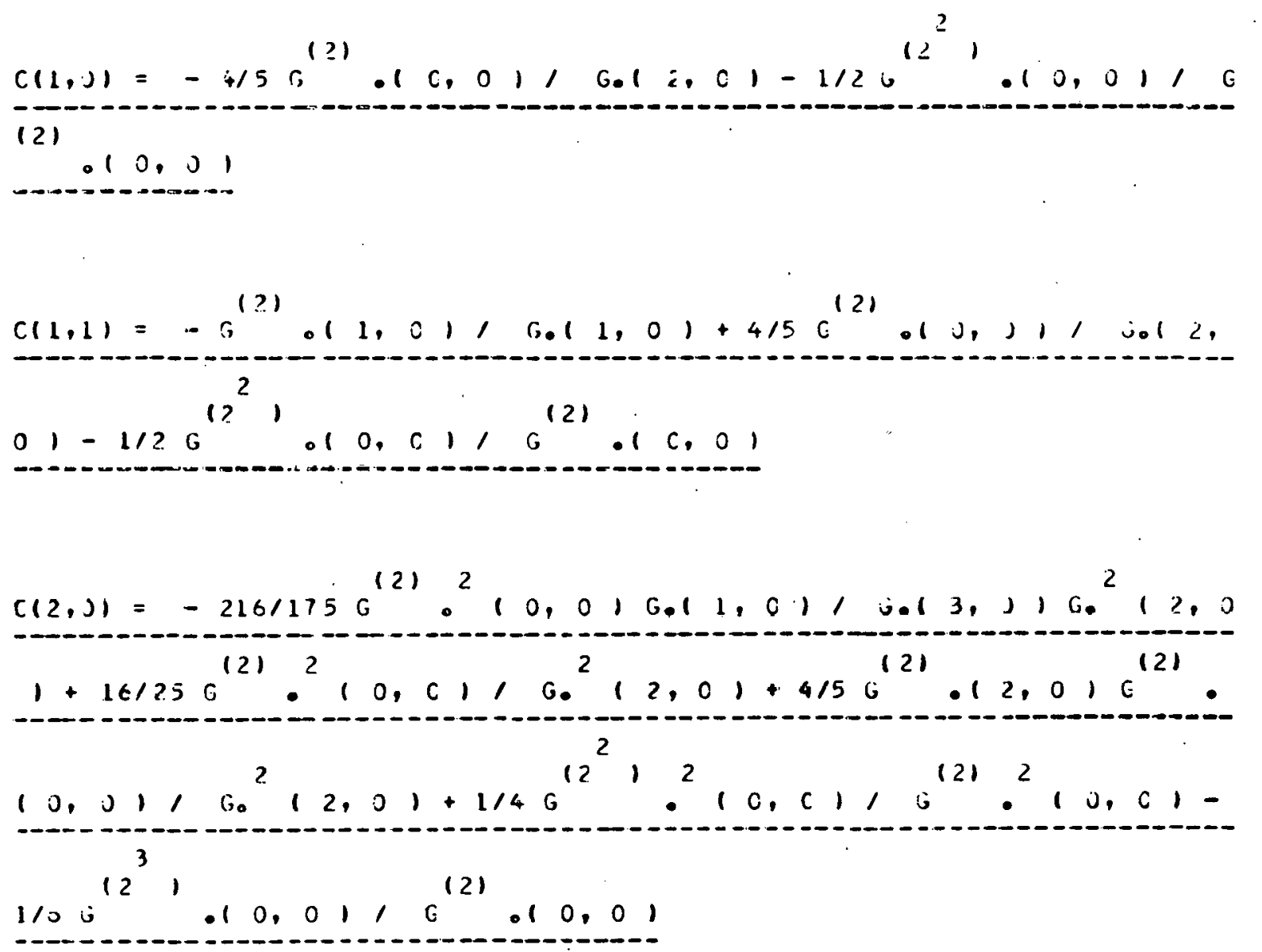

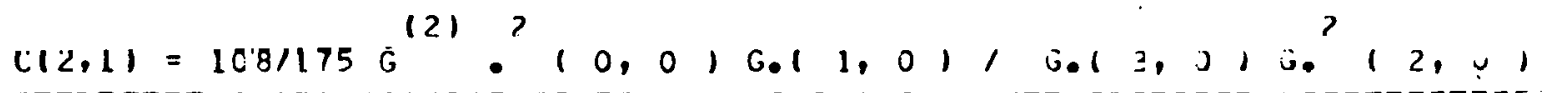

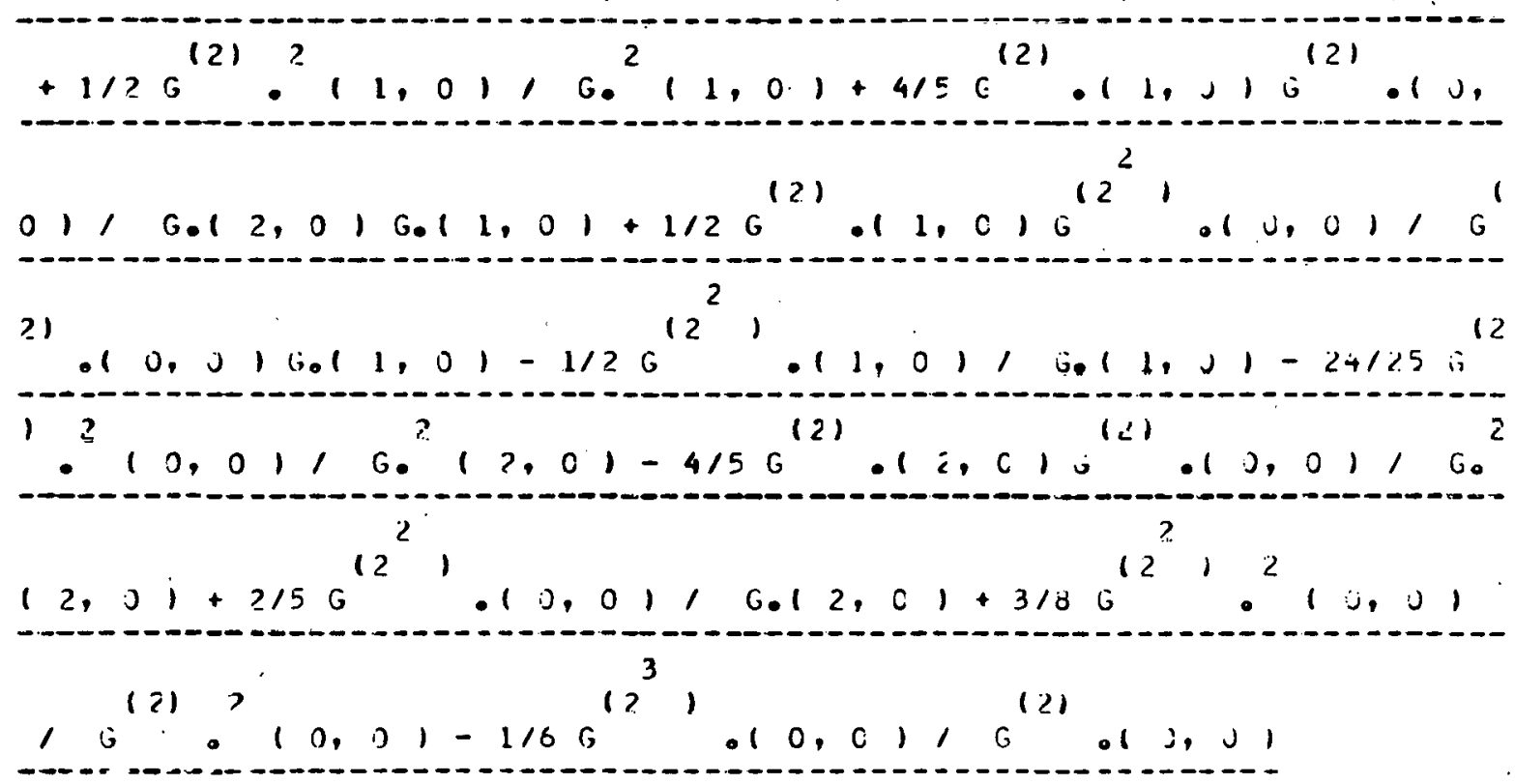


$C(2,2)=1 / 2 G^{(2)} \cdot 2,1,01, G^{2}, 1,01-4 / 5 G^{(2)} \cdot 11,01 G^{(2)}$ 2 $.10,01, G .12,0, G_{0}(1,0)+1 / 2 G^{(2)} .11,0, G^{(2)}, 10,01$

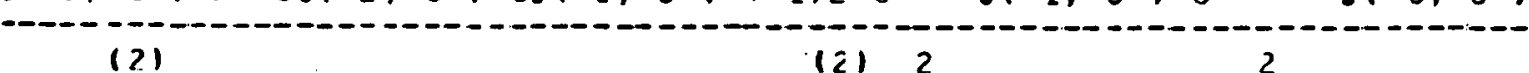
$16 \quad .10,010.11,01+8 / 25 G \quad \circ 10,011$ i. $12,01-$ ?
?

$2 / 50^{12} 1010,01,0.12,01+1 / 8 e^{12} 10^{2}, j, 01, G^{121}$.

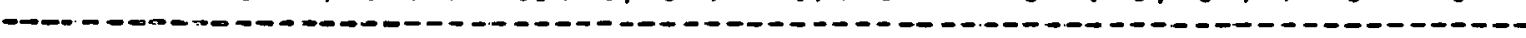
10,01

- -

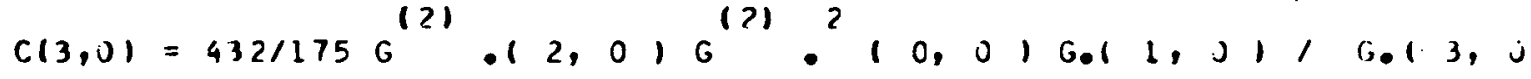
3 I G. $12,01+2552 / \varepsilon 75 G$ G $10,0,0.11, J 1,0.13, j, 6$. (15)

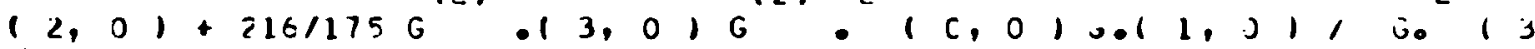
2

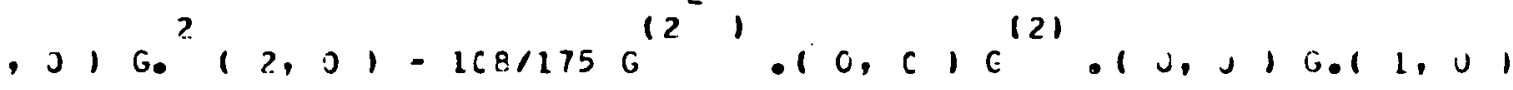

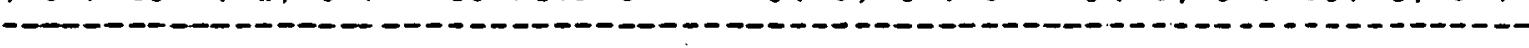
$2 \quad 121 \geq 2$

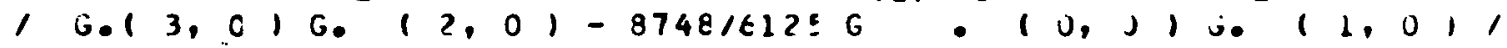
0

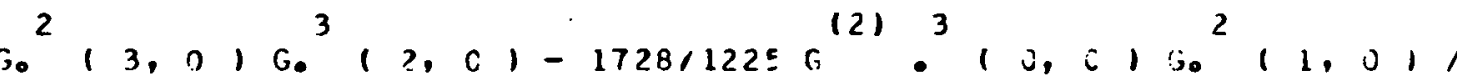
9.0

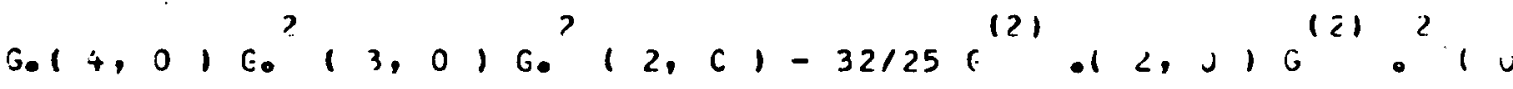
0 - $01 / G_{0}(2,01-64 / 125 G$. $13,01 /$ i. $(2, i)-4 / 5$ i

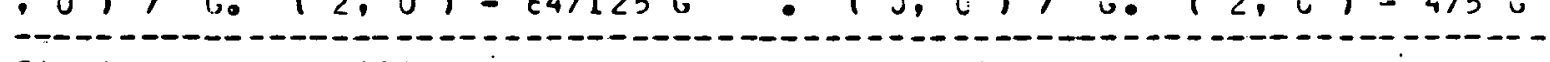
2121210121

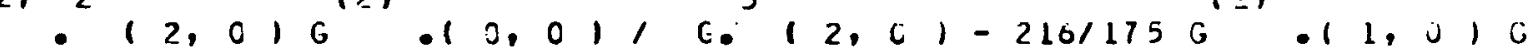
121
-

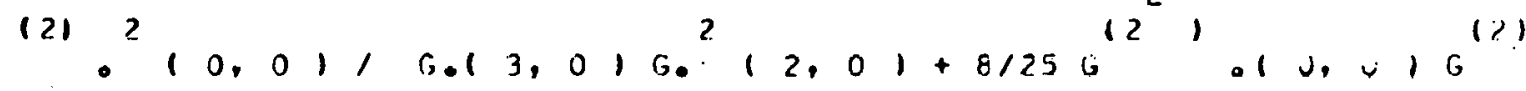

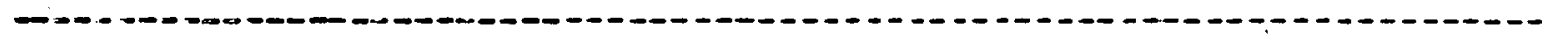
$212^{2}$,

$.10,01, G_{0}(2,0)+2 / 5 G^{2}, 12,01 G^{(2)}, 10,011$ i.

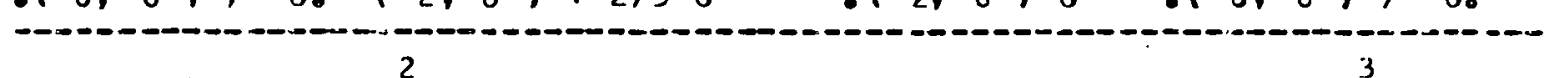
$12,01-1 / 3 \mathrm{G}^{12^{2}} 0^{3} 10,01, \mathrm{e}^{121} 0^{3} 10,01+112 \mathrm{G}^{12}, 10$

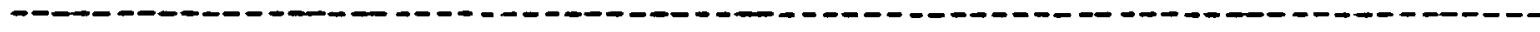
$\therefore 10^{12^{2}}, 010,01,0^{121} 0^{2} 10,01-1 / 2+0^{12^{4}} 1.10,0110^{1}$ 21 .$(0,0)$

- 
$(13,1)=-216 / 175 \mathrm{G}^{(2)} \cdot\left(2,0, \mathrm{G}^{(i)}, 2,0,0, \mathrm{G}\right)(1,1), 1, \mathrm{G}, 13$ 31213

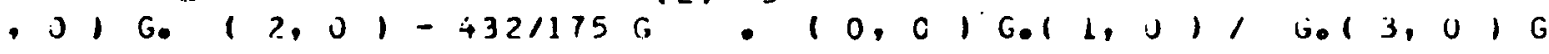
3

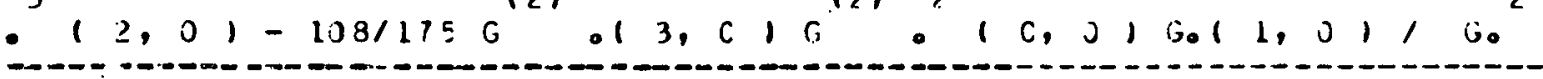
(

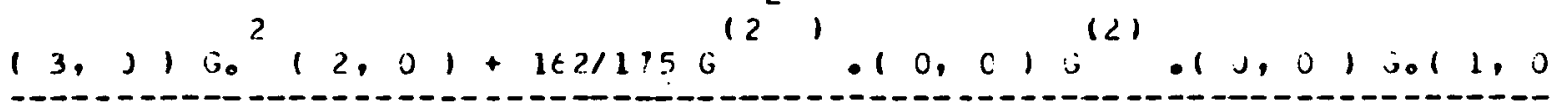

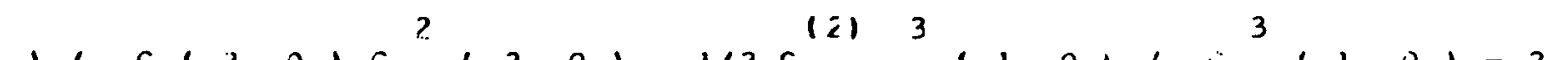

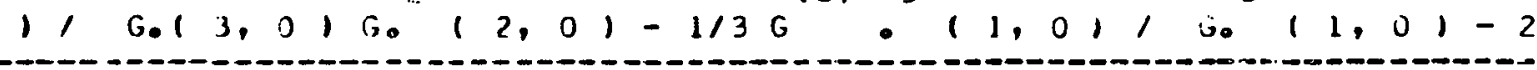
$121 ?(?)$ ? 121 $156.11,01 \%$ : $0,0,16012,0, G_{0} 11,01-1 / 46$ -

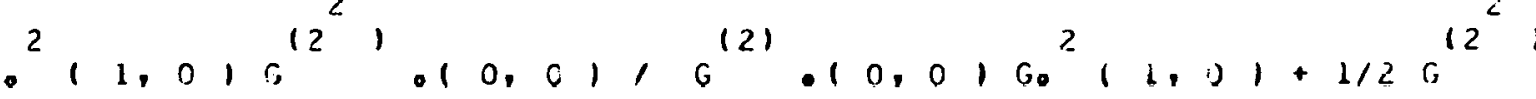

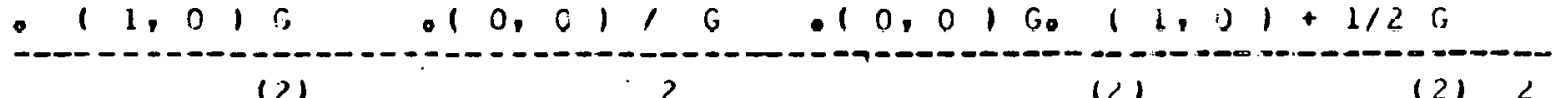
$.11,0, \mathrm{~g} 011,0,160,1,0,1=16 / 25 ; ; 01,0100$ - 1 (

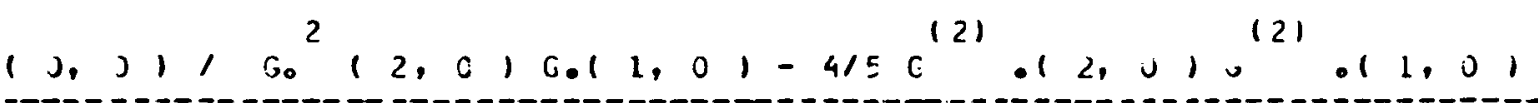

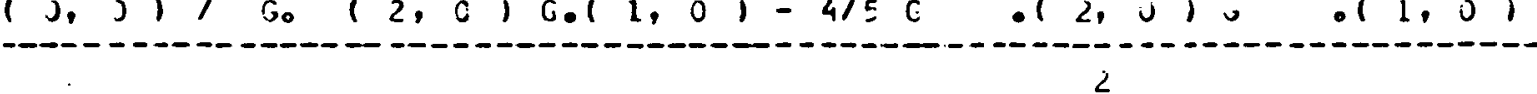

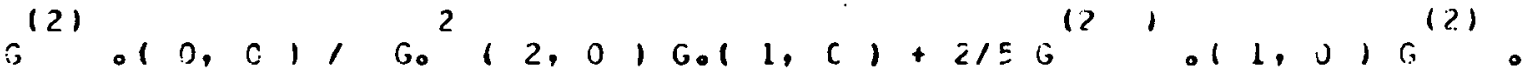
(

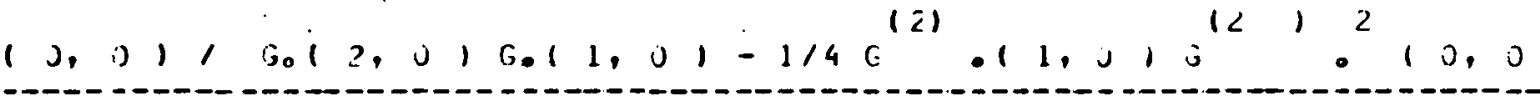

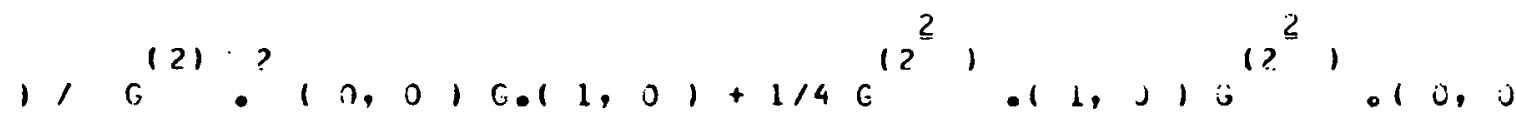

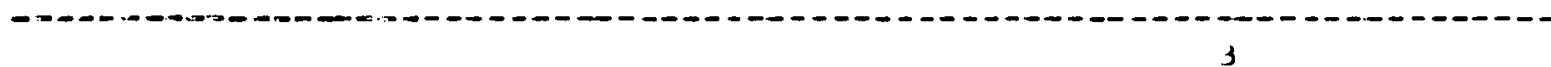

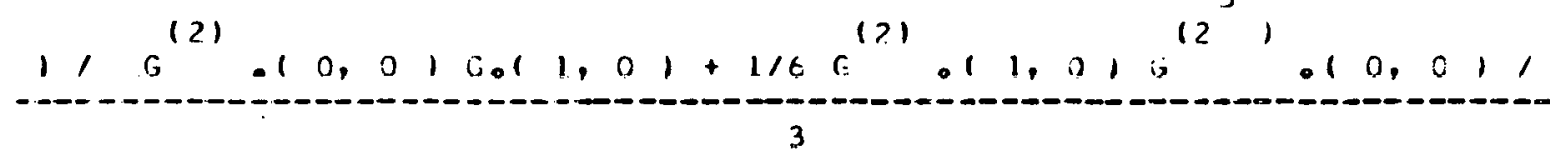
$G^{(2)} \circ(J, 0) \mathrm{G}, 1,1, \mathrm{~J},-1 / 6 \mathrm{C}^{12}$ $(1,01, \operatorname{lol} 1, j)+29101$

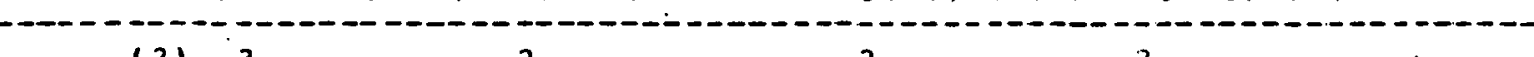

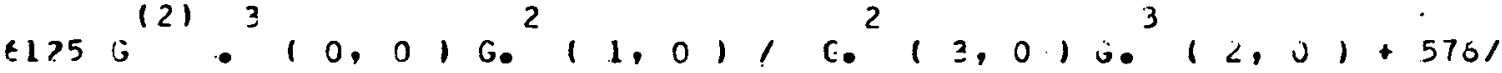
$\begin{array}{llll}121 & 3 & 2 & 2\end{array}$

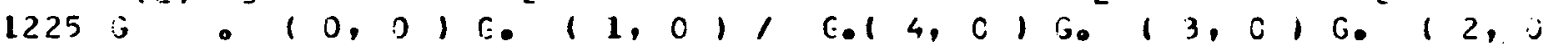
$(2)$ (2) $1+48 / 25 G \cdot 12,0) G(10,0), G_{0}(2,01+552 / 3756$ $13 \quad 3 \quad(i) \cdot 2 \quad(2)$

$-(0,01, G .(2,0)+4 / 5 G, \cdot 12,0100.10,011, G$ 3 121 $G^{(2)}{ }^{2}(0,0,1,0,13,0,10$ - $12.01+3241175 \mathrm{G}$. $01,01 \mathrm{G} \cdot 10,01,0.13,0160$ 
$\left(2,01-4 / 5 G^{12}, \cdot 10,01 G^{(2)} \cdot 10,01,0^{2}, 12,01-2 / 5 G^{12}\right.$ 2 $1.12,0, G^{(2)} .10,01, G^{2}, 2,0,-2 / 50^{(2)} .12,01 G^{12} 1$

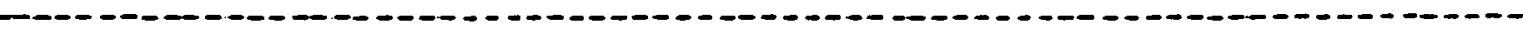
$10,01, G^{2} 12, c 1-1 / 10 G^{12} 12^{2}, 0,0,1,3^{121} 01000100$ $12,01+2 / 1500.10,01,6.12,01-11 / 4.3060$ 1513
3 $1, \mathrm{G}^{(2)} \cdot 3^{3}, 0,0,+1 / 4 \mathrm{G}^{12^{3}}, 10, \mathrm{c}, \mathrm{G}^{12^{2}}, 10,01, \mathrm{G}^{(2)}$

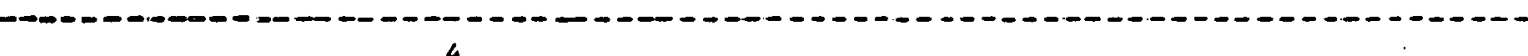

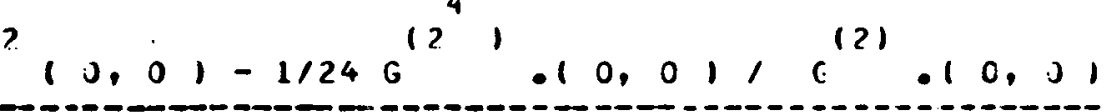

$C(3,2)=432 / 875 G^{(2)} 0^{3}, 0,0, G_{0}(1,0), G .13,0, G_{0}^{3}(2,0)$ $\frac{2}{2}$

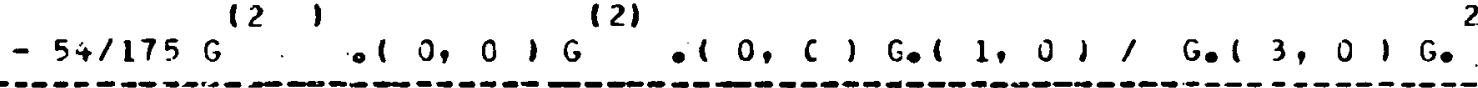

(2) 3 (2) 3 (2)

$(2,0)-1 / 2 G, \cdot(2,0) / G .(1,0)-1 / 2 G, \cdot(1,0) 6$ ( 2 (2) 2

$12^{2}, 10,01, G^{121},(0,0) G^{2}, 1,01+1 / 2 j^{12}, .11,01 G^{1}$

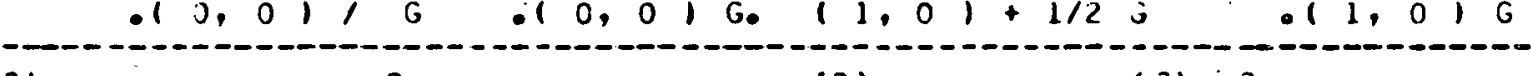
2) 2 (2) 1212 $.(1,0) /$ G. $(1,01+24 / 25 \mathrm{E}, 11,016 \cdot 10,011$ 2 ( 21 ( 121

G. $12,01,0,11,01+4 / 5 G$ o $12,0,0,01,1,1,0,01, j$

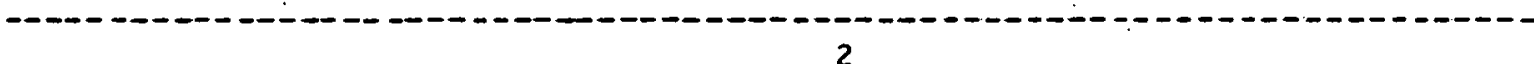
$1, G^{2}, 2,016.11,01-2 / 5 G^{(i)}, 011,010(2), 10,01,6$ -

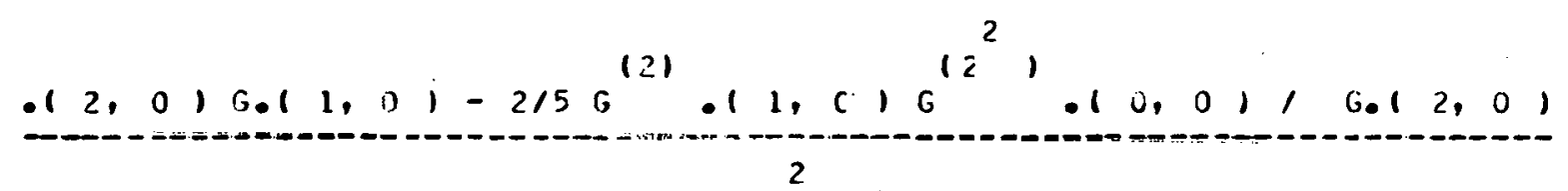
$0.11,01-3 / 8 G^{121} \cdot 11,01 G^{(2,12}, 0,01,0^{121} 0^{2}, 0,01$ 25 $0.11,01+11.4 G^{12} 1.11,0, G^{12}, .10,01, G^{(2)} .1 c_{0} 01 G_{0}$ 9. 


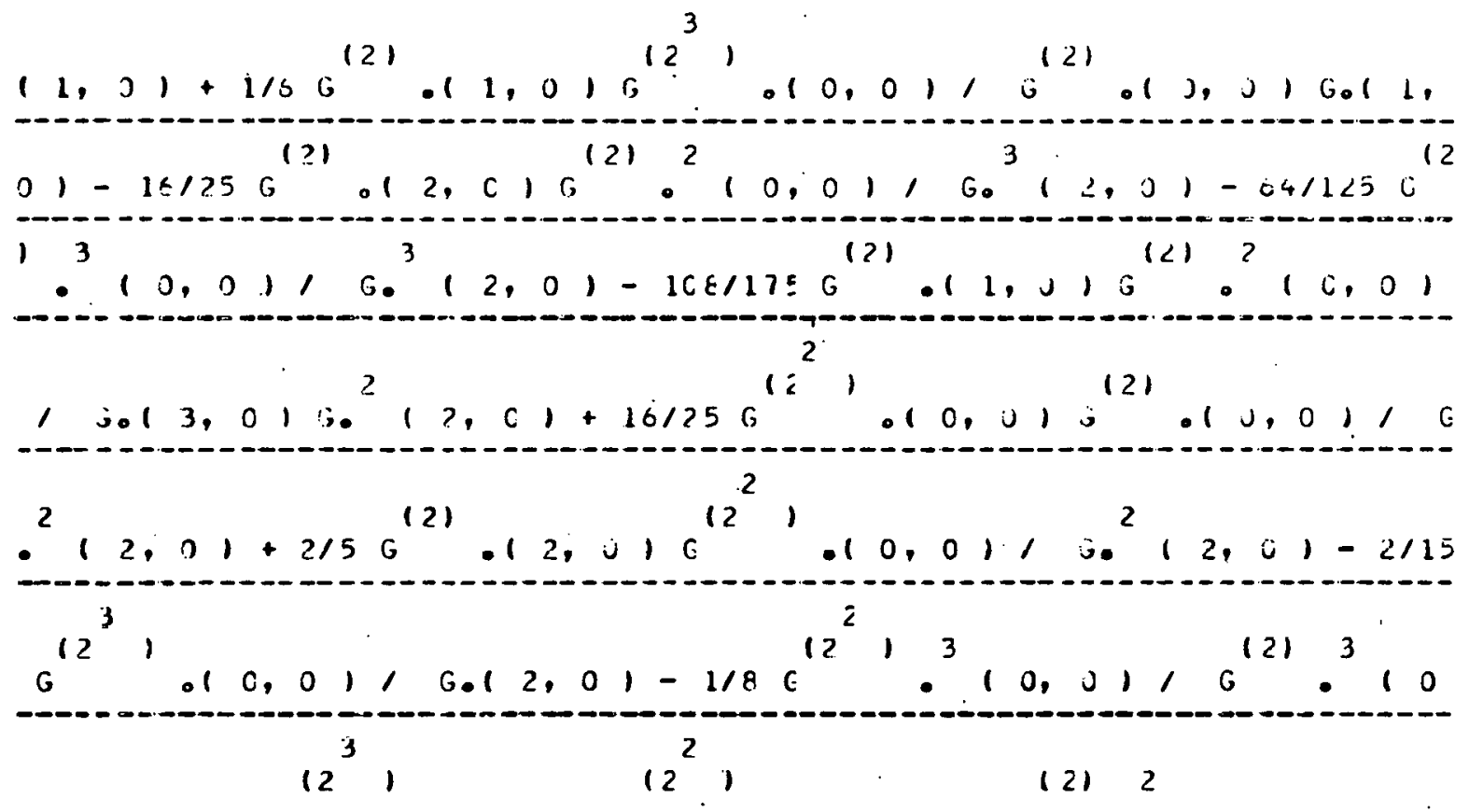

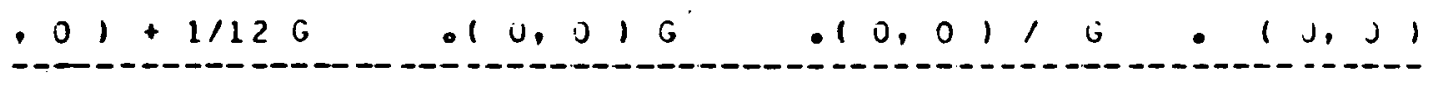

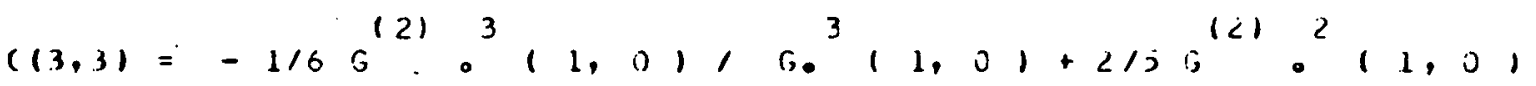
$(1.3,31$,

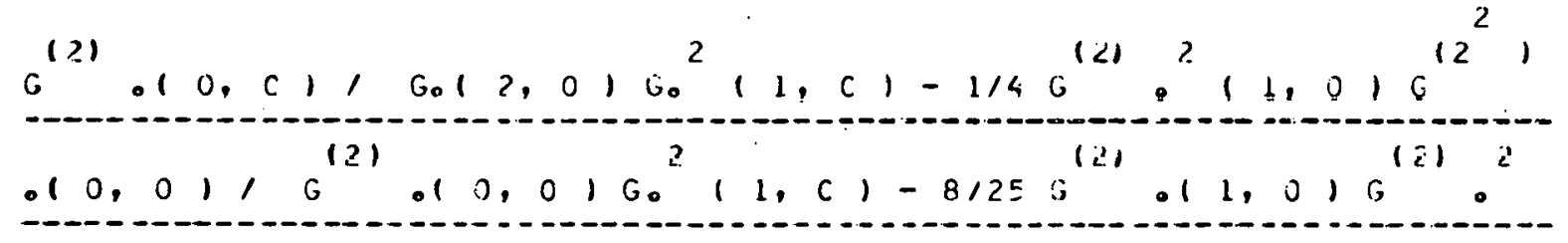

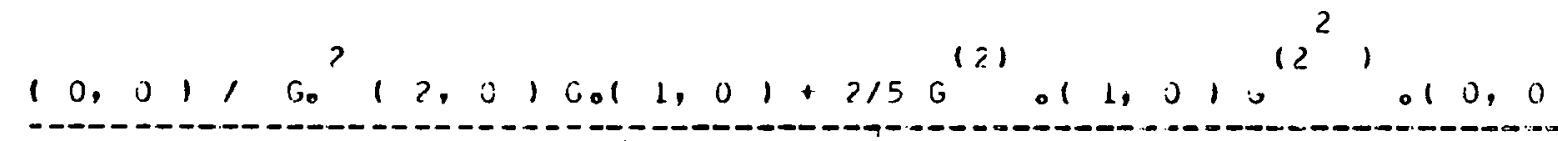
(

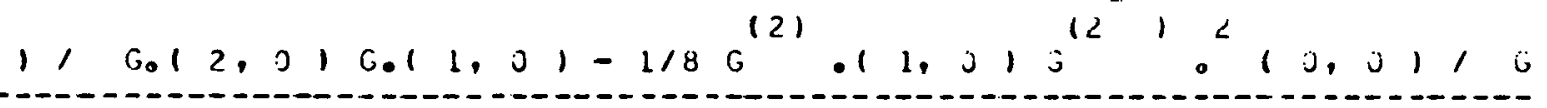

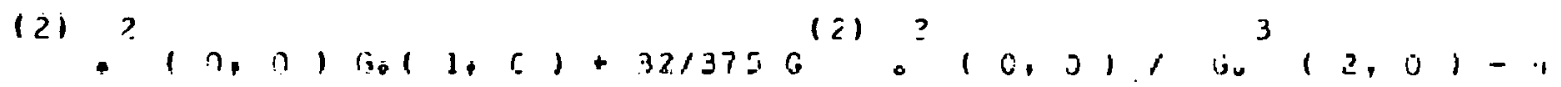

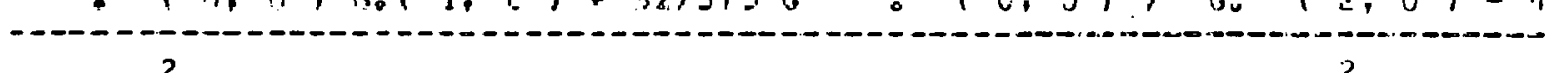

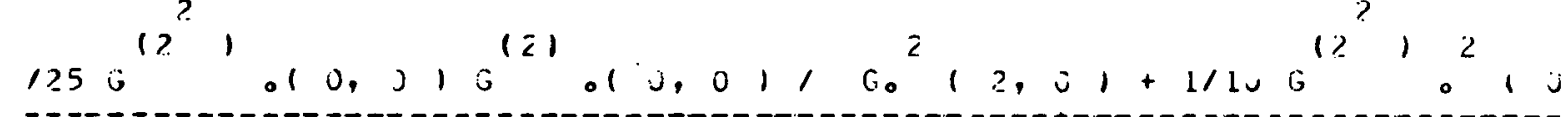

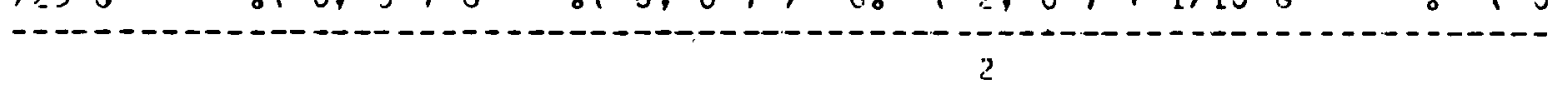

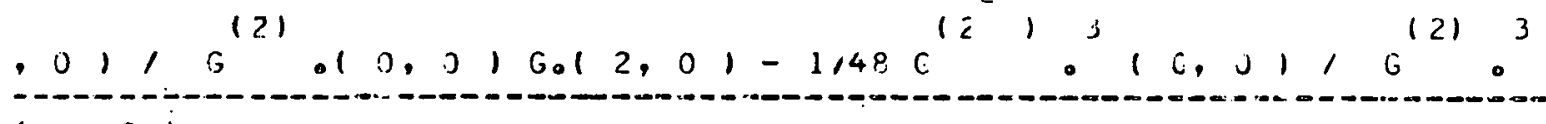
$1 \mathrm{~s} ; \mathrm{s}$ 


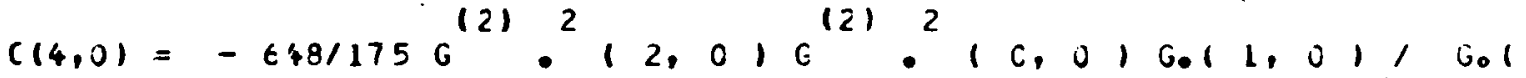

-

$3,0, G_{0}^{4}(2,0)-7776 / 875 G^{(2)} \cdot(2,0) G^{(2)} 0^{3}, J, J, G .11,0$

-

$1, G_{0}(3,0) G_{0}^{4}\left(2,01-20736 / 437 E G^{(2)} G^{4}, J, J, G_{0}(1,0)\right.$

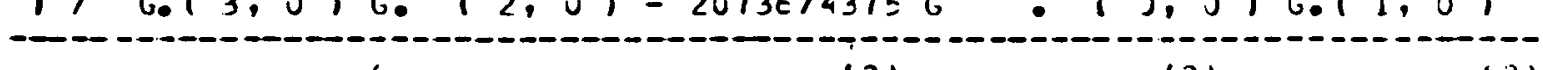

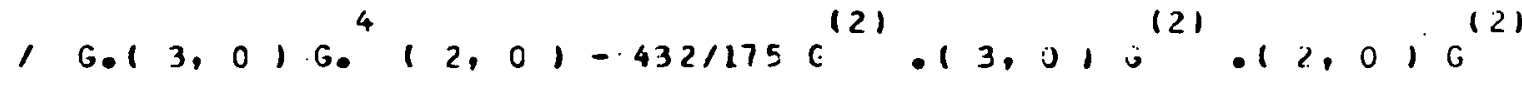

2

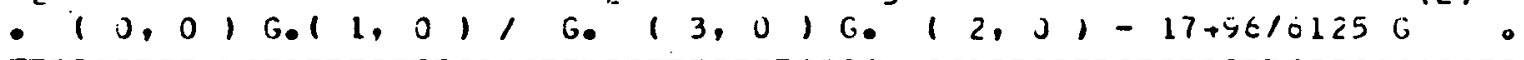

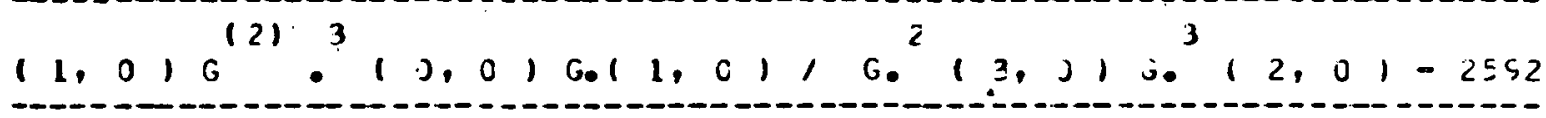

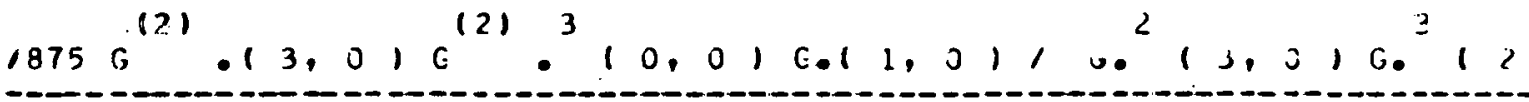

2 $01+259213756^{12}, .10,016^{121} 0^{2}, c, 010011,01,10013$, (3

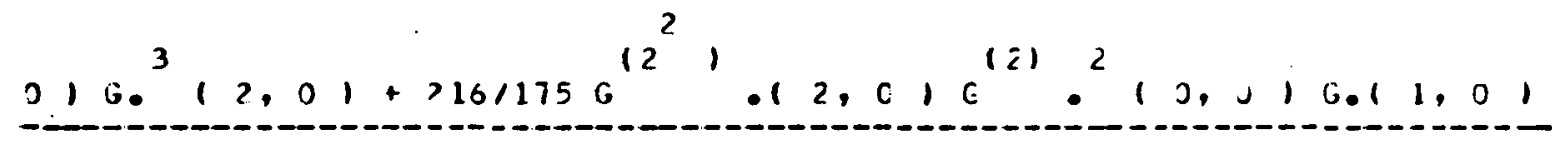

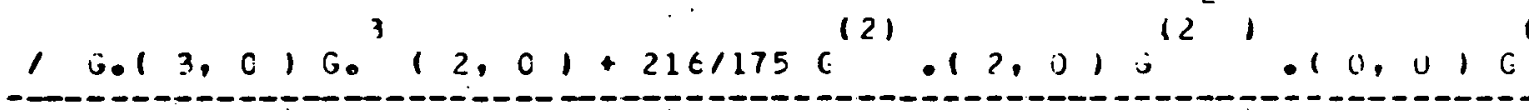
21 $21.10,0, G .11, c, 1, G .13,0) \epsilon^{3}(2,0)-2161115 G^{(2)} 0^{2} 13$ 0 $0, g^{121} 0^{2} 10,016011,0,1 e^{3}, 3,0,00^{2}(2,01-34551$

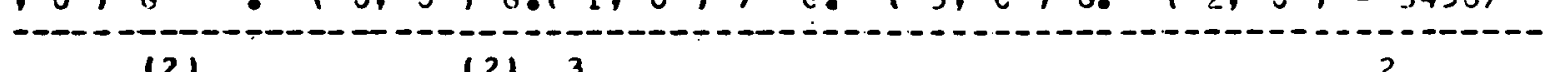
$1225 G^{(2)},(1,0) G^{(2)} 0^{3}(0,0) G .1,0,1,0.14, J, G_{0}, 3,0$ $1 G^{2} 12,01+108 / 175 G^{12}, \cdot 13,01 G^{121} G^{2} 10,016011,01$ -

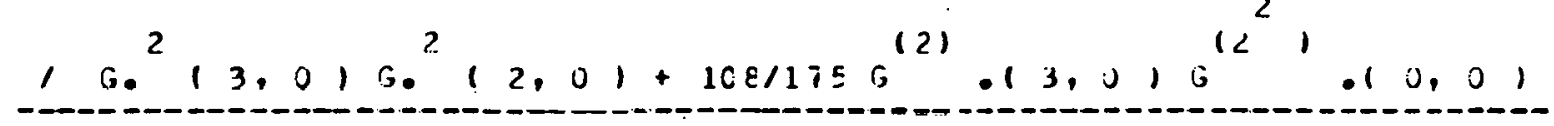

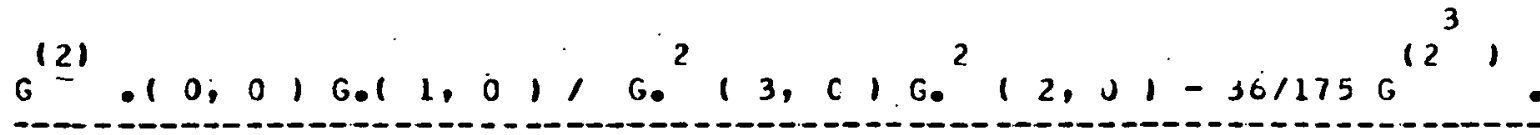
$(2)$

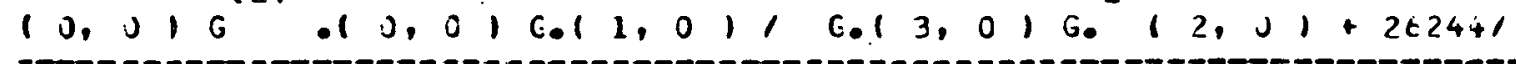
$10,1 \mathrm{~d}$

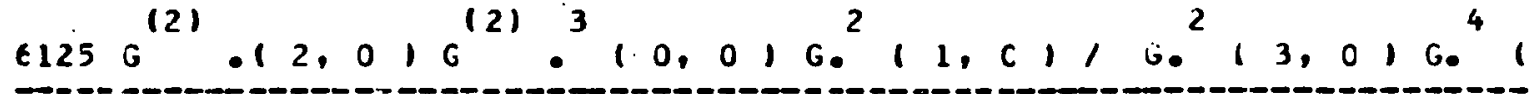

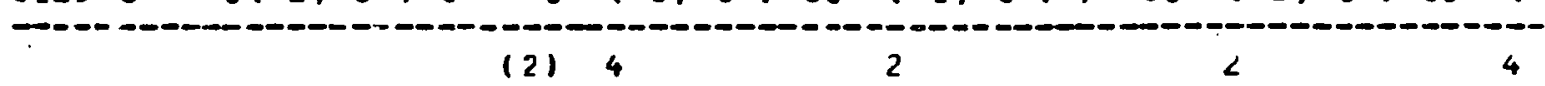

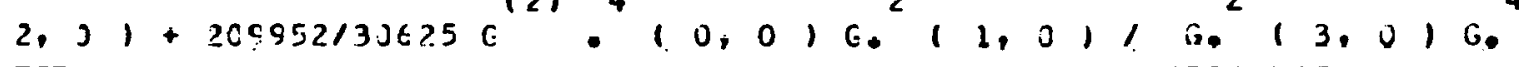


(2)

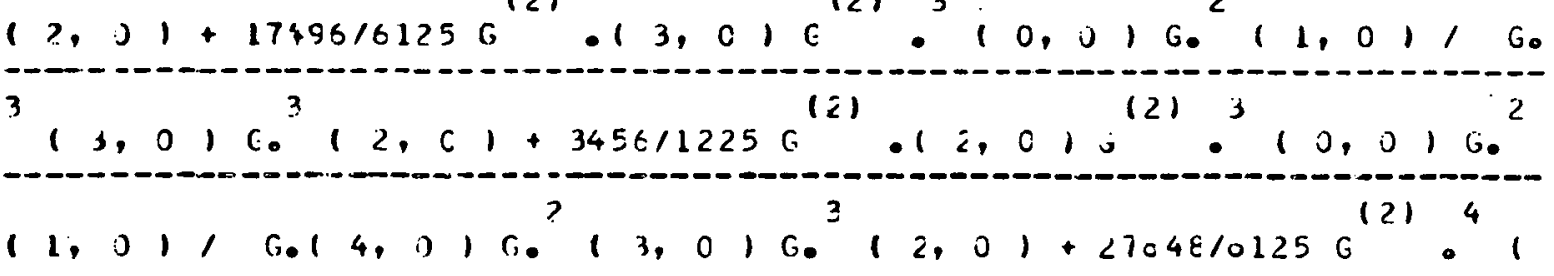

- - - -

$0,0, G_{0}^{2}\left(1,0,1, E_{0}\left(4,0, G_{0}^{2}\left(3,01 G^{3}(2, J)-\varepsilon 748 / 0125\right.\right.\right.$

-

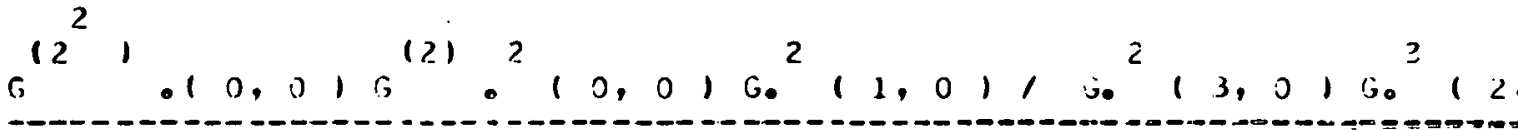
(

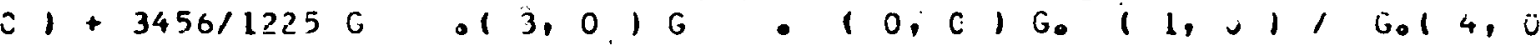

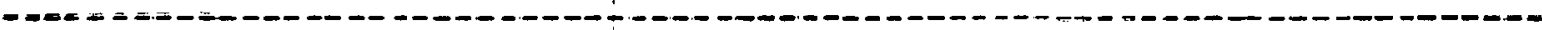
32 1213

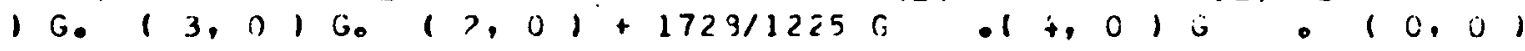
(-

$\mathrm{G}^{2}\left(1,0,1 \mathrm{G}^{2}\left(4,0, \mathrm{G}^{2}, 3,0, \mathrm{G}^{2} 12,01-1728 / 1225^{12}\right.\right.$ 6.

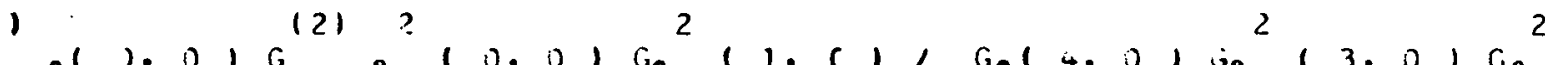

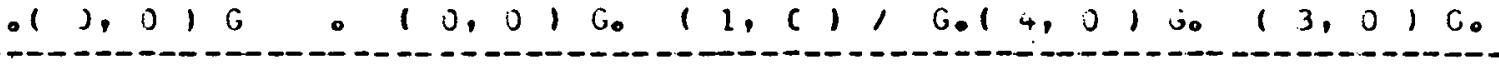

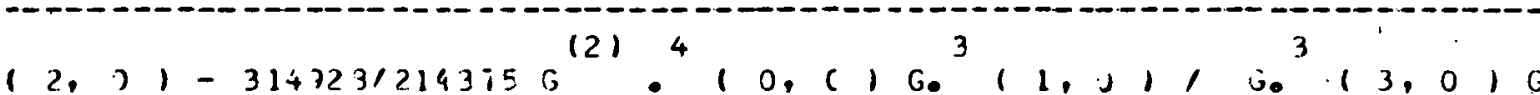

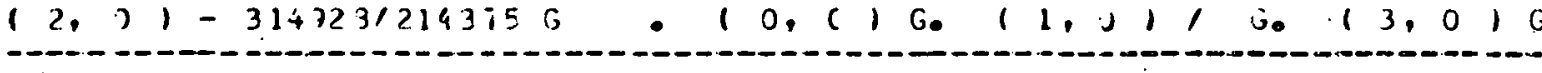
4 (2) 4

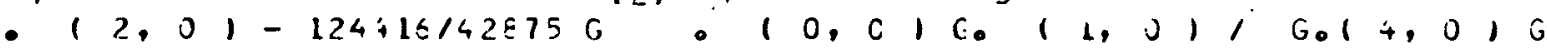

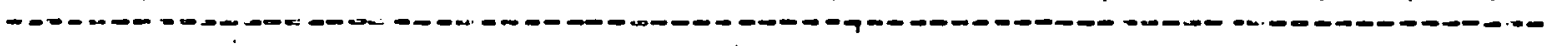
$3 \quad 3 \quad 12)_{1} \quad 1$

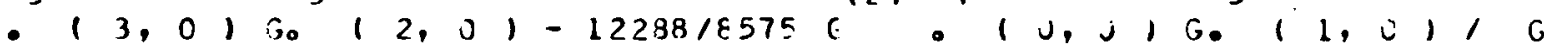
- - - - -

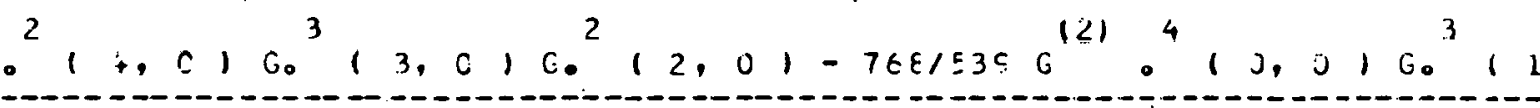
$, J, 1, G . i 5,0, G_{0}^{2}\left(4,0, G_{0}^{2}(3,0) G^{2}(2,0,1+48 / 25 G\right.$

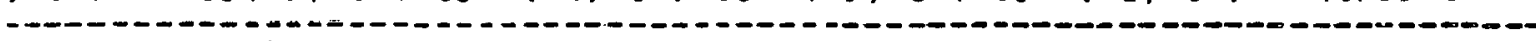
$2 \quad(2) 240121$ - $12,01 \mathrm{G}, 1,0,1 G_{0} 12,01+1921256,012,010$

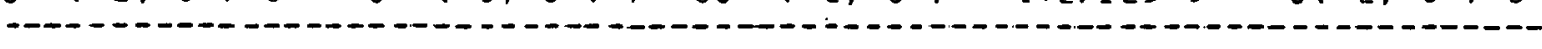
$21{ }^{3}\left(0,0,1 G_{0}^{4}\left(2,0,1+256 / 625 e^{(2)} e^{4}(J, 0,16,412,0\right.\right.$

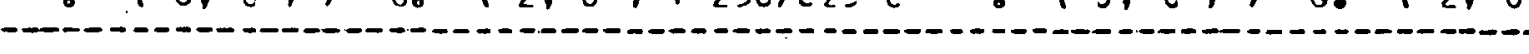
$\left.1+4 / 56^{(2)} 0^{3}, 2,0\right) 0^{(2)} \cdot\left(0,0,160^{4}(2,01+432 / 1756\right.$ (2) (2) 2 (

$.12,0, G$ G $1,0, G, 10,6,16.13,0, G_{0} 12,01+$

(1)

(2) (2) 3 (

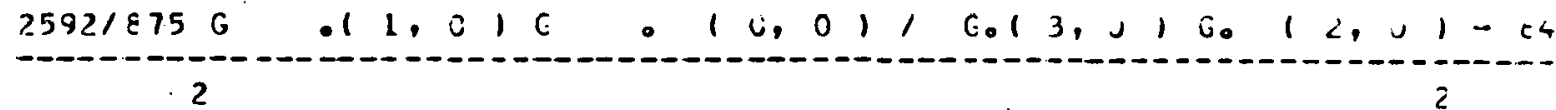

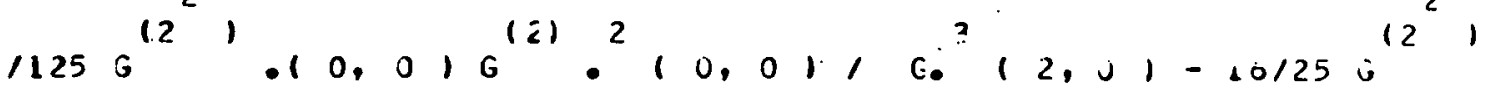
1125 G 


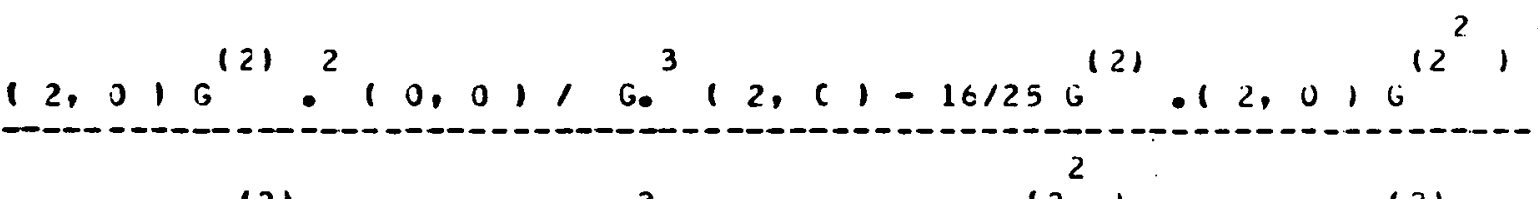
.$\left(0,0, \mathrm{G}^{(2)} \cdot\left(0,0,1 \mathrm{G}^{3}(2,0)-4 / 5 \mathrm{G}^{(2,}, 12,01 \mathrm{G} .1\right.\right.$

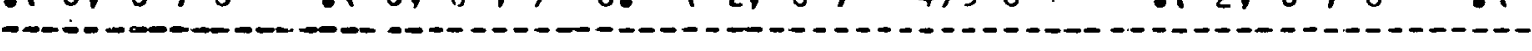
$2,0, G^{(2)} \cdot\left(0,0,1 G^{3}(2,0)+216 / 175 G^{(2)}, 13,01 G^{(2)} \cdot 11\right.$ (19) $.01 \mathrm{G}^{(2)} \cdot\left(0,0,1 \mathrm{G}^{2}\left(3,0, \mathrm{G}^{2}, 2, \mathrm{C},-138 / 175 \mathrm{G}^{12}\right.\right.$

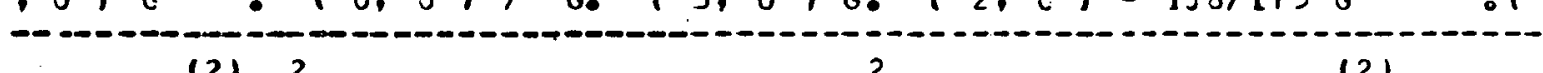

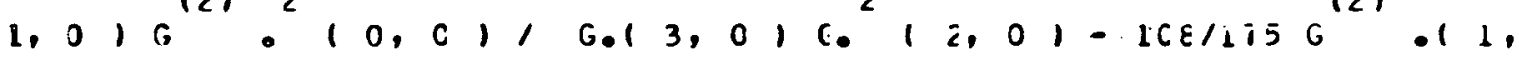
.

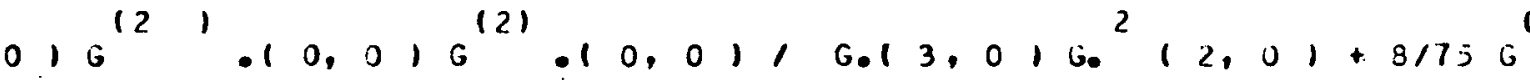

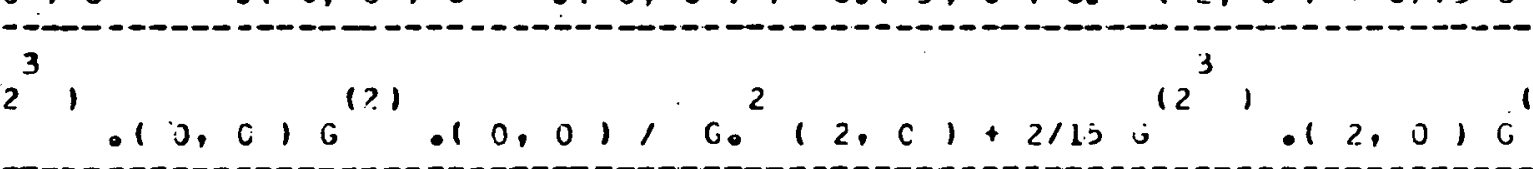

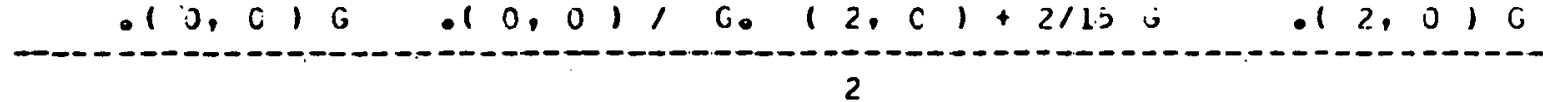

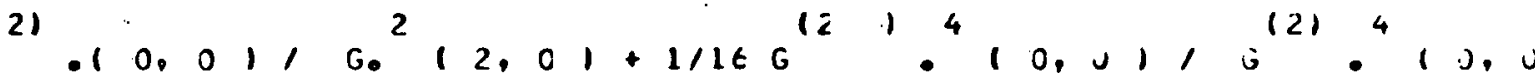
(3)

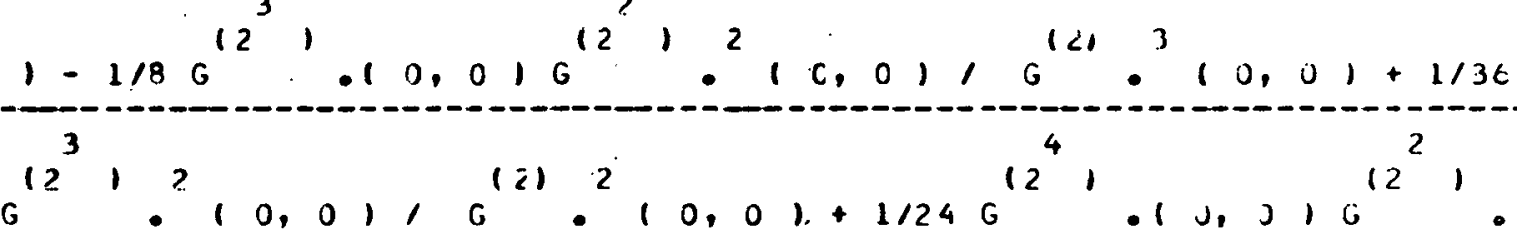

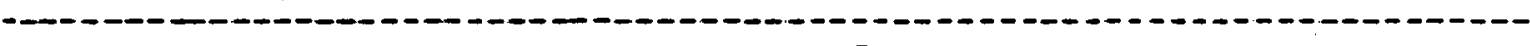

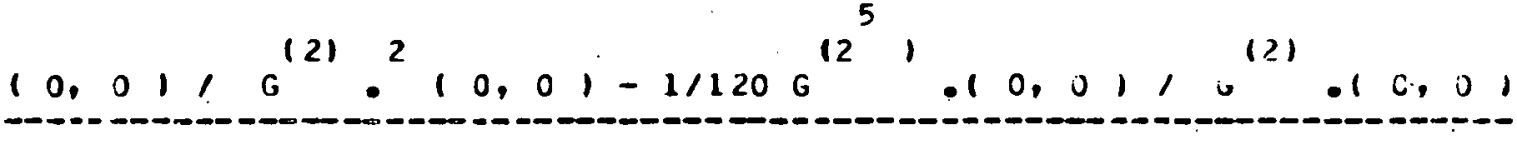
(2) 2

$C(4,1)=324 / 175 G G \cdot(2,0) G, 0(0,0) 0.11,0), G .13$,

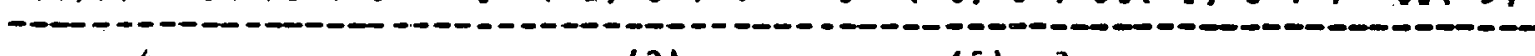
4

$01 G .12,01+1296 / 175 \mathrm{G}, 12,0, \mathrm{G} \cdot 10,016,11,01$ 0 4 $(2)^{4}, 1, J_{1}, \mathrm{G}, 1, \mathrm{C}, \mathrm{C}, 1$ I G. (3, C, G. $(2,01+22464 / 4375 \mathrm{G}, 1 \mathrm{~J}, \mathrm{C}, \mathrm{G}, 1 \mathrm{1}, \mathrm{C} 11$

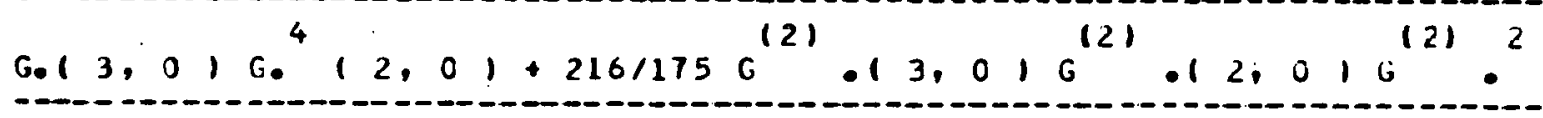
$\left(0,0, G_{0}\left(1,0,1, G_{0}^{2}\left(3,0, G_{0}^{2}(2, C)+\angle 516 / 1<2 j G(2), .1\right.\right.\right.$,

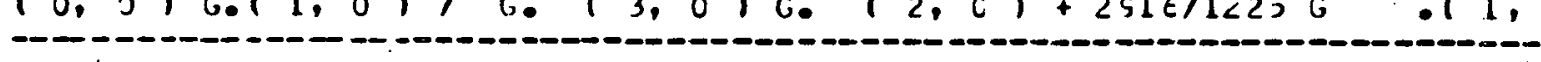
$G^{(2)} 0^{3}\left(0,0, G_{0}\left(1,0, / G_{0}^{2}(3,0) G^{3}(2, j)+432117 j G\right.\right.$

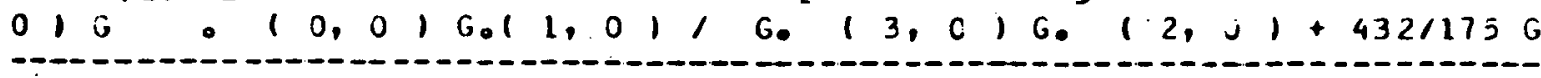
$(2)(2) \cdot 3 \quad 2 \quad 3$ $013,01 G+(C, 0,6.11,6,16.13,016.12,01$

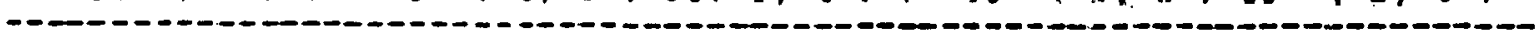




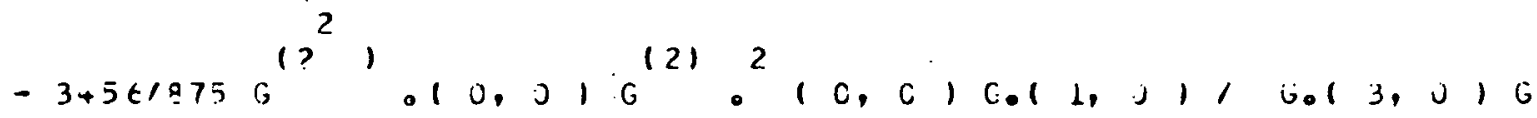

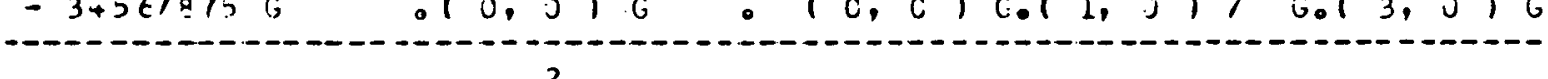

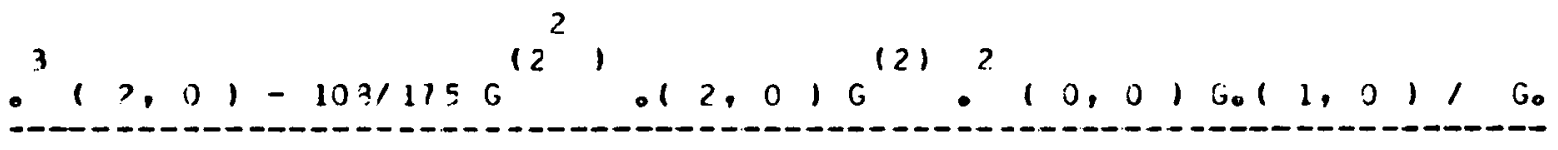
$\left(3, J, G_{0},(2,0)-324 / 175 G^{(2)} \cdot\left(2,0, G^{(2)}\right)\right.$

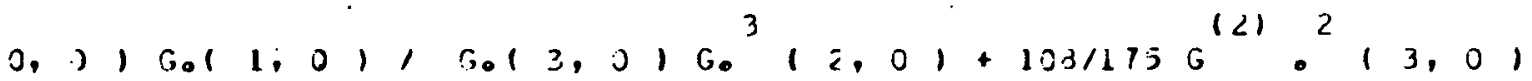
( $G^{(2)} 0^{2},\left(1,0,1,01, C, 1, G_{0}^{3}(3,0) G_{0}^{2}(2, J)+5761245 G^{12}\right.$ G-0.(.?) 3

$.11,0,6,0,0,0,11,0,1,6.14,0,6.13,016012$ -

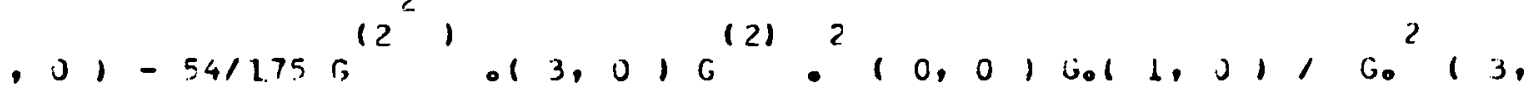
$\left.01_{0}^{2}(2,0)-162 / 175 G^{(2)} \cdot\left(3, c, G^{(2)}\right) .10,0\right) G^{(2)} \cdot 10,0$ ( 2 $10011.0,1,0^{2}(3,0) G^{2}\left(2, c 1+271175 G^{12}, 2,10,016\right.$

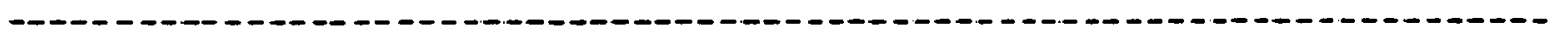

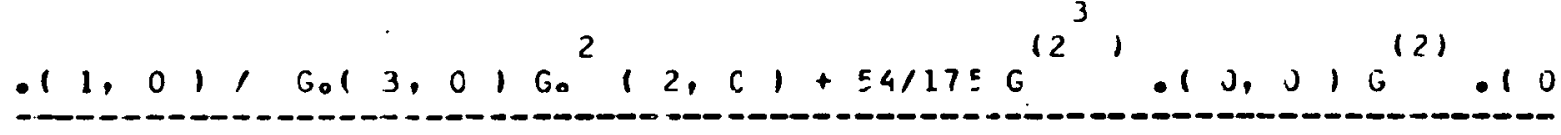

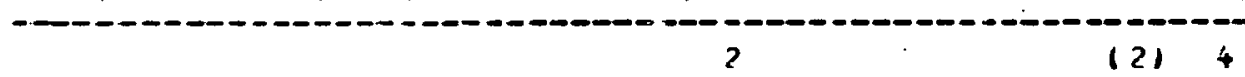

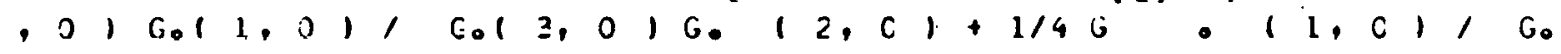

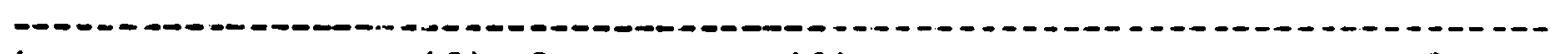

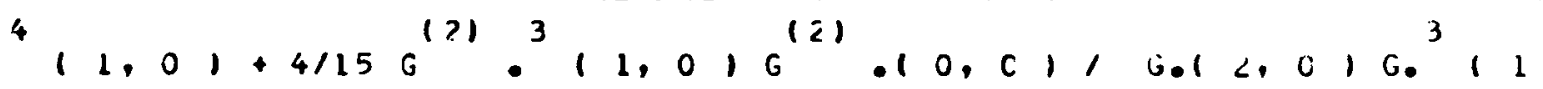

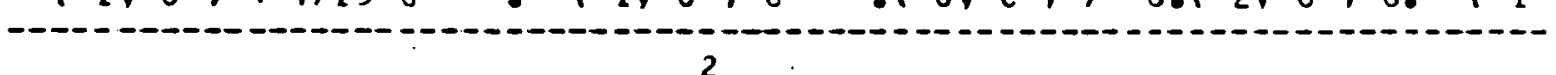

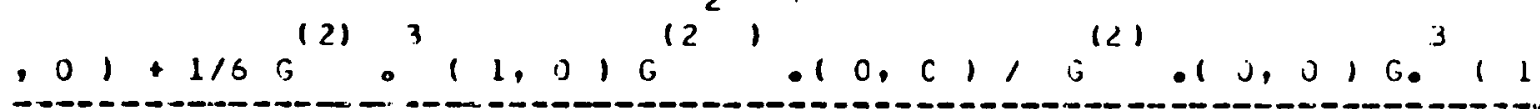

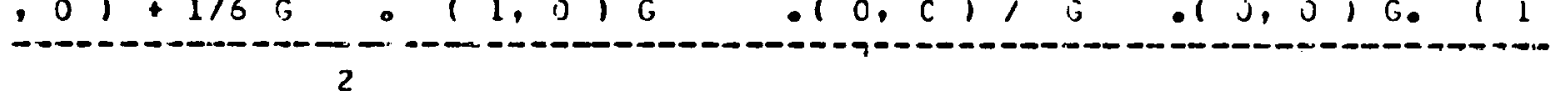

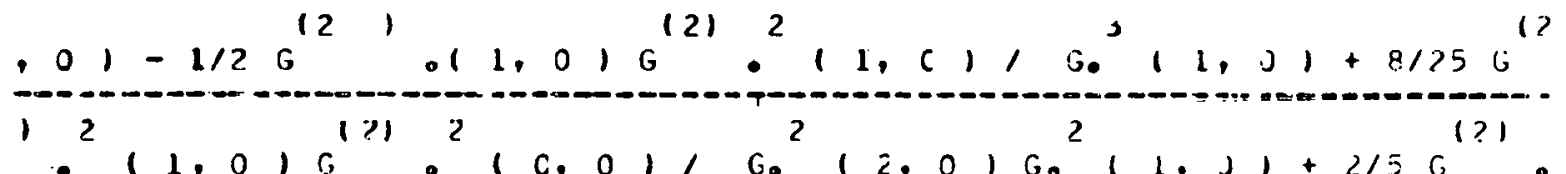

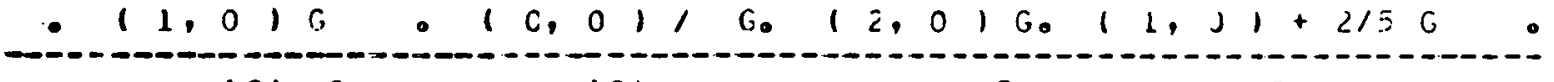
$\left(2, j, G^{(2)} G^{2}, 1,0, G^{121}, 10, c 1, c_{0}^{2} 12, J 1, j 011, i 1-\right.$ (

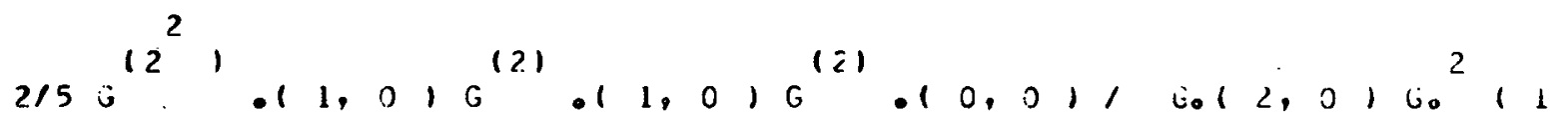

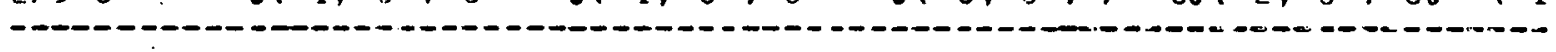

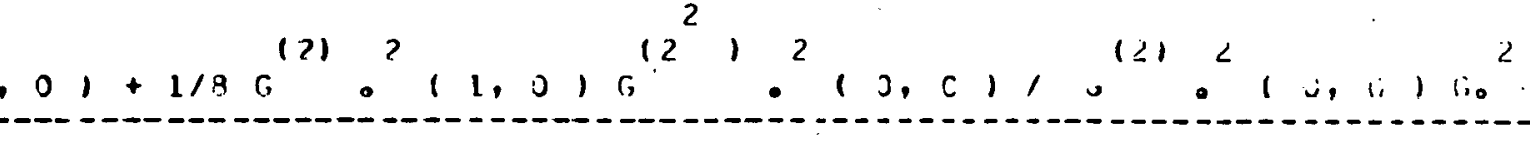


$\left(1,0.1-1 / 4 \mathrm{G}^{\left(2^{2}\right.}, \cdot 1,0, \mathrm{G}^{(2)} \cdot(1,0) \mathrm{G}^{(2}\right) \cdot\left(\mathrm{J}, \mathrm{c}, 1, \mathrm{G}^{(2)}\right.$

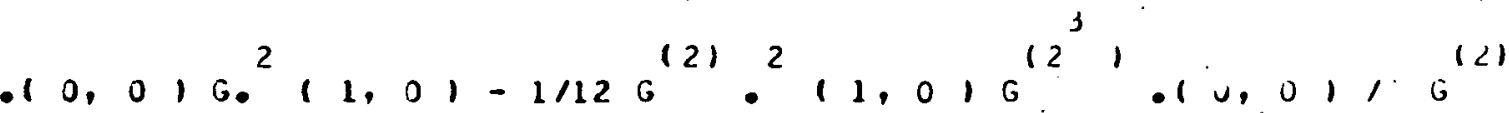
-

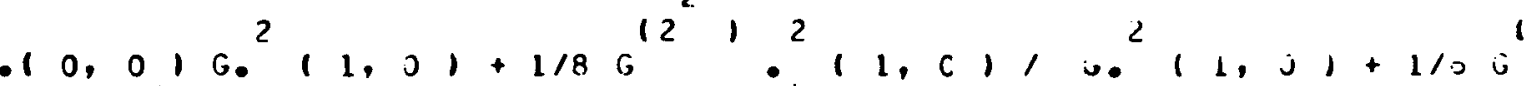
3 21 $(1,0) \mathrm{G}^{(2)} \cdot(1,0) \mathrm{G}^{2}(1, \mathrm{C})+32125 \mathrm{G}^{(2)}, 12101 \mathrm{G}$ 1.

$.11,0,6.10,0,16,12,016.11,01+54 / 1256$

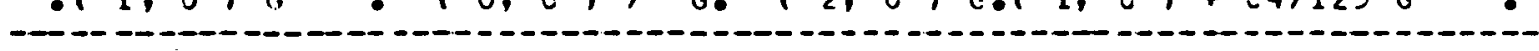
(2) $3 \quad 3 \quad(2) 2$

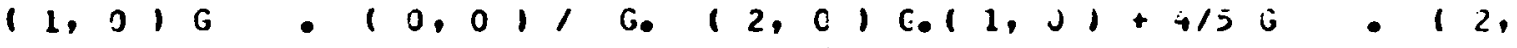

-

$0 \% G^{(2)} .11,0, G^{(2)} .\left(0,0,1, G^{3}, 2,0, G .11,01+108 / 1756\right.$ (19) $\left.(2)^{2}, 1,0, G^{(2)} 0^{2}, 0,0,1, G .13,0, G^{2}, 2,0\right) 6,1,1,01$ 0 $-8125 \mathrm{G}^{12^{2}}, 11,0, \mathrm{G}^{121}{ }^{2}\left(0,01, \mathrm{G}^{2}, 2,01,6.11,01,8\right.$

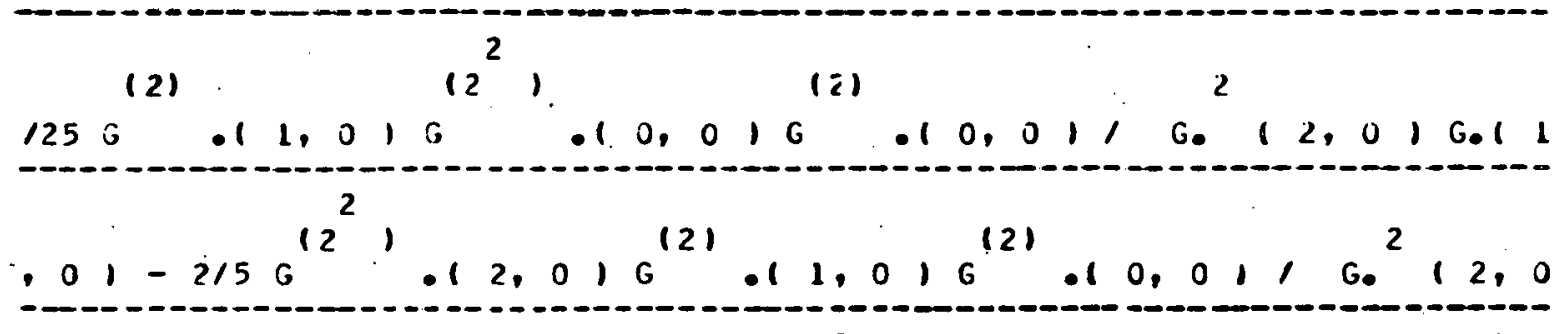

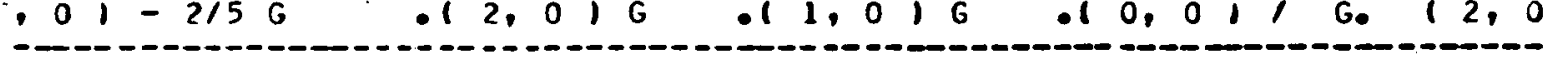
$1, G .11,01-2 / 5 \mathrm{G}^{(2)} \cdot(2,0) \mathrm{G}^{\left(2^{2}\right.}, \cdot\left(1,0, \mathrm{G}^{(2)} \cdot 10,011,6\right.$. ( $2\left(2,01 G_{0} 11,01+2 / 15 G^{\left.12^{3}\right)}\right.$ (2)

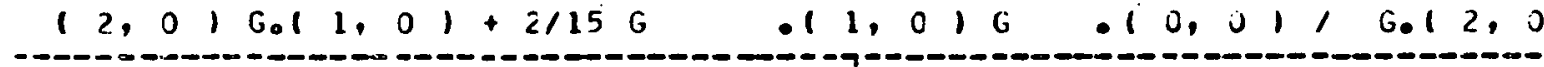

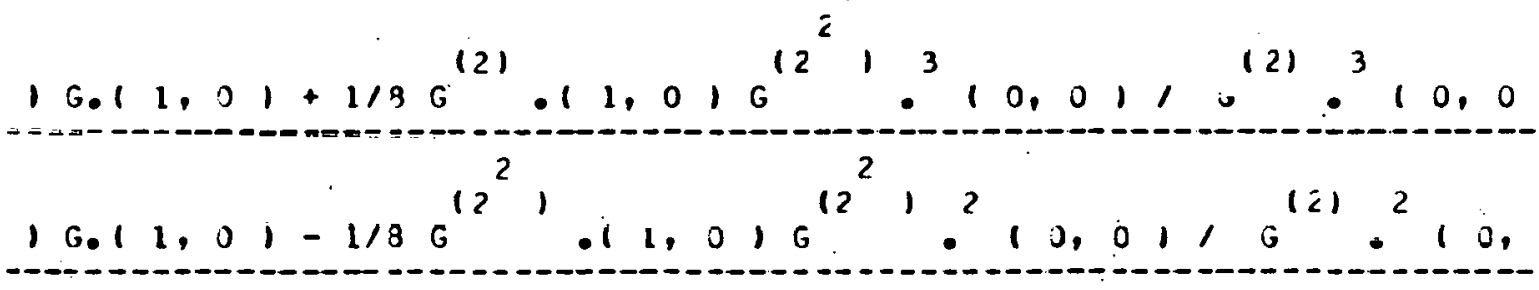

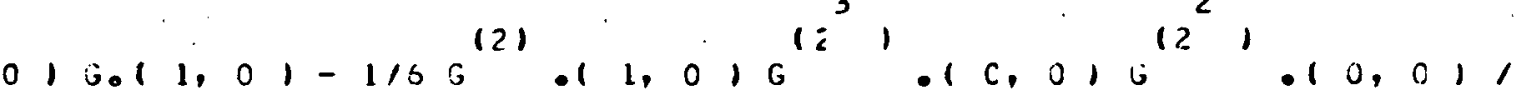

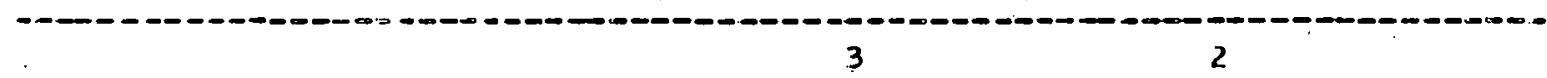
$\left.G^{(2)}\right)^{2}, C_{0}, 0, G, 11,01+1 / 12 G^{1}$ $1 i^{3}, 12,1$

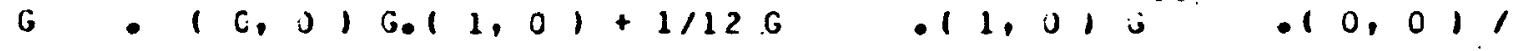




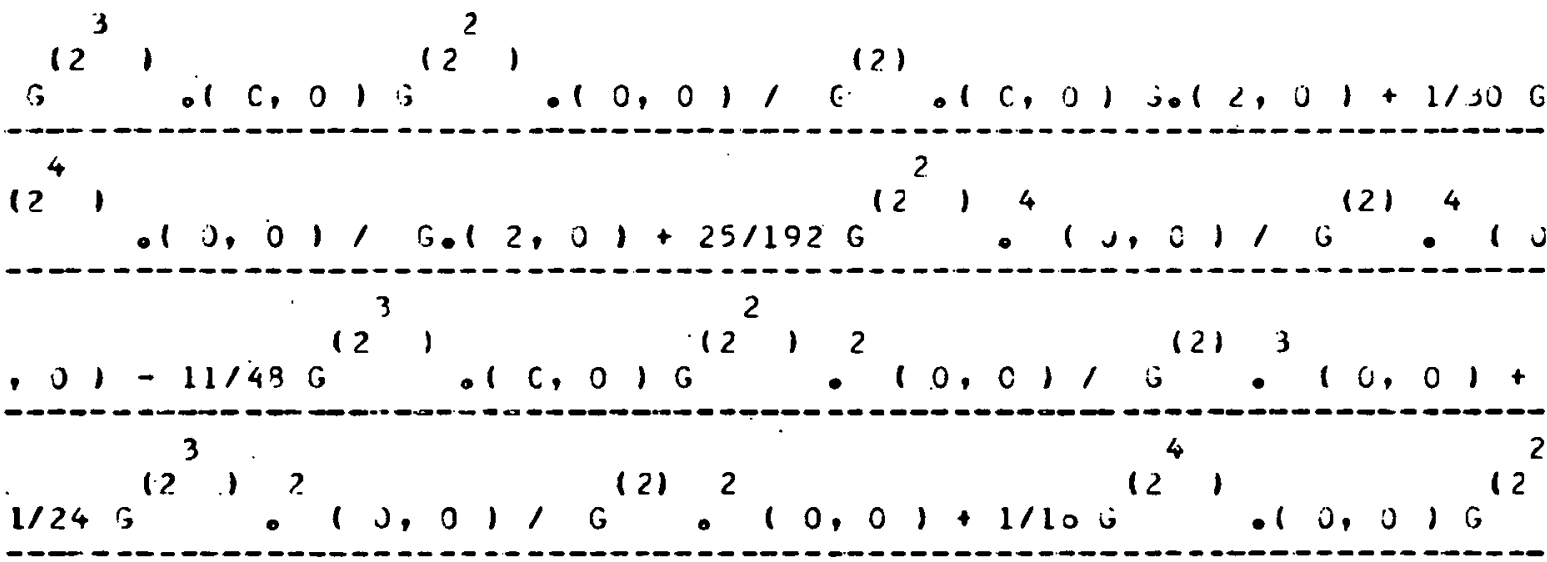

$1 \quad 12)^{2}\left(2^{5}\right)$

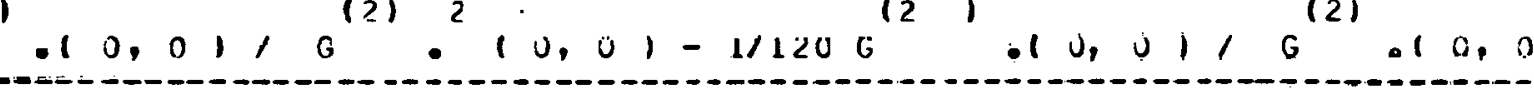
$C(4,2)=-1296 / 3756^{(2)} .12,0,0^{(2)} \cdot 10,010.11,01,0.13$ 4

, 01 G. $12,01-7776 / 4375 \mathrm{G}, 10$, C 1 G.1 $1,0,1$ G.1 3,01 $-10-0$ $G^{4}(2,0)-2916 / t 125 G^{(2)} \cdot(1, c) G^{(2)} \cdot 3(j, c), 0.11,011$ -

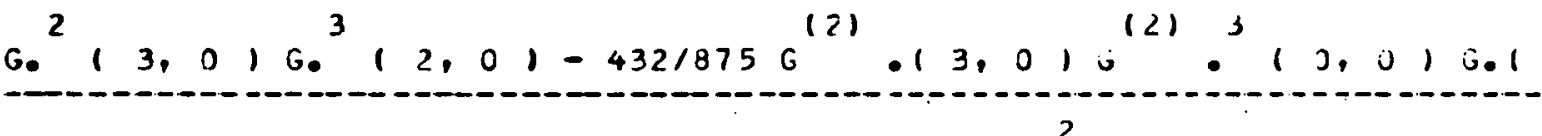

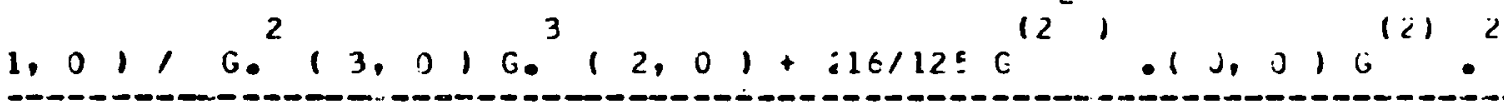
3 $(2)$

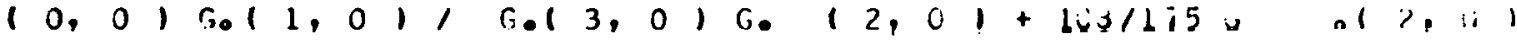

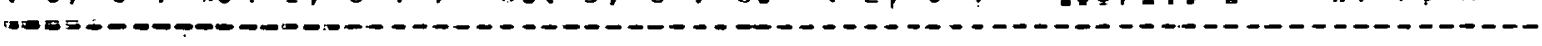
$2^{2}$

$G^{(2}, 10,0, G^{(2)} .10,0, G_{0} 1,0,0, G .13,0,0_{0}^{3}, 2, c 1$, 0 (2) $(2) 3$ ?

$576 / 12256$ o 1,016 o $10, c \mid c .11, c) / 0.1+0, c .1$ $3,01 G^{2}, 2,01+54 / 175 G^{(2)} \cdot 13,0, G^{\left(2^{2}\right.}, \cdot 10, j, G^{(2)} \cdot 1 j$, (3)

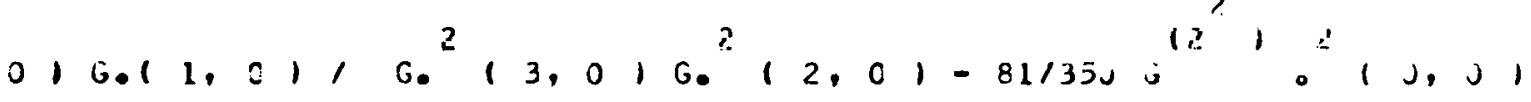
-

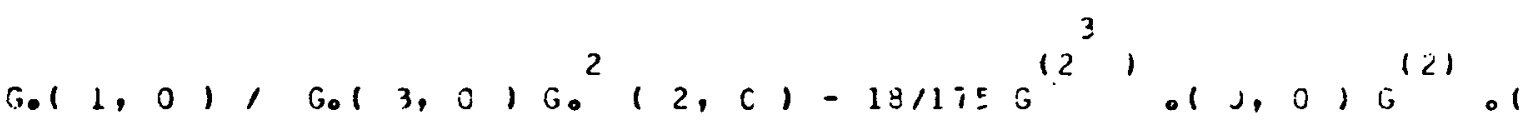
Gol $0,0, G .11,0,1, G .13,0,0_{0}^{2}, 2,0,+11 /<40_{0}^{(2)}+1, c 1$,

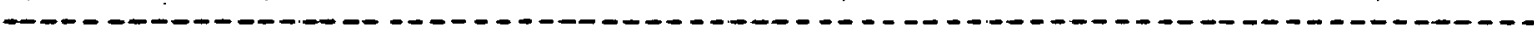
$G^{4}\left(1,01+2115 G^{(2)}{ }^{3}(1,0) c^{(2)} \cdot 10,3110.12,016_{0}^{3}\right.$ 
$1, G_{0}^{4} 12,01-64 / 75 G^{121}: 10,01, G_{0}^{4} 12,01-4 / 5 c^{121}$.

$3 \quad(2) \quad 4 \quad 0 \quad 121 \quad 01$

$12,016 \quad .1,0,01160,12,61-648 / 1756 \quad 012,0106$

(2) 2 (

$\because 1,0, G \quad(0,0), G 013,0) G 0,12,01-\$ 15218756$.

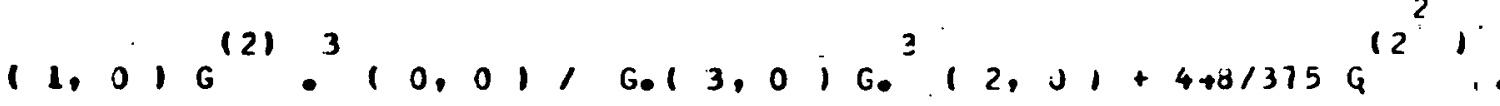
$\left(0,0, G^{(2)} e^{2}(0,0), G_{0}^{3}, 2,\left(1+24 / 25 G^{\left(2^{2}\right.}, \cdot 12,0, G^{(2)}\right.\right.$ (1) $\left.0^{2}(0,0), G^{3}(2,0)+8 / 5 G^{(2)} \cdot 12,0\right) G^{(2)}, 010,01 G^{(2)}$ $10,01,0_{0}^{3}\left(2,01+4 / 50^{12}, 012,0,0^{(2)} \cdot 12,016^{121} \cdot 10\right.$ $\begin{array}{lll}2 & 0\end{array}$ $.01, \mathrm{G}^{3}, 2,0,+2 / 5 \mathrm{G}^{121} 0^{2}, 2,0, \mathrm{G}^{12}, 10,011 \mathrm{G}^{3} 12$ $(2) \quad(2) \quad(2) \cdot 2 \quad 0$

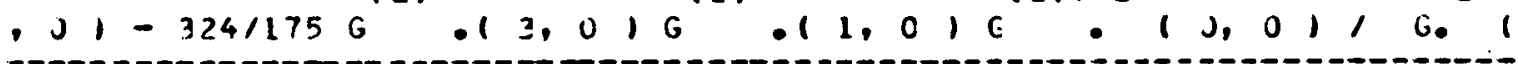

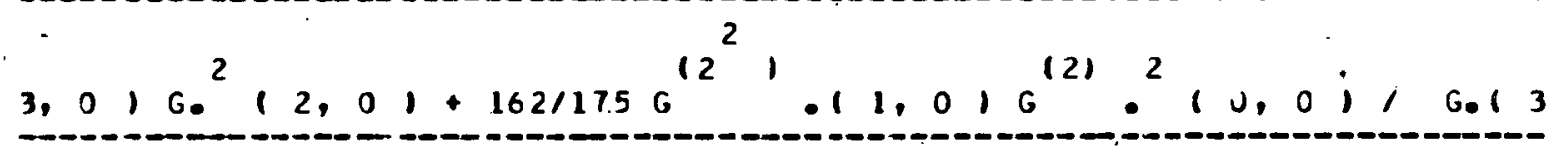
(1)

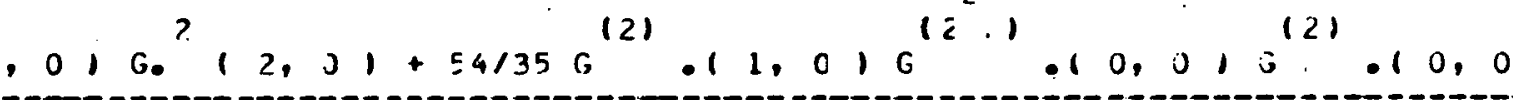

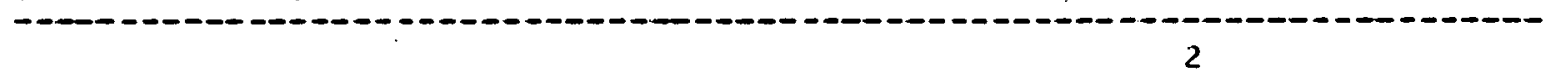

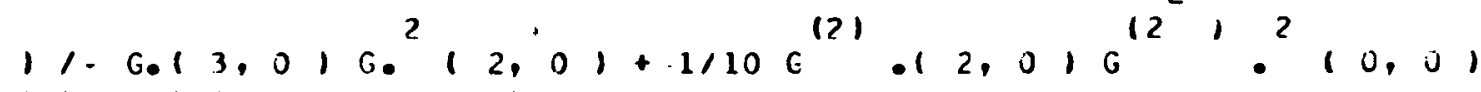

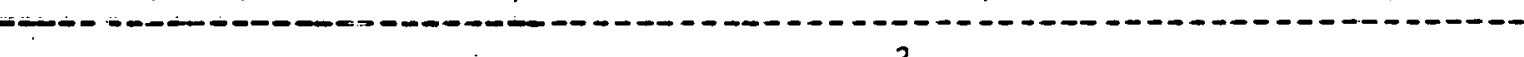

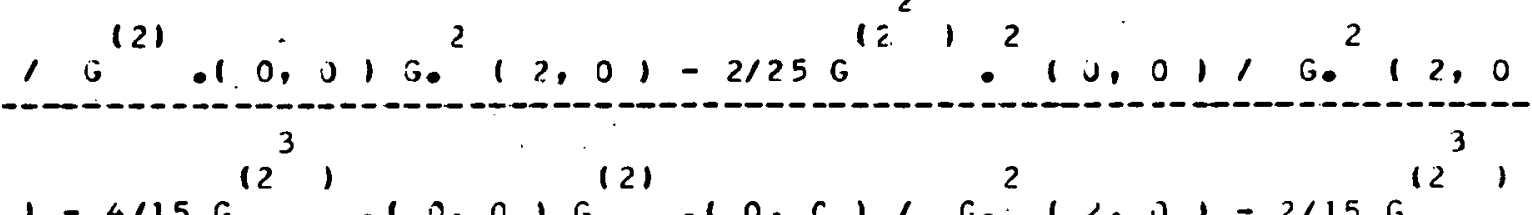
$1-4 / 15 G$ G 010,0$) G$ G $0, C, 16.1<, 01-2 / 15 G$

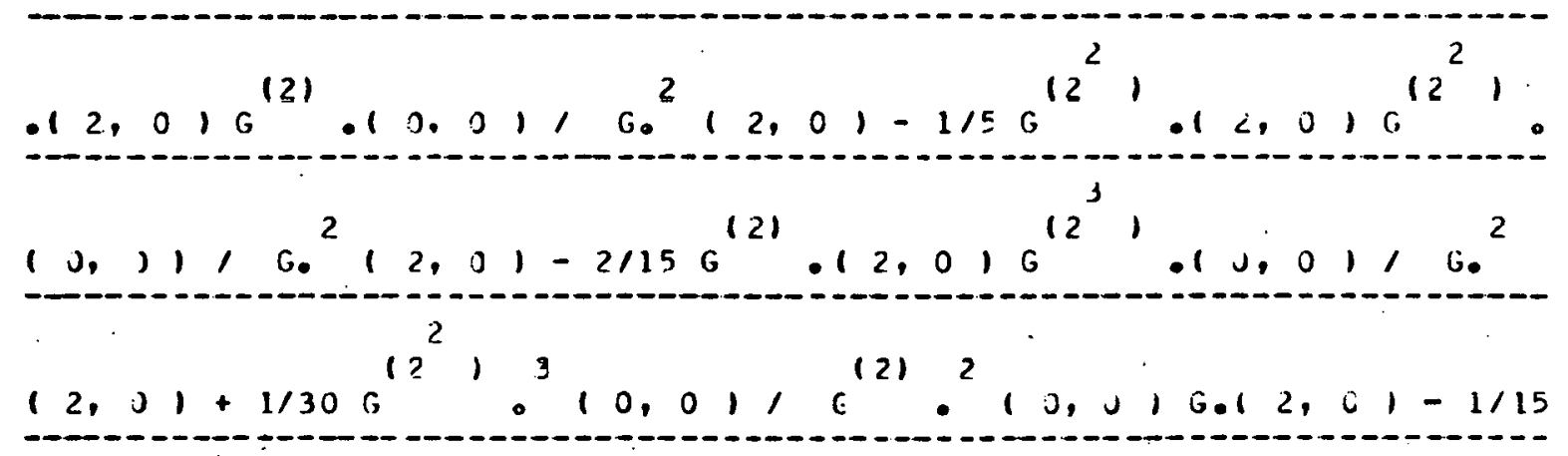




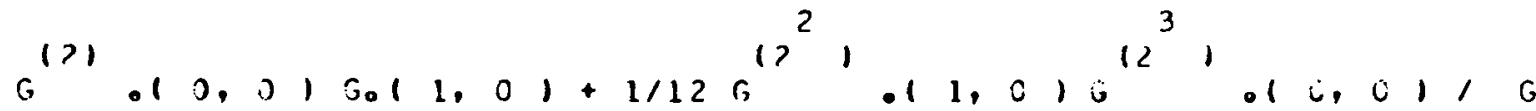
0

(2) (2) $\left.12^{4}\right)$

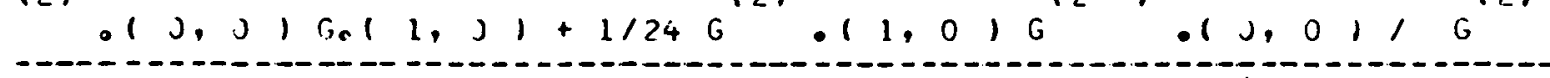

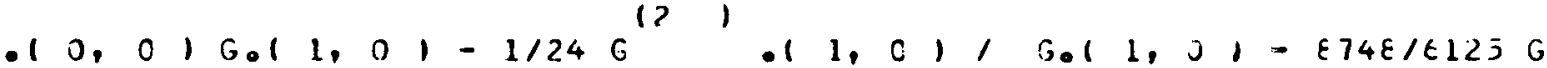
(1) (2) (?) 3 24 $01,0, G,(C, 0) G_{0}(1,0), G_{0}(3, j), G_{0}(2,0)$

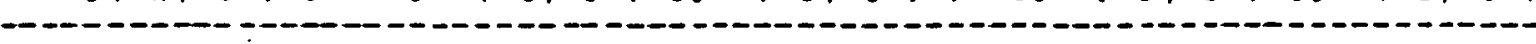
(2) 42024

$-1745 t / 4375 G$ G $10,0, G_{0}(1,0,1, G .1,3, J, G, 12,01$

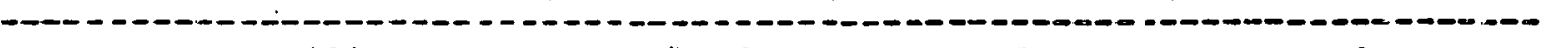
(2) (?) 3 3

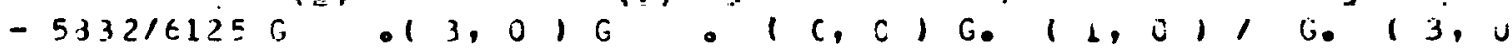

$\begin{array}{cccc}3 & (3) \\ 3 & (z) & 3\end{array}$

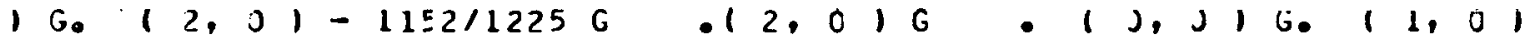
$2 \quad 3 \quad 12144$

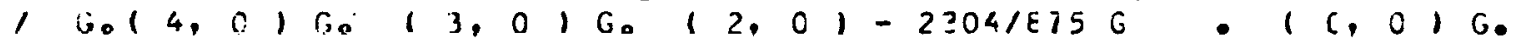
(-)-

$\left(1,0,1,0,14,0, G_{0}, 13,0, G_{0}^{2}(2,0)+145 E / 1<25 G^{12}, 010\right.$ ( $1{ }^{\circ}$ 0 1 / $0, G^{121}{ }^{2}\left(0,0, G^{2}\left(1,0,1 / G_{0}^{2} 13,0, G^{3}(2,011-11521\right.\right.$

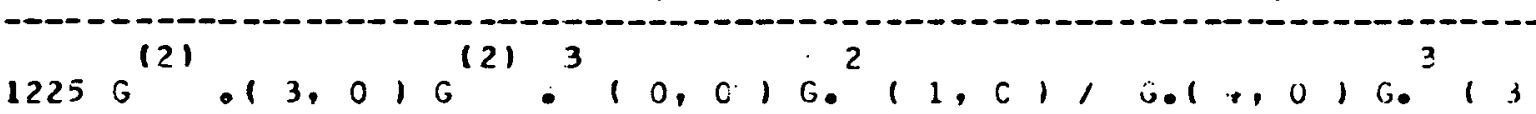

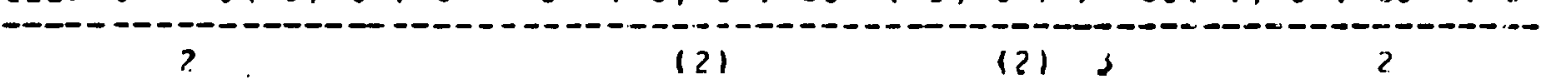
, 01 G. $(2,0,-576 / 1225 \mathrm{G}, 14,0, \mathrm{G}, 10, J 16,11, i$ ($2,2,2,121$ $1 /$ G. $\left(4,0, G_{0}\left(3,0, G_{0}(2,0)+2 \varepsilon \varepsilon / 24 j G, 010,01\right.\right.$ $\begin{array}{lll}(2) & \text { ? }\end{array}$ G $\quad\left(0,0, G_{0}, 1,0,1,0.14,0, C_{0}(3, j), 0,12,01+\right.$ (2) 4303

$79732 / 214375 G$ G $\quad 0,0)$ G. $\left(1,0,1, G_{0}(3, J), 0.12,01\right.$

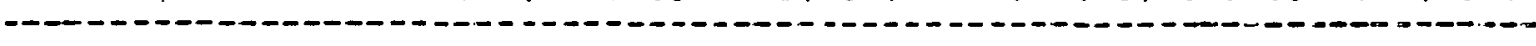

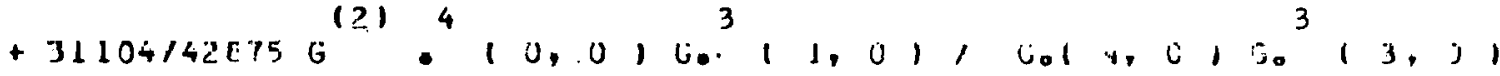

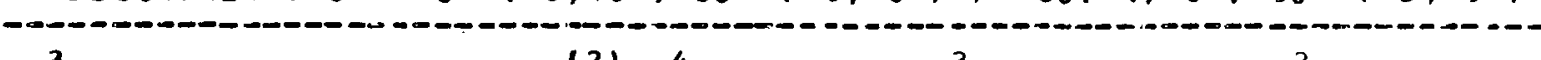

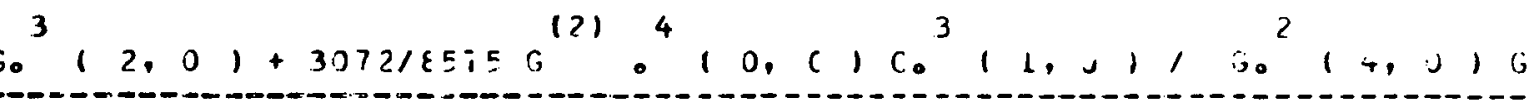
$0^{3}\left(3,0, G_{0}^{2}(2,0)+192 / 5390^{(2)} 0^{4}(0,0) 0_{0}^{3}(1,0), G_{0}\right.$

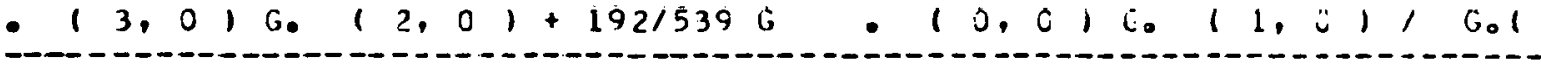

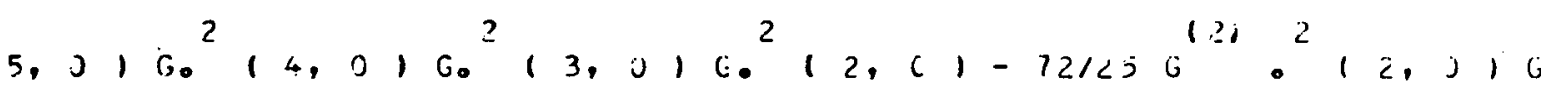
-

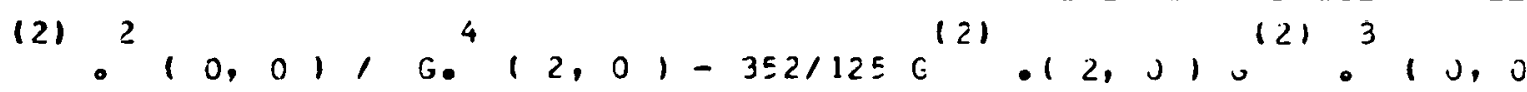




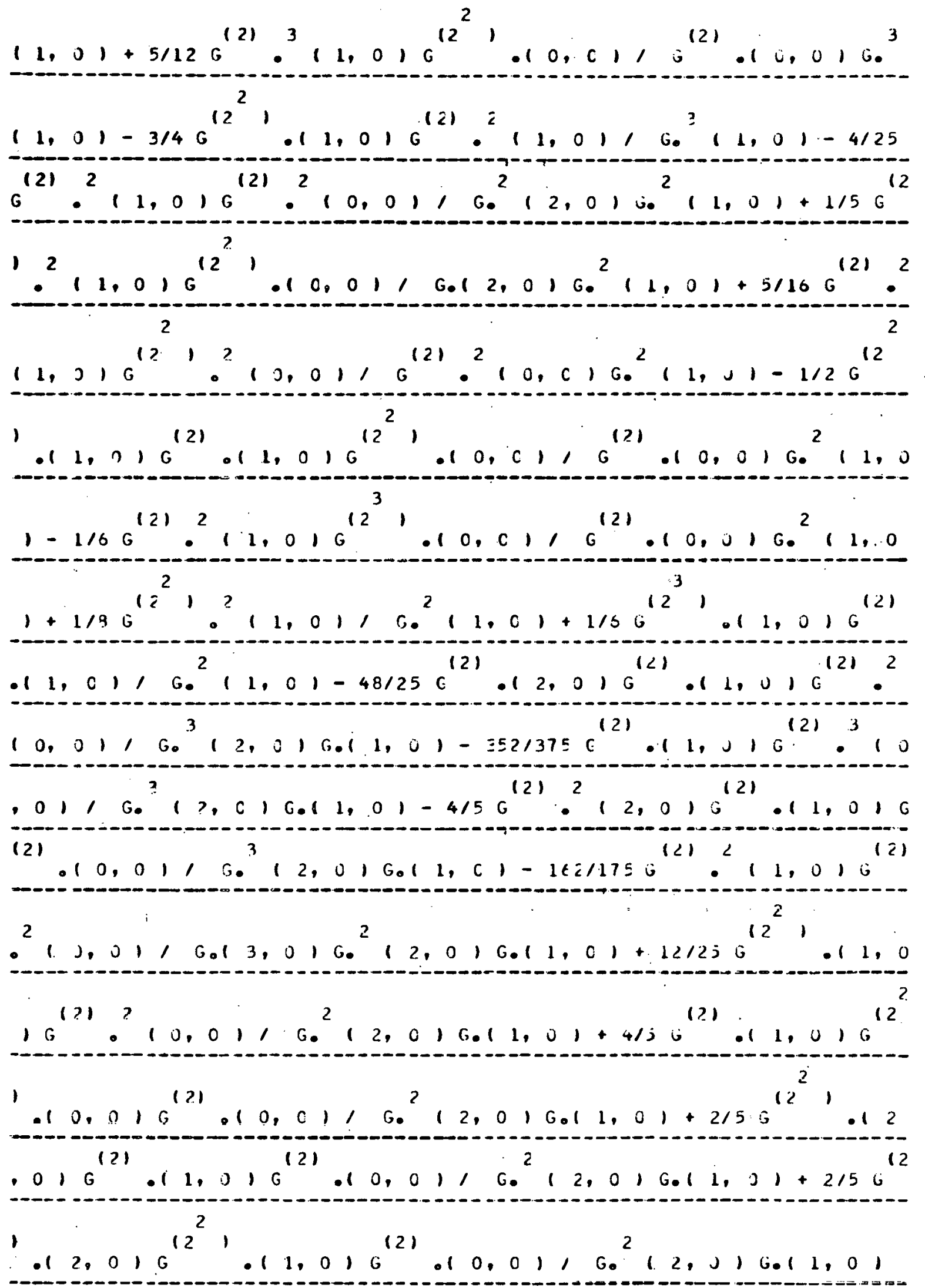




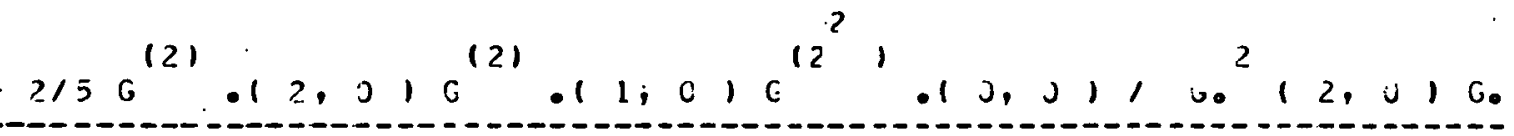
2

$(1,0)+1 / 10 g^{(2)} \cdot 11,0, G^{(2)} 0^{2}, 0,0,16^{(2)} \cdot 10,01,0.1$ (10.-

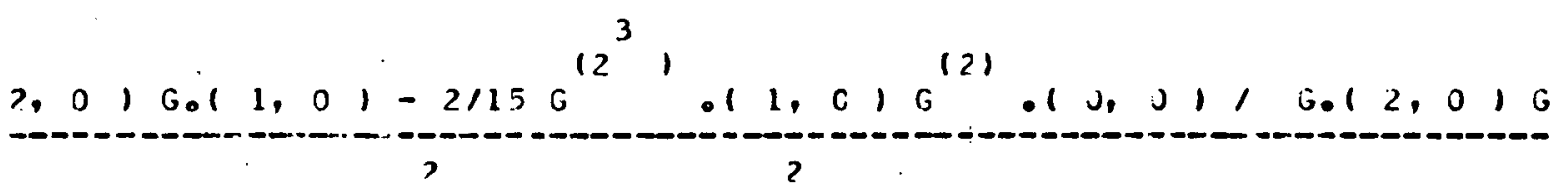
$121 \quad 121$

$.11,01-1 / 5 G^{\circ} \quad .11,01, G \quad .10, C 1,0.12,01,6011,0$

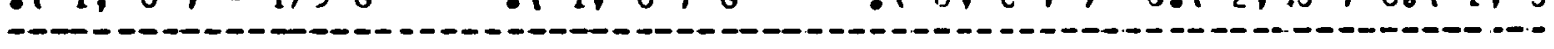
3

$1-2 / 15 \mathrm{G}^{(2)} \cdot 11,0, \mathrm{G}^{12}, .10, \mathrm{C}, 1, \mathrm{G}, 12,0,1 \mathrm{k}, 1,01+111$ 1

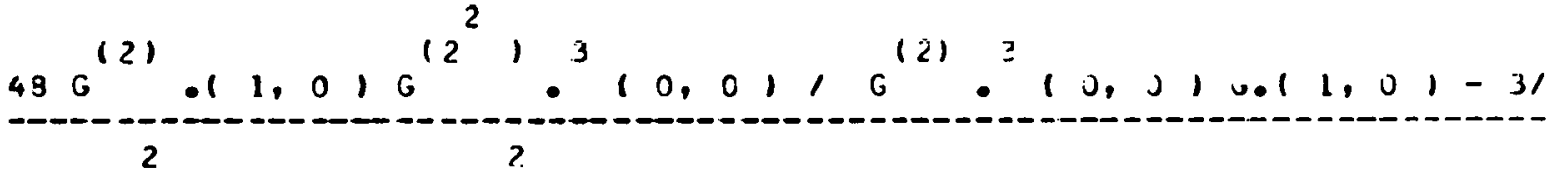

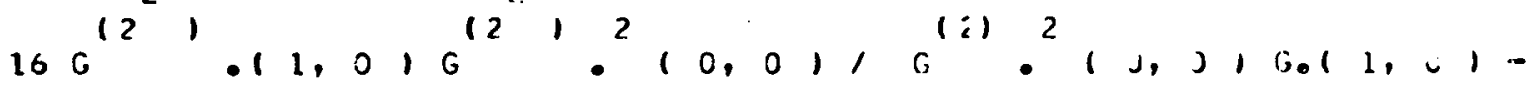
16 $1 / 4 G^{(2)} \cdot 11,01 G^{12^{3}}, \cdot 10,01 G^{12^{2}} 1,10,01,0^{121} 0^{2}, 0,01$

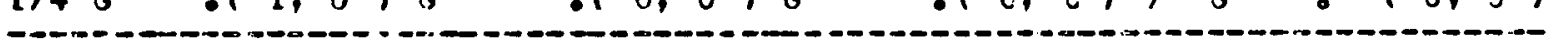
(1) $0.11,01+11120^{12}, 11,0.10^{1 i^{2}}, 010,01, j^{121} .10,010$ G $12^{2}, 12^{3} j 121$ $.11,01+1 / 12 G \cdot .11,0,0,010,0,1, G \quad 010,010$ -

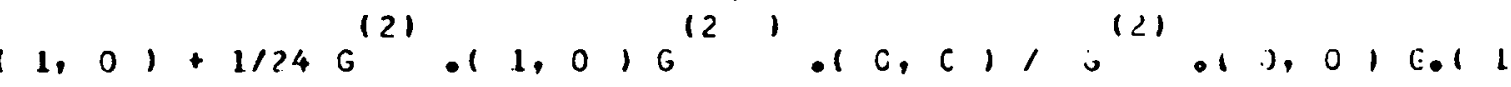

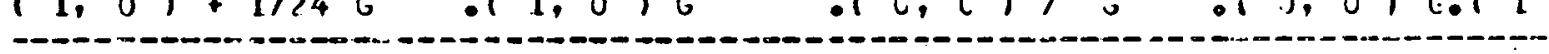

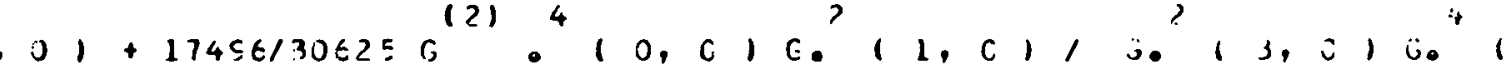

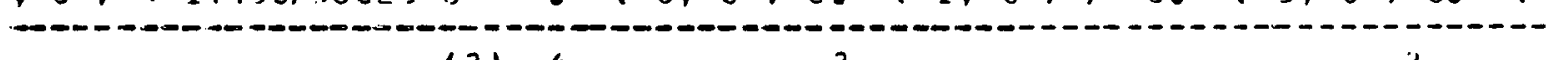
$2, J 1+2304 / \in 125 G^{(2)} G^{4}, 0,0, G_{0}^{2}, 1,0,1, G_{0} 1+0, G_{0}^{2} 13$, -

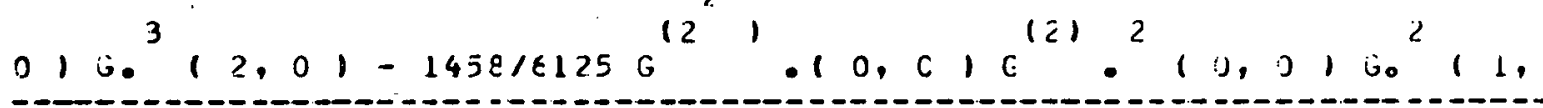

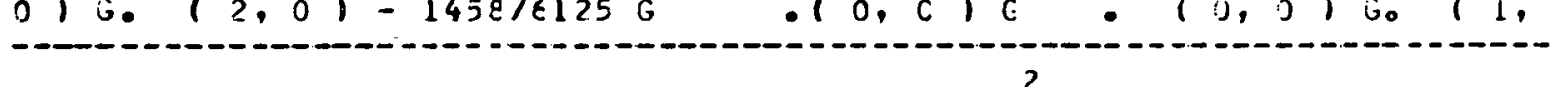
$c 1, G_{0}^{2}, 3,0, G_{0}^{3}\left(2,0,-288 / 122 \leq e^{(2,1}, 1 j, J 1 i^{(2)} e^{2}\right.$,

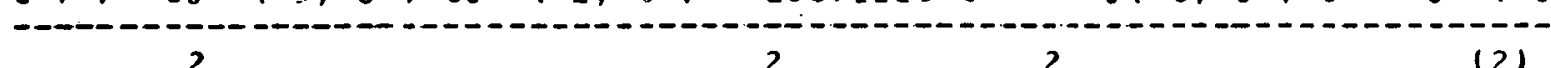
, $01 G_{0}(1,0), G .(4,0) G_{0}(3,0) G_{0}(2,0)+24 / 25 G$ - 


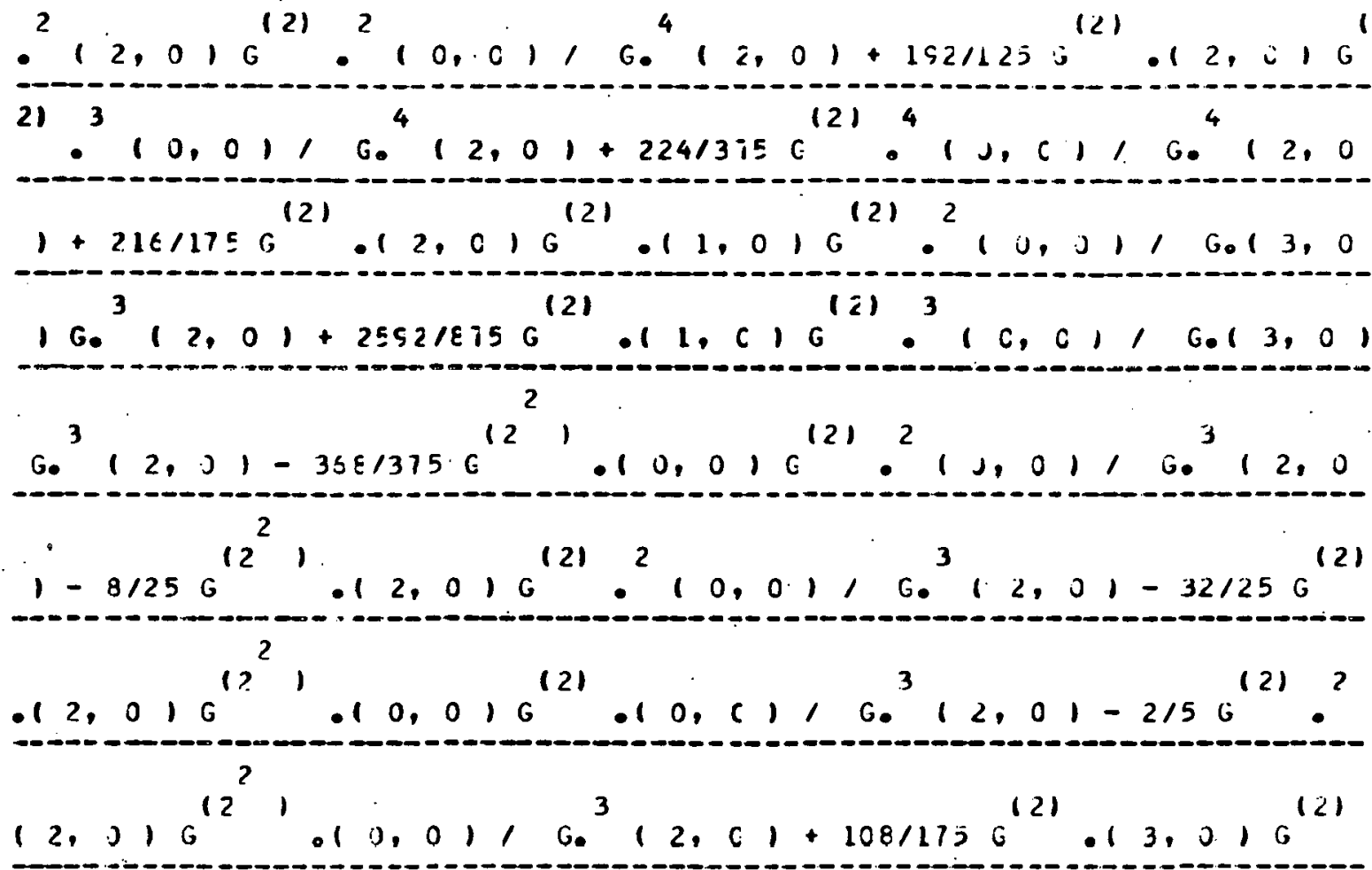

$.11,0) G^{(2)} 0^{2}, 0,01 / G^{2}\left(3,0, G^{2}(2, c)-54 / 175 G^{12}\right.$,

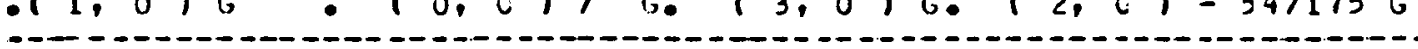
$\begin{array}{llll}121 & 2 & 2 & 121\end{array}$ $.11,01 G \bullet 10,01 / G .13,0, G_{0} 12,01-216 / 175 G \quad .1$ $12^{2}$,

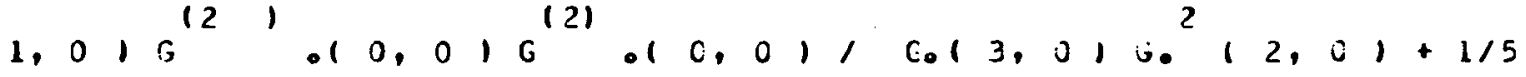
$\begin{array}{ll}15 & \\ & \end{array}$ $G^{12} 0^{2}, 0,01, G_{0}^{2}\left(2,01+16 / 75 G^{12} 1.10,01 G^{121} \cdot 10,0\right.$

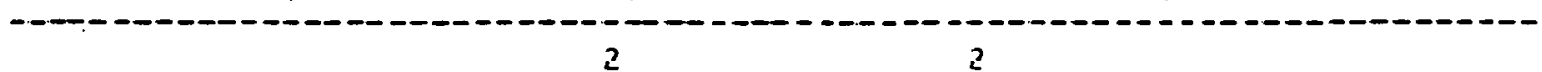
$1, G^{2} 12,01+1 / 5 G^{12^{2}}, \cdot 12, C, G^{12^{2}}, 010,0,1, G 0^{2} 12,0$ 1
3 $1+2115 \mathrm{G}^{121}+12,0, \mathrm{G}^{12},(0, c), 0_{0}^{2} 12,01+1100 \mathrm{G}^{(2)}$

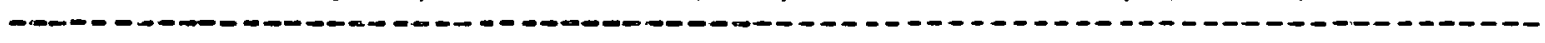
$0^{3}(0,0), G^{(2)} e^{2}, 0,0,6.12,01-1 / 300^{12^{4}} 1$

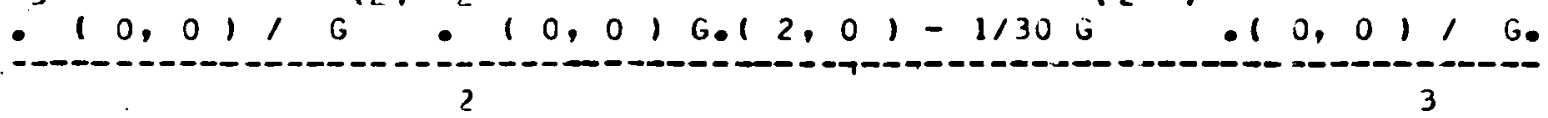
$12,01+251384 G^{12} 0^{4}, 0,01, G^{121} 0^{4}\left(J, 01-1 / 8 G^{12} 1\right.$.

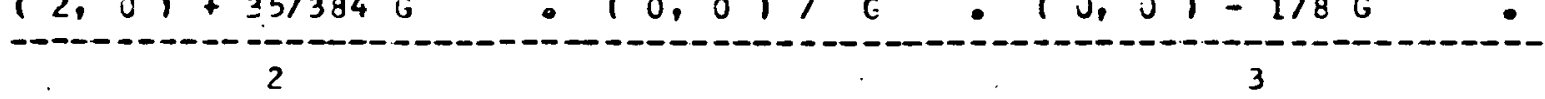
$(0,0)^{1} \mathrm{G}^{12}, 0^{2}, 0,1, \mathrm{G}^{121} 0^{3}, 0, \mathrm{C},+1 / 72 \mathrm{G}^{1 / 1} 0^{2} 10,0$ 1000 


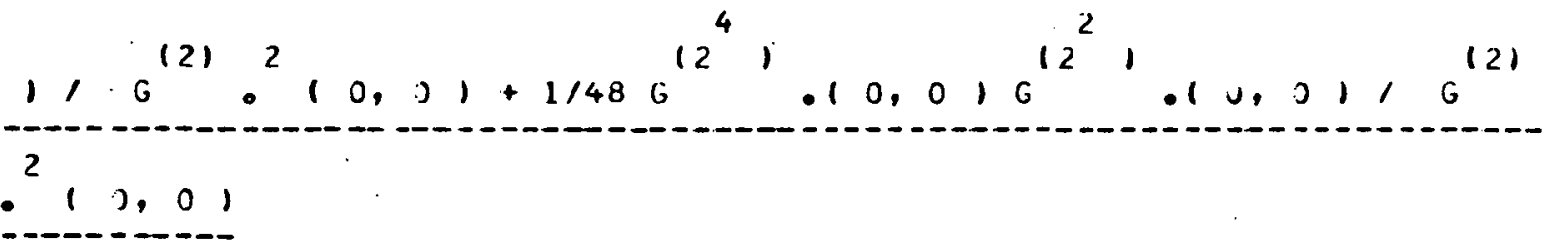

$\left.C(4,3)=36414375 G^{(2)} \cdot 4,0,0, G .11,0,1,0.13,\right), G, 12,01$ ( $-216 / 875 \mathrm{G}^{12^{1}}$ (2) 2

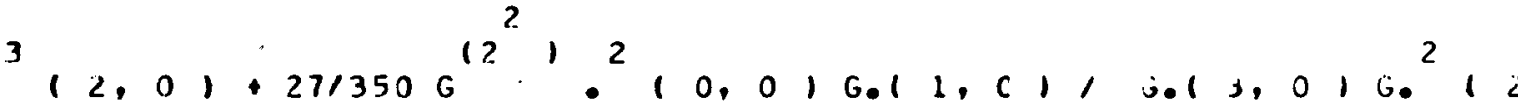
$121: 4 \quad 1213312$ , $01+1 / 4 \mathrm{G} \cdot\left(1,01, G_{0}, 1,01-4 / 15 G \mathrm{G} 11, i 16\right.$

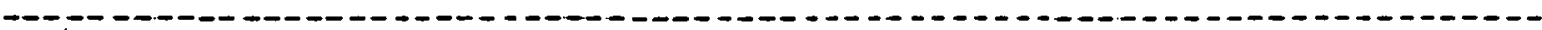
$1.1,0,1, G .12,01 G^{3}, 1,01+1 / 3 G^{(2)}+1,1,1, G^{2} 12^{1}$ -- - - - - - - - - - - - - - - - -

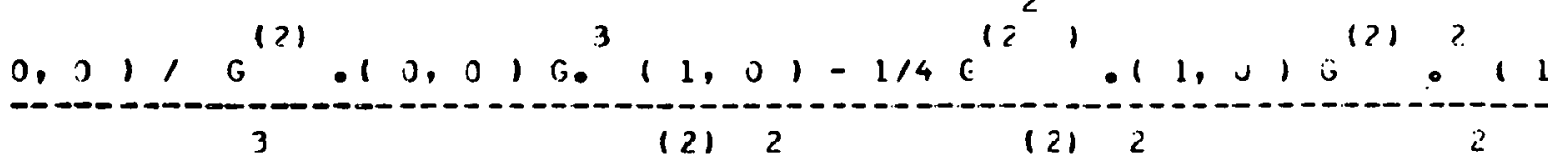

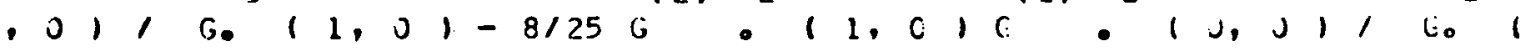

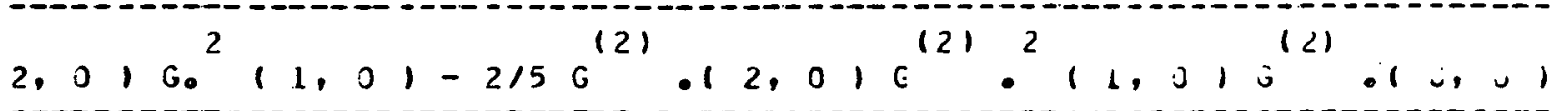
2 .

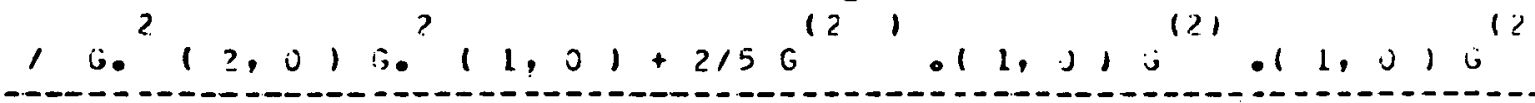

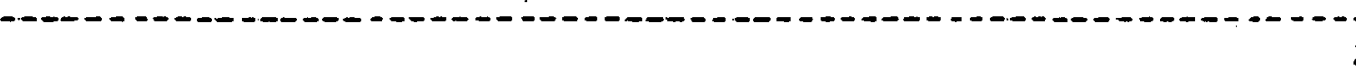

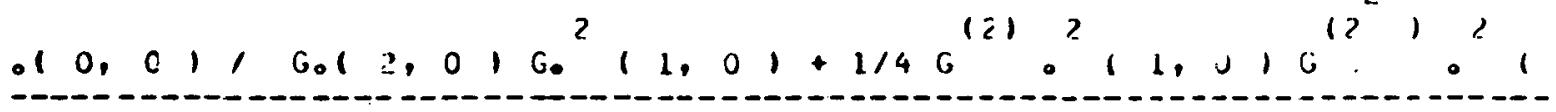

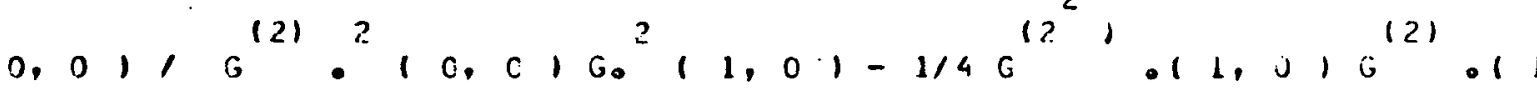
0

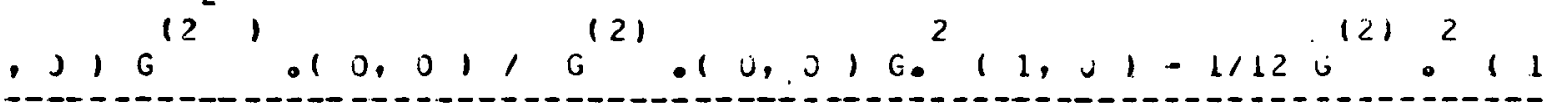

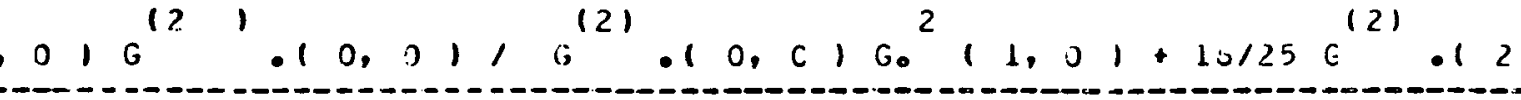
( 21 (

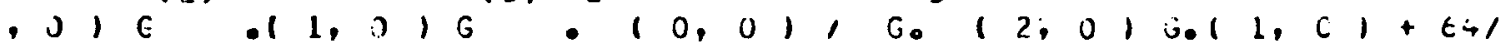

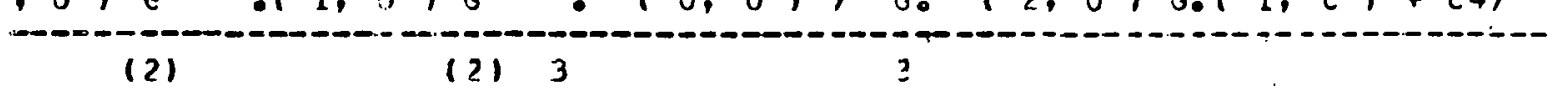

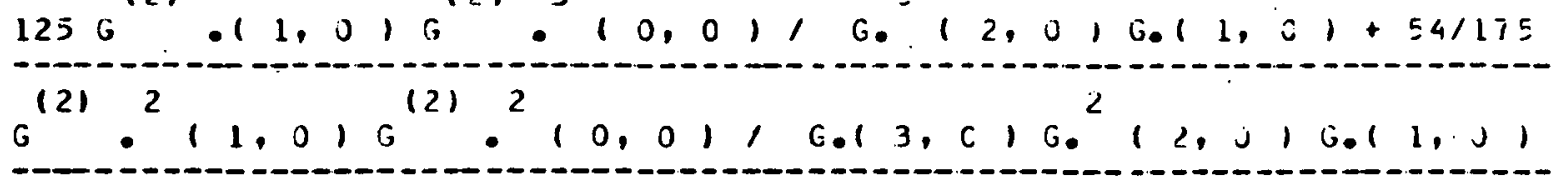


$-4 / 25 G^{12^{2}}, .11,0, G^{121} 0^{2}, 0, c, 1, G^{2}, 2,0, G 01,0,1,-$

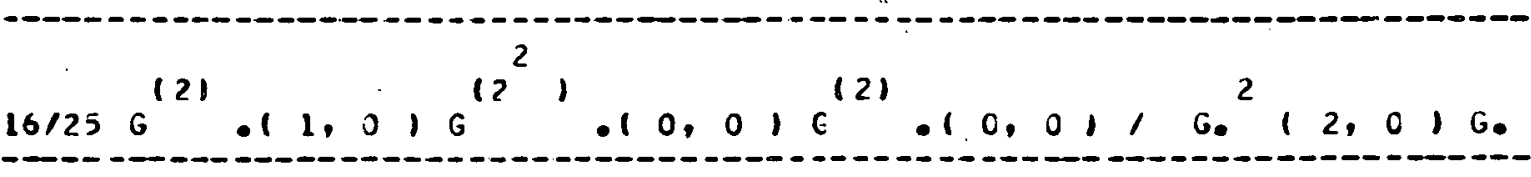
(16)

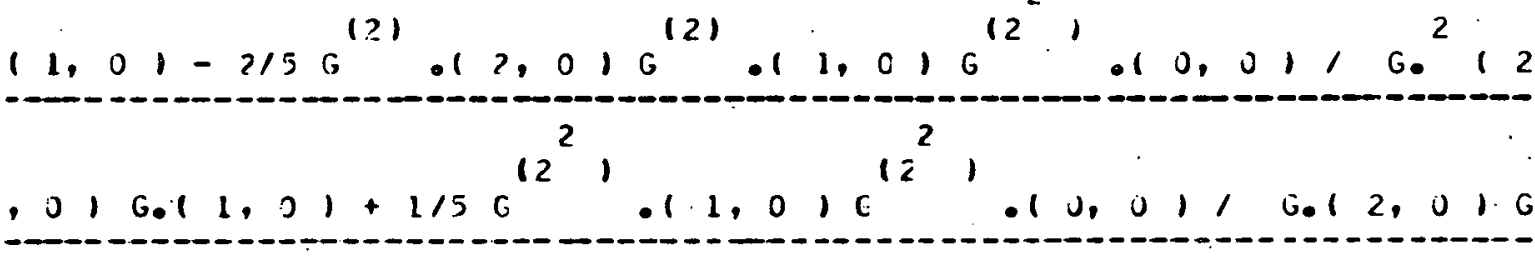

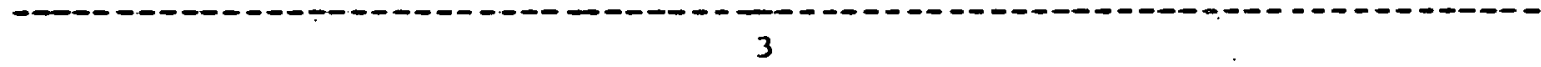

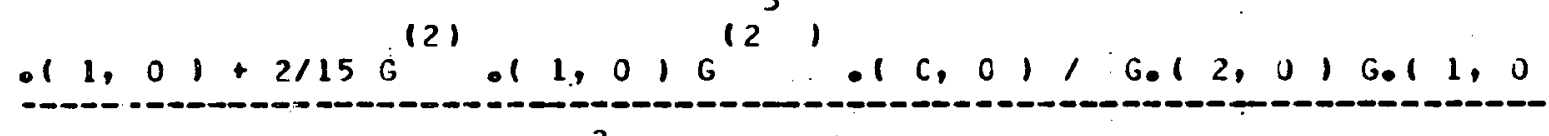

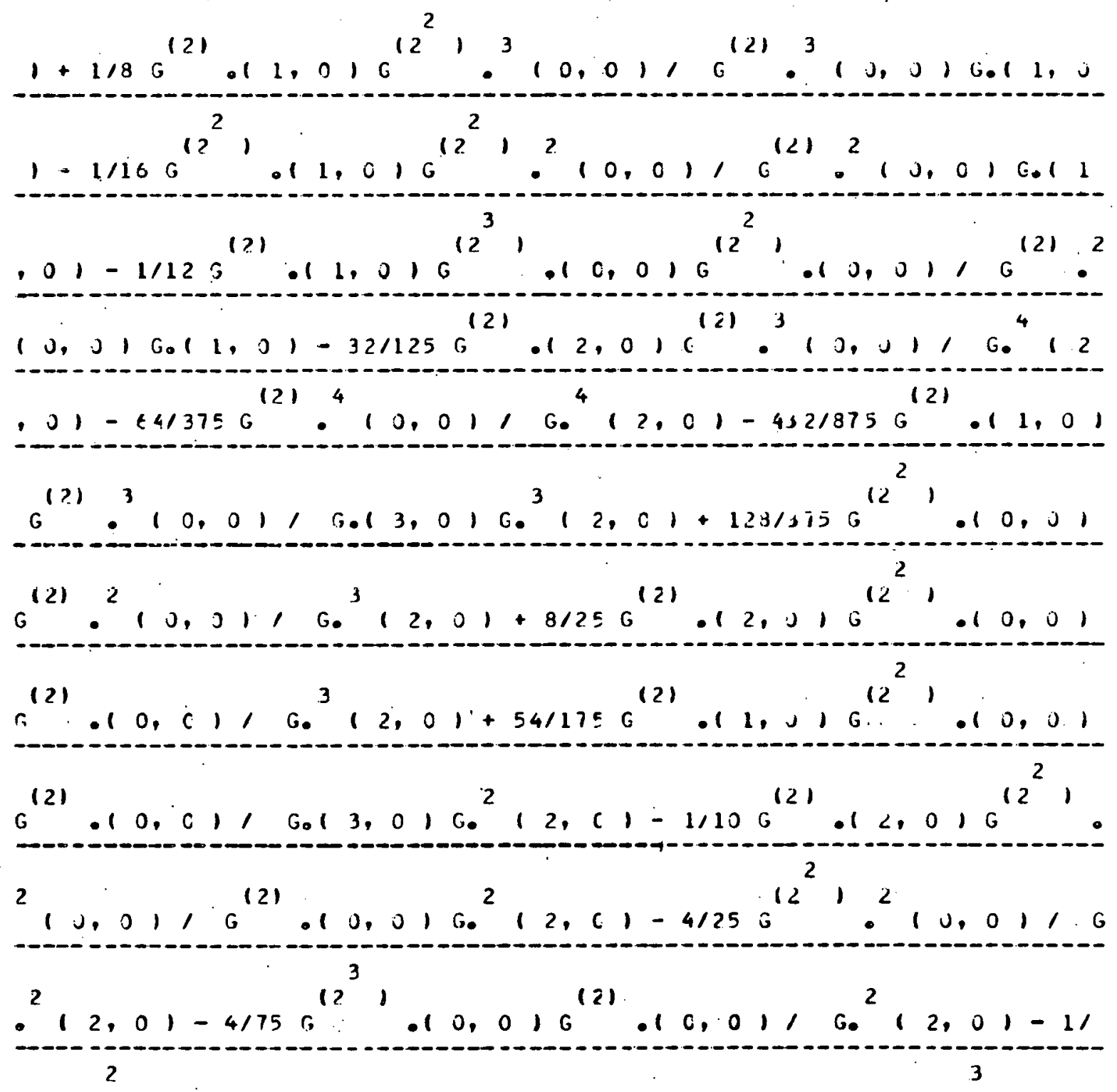




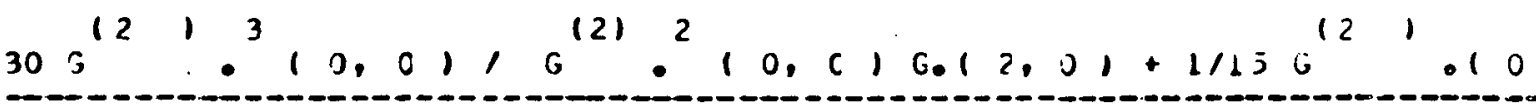

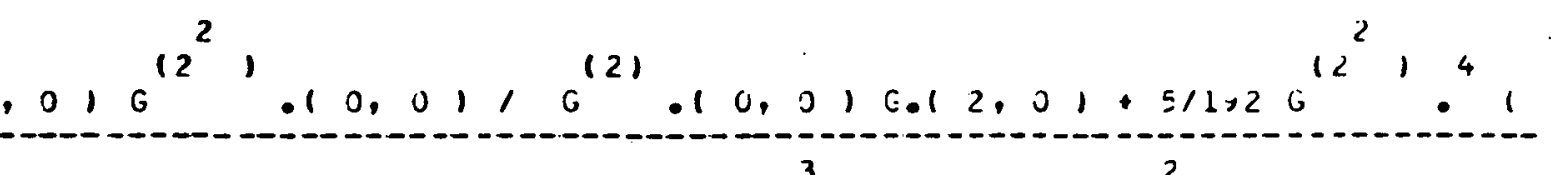

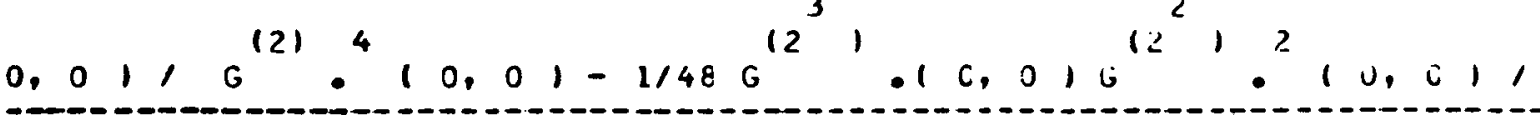

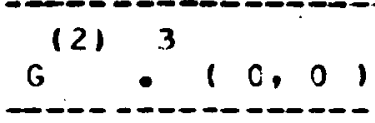

$c(4,4)=1 / 24 G^{(2)} 0^{4}, 1,01, G^{4} 11,01-2 / 156^{(2)} 0^{3} 11,016$

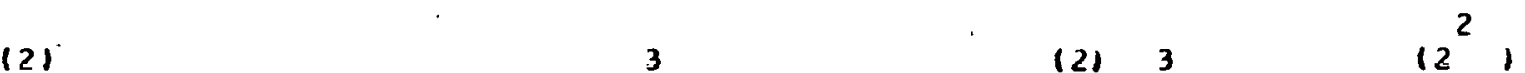

$(2) .10,01,0,12,0, G^{3}(1,0)+1 / 12 G^{(2)} 0^{3}, 1,0, G^{3}$

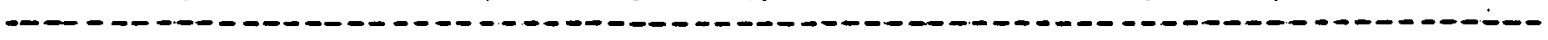

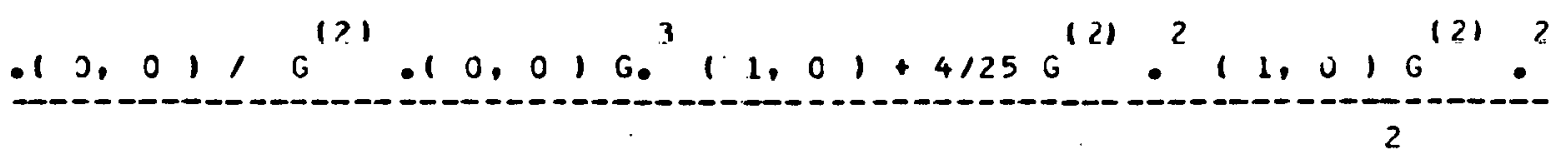
$\left(0,01, G_{0}^{2} 12,0, G_{0}^{2}, 1,01-1 / 5 c^{(2)} e^{2}, 1,01 G^{12}, 10\right.$ (

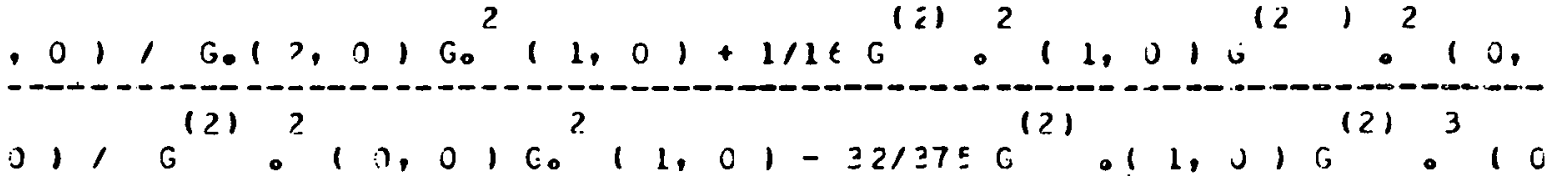
(-)$01, G_{0}^{3} 12,1,6011,01+4 / 2500^{121} 011,510^{12} 1.10,01$ (1)

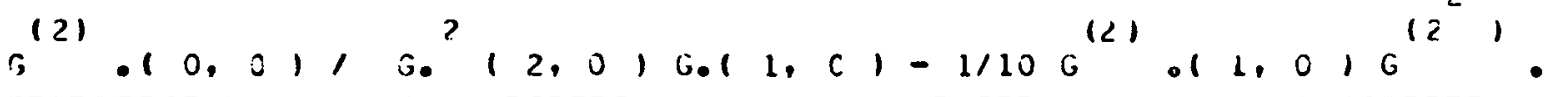

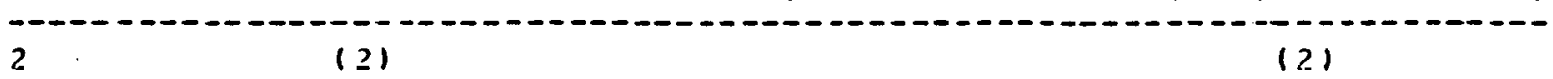

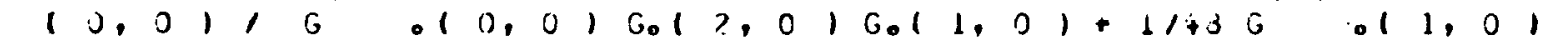
1
5

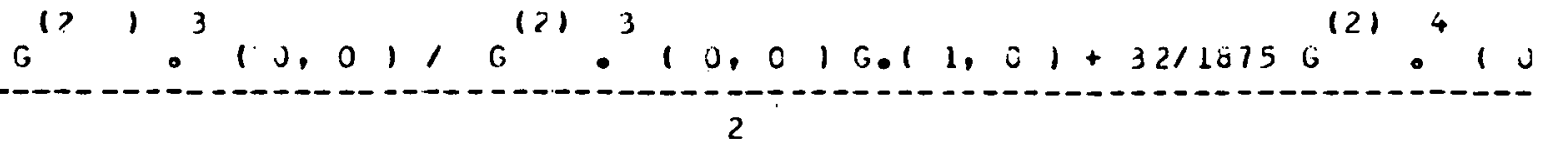

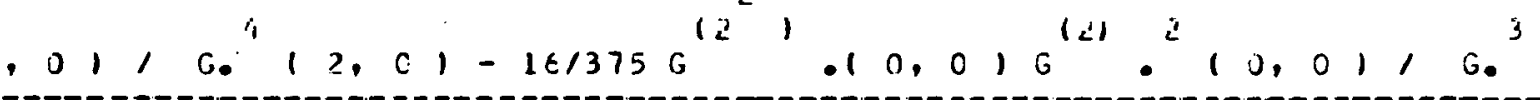

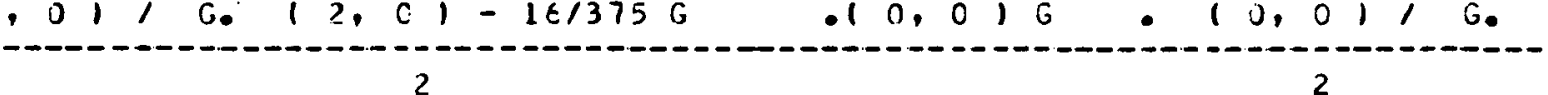
$(2,0)+1 / 250^{12} 0^{2} 10,01, c^{2}\left(2,01-1 / 600^{12} 0^{3} 10\right.$

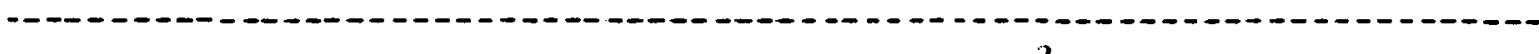
$\frac{01 ; G^{(2)}{ }^{2}(0,0, G, 12, c)+1 / 384 G^{(2)}+10,01, G^{(2)}}{410,01}$ 
$C(5,0)=864 / 175 \mathrm{G}^{(2)} \bullet^{3}\left(2,0, \mathrm{G}^{(2)} \bullet^{2}(\mathrm{C}, 0) \mathrm{G}, 1, \mathrm{~J}, 1, \mathrm{G}, 1\right.$,

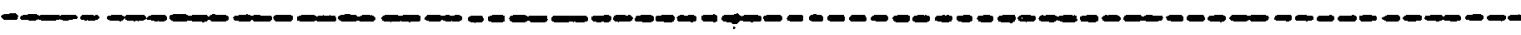
5 (2) 2 (2) 3

$016.12,0,+15552 / 875 \mathrm{G}, 12,0, \mathrm{G}, 1,0,0, \mathrm{G}, 1,1, J$ -

$(2)$ (2) 4

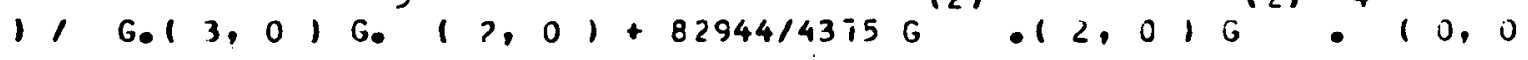
5

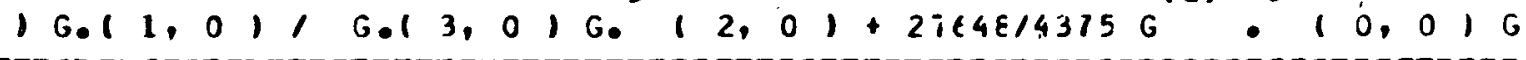

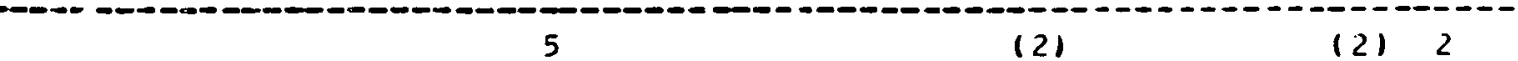

$.1,0,1, G .13,0) G,(2,0)+648 / 1 i 5 C$ $\bullet 13,0) \mathrm{G}$

$\begin{array}{ll}(2) & 2 \\ 2 & 0\end{array}$

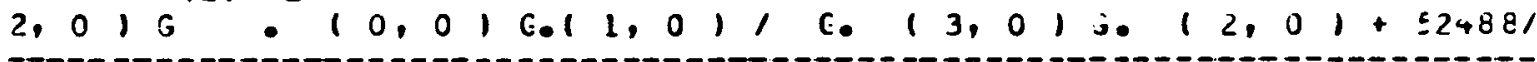
$(2) \quad(2) \quad(2) \equiv$

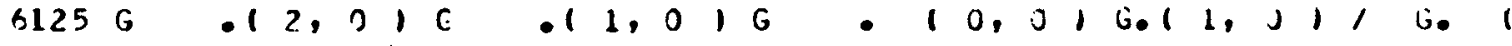

- - - -
$3,0, G e^{4}(2,0)+7776 / 875 \mathrm{G}^{(2)} \cdot 13, \mathrm{C}, \mathrm{e}^{(2)} \cdot\left(2, v 1 \mathrm{G}^{(2)} 0^{3} 10^{(2)}\right.$

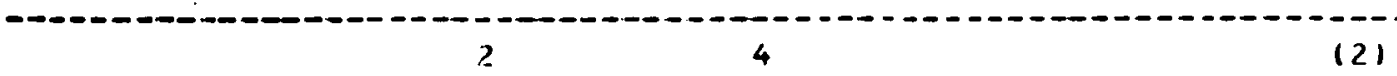

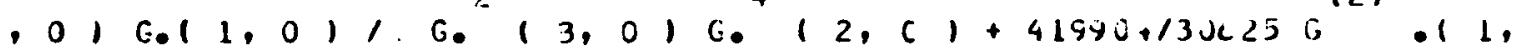

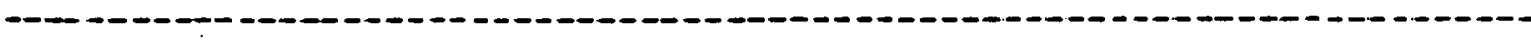
$0, G^{(2)} G^{4}, 0,0, G .(1,0), G_{0}^{2}\left(3,0, G_{0}^{4}(2, J)+207361\right.$

-

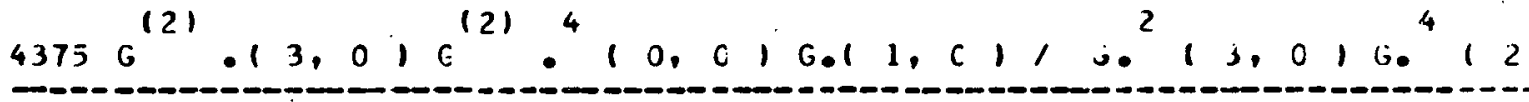

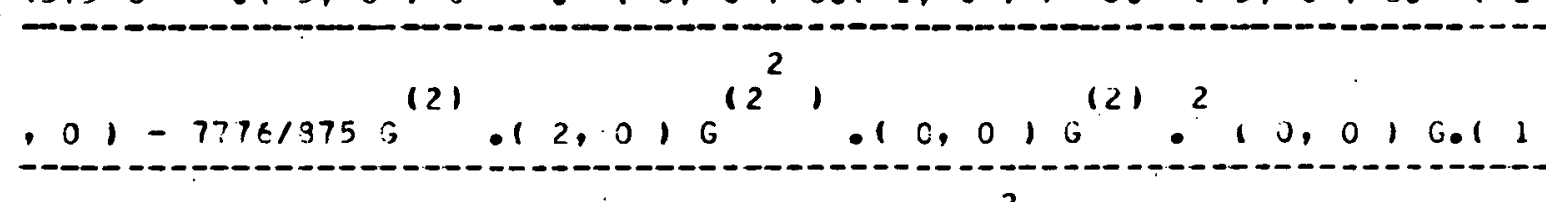

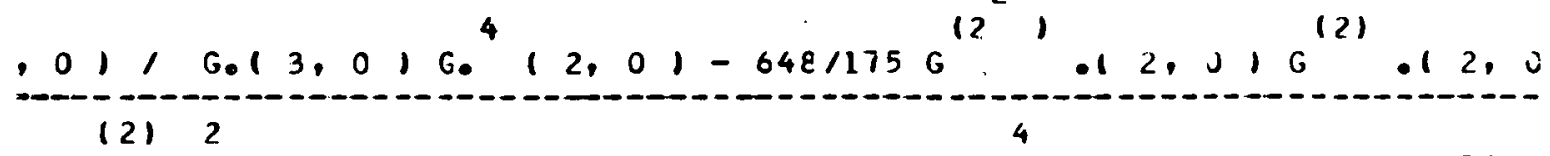

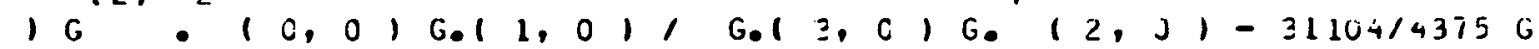
2

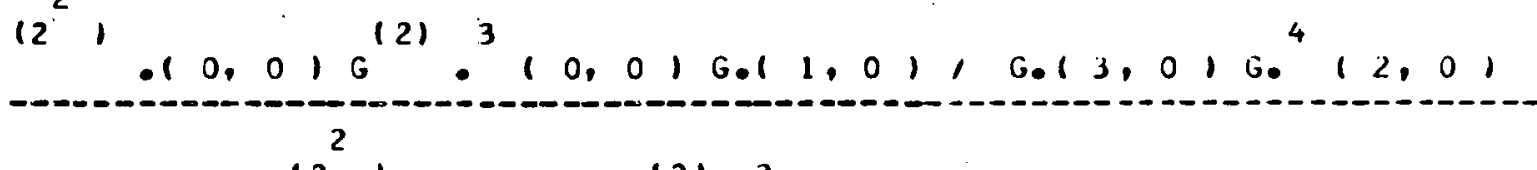
$-38881875 \mathrm{G}^{12}, .12,0, \mathrm{G}^{121} \bullet^{3}, 0,0, \mathrm{G}, 1,1,0,1, \mathrm{G}, 13,01 \mathrm{G}$

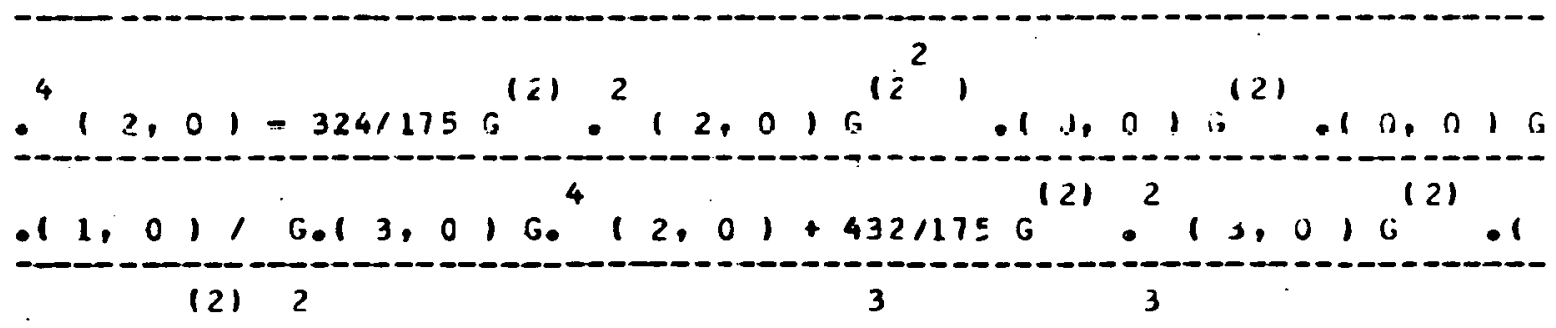


$2,010 \quad 010,01$ G.1 $1,0,1 /$ G. $12,0,0012,01+25921$ $275 G^{(2)} \cdot\left(3,0, G^{(2)} \cdot 3\left(0,0, G_{0} 1,0,1, G^{3} 13,0, G^{3} 12\right.\right.$

0
. $01+3499216125 \mathrm{G}^{12 J}$ $(2) .13,0, \mathrm{G}^{(2)} \cdot 11,01 \mathrm{G}^{(2)} \mathrm{G}^{3} \mathrm{1}, 0, \mathrm{G}, 1 \cdot 1$

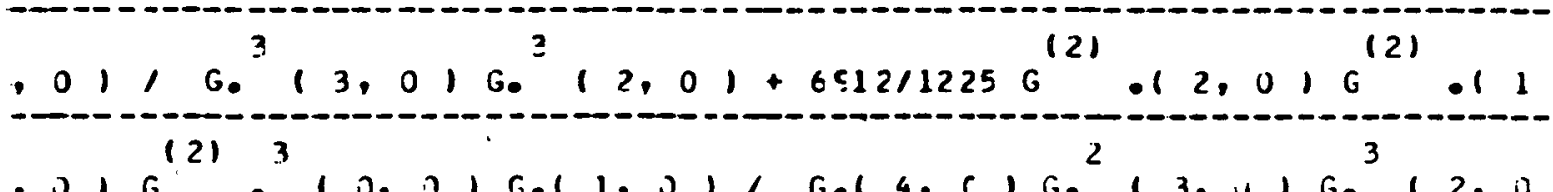

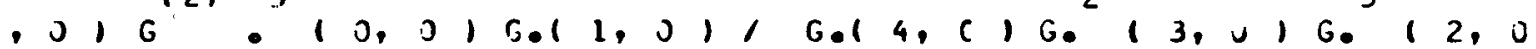
(2) $1+55256 / t 125 \mathrm{G}$ $.11,0, G, 10,0, G .11,0,1, G .14,01$ -

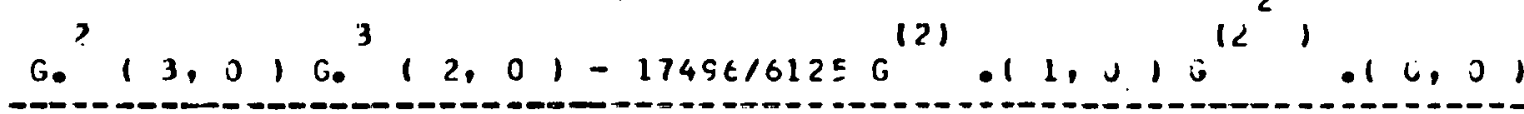

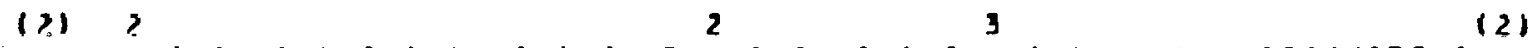
$G \quad(J, J) G, 11,0,1$ G. $(3,0)$ G $12,01,259218756$ G-2

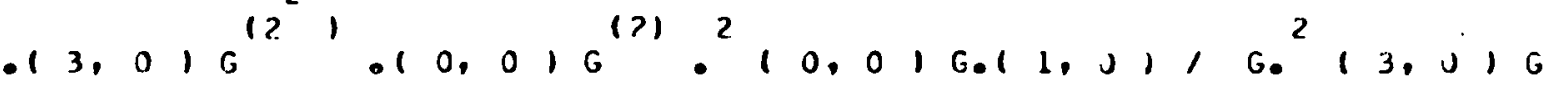
(1)

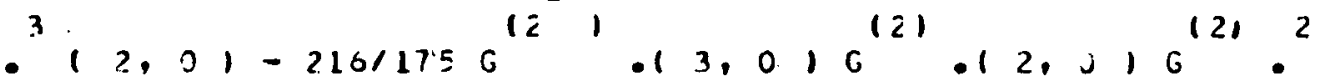

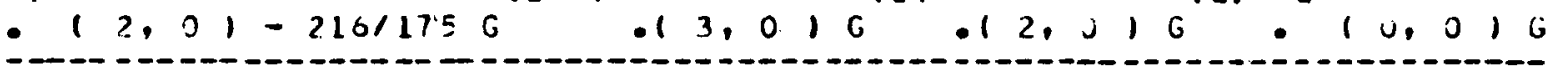
$.11,01,0^{2}, 3,0, G^{3}\left(2,01-216 / 175 G^{(2)}, 13,0, G^{12} 1\right.$,

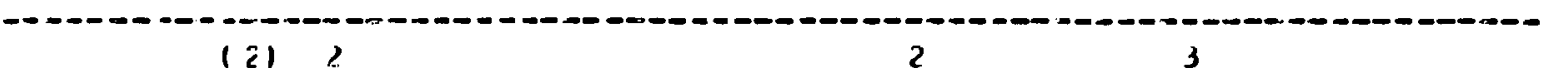

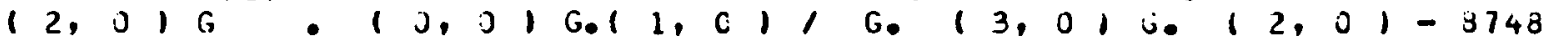
${ }_{2}$

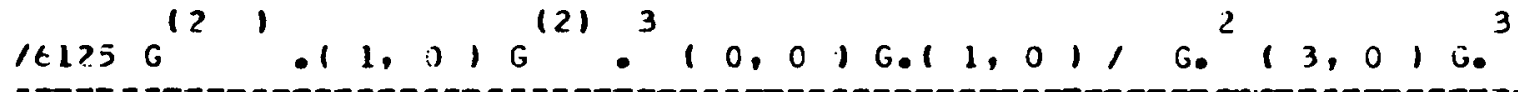
2
2

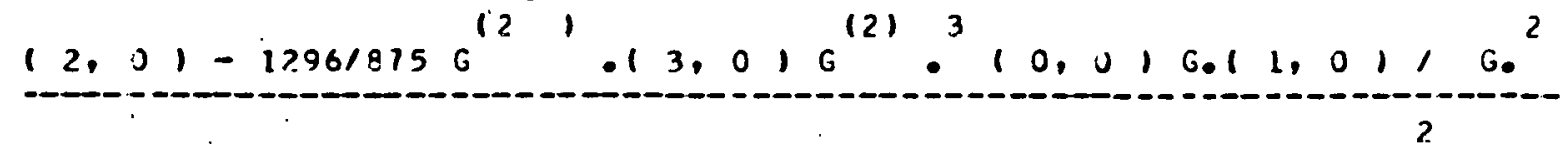

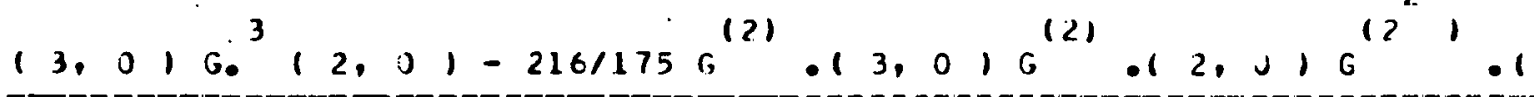

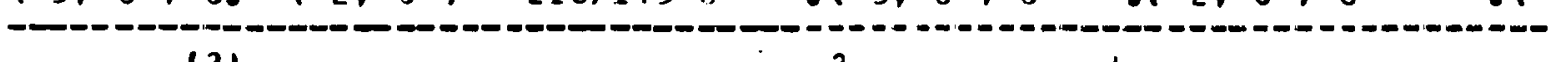
$0,0, \mathrm{G}^{0}, 0,0, \mathrm{C}_{0}\left(1,0,1, \mathrm{G}^{2}, 3,0, \mathrm{G}_{0}, 2,0,1+864 / 375\right.$

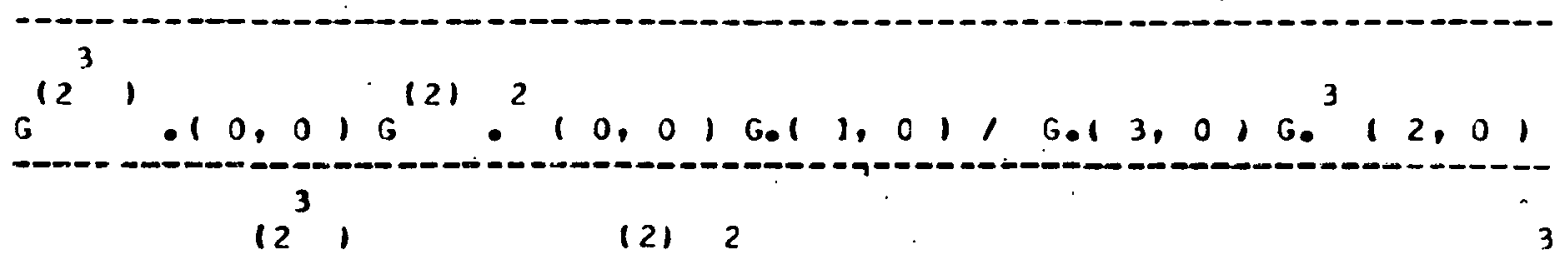




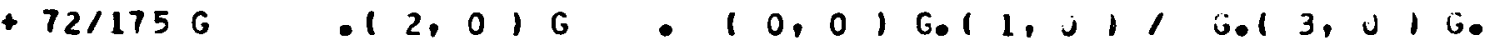
$12,01+648 / 875 G^{12} 12,0,0,0^{121}$ 30

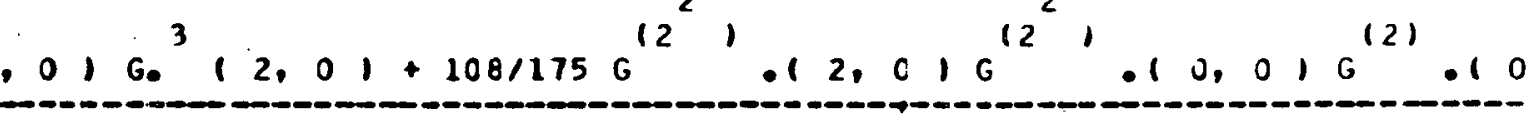
3

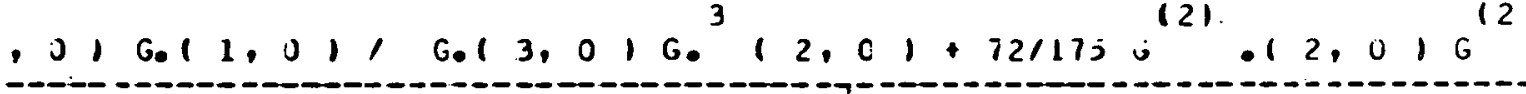

1
$.10,0, G^{0},(0,0) G .11,0,1, G .13,0, G_{0}^{3}(2,0)+2161$

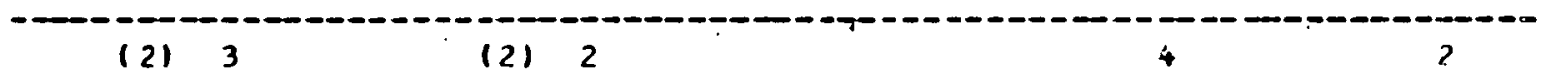

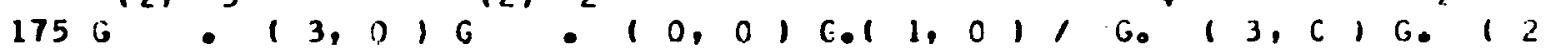
$175 \mathrm{G}$ $\left..01+691211225 G^{(2)} .(3,0) G^{(2)} \cdot 11, C\right) G^{(2)} G^{3}(0,0), G .11$, - 0 $01, G .14,0, G 0^{3}, 3,01 G^{2}, 2,01-216 / 115 \epsilon^{12}, .13,016$ 0 121 $(2){ }^{2}, 0,0, G_{0}\left(1, C, 1, G_{0}^{3}, 3, C, G_{0}^{2}, 2,01\right.$

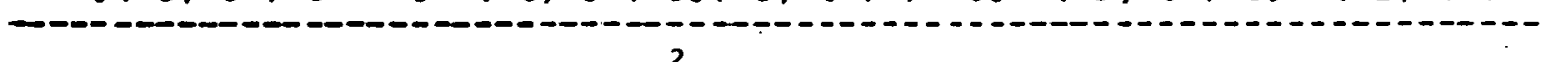

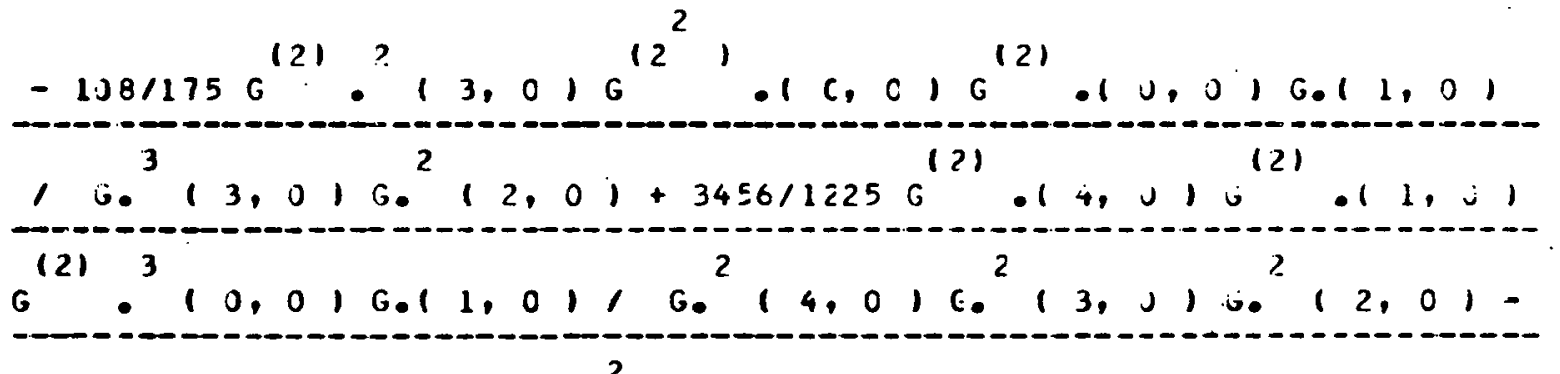
$3456 / 1225 \mathrm{G}^{(2)} \cdot 11,01 \mathrm{G}^{(2)}, 10, \mathrm{C}, \mathrm{G}^{(2)} \mathrm{C}^{2}, 0,0, \mathrm{G}, 11,01$, (1) $G_{0} 14,0, G_{0}^{2}\left(3,0, G_{0}^{2}, 2,01-1728 / 12250^{12} 1,11,01 G^{121}\right.$ ${ }^{3}\left(0,0, G_{0}\left(1,0,1, G_{0}\left(4, C, G_{0}^{2}, 3, c i b_{0}^{2}, 2,01+36 / 175\right.\right.\right.$ 13

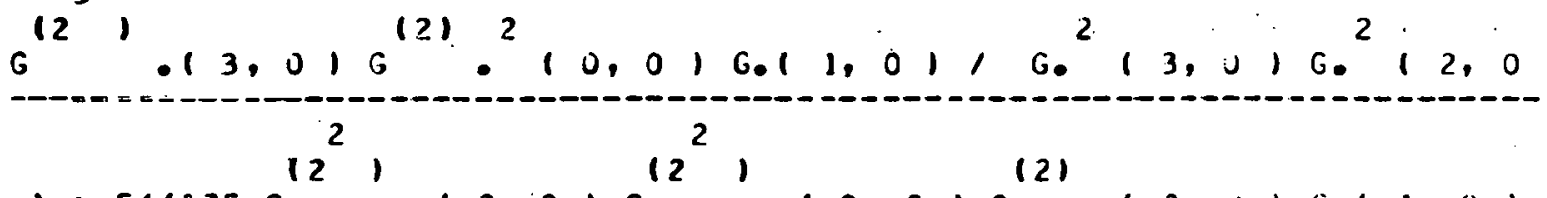

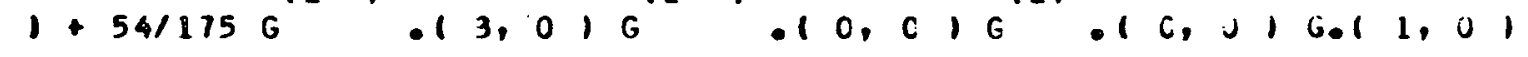
- 
$10^{2}\left(3,01 G_{0}^{2}, 2,01+36 / 175 G^{121}, 13,016^{12}\right) .10,016$

(2)

$.10,0, G .11,0,1 G^{2}, 3,0, G_{0}^{2}, 2,0,-9 / 175 G^{4} 2^{4}$

$G^{(2)} \cdot\left(0,0, G .11,0,1, G .13,0, G_{0}^{2}, 2,0,-\$ 248816125\right.$

0.016

$(2) 2$ ( 21 ?

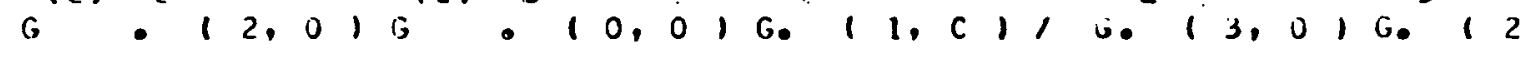

$(2) \quad(2) 440$

. $01-8378 \mathrm{C} 8 / 30625 \mathrm{G}$ $.12,0, G+10,01 G,(1, C), G$

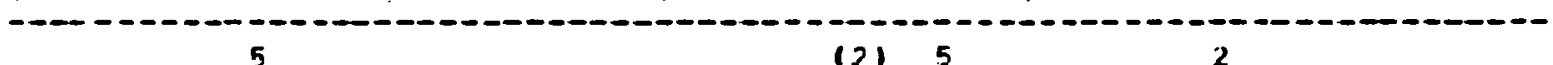

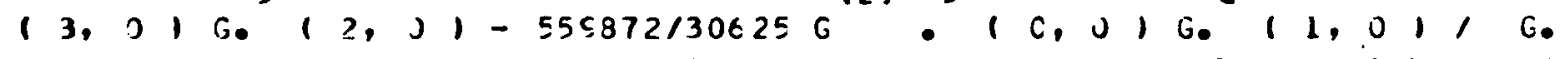
? $550121 \quad(2) \quad(2)$ (. $3,0, G_{0}(2,0,-524 \varepsilon \varepsilon / 6125 \mathrm{G} .1 \pm .01 \mathrm{G}, 12,01 \mathrm{G}$

3 ( 320

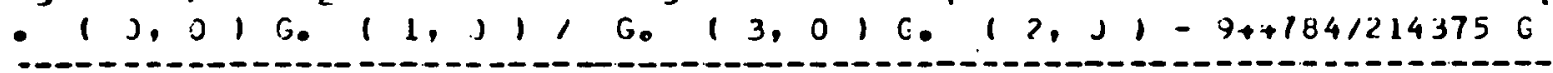
$21 .\left(1,0, G^{121} \mathrm{G}^{4}\left(0,0, G^{2}, 1,0,1, G_{0}^{3}\left(3,0, G_{0} 12,01\right.\right.\right.$

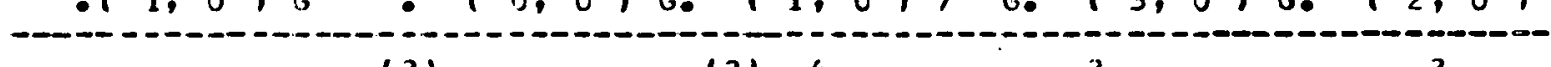

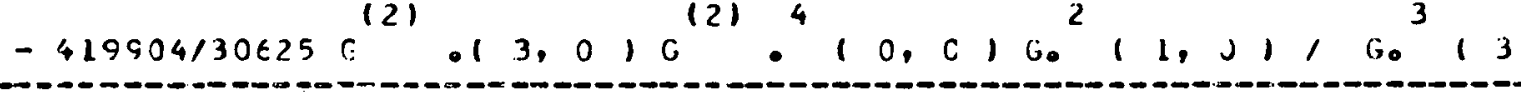

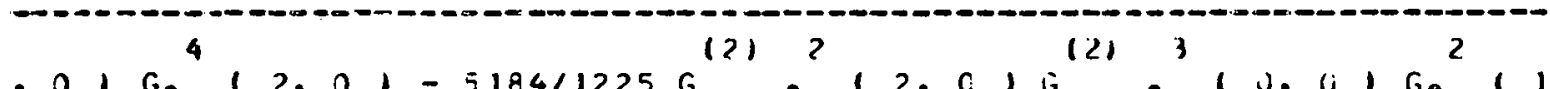

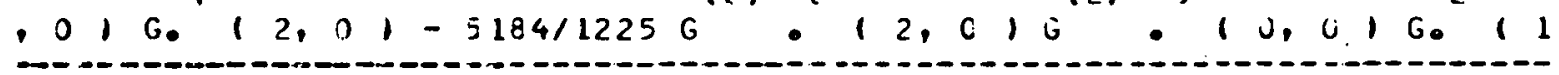

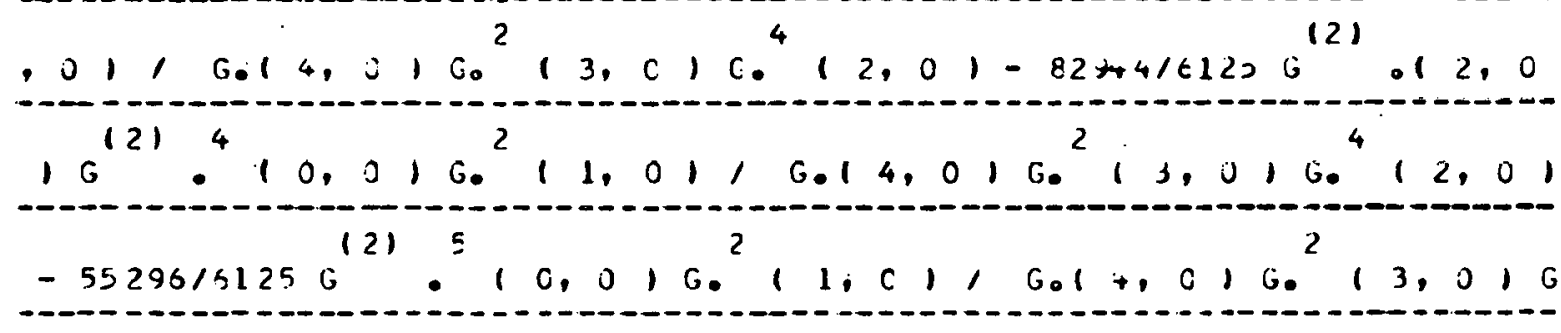

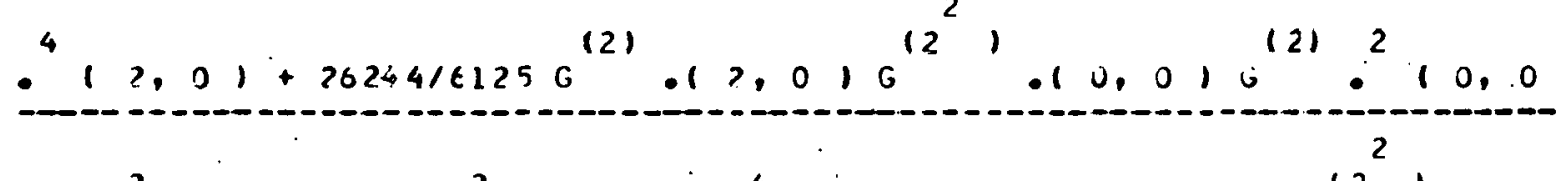

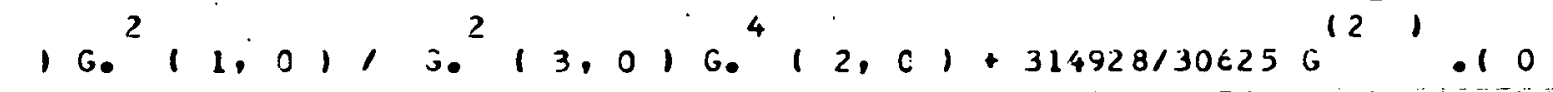
1 , $01 G^{121} \cdot(0,0) G^{2}(1, C) / G_{0}^{2}\left(3,0, G^{4}(2,01+13122\right.$ \begin{tabular}{cc}
2 \\
\hline
\end{tabular}

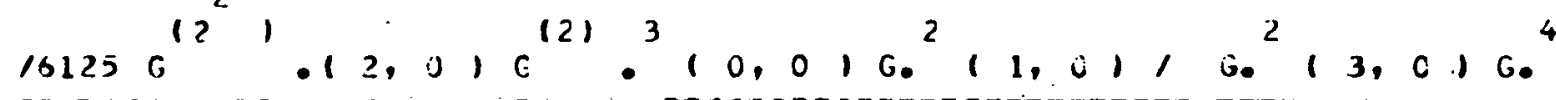

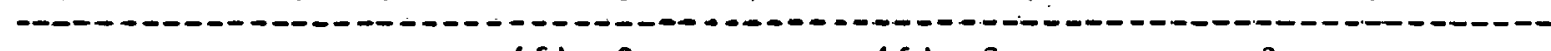

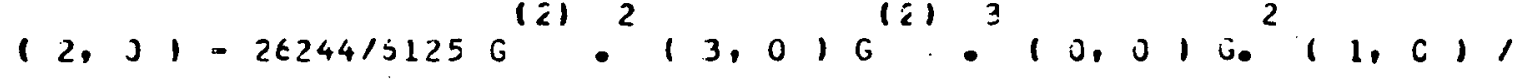




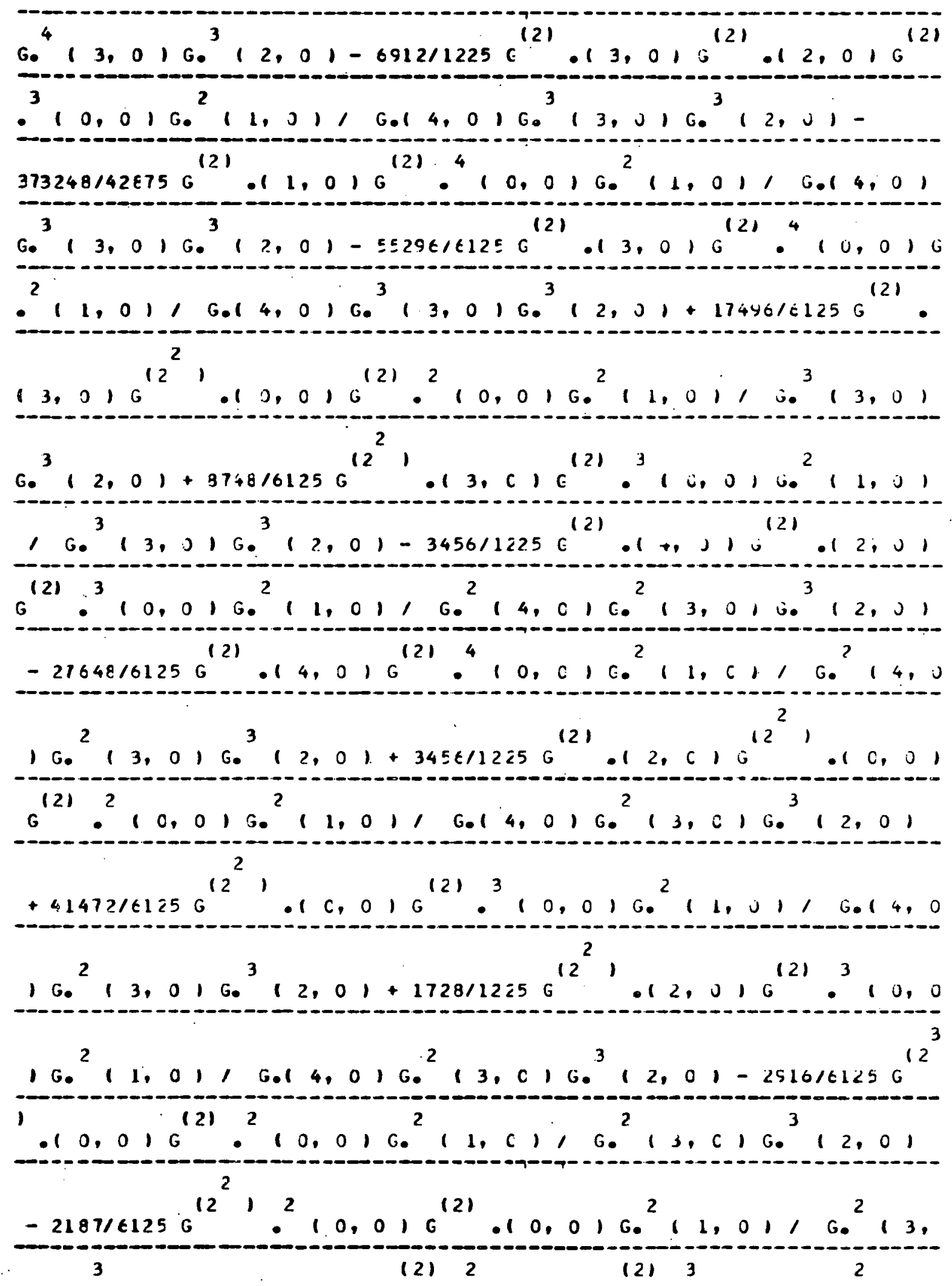


$01 \mathrm{G} .12,31.5184 / 1225 \mathrm{G} \cdot 13,01 \mathrm{C} .1 \mathrm{~J}, 016011$, 42 (2)

$01 /$ Gol 4, 01 G. 13,0, G. $12,0,1-3456 / 1225 \mathrm{G} \quad .14,016$ (2) $\quad \begin{array}{lllll}2 & 3 & 2 & 2 & 3\end{array}$

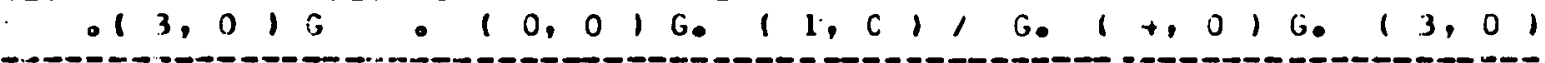
(?) G. $(2,0)-35854 / 85 i 5 G \quad 0(1, C) C$ ( $)$

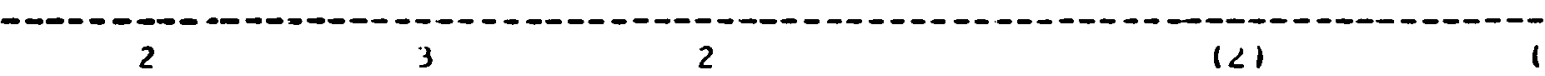

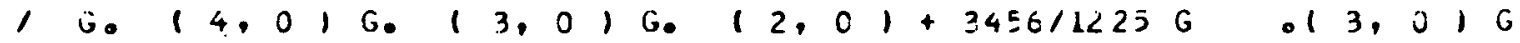
2

$2^{2}, 10,01 G^{(2)} 0^{2}, 0,0,0^{2}, 1,01,6.14,0,60^{3}, 3,01$
$G^{2}(2,0)+1739 / 1225 G^{(2)},(3, C) G^{(i)} G^{2}\left(j, j, G^{2}, 1, J 1\right.$

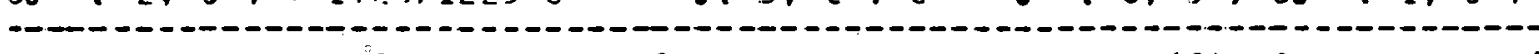
$, \quad 0014,0, G_{0}^{3}(3, C) G_{0}^{2}(2,0)-1723 / 12 \pi j j^{(2)} 0^{2}(4,0) G$

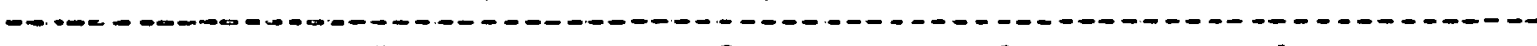
23 ? 3 ? - $\left(0,0, G_{0}(1,0), G_{0}(4,0) c_{0}(3, J) 0_{0}(2,0)-\right.$

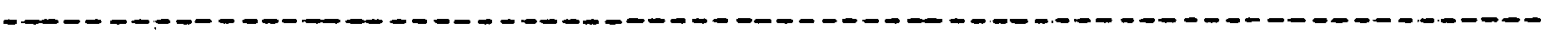

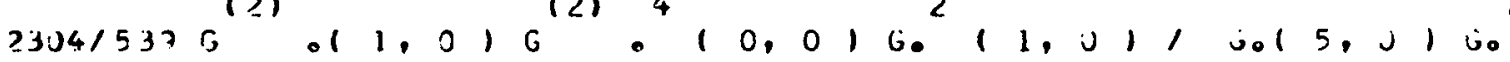
- - - - - - - -

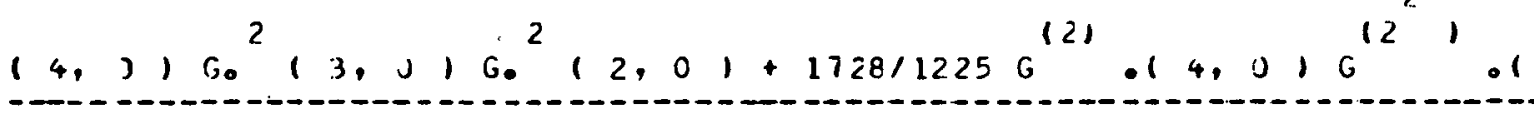
121 ?

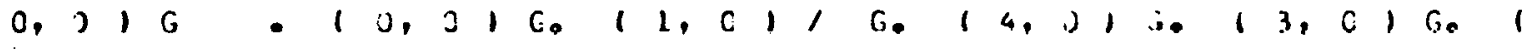
0.--

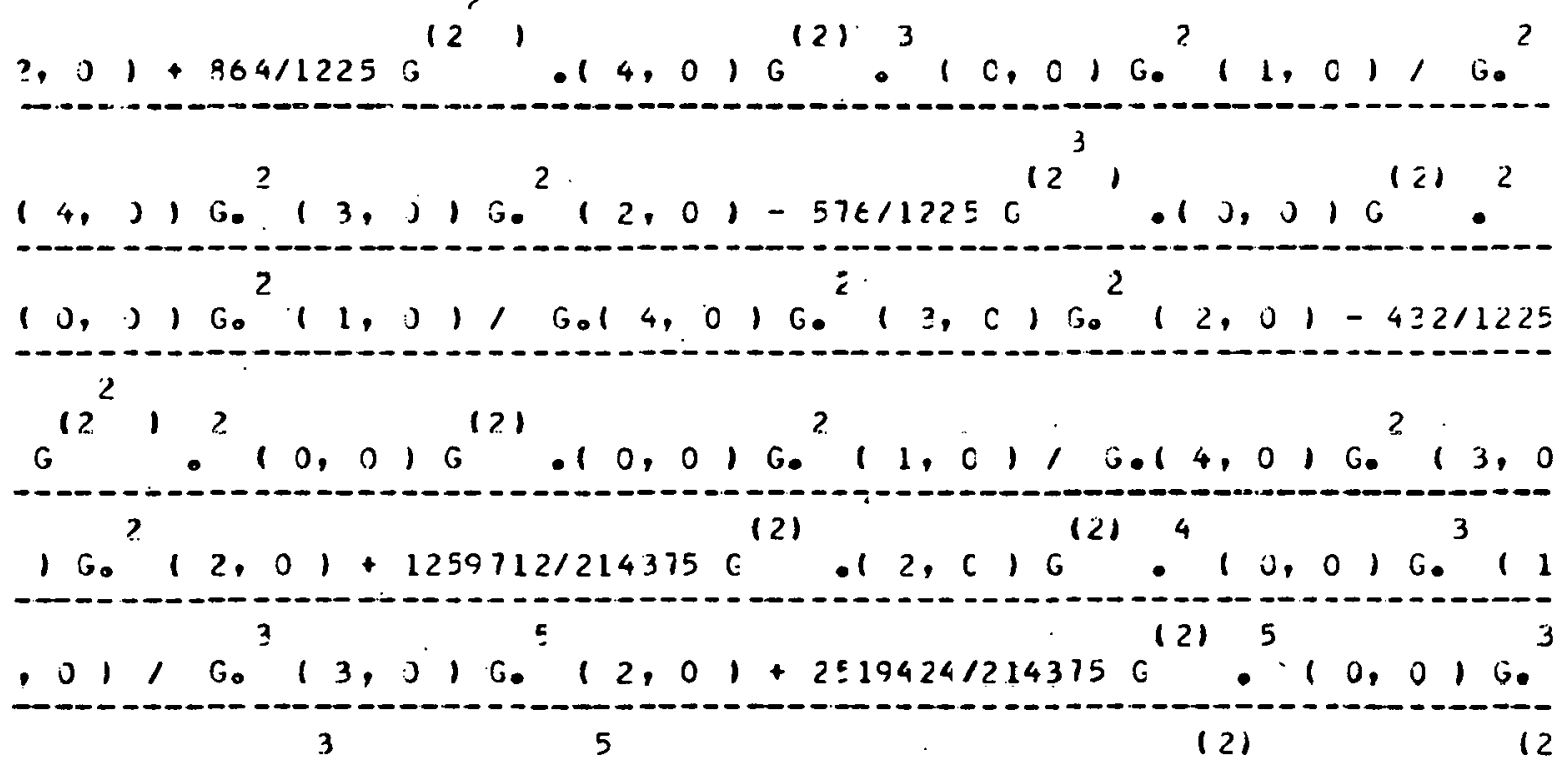




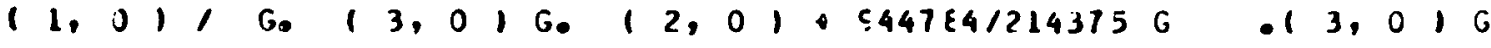

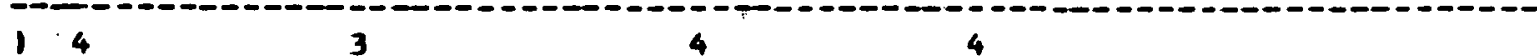

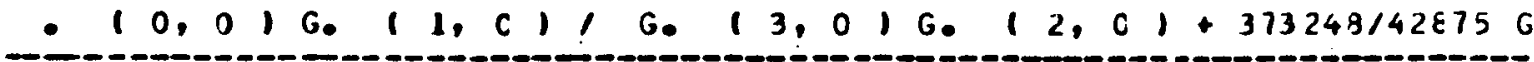
(2) 121443 $.12,0, G, \circ(0,0, G, 1,1, C, 1, G, 1+C, G, 13, J 1 G$

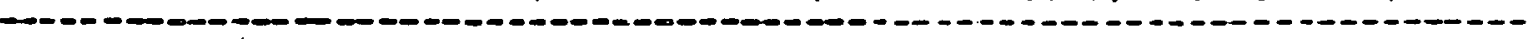
4 (2) 5 3

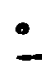

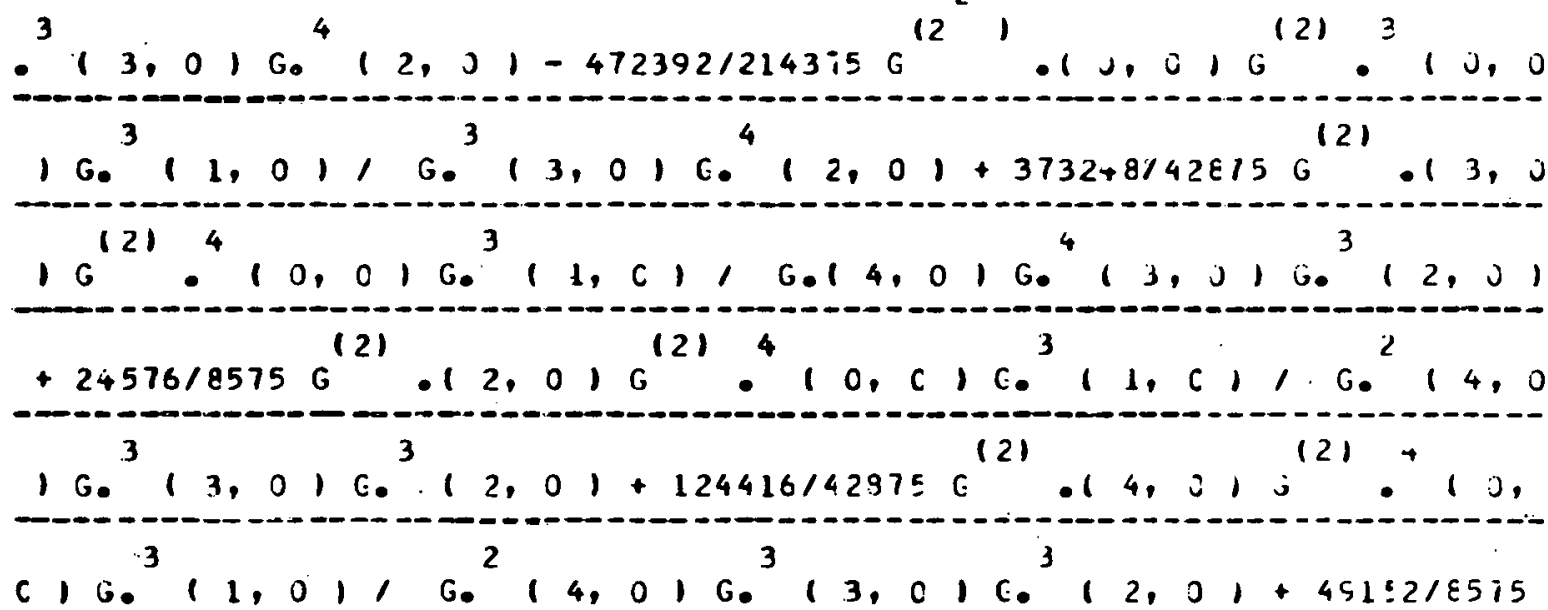

С 1 G.
$G^{121}{ }^{5}\left(0,0, G^{3}, 1,0,1, G^{2}\left(4,0, G_{0}^{3}(3), 0012,01\right.\right.$, G--

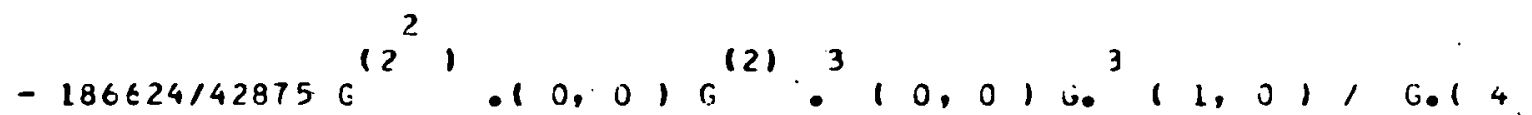

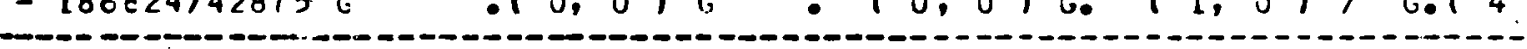
3 (2) $(2) 4,0,0$ 101 G $(3,0, G, 12,0,+15361539 \mathrm{G}, 12,0100$

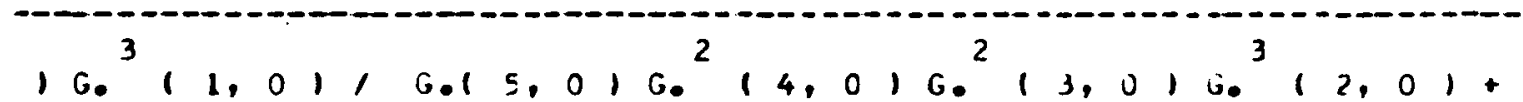

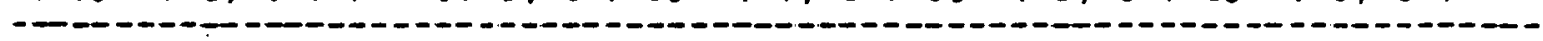
$3072 / 539 G^{121} G^{5}\left(0,0, G^{3}, 1,0,1, G .15,0,6.14,016.1\right.$ $30727539 \mathrm{G}$ 3 (2)

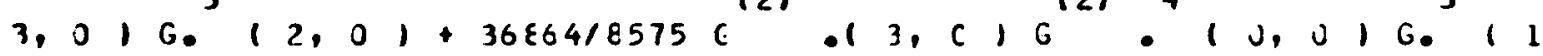
(240121

$.01 /$ G. 14,0, G. $(3,0, G, 12, C)+24576 / 8575 G$, 14.

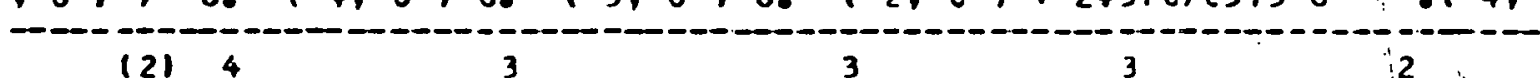

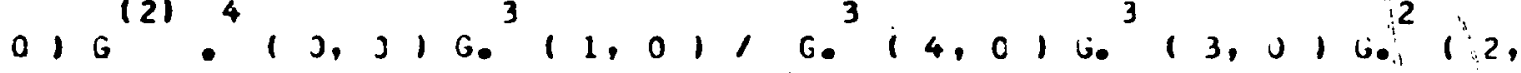
$01+1536 / 539 \mathrm{G}^{(2)} \cdot 13,01 \mathrm{G}^{(2)} \cdot(0,0) \mathrm{G}^{3}(1,0,1, \mathrm{G}, 15,0$

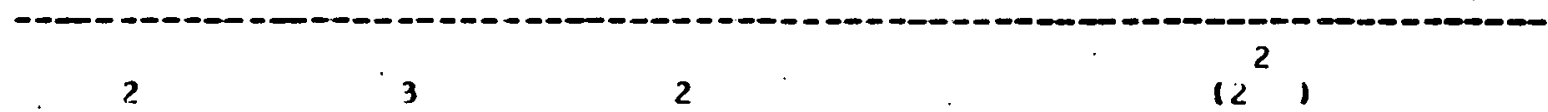


I G. 14,01 G. 13,0, G. $12, C,-1843218573 G \quad 010,01$ 0 (2) $323 \quad 2 \quad 3 \quad 2$

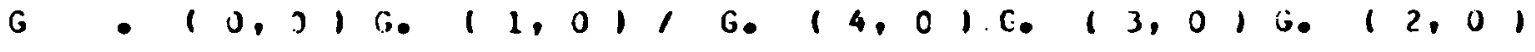

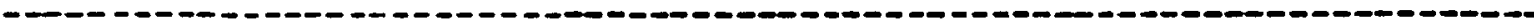
$+15361539 \mathrm{G}^{121} \circ(4,0) \mathrm{G}^{121} \mathrm{G}^{4} \mathrm{l} 0,0, \mathrm{C}^{3}(1,01, \mathrm{G}, 15,01 \mathrm{G}$ $3 \quad 2 \quad 2 \quad 0 \quad(2) \quad 1214$

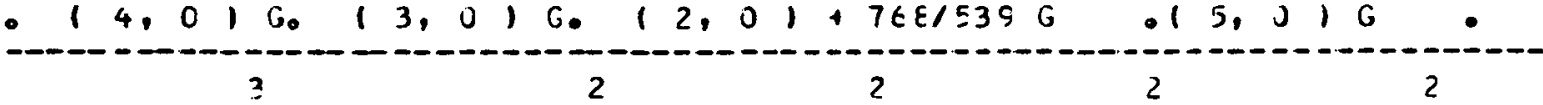
$(0),, G_{0}\left(1,0,1, G_{0}(5,0) C_{0}(4,0,1,0,3,016012\right.$ (--- -

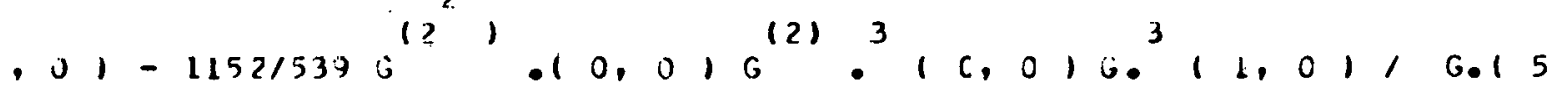
- - =-

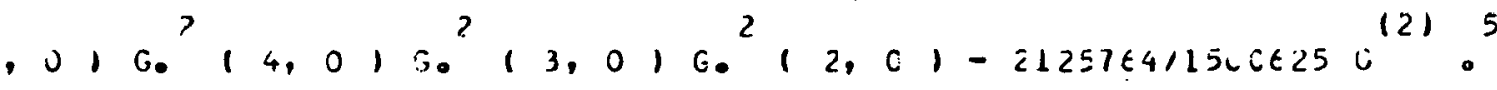
$10,2, G^{4}\left(1, j, 1, G^{4}, 3,0, \epsilon^{5}\left(2,01-12537121360125 G^{121}\right.\right.$ (-- - $5,1,0, G_{0}^{4}\left(1,0,1, G_{0}\left(4,0, G_{0}^{4}, 3,0, E^{4}, 2,01,-\right.\right.$

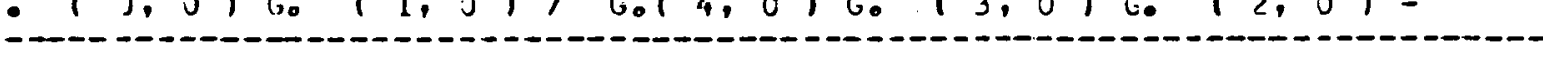

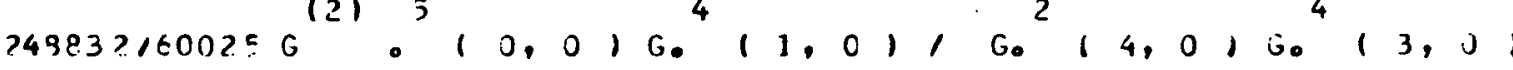

--- - -
$3(2) 504$

Go $12,01-13363 / 3773 \mathrm{G} \bullet 10, \mathrm{C}, \mathrm{G}, 1,1,01,0_{0}(5, i 16$. $21+, j, C_{0}^{3}\left(3,0, G_{0}^{3}, 2,0,-16364 / 12 \cos j^{(2)}, 510,010,4\right.$ $1+, 0) C_{0}(3,0, G, 12,01-16364 / 12605 j \quad 010,0100$ $11,0,1 G^{3}, 4,0, G_{0}^{4}, 3,010^{2}, 2,01-10240 / 3 i 73 G^{5}$ $11,06014,0$ Go 4

$10,0, G_{0}, 1,0,1, G_{0}\left(5,0, G_{0}, 4, C, G_{0}, 3,0, c_{1} 12,0\right.$

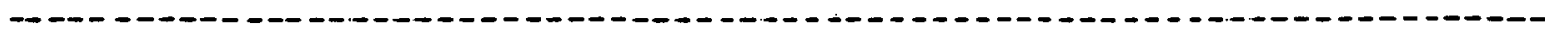
(2) 5403

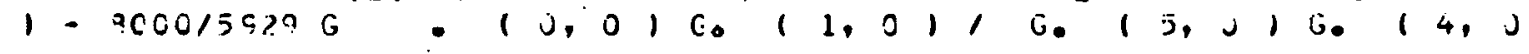

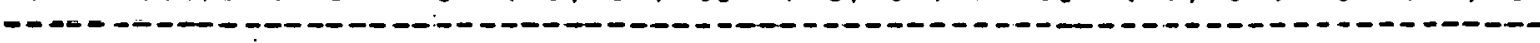
$2 \quad 2 \quad 1215$

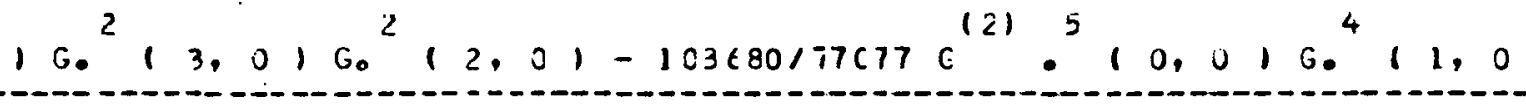
$1, G_{0}(j, 0) G^{2}\left(5,0, G_{0}^{2}\left(4,0, G_{0}^{2} 13,0, j_{0}^{2} 12,01-\right.\right.$

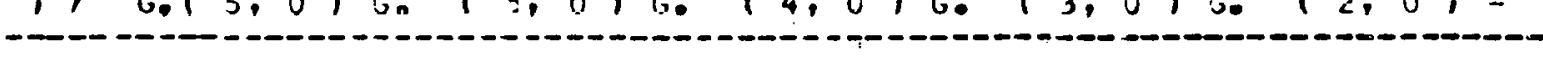

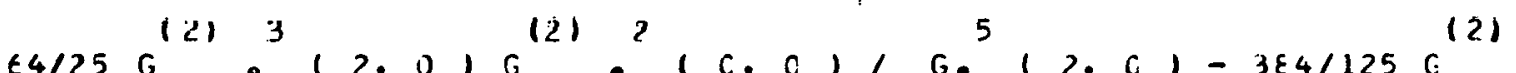
$64 / 25 \mathrm{G}: 12,0, \mathrm{G} \bullet \mathrm{C}, \mathrm{C}, \mathrm{G}, \mathrm{G} 2, \mathrm{C},-3 \varepsilon 4 / 125 \mathrm{G}$

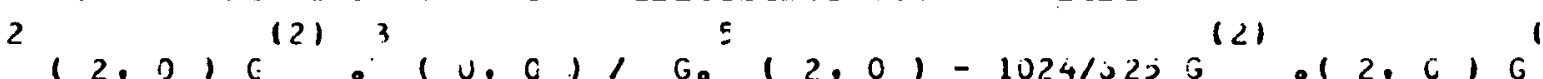
(1) $21+\left(0,0,1, G_{0}(2,0)-102413125 G^{12}\right)^{5}(0,011,6012$, 
$0,-415 \mathrm{G}^{(2)} \mathrm{G}^{4}\left(2,0, \mathrm{G}^{(2)} \cdot(0, \mathrm{C}), \mathrm{G}^{5}(2,01-64 \varepsilon / 1756(2)\right.$

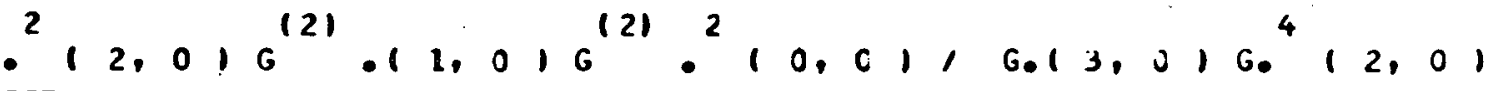

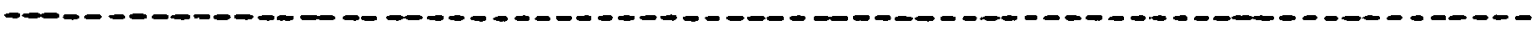
$-7776 / \varepsilon 75 \mathrm{G}^{(2)} \cdot\left(2,0, \mathrm{G}^{(2)} \cdot(1, \mathrm{C}) \mathrm{G}^{(2)} \cdot \mathrm{G}^{3}(0,0,1,6.13,01\right.$ G. $12,01-20736 / 4375 \mathrm{G}^{121} \cdot\left(1, \mathrm{C}, \mathrm{G}^{121} \cdot{ }^{4}(0,011,6.13,011\right.$ Go-

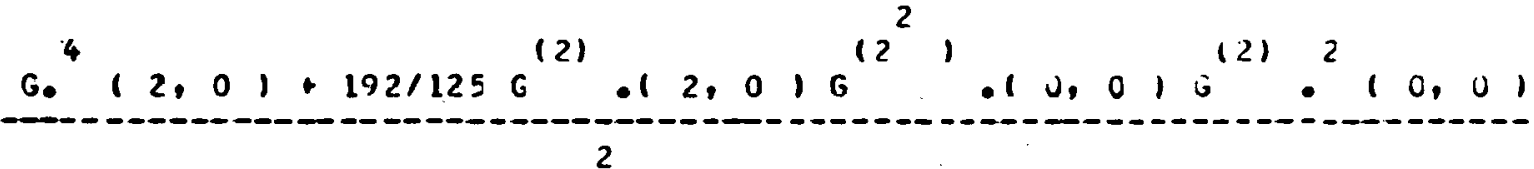

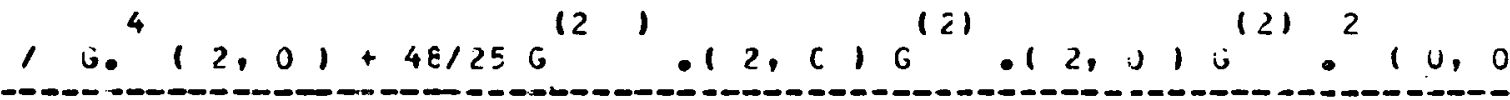

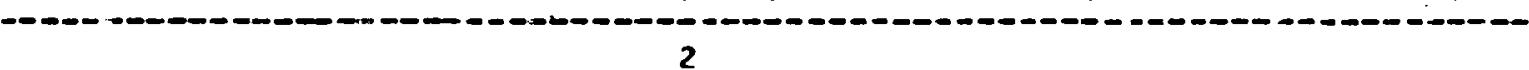
$1, G_{0}^{4} 12,01+384 / 625 \mathrm{G}^{12^{2}}, \cdot 10,0, \mathrm{e}^{121} \cdot 3,10,01,0.4$

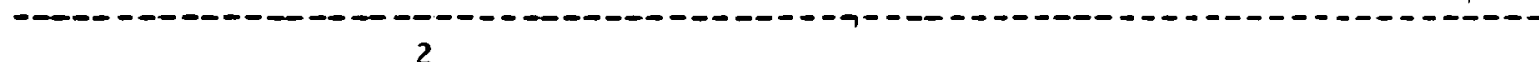

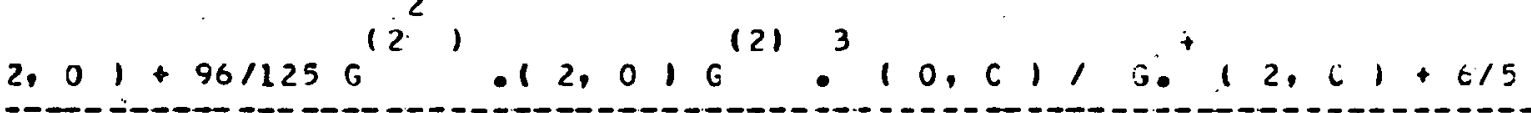
${ }^{2}$

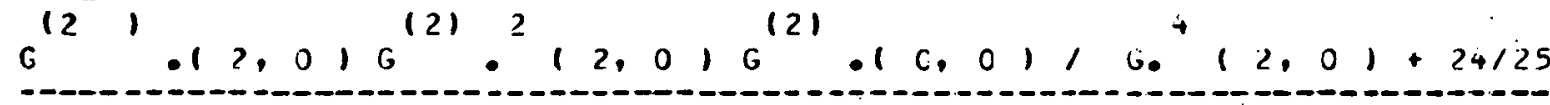
151
5

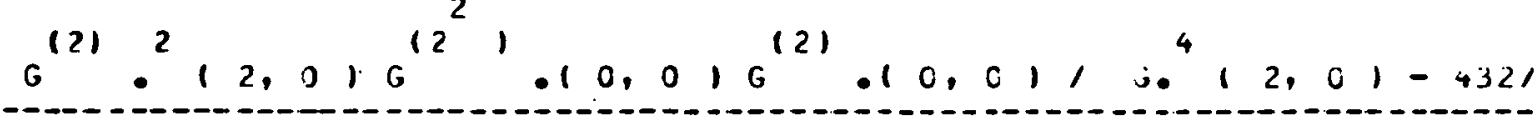
12). $(2)(2) \quad(2) 2$

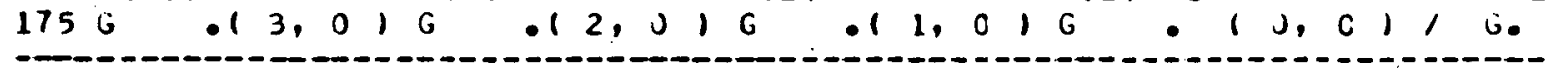

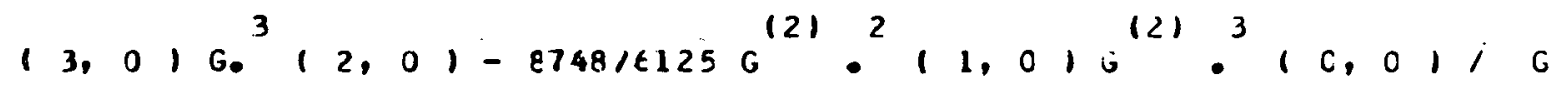

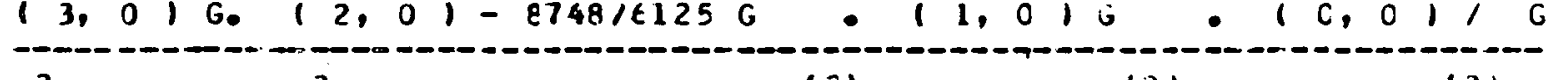

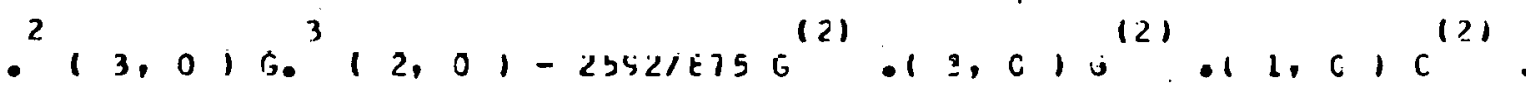

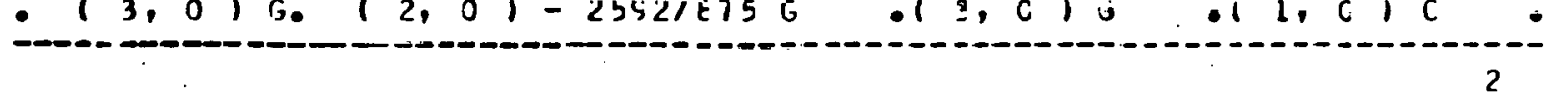
${ }^{3}\left(0,0,1, G^{2}\left(3,0, G_{0}^{3}(2,0)+2592 / 975 j^{(2)}, 11,01 c^{12} 1\right.\right.$ $(0,0)$, G. $(3,0)$ G. $(2,0)+2592 / 875 \mathrm{i}, 011,010 \mathrm{C}$

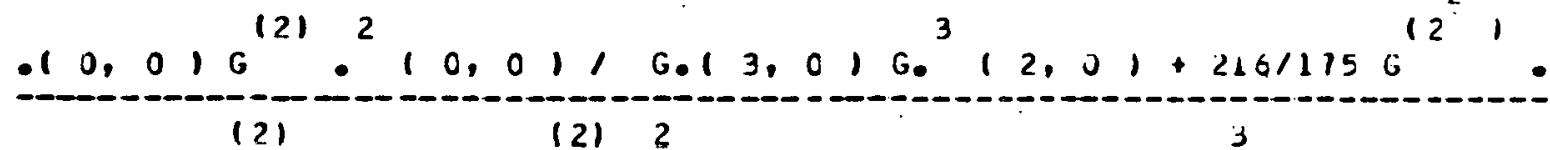
(2, $01 \mathrm{G} .11,0, \mathrm{G} \quad \bullet \quad(0, \mathrm{C}, 1, \mathrm{G}, 13, \mathrm{~J}, \mathrm{G}, 12,01$, $-$ $216 / 175 \mathrm{G}^{(2)} \cdot(2,0) \mathrm{G}^{(2)}, 11,0, \mathrm{G}^{(2)} \mathrm{G}^{2}(0, J 1 / \mathrm{G}, 13,01 \mathrm{G}$ (196/1 


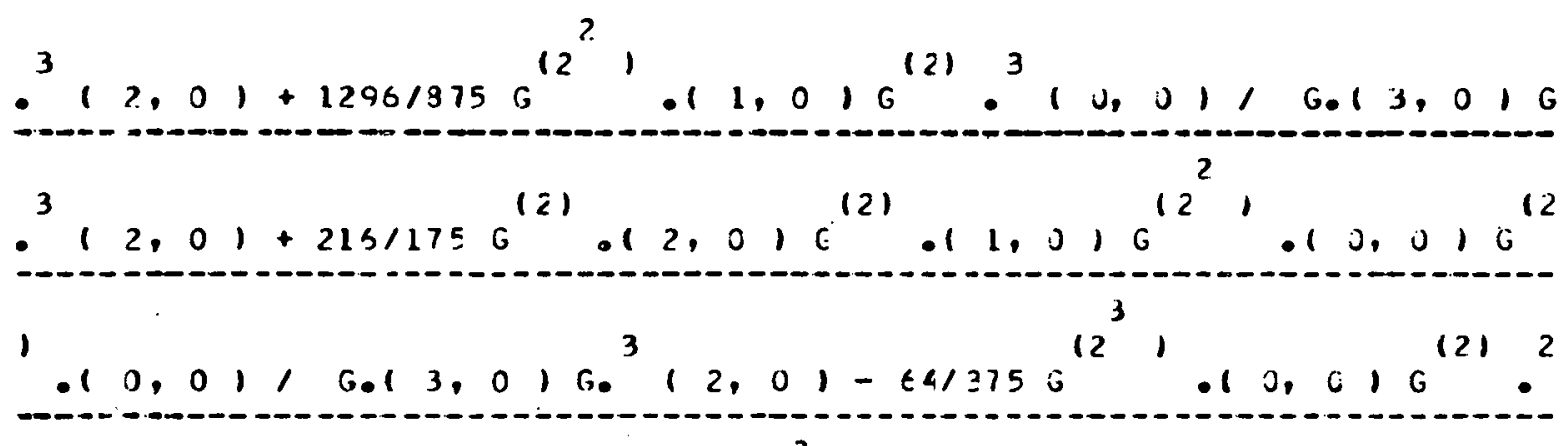

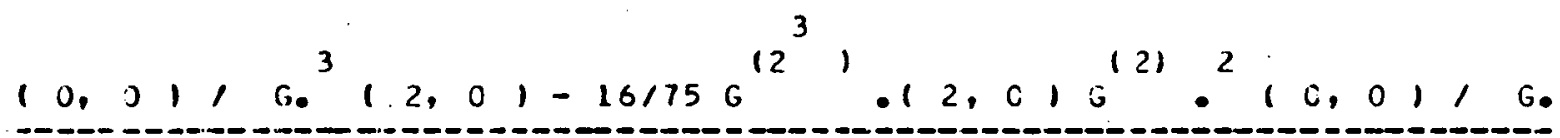
2
1

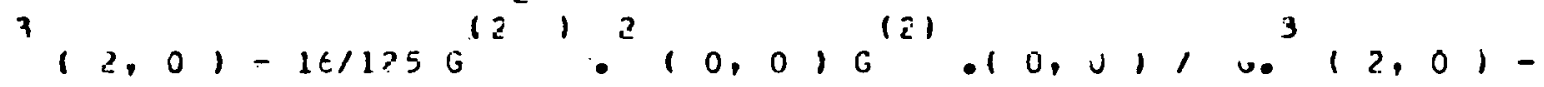

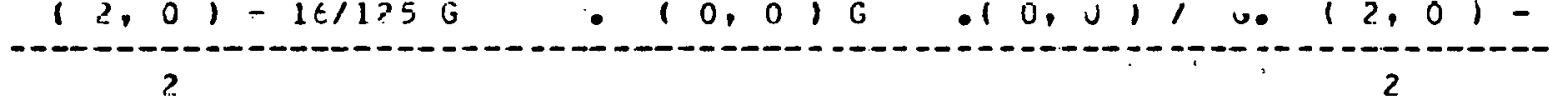

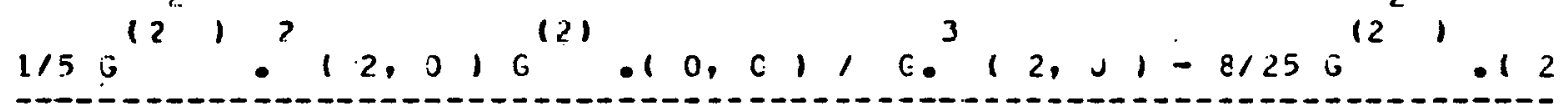

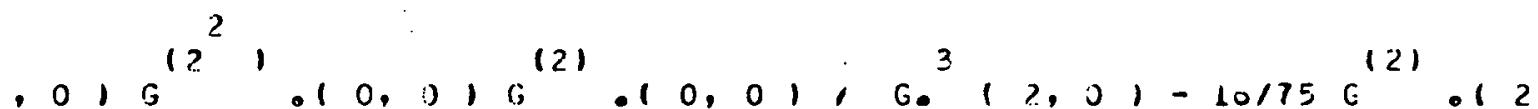
10 (

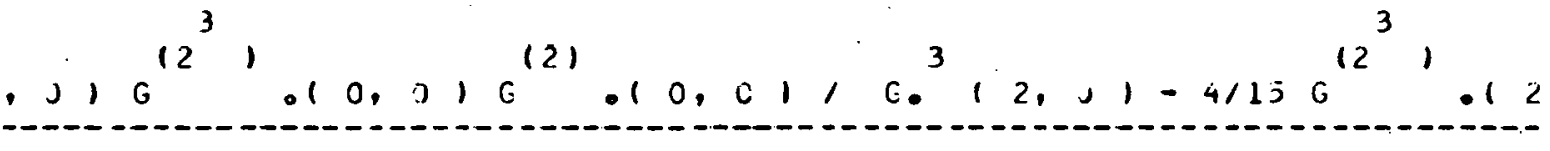
$G^{(2)} \cdot 12,0, G^{(2)} .10,0,1 G^{2},(2,0)-215 / 175 \mathrm{C}$ (2) 2

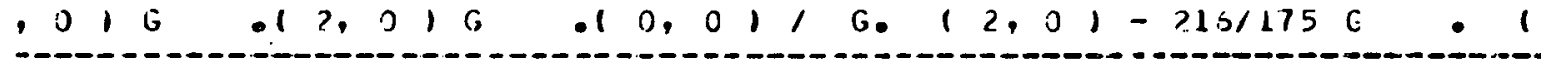

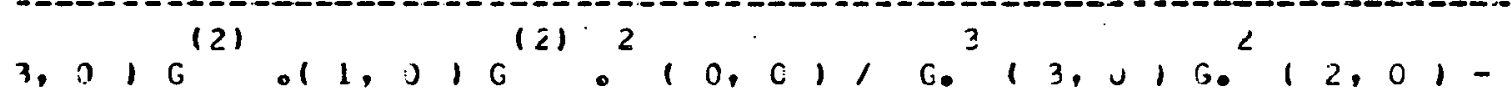

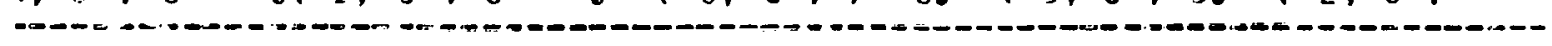
$1729 / 1225 \mathrm{G}^{(2)} \mathrm{G}^{2}, 1,0, \mathrm{G}^{(2)} \mathrm{B}^{3}(0, \mathrm{C}, 1 \mathrm{G}, 1+1,1 \mathrm{G}, 13,01 \mathrm{G}$ (1)

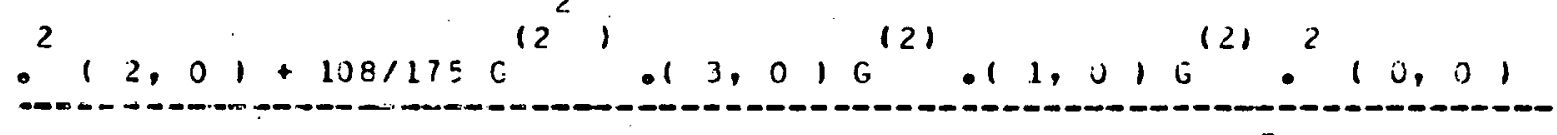

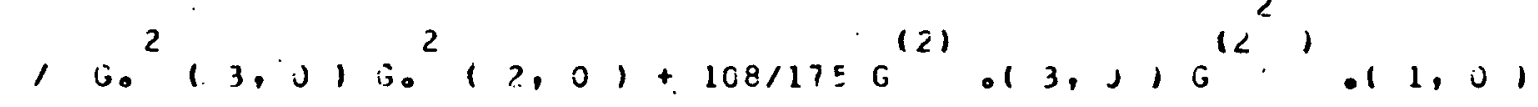
( 2$)^{2} 2$ $\mathrm{G}^{2}\left(0,01, \mathrm{G},\left(3,0, \mathrm{G}_{0}^{2}(2,0)+108 / 175 \mathrm{G}, 1,1,016\right.\right.$

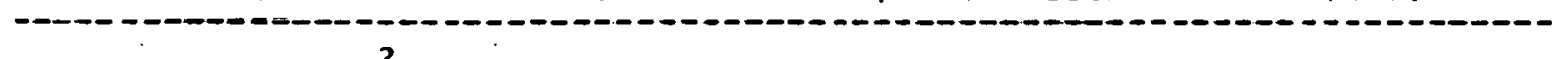

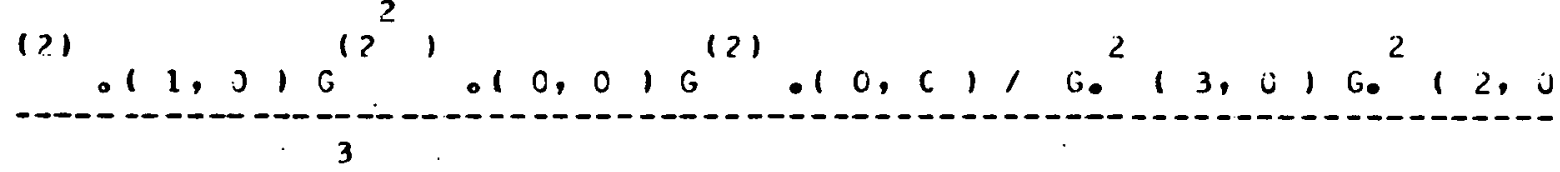
$1-3 t / 175 \mathrm{G}^{(2,}, 11,01 \mathrm{G}^{(2)} \mathrm{G}^{2}, 0,0,1 \mathrm{G}, 13,0, \mathrm{G}^{2}(2,0)$

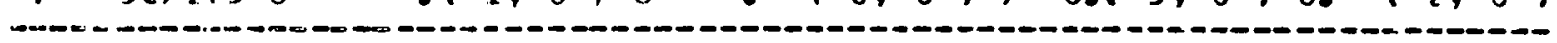


$-541175 G^{12^{2}}, .11,0, G^{12^{2}}, .10,0, c^{12)} \cdot 10,0,1, G .13,01$

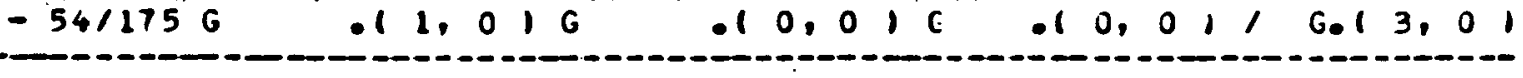

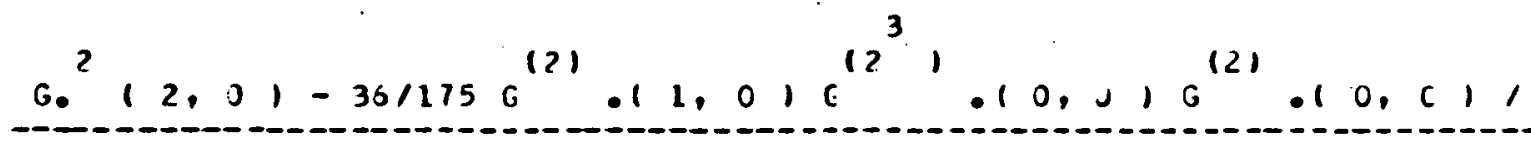
(6) $G_{0} 13,01 G_{0}^{2}, 2,01+2175 G^{12} 1,10, c 1 G^{(2-1}, 10,011 G^{2}$ $12,01+11306^{12}, 012,01 c^{121} \cdot 10,0110012,01-1 / 320$ 2 .

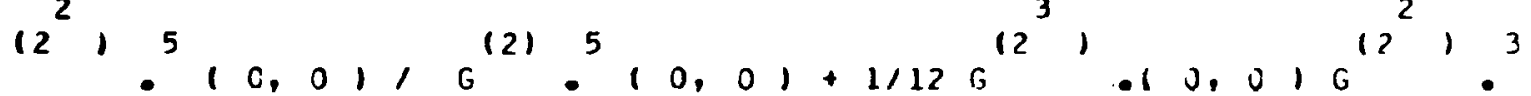
0

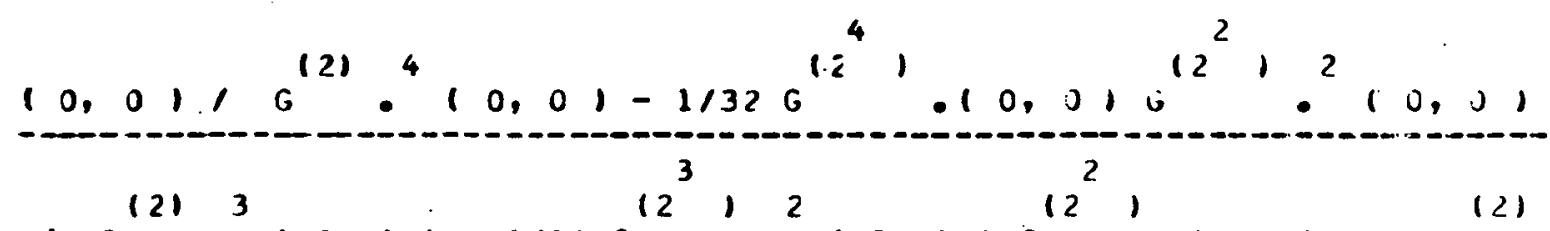

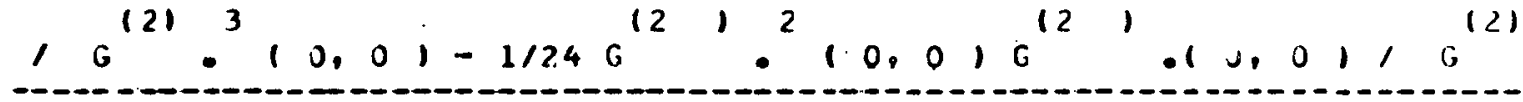
(5

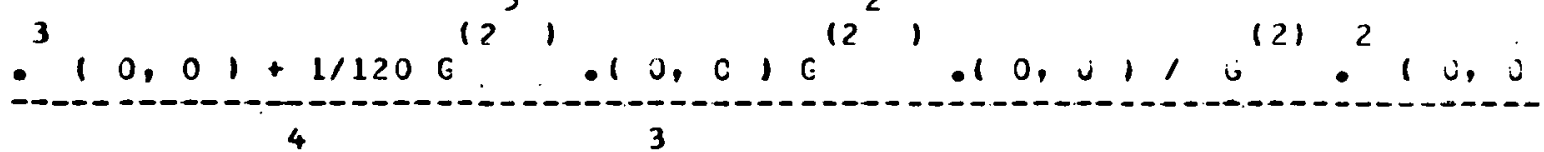
$1+1 / 72 G^{12}, \cdot 10, C 1 G^{12^{3}}, 10,01, G^{121} 0^{2}, 0,01-1 / 720$ 0 $\mathrm{G}^{\left(2^{6}\right)} \cdot 10,01, \mathrm{e}^{121} \cdot 10,01$

$c(5,1)=-4321175 G^{(2)} e^{3}(2,0) c^{(2)} 0^{2}\left(0,0, G_{0}(1,0), G, 1\right.$

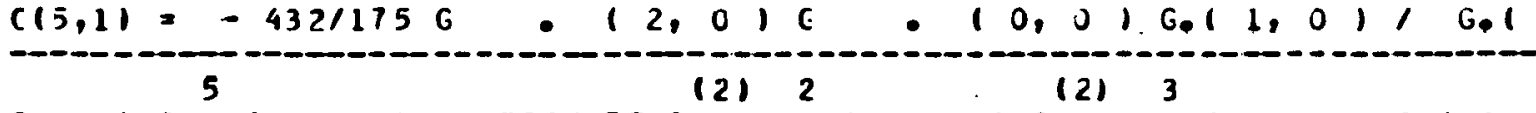
$3,01 G^{5}(2,0)-25921175 G^{2}, 12,01 G^{3}, 10,016.11$,

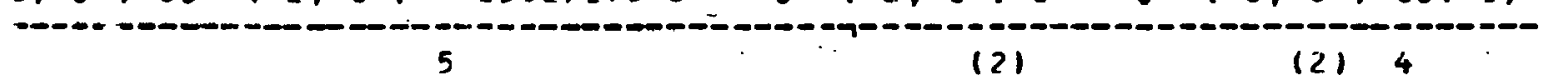
$01 / G .13,01$ G. $12,01-89856 / 4375 \in$ G 2,016 G 10 , 0 $010.11,0,1, G,(3,0) G_{0}^{5}(2, C)-25344 / 3125 G^{(2)} e^{5}, 0,01$

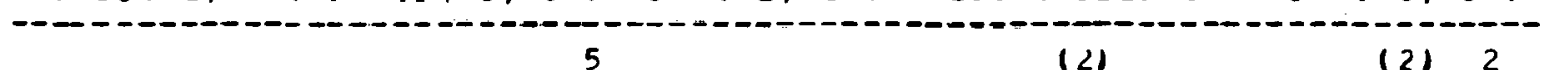

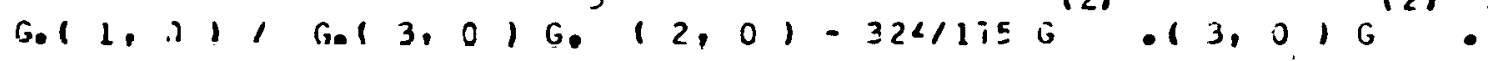

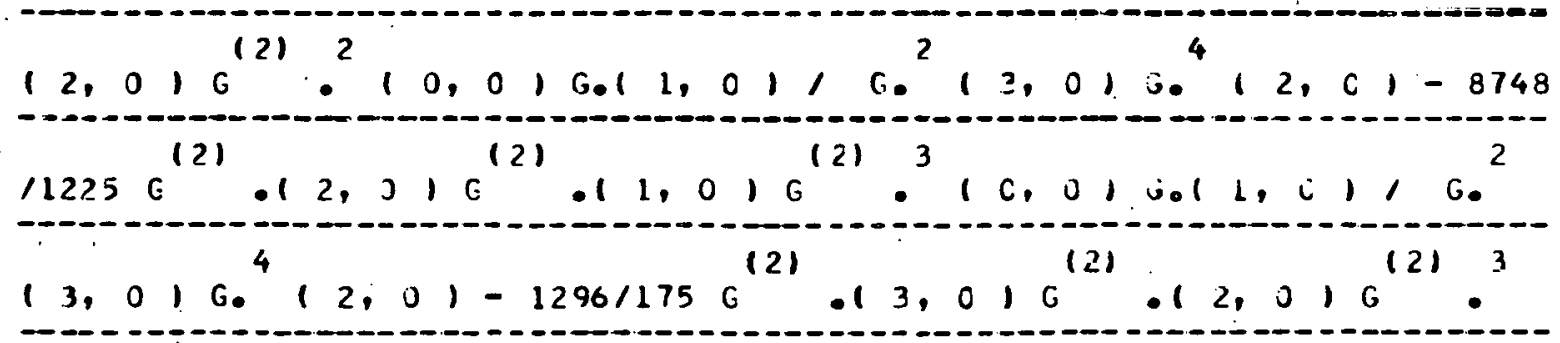




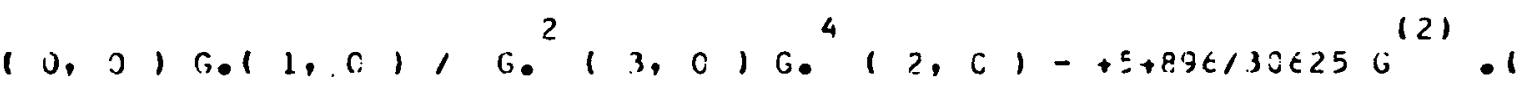

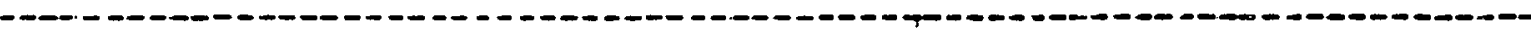

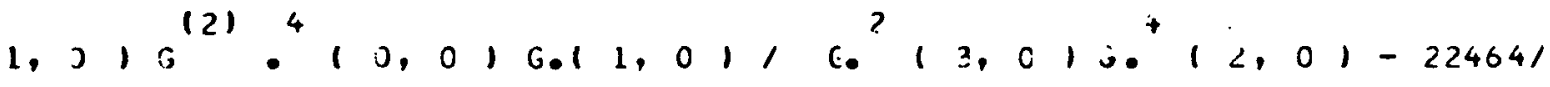
$(?)$

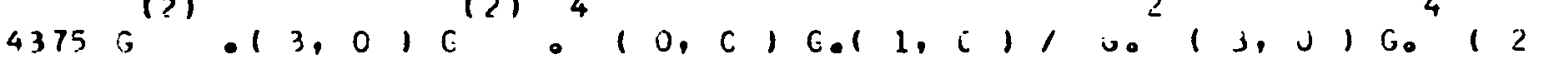
4372 6

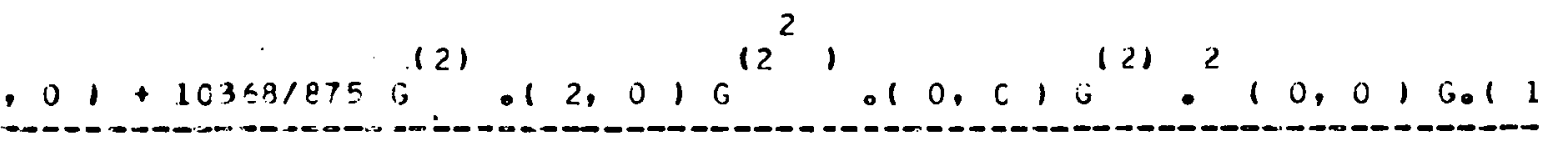

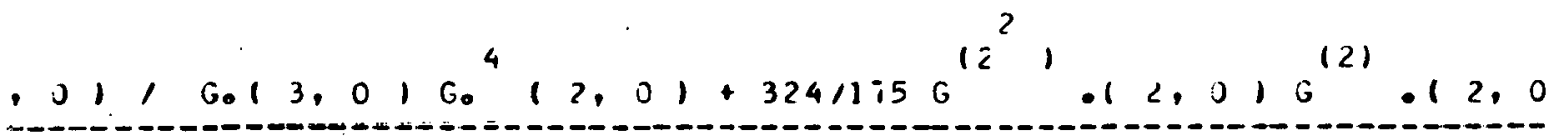
$10^{(2)} 2,0,0, G_{0}\left(1,0,1, G_{0}\left(2,0, G_{0}^{4}(2,0,+44054143756\right.\right.$ ( $12^{2} 1$

$$
.10,0, \mathrm{G}^{(2)}{ }^{3}(0,0) \mathrm{G}, 1,0,1,1, \mathrm{G}, 1,3,0,0.4,2,01
$$

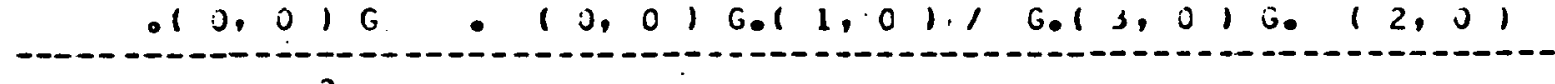

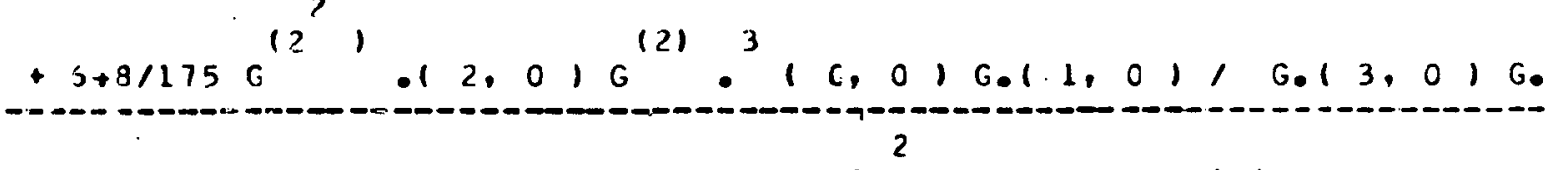
$4(2,0)+486 / 175 \mathrm{G}^{(2)} \mathrm{G}^{2}(2,0) \mathrm{c}^{\left(2^{2}\right.}, \cdot(0, \mathrm{~J}) \mathrm{G}^{(2)} \cdot 10,01 \mathrm{G}$ ( 4 ( $11,0,1, G .13,0, G_{0}, 2,0,-216 / 17 E G^{4}, 13,0, G, 012$

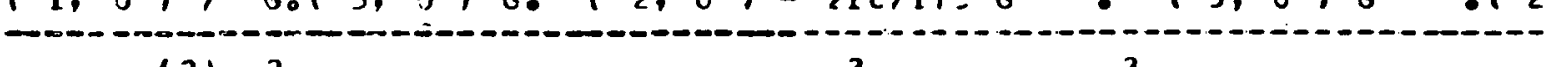
12123.3

. $01 \mathrm{G}, 1 \mathrm{C}, \dot{0}, \mathrm{G}, 1,0,1, \mathrm{G}, 13, \mathrm{C}, \mathrm{i}, 12,01-4321175$

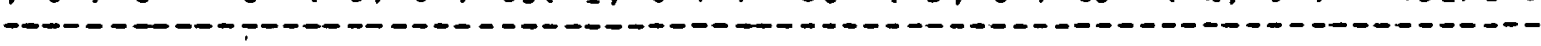

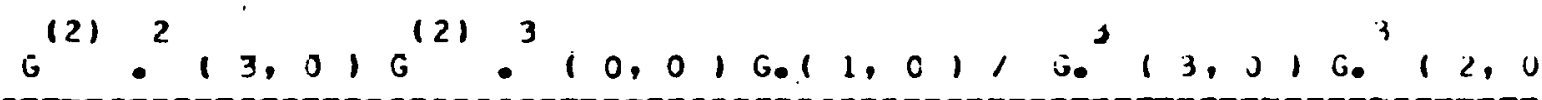
$1-583211225 \mathrm{G}(2) \cdot\left(3,0, \mathrm{G}^{(2)}, 1 \mathrm{l}, \mathrm{C}\right) \mathrm{G}(2) \mathrm{G}(\mathrm{c}, \mathrm{c}) \mathrm{G}, 1,1,0,1$ $\begin{array}{llll}3 & (2) & (2)\end{array}$

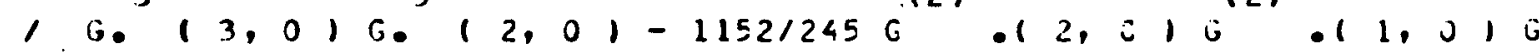

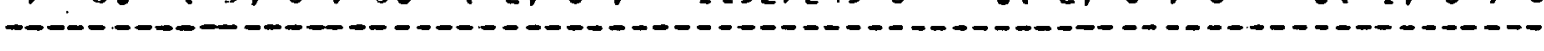
(2) 3

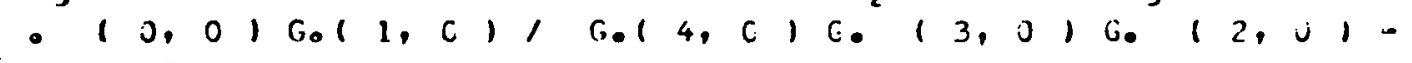
-

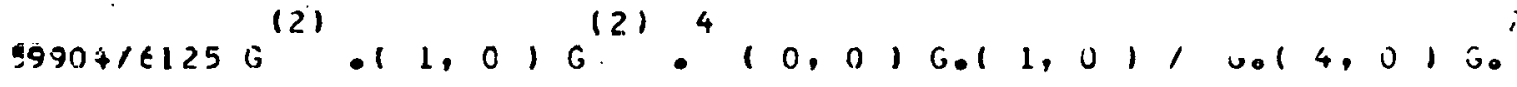

0

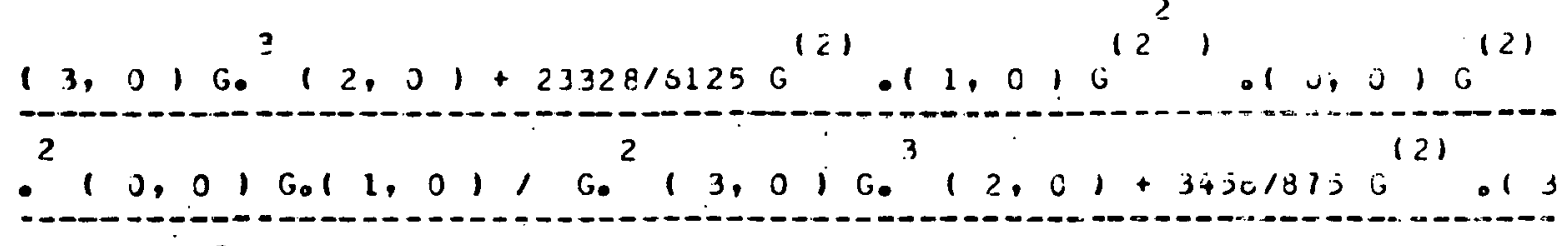

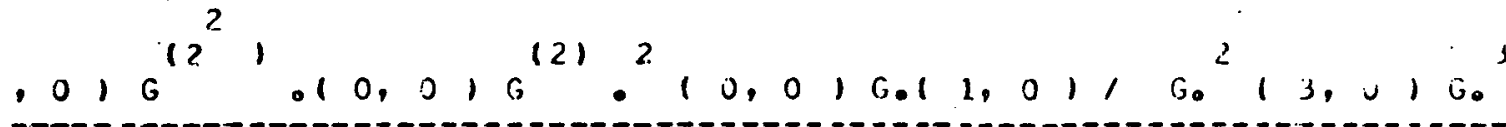
0 


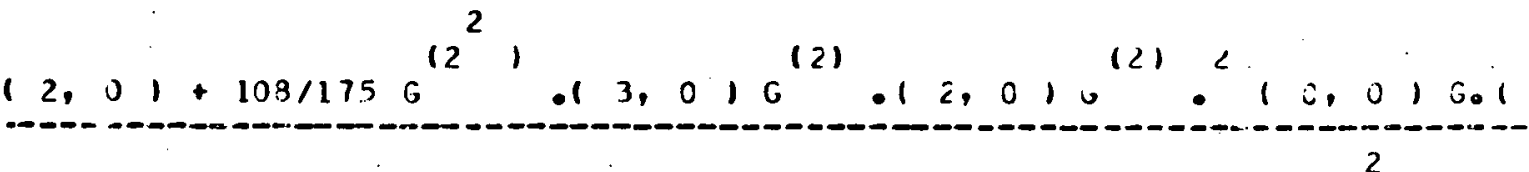

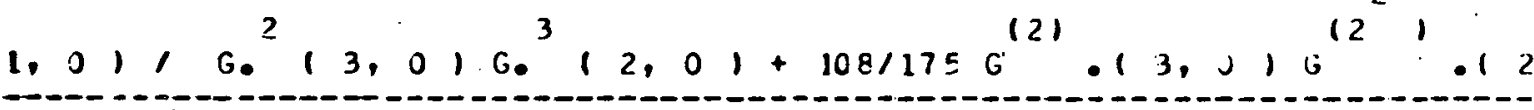
$, 01 G^{|2|} \mathrm{G}^{2}, 0,0, \mathrm{G}, 1,0,1, G^{2}, 3,0, \mathrm{U}^{3}(2,0,1+14581$ (

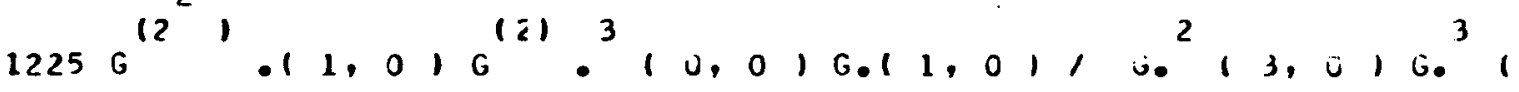

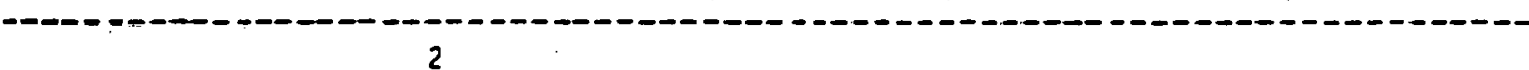

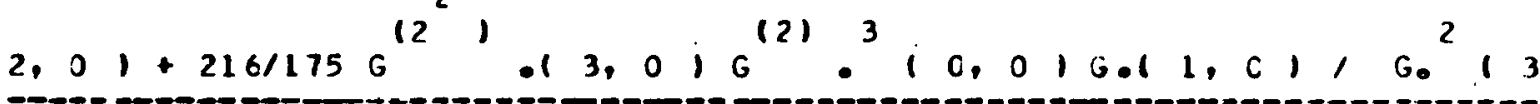
$, 01 G^{3} 12,01+324 / 175 G^{(2)} \cdot 13, C, G^{(2)}, 12,0, G^{(2)}, 10$, (2) $01 G^{\prime} 0,0, G, 1,0, / G^{2}, 3, C, G^{3}(2,0,-1152 / E 75 G$

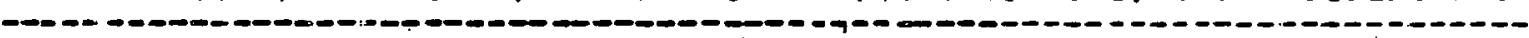
3

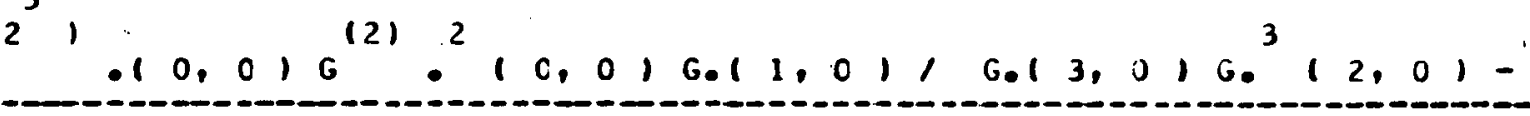
$\left(2^{3} 1 \quad(2) 22\right.$

$36 / 175 G \quad .12,0, G \quad \bullet \quad 0,0, G .11,0,1, G .13,0,6,1$ $2,01-541175 \mathrm{G}^{(2)} \cdot\left(2,0, \mathrm{G}^{12^{2}, 2^{2}}(0,0) \mathrm{G}, 1,01,1, \mathrm{G}, 13,0\right.$

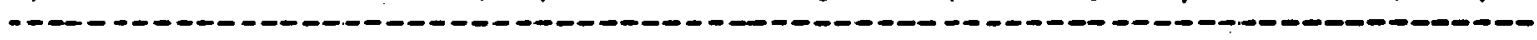

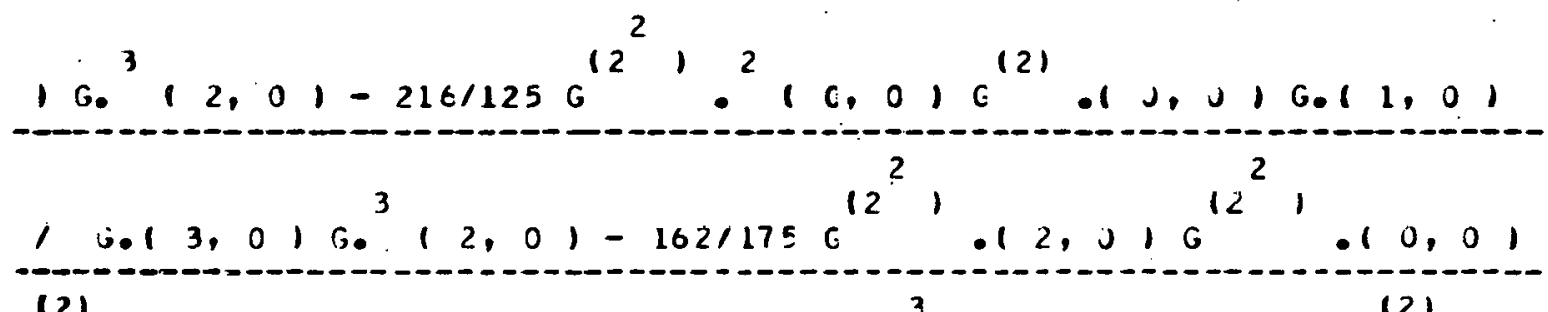
(2) $\mathrm{G} .10,0, \mathrm{G}, \mathrm{i} 1, \mathrm{C}, 1 \mathrm{G}, 13,0, \mathrm{G}, 12,01,108 / 175 \mathrm{G} .12$ 3

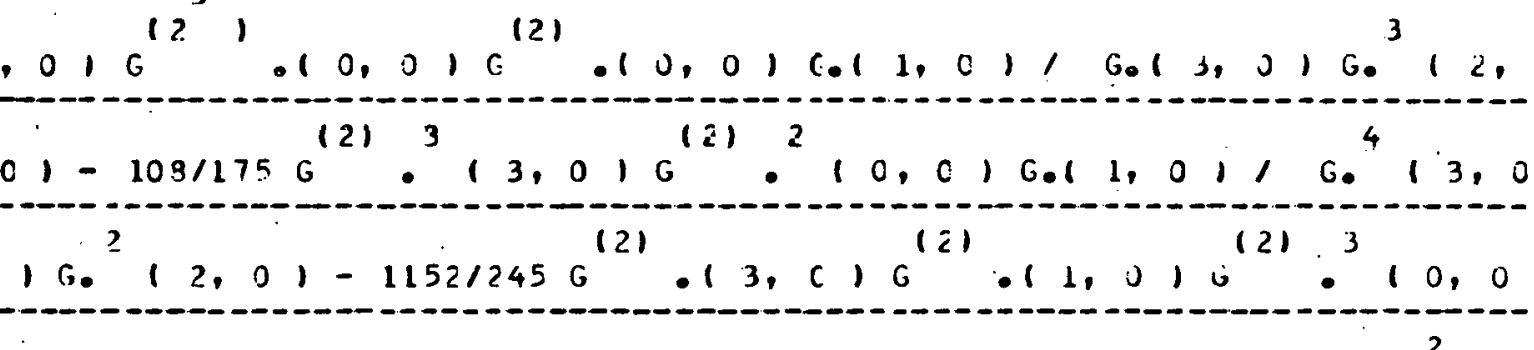

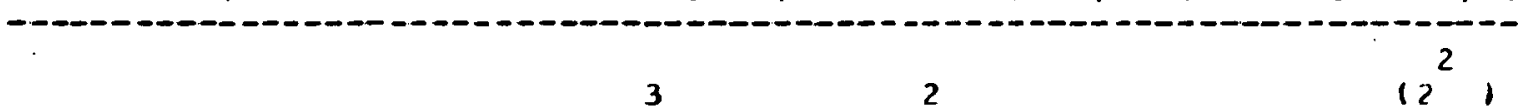
$16.11,0,16.14,0, G_{0}, 3,0,6.12,01+1 j 8 / 175 G$

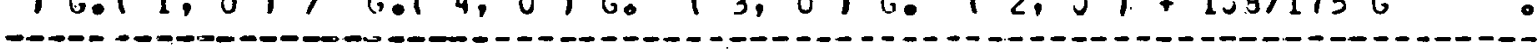




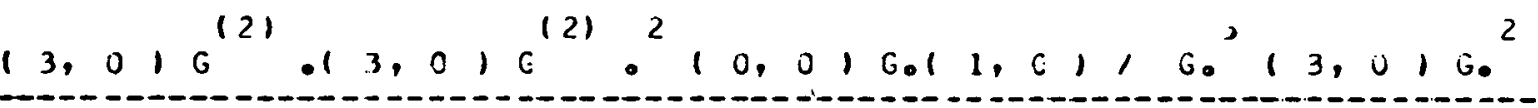

$12,01+16211750^{121} \cdot 13,0,6^{(z)} .10,016^{121} .10,01601$

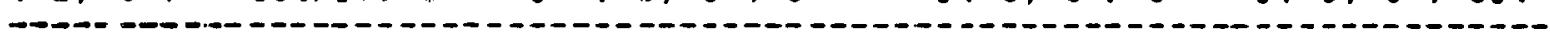

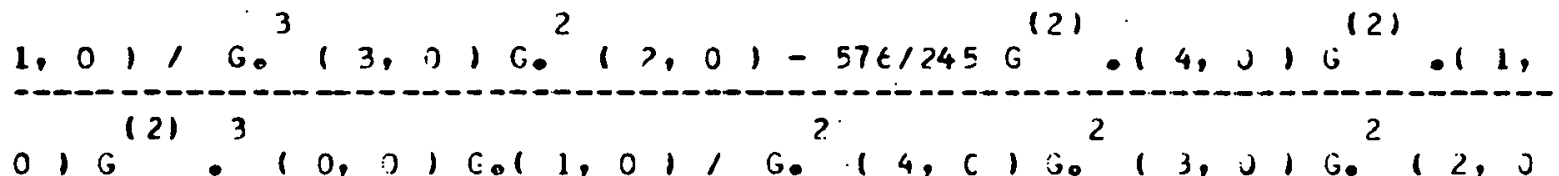

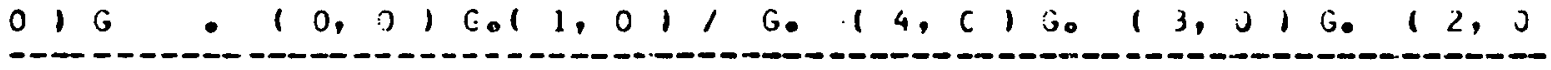
( $1+4608 / 1225 \mathrm{G}^{(2)} \cdot(1,0) \mathrm{G}^{(2)} \cdot(-0,0) \mathrm{G}^{(2)} \cdot 1,0,016.11 .0$ 9

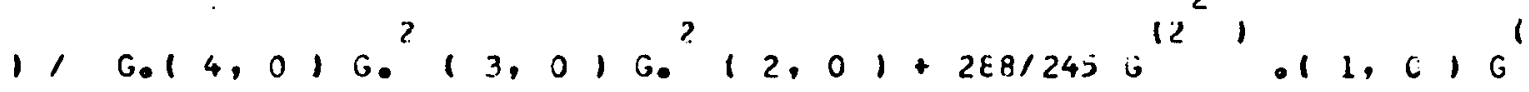
2) 3 . - $(0,0, G .11,0,1,0.14,0,6,12,010.12,01-181$ 3

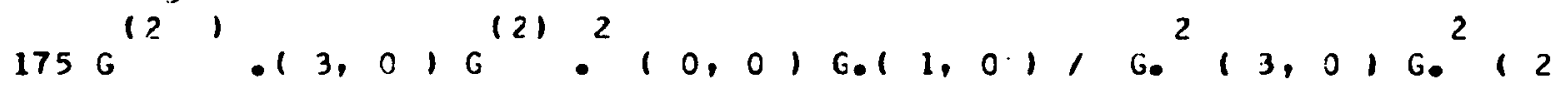
(175 $.01-27 / 175 \mathrm{G}^{(2)} \cdot(3,0) \mathrm{G}^{(2)} \bullet^{2}\left(\mathrm{c}, 01 \mathrm{G}, 11, \mathrm{~J}, \mathrm{G}^{2} 13\right.$,

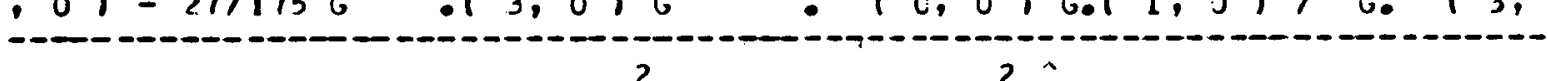

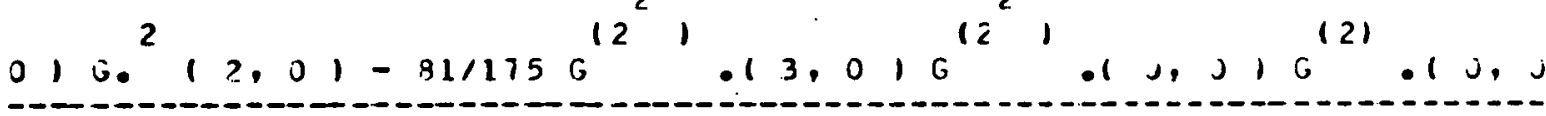
(1)

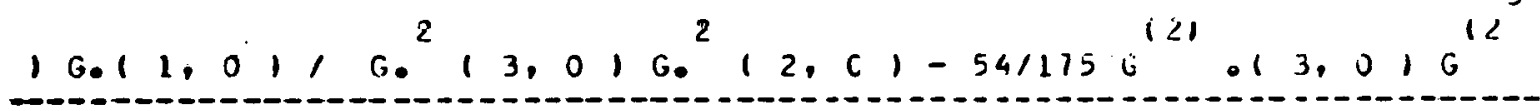

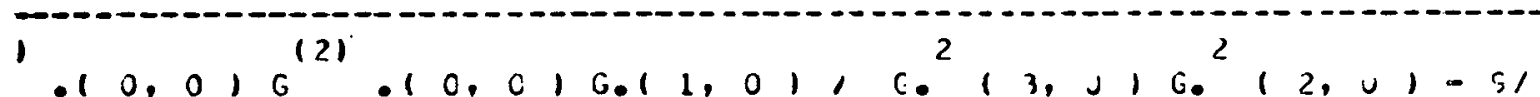

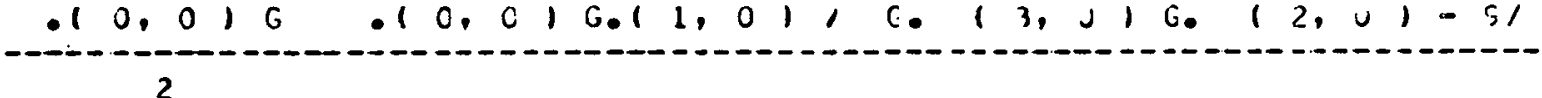

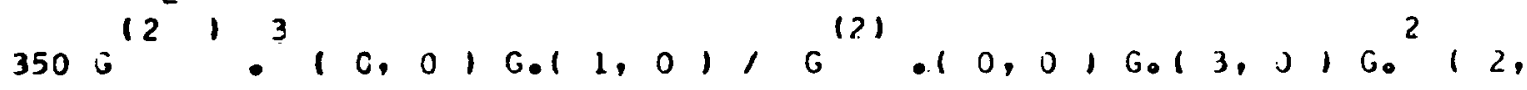
-

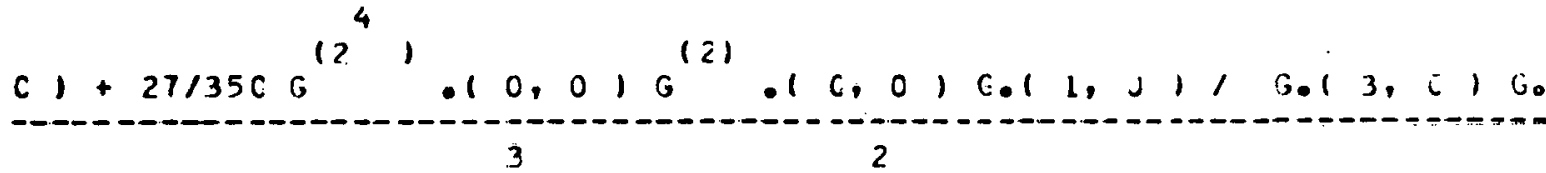
2

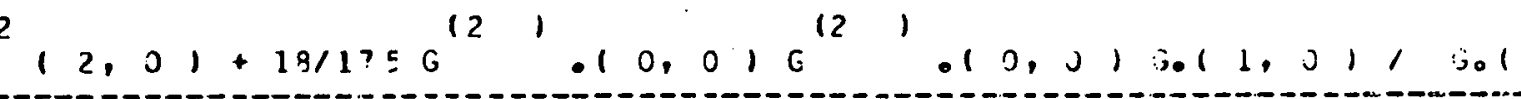

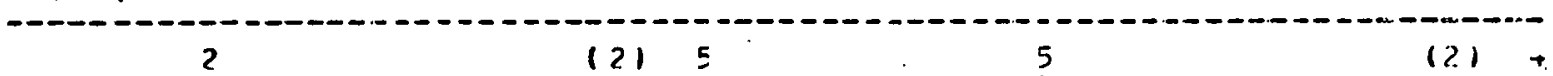

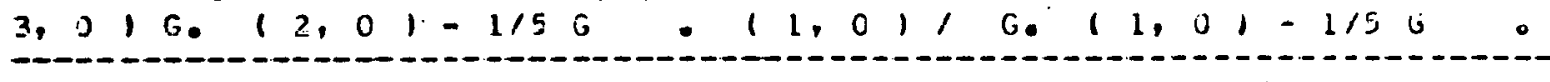

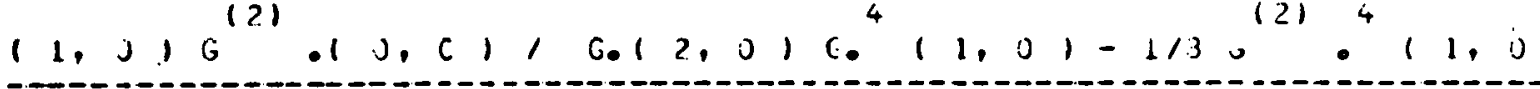

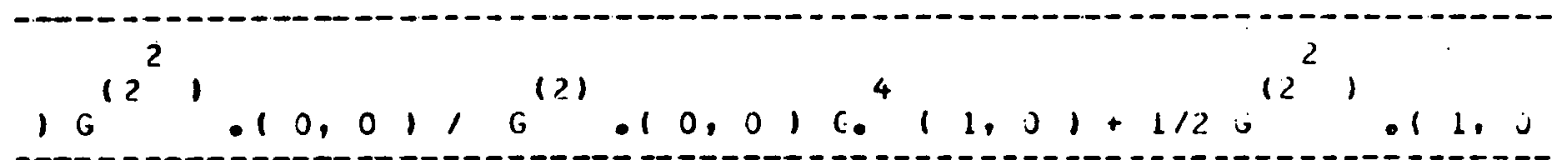




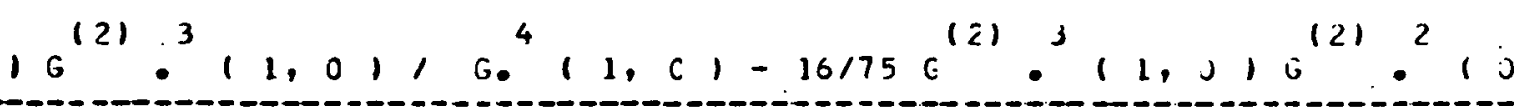
$, 01, G_{0}^{2}, 2,0, G^{3}, 1,0,-4 / 15 \epsilon^{(2)}, 12, J, 0^{(2)} e^{3}, 1,0$

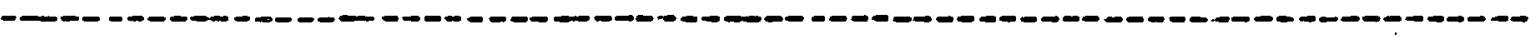
$, G^{12 i} \cdot 10,01, G^{2}\left(2,0, G^{3}, 1,0,+2 / 3 j^{12}, 011,016\right.$ 2)

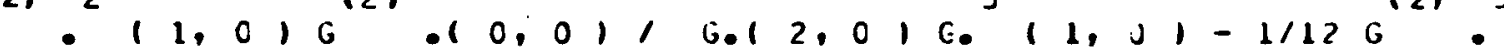

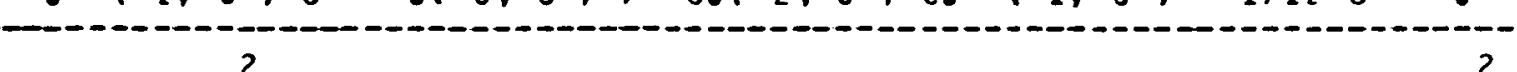

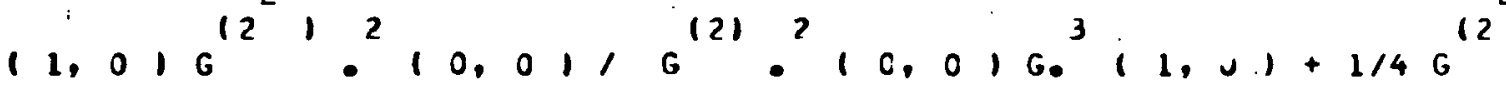
( 10

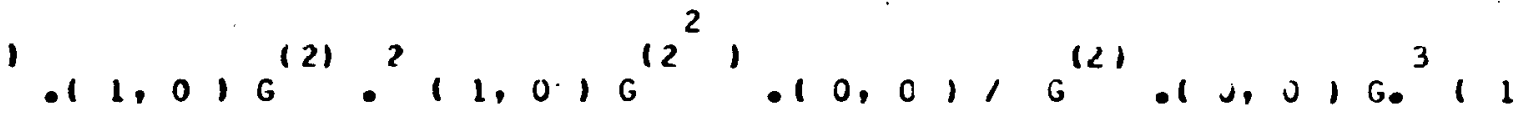

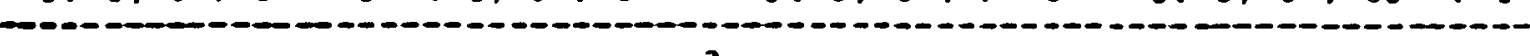

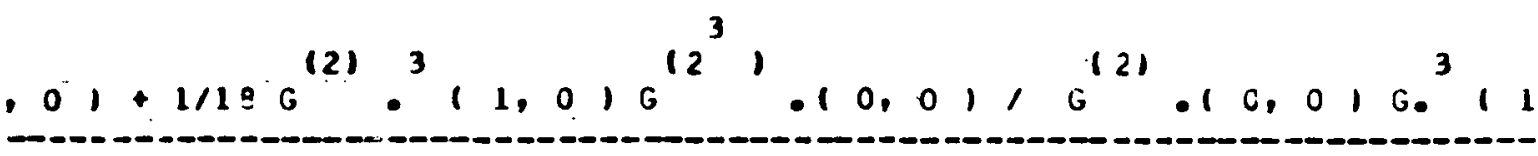

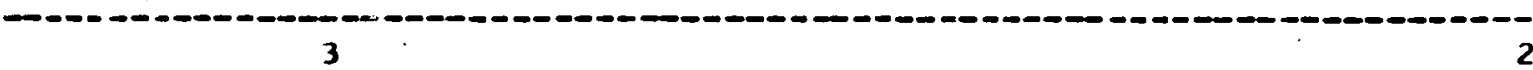

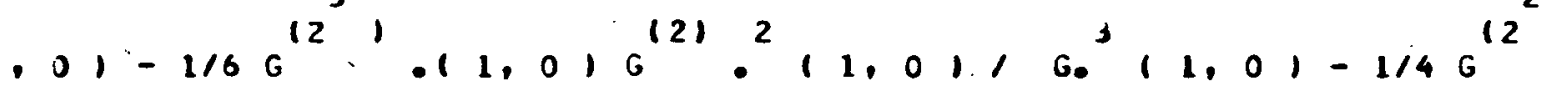
12 (2)

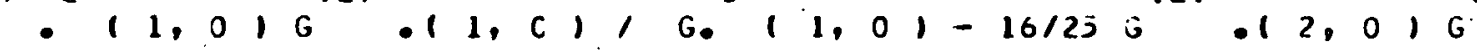
2 $\cdot 11,0, G^{2}\left(0,0,1, G^{3}, 2,0, G_{0}^{2}(1,0,-32 / 1256\right.$

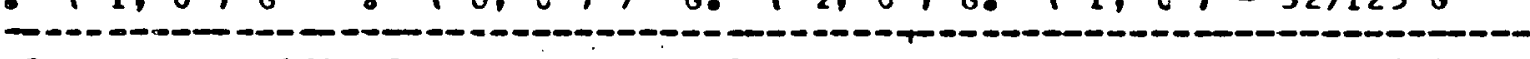

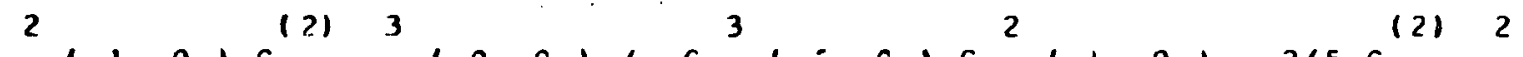

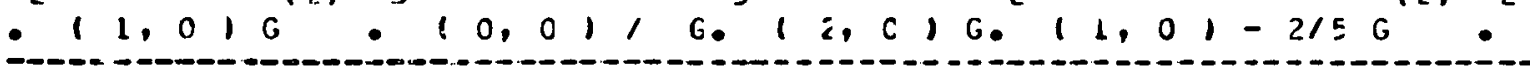
$12,01 G^{(2)} \cdot\left(1, C, G^{(2)} \cdot(0,0), G^{3}(2,01,1011,01,-\right.$ 1210

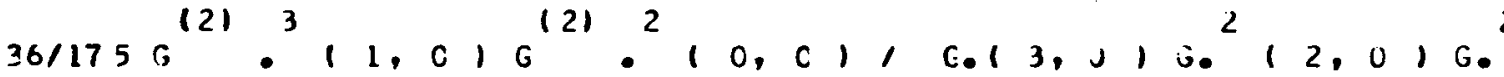
-

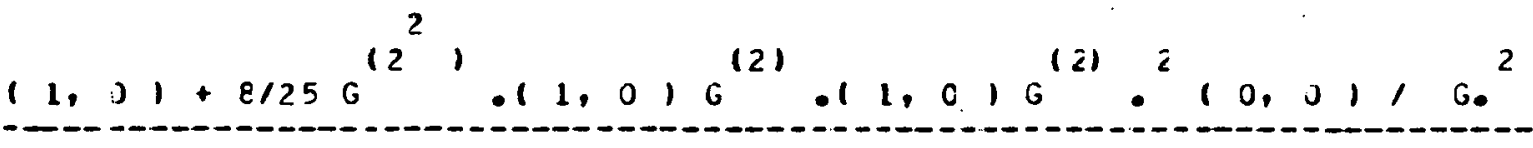
(1)

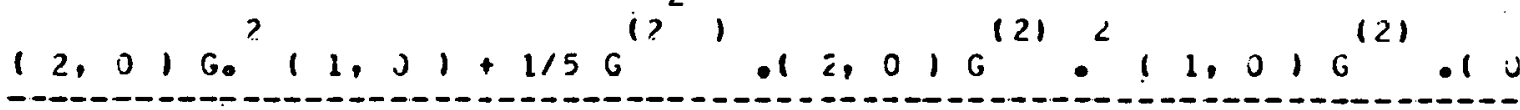
(

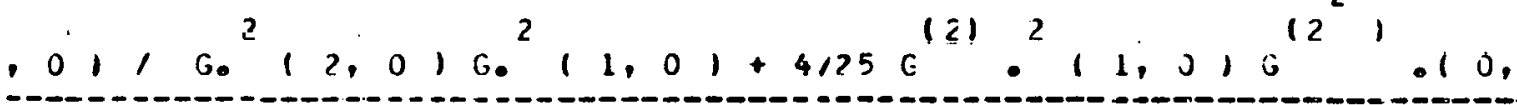
$(2) \quad 21,2512$

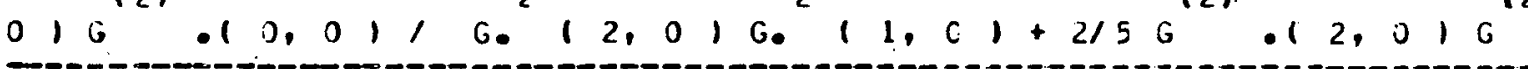
2. $1.11 .01 \mathrm{G}^{(2)} \cdot(1,0) \mathrm{G}^{(2)} \cdot\left(0,01, \mathrm{G}^{2}, 2,0, \mathrm{G}^{2} 11,01\right.$

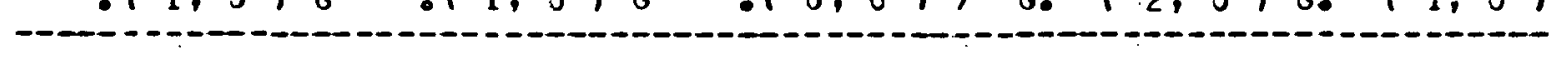




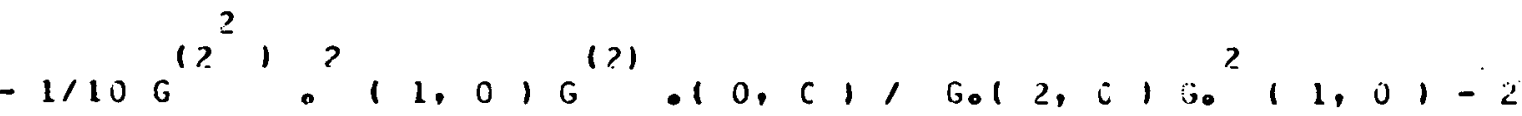

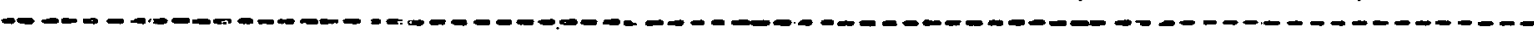
3

$115 j^{(2)}, 11,0, G^{(2)} \cdot 1,0, G^{(z)}, 10,01, G .12,0, G_{0}^{2}, 1$ -

$, J 1-1 / 1 \in G^{(2)} \cdot(1,0) G^{12} e^{2}(0,0), 0^{121} \cdot 3\left(0,010^{2}\right.$ (5) $11,01+1 / 9 G^{12} 1011,01 G^{121} \cdot 11,01 G^{12}, 2^{2}, 0, c 1, G^{12}$ . $10^{2}\left(0,0, G_{0}^{2}\left(1,01+1 / 12 G^{121} 0^{2} 11,010^{12}, 010,01 G^{12}\right.\right.$ $-0$

$1.10,01, G^{(2)} 0^{2}, 0,010^{2}, 1,01-1 / 160^{12} 0^{2}(1,0) 0$ ${ }^{2}$ $12^{2} 1(2) \quad 2121$

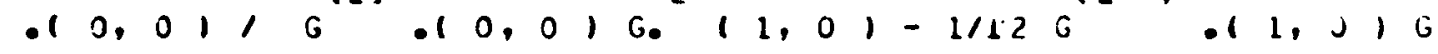
(2) $.11,0, \mathrm{G}^{\left(2^{2}\right.}, \cdot 0,0,1 \mathrm{G}^{(2)} \cdot 10,0,0<11,01-1 / 126^{12}$

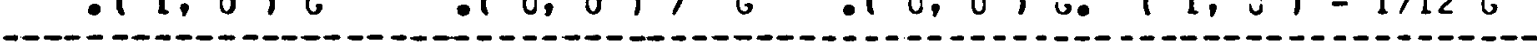
2

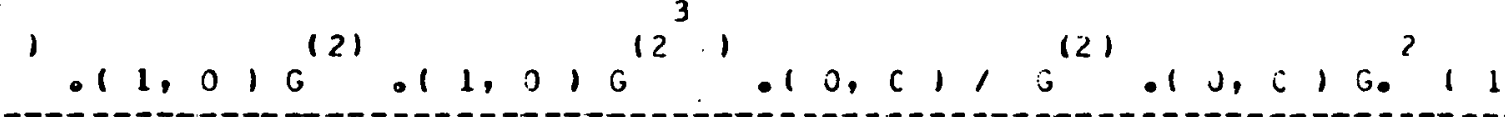

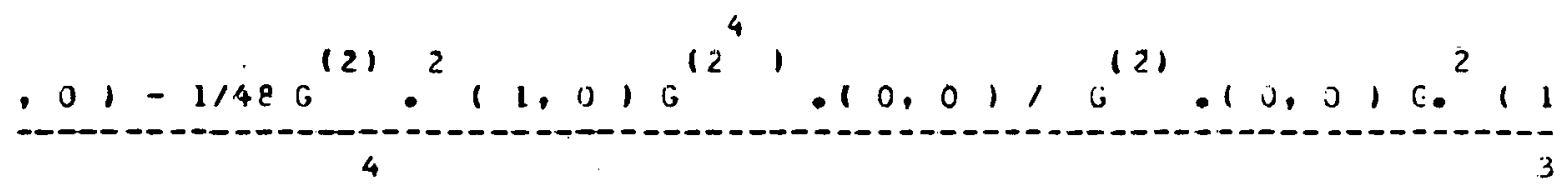
$.01+1 / 24 r^{12}+11,01 G^{12}+11, c 1, G^{2} 11,01+1 / 120^{12}$

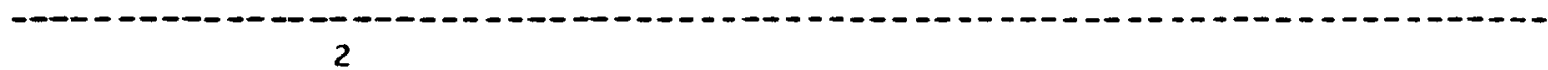
$\left.1.11,0, G^{12}, .11,01, G^{2}, 1,01-43 / 200^{(2)}\right)^{2}, 2,010^{1}$ 2) 12$)^{2}$ $21.11,0, G^{(2)} 0^{2}, 0,0,10^{4}, 2,0,6.11, j 1-19211256$ -

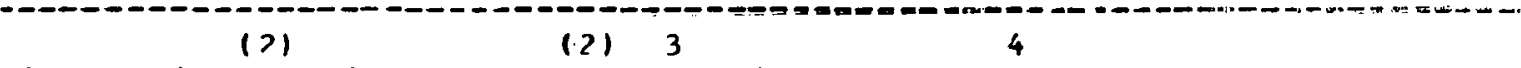

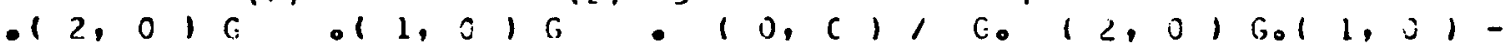

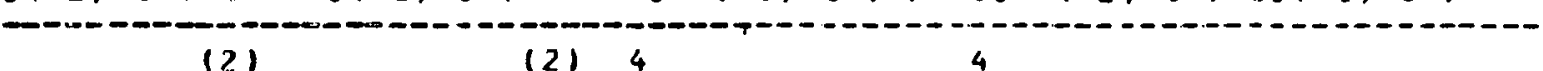

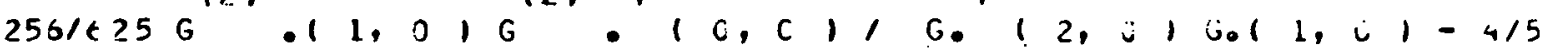

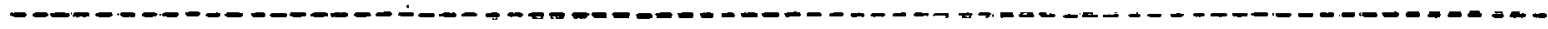
$\left.G^{(2)} 0^{3}(2,0) G^{(2)} \cdot 11,0, G^{(2)}, 10, c\right), 0^{+} 1<01,0.11,0$

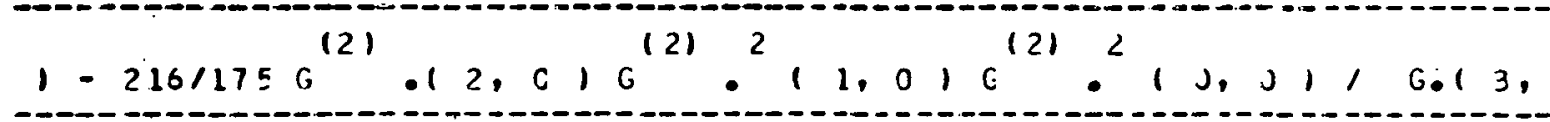


$0100^{3}\left(2,0,9011,01-1256 / 875 \mathrm{G}^{(2)} 0^{2}, 1,0,0^{121} 0^{3}(0,01\right.$ (-)$\left., G .13,0, G^{3}, 2,0, G, 11,0,+64 / 125 G^{121}, 11,0, G^{12}\right)$

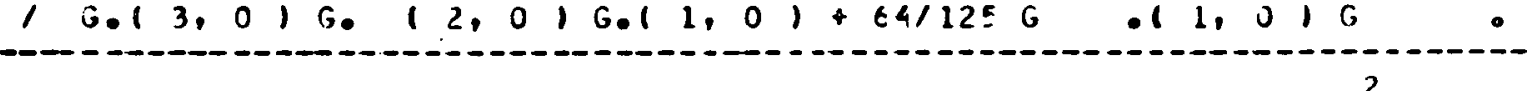
$10,01 G^{121} 0^{2}, 0,01, G^{3}\left(2,0, G 011,01+16 / 25 G^{12}, 12\right.$ (2) 1212

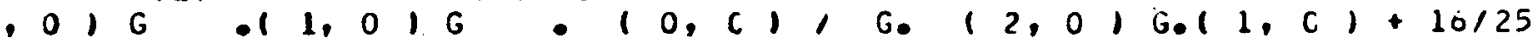
-

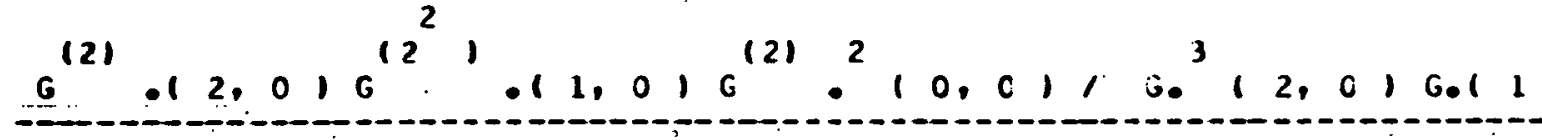

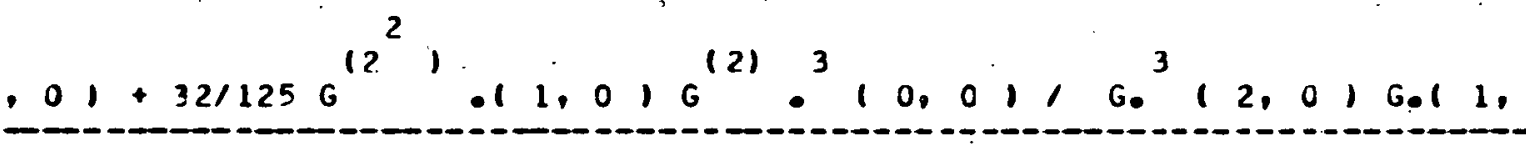
$01+16 / 25 G^{(2)} \cdot 12,01 G^{(2)} \cdot 11,01 G^{12}, 010,016^{(2)} \cdot 10,0$ (1)

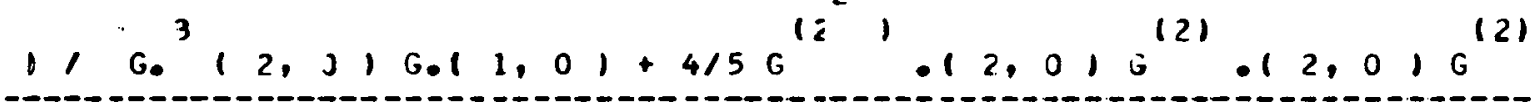
$\left.\| 1,0, G^{(2)} \cdot(0, C), G^{3}, 2,0, G, 11,0,+2 / 5 G^{(2)}\right)^{2}(2,0$ $-0$

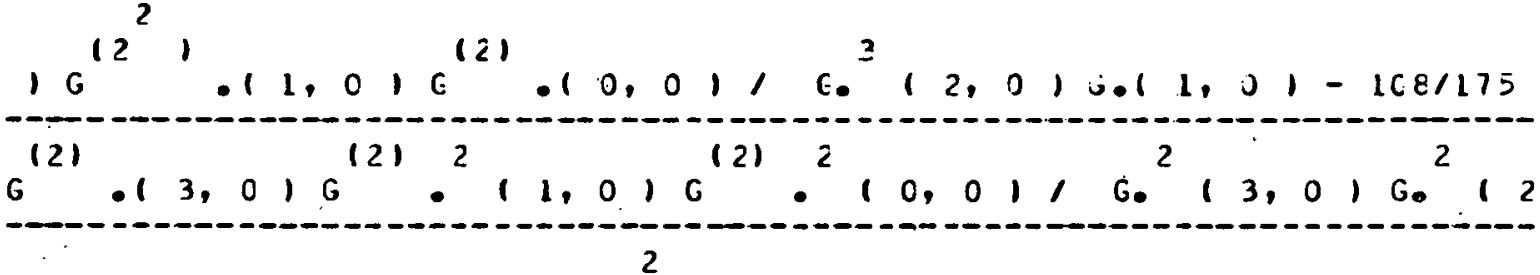
$.01 G 011,01+103 / 175 G^{(2)}, 11,010^{121} \cdot 11,010^{121} 0^{2} 11$

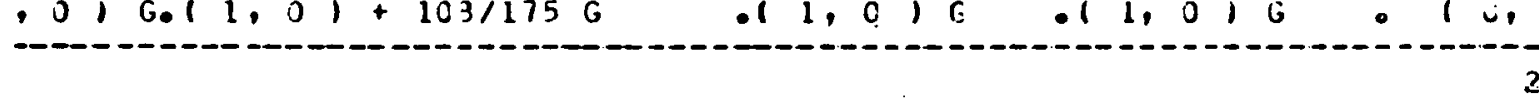

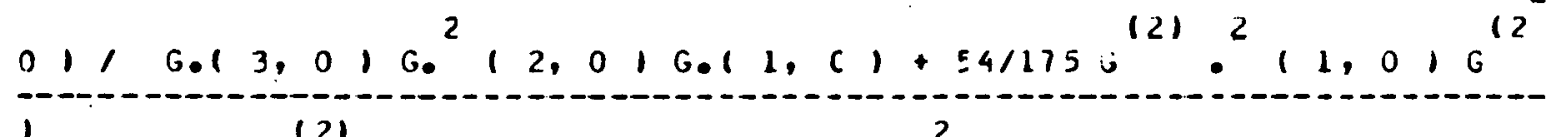
$1.10,0, \sigma^{(2)} .10,0,1,0.13,0, c^{2}, 2,0,0.11,01-8 / 75$ 3

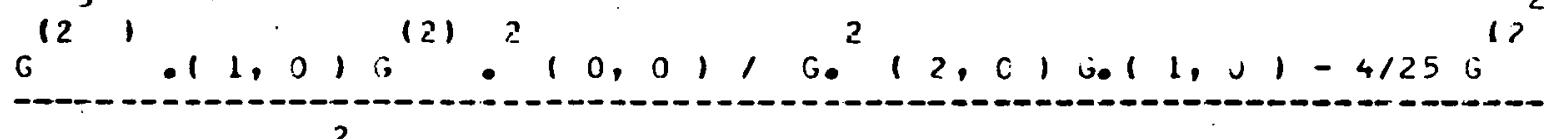
$\left.1.1,0, G^{(2,}, 10,0, G^{(2)}, 10,0,1, G^{2}, 2, J, G .1,0\right)$

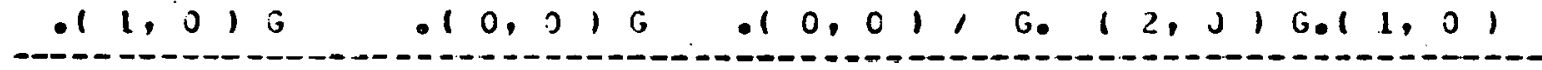
$-8175 \mathrm{G}^{(2)} \cdot 11,01 \mathrm{G}^{12^{3}}, \cdot 10,01 \mathrm{G}^{(2)} \cdot 10,0110^{2}(2,016$ 


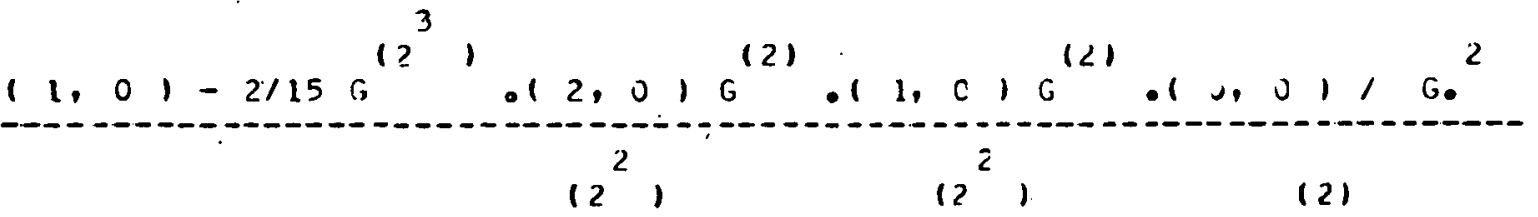

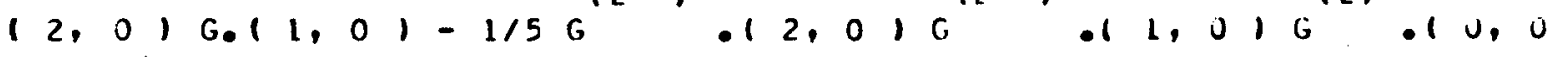
1
3 $1, G_{0}^{2}\left(2,0, G_{0} 1,01-2115 G^{12}, 12,0, G^{12}, \cdot 1,01 G^{12}\right.$ $1,0,0, G^{2}\left(2,0, G .11,01+1 / 30 \mathrm{c}^{12^{4}}, .11,01 \mathrm{G}^{121} .10\right.$

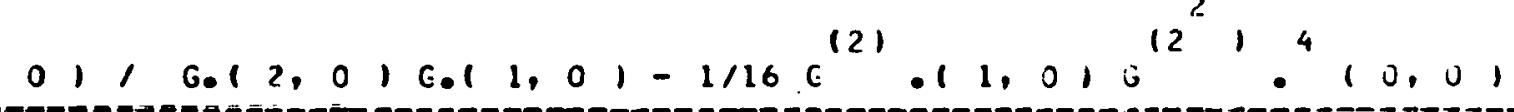
-

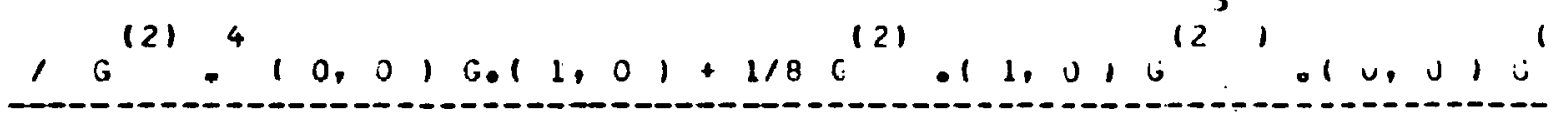
$2^{2}, 2$ (द) 3 (2)

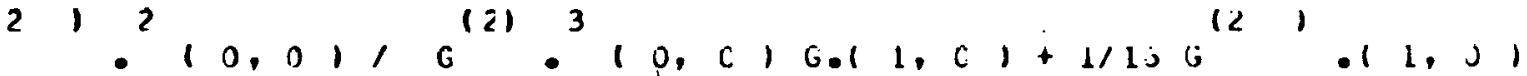
25 $G^{12}, 2^{3}, 0,01, G^{121} 0^{3}, 0,0016.11,0,1-1 / 210^{1}, .11,0$

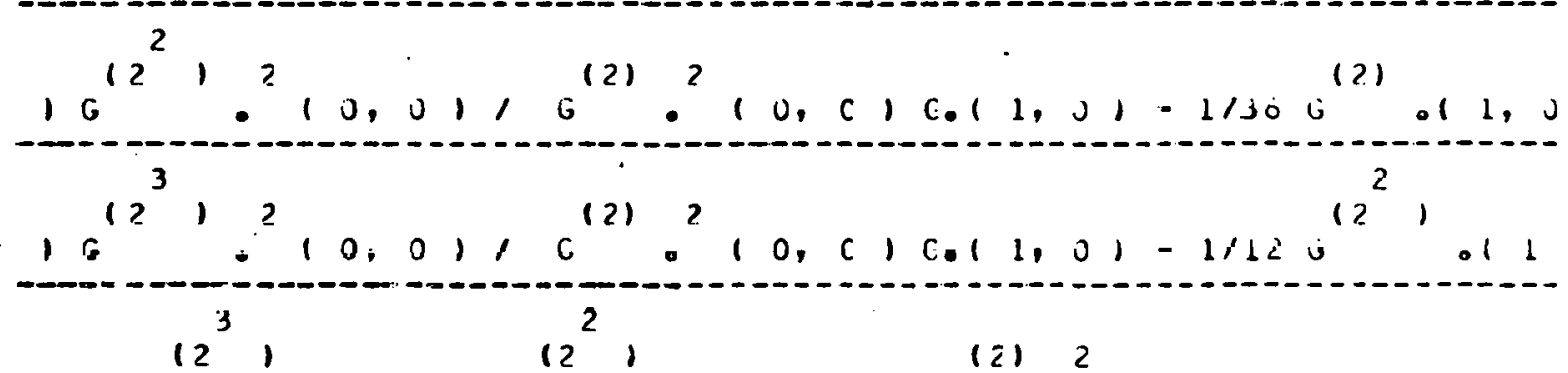

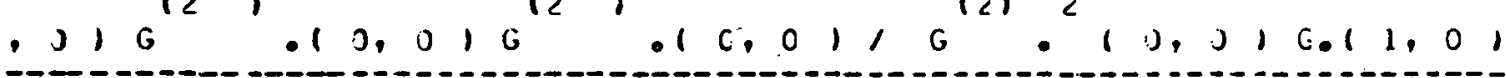

4
4

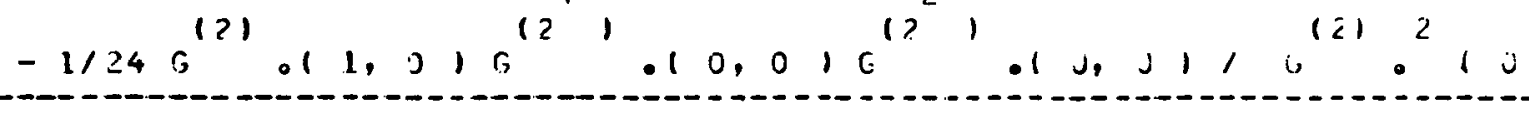

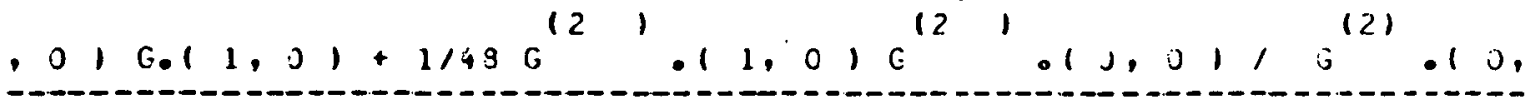

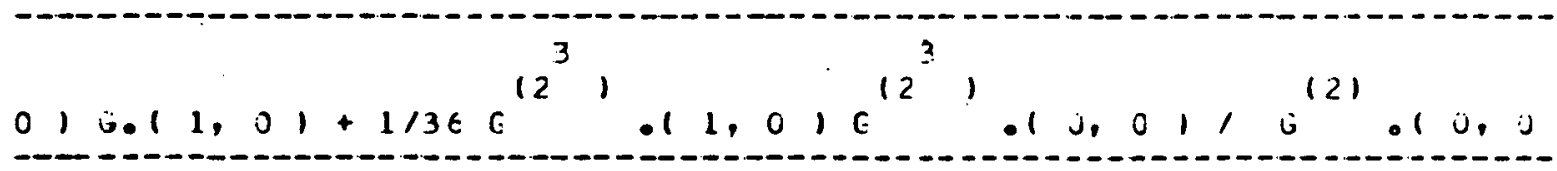

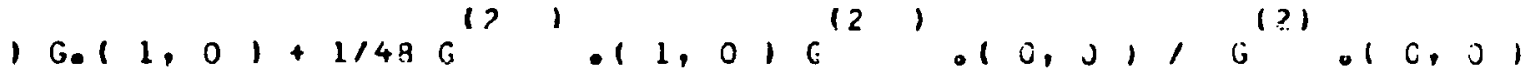
( $\mathrm{G}, 11,01+1 / 120 \mathrm{G}^{121} \cdot 11,0, \mathrm{G}^{12}, 10,01, \mathrm{G}^{121} \cdot 10,01 \mathrm{G}$ 


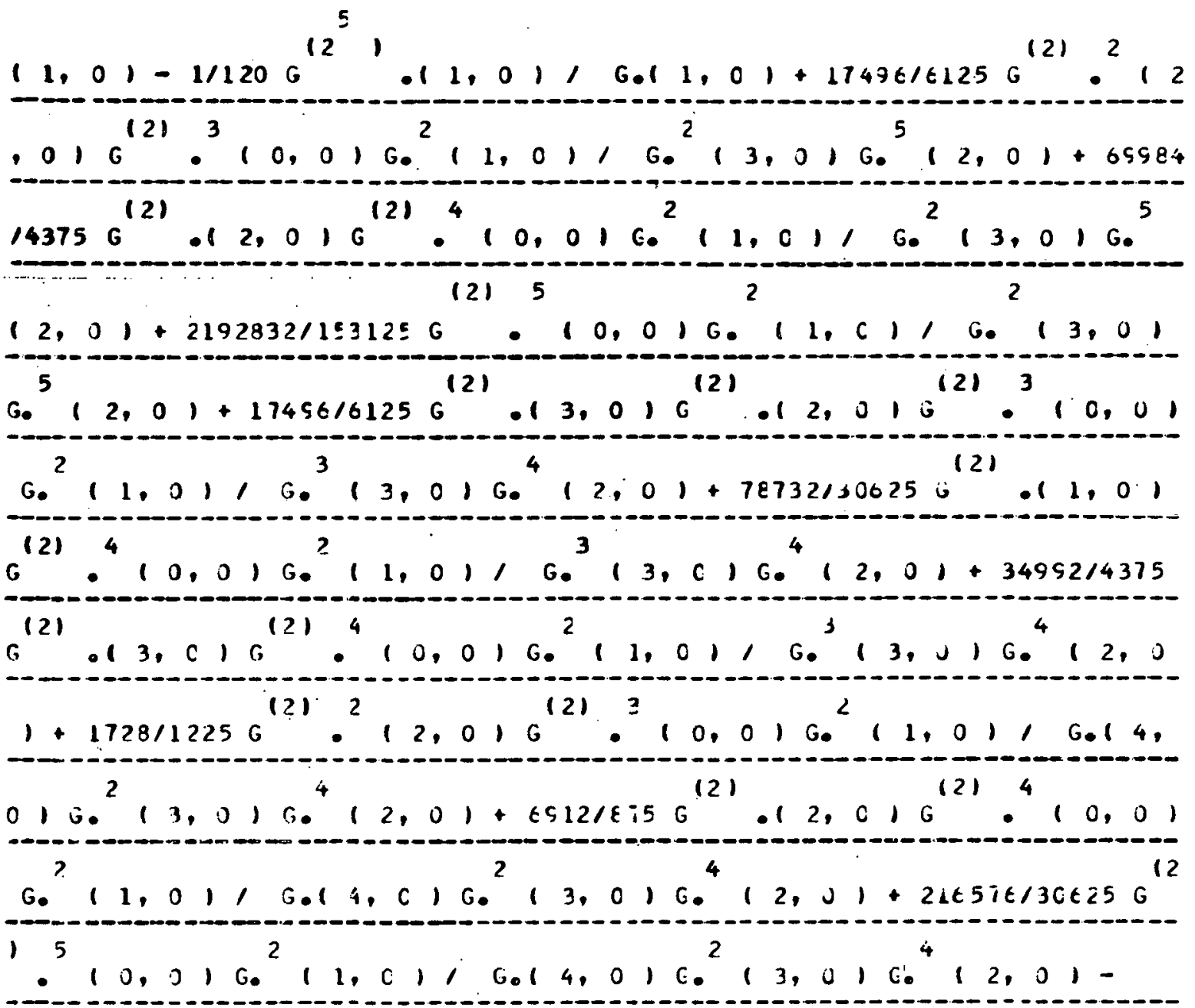

$4374 / 1225 G^{(2)} \cdot(2, J) G^{(2)}, \cdot 10, C, G^{(2)} G^{2}, J, 0 ; G^{2}, 1,01$

40

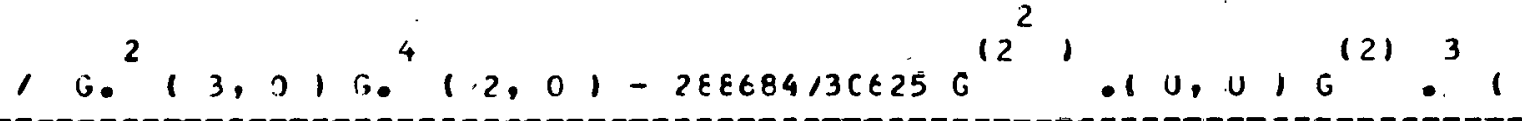

$0,0, G_{0}^{2}, 1,0,1 G^{2}\left(3,0, G_{0}^{4} \mid 2,0,-+374 / 6125 G^{12} 1.12\right.$ $G^{(2)} 0^{3}(0,0) G^{2}(1,0), G_{0}^{i}(3,0) G_{0}^{4} \mid 2,01+87481$ \begin{tabular}{ll}
$01 G$ & $G$ \\
\hline
\end{tabular} $\epsilon 125 G^{(2)} 0^{2}, 3,0, c^{(2)} 0^{3}, 0,0, G_{0}^{2}, 1,0,1 G_{0}^{4}, 3,0, G_{0}^{3}$

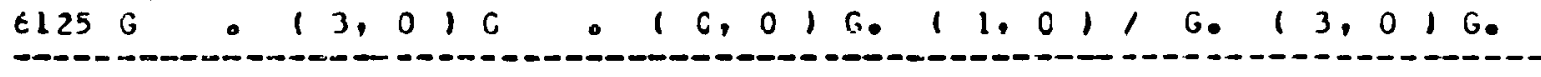
$12,01+2304 / 1225 \mathrm{G}^{(2)} \cdot 13,01 \mathrm{G}^{(2)} \cdot 12,01 \mathrm{G}^{(2)} \mathrm{G}^{3} 10,01 \mathrm{G}^{2}$

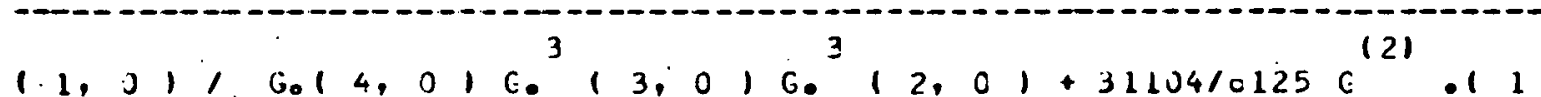
1. $1,01,6.14,016013,0,6012,01+31104 / 01256,011$

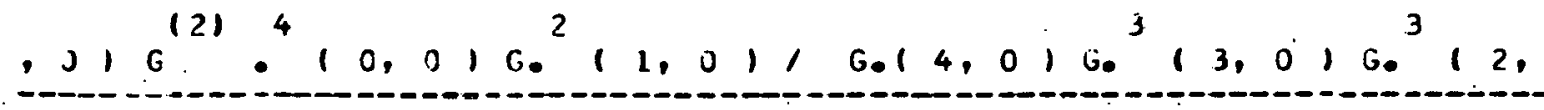


$01+46 C 9 / \varepsilon 75 G^{(2)} .13,0, G^{(2)} \cdot 4(0,0) G^{2}(1,0), G .14,0$ $\mathrm{G}^{3}\left(3,0, \mathrm{G}_{0}{ }^{3}(2,0)-2916 / 1225 \mathrm{G}^{121} \cdot 13,01 \mathrm{G}^{(2)} 2^{2} \cdot 0,01\right.$ $\mathrm{G}^{(2)}{ }^{2}\left(0,0, \mathrm{G} 0^{2}, 1,0,1, G^{3}, 3,0, G^{3}, 2,0,-291016125\right.$

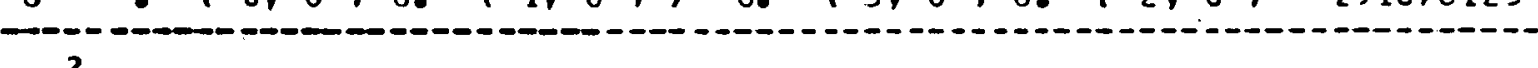
$12^{2} 1$

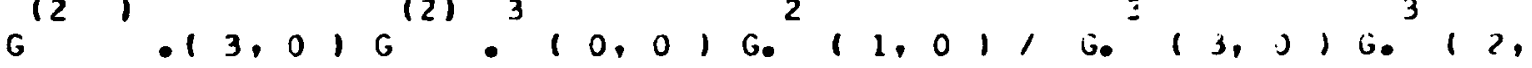

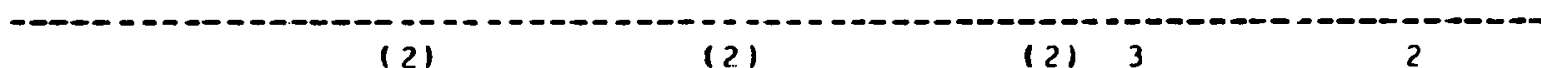
$01+1152 / 1225 \mathrm{G},(4,0, \mathrm{G}, 12, \mathrm{C}, \mathrm{G} \bullet 10,01 \mathrm{G}, 11$,

-
230 (?)

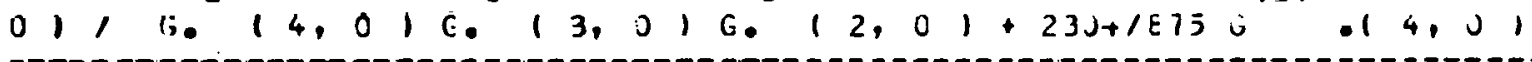

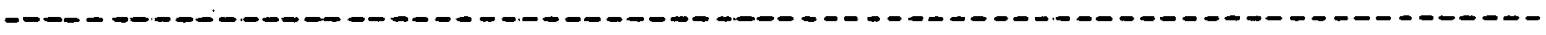
$G^{(2)}{ }^{4}\left(0,0, G_{0}^{2}, 1,0,1 \epsilon^{2}, 4, c, G_{0}^{2}, 3, j, G_{0}, 2, j 1\right.$ G-2

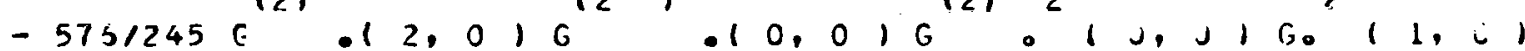

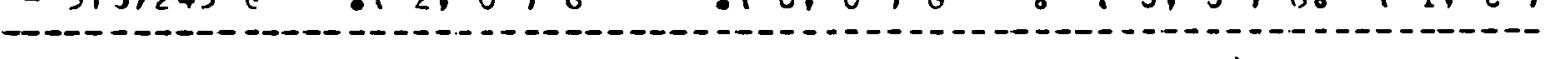
$2 \quad 3 \quad 12^{2} 1$

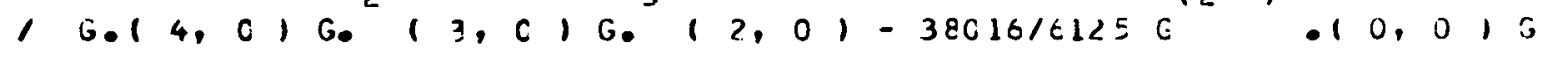
151

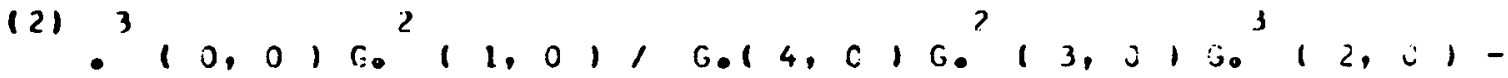

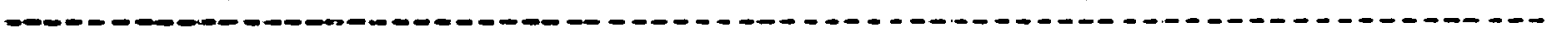
$2^{2} 1$ (द) 3 .

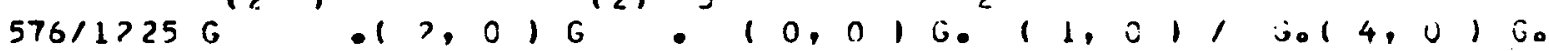
(-)$2,3,0, G^{3}(2,0)+486 / 1225 G^{12}, 010,0, e^{(2)} a^{2}, 0,0100$ $\left(1,0,1, G_{0}^{2}, 3, C, G^{3}, 12, C 1+\varepsilon C 19112250 j^{12} 12,10,010\right.$ 21 $.10,0, G_{0}(1,0,1, G,(3, C) G .12, J)+172 \varepsilon / 1225 G$ $2 \quad(2)^{3} \quad 2 \quad+\quad 2$

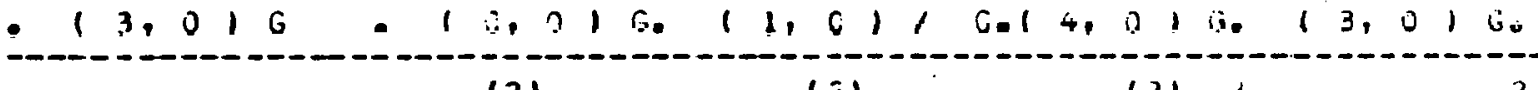

$\left(2,01+115211225 \mathrm{G}^{(2)}\right.$

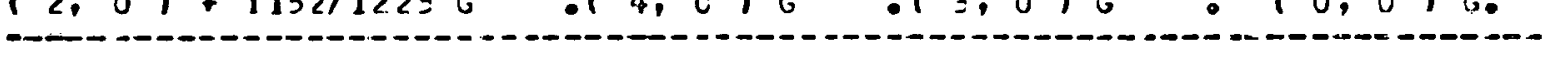
23 ? 321

$11,01,6014 ; 0,0,13,0,0_{0} 12,01,3672 / 12256,011$

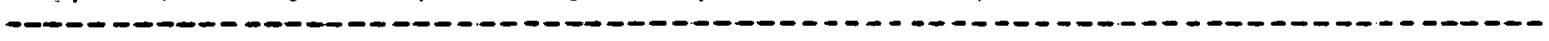
$.01 G^{121} G^{4}\left(0,0, G^{2}, 1,0,1, G_{0}^{2}, 4,01 G_{0}^{2}, 3,0, G^{2} 12\right.$ 0 


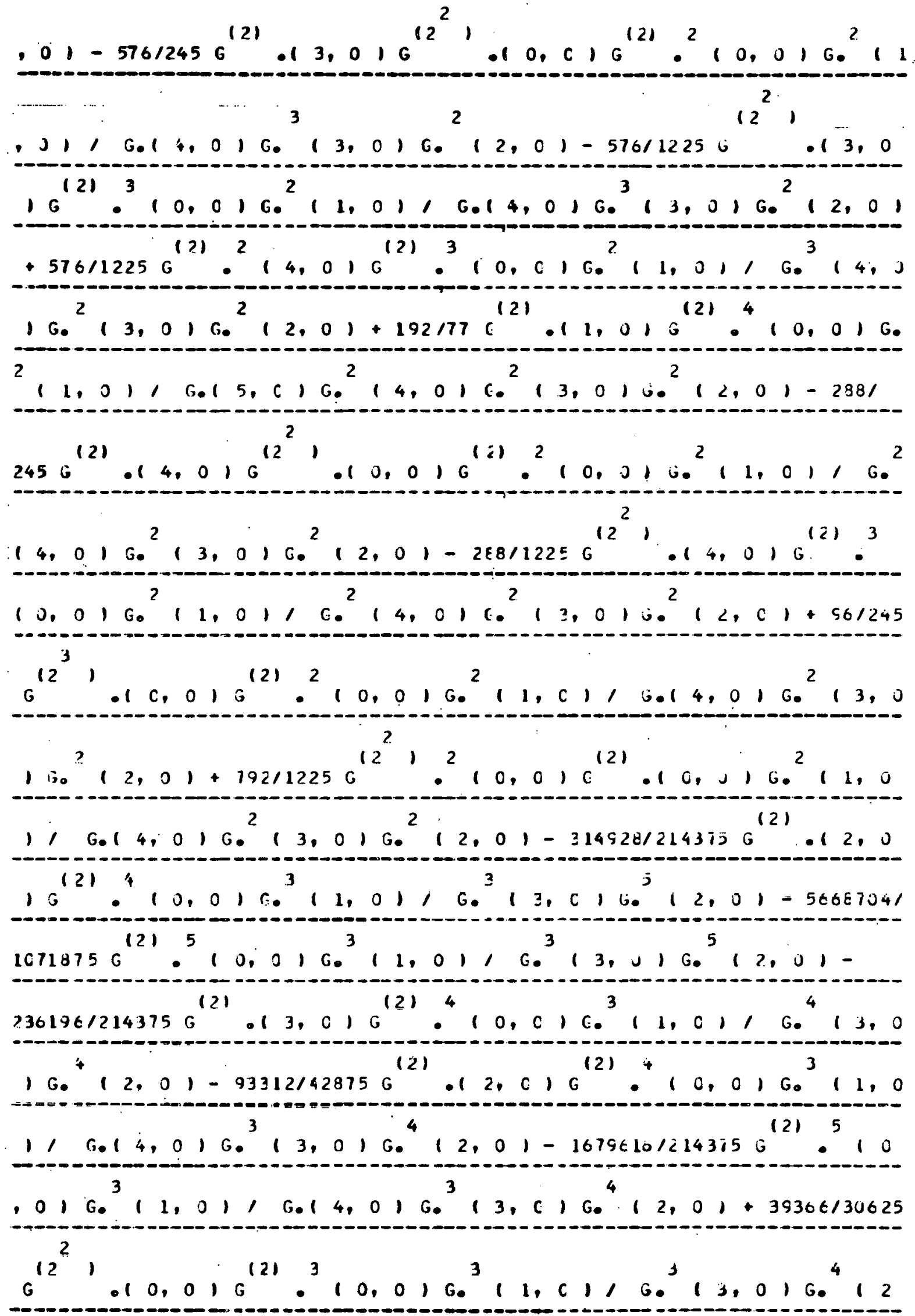


(2)

- $01-93312 / 428750$

. $13,0,0$

$(2) \cdot 10,0, G^{3}, 1,01,0.1$

$4,0, G_{0}^{4}\left(3,0, G_{0}{ }^{3}(2,0)-6144 / 2575 G^{(2)} \cdot 12, J\right) G^{(2)} \cdot 10$

, 0, G. $11,01 /$ G. $14,0, G_{0} \mid 3, c, 6012,01-311641$

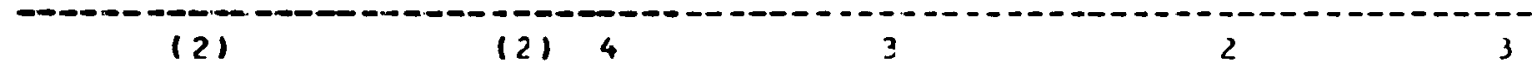

42875 (2)

$(2) 0^{4}(0,0) 0^{3}, 1,01,0_{0}^{2}, 4,0,0$

487 G 3 (2) 5 ( 3

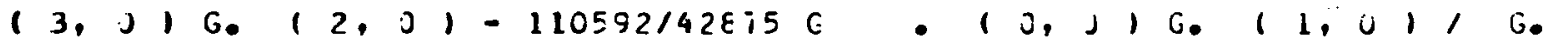
( $7 \quad 3 \quad 12^{2} 1$

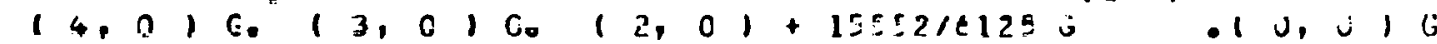
3

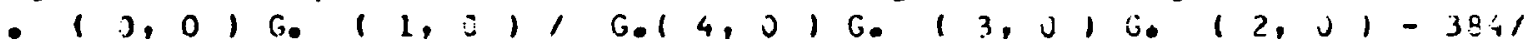

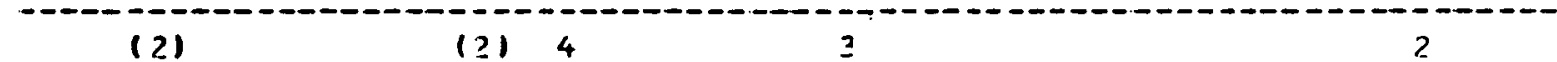

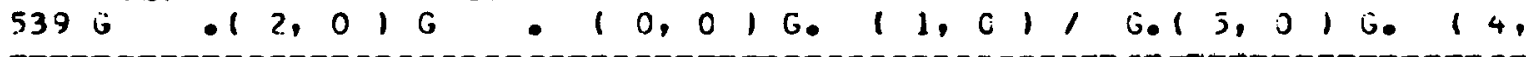
30

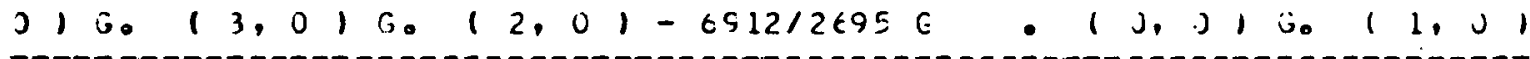
 $, G .15, c, G_{0}^{2}\left(4,0, G_{0}^{2}, 3,0, G_{0}^{3}, 2, j,-s<16 / \varepsilon 575 G\right.$

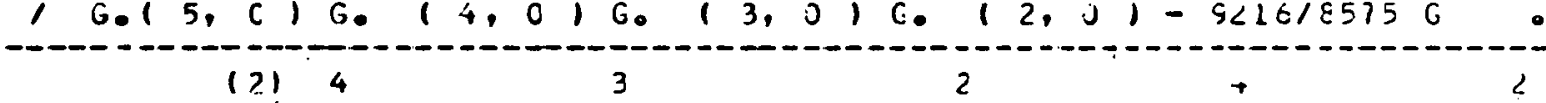
$13,016,0,0,0) G_{0}(1,0) / G_{0}(4, J), G_{0}(3,0), G_{0}$ $12,01-6144 / 8575 e^{(2)}(4,0)(a) 4, c_{0}, 3$

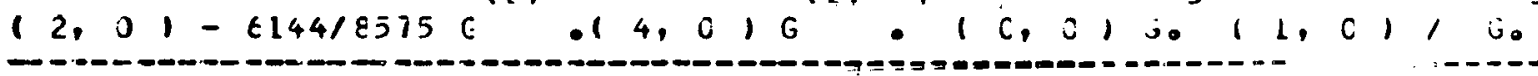

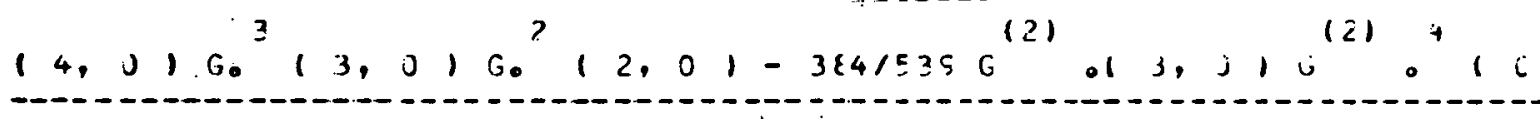

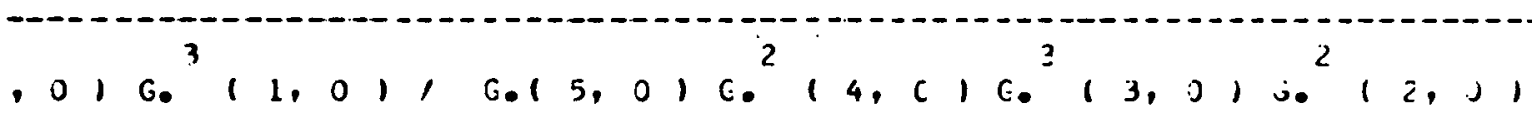
? $+1536 / 1225 \sigma^{12}, 0^{2} 0,0, G^{121} \cdot 10,01 G^{3} 11001, G_{0}^{2}, \rightarrow$ -

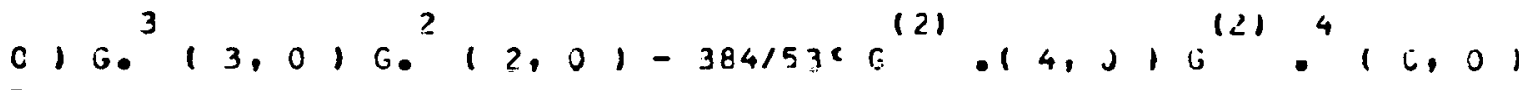

(-0.0
$G^{\prime}(1, J), G_{0}\left(\leq, 0, G_{0}^{3}, 4,0, G_{0}^{2}, 3, J, 0_{0}^{2}, 2,0,-1721\right.$ G. $539 G^{(2)} \cdot 15,0, G^{(2)} G^{4}, 0,0, G^{2}\left(1,0,1 G^{2}\left(5,0, G^{2} 1+\right.\right.$ G $01 G^{2}\left(3,0, G^{2}(2,0)+56 / 7 e^{12} 1010,01 e^{121} e^{3} 10,0\right.$ $1 G^{3}\left|1,0,1, G_{0}\right| E, 0, G_{0}^{2}\left(4,0, G_{0}^{2}, 3, c, G_{0}^{2}, 2,01+\right.$ 
$2125764 / 7503125 \mathrm{e}^{(2)} \mathrm{E}^{5} \mathrm{O}, 0, \mathrm{G}^{4}(1, \mathrm{c}), \mathrm{G}^{+} 13,01 \mathrm{G}_{0} 12,0$

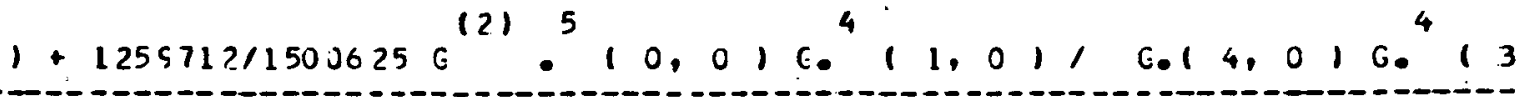
$4{ }_{4}$ $\therefore 01 G_{0}(2,01+2488321300125 \mathrm{G}, 0.0,010011,011 \mathrm{G}$ 403012150

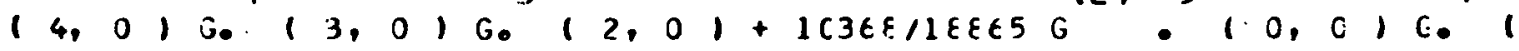
$1,0,1, G 015,0, \epsilon_{0}^{2}\left(4,0, G_{0}^{3}, 3,0, G_{0}^{3}, 2,0\right)+153841$ 0

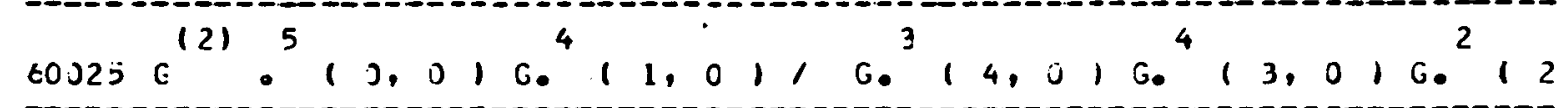
(1000

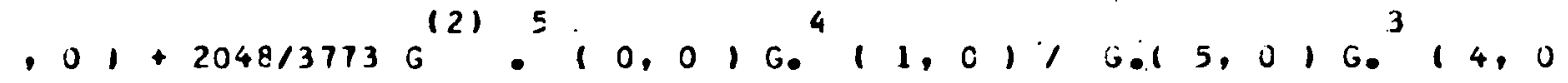
0 $1 G^{3}\left(3,0, G_{0}^{2}\left(2,01+1600 / 5 s=9 G^{(2)}{ }^{5} 1, \mathrm{~J}_{1} \mathrm{G}_{0}(1,0)\right.\right.$ $, G 0^{2}, 5,0, G 0^{3}, 4,01 G^{2}, 3,0, G^{2} 12,01+20736177077 G^{1}$ $2)^{5} 0_{4} G_{0}$

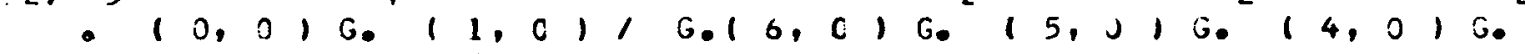

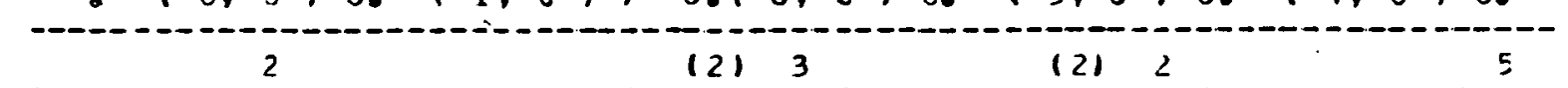
$\left.(3,0) G_{0}(2,0)+56 / 25 G \quad \bullet 12,0\right) G \bullet 1,011 G_{0}$ $12)^{2}(2) ?$

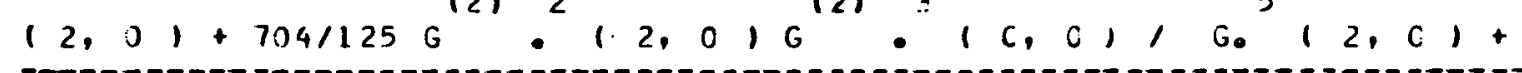
121 $256 / 75 \mathrm{G} .12,01 \mathrm{G}^{\circ}, 0,01 / \mathrm{G}, 12, \mathrm{~J}, 05072146875 \mathrm{G}$ 256175 G 15 (2) 4 (2)

$\circ(0,0)$, G. $(2,0)+4 / 5 G \cdot 12,0100,10,0116$ 0 5 (द) 2 (2) 1212

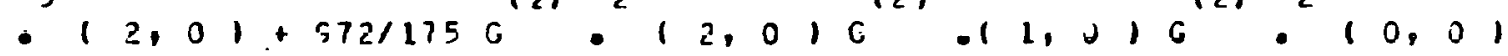
11290 1

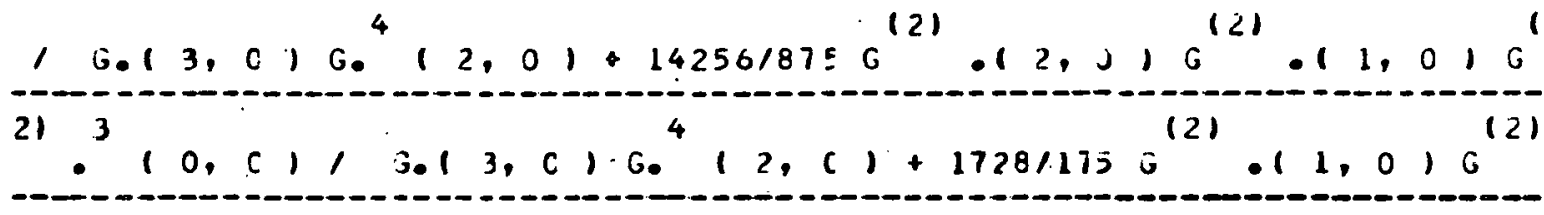
4

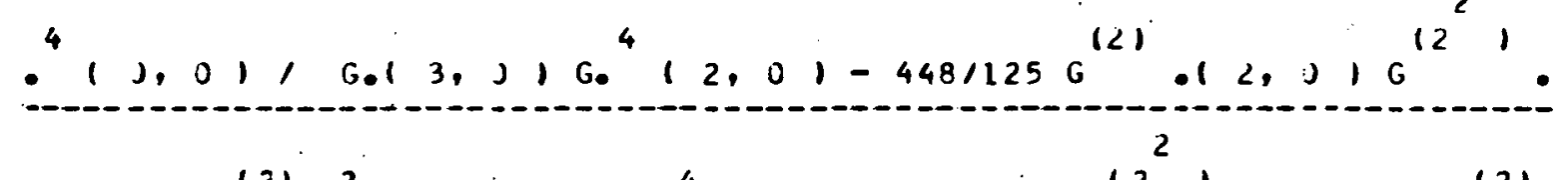
$\left(0,01 G^{(2)} 0^{2}, 0,0\right), G_{0}^{4}, 2, C 1-72 / 25 G^{12}, 012,01 G^{121}$ 10,0 10 
$.12,01 \mathrm{G}^{(2)} 0^{2}, 0,01, G^{4}, 2,01-52816250^{12} 10.10,01 G^{1}$ (2) 3 (

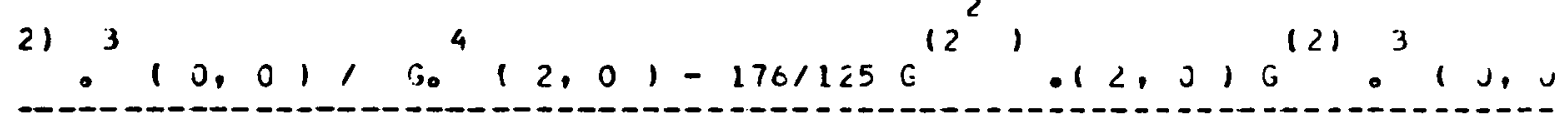

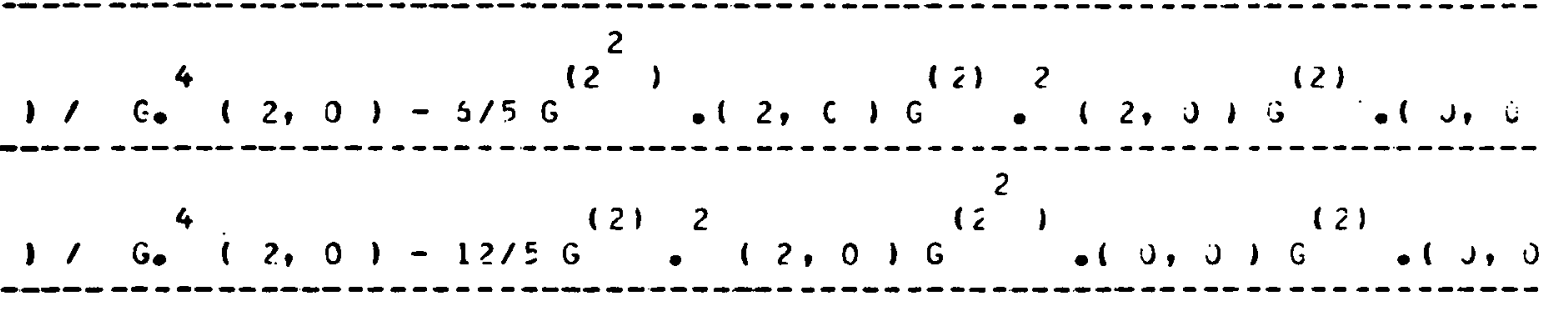

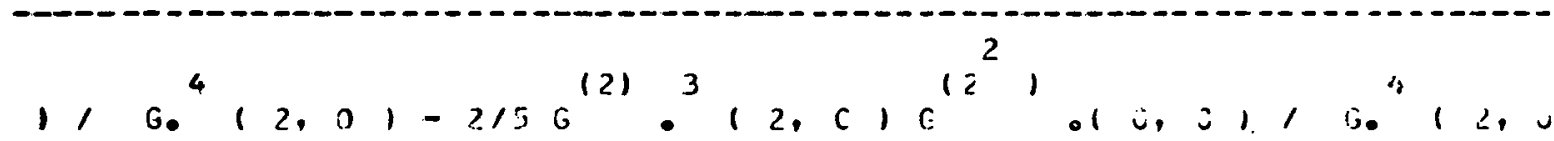
000 $1+648 / 17 E^{(2)} \cdot(3,0) G^{(2)} \cdot\left(2,01 G^{(2)} \cdot 11,0\right) G^{(2)} G^{2}, J, J$

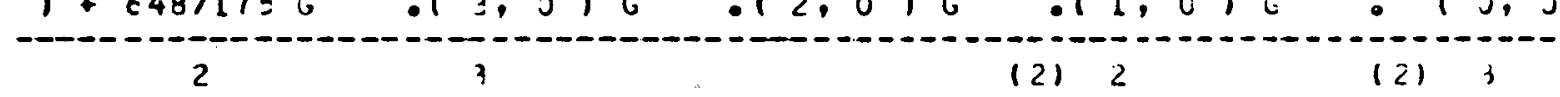
$11 G_{0}(3,0, G, 12,01+1 \in 03 \varepsilon 1 \in 12560,11,010,01$ $0,0,1 G_{0}\left(3,0, G_{0}^{3}(2,01+47521875 G\right.$ G $.13, j, 0.01$ 0

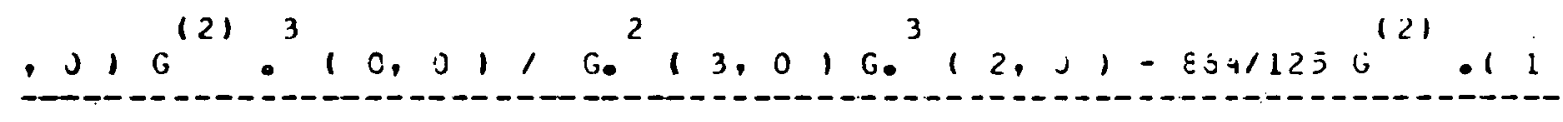
$, 016^{12^{2}}, 10,010^{(2)} 0^{2}, 0, c 1,0.13, j 1 c_{0}^{3} 12,01-224$ 15
1 $11756^{12^{2}} ! \cdot 12,0, G^{121} \cdot 11,016^{(2)} 0^{2} 10,011,0.13,010^{3}$ -

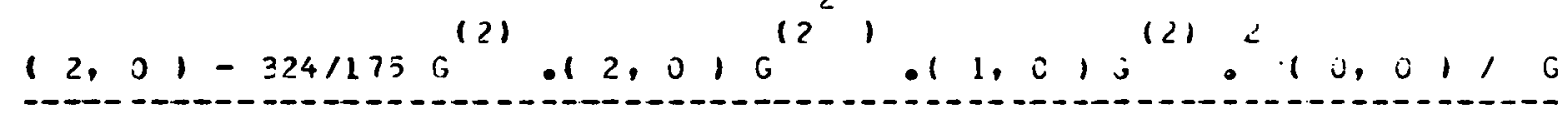

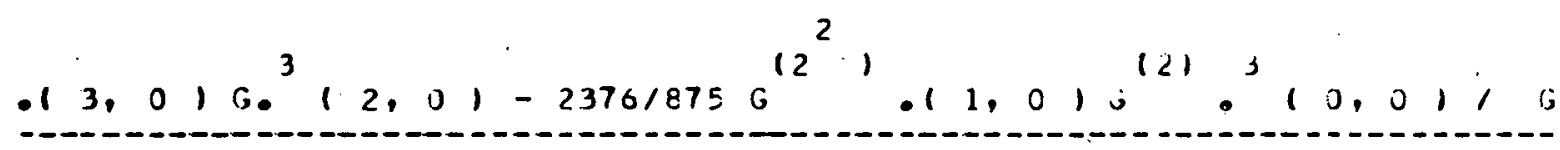

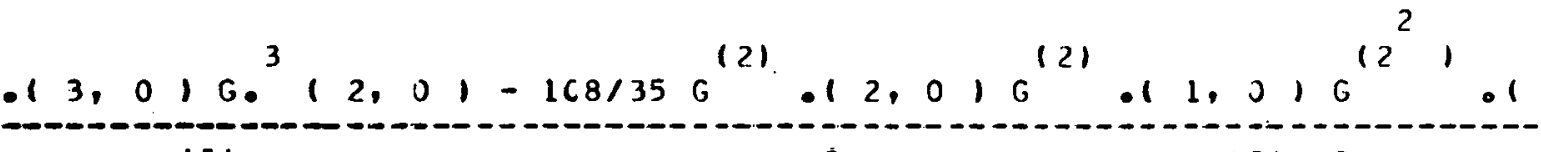

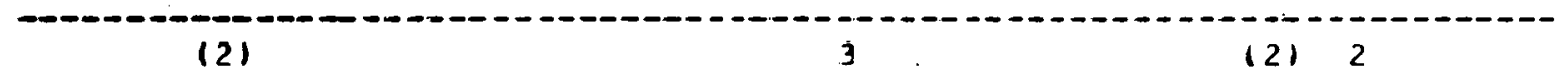
$0,0, G, 10,0), G .13,0, G_{0}(2,0,-1 / 106,02,0)$

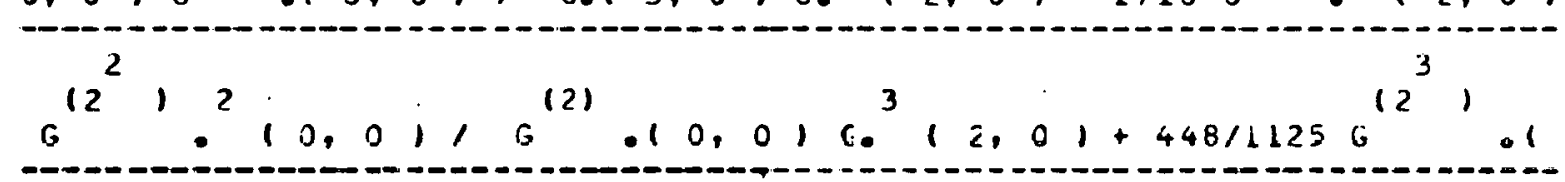


$0,0, \mathrm{G}^{(2)}{ }^{2}\left(0,01, \mathrm{G}^{3}\left(2,01+8 / 25 \mathrm{G}^{(2)} 1,12,01 \mathrm{G}^{(2)} 0^{2}\right.\right.$

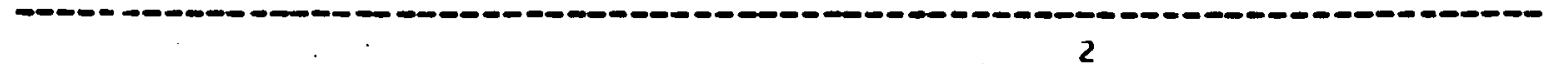
$10,01, G_{0}^{3}(2,0)+4 / 25 G^{121} \cdot 12,01 G^{12} 122^{2}, 0,01, G^{3}$

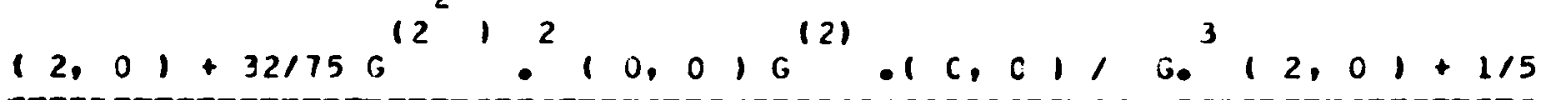
2

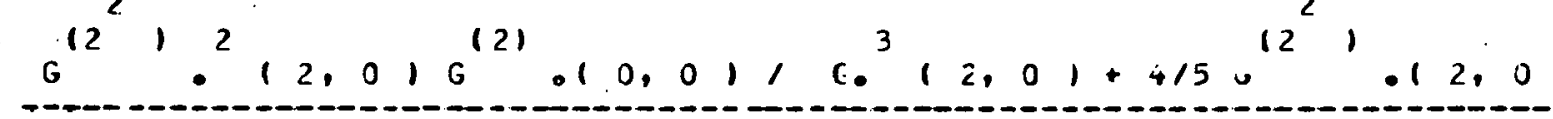
$12^{2} 1 \quad(2) \quad 3 \quad(2)$

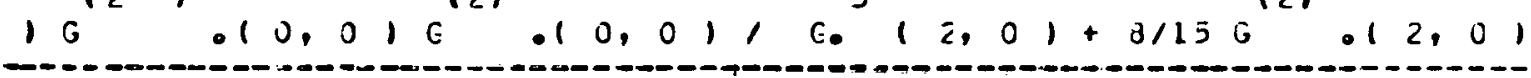
(-

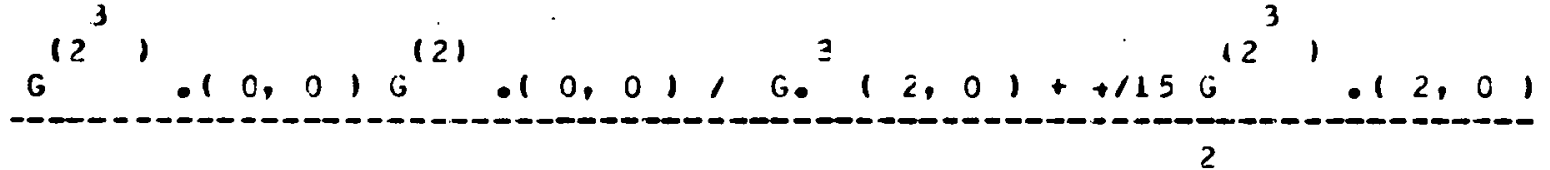

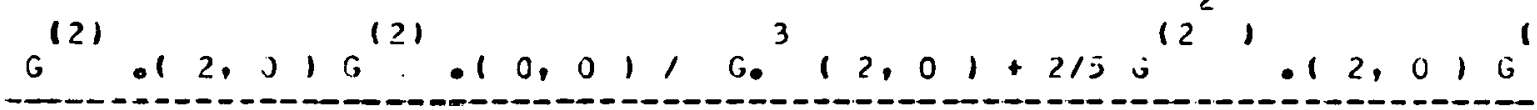
-

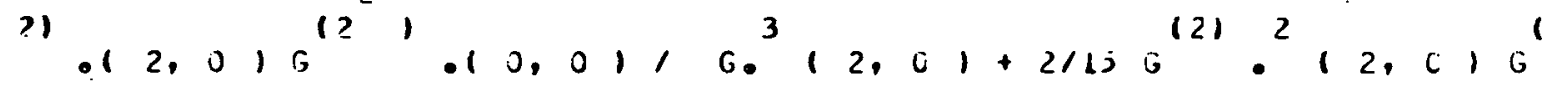

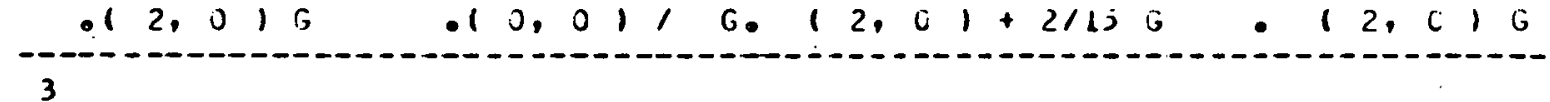
$2^{3} 1$ 3

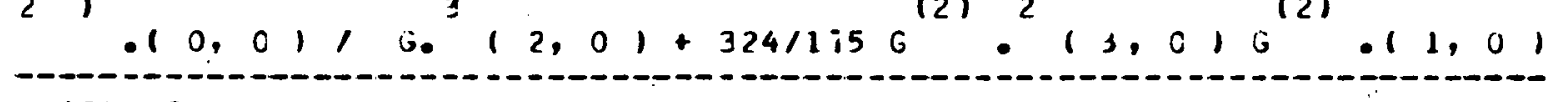

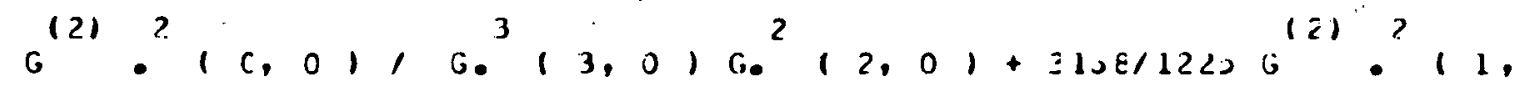

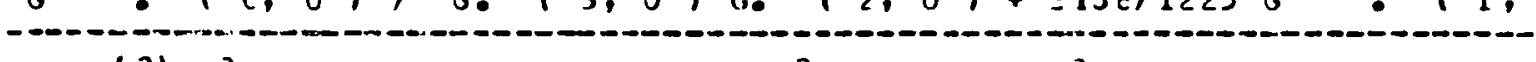
$010^{(2)} \cdot\left(0,0,1, G .14,0, G_{0}^{2}, 3, C, G e^{2}(2, J 1-16211756\right.$

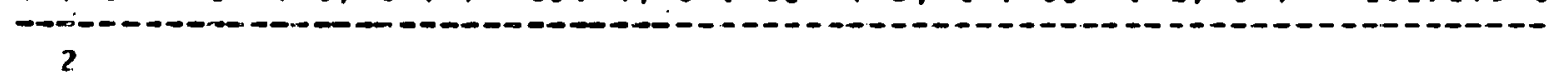

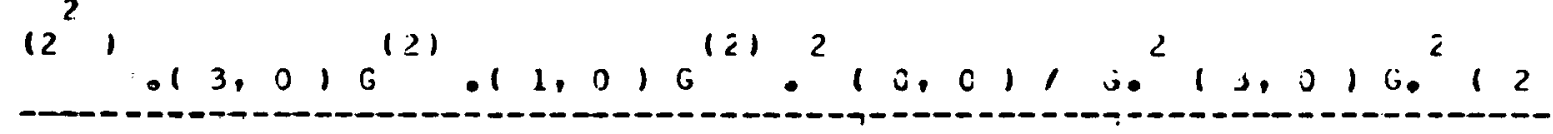

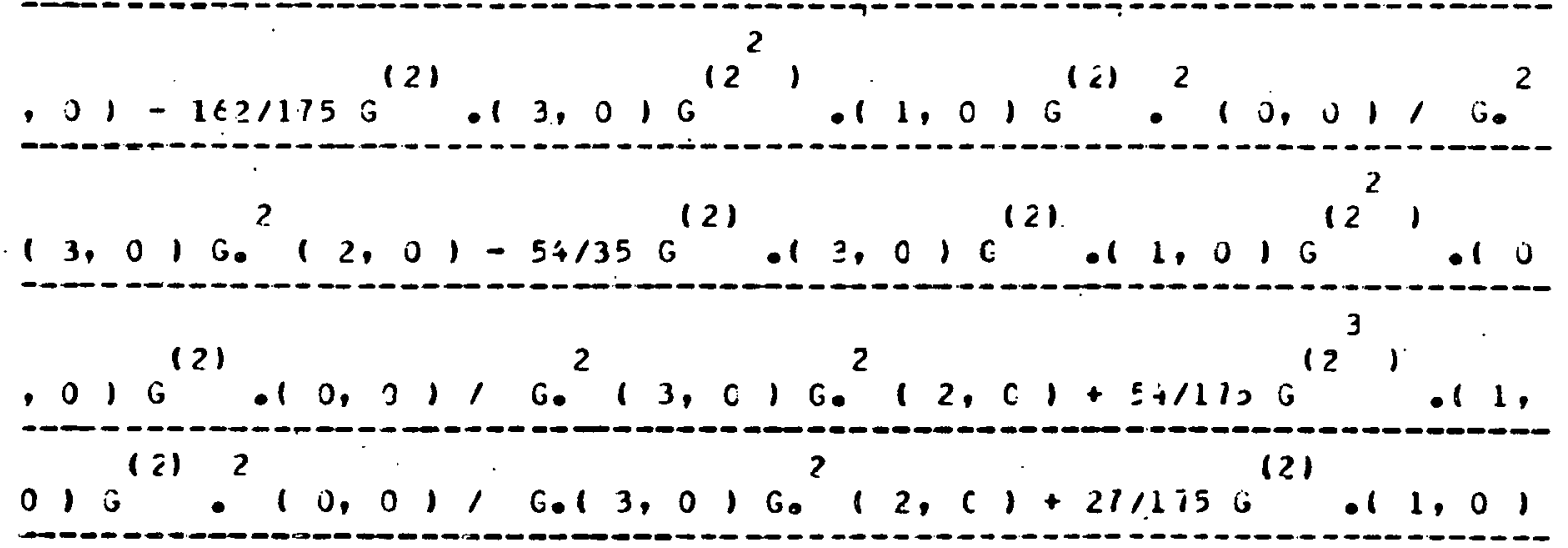




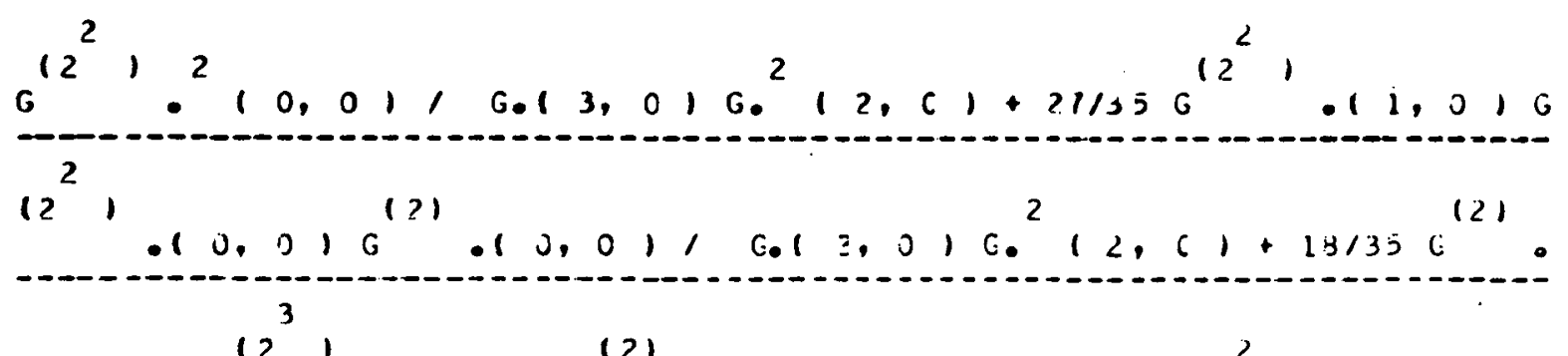

$11,0, G^{12}, 010,01 G^{121}, 10,01, G_{0} 13,01 G^{2}, 2, i 1,11$

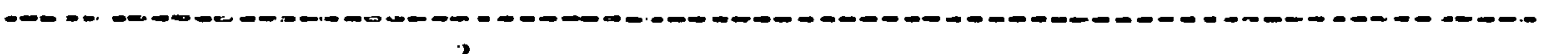

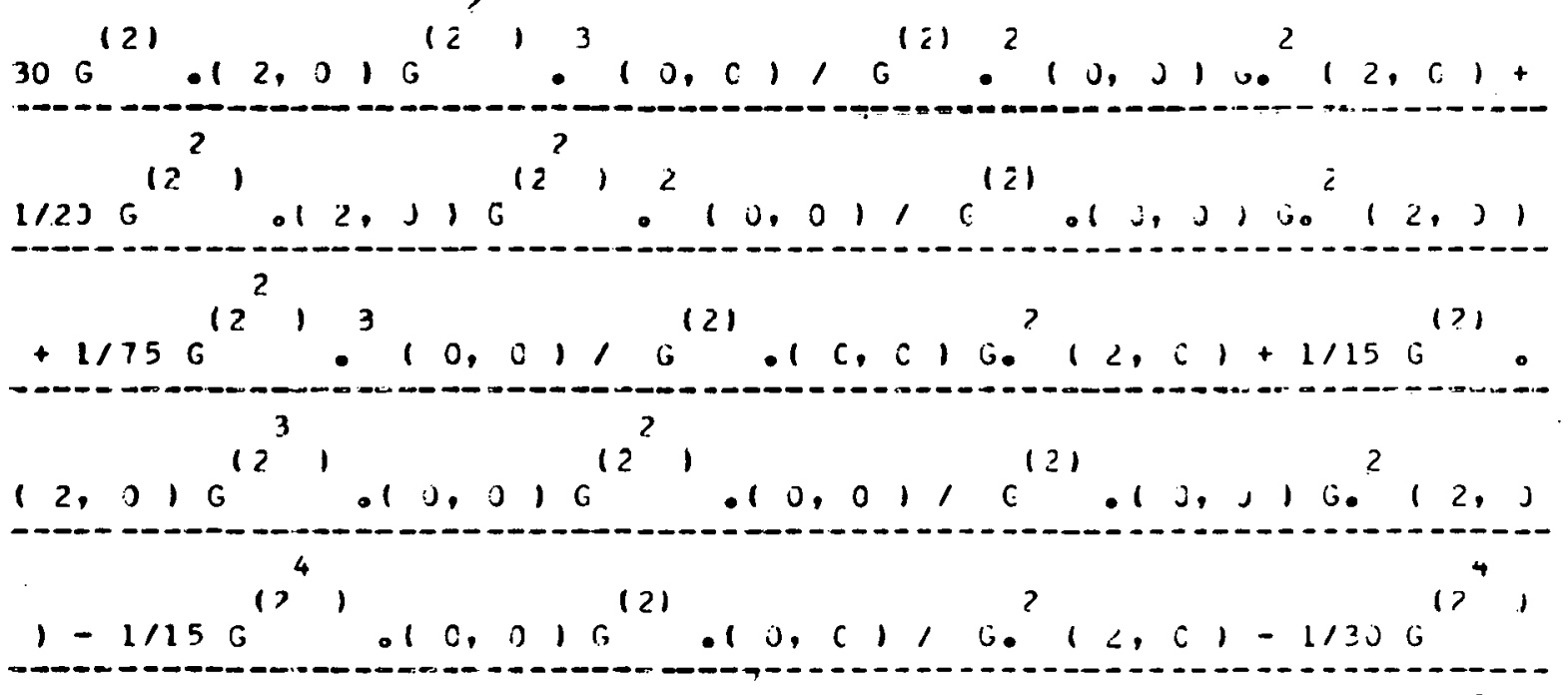

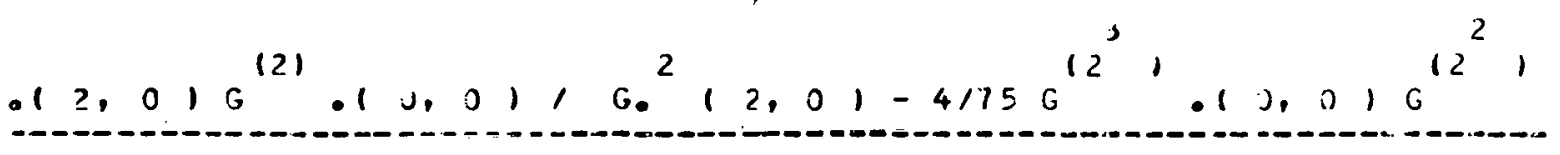

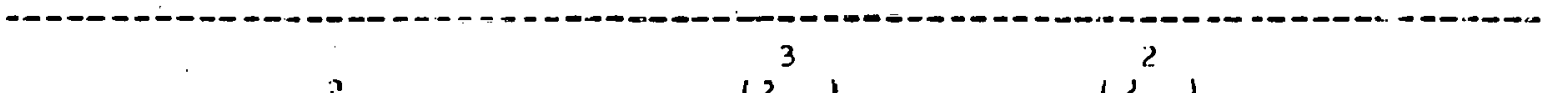
$.10,01,0_{0}^{2} 12,01-1 / 150^{12}, 12,011^{12} 12^{2} 0100110$.

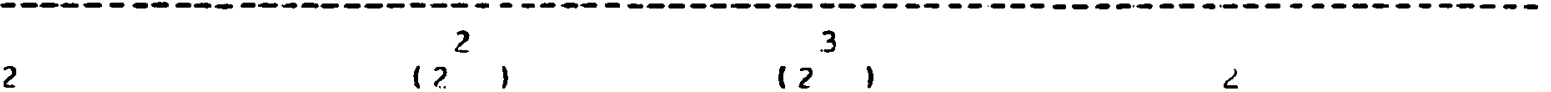

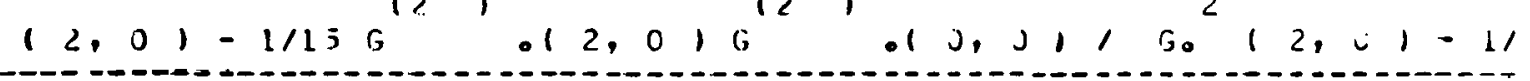
1
1 $30 \mathrm{G}^{121} \cdot 12,0, \mathrm{G}^{12^{4}}, 10,01, \mathrm{C}^{2}, 2,01-1 / 30 \mathrm{G}^{12^{2}},{ }^{4} 10$

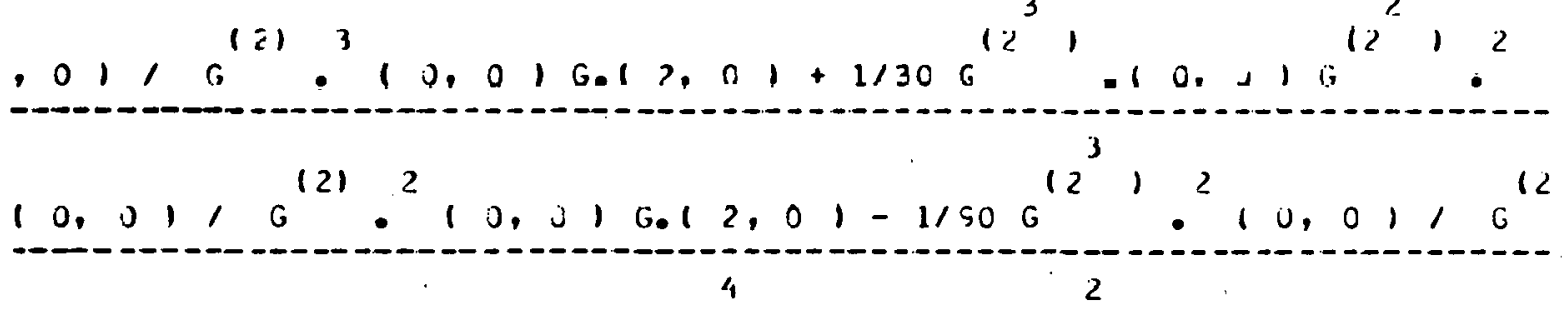
$\left.1.10,0, G .12,01-1 / 60 G^{12}\right) .10,01 G^{12}, .1,01 a^{(2)}$

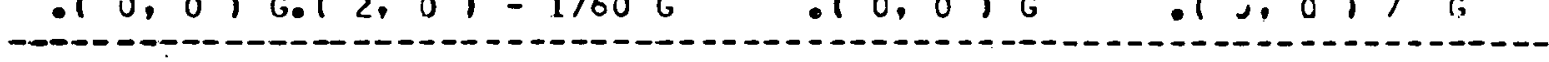




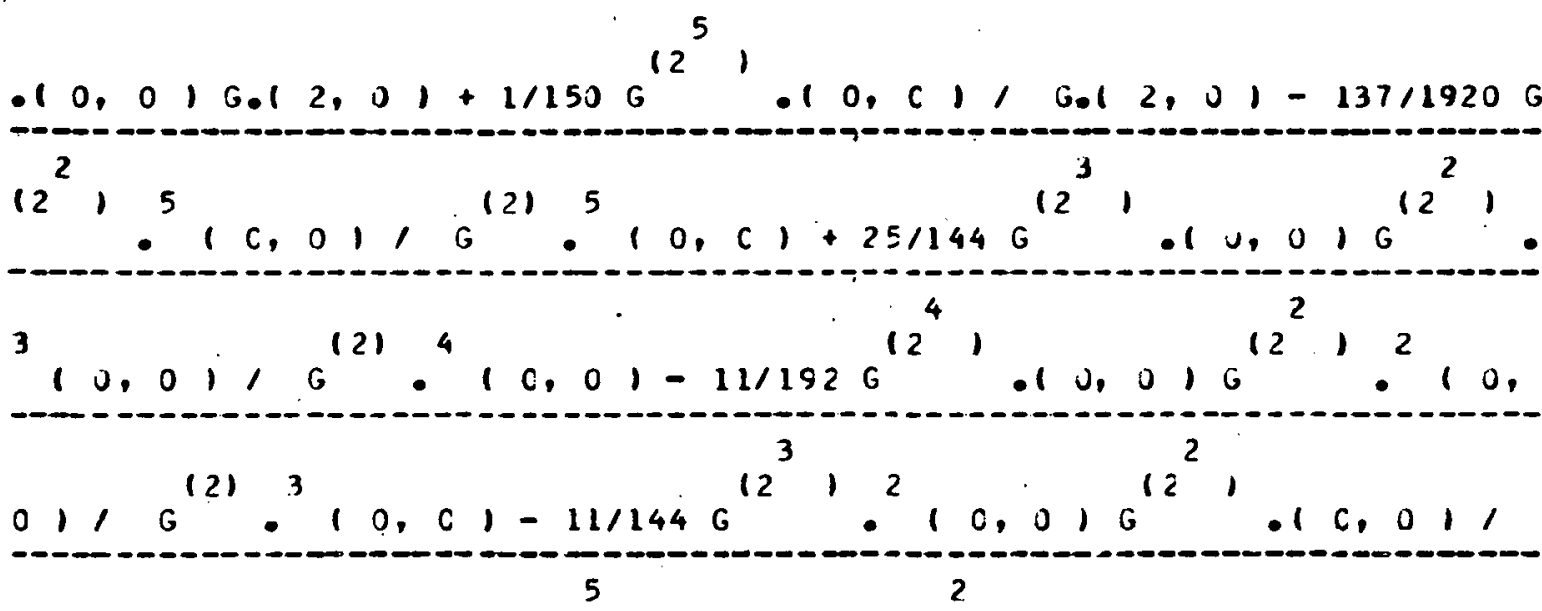

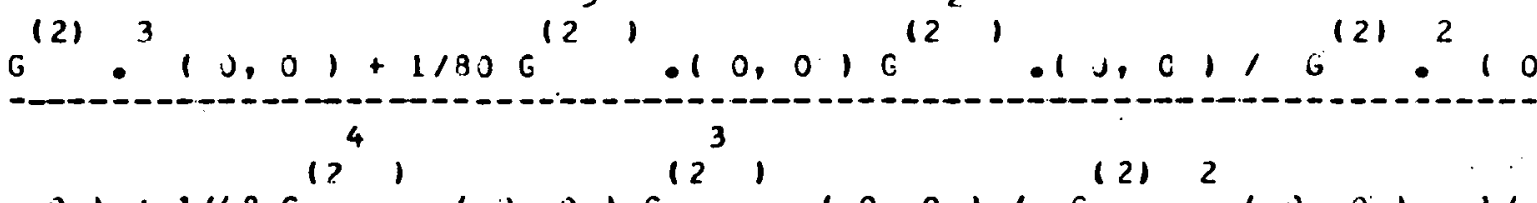

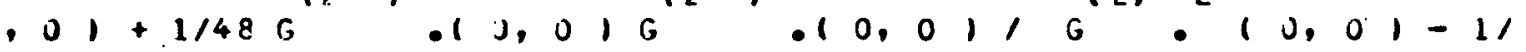
60 $720 \mathrm{G}^{12^{6}}, .10,0, \mathrm{G}^{(2)} .10,01$

$(2) 2$

$C(5,2)=25921875 G^{2} \cdot 12,0, G^{2} \cdot 10,016.11,0116.13$

C

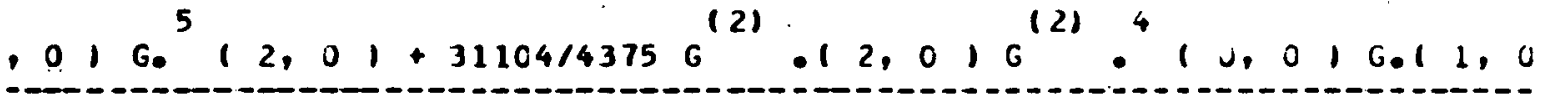

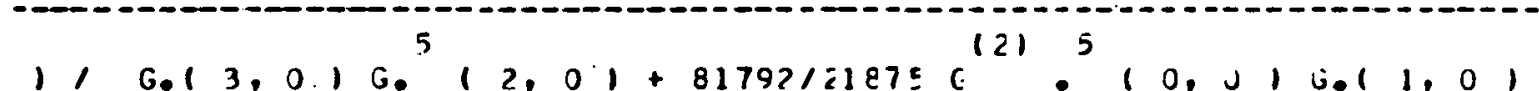
$-0$

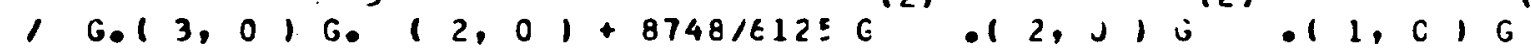
213 $\because\left(0,0, G, 1,0,1, G_{0} \mid 3, C, C_{0}, 2, J 1+1253 / E 75 G\right.$. $13,0,6^{121}+\left(2,0,6^{(2)} 0^{3}(0,0) 6.1,1,0,1,60^{2}, 3,0,6^{4}\right.$

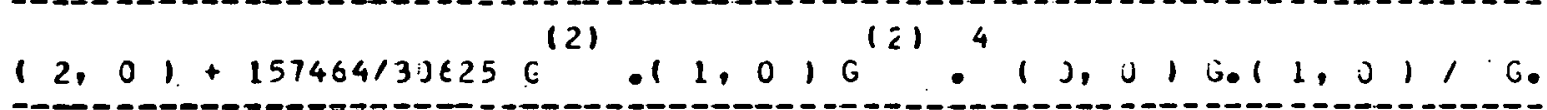
$2-121 \quad 121+0_{0}$ $213,01 \epsilon_{0}(2,0)+7776 / 4375 \mathrm{G} \bullet 13,01601010,01001$ $1,0,1 G_{0}^{2}, 3,0, G^{4}, 2,01-648 / 125 G^{(2)}, 12,0, G^{(2)} 1.10$ 1. 0 1 0 ( $.01 G^{2}, 10,0, C_{0}\left(1,0,1, G .13, c, G_{0}^{+}, 2, J 1-223501\right.$

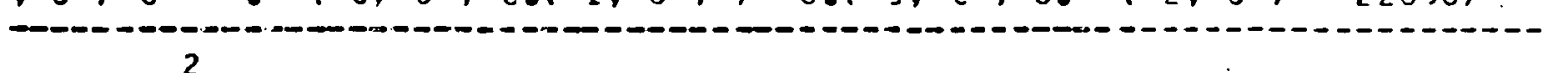
$4375 G^{12}, 010,0, G^{(2)} 0^{3}, 0,010.11,01,0.1,0,0.42$ 0 


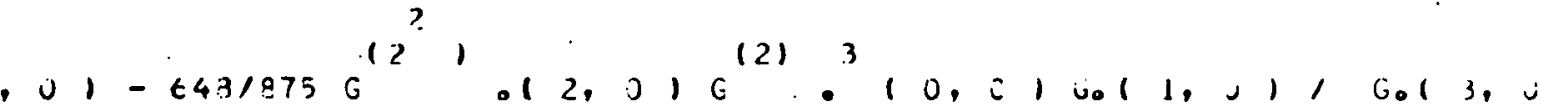
0

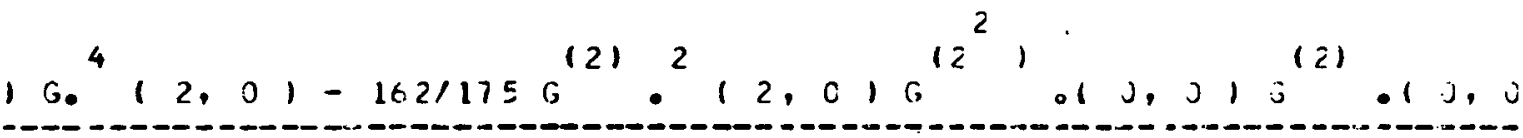

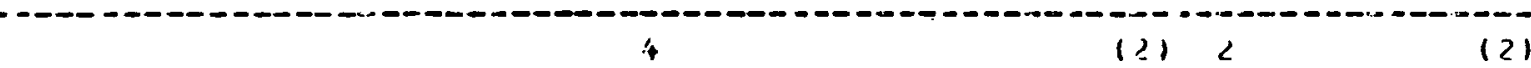

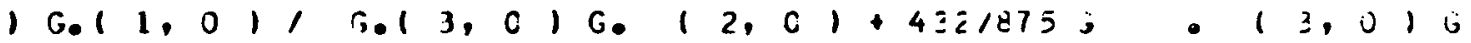
10 $3 \quad 3 \quad 2 \quad 3 \quad(2)$

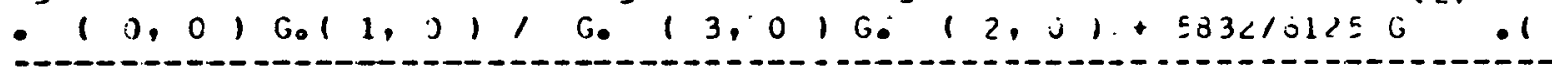
$3,0, G^{121} \cdot 1,0, G^{121} 0^{3}, 0,0, G, 1,0,1,00^{3}, 3,0, G^{3} 1$ 3. 0 10

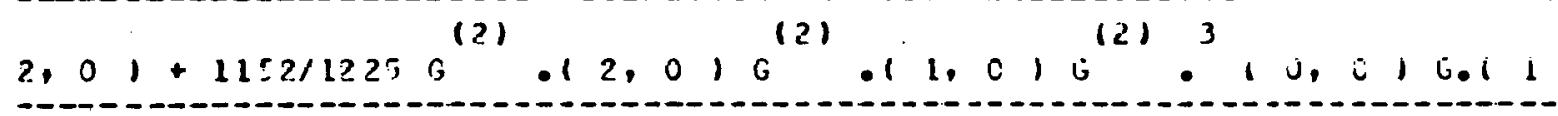

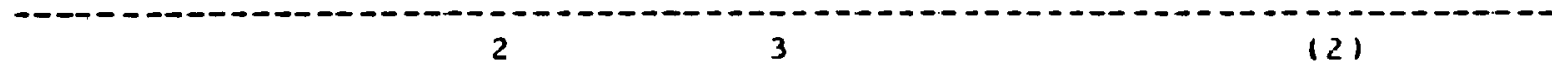
$.01,6.14,0, G_{0}(3,0,6,12,01+26756 / 61226$. 1,6 $1 G^{121} 0^{4}\left(0,0, c_{0}\left(1,0,1, G .14, c, G_{0}^{2}, 3,0, G_{0}^{3}(2,0)\right.\right.$ (

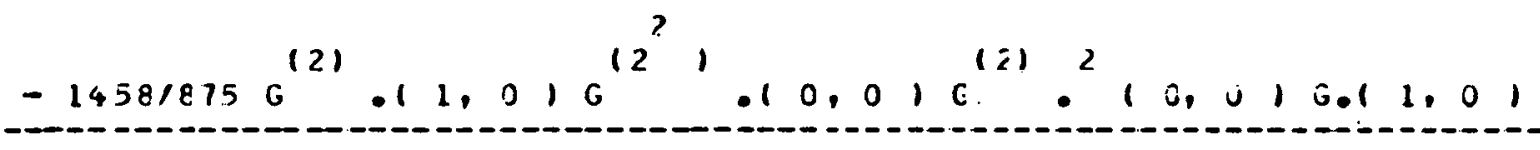
, jo ${ }^{2}, 3,0, G^{3}, 2,0,-215 / 125 G^{(2)}, 13, j, G^{\left(2^{2},\right.}, 10,01$

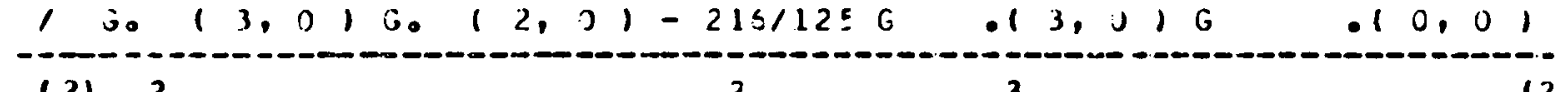
$G^{(2)}{ }^{2}, 0,0, G 011,01, G^{2}, 3,0, G^{3}, 2,0,-145 \varepsilon / 6125 G^{12}$

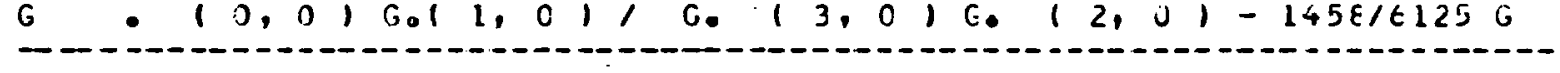
?

$1.11,0, G^{(2)} 0^{3}\left(0,0, G .11, c 1, G^{2}(1,0) 0^{3}(2,0)\right.$ $0.12,010$ $-2161875 e^{12^{2}}, 013,01 G^{(2)} 0^{3}, c, 0, G, 1,0,1, G_{0}^{2}, 3,01$

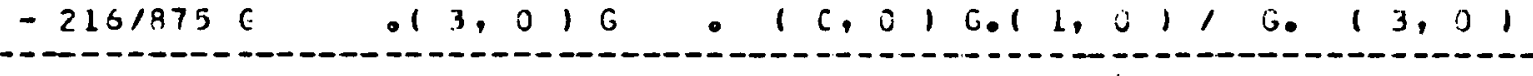
$G^{3} 12,0,-10811750^{(2)} \cdot 13,01 e^{(2)} \cdot 12, J 10^{12} 1010, c 1 c^{1}$ 2)

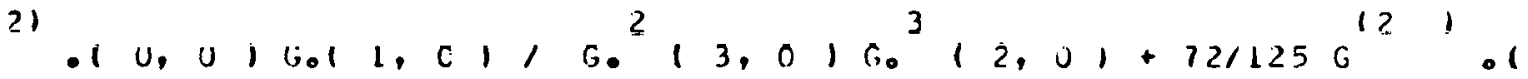

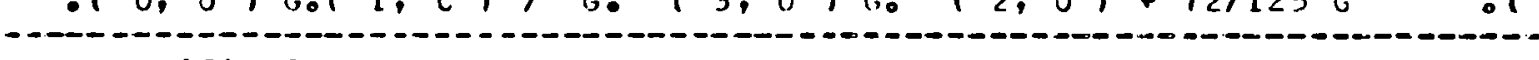
$0,0, G, 010,0, G_{0} 1,0,1, G_{0}, 3,0,0_{0}, 2,01+31 / 1730$ (-) $(2) .12,0, G^{12}, 0^{2}, 0,0,0.11,01,0.1,0,0,12, j 1$ ( $12421 \varepsilon 75 \mathrm{G}^{12^{2}} 12,01010$

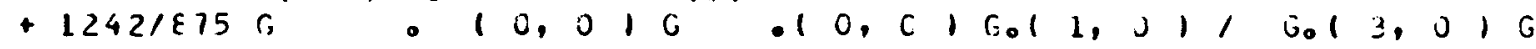




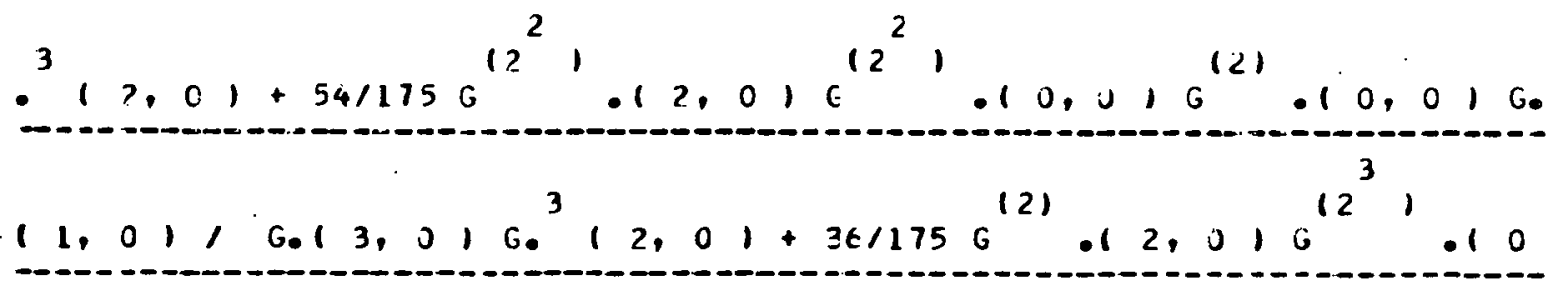
(2) 3

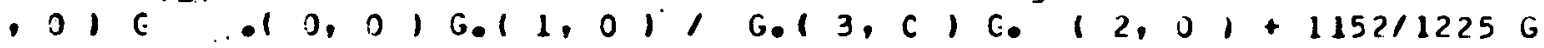
(5) 121 (2) (2) 3

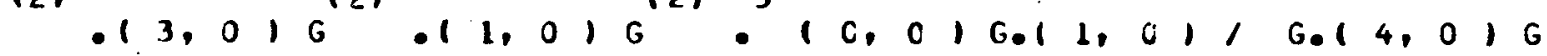

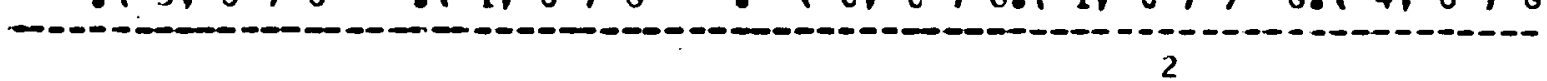

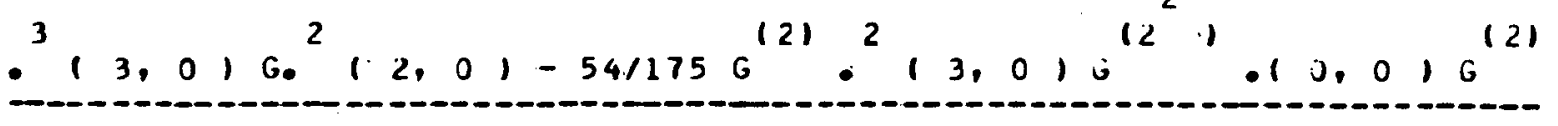
$.10,0, G .11,0,1 G_{0}^{3}\left(3,0, G_{0}^{2}, 2,0,+516 / 1225 G(2), 14\right.$, $(2) \quad(2) 3$

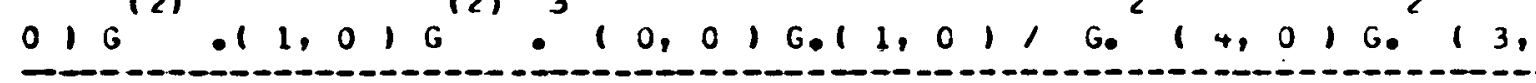

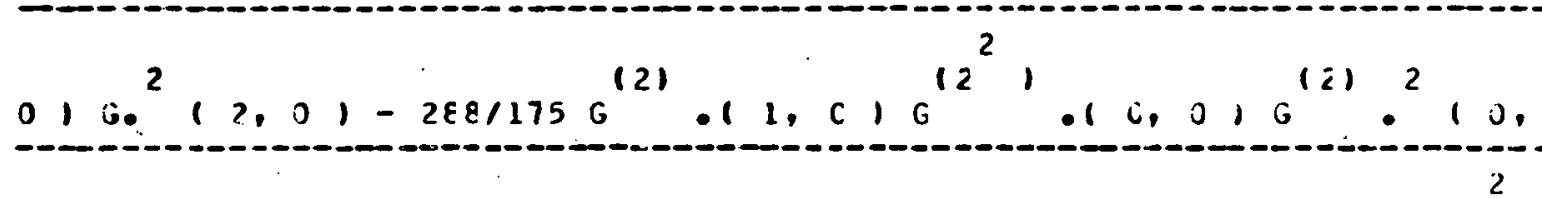

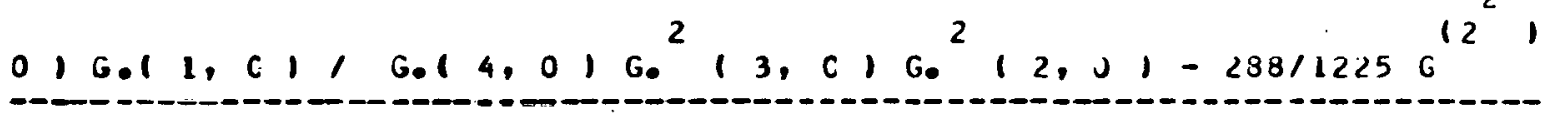
1213 $.11,0, G^{\circ}, 0,0, G_{0}\left(1,0,1, G, 14,0, G_{0}, 3,0,6.12\right.$ -

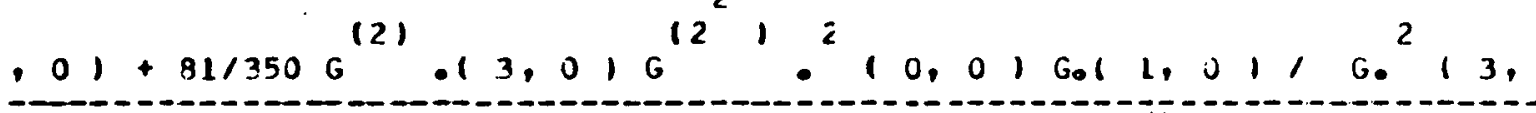

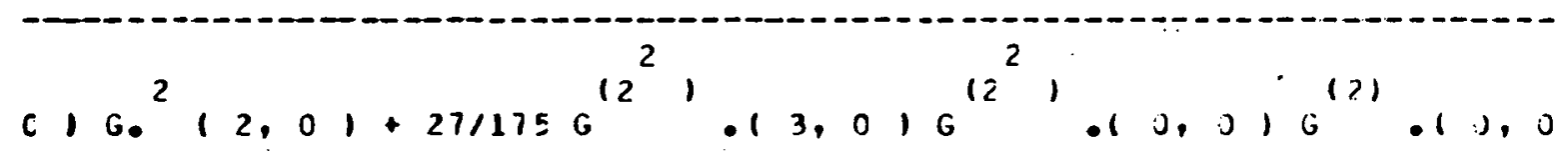
(

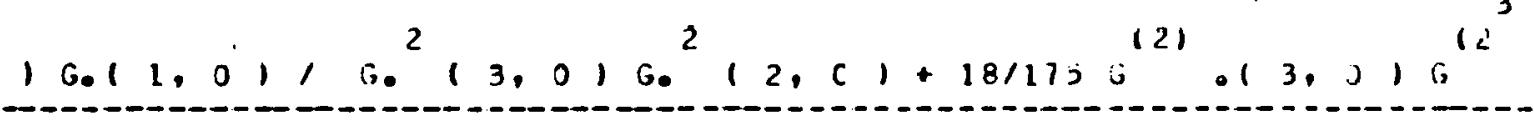
(2)

$.10,0, G^{1}, 0,0, G .11,0,1, G, 13, J, G, 12,01+91$ 0

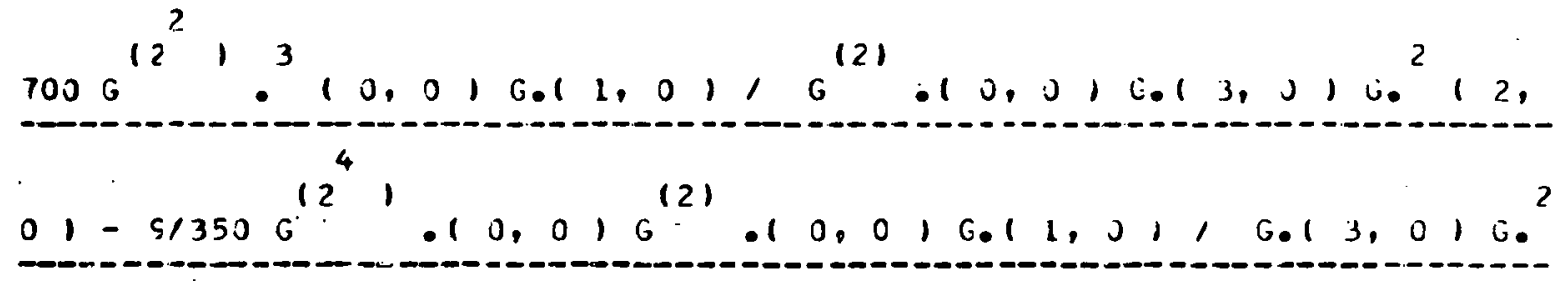
$\begin{array}{ll}3 & \\ 3 & \end{array}$

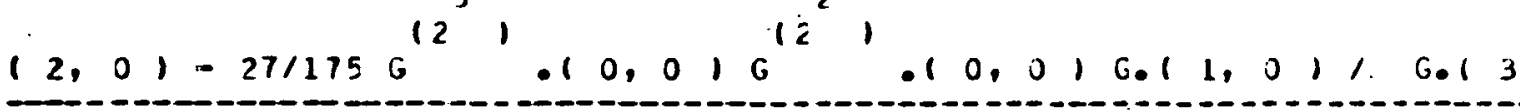

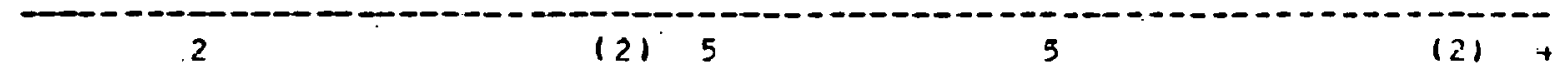

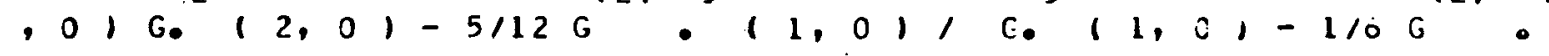




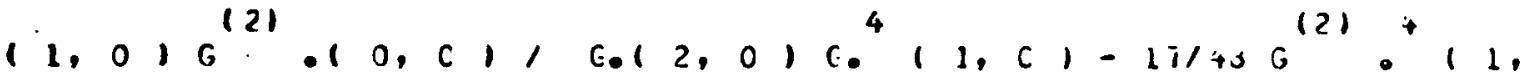

1
1 $0, G^{12}, .10,0,1 G^{121} \cdot 1,0,0, G^{4}\left(1,01+11112 r^{12}, 011\right.$

$(2) 33(2)(2) 3$

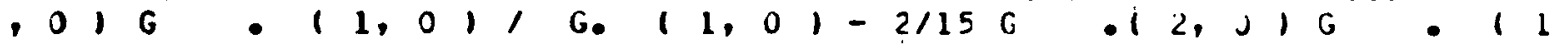
-

(2) 2

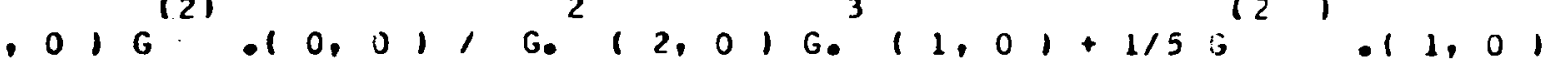
$(2)^{2}{ }^{2}(2)$ $G .11,0, G .10,01, G .12,0, G, 11,01-2115 G$

G-..-

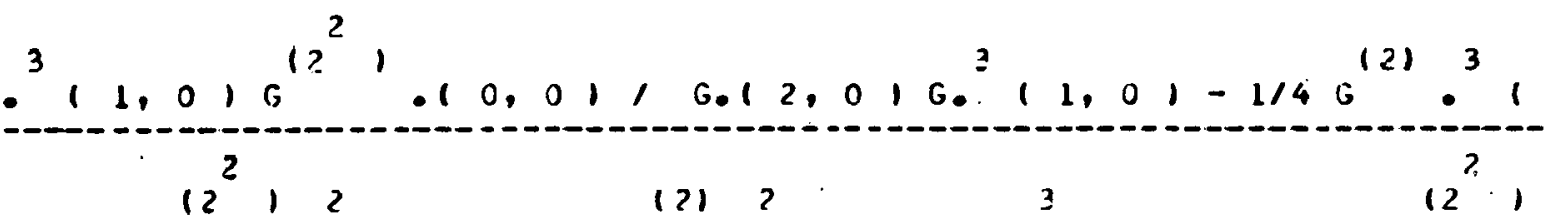
$1,016^{2}, 0,0,1 G^{(2)} e^{2}, 0,0,0^{3}, 1,0,1+5 / 86$ 10 $\left(1,0, G^{12)} \geq 1,0, G^{12}, 010,01, G^{121}\right.$ ol $3,0, G_{0}^{3} 11,0$ 3 ( 3210

$1+5 / 36 G^{(2)}:^{3} 11,0, G^{12} 1,10,0,1, G^{(2)} .1,1,1, G^{3}, 1,0$

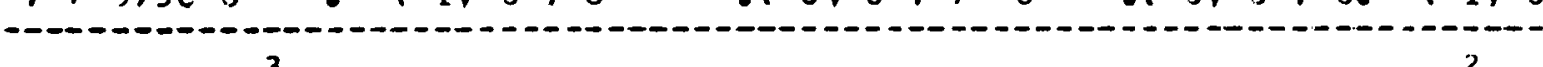

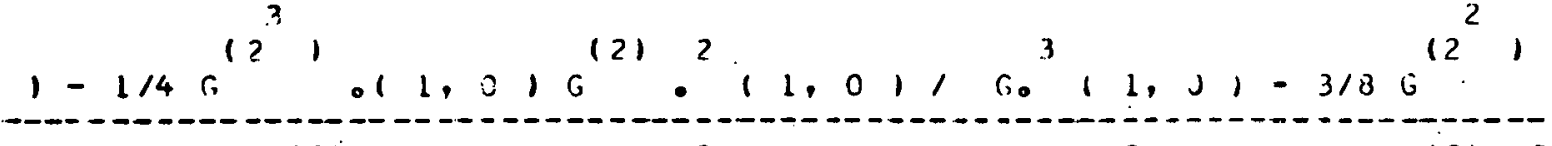
$2,1,0, G^{121} \cdot 1,01,0_{0}^{3} \mid 1,01+\varepsilon / 250^{121} \cdot 12,0,6^{(2)} 0^{2}$

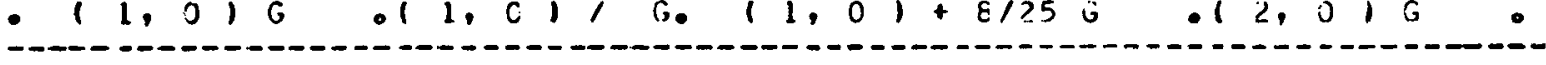
$\left(1,0, G^{(2)} 0^{2}(0, C), G_{0}^{3}(2, C) G_{0}^{2}(1, J)+16 / 75 G_{0}^{(2)}<\right.$ $1,1,0$ r 6

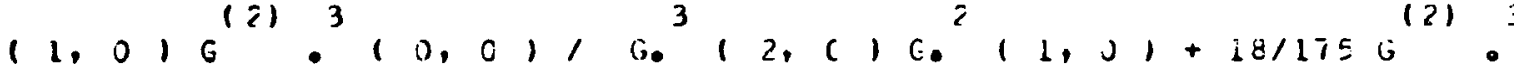

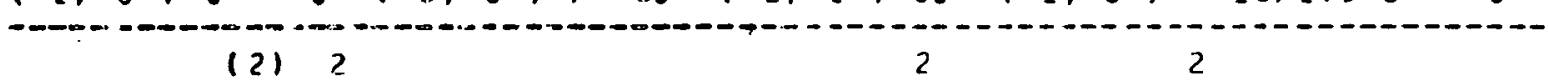

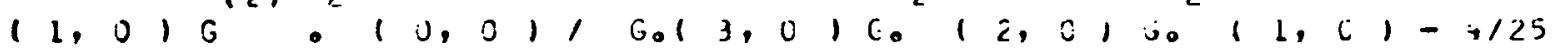
\begin{tabular}{l}
15 \\
\hline
\end{tabular}

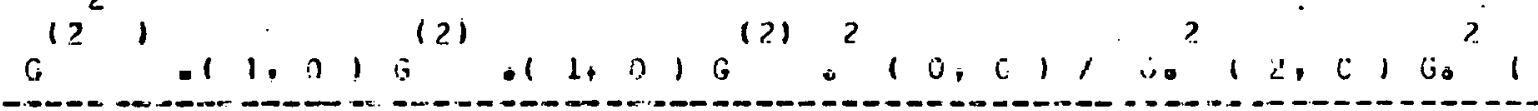
(121 $1,01-6 / 25 G^{121} 0^{2}\left(1,0,0^{12} 1010,0, G^{121} \cdot 10,01, c^{2} 1\right.$

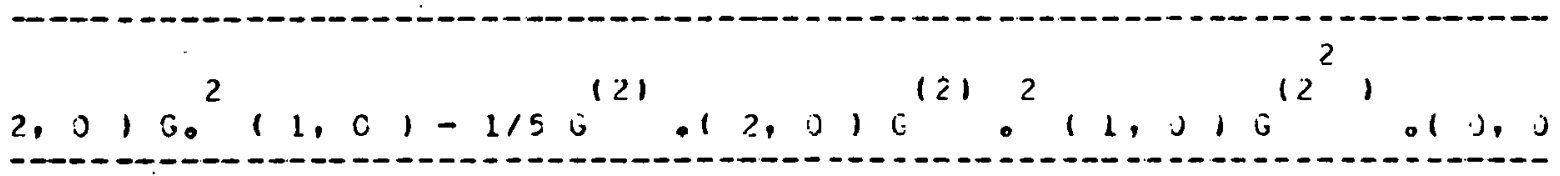

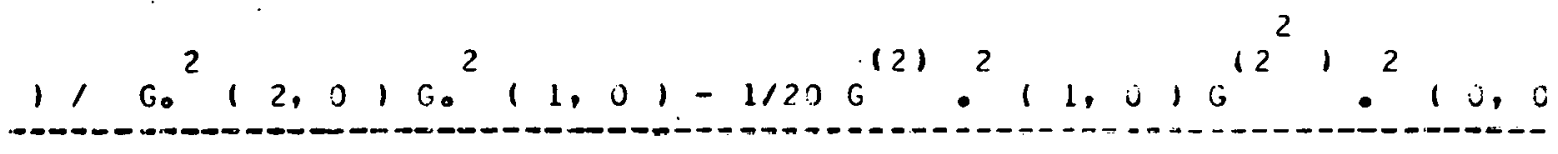


$1 G^{121} \cdot 10,016.12,010^{2}, 1,01+1 / 50^{(2)} 1.11,016^{(2)}$

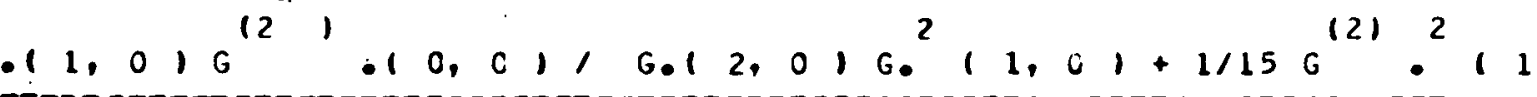
3

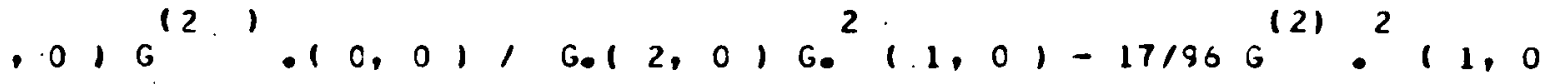
-

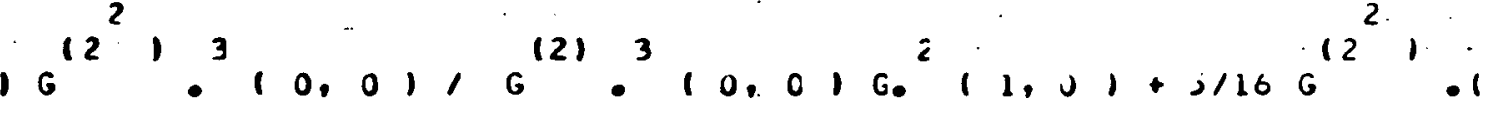
$1,01 G^{121} \cdot 11,01 e^{12}, 0^{2}, 0,01, e^{121} 0^{2}, 0,010^{2}, 1,0$

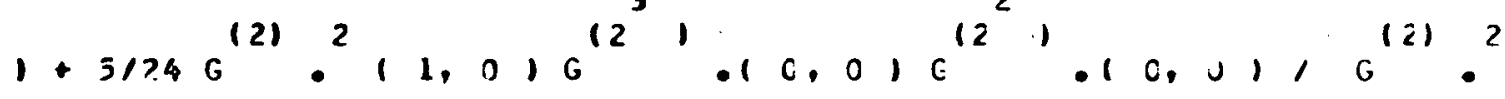
20 $10,01 G_{0}^{2}, 1,0,-1 / 8 G^{12} G^{2}, 1,01 G^{12}, 10,01,6^{(2)}$

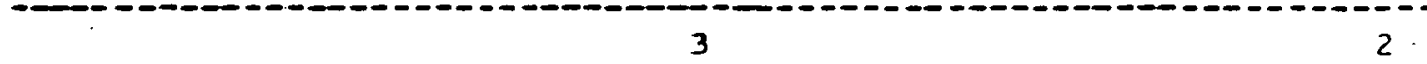
$.10,0100^{2}, 1,01-1 / 6 \mathrm{G}^{12}, .11,0, \mathrm{c}^{(2)}, 11,01 \mathrm{G}^{12}, 01 \mathrm{j}$

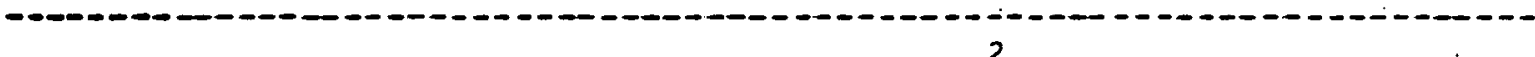

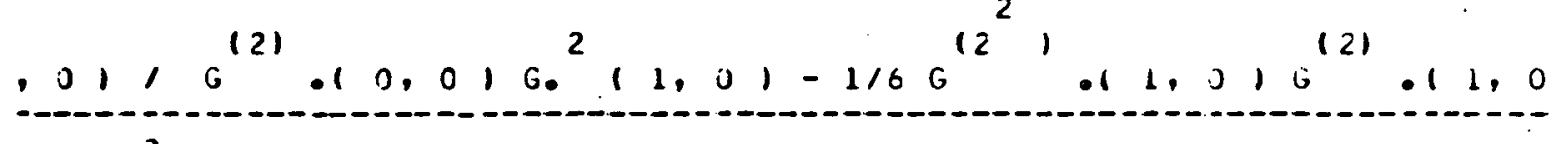

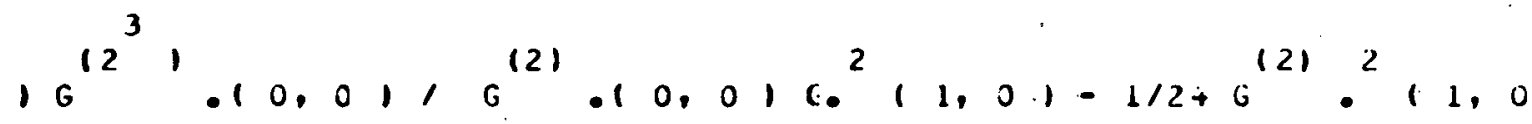
-

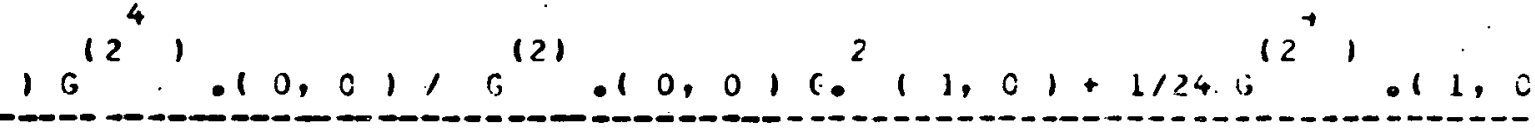
$1 G^{(2)} \cdot 11,01, G 0^{2}\left(1,01+1 / 12 G^{12^{2}}, 011,010^{12^{2}}, .11,0\right.$

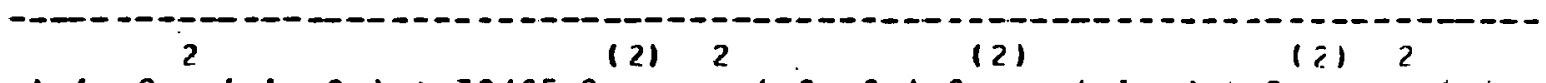

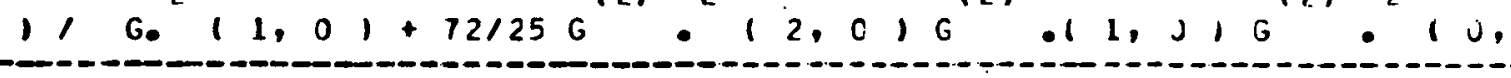

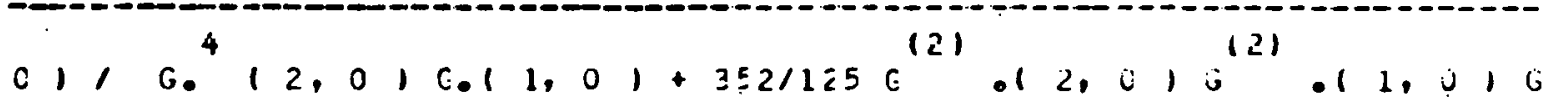

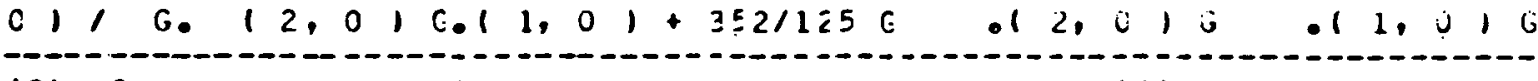
(2) $\left.3(0,0), G_{0}(2,0) 0_{0} 1,0\right)+64 / 750121$ $-10,011$ G. $(2,0) 6011,01+64 / 7500011,016$ 4 . 40 (2) 3 (2) $i J, 01$, G. $12,016011,01+4 / 5 C, 012, j 160.11$, 121

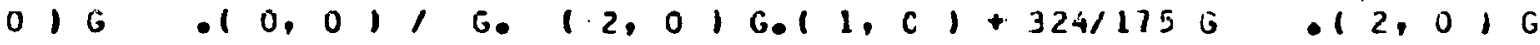
- 
$1212(2) 2$

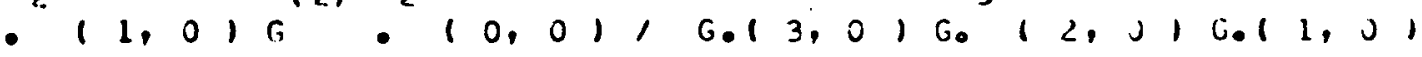
(2) $2(2) 3 \quad 3$

$+2376 / 875 \mathrm{G} \bullet 11,0, \mathrm{G} \cdot(0, \mathrm{C}, 1 \mathrm{G}, 1,0,16.12,01$

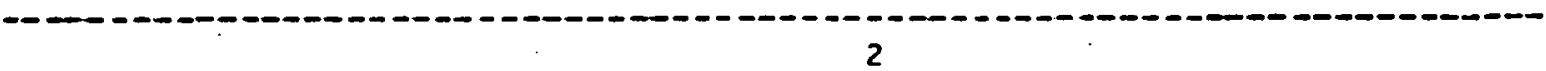

(2) 12101212

G.1 1. 0 1 - $148 / 375 \mathrm{G} .11,01 \mathrm{G}, .10,01 \mathrm{G}, 10,01$

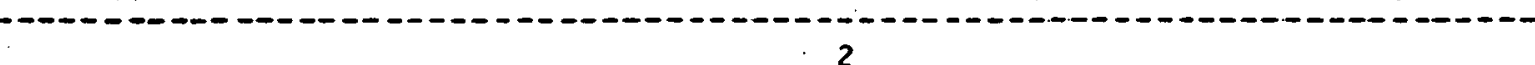

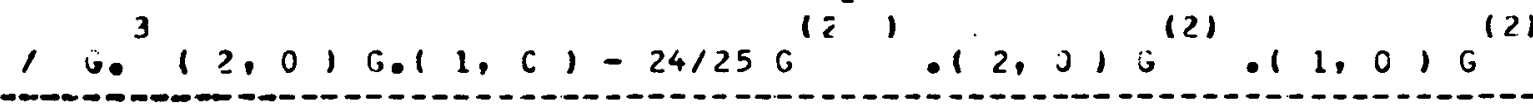

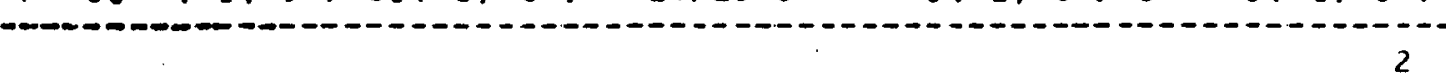

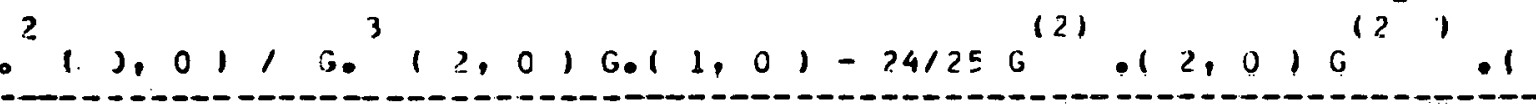

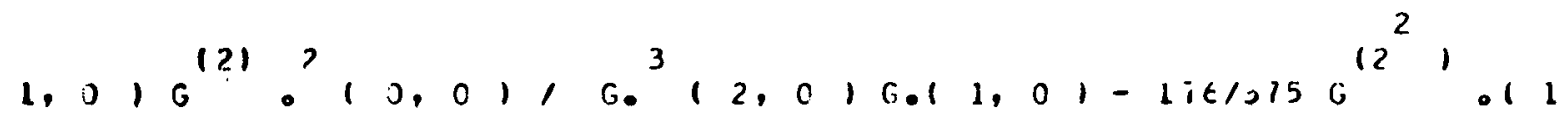
10
1 $1 ? 13 \cdot 3 \quad(2)$

$.0, G \circ 10,0,1, G,(2,0, G .11,0)-8 / 56,012,016$

---

$(2) \cdot\left(1,0, \mathrm{G}^{12^{2}}, 0(\mathrm{c}, 0) \mathrm{G}^{(2)} \cdot\left(0, \mathrm{c}, 1 \mathrm{G}^{3}, 2,0, \mathrm{G}, 1,01\right.\right.$,

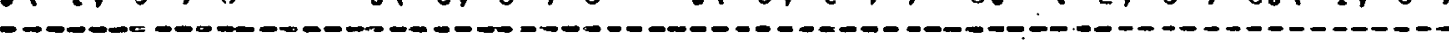

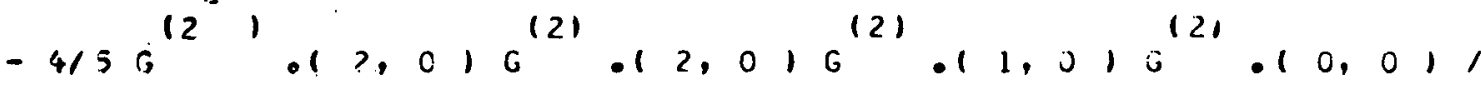

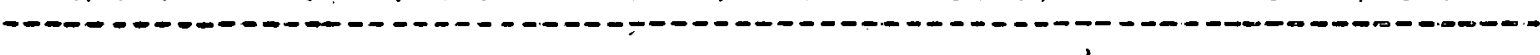

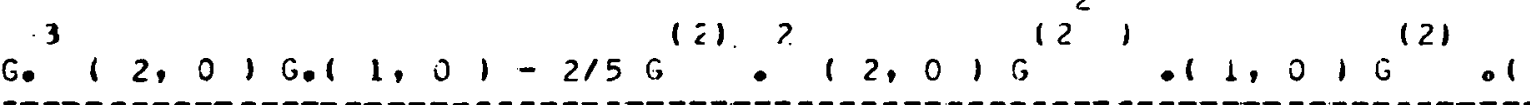

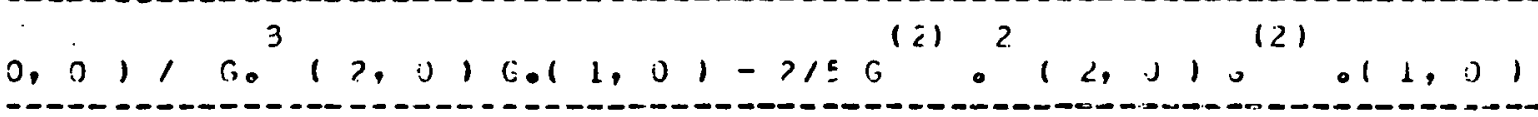
$212^{2}$

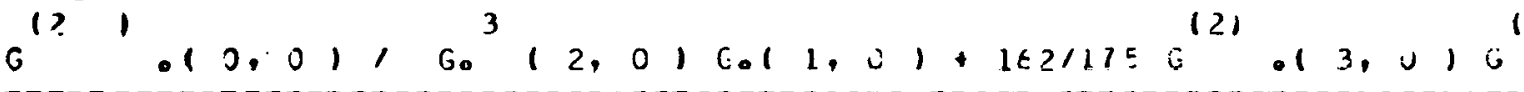
(1)$12\left(1,0, G^{(2)} 0^{2}(0,0), G_{0}^{2}, 3,010,12, j, 0,1,1,01\right.$

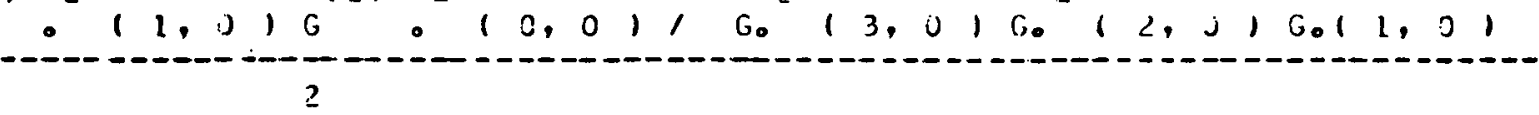

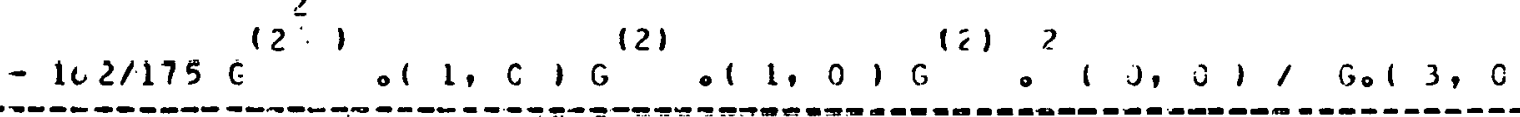

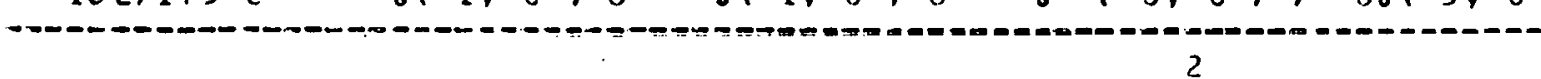

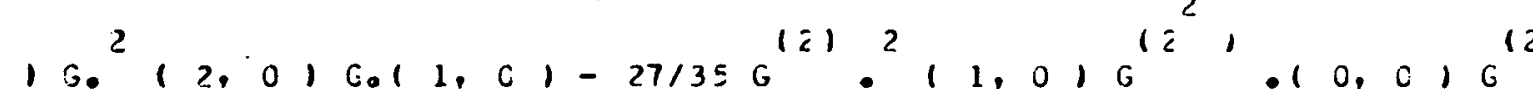

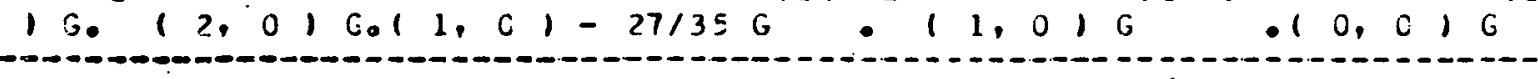
125 (2)

ol $3,0,16013,0,6,12,0,6.11,0,-1 / 130,0(2,0)$ $G^{(2)} \cdot 1.1,0, G^{12^{2}} 0^{2}\left(0,01 / G^{(2)}, 10,01 G^{2}(2,0) G .11\right.$,

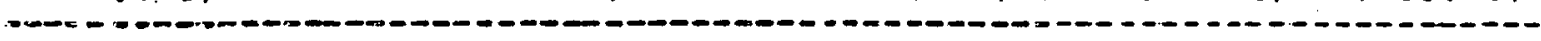




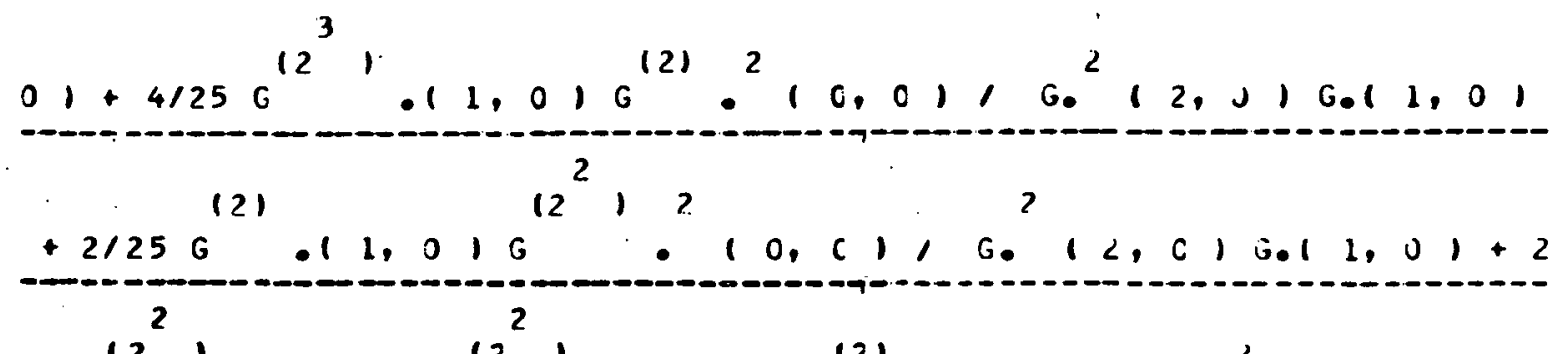

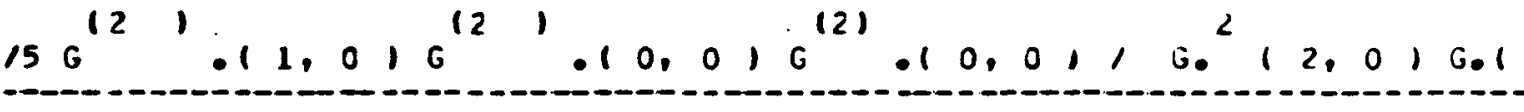
3

$\left.1,01+4 / 25 G^{(2)} \cdot(1,0) G^{(2)}, 10,0\right) G^{(2)} \cdot 10, j, 1, G^{2} 12$

19-0.-3

$.01 G .11,01+2115 G^{(2)}, .12,01 G^{(2)} .11,010^{(2)} .10,01$

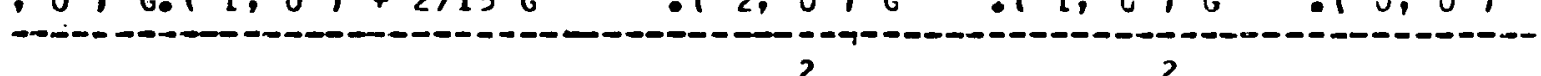

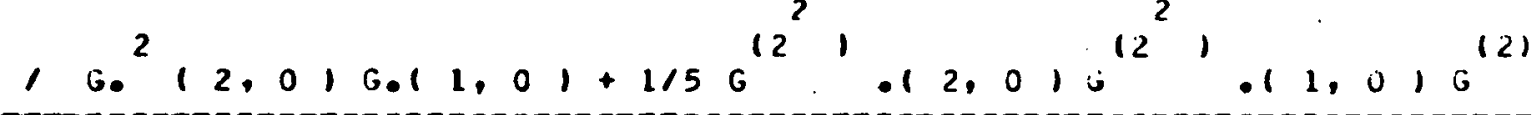

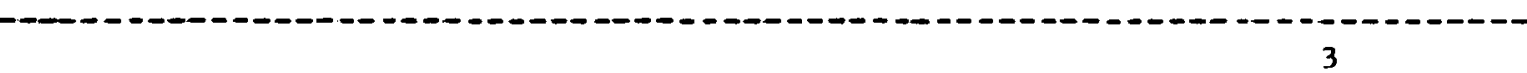

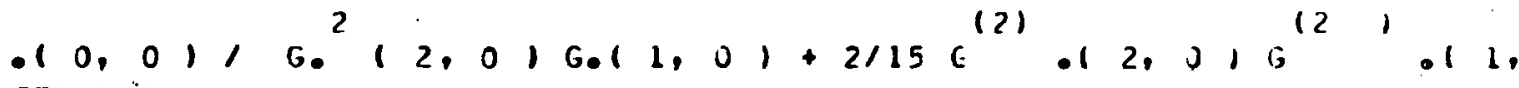
(121 $\left.12121 c^{2}\right)$

$0.16 .10,01, G,(2,0, G, 11,0,+1 / 5 G \quad .12,0, G$ 0 $1.1,0, G^{12} 1,10,01, G^{2}, 2,01 G 011,01+1 / 5 G^{121} .12$

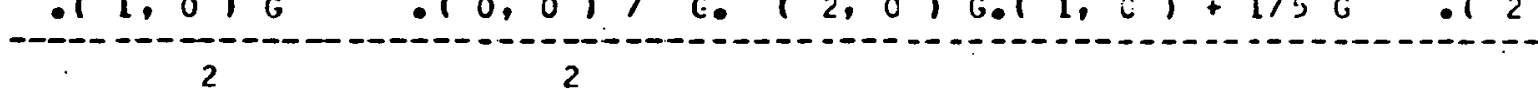
$, 016^{12^{2}}, .11,0, G^{12^{2}}, .10,0,1,0^{2}, 12,0,0.11,0,+21$ (0)--

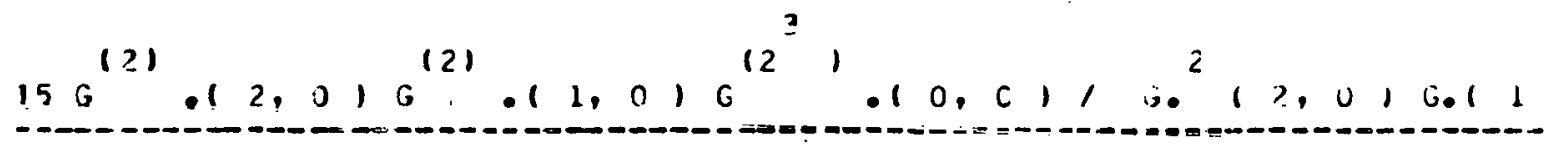
$, 01-1 / 30 G^{(2)} \cdot 11,010^{12}, 2^{3}, 0,01,0^{121} 0^{2}, 0,010.12$ 0

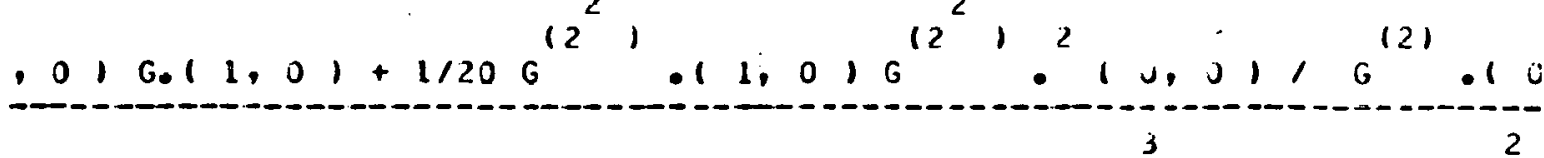
$.01 \mathrm{G} .12,01 \mathrm{G}, 11,01+1 / 15 \mathrm{G}^{121} \cdot 11,01 \mathrm{G}^{12}, 10,010^{12}$ $\left.1,10,0,1 G^{(2)}, 10,0, G, 12,0, G, 1,0,-1 / 3, G^{12}\right)$ $.10,01 / G \quad .10,0, G, 12,0,6.11,01-1 / 3 J G \quad .11$, (1) $016^{121} \cdot 10,01,0.12,016.11,01-1 / 156^{12} 1,11,016^{12}$ 
I

$12^{2} 1 \quad 12^{3} 1$

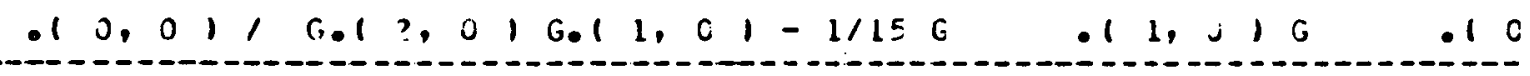
i $, 01, G_{0}\left(2,0, G 011,01-1 / 30 \mathrm{G}^{(2)} \cdot 1,1,010^{(2,1,0,0,1}\right.$ 2
2

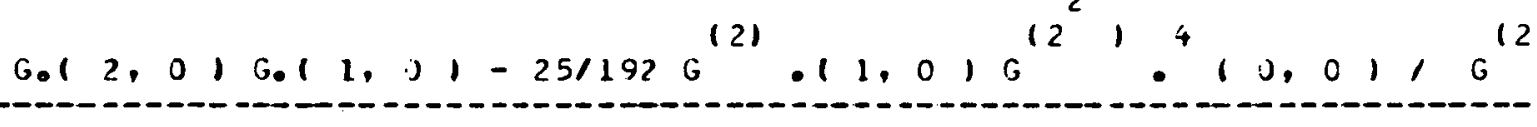
14 14 (2)

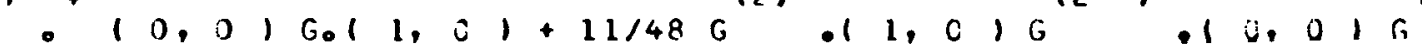

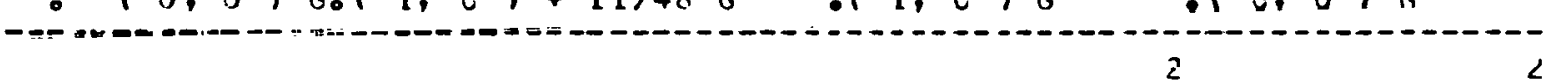

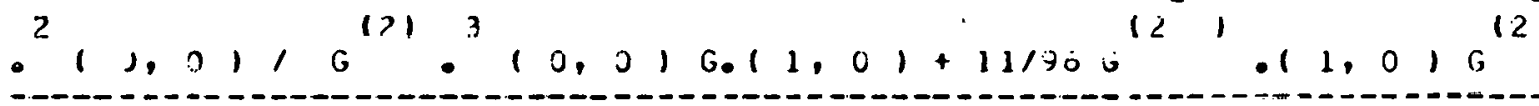
1
3

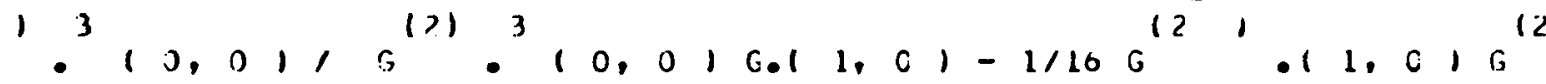
? 1212121212012

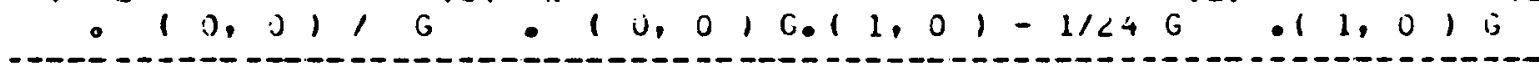
3

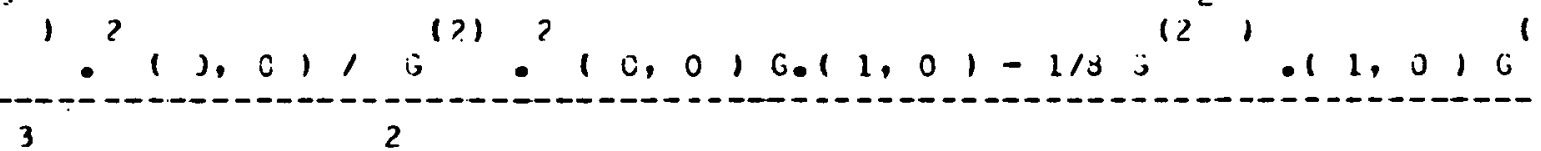

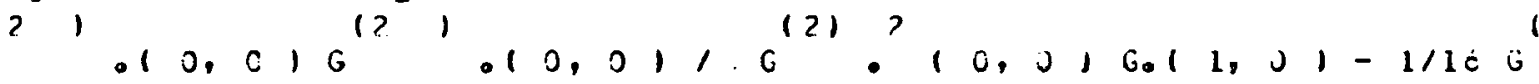

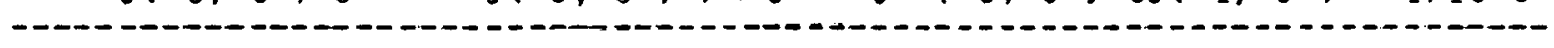

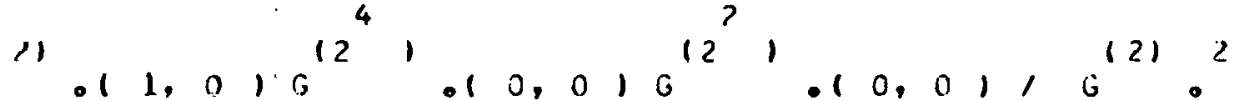

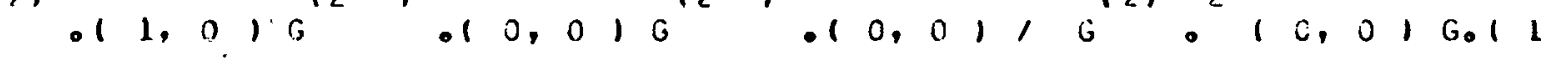

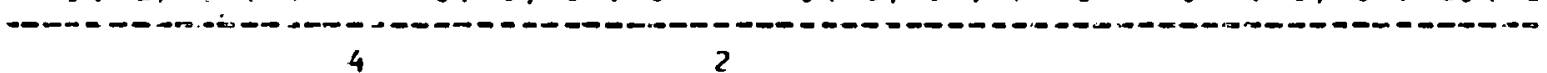

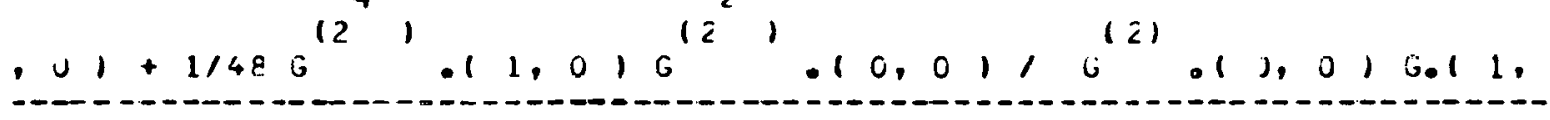

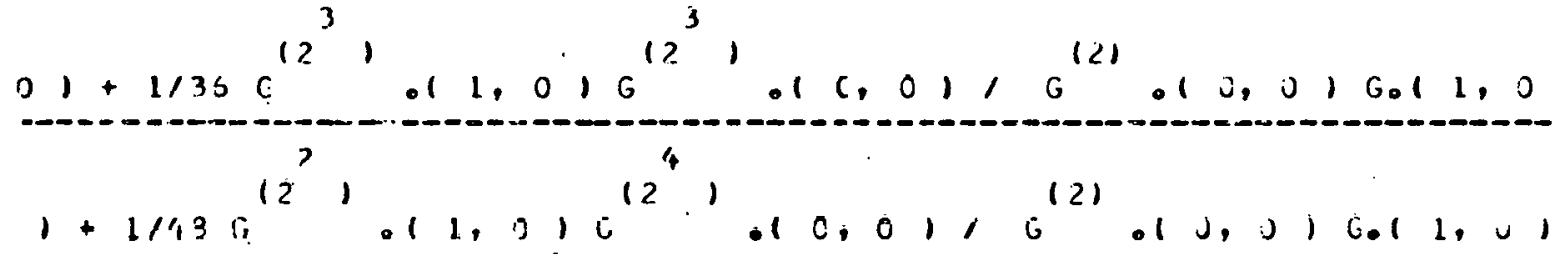

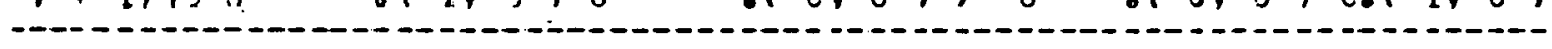

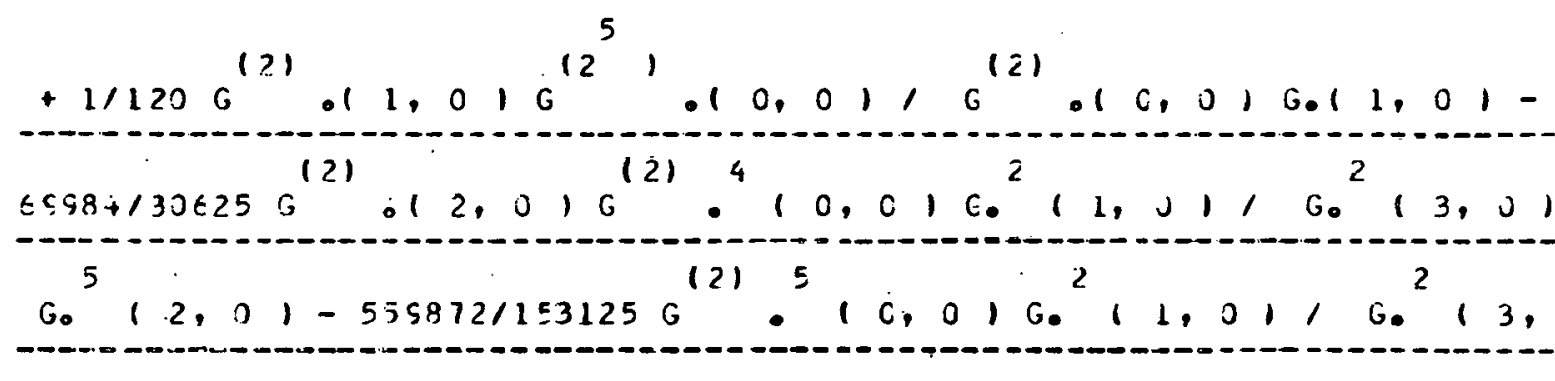




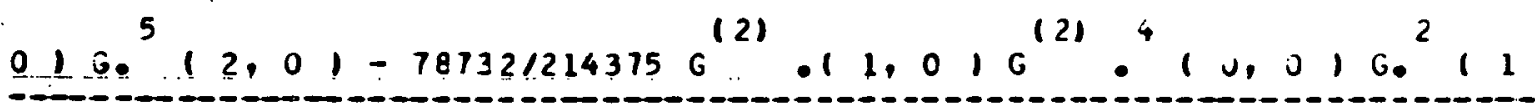
, $01 / G_{0}^{3}\left(3,0, G_{0}^{4}(2,0)-3499213 c 625 G^{(2)}, 13,0, G^{(2)}{ }^{4}\right.$

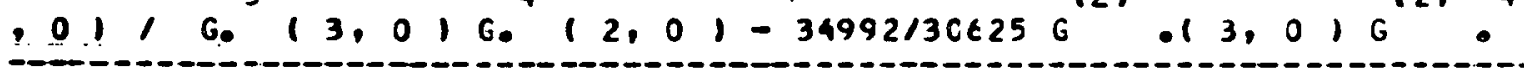
$\left.(0,0) G_{0}^{2}(1,0), G_{0}^{3}(3,0) G_{0}^{4} \mid 2,0\right)-051216125 G^{(2)}, 12$ 1214 $, 01 G^{\circ}: 0,01 G_{0}\left(1,0,1, G_{0}\left(4,0, G_{0}\left(3,0, G_{0} 12\right.\right.\right.$,

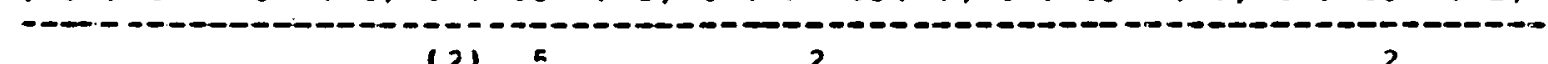

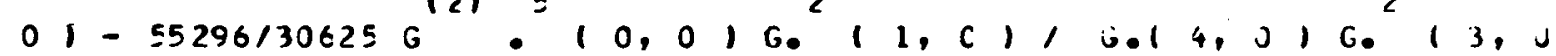
(-) - -

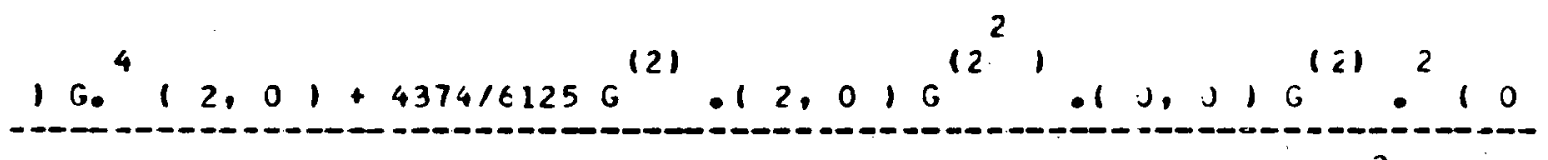
$.01 G_{0}^{2}\left(1,01, G_{0}^{2}\left(3,0, G^{4}(2,0)+174 j 610125 G^{12} 1,10\right.\right.$ $, 01 G^{(2)} 0^{3}, 0,01 \varepsilon^{2}, 1, c, 1, G^{2}, 3,010^{4}, 2, c 1-31104$

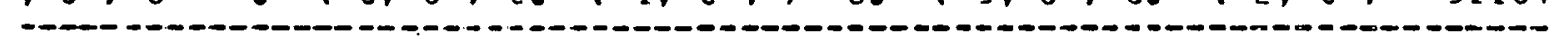
$142375 G^{(2)}+11,01 G^{(2)} 0^{4}, 0,01 G^{2}, 1, C, 10.14, C 1 G^{3} 1$ 30

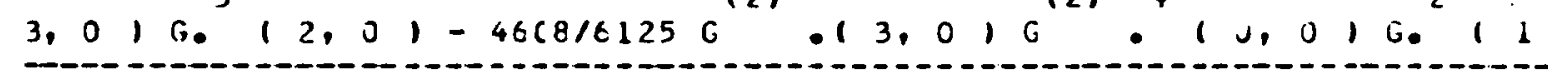
$.01, G_{0} 14,0, G_{0}^{3}\left(3,0, c^{3}(2,0)+291010125 G^{(2)} .12,0\right)$ ${ }^{2}$ $G^{12^{2}} \cdot .10,01 G^{121} 0^{2}, 0,01 G^{2}, 1, c 1,0^{3} 13,01 G^{3} 12$ 0

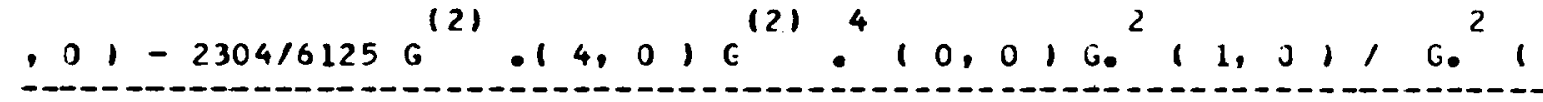
$4,0, G^{2}, 3,0, G^{3}, 2,01+57 t 112250^{121}, 12,0,6^{12}, 010$

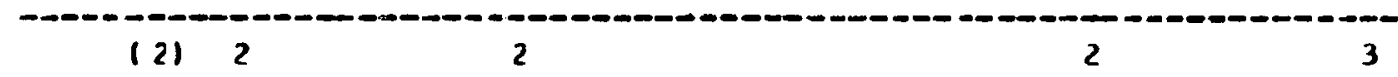
$016 \circ(0,0) 6,(1,0), G, 14, C) G, 13, J 16.12,0$

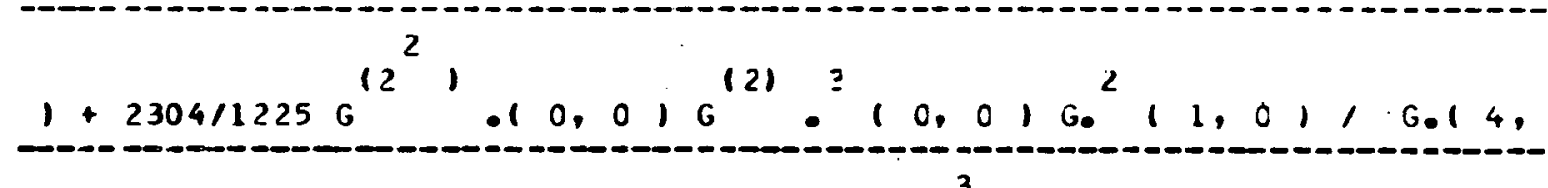
$010^{2}(3,0) 0^{3}\left(2,01-486 / 6125 G^{12^{3}} 1010,01 G^{(2)} 0^{2} 10,0\right.$

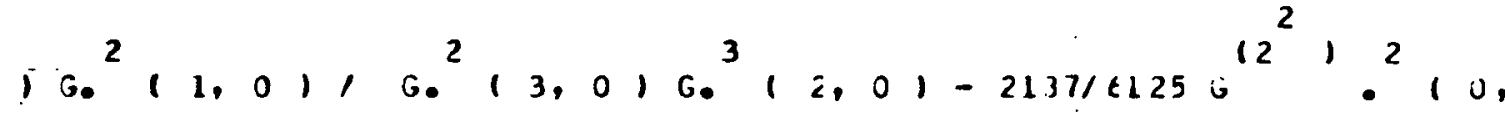


$0, G^{(2)} \cdot(0,0) G^{2}(1,0), G^{2}\left(3,0,60^{3} 12,0,-3072185 i 5\right.$ $121)(2) 4 \quad 030$

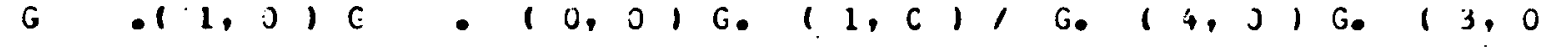
G IG. $\left.{ }^{2}(2,0)+57 \in / 1225 \mathrm{G}^{(?)}, 13, \mathrm{C}, \mathrm{G}^{\left(2^{2}\right.}\right)$

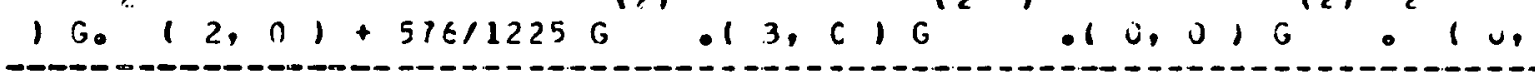

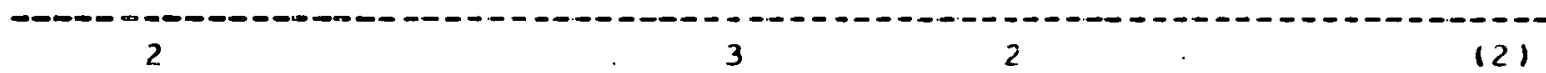
$0,00(1, J) /$ G. $14,0, G 0(3,0)$ Go $(2, J 1-1921539 G$ 0 (2) 4 ? 2 $011,016.10,016,11, C) / G .15,016014,0160$

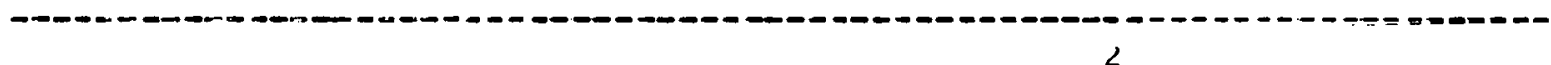

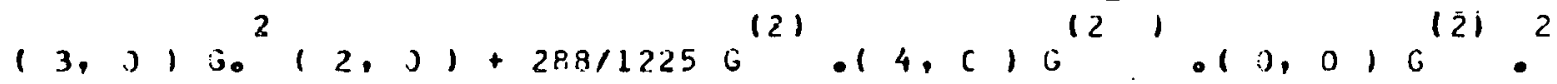
1 $\left(1,0,0, G_{0}^{2}, 1,0,1,0^{2}, 4,0, c_{0}^{2}(3,0,0.2,1<1-561\right.$

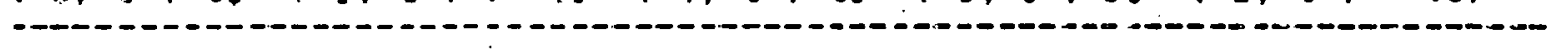

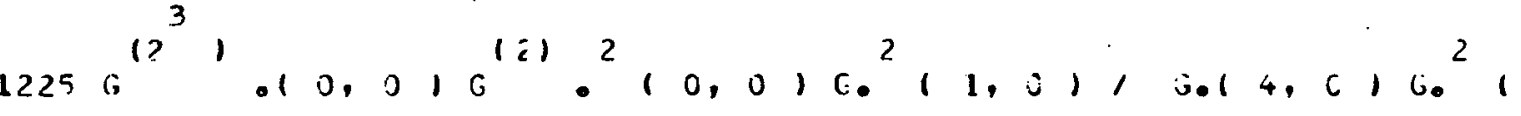
-

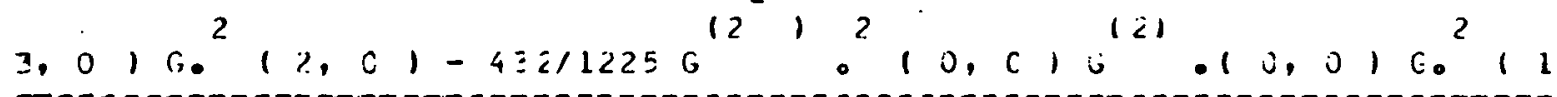
בn $001, G_{0} 140,0, G^{2}\left(3,01 G^{2}\left(2,01+62+350 / 1011 \varepsilon 75 G^{(2)}{ }^{5}\right.\right.$

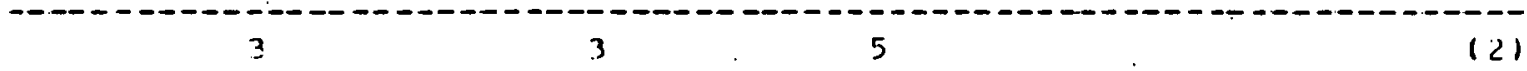
$(j),, G_{0}(1,0) / G_{0}(3,0) C_{0}(2,0)+1 \varepsilon i \in 24 / 2143 i 56$ $0^{5}\left(0,0, G_{0}^{3}, 1, j, 1, G_{0}\left(4,0, \epsilon_{0}^{3}\left(3,0, \epsilon^{4}, 2,0,-3536 \epsilon\right.\right.\right.$ 15
0 $1214375 G^{12}$, o $J, J, G_{0}^{(2)} 0^{3}, 0, c, G_{0}^{3}, 1, J 1, j^{3}, 3,01 G$ 0

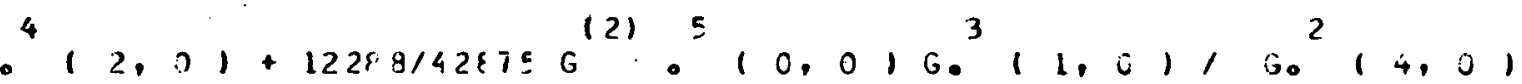

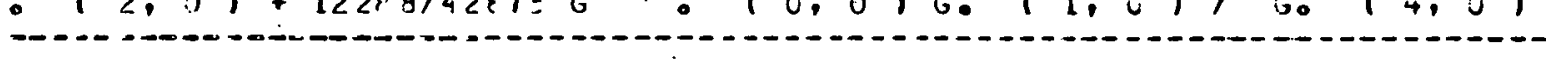

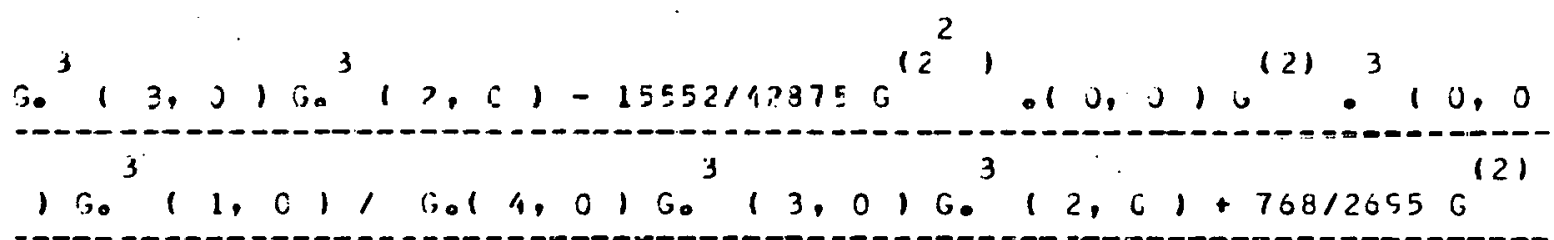

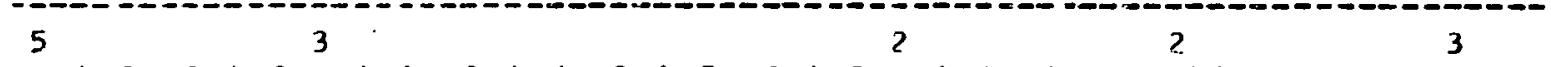
- $\left(0,01 G_{0}\left(1,0,1 / G_{0}(5,0) G_{0}(4,0) G_{0}(3,0016012\right.\right.$

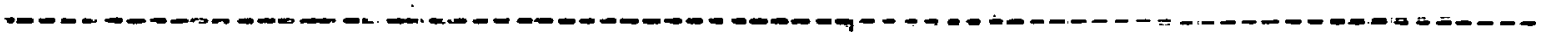

$12^{2}, \quad(2) \quad 3.32$




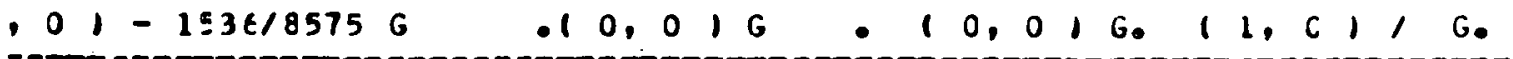

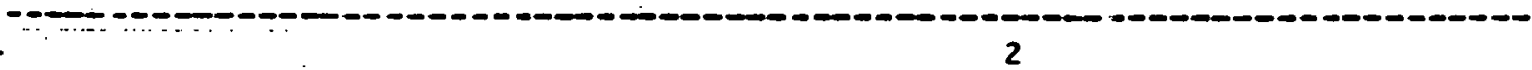
$14,01 G_{0}^{3} 13,0,6^{2}, 2,0,1-56 / 539 \mathrm{c}^{12} 1010,01 \mathrm{G}^{(2)} 0^{3} 10$ 3

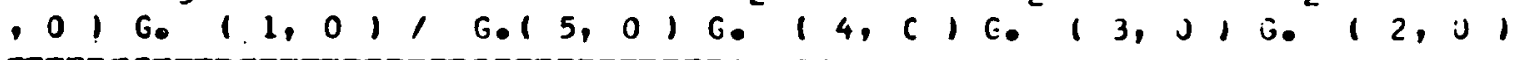
121331212 $-32 / 25 \mathrm{G}: 12,01 \mathrm{G}: 10,01 / 6012,0,-384 / 125 \mathrm{G}$ 12 (2) 3 ( 121

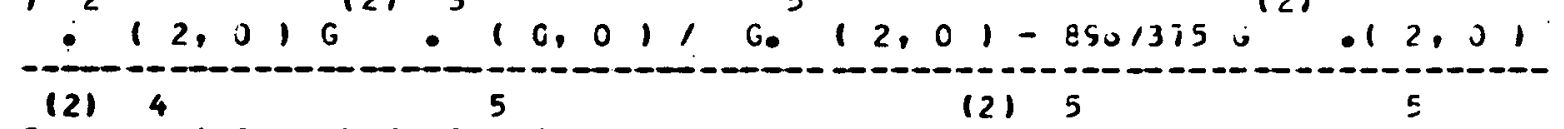

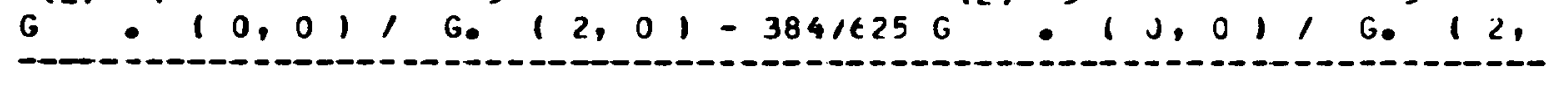
$01-3241175 G^{(2)} \mathrm{G}^{2}, 2,0, \mathrm{G}^{(2)} \cdot 11,0, \mathrm{e}^{(2)} e^{2}, 0,01,6.13$

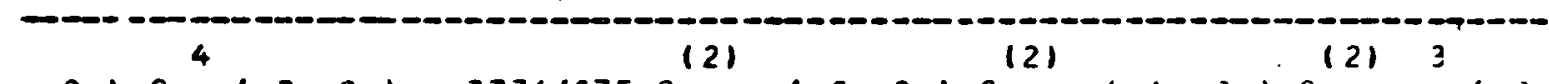

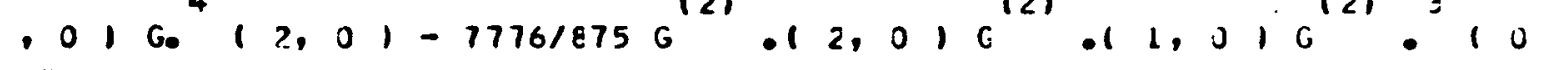

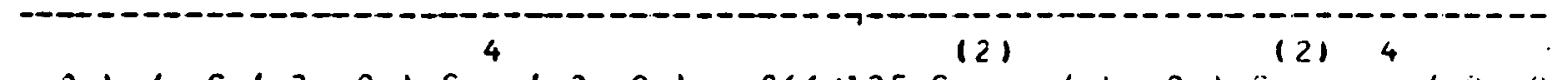

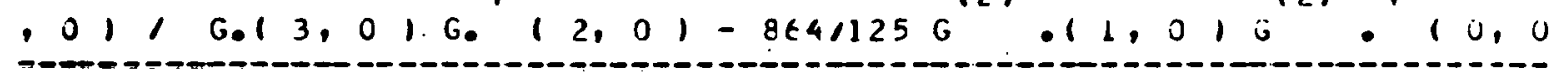
$4(\pi) \quad\left(\alpha^{2}\right)$

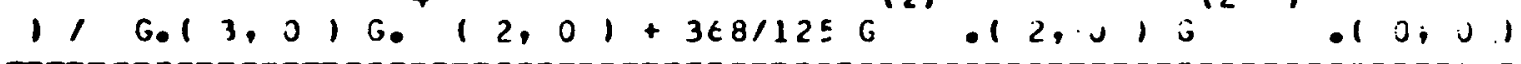

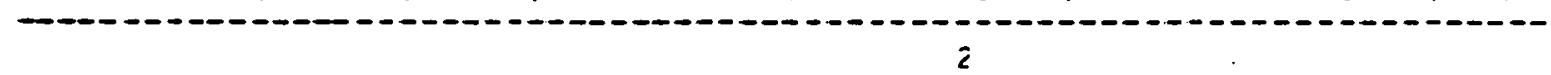

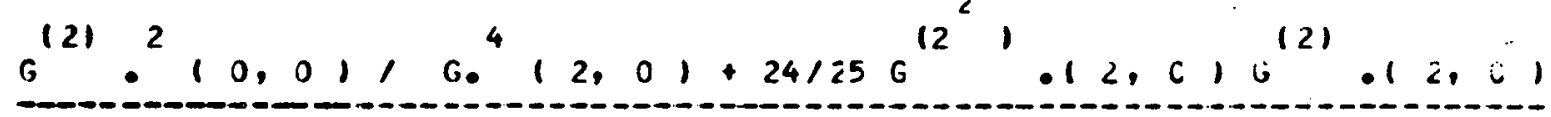

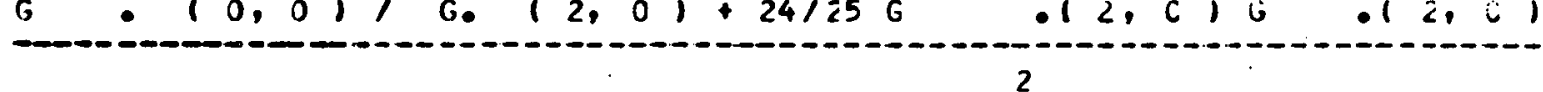

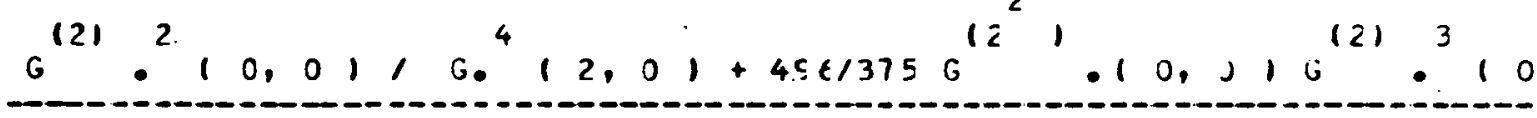
20

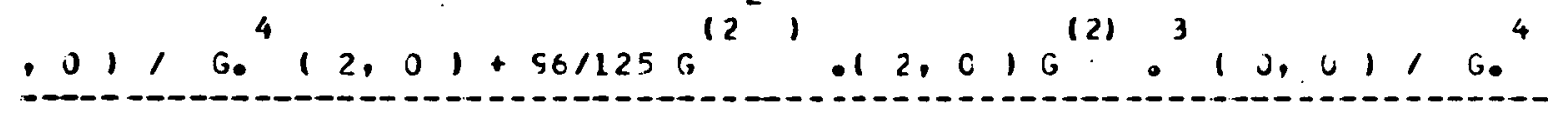

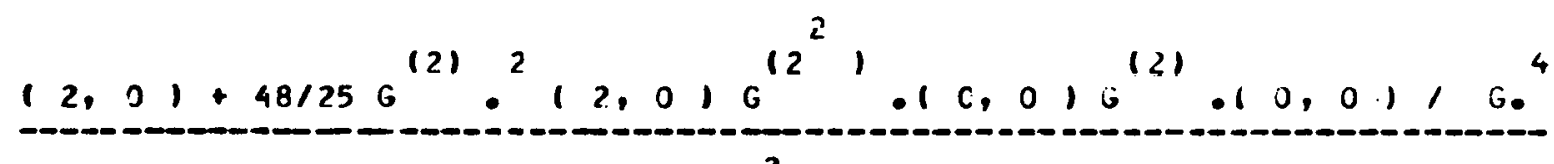

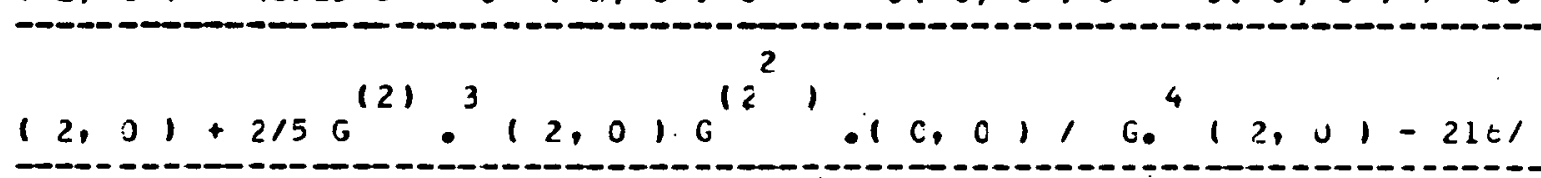

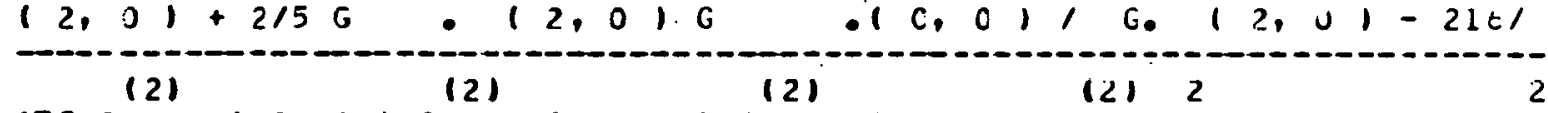

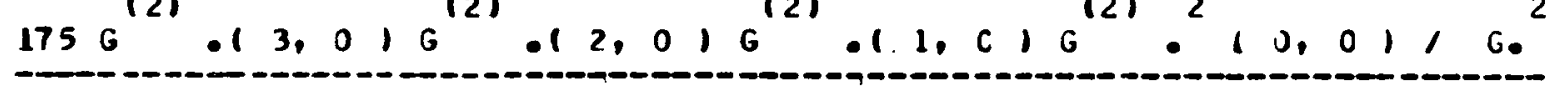

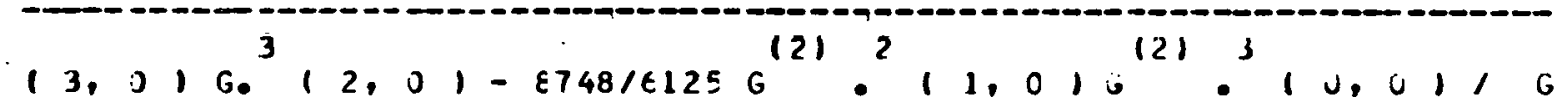

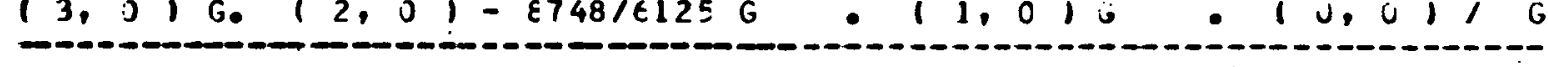

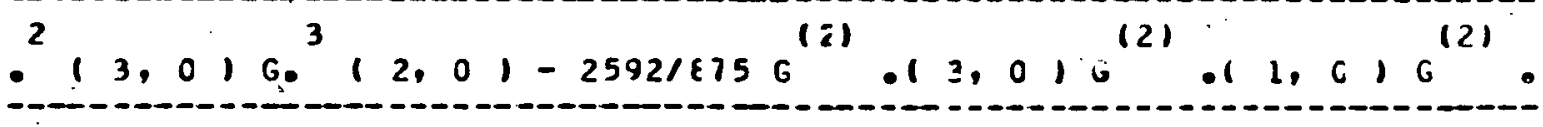


$3,1,0,1 G_{0}^{2} 13,01 G^{3} 12,01+45 \in \varepsilon / \varepsilon 750^{(2)} \cdot 11,010^{12^{2}} 1$ - - - -

(2), 5 3 $3012^{2}$,

$01,016: 10,01,6.13,01 E_{0}, 2,01+1 J 311756,0$

(2) 2

$12,01 G$, $1,0, G \circ(0, C), G_{0}(3, J)$ b. $12,01+$

(-

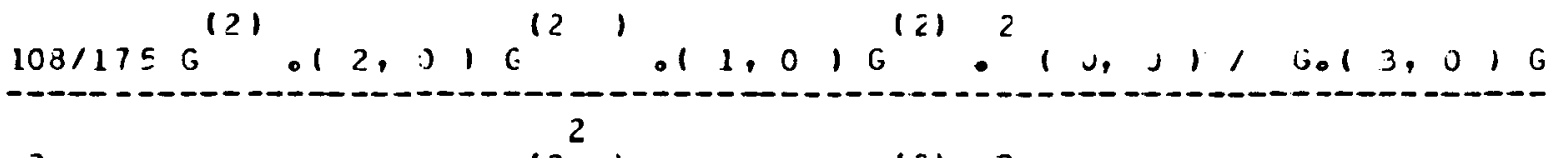

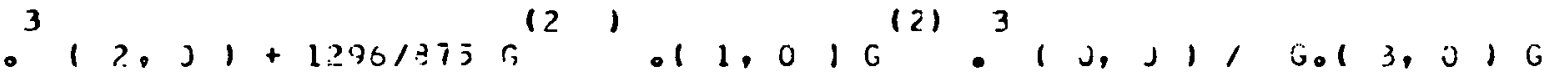

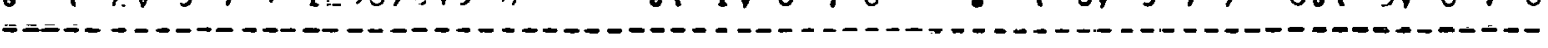

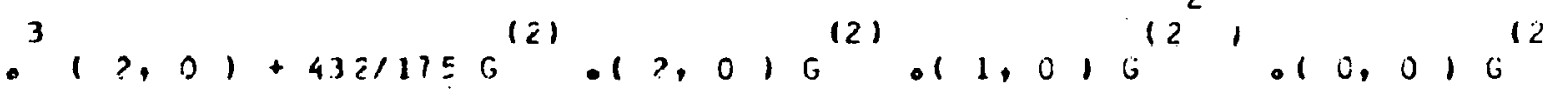
$1010,0,1,0,13,0, G_{0}^{3}, 2,0,-3 \in 8 / 11250^{12}, 010,0, g^{121}$ -

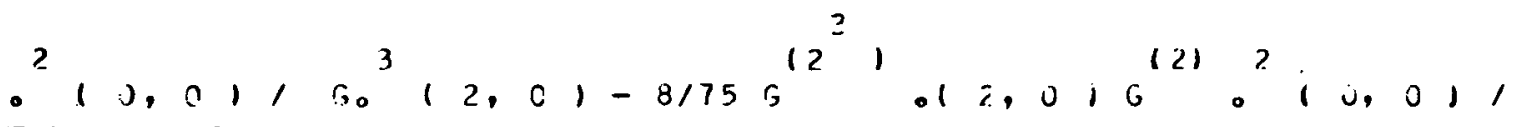

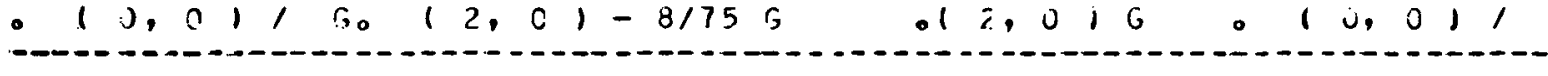

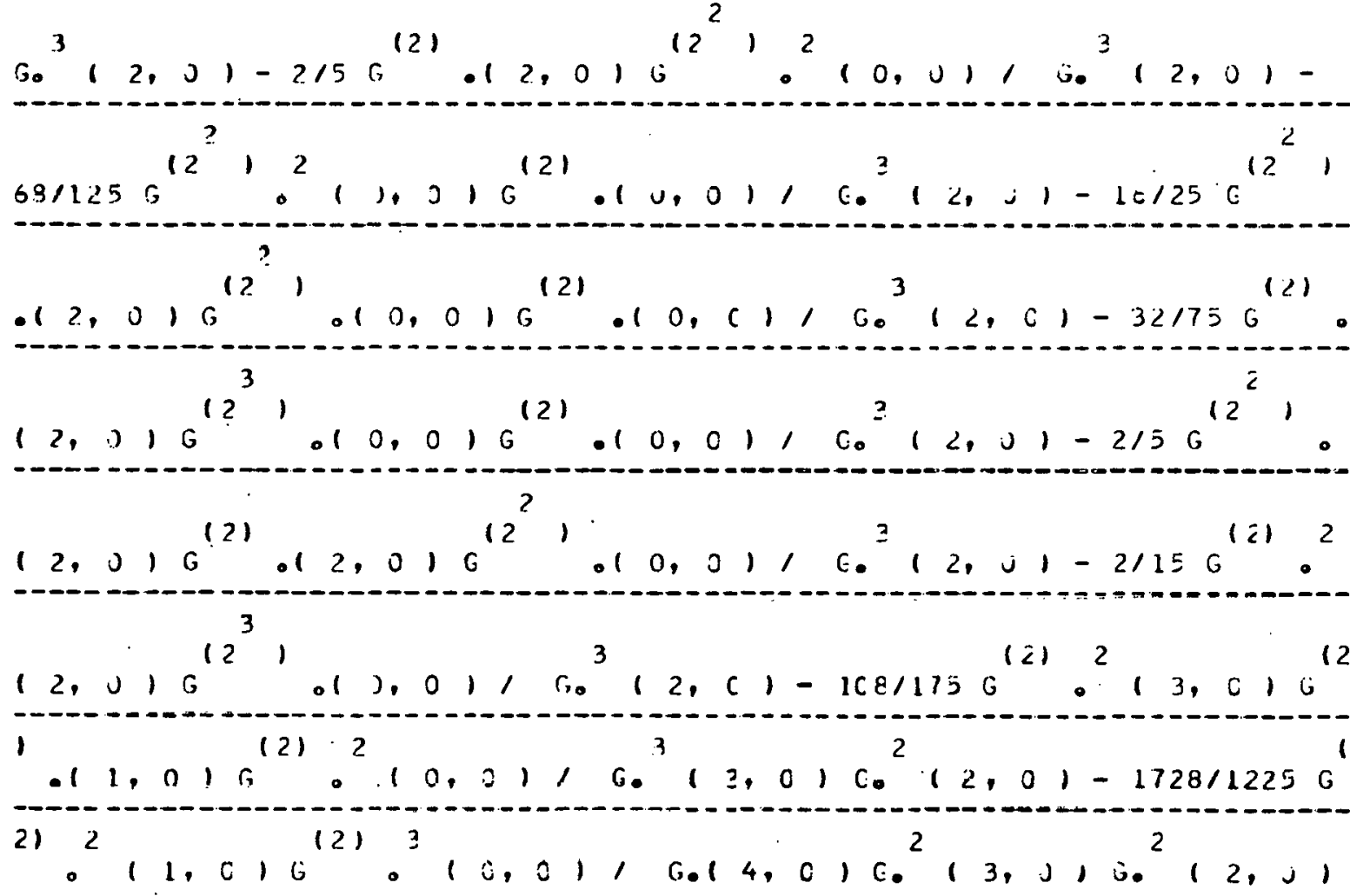




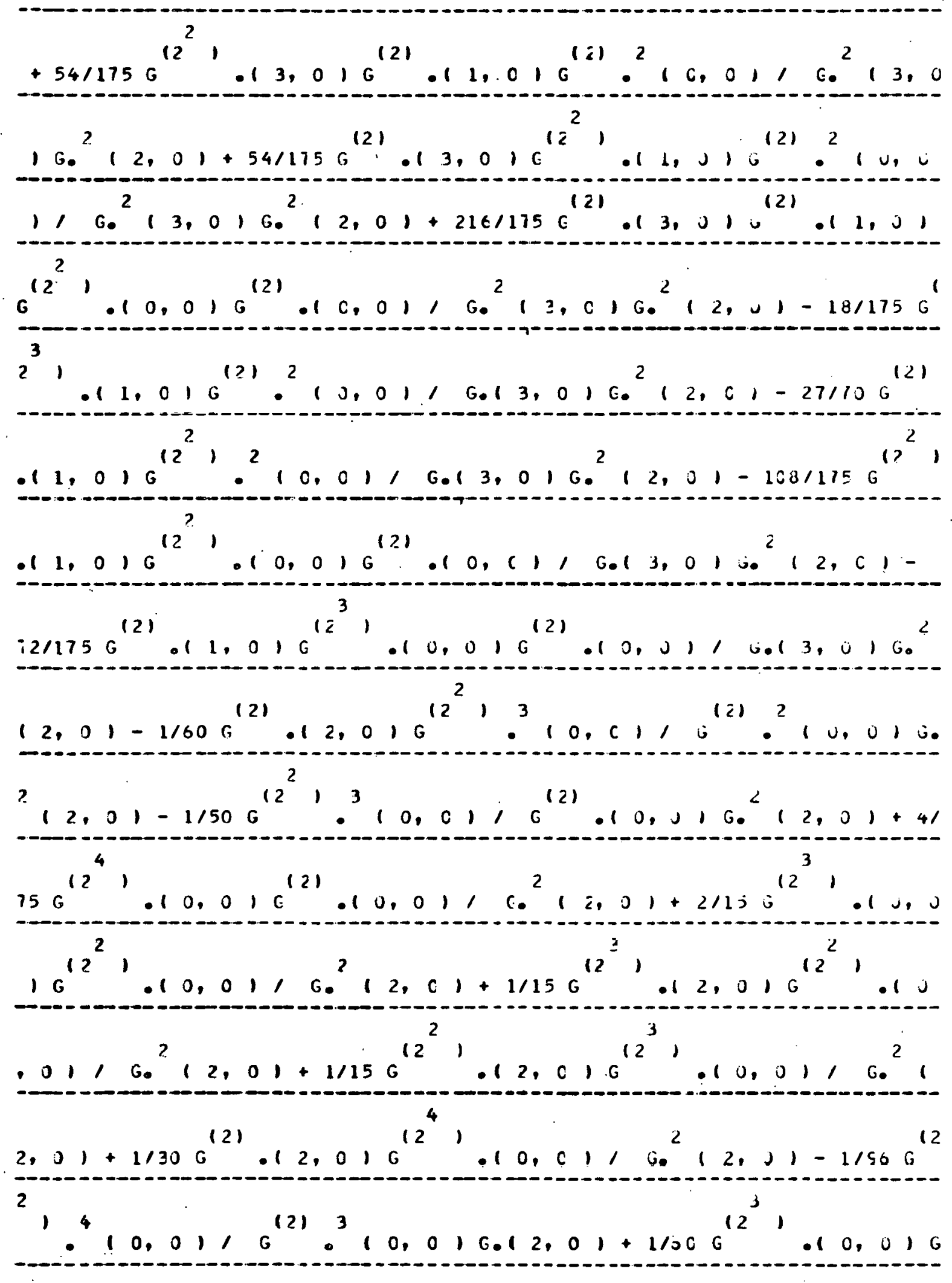




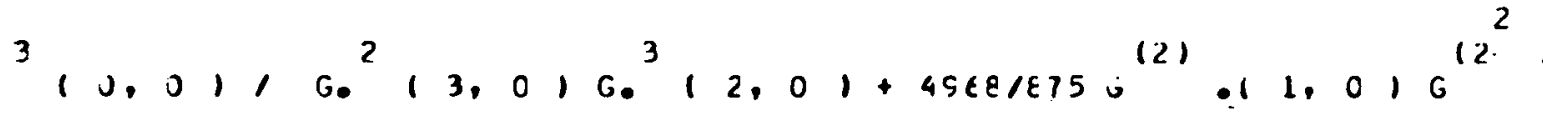

(

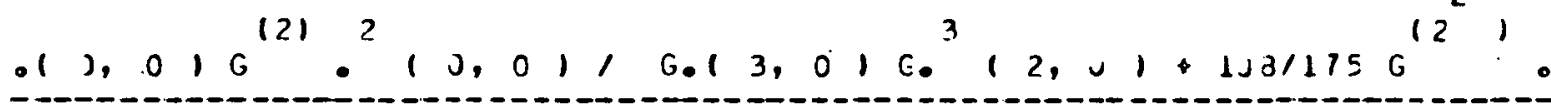

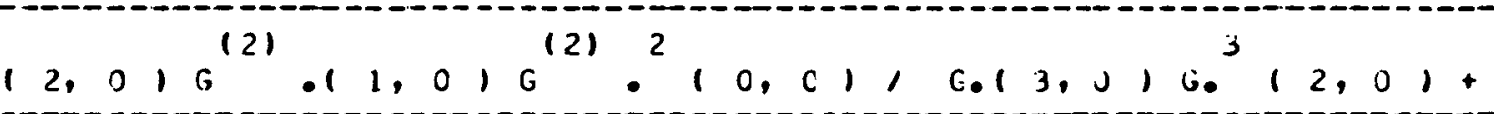
-

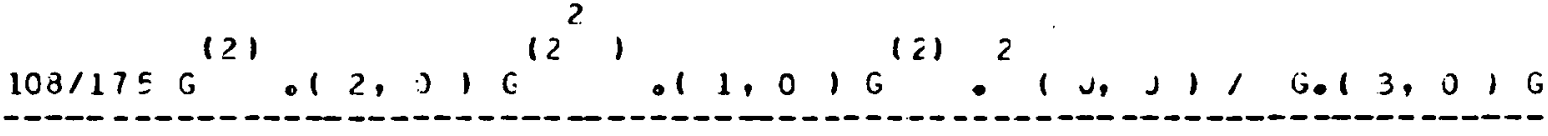

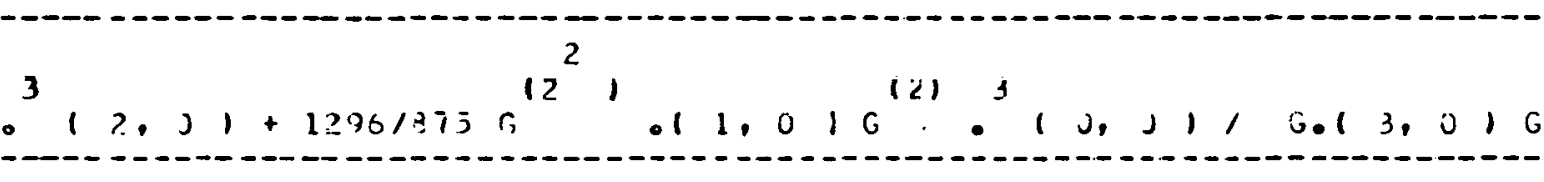

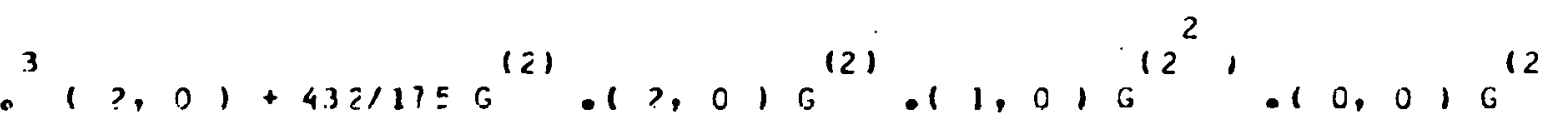

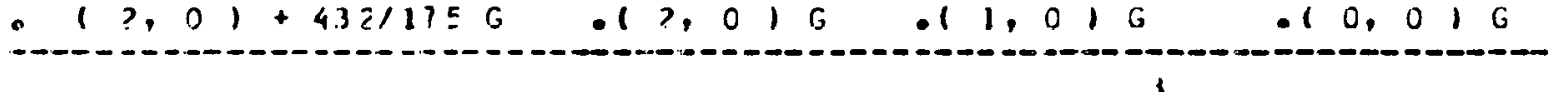
$1010,0,1, G 013,0, G^{3}, 2,0,-368 / 11256^{12}, 010,01 G^{121}$

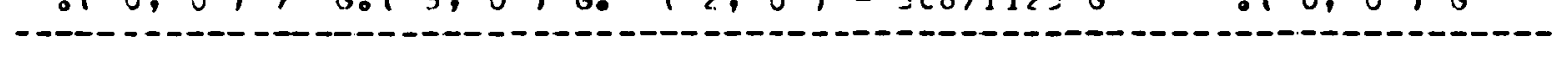

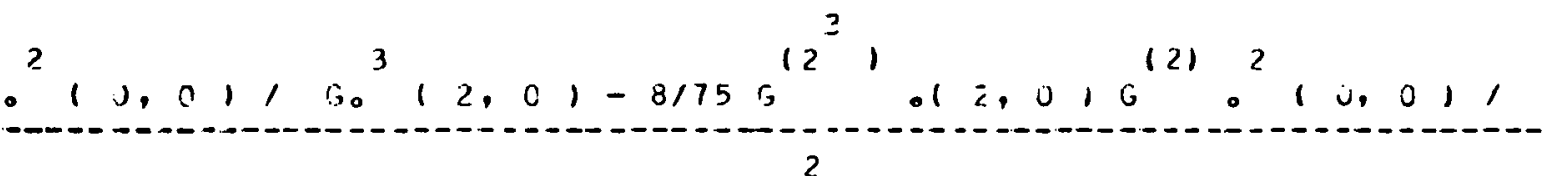

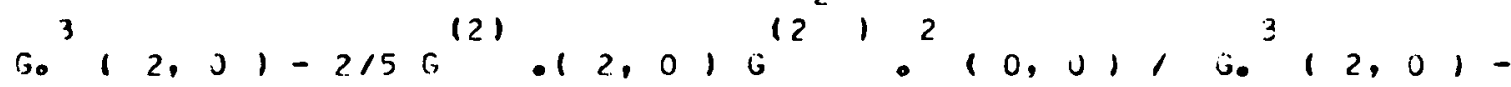
$\mathrm{s}$
$\mathrm{s}$

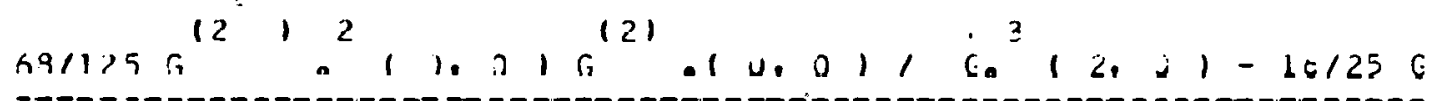
-

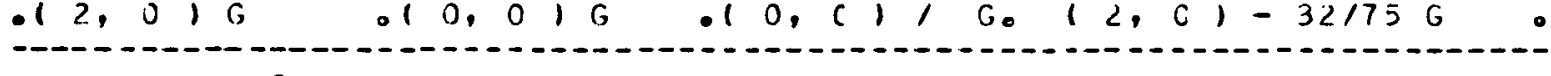

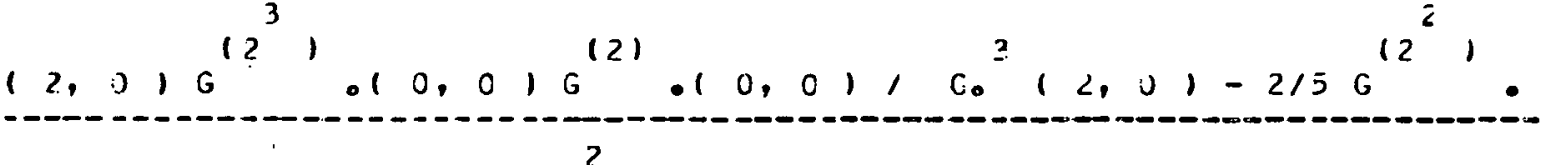
$12,0, G^{(2)} \cdot(2,0) G^{(2)} \cdot(0,0), G_{0}^{2}(2, j)-2 / 15 G^{(2)} 0^{2}$ 3
3

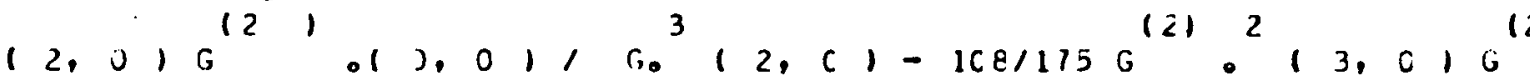
$\begin{array}{ll}1 & (5) \cdot 5 \\ 1 & 0\end{array}$ $.1,0, G_{0}, 1,0,0,1, G_{0}, 2,0, G_{0}(2,01-1728 / 1225 G$ 212 (2) 320

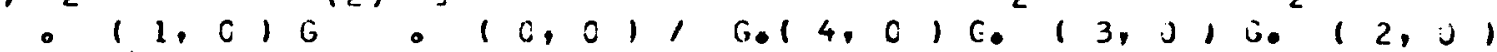


$+541175 \mathrm{G}^{12^{2}}, .13,0, \mathrm{G}^{(2)} \cdot 11,0, \mathrm{G}^{(2)} \mathrm{C}^{2} \mathrm{G}, 01, \mathrm{G}^{2} 13,0$

(1)

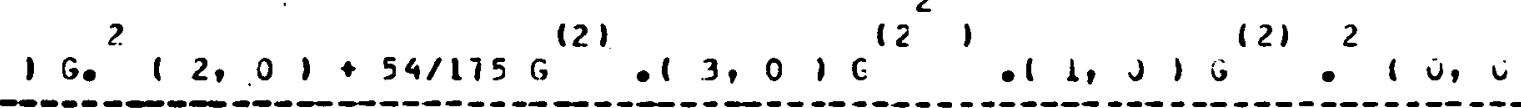
$1, G^{2}\left(3,0, G^{2}, 2,01+216 / 175 e^{(2)}-13,010^{(2)} .11,01\right.$ $-\cdots$ $\left.G^{\left(2^{2}\right)} \cdot(0,0) G^{(2)}-(c, 0), G^{2}, 3, c\right) G^{2}(2,0)-181175 G^{1}$ 3

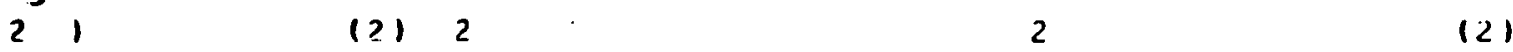
$.11,0, G, 10,01$, G.1 3,0, G. $12,01-27170 \mathrm{G}$ ${ }^{2}$ $.11,0, G^{12}, 2^{2}, 0,01, G .13,0, G^{2}, 2,01-1001175 G^{12^{2}} 1$

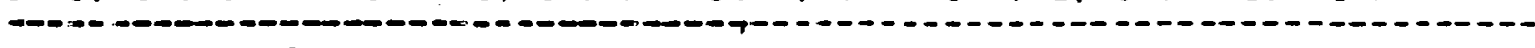

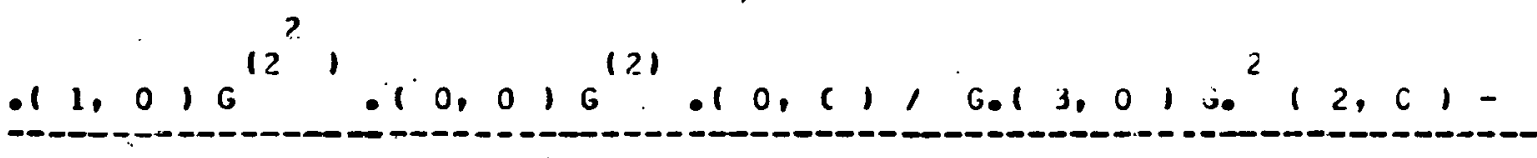

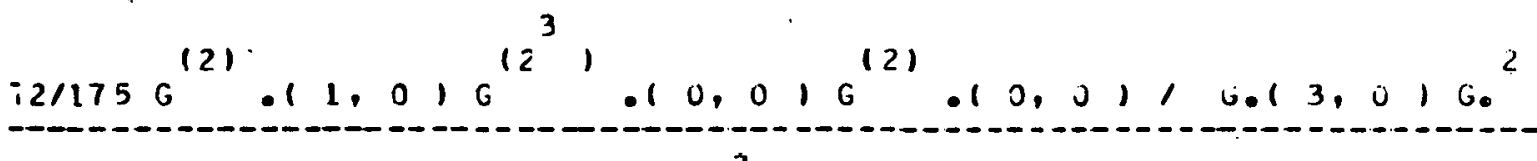

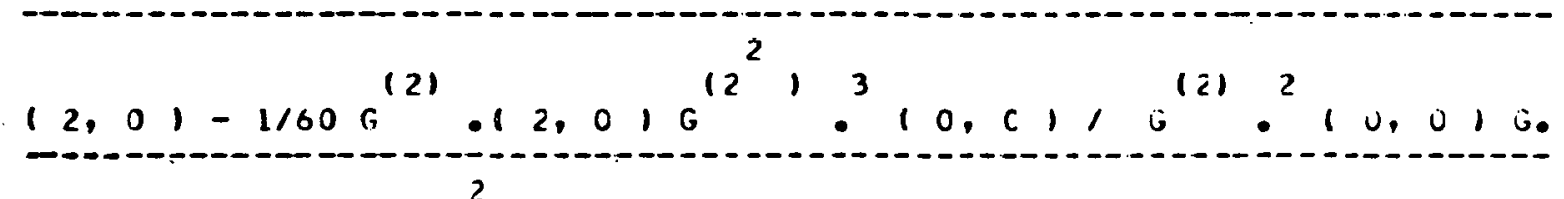
? $12,01-1 / 50 \mathrm{G}^{12^{2}},{ }^{3}, 0,0,1 \mathrm{G}^{(2)}, 10, \mathrm{~J} 1 \mathrm{G}^{2}, 12,01+41$

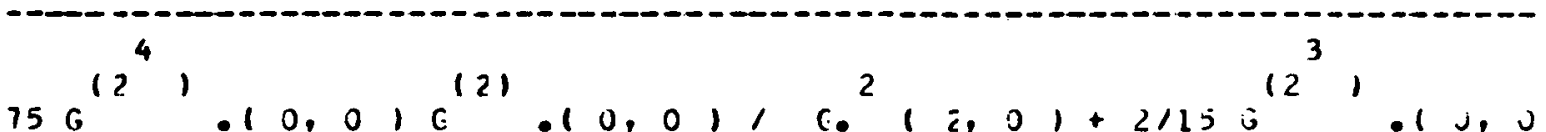

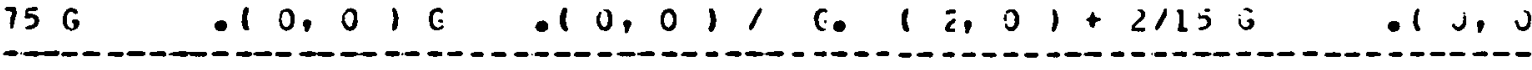

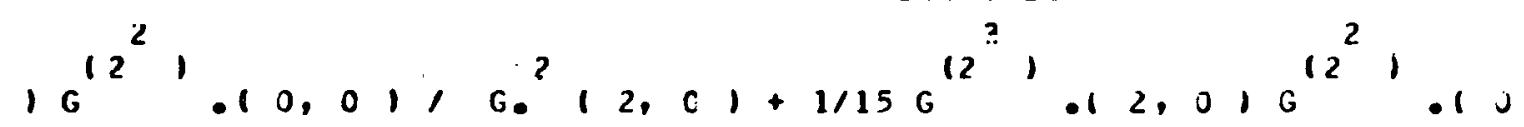

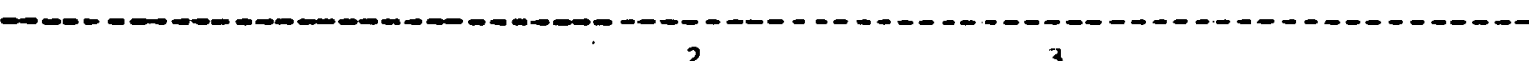
$01, G_{0}^{2} 12,01+1 / 156^{12^{2}} 1,12,016^{12^{3}}, 10,0110_{0}^{2} 1$ $2,01+1130 \mathrm{G}^{(2)} \cdot 12,0, \mathrm{G}^{12} \mathrm{1}, 10, \mathrm{c}, 1 \mathrm{G}^{2}, 2, \mathrm{~J},-1 / 5 \mathrm{G}^{12}$ 2 $14(2) 3121$

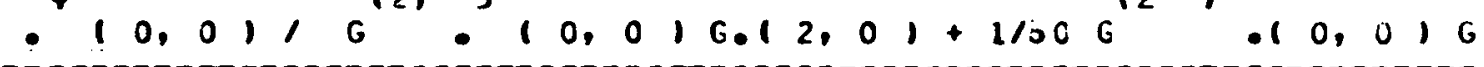




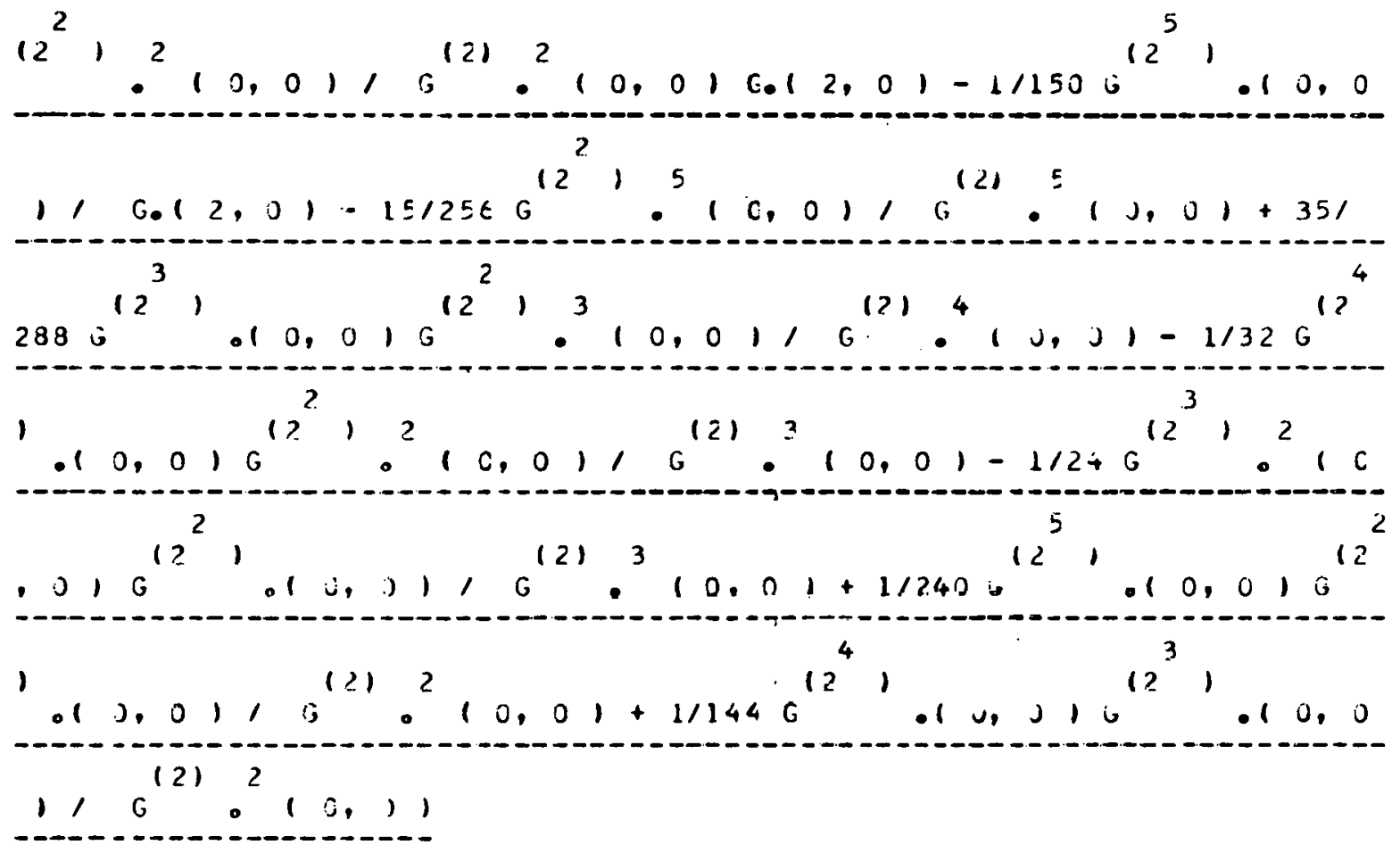

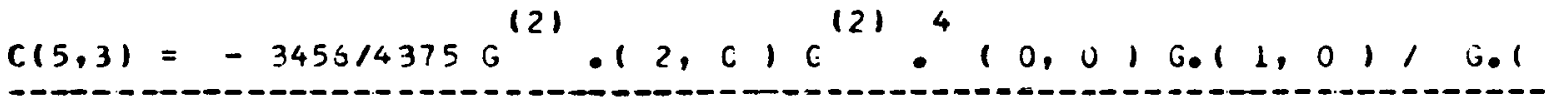

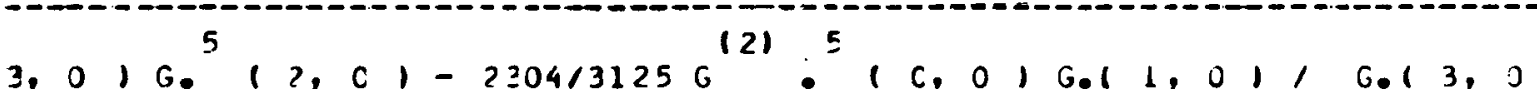
3. 0 1 G

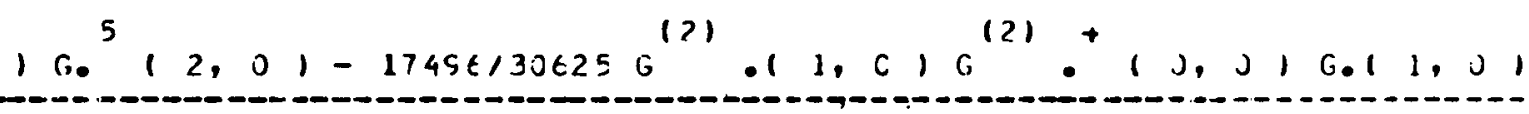

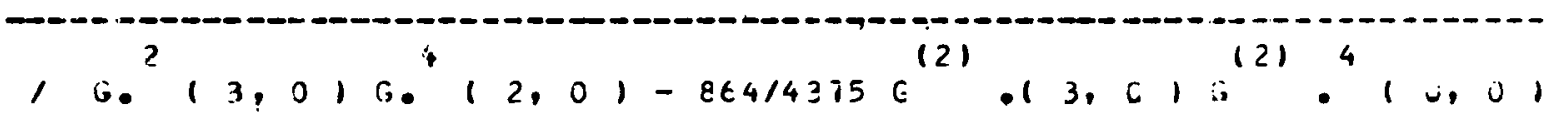

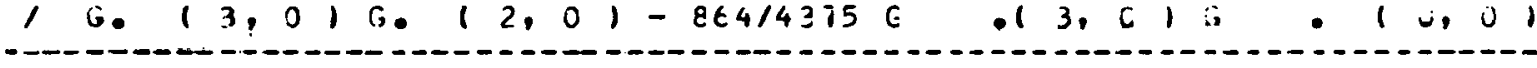

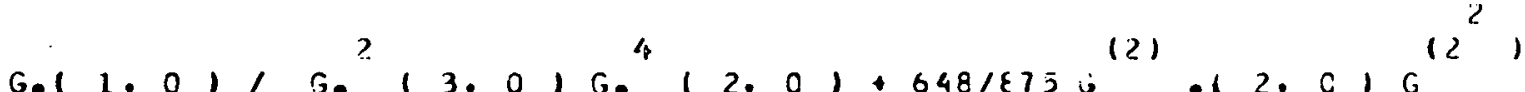

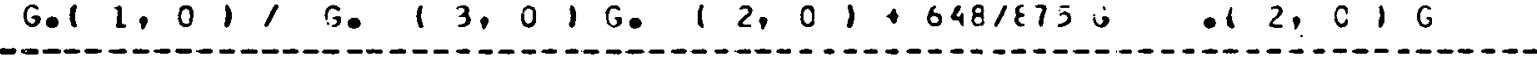

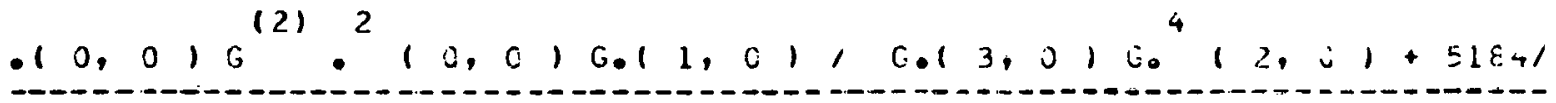
2
2 $\left.4375 \mathrm{G}^{12}, \cdot 10,0, \mathrm{G}^{(2)}\right)^{3}(0,0, \mathrm{G} .11, \mathrm{C}), 1,013,0100412$ (2) $121 \quad 4$

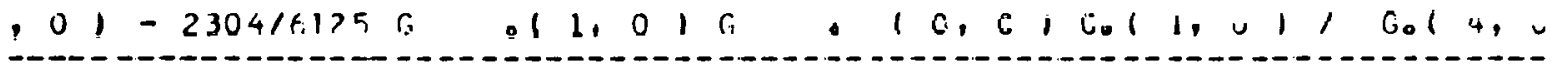

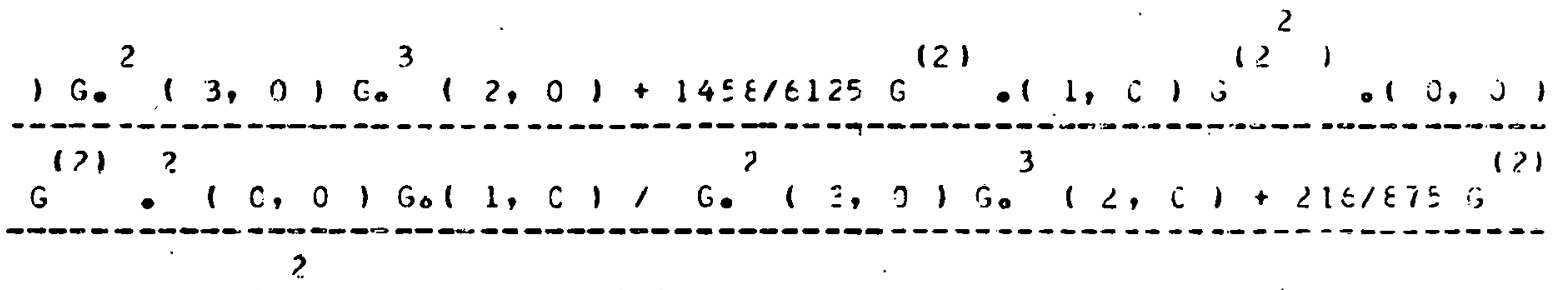
$\left.\because 3,0, G^{12}\right)$ 
${ }^{3}(2,0)-72 / 875 \sigma^{\left(2^{2}\right.}, .10,01 e^{(2)} \cdot(0, J) G .11,01, G_{0}$ $13,0, G_{0}^{3}, 2,01-27 / 175 G^{(2)}$ (2) 12,2

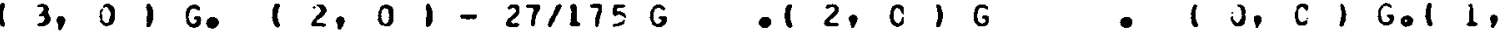
(1) $01, G .13,01 G^{3}(2,0)-432 / \varepsilon 7 G^{12} G^{2}, 0,010^{121} \cdot 10,0$ $1 G, 11,01,6.13,0, G_{0}^{3}(2,0)+288 / 1225 G^{121} \cdot 11,01 G^{12}$ $1 G, 11,01, G .13,0, G_{0}^{3}(2,0)+288 / 1225 G^{(2)} \cdot 11,01 G^{12}$ 2 1 $.10,0, G .10,0, G .11,0,1, G .14,0, G .13,016$. 0 (2., $0,1-27 / 350 G^{121} \cdot 13,01 G^{12} 10^{2}(0,0) G 011,01, G^{2}$ (12 $3,0, G_{0}^{2}\left(2,01.5 / 350 G^{12}, 310,0, G .11,0,1 G^{(2)}, 10\right.$, (3) $01 G .13,0, G^{2}, 2,01+9 / 175 \mathrm{G}^{12} 1,10,010^{12} 1,10,010_{0}$

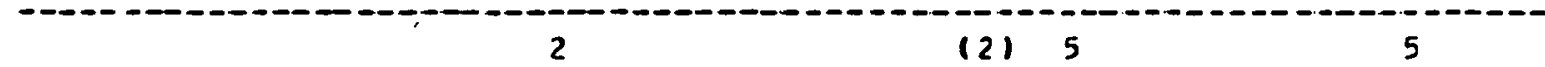
$11,01, G, 13,0, G_{0} 12,01-7 / 24 G, 011,00116011$, $0.121)^{1}$

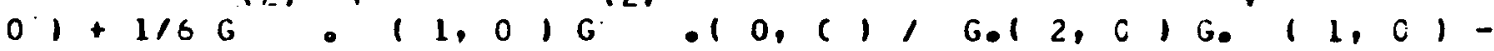

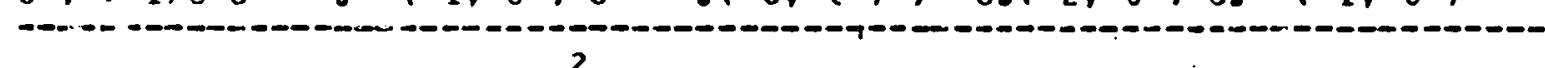

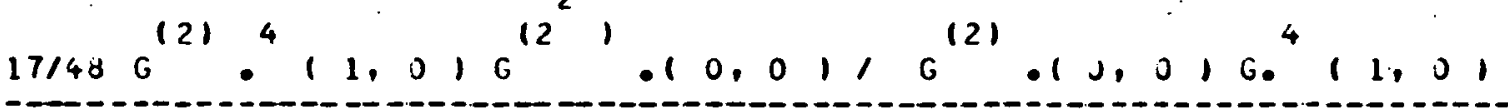
$\begin{array}{r}15 \\ \hline\end{array}$ $+1 / 2 G^{12} 1.11,01 G^{(2)} 0^{3}(1,0) 1 G^{4}(1,0)+4115 G^{(2)} 0^{3}$ 0 $\left(1,01 G^{(2)} 0^{2}, 0,0,1 G^{2}\left(2, c 1 \varepsilon^{3} \mid 1,01+115 G^{(2)} .12\right.\right.$

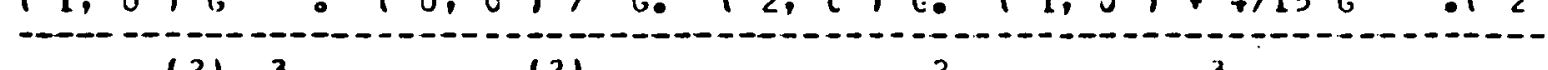
$, 016^{(2)} 0^{3}, 1,01 e^{(2)} \cdot(0, c), G^{2}, 2, u, c^{3} \mid 1,01-215$ 1
2

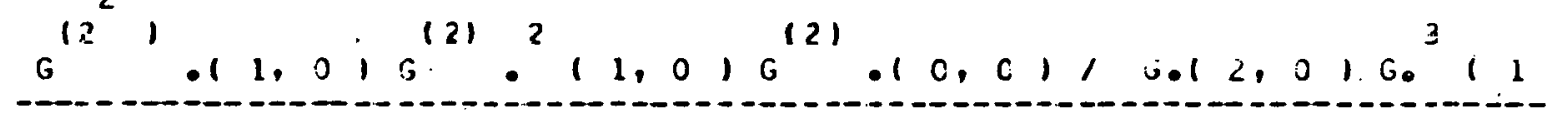

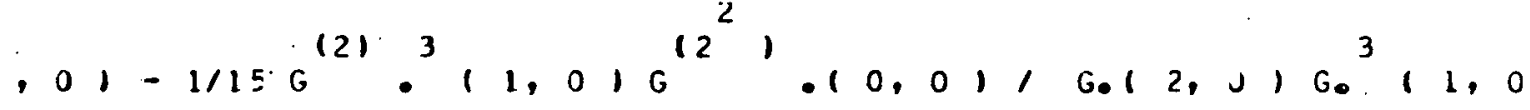
(

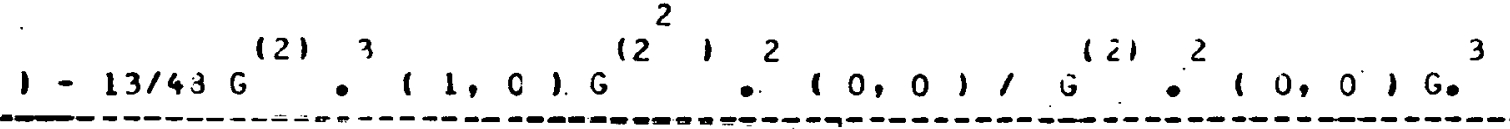


$11,01+1 / 20^{12^{2}} 1011,010^{(2)} 0^{2} 11,010^{12}, 01,0,1,0^{12}$ -

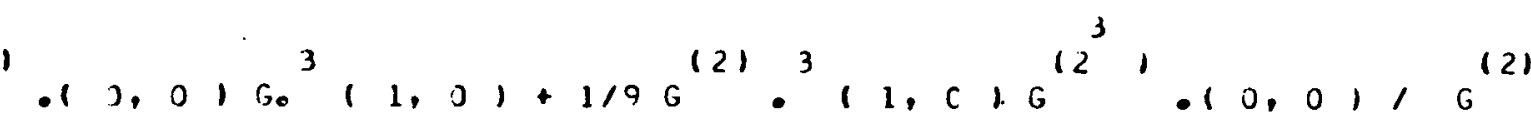

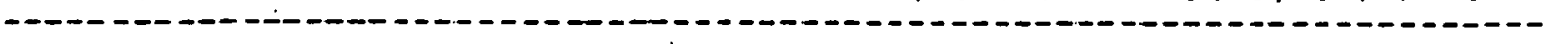

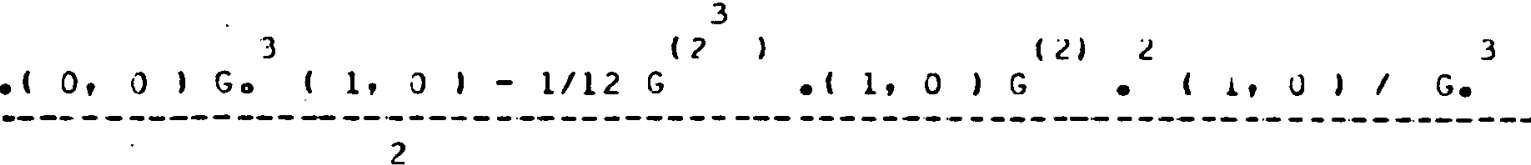
$(1,0)-1 / 9 \mathrm{G}^{12} \mathrm{G}^{2}\left(1,0, \mathrm{G}^{(2)}, 11,01 / \mathrm{G}^{3}(1,0) 1+16 / 25\right.$ (2) (2) 2 (2) 2

3
$\mathrm{G}: 12,0, \mathrm{G}:(1,0, \mathrm{G}: 10,0) 16,12,0, \mathrm{G}, 1$

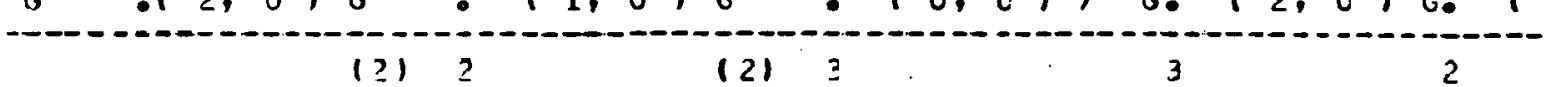
$1,01+16 / 75 G, 11,0, G, 1,0,0,1,6,12,010,11$

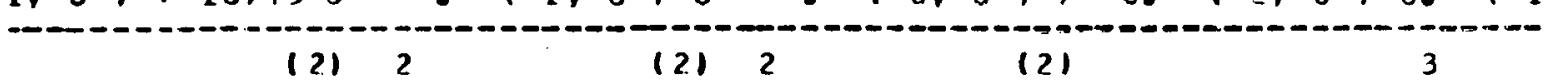

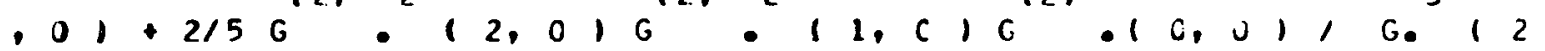
2
2

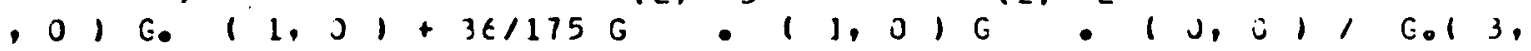
-

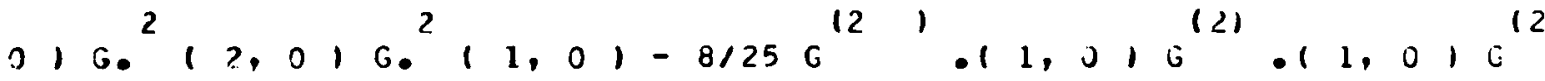

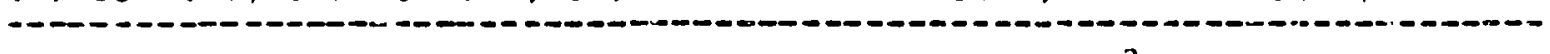
12201212121

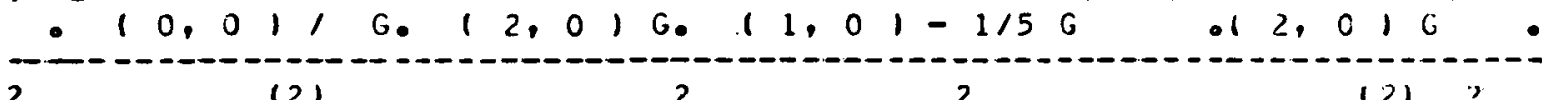
2

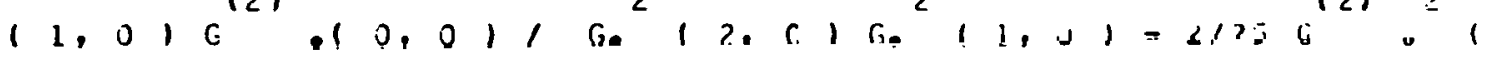
?
, 15 $1,0, G^{12}, .10,0, G^{(2)} \cdot 10,0,1, G_{0}^{2} 12,0,10.11,01-21$

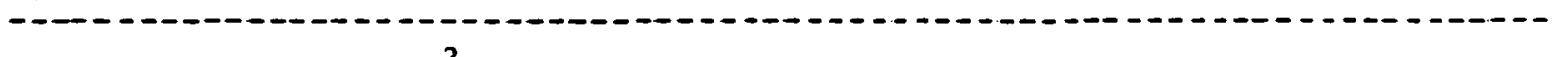

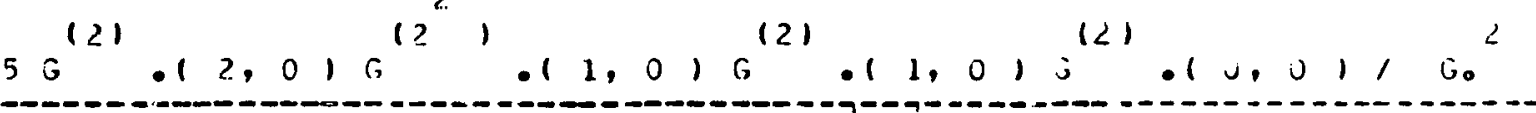
2
2

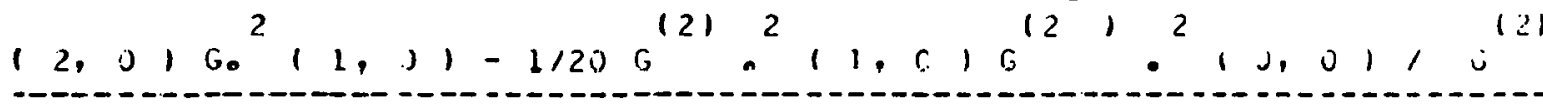
-

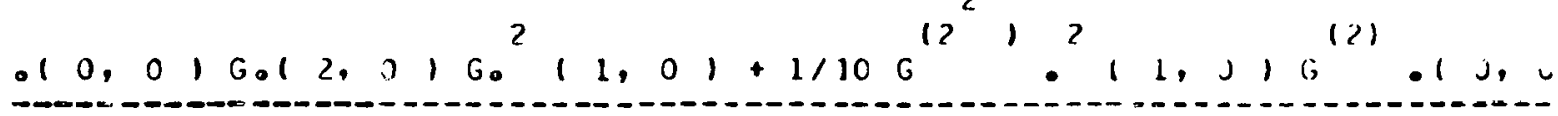

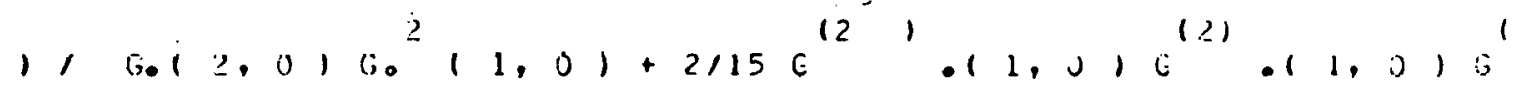
-

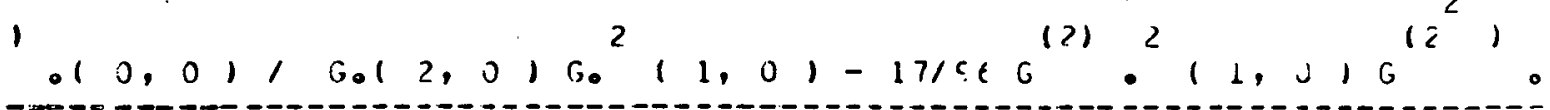




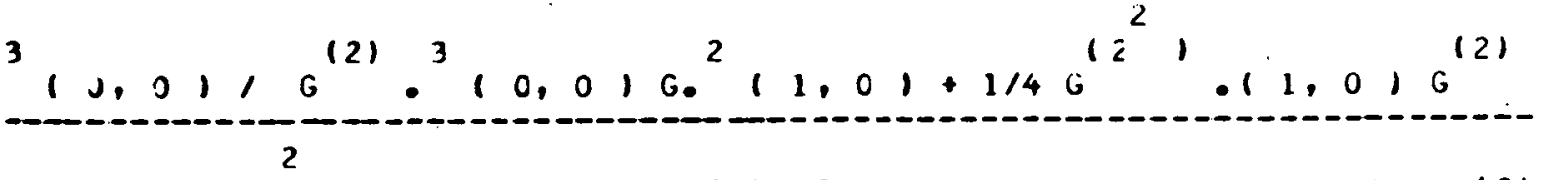
$.11,01 \mathrm{G}^{12} 0^{2}(0,0), \mathrm{G}^{(2)} 0^{2}, 0,0, \mathrm{G}^{2}, 1,01+1 / 0 \mathrm{G}^{121}$ (1 10

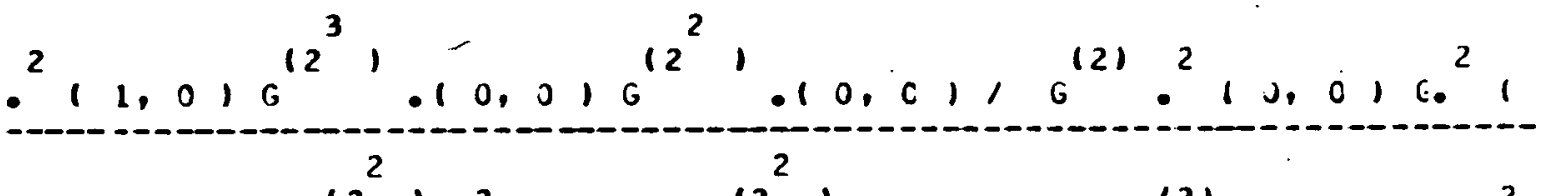

$1,01-1 / 166^{12^{2}}, 0^{2}, 1,0,6^{12}, 010,01,0^{121}, 10,0100^{2}$

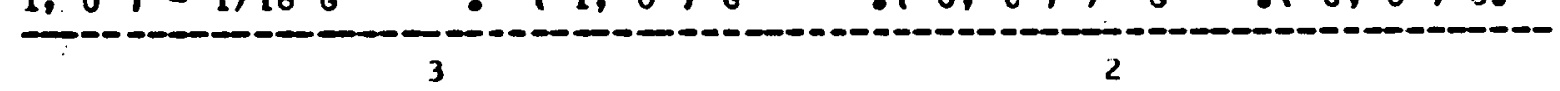

$11,31-1 / 12 \mathrm{G}^{12}, \cdot 11,0, \mathrm{c}^{121} \cdot 11, \mathrm{c}, \mathrm{G}^{12}, .1,1,01, \mathrm{G}^{121}$

$\left.12^{2}\right)(2) \quad 12^{3} 1$

$\therefore 0,01 G^{2}, 1,01-1 / 12 \mathrm{G}^{12} 1,11,01 \mathrm{G}^{(2)} \cdot 11, \mathrm{~J}, \mathrm{G}^{12} 1$

(1)

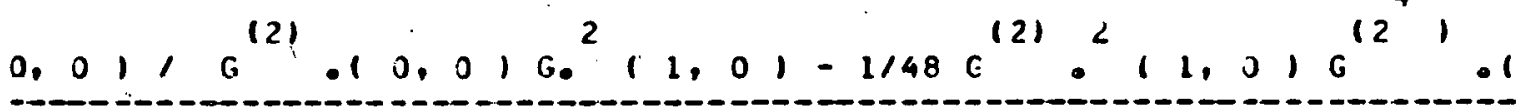
$\left.0,0, G^{(2)}, 10,0, G^{2}, 1, G,-24 / 25 G^{12}\right)^{2}, 2,0, G^{12}, 11$ $.01 G^{121}{ }^{2}, 0,0, G_{0}^{4}, 2,01 G 011,01-1921125 G^{121} .12,0$ $1 G^{121} .11,0, G^{121} 0^{3}\left(0,01, c^{4}, 2,010.11,0,-2241375\right.$

$121 \quad 121^{4}(12$ $6.11,01 G \quad 010,01, G, 12, C 16.11,01-10811756$

100
$1.12,01 G^{121}{ }^{2}, 1,01 G^{121}{ }^{2}, c, c, 1, G .13, j, G^{3} 12,01$

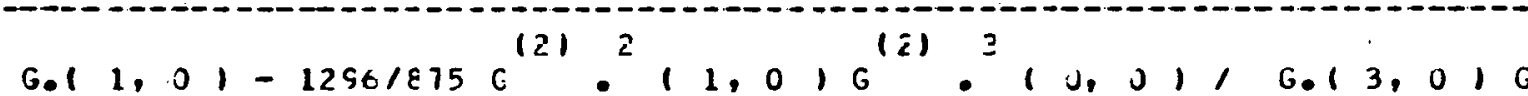
(6. ${ }^{3} 12,016011,01+368 / 375 G^{121} .11,01 G^{(2)} \cdot 10,016^{(2)} .^{2}$

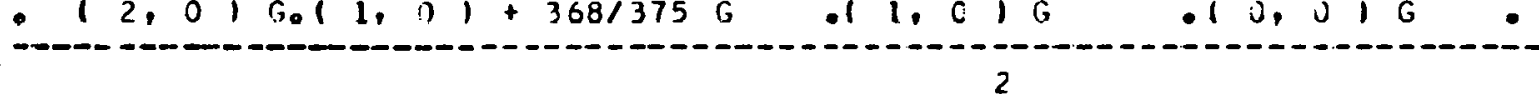
$\left(J, J 1, G 0^{3}, 2,01 G 0(1,0)+\varepsilon / 25 G^{(2)}, 12,0\right) G^{(2)} \cdot 11, c$ $151 ?$

$1 G \cdot 10,01, G,(2,0) G 011,0,+\varepsilon / 25 G \quad 012,01 G$ ?

$1 \quad 12120 \quad 3 \quad 12$ $\because 11,01 G$ G 010,011 G. $12,01, G 011,61+321125 \mathrm{G}$ $\begin{array}{llllll}1 & 121 & 3 & 3 & 121\end{array}$ $.1,0, G, 10,01, G, 12,0,6.1, c 1,221256$ 


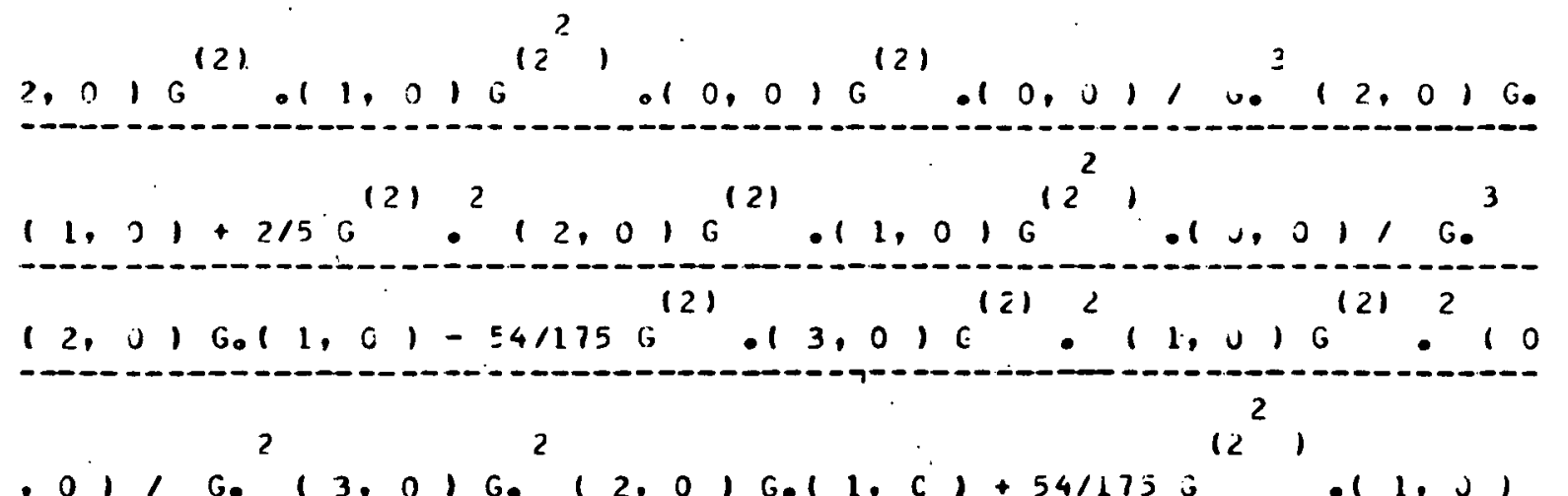

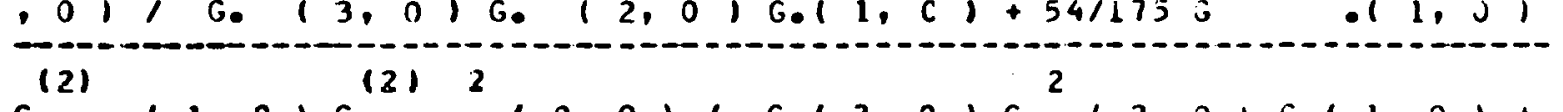
$\mathrm{G}^{\circ 1}, 0, \mathrm{G}^{\circ}, 10,0,1 \mathrm{G}, 13,0, \mathrm{G}, 12,0,6.11,01+$ 5
2

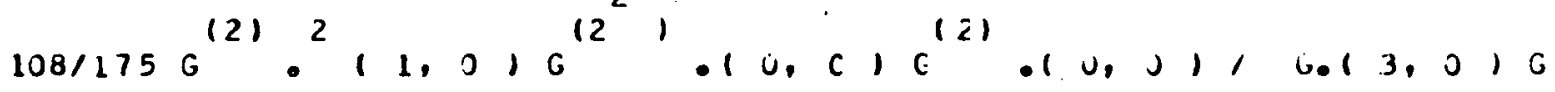
108/

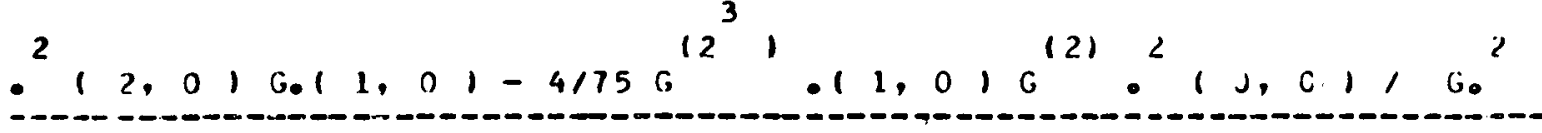

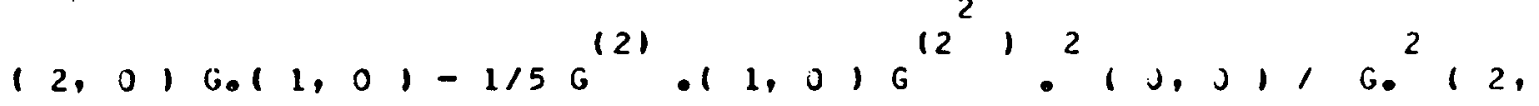

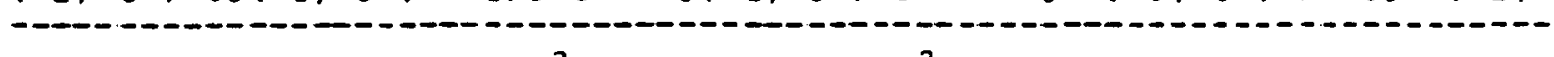

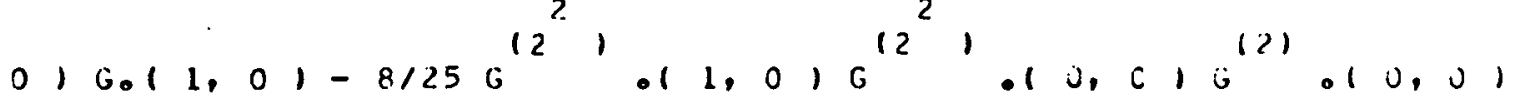

0--

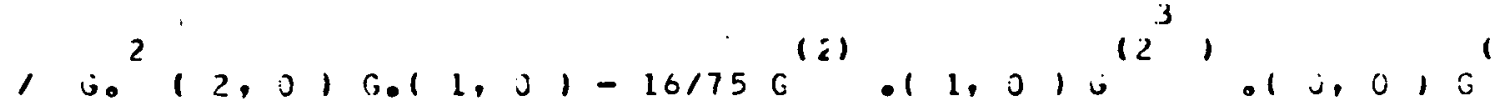

- - - -

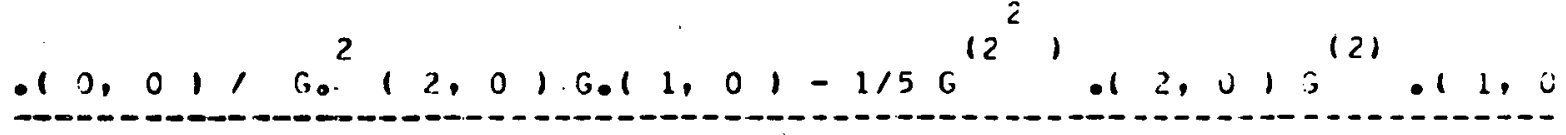
5
5 $j 0^{12^{1}}, 10,0,1,0^{2}, 2,0,0.11,01-1 / 50^{121}, 12,010012$

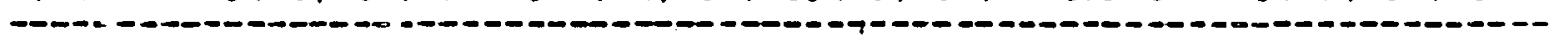
$1 \quad 12^{2}, \quad 2 \quad(2)$

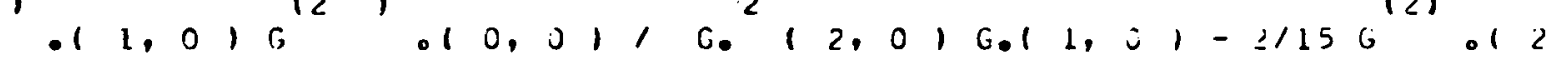
-

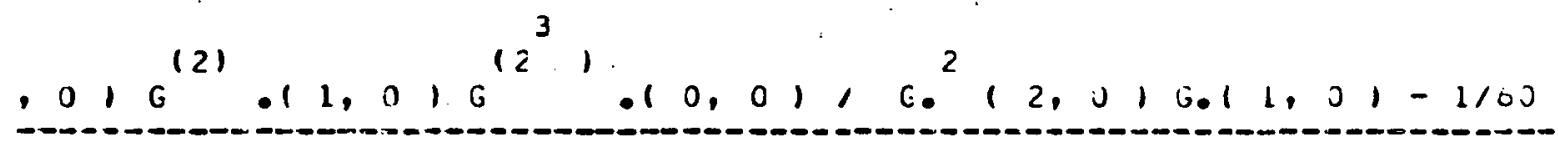

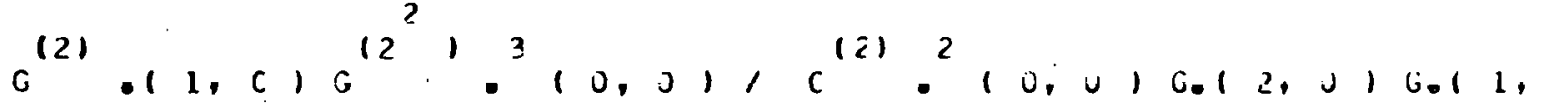

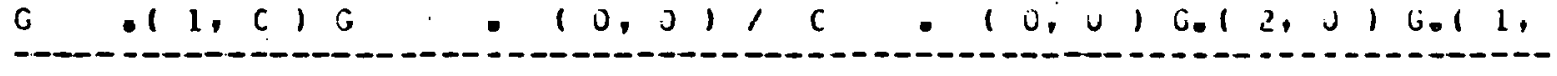

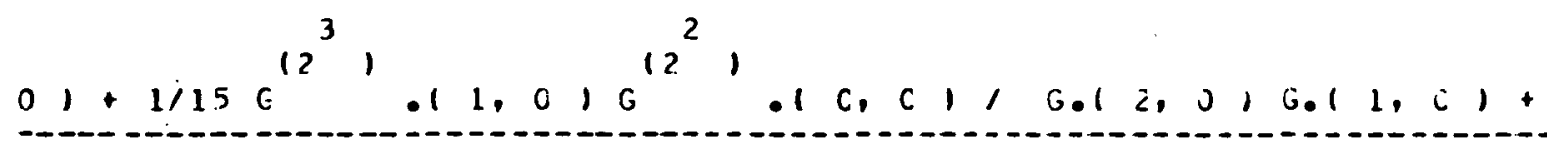


$1 / 15 G^{12^{2}} 1.11,0, G^{12^{3}}, \cdot 10,0,1, G_{0}(2,016.11,01+1 / 30 G$

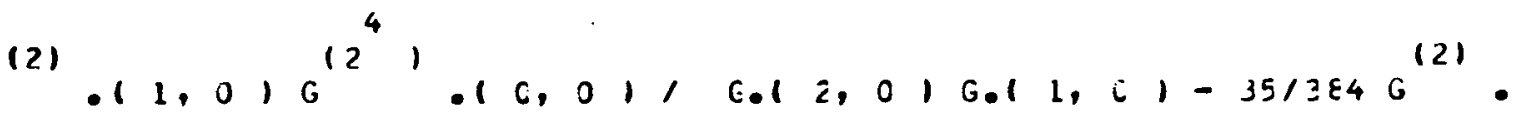
12

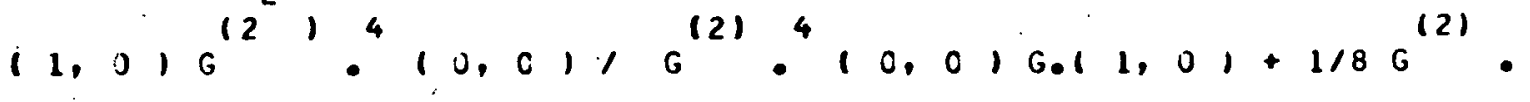

-

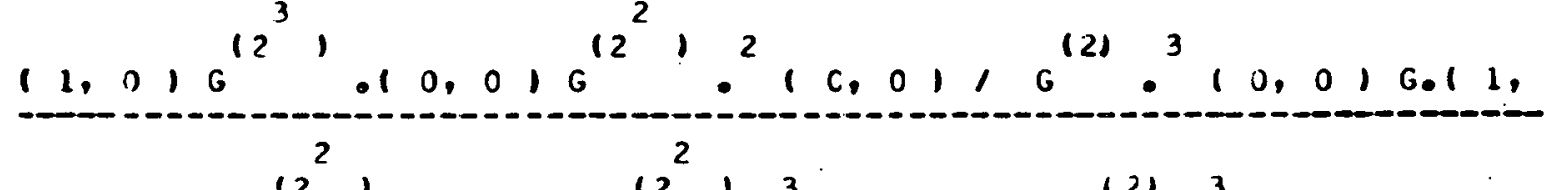
$01+1 / 160^{12} 1.11,016^{12} 10^{3}, 0,01,6^{121} 0^{3} 10,016.11$ ${ }^{3}$
-

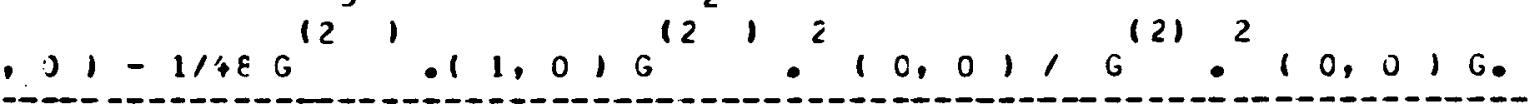

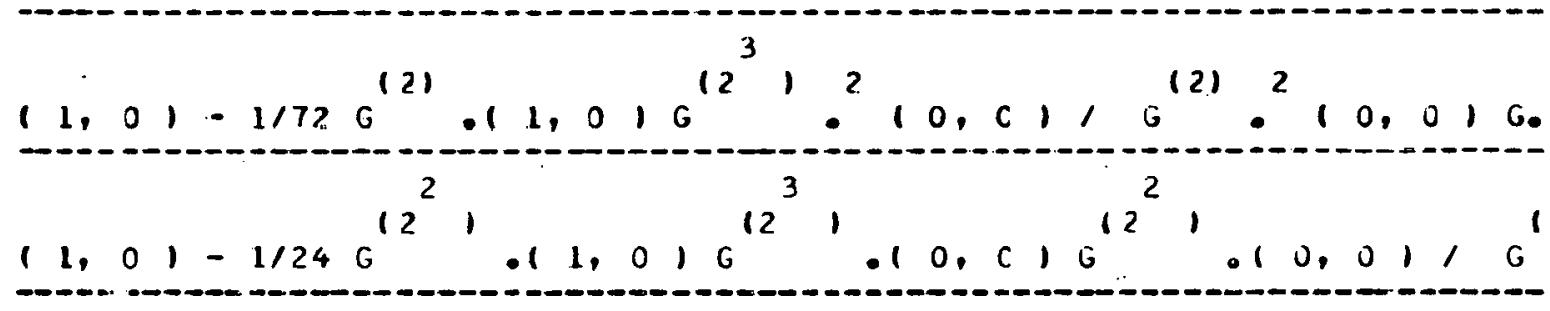
(1) $21210,01,0.11,01-1 / 48 G^{121}, 11,0, G^{12}, .10,016^{1}$

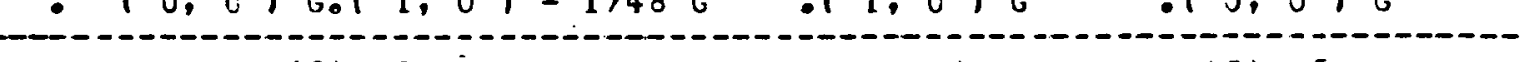
$.10,01, \mathrm{G}^{(2)} \cdot{ }^{2}(0,0) 1 \mathrm{G}, 11,01+4 t 656 / 1 \leq 3125 \mathrm{G}^{(2)} 0^{5} 14,01$ $2,2,5,2,1215$

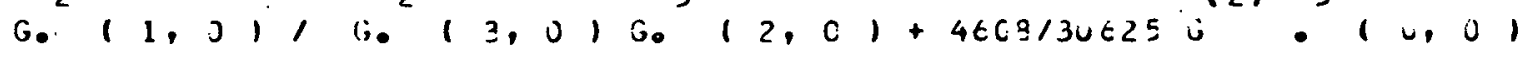
$G^{2} i 1,0,1, G 014,0, G_{0}^{2}, 3,0, G^{4}, 2,01-8748 / 306256^{12}$ 10121302040

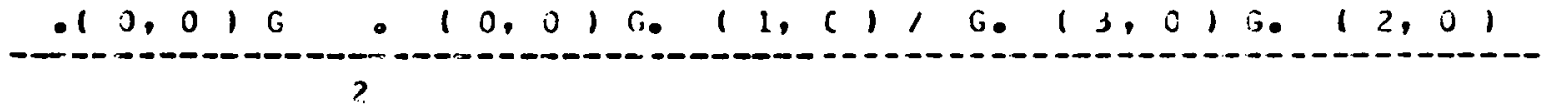

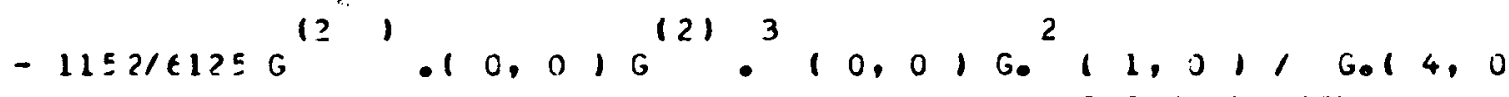

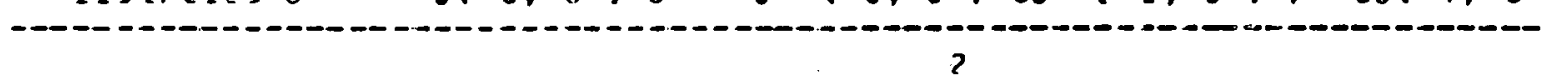

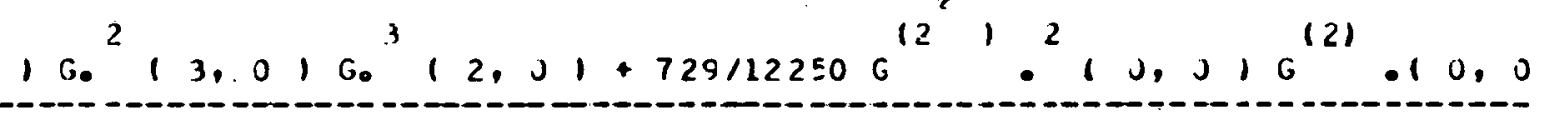
(1 $10^{2}, 1,0,1,0^{2}, 3,0 i 0^{3} 12, c 1+72 / 12250^{12} 0^{2} 10,0$

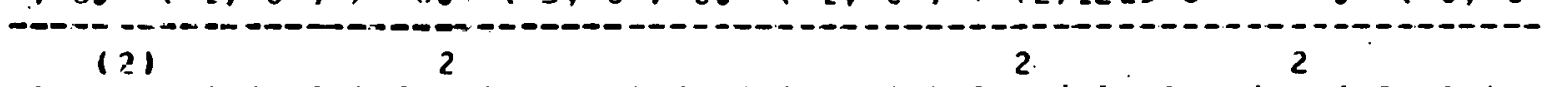
$1 \mathrm{G} .10,01 G_{0}\left(1,0,1, G_{0}\left(4,0, G_{0}(3,0,1\right.\right.$ b. 12,01 
$+64 / 1250^{(2)} 0^{2}(2,0) G^{(2)} 0^{3}(0, C), G 0^{5}\left(2,01+256 / 375 G^{1}\right.$

0

2)

(2) 4

(2) 5

.$\left(2,01 G, 10,0,1 G_{0}(2, C)+2176 / 53750,010,0\right.$

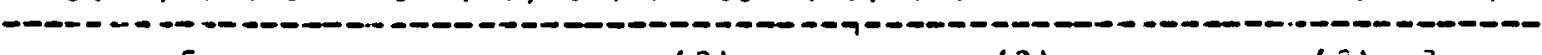
5

(2)

(2)

(2) 3

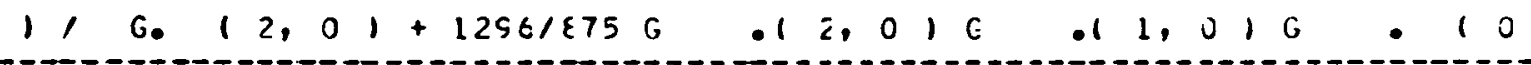

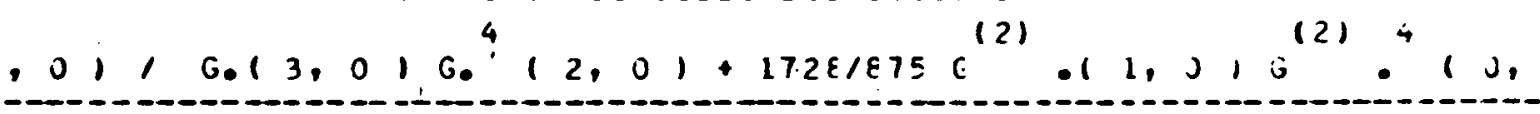
$\left.4 \quad(2) \quad 0 \quad 0 \quad 0^{2}\right)$ $\left.01, G .12,0, G_{0}^{4}(2,0)-128 / 125 G^{(2)}-12,010^{(?)}, 10, i\right)$

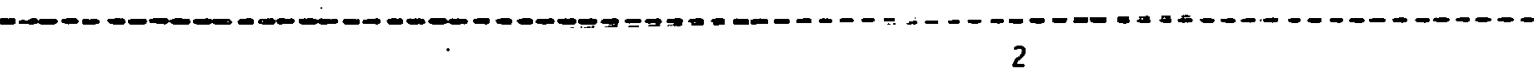
$\left.G^{(2)}: 10,01,60^{4}, 2,0,-20 \varepsilon / 37 \leq e^{12},(c, j) 0^{(2)} 0^{3} 1\right)$

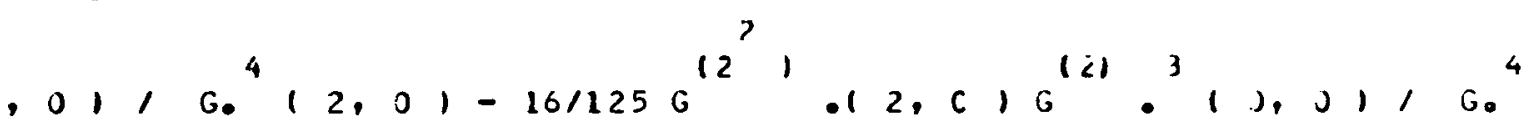

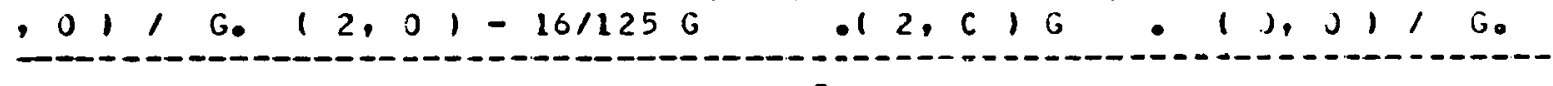

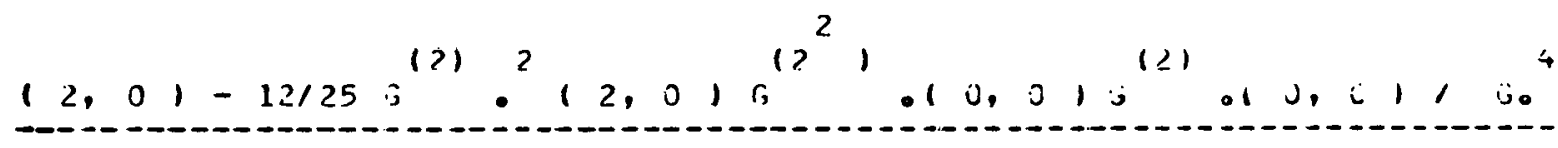
$(2)^{2}\left(\begin{array}{lll}3 & \end{array}\right.$

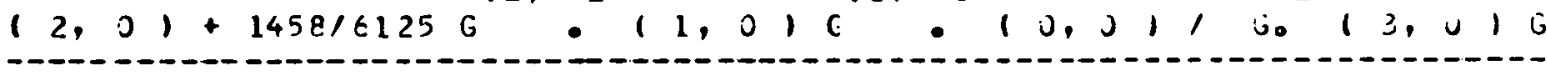
$0^{3} 12,01+432 / \varepsilon 75 G^{(2)} \cdot(3,0) \epsilon^{(2)} \cdot\left(1,0, G^{(2)} 0^{3}, 0,0\right)$,

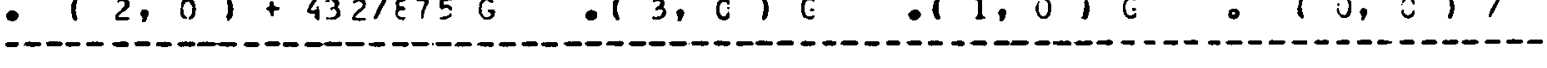

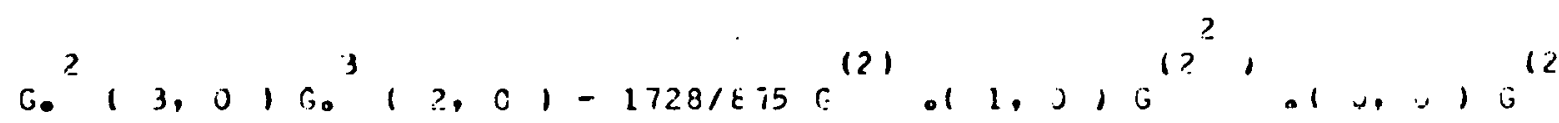

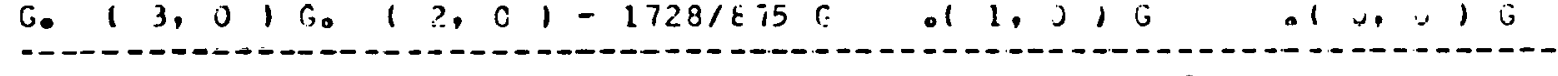
$123\left(0^{2}\right.$,

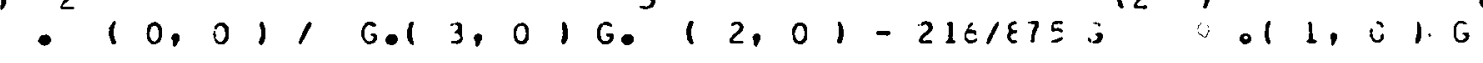
3 $-1,01, G 013,0, G_{0} 12,01-168 / 17500012,01001$ $\begin{aligned} r^{2} & \end{aligned}$

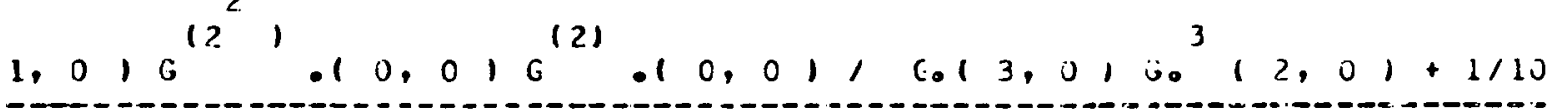
2

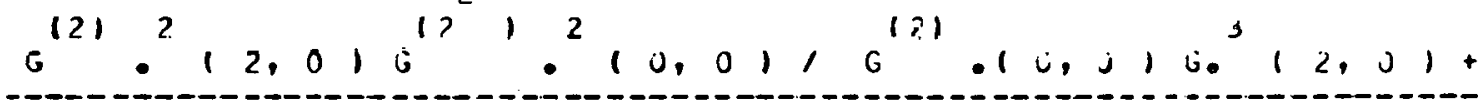
(-0.-0.

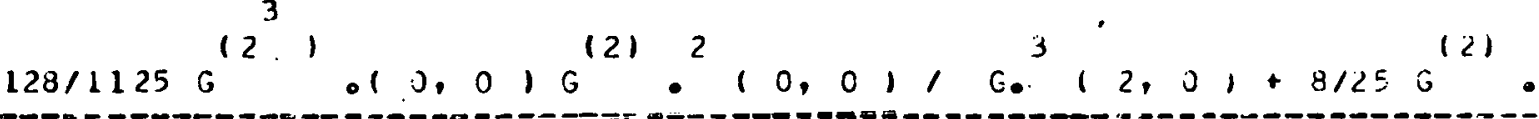

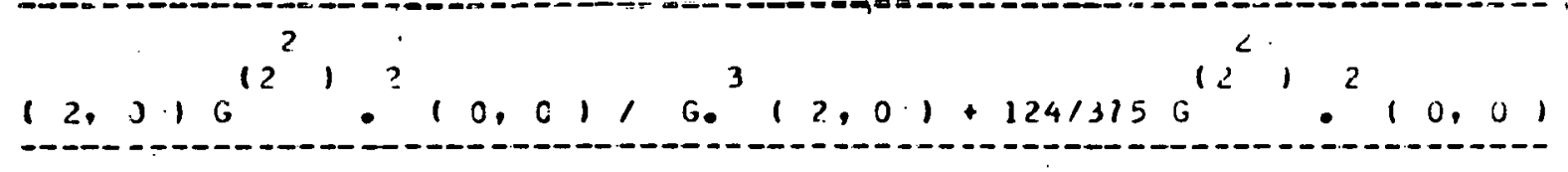




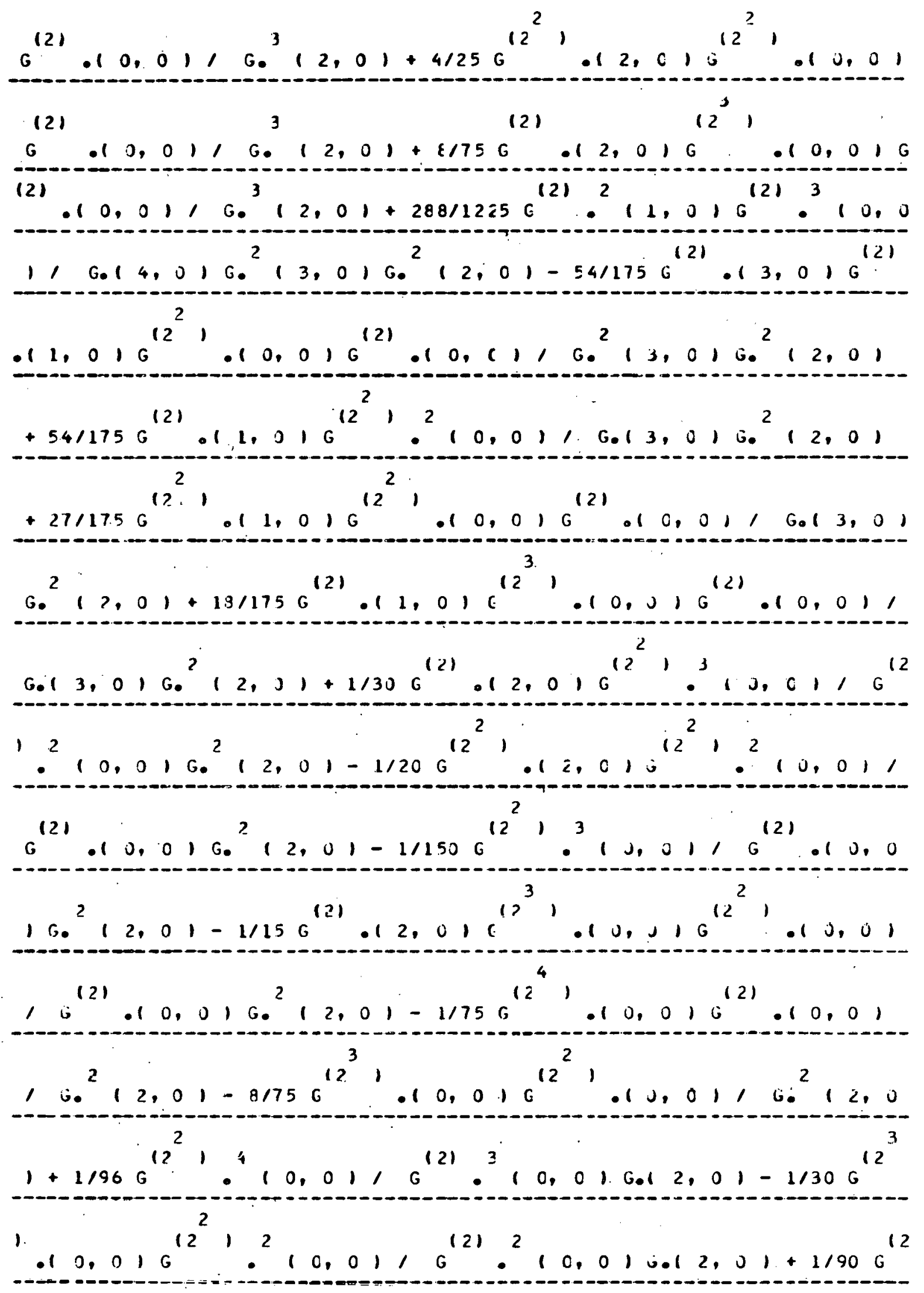


3

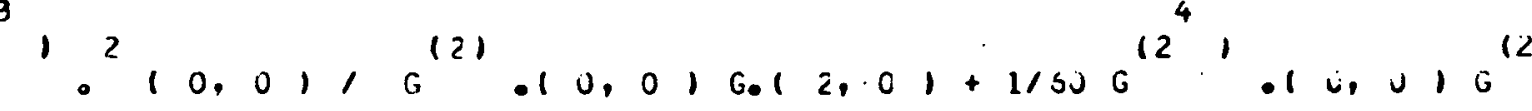

2

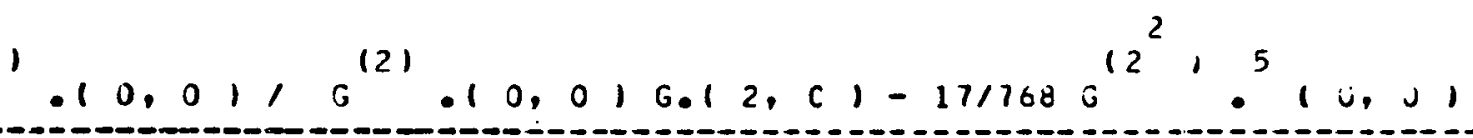

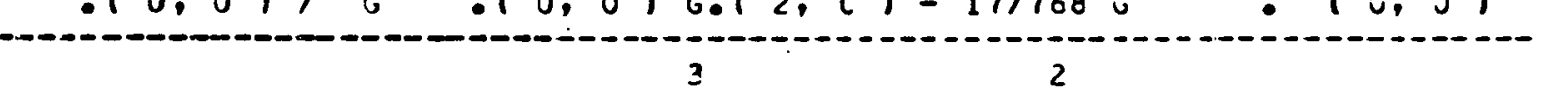

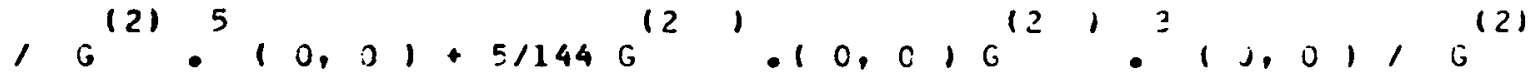

-

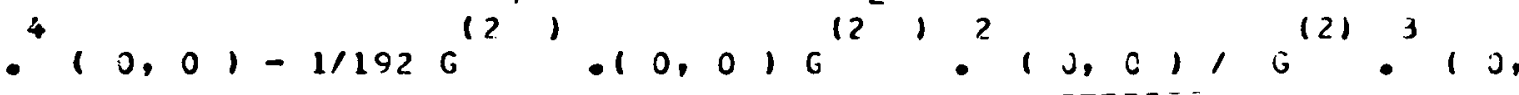

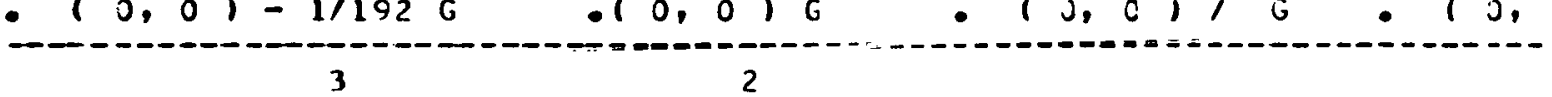

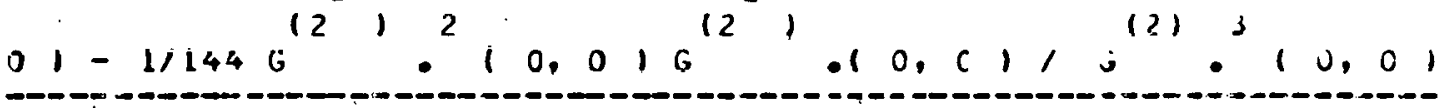

(

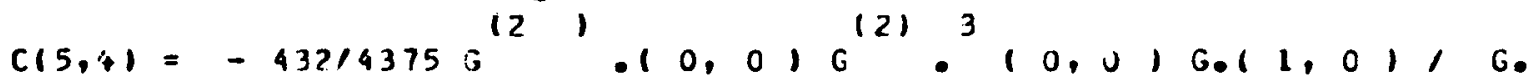

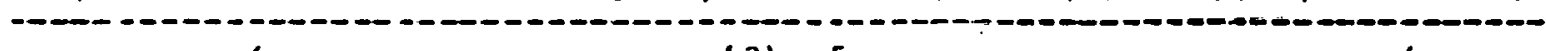
$(3,0) G_{0}^{4}(2,0)+128 / 625 G^{(2)} G^{5}\left(0, C, G_{0}(1,0,1, G .12\right.$, 1

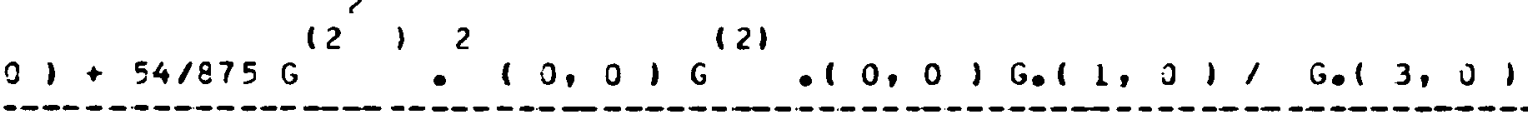
20

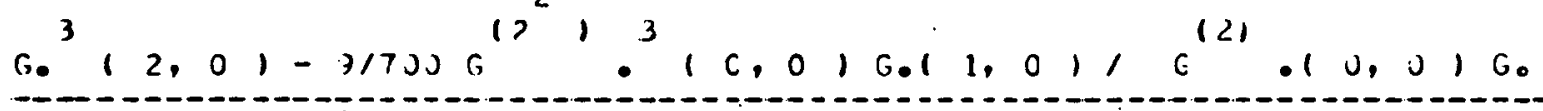
2 (2) 5 (2) (3, $0, G_{0}(2,0,-1 / 12 G, \cdot 11,0,1, G,(1, J)+1 / 6$

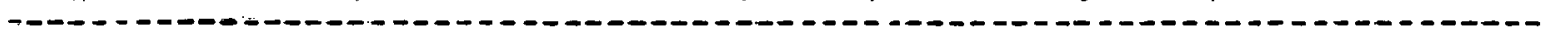
$4 \quad(2) \quad 4 \quad 6 \quad 12) .4$ - $11,01 G$ G $0,0,1, G .12,016011,01-7 / 40 G, 1$ 12

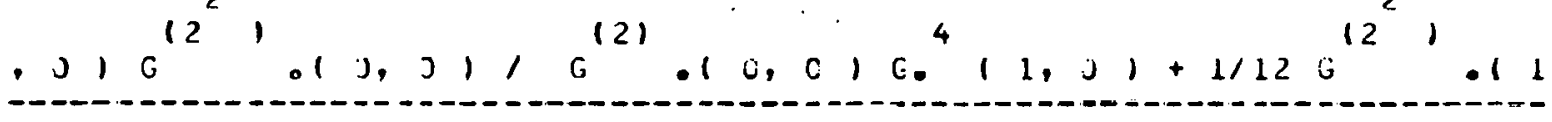

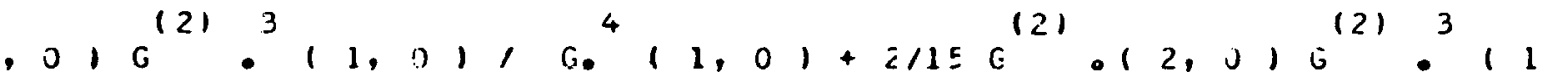
$\div 0$

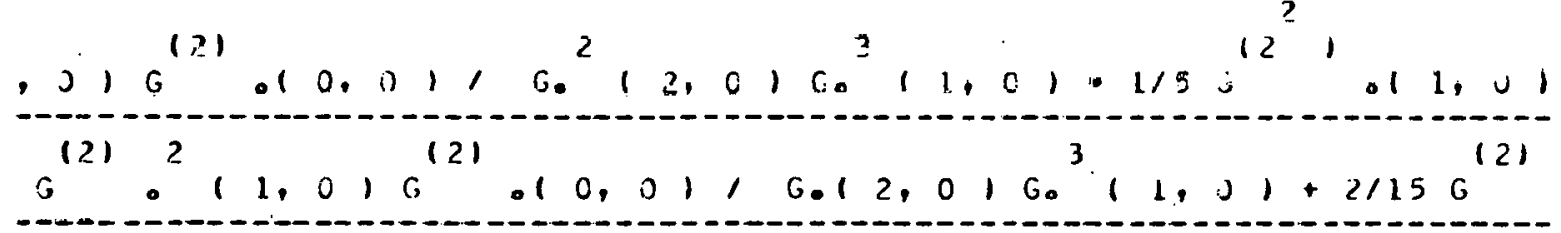

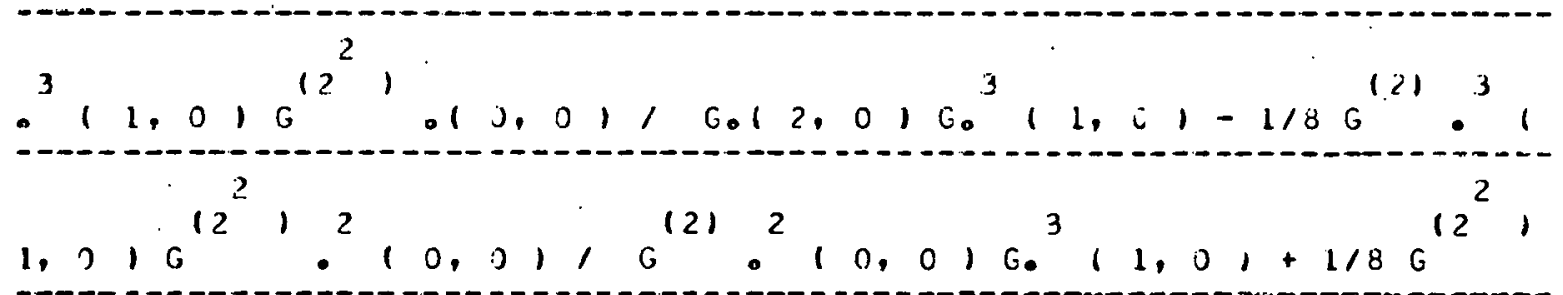




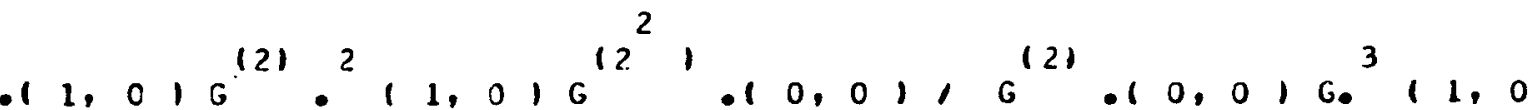
( 10

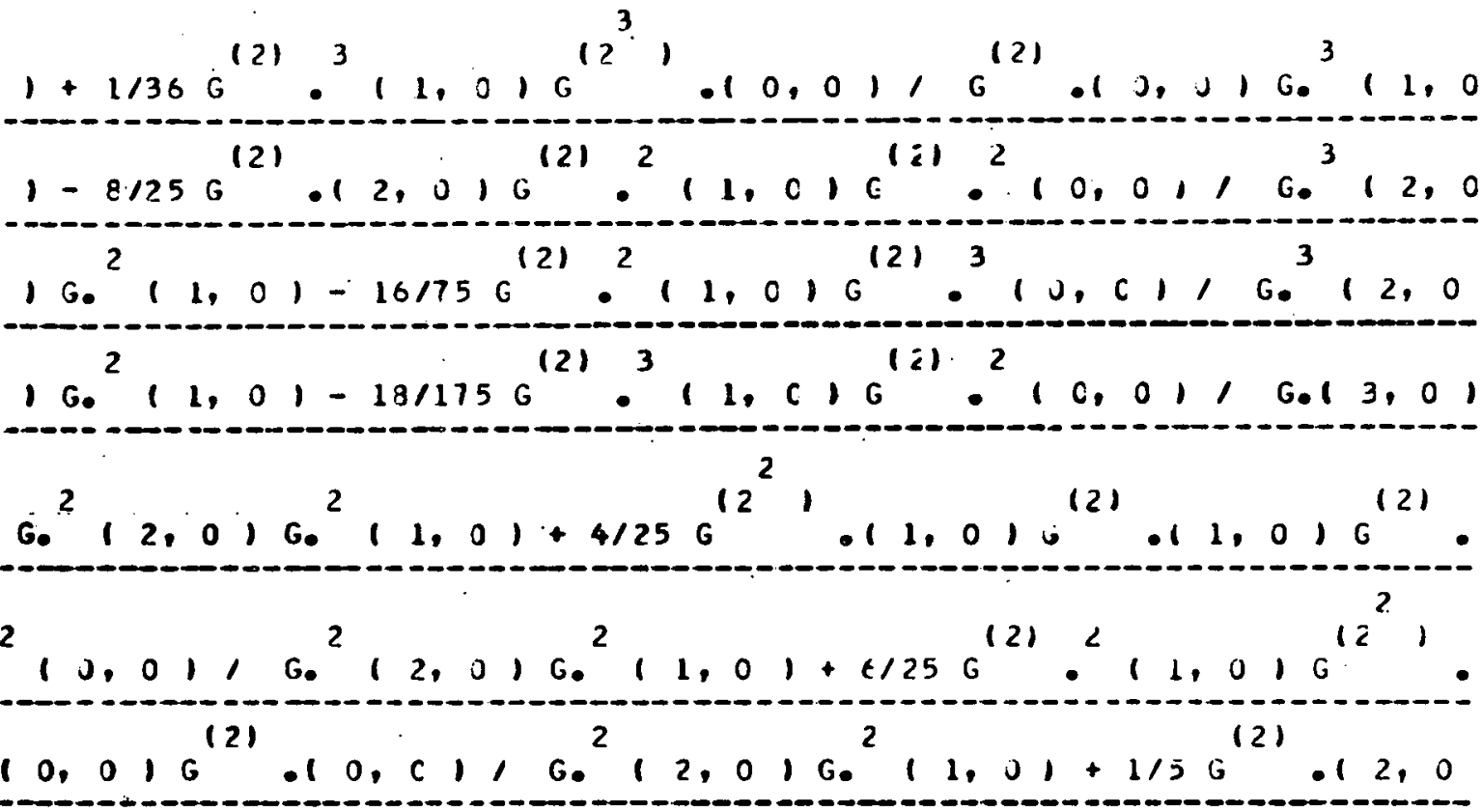

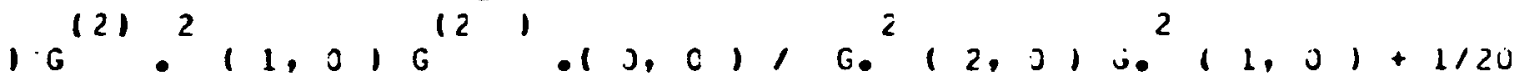

0

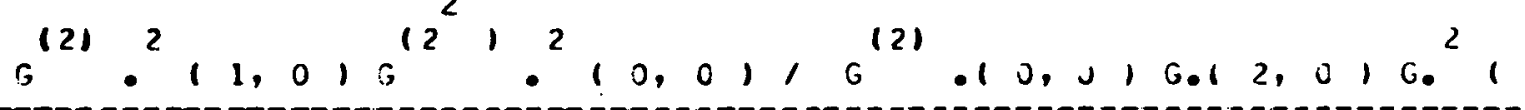

$1,01-11 \mathrm{G}^{12^{2}}, \cdot 11,0, \mathrm{G}^{12} \cdot 11,0, \mathrm{G}^{12^{2}}, \cdot 10,01, \mathrm{G}, 121$ $21,122^{2}, 1,12^{3} 1$

$016.11,01-1 / 15 G$ G $11,01 G$ G 1,011 Gol $2, J 1$ - - - - - $G^{2}, 1,01-7196 G^{121} 0^{2}, 1, \mathrm{C}, \mathrm{G}^{12} 0^{3}, 0,01, \mathrm{G}^{121} 0^{3} 10$, 0

$01 G 0^{2} 11,01+1 / 16 G^{12}, 011,01 G^{(2)} \cdot 11,0,3^{12}, 3^{2}, 1,0$

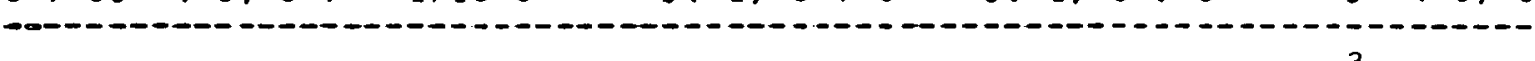
$1,0^{(2)} 0^{2}, 0,0,0^{2}(1,0)+1 / 24 G^{(i 2)} 0^{2}, 1,010^{1.2^{3}}, .10$ 15 $.01 G^{12} \cdot 10,01, G^{(2)} 0^{2}(0,0) G^{2}(1,0)+321125 G^{(2)}$.

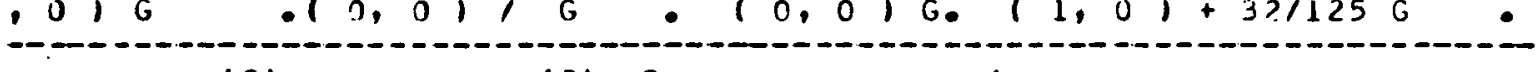
$\left.(2,0) G^{(2)},(1,0) G^{(2)},(0,0), G_{0}^{4}, 2, J 1 \mathrm{bel}, 1,0\right)+\epsilon_{4}$ 1 $2,1 \mathrm{~g}$ 
$1375 \mathrm{G}^{(2)} \cdot 11,010^{(2)} 0^{4}\left(0,0,1, c^{4}(2,0), 0.1+0\right)+2101$

-
$\varepsilon 75 G^{(2)} e^{2}\left(1,0, G^{(z)} e^{3}(0,0), E_{0} 3,0, G^{3}(2,0) 0.11\right.$, (-10.--

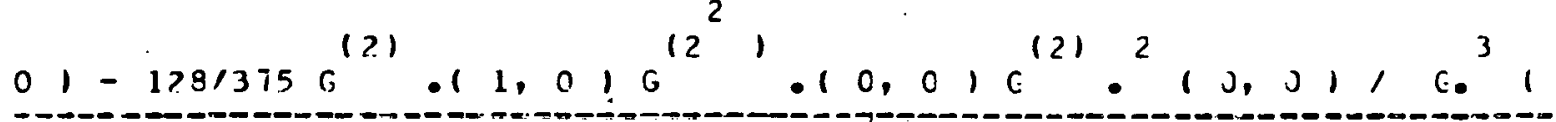
$12^{2}, \quad 121 \geq$

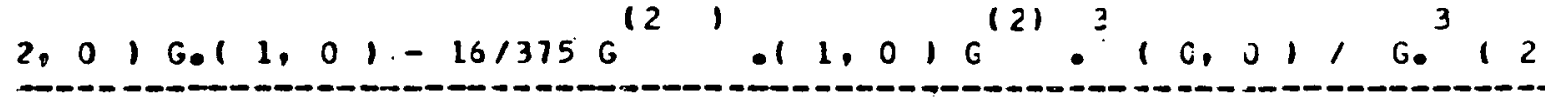
$, 01 G 011,01-3 / 25 G^{(2)}-12,01 G^{(2)} .11,0,6^{12} 1,-10.010$ 181. (2). $\left(0,0,1,0^{3}(2,0,601,0)-2711750^{(2)} 0^{2} 11,0,6^{12}\right.$ 1 $.10,0, \mathrm{G}, 10,0,1, \mathrm{G}_{0}, 3,0, \mathrm{G}_{1,12,0,1,1,1,0,1,10}$ -

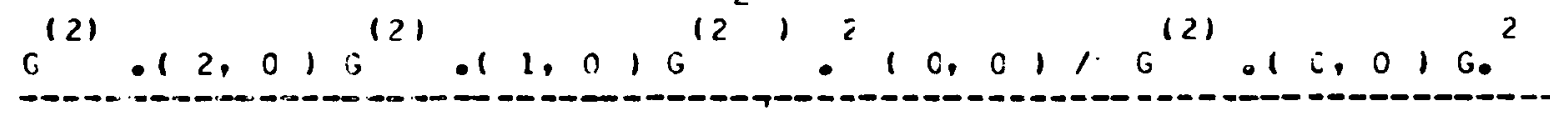

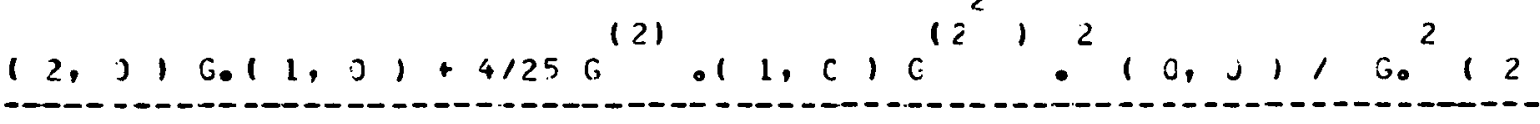

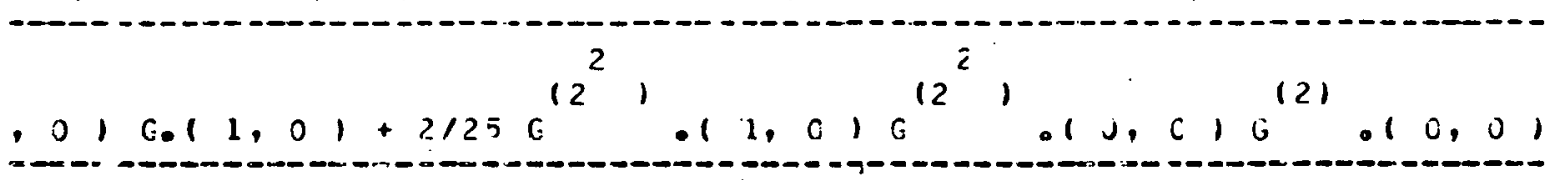
$100^{2}\left(2,010011,01+41750^{121} \cdot 11,010^{12}, 010,010^{12}\right.$ - - - -

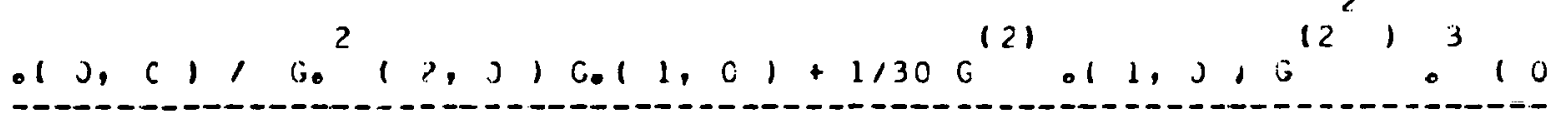

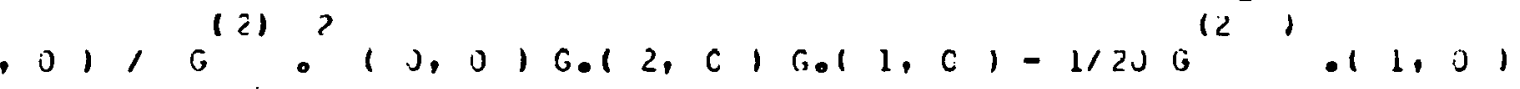
3
3

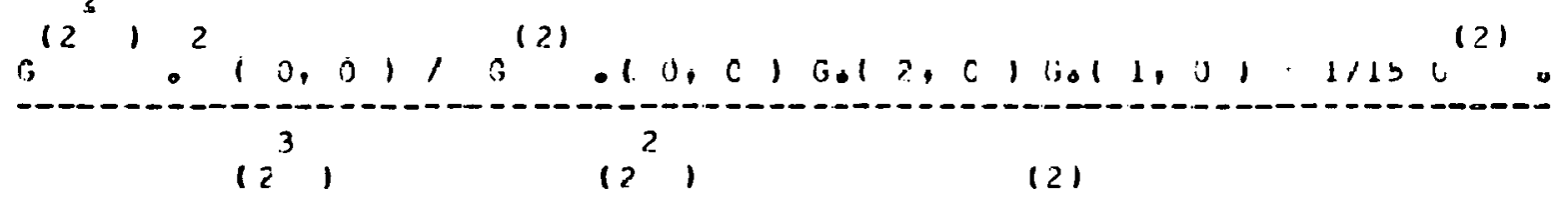

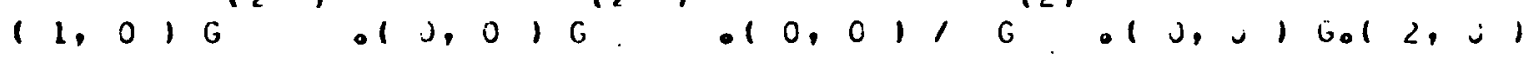
-

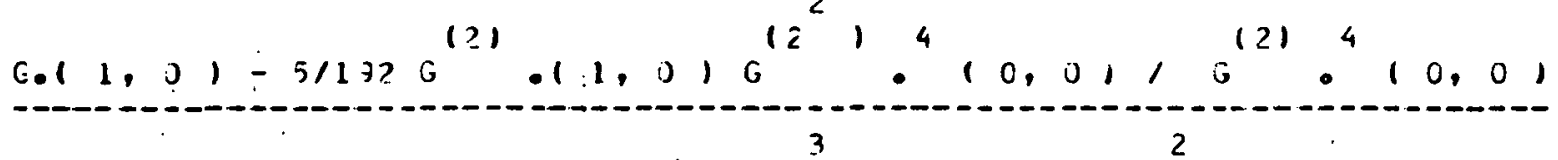

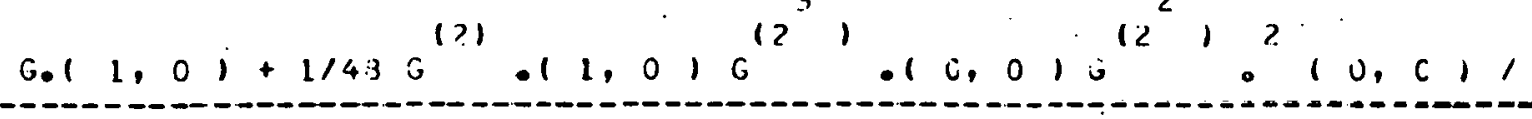




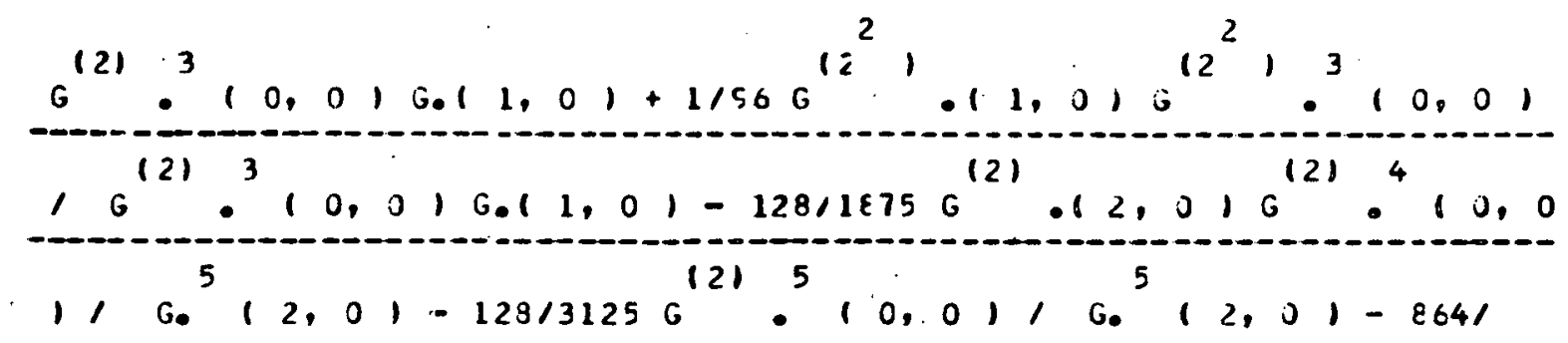

$4375 G^{(2)}, 11,0, G^{(2)} \cdot(0, C), G, 13,0,6.4(2,0)+16 / 125$

4

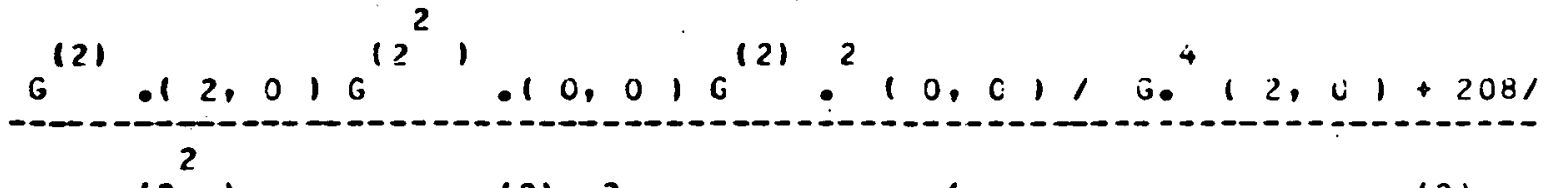
$1875 G^{(2)} \cdot 10,01 G^{(2)} \cdot 0^{3}, 0,0,1 G_{0}^{4}(2, J)+\angle 15 / E 75 G^{(2)}$. (1875 G $1,016^{12^{2}}, 10,016^{(2)} 0^{2}(0,0), 6.13,0100^{3}(2,01$, $16 / 1125 G^{12^{3}}, 10,01 G^{(2)} 0^{2} 10, c 1, G_{0}^{3} 12,01-2125 G^{121}$.

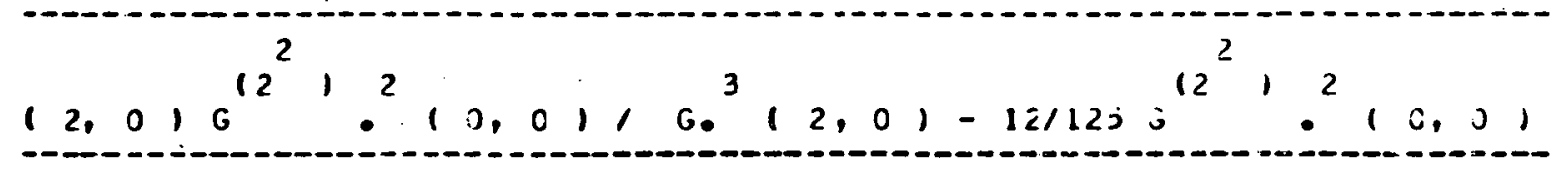
(1)

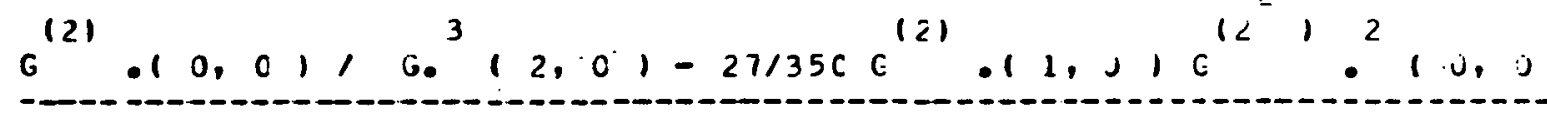
G $1, G .13,0, G^{2} 12,01+1160 G^{121} \cdot 12,0, G^{12}, 0^{3}, 0,01$

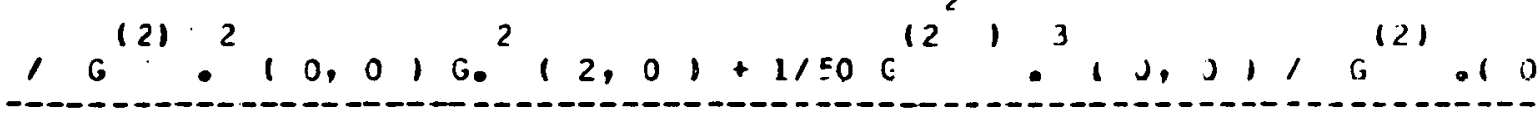
30

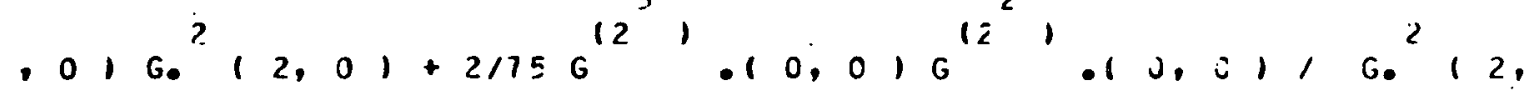

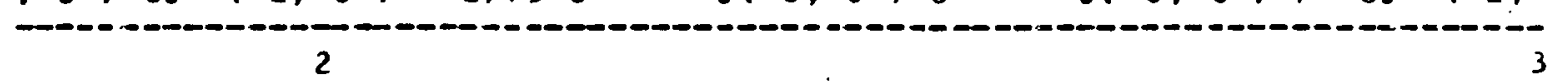
$\left.\left.01+1 / 96 G^{12}\right)^{4}(0,0), G^{(2)} e^{3}, 0, c\right) 0.12,01-1 / 60 G^{12}$ ( $1.10,0, \mathrm{G}^{12}, 0^{2}, 0,01, \mathrm{G}^{(2)} 0^{2}, 0,010012,01-1 / 250 \mathrm{c}^{1}$

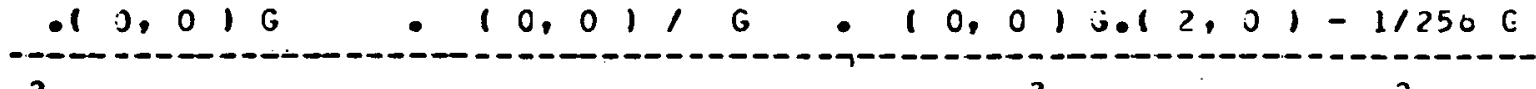
$2^{2}{ }^{5}{ }^{5} 10,01, \mathrm{G}^{(2)}=10,01+1128 \mathrm{G}^{12^{3}}, 01 \mathrm{~J}, 01 \mathrm{G}^{12^{2}} \mathrm{G}^{3}$ $10,01, a^{(2)} * 10,01$ 
$c(5,5)=-1 / 120 G^{(2)} 0^{5}, 1,01, c^{5}\left(1,01+1 / 30 G^{(2)} \cdot 41,0\right.$ $(2)$ $16.10,01,6.12,016011,01-1 / 486,011,016$ 2

$1 \quad(\geq), 4 \quad 12$ $.10,01 / G, .10,016.11,01-4 / 75 G, 011,016$ (1) $12,0,01, G_{0}^{2} 12,0, G^{3}, 1,01+1 / 156^{(2)} 0^{3}, 1,01.6^{12}$

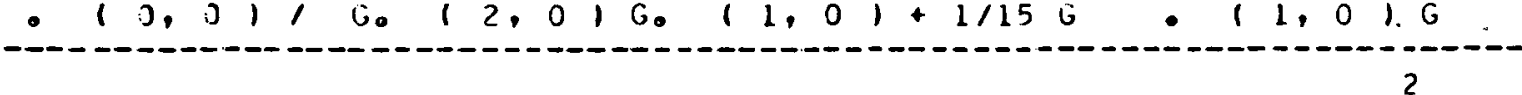

$1, G^{3}, 1,01-1448 G^{121} 3,1,010^{12} 2^{3}$ $.1,0,01 / G_{0}, 2,0, G_{0}(1,0,1-1148, G, 011,0160$ $10,01, \mathrm{G}^{(2)} 0^{2}\left(0,0, \mathrm{G}^{3}(1, \mathrm{C})+1613750^{121} 0^{2}\left(1,01 \mathrm{G}^{121}\right.\right.$

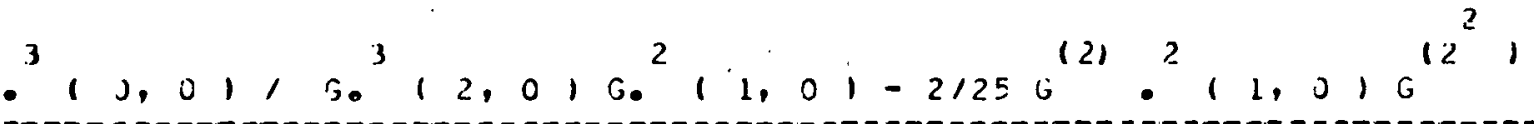

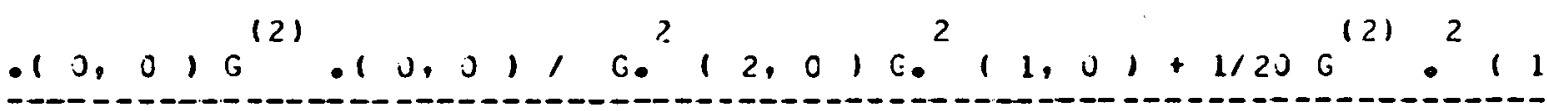

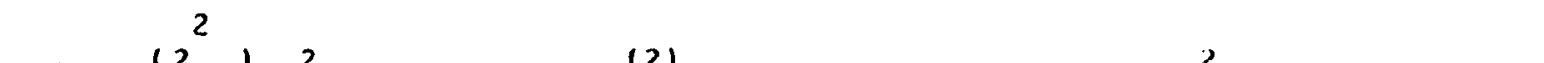
$, 01 G^{2}, 0,0, G^{(2)} \cdot 10,0,10.12,0, G_{0}^{2} 11,01-11$ - 0

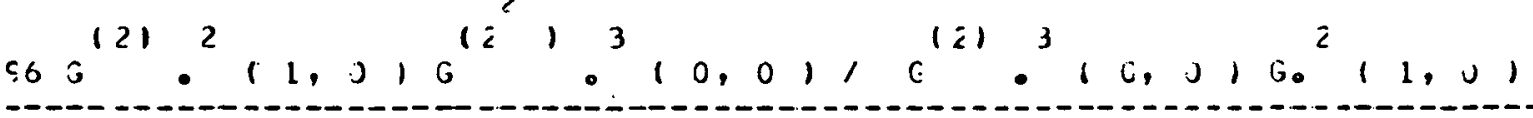

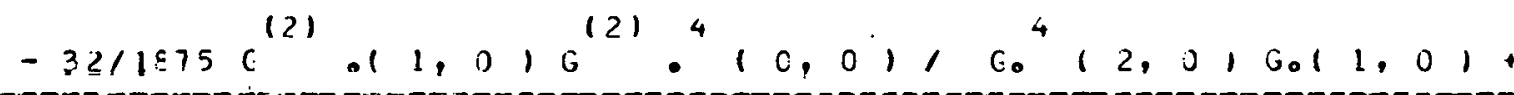
$-3 \leq 11675$ e

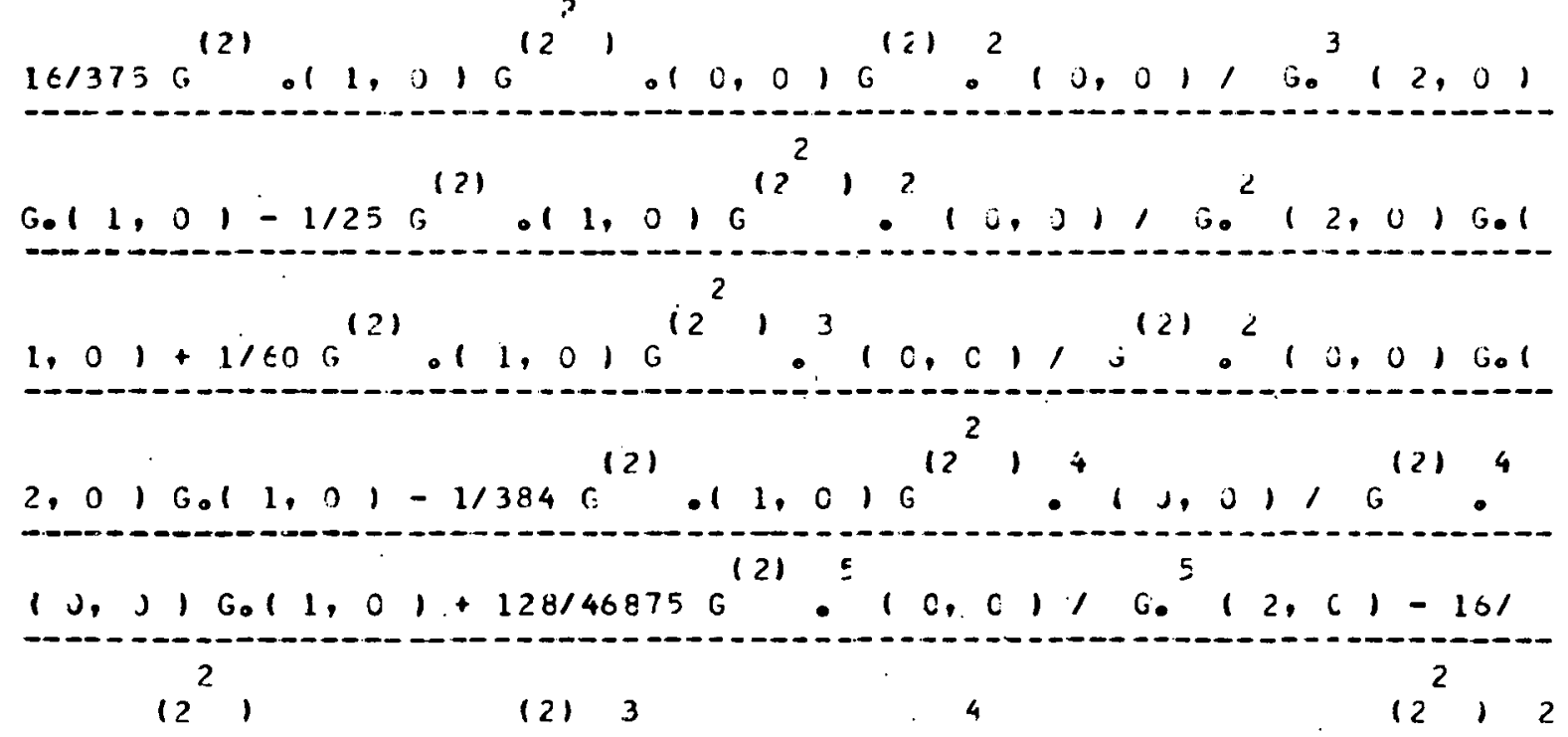




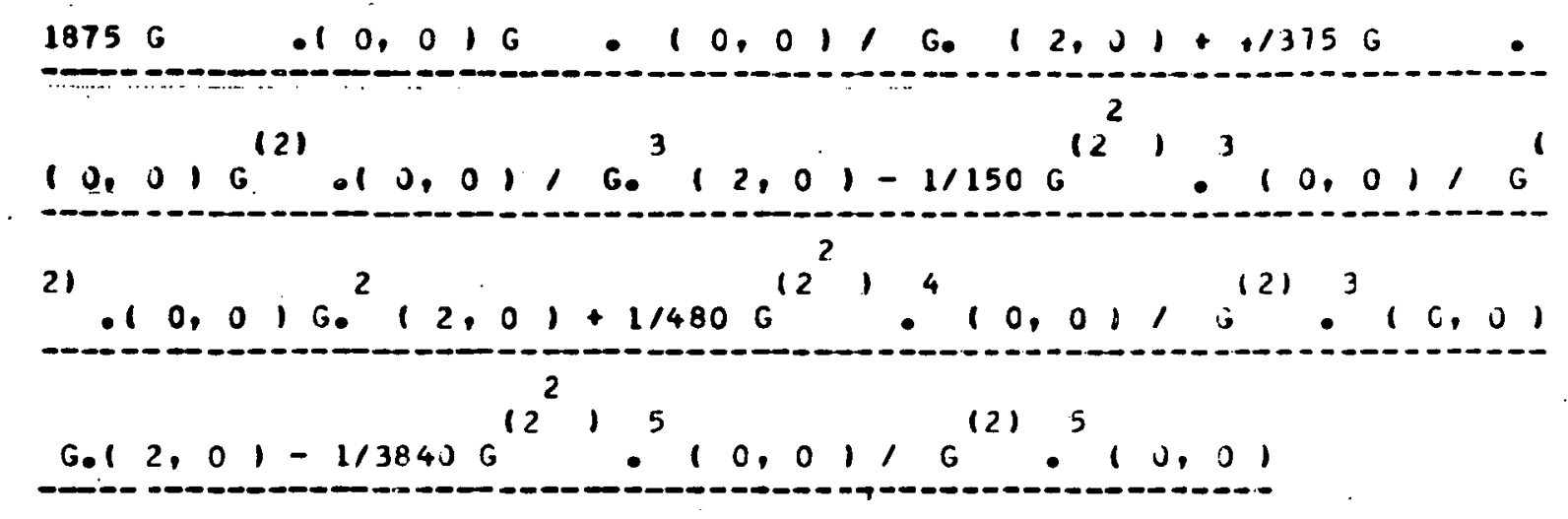




\section{REFERENCES}

1. R. E. Marshak, "Theory of the Slowing Down of Neutrons by Elastic Collision with Atomic Nuclei," Rev. Mod. Phys., 19, No. 3, 185 (1947).

2. M. Verde and G. C. Wick, "Some Stationary Distributions of Neutrons in an Infinite Medium," Phys. Rev., 7l, 852 (1947).

3. I. Waller, "On the Theory of the Diffusion and the Slowing Down of Neutrons," Ark. Mat. Astr. Fys., 34A, No. 3 (1946) and No. 5 (1946).

4. G. C. Wick, "On the Space Distribution of Slow Neutrons," Phys. Rev., 75, No. 5, 738 (1949).

5. H. A. Bethe, L. Tonks, H. Hurwitz, Jr., "Neutron Penetration and Slowing Down at Intermediate Distances through Medium and Heavy Nuclei," Fhys. Rev., 80, No. 1, 11 (1950).

6. G. Placzek, "The Spatial Distribution of Neutrons Slowed Down by Elastic Collisions," Manhattan Project Report A-25. Apparently it was never published, but copies can be obtained from Technical Information Center, ERDA, Oak Ridge, Tenn.

7. G. Holte, Ark. for Math, Astr., och Fys., Band 35A, №. 36, 1 (1948).

8. G. Holte, "On the Space Energy Distribution of Slowed-Down Neutrons: I," Ark. for Fys., Band 2, No. 48 (1950).

9. B. Davison, Neutron Transport Theory (Oxford University Press, London, 1958).

10. A. M. Weinberg and E. P. Wigner, The Physical Theory of Neutron Chain Reactors (The University of Chicago Press, Chicago, 1958).

11. J. J. McInerney, Nucl. Sci. Eng., 22, 215 (1965).

12. H. J. Amster, Nucl. Sci. Eng., 34, 313 (1967).

13. H. J. Amster and K. C. Chan, Nucl. Sci. Eng., 61, 388 (1976).

11. K. M. Case, Aror. Pliyso, 9, 1 (7960).

15. K. M. Case and P. F. Zweifel, Linear Transport Theory (AddisonWesley, Reading, Mass., 1967).

16. K. M. Case, J. Math. Phys., 15, 974 (1974).

17. E. W. Hobson, The Theory of Spherical and ElZipsoidal Harmonics (Cambridge University Press, 1931). 
18. J. R. Mika, Nucl. Sci. Eng., 11, 415 (1961).

19. R. Zelazny, A. Kuszell and J. Mika, Ann. Phys., 16, 69 (1961).

20. R. Zelazny and A. Kusze11, Ann. Phys., 16, 81 (1961).

21. C. E. Siewert and P. F. Zweifel, Ann. Phys., 36, 61 (1966).

22. D. R. Metcalf and P. F. Zweifel, Nucl. Sci. Eng., 33, 3071 (1968).

23. P. Silvennoinen and P. F. Zweifel, TTSP, 1, 239 (1971).

24. R. Zelazny and A. Kusze11, Proc. of the Symp. on Physics of Fast and Intermediate Reactors, Vol. 2 (Vienna, 1962), pp. 55.

25. C. E. Siewert and P. F. Zweifel, J. Math. Phys., 7, 2092 (1966).

26. T. Yoshimura and S. Katsuragi, Nucl. Sci. Eng., 33, 297 (1968).

27. J. K. Shultis, Nucl. Sci. Eng., 38, 83 (1969).

28. P. Silvennoinen and P. F. Zweifel, J. Math. Phys., 13, 1114 (1971).

29. P. Silvennoinen, TTSP, I, 263 (1971).

30. R. J. Bednarz and J. Mika, J. Math. Phys., 4, 1283 (1963).

31. A. Leonard and J. Ferziger, Nucl. Sci. Eng., 26, 170, 181 (1966).

32. J. R. Mika, Nucl. Sci. Eng., 22, 235 (1965).

33. J. C. Steward, I. Kuscer and N. McCormick, Ann. of Phys., 40, 321 (1966).

34. I. Kuscer in Recent Development in Transport Theory, edited by

P. F. Zweifel and E. Inönü (New York, N.Y., 1967).

35. B. Nicolaenko, J. Math. Firys., 11, 174 (1970).

36. P. Jauho and M. Rajamaki, Nuct. Sci. Eng., 43, 145 (1971).

37. R. L. Bowden and C. D. Wiliams, J. Math. Phys., 5, 1527 (1964).

38. I. Kuscer and P. F. Zweifel, J. Math. Phys., 6, 1125 (1965).

39. P. A. Newman and R. L. Bowden, J. Math. Phys., 11, 2445 (1970).

40. E. Inönü, J. Math. Phys., 11, 568 (1970).

41. N. I. Muskelishvili, Singular Integral Equations (Noordhof, Groningen, 1972). 
42. B. W. Roos, Analytic Functions and Distributions in Physics and Engineering (John Wiley \& Sons, New York, 1969).

43. E. E. Burniston, C. E. Siewert, P. Silvennoinen and P. F. Zweifel, Nucl. Sci. Eng., 45, 331 (1971).

44. E. W. Larsen and G. J. Habetler, Commun. Pure App Z. Math., 26, $525(1973)$.

45. E. W. Larsen, Commun. Pure Appl. Math., 27, 523 (1974).

46. E. W. Larsen, S. Sancaktar and P. F. Zweifel, J. Math. Phys., 16, $1117(1975)$.

47. Edward W. Larsen, Commun. Pure Appz. Math., 28, 729 (1975).

48. William Greenberg and Paul F. Zweifel, J. Math. Phys., 17, No. 2, $163(1976)$.

49. R. L. Bowden, S. Sancaktar and P. F. Zweifel, J. Math. Phys., 17, No. $1,76,82$ (1976).

50. R. L. Bowden, W. L. Cameron and P. F. Zweifel, J. Math. Phys., 18, No. 2, 287 (1977).

51. Jan N. Sneddon, The Use of Integral Transforms (McGraw-Hi11, New York, 1972).

52. G. Doetsch, Guide to the Application of the Laplace and Z. Transforms (Van Nostrand, Princeton, 1971).

53. D. W. Widder, The LapZace Transform (Princeton University Press, Princeton, 1972).

54. A..H. Zemanian, Generalized Integral Transformations (John Wiley \& Sons, Inc., Sydney, Toronto, 1968).

55. N. I. Akhiezer, The Classical Moment Problem (01yver and Boyd Ltd., Edinburgh and London, 1965).

56. G. Szegö, Orthogonal Polynomials; Am. Math. Soc., Vol. XXIII, 4th ed. (1975).

57. G. Freud, Orthogonalo Polynome (Birkhäuser Verlag, Basel, 1969),

58. H. S. Wa11, Analytic Theory of Continued Fractions (Van Nostrand, Princeton, N.J., 1948).

59. T. Muir, A I'reatise on the Theory of Determinants (Longman, freen \& Co., New York, London and Toronto, 1933). 
60. PL/1 - FORMAC Symbolic Mathematic Interpreter, IBM Corp. 360-03.3.004 (1971).

61. For an exposition of some basic ideas, see, for ex., C. L. Liu, Introduction to Combinational Mathematics (McGraw-Hill Book Co., New York, 1968).

62. K. M. Case, F. de Hoffman and G: Placzek, Introduction to the Theory of Neutron Diffusion, Vol. 1 (U.S. Government Printing Office, Washington, D.C., 1953).

63. K. M. Case, Phys. Fluids, 16, 1607 (1973).

64. G. Placzek, Phys. Rev., 69, 423 (1946).

65. T. Teichmann, Nucl. Sci. Eng., 7, 292 (1960).

66. A. H. Zemanian, Distribution Theory and Transform Analysis, (McGraw-Hill, New York, 1970).

67. G. Polya and G. Szegö, Problems and Theorems in Analysis, Vol. $Z$ (Springer-Verlag, Berlin, 1972) (Revised and enlarged translation of "Aufgaben and Lehrsätze aus der Analysis I," 4th ed., 1970.)

68. A. I. Markushevich, Theory of Functions of a Complex Variable, Vols. 1-3 (Prentice-Hall, Englewood Cliffs, N.J., 1965).

69. E. Hille, Analytic Function Theory, Vols. 1-2 (Ginn and Co., Boston, 1959).

70. T. J. I' a. Bromwich, An Introduction to the Theory of Infinite Series (Macmillan and Co., London, 1947).

71. L. Y. Huang, Private Communication (1974).

72. L. K. Kim, High Order Spherical Harmonics Method for Neutron Deep Penetration (Doctoral Thesis, Columbia Univ., 1975).

73. E. Oblow, K. Kin, H. Goldstein and J. J. Wagschal, NucZ. Sci. Eng., 54, $72(1974)$.

74. R. B. Dingle, Asymptotic Expansions: Their Derivation and Interpretation (Academic Press Inc., London, 1973).

75. G. A. Baker, Jr., Essentials of Padde Approximants (Academic Press, New York, 1975).

76. K. C. Chan and H. J. Amster, Nucl. Sci. Eng., 61, 434 (1976).

77. I. S. Gradshtein and I. M. Ryzhik, Tables of Integrazs, Series and Products (Academic Press, New York, 7th printing, 1973). 
78. J. P. Price, A.E.R.E., T/R 523, April 1950.

79. A. M. Ostrowski, Solution of Equations and Systems of Equations (2nd Ed.; Academic Press, New York, 1966).

80. D. G. Cacuci and H. Goldstein, Neutron Slowing Down and Transport in a Medium of Constant Cross Section. I: Spatial Moments, J. Math. Phys., 18, 2436 (1977) 
ORNL/TM-6378

\section{Internal Distribution}

1. L. S. Abbott

2. R. G. Alsmiller, Jr.

3. D. E. Bartine

4-15. D. G. Cacuci

16. G, de Saussure

17. G. F. Flanagan

18-20. H. Goldstein (Consultant)

21. R. F. Hibbs

22. F. C. Maienschein

23. J. H. Marable

24. E. M. Oblow

25. P. Patriarca

26. R. W. Peelle

27. F. G. Perey

28. R. B. Perez

29. H. Postma

30. RSIC
31. R. W. Roussin

32. D. B. Trauger

33. C. R. Weisbin

34. J. E. White

35. G. E. Whitesides

36. R. Q. Wright

37. A. Zucker

38. P. Greebler (Consultant)

39. W. B. Loewenstein (Consultant)

40. R. E. Uhrig (Consultant)

41. Richard Wilson (Consultant)

42-43. Central Research Library

44. ORNL Y-12 Technical Library Document Reference Section

45. Laboratory Records Department

46. Laboratory Records ORNL RC

47. ORNL Patent Office

\section{External Distribution}

48-52. Columbia University Dissertation Secretary, 105 Low Memorial Library, 520 West 120th Street, New York, New York 10027.

53. Prof. W. W. Havens, Jr., Columbia University, 520 West 120th Street, New York, New York 10027.

54. W. Patton, Columbia University, 520 West 120th Street, New York, New York 10027.

55. Prof. L. J. Lidofsky, Columbia University, 520 West 120th Street, New York, New York 10027.

56. Prof. J. P. Felvinci, Columbia University, 520 West 120 th Street, New York, New York 10027.

57. Prof. E. Melkonian, Columbia University, 520 West 120th Street, New York, New York 10027.

58. Prof. G. C. Wick, Columbia University, 520 West 120th Street, New York, New York 10027.

59. R. S. Johnston, Columbia University, 520 West 120 th Street, New York, New York 10027.

60. U.S. Department of Energy, Oak Ridge Operations, Research and Technical Support Division: Director.

61. U.S. Department of Energy, Oak Ridge Operations, Reactor Division: Director.

62-63. U.S. Department of Energy, Division of Reactor Research and Development, Washington, D.C. 20545: Director.

64-90. Technical Information Center (TIC). 